\title{
SHEAR BEHAVIOR OF STEEL FIBER REINFORCED CONCRETE BEAMS WITHOUT STIRRUP REINFORCEMENT
}

by

\author{
Hai H. Dinh \\ A dissertation submitted in partial fulfillment \\ of the requirements of the degree of \\ Doctor of Philosophy \\ (Civil Engineering) \\ in The University of Michigan \\ 2009
}

Doctoral Committee:

Associate Professor Gustavo J. Parra-Montesinos, Co-Chairman

Professor James K. Wight, Co-Chairman

Professor Antoine E. Naaman

Associate Professor John A. Shaw

Professor Frank J. Vecchio, University of Toronto 
To my wife, Dieu-Thanh, my daughter, Tue-My, and my beloved family 


\begin{abstract}
AKNOWLEDGEMENTS
This research report was submitted by the author in partial fulfillment of the requirements of Doctor of Philosophy in Civil Engineering at The University of Michigan. The author would like to acknowledge the support from Associate Professor Gustavo J. Parra-Montesinos and Professor James K. Wight, who provided the funds for this research. The grant from Vietnam Education Foundation (VEF) to the author during the period from September 2004 to May 2006 is also acknowledged.

The author would like to express his sincerest gratitude to Associate Professor Gustavo J. Parra-Montesinos and to Professor James K. Wight, the Co-Chairmen of his doctoral committee, who supported and mentored the author with love and care throughout the course of this research. The author would also like to extend his appreciation to the other members of his doctoral committee, Professor Antoine E. Naaman, Associate Professor John A. Shaw, and Professor Frank J. Vecchio for their review of and helpful suggestions to this research report.

The author also recognizes the contribution of Carlos M. Padilla and Alexander L. Libbrecht, who constructed and tested several beams of this research. Appreciation is also extended to Robert M. Spence, Robert G. Fischer, and Jan E. Pantolin, the technicians of The University of Michigan Structural Engineering Laboratory, and to the fellow undergraduate and graduate students, who offered helps during the experimental phase of this research.

The author is deeply indebted to his wife, Dieu-Thanh, his daughter, Tue-My, and his beloved family for their unyielding support and encouragement.
\end{abstract}




\section{TABLE OF CONTENTS}

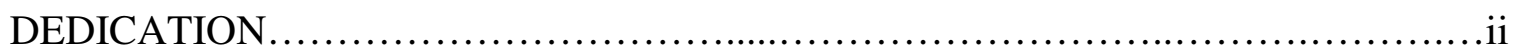

ACKNOWLEDGEMENTS.................................................... iii

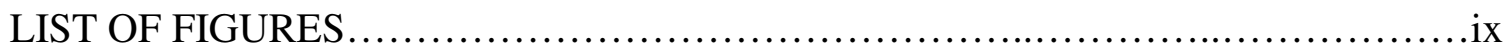

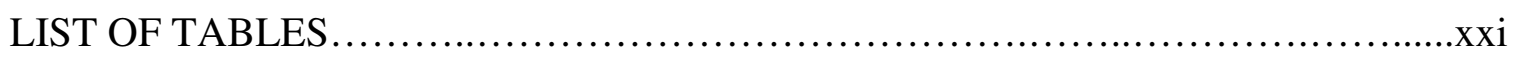

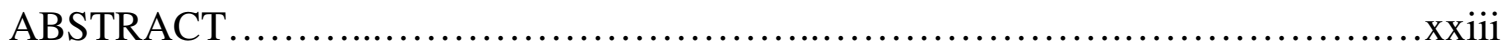

CHAPTER

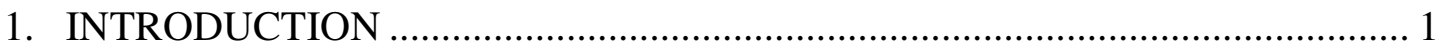

1.1 BACKGROUND AND MOTIVATION ................................................. 1

1.2 OBJECTIVES AND SCOPE OF THE RESEARCH ............................... 4

1.3 ORGANIZATION OF THE THESIS ................................................... 6

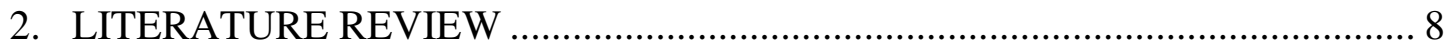

2.1 MECHANICAL PROPERTIES OF SFRC ........................................... 8

2.1.1 Types of steel fibers and bond between steel fibers and concrete ........ 8

2.1.2 SFRC direct tensile strength .................................................... 13

2.1.3 SFRC flexural tensile strength ................................................... 19

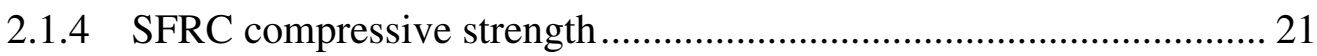

2.1.5 SFRC direct shear strength ................................................ 23

2.1.6 Bond between reinforcing bars and SFRC ................................. 24

2.2 SHEAR FAILURE MECHANISM AND TEST RESULTS OF SFRC

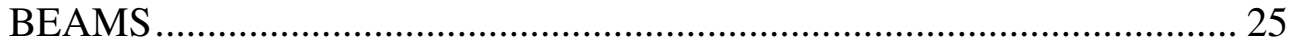

2.2.1 Stress distribution and failure of plain concrete beams ................... 25 
2.2.2 Failure of RC beams without stirrup reinforcement ...................... 26

2.2.3 Roles of stirrup reinforcement in $\mathrm{RC}$ beams................................. 29

2.2.4 Failure of SFRC beams without stirrup reinforcement..................... 30

2.2.5 Tests of SFRC beams without stirrup reinforcement....................... 31

2.3 PREDICTION OF SHEAR STRENGTH OF SFRC BEAMS .................. 36

2.4 SHEAR THEORY OF RC BEAMS ................................................. 42

2.4.1 Shear strength models based on the contribution of the compression

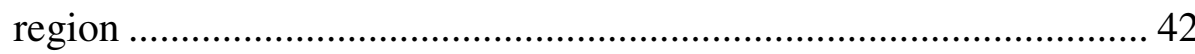

2.4.2 Shear strength models based on the contribution of the aggregate interlock ........................................................................... 45

3. EXPERIMENTAL PROGRAM ................................................................. 49

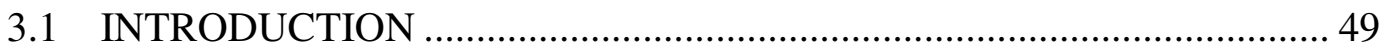

3.2 DESIGN OF BEAM SPECIMENS ................................................... 50

3.2.1 Fixed parameters ............................................................... 51

3.2.2 Varied parameters .............................................................. 54

3.3 FABRICATION OF REINFORCEMENT CAGE .................................. 64

3.4 PROPORTIONING AND MIXING OF SFRC - CASTING AND CURING

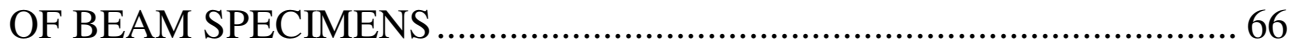

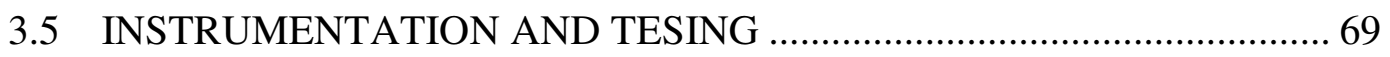

3.6 MATERIAL TESTING AND PROPERTIES ....................................... 75

3.6.1 Reinforcing bars .................................................................. 75

3.6.2 SFRC compressive strength ............................................... 77

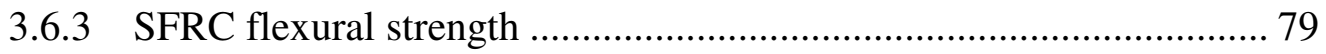

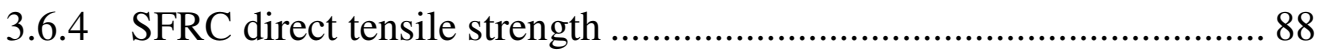

4. RESULTS OF THE EXPERIMENTAL PROGRAM ...................................... 91

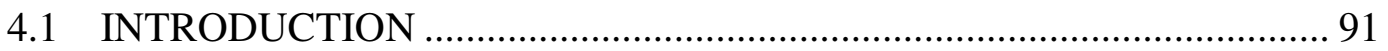




\subsection{PROCESS OF OPTOTRAK DATA AND CALCULATION OF}

AVERAGE CONCRETE STRAINS....................................................... 91

4.2.1 Transformation of coordinates ........................................................... 91

4.2.2 Displacements of markers.................................................................. 94

4.2.3 Calculation of concrete strains ......................................................... 95

4.2.4 Calculation of principal strains and principal directions ...................... 96

4.3 BEHAVIOR OF BEAMS IN SERIES B18 …………............................. 97

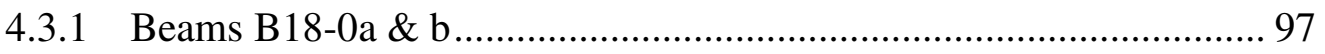

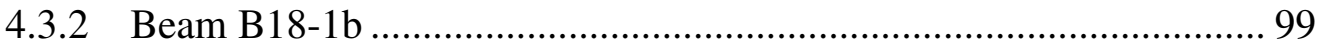

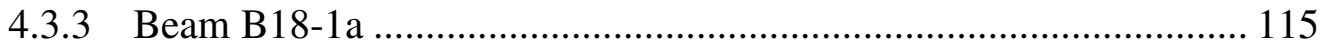

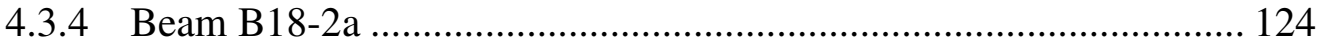

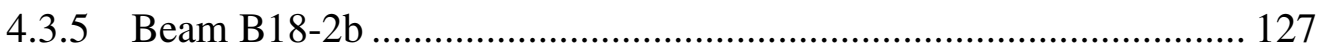

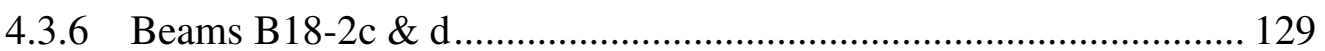

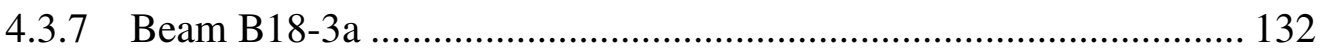

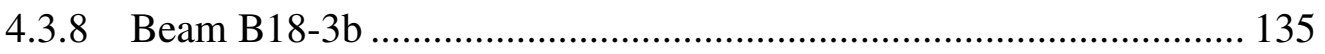

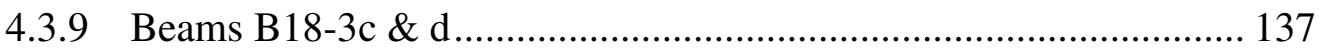

4.3.10 Beams B18-5a \& b .......................................................................... 141

4.3.11 Beam B18-7a ………………………………........................... 145

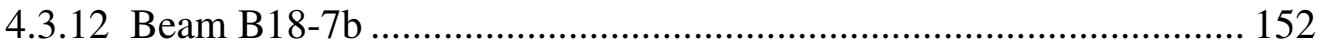

4.4 BEHAVIOR OF BEAMS IN SERIES B27 ………................................ 157

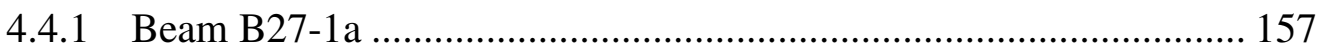

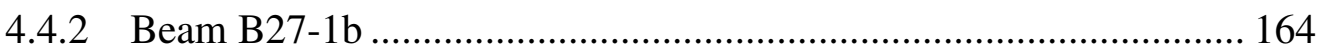

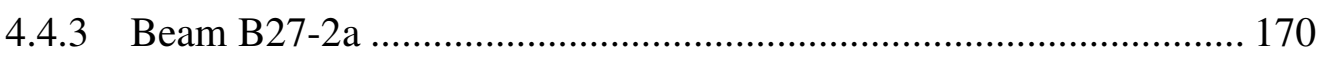

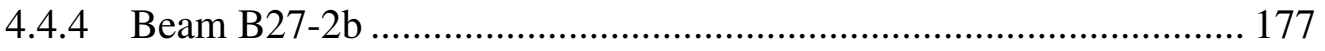

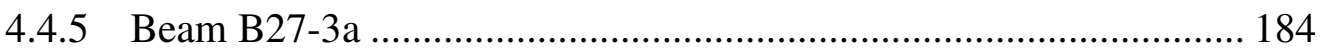




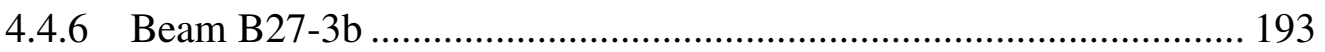

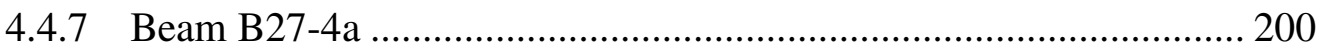

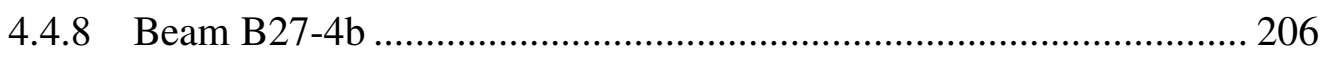

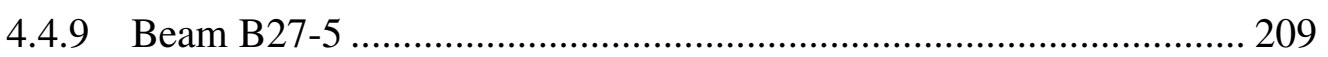

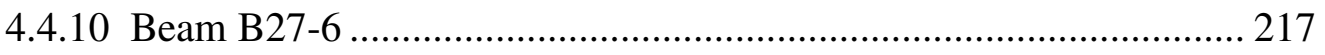

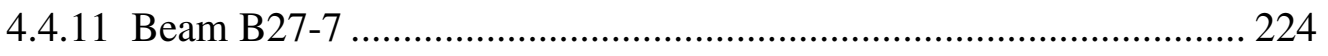

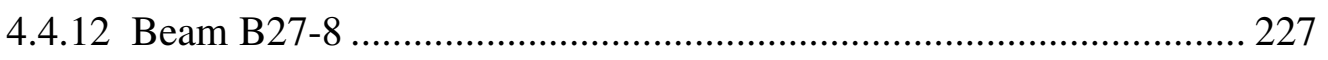

5. ANALYSIS OF EXPERIMENTAL RESULTS ................................................ 232

5.1 OVERALL BEHAVIOR OF RC AND SFRC BEAMS ………………...... 232

5.1.1 Shear stress and normalized shear stress ......................................... 232

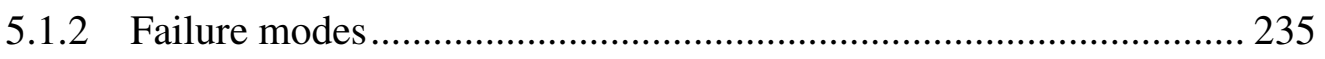

5.1.3 Crack patterns ............................................................................ 238

5.1.4 Horizontal spacing of inclined cracks ............................................. 241

5.1.5 Angles of critical inclined cracks..................................................... 244

5.2 EFFECT OF STUDIED PARAMETERS …………………………........ 245

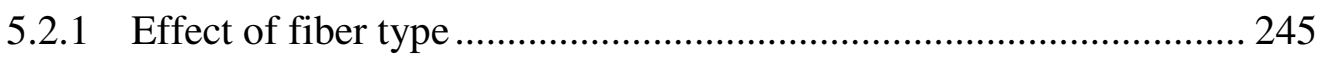

5.2.2 Effect of fiber volume fraction .................................................... 246

5.2.3 Effect of longitudinal reinforcement ratio ...................................... 247

5.2.4 Effect of effective beam depth ...................................................... 249

5.2.5 Replacement of minimum shear reinforcement ............................... 249

\subsection{PREDICTION OF SHEAR STRENGTH OF SFRC BEAMS .................. 250}

5.3.1 Shear prediction of SFRC beams without stirrups from previous

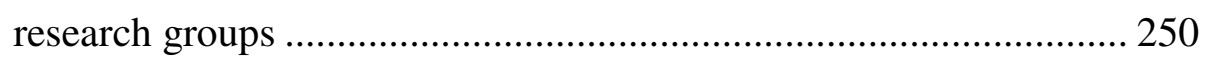

5.3.2 A mechanics-based model for shear prediction of SFRC beams

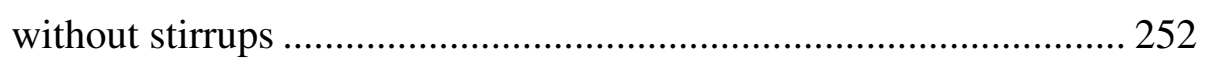

5.3.3 Design recommendation for average tensile stress ............................ 268 
5.3.4 Validation of the proposed method for estimating the shear strength of SFRC beams with data obtained from previous experimental programs

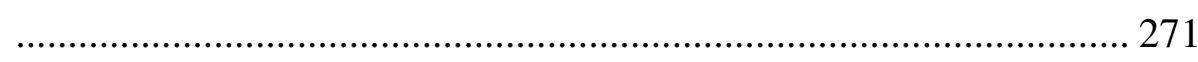

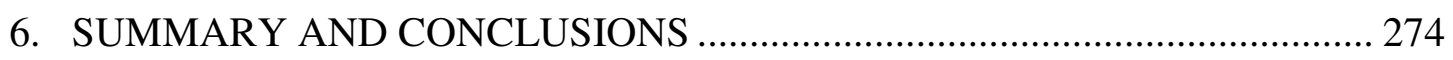

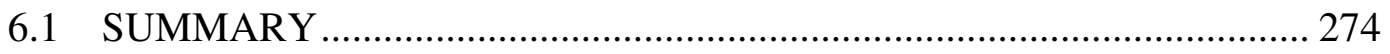

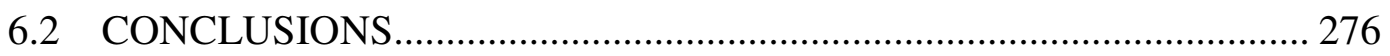

REFERENCES........................................................... 278 


\section{LIST OF FIGURES}

Fig. 1-1: Shear failure of reinforced concrete beams in the U.S. Air Force Warehouse (from Wight and MacGregor, 2009) …......................................................................... 2

Fig. 1-2: Different types of steel fibers ........................................................................... 2

Fig. 2-1: Effect of matrix strength and fiber embedment length on pullout behavior (from

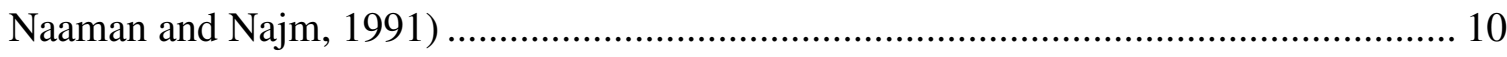

Fig. 2-2: Effect of fiber inclination for hooked steel fiber in normal-, mid-, and highstrength concrete (from Banthia and Trottier, 1994) ..................................................... 12

Fig. 2-3: Direct tensile stress-strain curves for different types of SFRCs (from ACI Committee 544, 1988) 13

Fig. 2-4: Load-extension relationship for different SFRC mixes (from Lim et al., 1987) 14 Fig. 2-5: Tensile stress-crack opening relationship for different SFRC mixes (from Noghabai, 2000) 15

Fig. 2-6: Different stress-strain curves for brittle matrix composites 18

Fig. 2-7: Effect of hooked and straight steel fibers on flexural performance of concrete 20 Fig. 2-8: Examples of compressive stress-strain relationships with different fiber types and fiber aspect ratios (from Soroushian and Bayasi, 1991)

Fig. 2-9: Model of SFRC compressive stress-strain relationship (from Fanella and Naaman, 1985) 22

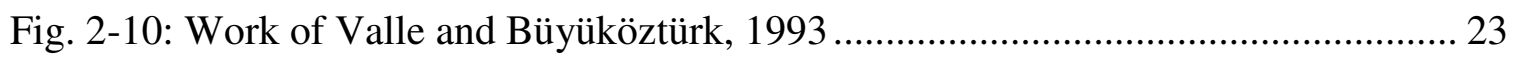

Fig. 2-11: Plain concrete beam subjected to a concentrated load .................................. 26

Fig. 2-12: Behavior of RC beam without stirrup reinforcement................................... 27

Fig. 2-13: Beam failure modes (from ACI-ASCE Committee 426, 1973) ...................... 28

Fig. 2-14: Shear resistance in RC beams with stirrup reinforcement ............................. 29

Fig. 2-15: Effect of shear span-to-effective depth ratio on SFRC beam strength from previous investigations. 
Fig. 2-16: Stress distribution in RC beams (from Bresler and Pister, 1958) ................... 43

Fig. 2-17: Free body diagram at a crack (from Tureyen and Frosch, 2003) .................... 44

Fig. 2-18: Stress versus strain relationships for reinforced concrete in the Modified

Compression Field Theory (from Vecchio and Collins, 1986)..................................... 46

Fig. 3-1: Basic dimensions, load, and boundary conditions for a test beam.................... 51

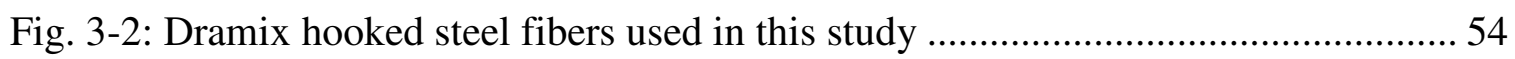

Fig. 3-3: Reinforcement details and strain gauge placement for beams in Series B18 .... 59

Fig. 3-4: Reinforcement details and strain gauge placement for beams in Series B27 .... 63

Fig. 3-5: Reinforcing cage ready for placement and laboratory site ready for casting.... 65

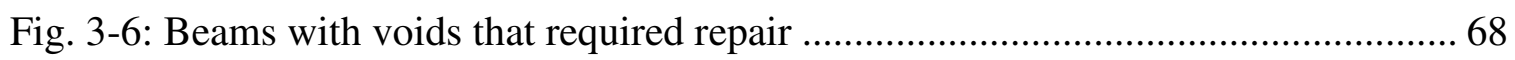

Fig. 3-7: Devices in an active infrared optical tracking system.................................. 70

Fig. 3-8: Characteristic volume of close-focus OptoTRAK Certus sensors (from

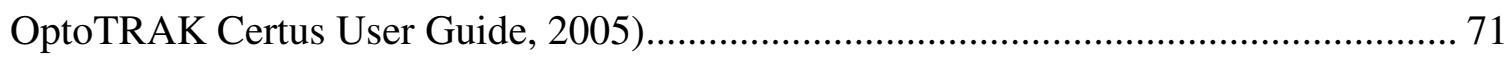

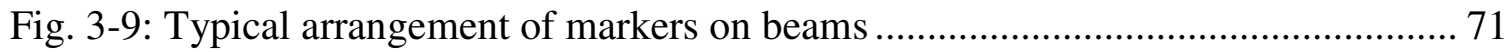

Fig. 3-10: Test setup for beams in Series B18 with 115-kip Instron machine (INS115) 72

Fig. 3-11: Test set-up for beams in Series B18 with 235-kip hydraulic actuator (ACT235)

Fig. 3-12: Test setup for beams in Series B27 with 500-kip hydraulic cylinder (CYL500)

Fig. 3-13: Stress versus strain relationships of reinforcing bars .................................. 76

Fig. 3-14: Test setup for four-point bending tests................................................ 79

Fig. 3-15: Distribution of compression region depth, crack width, and crack location at a

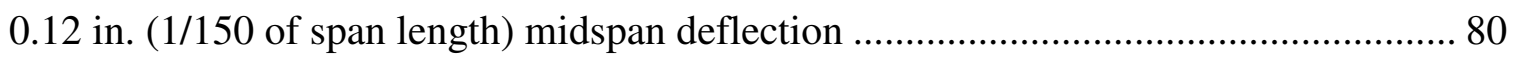

Fig. 3-16: Equivalent bending stress versus midspan deflection relationship from four-

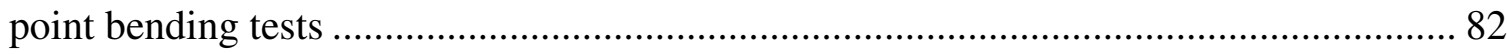

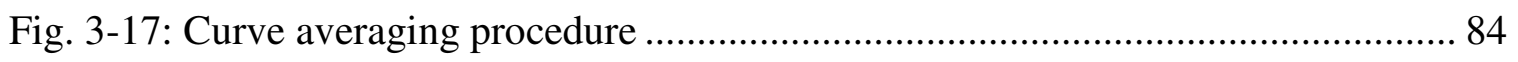

Fig. 3-18: Two different equivalent bending stress versus deflection responses and important points on the curves

Fig. 3-19: Different equivalent bending stresses versus fiber reinforcing index

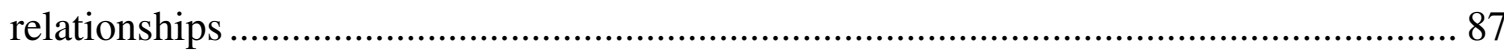

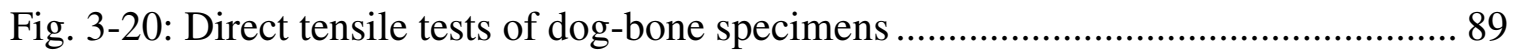




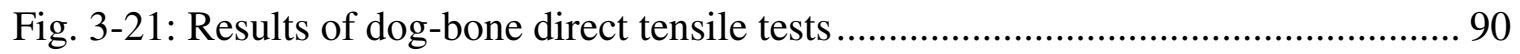

Fig. 4-1: Transformation of coordinates of markers .................................................. 92

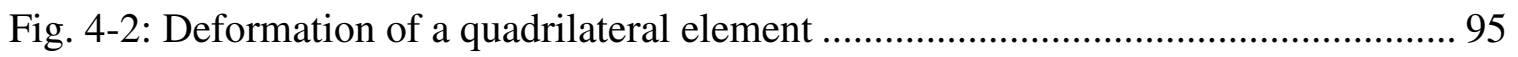

Fig. 4-3: Load versus deflection relationships - Beams B18-0a \& b ............................. 98

Fig. 4-4: Crack pattern after failure - Beam B18-0a .................................................. 98

Fig. 4-5: Crack pattern after failure - Beam B18-0b ............................................ 98

Fig. 4-6: Load versus deflection and reinforcement strain relationships - Beam B18-1b 105

Fig. 4-7: Crack pattern prior to failure - Beam B18-1b ........................................... 105

Fig. 4-8: Crack pattern after failure - Beam B18-1b ............................................ 105

Fig. 4-9: Numbering of markers, elements, and strain gauges - Beam B18-1b ............ 106

Fig. 4-10: Reinforcement strains along the beam axis - Beam B18-1b ....................... 106

Fig. 4-11: Model of direct tensile stress versus strain relationship for reinforcing bars 106 Fig. 4-12: Bond along the lower reinforcing bars at various applied loads - Beam B18-1b

Fig. 4-13: Bond along the upper reinforcing bars at various applied loads - Beam B18-1b 107

Fig. 4-14: Truss model 107

Fig. 4-15: Distribution of longitudinal strains at various applied loads - Beam B18-1b 108 Fig. 4-16: Comparison of reinforcement and average concrete strains at the lower level of

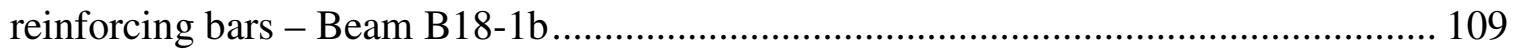

Fig. 4-17: Distribution of average concrete strains - Beam B18-1b ........................... 110

Fig. 4-18: Distribution of transverse strains at various applied loads - Beam B18-1b .. 110 Fig. 4-19: Concrete strains in the parallelogram region shown in Fig. 4-18 - Beam B18$1 \mathrm{~b}$.

Fig. 4-20: Distribution of shear strains at various applied loads - Beam B18-1b ......... 112 Fig. 4-21: Distribution of transverse and shear strains averaged over the beam depth Beam B18-1b

Fig. 4-22: Principal strain field at first diagonal cracking (a) and peak load (b) - Beam B18-1b. 
Fig. 4-23: Rotation of principal angles from first diagonal cracking (continuous lines) to maximum load (dashed lines) - Beam B18-1b 114

Fig. 4-24: Development of strains along the critical diagonal crack around the peak load Beam B18-1b 114

Fig. 4-25: Load versus deflection relationship - Beam B18-1a 117

Fig. 4-26: Crack pattern prior to failure - Beam B18-1a 117

Fig. 4-27: Crack pattern after failure - Beam B18-1a 117

Fig. 4-28: Numeration of markers, elements, and strain gauges - Beam B18-1a .......... 118

Fig. 4-29: Load versus reinforcement strain relationships - Beam B18-1a 118

Fig. 4-30: Reinforcement strains along the beam axis - Beam B18-1a.... 118

Fig. 4-31: Average bond stress in the lower tension reinforcement - Beam B18-1a ..... 119 Fig. 4-32: Average bond stress in the upper tension reinforcement - Beam B18-1a ..... 119 Fig. 4-33: Distribution of longitudinal, transverse, and shear strains at peak load - Beam B18-1a. 120

Fig. 4-34: Comparison of reinforcement and average concrete strain at the level of lower and upper reinforcing bars - Beam B18-1a 121

Fig. 4-35: Distribution of average concrete strain at various applied loads - Beam B18-1a

Fig. 4-36: Distribution of transverse and shear strains averaged over the beam depth -

Beam B18-1a 122

Fig. 4-37: Load versus concrete strain relationships in the parallelogram region shown in

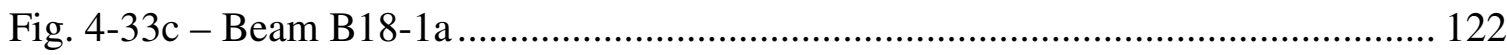

Fig. 4-38: Principal strain field at 99.2 kips - Beam B8-1a ..................................... 123

Fig. 4-39: Development of principal tensile strain versus time - Beam B18-1a........... 123

Fig. 4-40: Crack pattern prior to failure - Beam B18-2a....................................... 125

Fig. 4-41: Crack pattern after failure - Beam B18-2a ............................................. 125

Fig. 4-42: Load versus deflection and reinforcement strain relationships - Beam B18-2a

Fig. 4-43: Reinforcement strains along the beam axis - Beam B18-2a....................... 126

Fig. 4-44: Average bond stress along the beam axis - Beam B18-2a ......................... 126

Fig. 4-45: Crack pattern prior to failure - Beam B18-2b 127 
Fig. 4-46: Load versus deflection and reinforcement strain relationships - Beam B18-2b

Fig. 4-47: Reinforcement strains along the beam axis - Beam B18-2b ....................... 128

Fig. 4-48: Average bond stress along the beam axis - Beam B18-2b .......................... 128

Fig. 4-49: Load versus deflection and reinforcement strain relationships - Beam B18-2c

Fig. 4-50: Reinforcement strains along the beam axis - Beam B18-2c....................... 130

Fig. 4-51: Average bond stress along the beam axis - Beam B18-2c .......................... 130

Fig. 4-52: Load versus deflection and reinforcement strain relationships - Beam B18-2d

Fig. 4-53: Reinforcement strains along the beam axis - Beam B18-2d ...................... 131

Fig. 4-54: Average bond stress along the beam axis - Beam B18-2d

Fig. 4-55: Load versus deflection and reinforcement strain relationships - Beam B18-3a

Fig. 4-56: Crack pattern after failure - Beam B18-3a

Fig. 4-57: Reinforcement strains along the beam axis - Beam B18-3a....................... 134

Fig. 4-58: Average bond stress along the beam axis - Beam B18-3a

Fig. 4-59: Load versus deflection and reinforcement strain relationships - Beam B18-3b

Fig. 4-60: Crack pattern after failure - Beam B18-3b ............................................ 136

Fig. 4-61: Reinforcement strains along the beam axis - Beam B18-3b ....................... 136

Fig. 4-62: Average bond stress along the beam axis - Beam B18-3b ......................... 137

Fig. 4-63: Load versus deflection and reinforcement strain relationships - Beam B18-3c

Fig. 4-64: Crack pattern after failure - Beam B18-3c .......................................... 138

Fig. 4-65: Reinforcement strains along the beam axis - Beam B18-3c....................... 139

Fig. 4-66: Average bond stress along the beam axis - Beam B18-3c .......................... 139

Fig. 4-67: Load versus deflection and reinforcement strain relationships - Beam B18-3d

Fig. 4-68: Crack pattern after failure - Beam B18-3d ............................................. 140

Fig. 4-69: Reinforcement strains along the beam axis - Beam B18-3d ....................... 140 
Fig. 4-70: Load versus deflection and reinforcement strain relationships - Beam B18-5a 142

Fig. 4-71: Crack pattern after failure - Beam B18-5a .............................................. 142

Fig. 4-72: Reinforcement strains along the beam axis - Beam B18-5a........................ 143

Fig. 4-73: Average bond stress along the beam axis - Beam B18-5a ......................... 143

Fig. 4-74: Load versus deflection and reinforcement strain relationships - Beam B18-5b

Fig. 4-75: Crack pattern after failure - Beam B18-5b ............................................. 144

Fig. 4-76: Reinforcement strains along the beam axis - Beam B18-5b ....................... 144

Fig. 4-77: Average bond stress along the beam axis - Beam B18-5b ......................... 144

Fig. 4-78: Crack pattern after failure - Beam B18-7a .............................................. 147

Fig. 4-79: Load versus deflection relationship - Beam B18-7a ................................. 147

Fig. 4-80: Numbering of markers, elements, and strain gauges - Beam B18-7a .......... 148

Fig. 4-81: Load versus reinforcement strain relationships - Beam B18-7a ................. 148

Fig. 4-82: Reinforcement strains along the beam axis - Beam B18-7a....................... 148

Fig. 4-83: Average bond stress along the beam axis - Beam B18-7a ......................... 149

Fig. 4-84: Distribution of longitudinal, transverse, and shear strains - Beam B18-7a... 149

Fig. 4-85: Distribution of transverse and shear strains averaged over the beam depth -

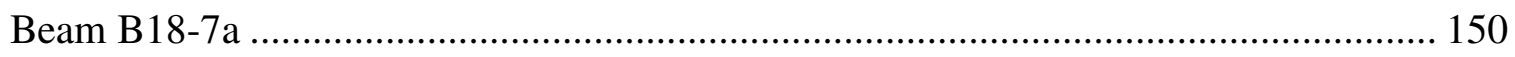

Fig. 4-86: Principal strain field at 112 kips - Beam B18-7a .................................... 151

Fig. 4-87: Development of principal tensile strain versus time - Beam B18-7a.......... 151

Fig. 4-88: Crack pattern after failure - Beam B18-7b …...................................... 153

Fig. 4-89: Load versus deflection and reinforcement strain relationships - Beams B18-7b

Fig. 4-90: Reinforcement strains along the beam axis - Beam B18-7b ...................... 153

Fig. 4-91: Average bond stress along the beam axis - Beam B18-7b ......................... 154

Fig. 4-92: Distribution of longitudinal, transverse, and shear strains - Beam B18-7b... 154

Fig. 4-93: Principal strain field at 110 kips - Beam B18-7b .................................... 155

Fig. 4-94: Distribution of transverse and shear strains averaged over the beam depth -

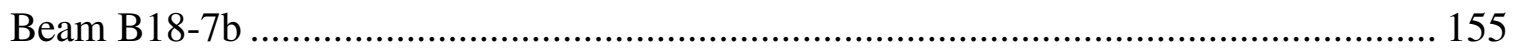

Fig. 4-95: Development of principal tensile strain versus time - Beam B18-7b........... 156 
Fig. 4-96: Crack pattern after failure - Beam B27-1a

Fig. 4-97: Load versus deflection and reinforcement strain relationships - Beam B27-1a

Fig. 4-98: Numbering of markers, elements, and strain gauges - Beam B27-1a .......... 160

Fig. 4-99: Reinforcement strains and average bond stress along the beam axis - Beam B27-1a 160

Fig. 4-100: Distribution of longitudinal, transverse, and shear strains at 204 kips - Beam B27-1a. 161

Fig. 4-101: Comparison of reinforcement and concrete strains - Beam B27-1a. 162

Fig. 4-102: Distribution of transverse and shear strains averaged over the beam depth Beam B27-1a 162

Fig. 4-103: Principal strain field at 204 kips - Beam B27-1a 162

Fig. 4-104: Development of principal tensile strains along the critical inclined crack Beam B27-1a 163

Fig. 4-105: Crack pattern after failure - Beam B27-1b 165

Fig. 4-106: Load versus deflection and reinforcement strain relationships - Beam B27-1b 166

Fig. 4-107: Reinforcement strains and average bond stress along the beam axis - Beam B27-1b 166

Fig. 4-108: Numbering of markers, elements, and strain gauges - Beam B27-1b ........ 166 Fig. 4-109: Distribution of longitudinal, transverse, and shear strains at $188 \mathrm{k}-$ Beam B27-1b

Fig. 4-110: Distribution of transverse and shear strains averaged over the beam depth Beam B27-1b 168

Fig. 4-111: Principal strain field at 188 kips - Beam B27-1b 168

Fig. 4-112: Development of principal tensile strains along the critical inclined crack Beam B27-1b 169

Fig. 4-113: Crack pattern after failure - Beam B27-2a 171

Fig. 4-114: Load versus deflection and reinforcement strain relationships - Beam B27-2a 172

Fig. 4-115: Numbering of markers, elements, and strain gauges - Beam B27-2a ..... 172 
Fig. 4-116: Reinforcement strains along the beam axis - Beam B27-2a..................... 173

Fig. 4-117: Average bond stress along the beam axis - Beam B27-2a ....................... 173

Fig. 4-118: Distribution of longitudinal, transverse, and shear strains at $196 \mathrm{k}$ - Beam

B27-2a

Fig. 4-119: Distribution of transverse and shear strains averaged over the beam depth -

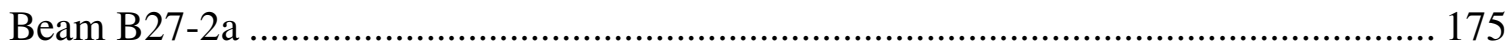

Fig. 4-120: Comparison of reinforcement and concrete strains - Beam B27-2a........... 175

Fig. 4-121: Principal strain field at 196 kips - Beam B27-2a .................................... 175

Fig. 4-122: Development of principal tensile and compressive strains along the critical

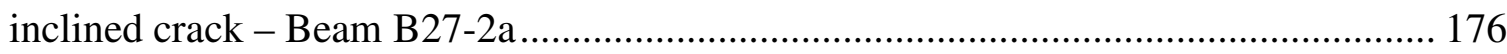

Fig. 4-123: Load versus deflection and reinforcement strain relationships - Beam B27-2b

Fig. 4-124: Crack pattern prior to failure - Beam B27-2b ....................................... 179

Fig. 4-125: Crack pattern after failure - Beam B27-2b ............................................ 179

Fig. 4-126: Reinforcement strains along the beam axis - Beam B27-2b ..................... 180

Fig. 4-127: Average bond stress along the beam axis - Beam B27-2b ....................... 180

Fig. 4-128: Distribution of longitudinal, transverse, and shear strains at $192 \mathrm{k}-$ Beam

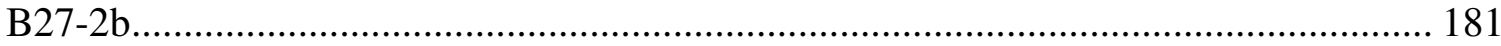

Fig. 4-129: Numbering of markers, elements, and strain gauges - Beam B27-2b ........ 181

Fig. 4-130: Comparison of reinforcement and concrete strains - Beam B27-2b .......... 182

Fig. 4-131: Distribution of transverse and shear strains averaged over the beam depth -

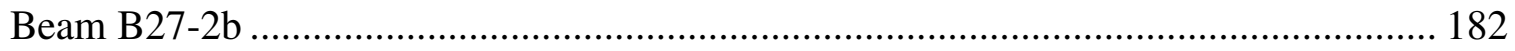

Fig. 4-132: Principal strain field at 192 kips - Beam B27-2b ................................... 183

Fig. 4-133: Development of principal tensile strains along the critical inclined cracks -

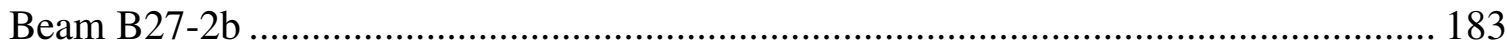

Fig. 4-134: Crack pattern after failure - Beam B27-3a ............................................. 186

Fig. 4-135: Load versus deflection relationship - Beam B27-3a ............................... 187

Fig. 4-136: Reinforcement buckling (a) and transverse expansion (b) - Beam B27-3a. 187

Fig. 4-137: Load versus reinforcement strain relationships - Beam B27-3a ............... 187

Fig. 4-138: Numbering of markers, elements, and strain gauges - Beam B27-3a ........ 188

Fig. 4-139: Reinforcement strains along the beam axis - Beam B27-3a...................... 188 
Fig. 4-140: Average bond stress along the beam axis - Beam B27-3a 188

Fig. 4-141: Distribution of longitudinal, transverse, and shear strains at peak load - Beam B27-3a.

Fig. 4-142: Distribution of longitudinal, transverse, and shear strains at failure load -

Beam B27-3a 190

Fig. 4-143: Distribution of transverse and shear strains along the beam axis averaged over the beam depth - Beam B27-3a .......................................................................... 191

Fig. 4-144: Principal strain field at peak and failure load - Beam B27-3a ................... 191

Fig. 4-145: Development of principal tensile and compressive strains along the critical inclined crack - Beam B27-3a ....................................................................... 192

Fig. 4-146: Crack pattern after failure - Beam B27-3b ........................................... 195

Fig. 4-147: Load versus deflection relationship - Beam B27-3b ............................... 195

Fig. 4-148: Numbering of markers, elements, and strain gauges - Beam B27-3b ........ 195

Fig. 4-149: Load versus reinforcement strain relationships - Beam B27-3b ................ 196

Fig. 4-150: Reinforcement strains along the beam axis - Beam B27-3b ..................... 196

Fig. 4-151: Average bond stress along the beam axis - Beam B27-3b ........................ 197

Fig. 4-152: Distribution of longitudinal, transverse, and shear strains at peak load - Beam B27-3b

Fig. 4-153: Distribution of longitudinal, transverse, and shear strains at failure load -

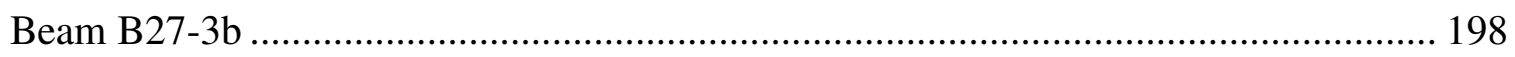

Fig. 4-154: Distribution of transverse and shear strains averaged............................... 199

Fig. 4-155: Development of principal tensile and compressive strains along the critical

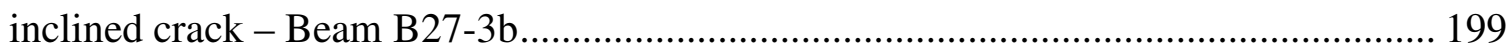

Fig. 4-156: Crack pattern after failure - Beam B27-4a .............................................. 202 Fig. 4-157: Load versus deflection and reinforcement strain relationships - Beam B27-4a 203

Fig. 4-158: Numbering of markers, elements, and strain gauges - B27-4a.................. 203

Fig. 4-159: Distribution of longitudinal, transverse and shear strains at $149 \mathrm{k}-$ Beam

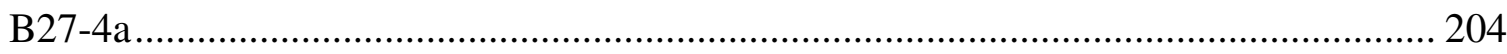

Fig. 4-160: Principal strain field at 149 kips - Beam B27-4a .................................... 204 
Fig. 4-161: Development of principal tensile strains along the critical inclined crack Beam B27-4a 205

Fig. 4-162: Load versus deflection and reinforcement strain relationships - Beam B27-4b 207

Fig. 4-163: Crack pattern after failure - Beam B27-4b .............................................. 207

Fig. 4-164: Numbering of markers, elements, and strain gauges - Beam B27-4b ........ 207

Fig. 4-165: Distribution of longitudinal, transverse, and shear strains at $125 \mathrm{k}-$ Beam

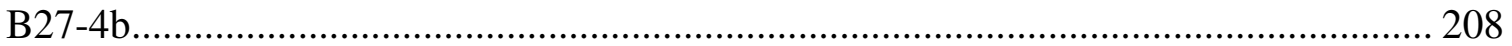

Fig. 4-166: Principal strain field at 125 kips - Beam B27-4b .................................. 208

Fig. 4-167: Development of principal tensile strains along the critical inclined crack -

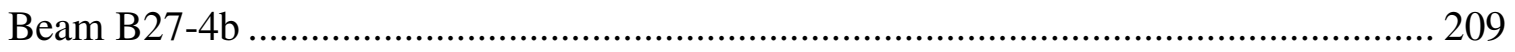

Fig. 4-168: Crack pattern after failure - Beam B27-5 ........................................... 211

Fig. 4-169: Load versus deflection relationship - Beam B27-5 …........................... 212

Fig. 4-170: Numbering of markers, elements, and strain gauges - Beam B27-5 .......... 212

Fig. 4-171: Load versus reinforcement strain relationships - Beam B27-5 ................. 212

Fig. 4-172: Distribution of longitudinal, transverse, and shear strains at peak load - Beam

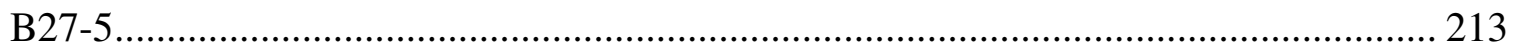

Fig. 4-173: Distribution of longitudinal, transverse, and shear strains failure load - Beam B27-5

Fig. 4-174: Transverse and shear strains averaged over the beam depth - Beam B27-5 215 Fig. 4-175: Development of principal tensile and compressive strains along the critical

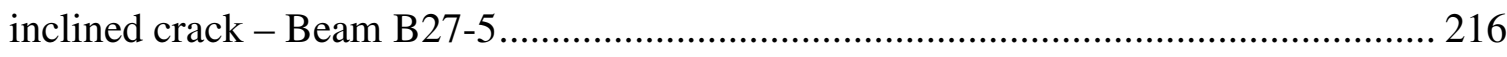

Fig. 4-176: Load versus deflection and reinforcement strain relationships - Beam B27-6 218

Fig. 4-177: Numbering of markers, elements, and strain gauges - Beam B27-6 .......... 218

Fig. 4-178: Crack pattern prior to failure - Beam B27-6 ........................................... 219

Fig. 4-179: Crack pattern after failure - Beam B27-6 …........................................ 219

Fig. 4-180: Transverse and shear strains averaged over the beam depth - Beam B27-6219

Fig. 4-181: Distribution of longitudinal, transverse, and shear strains at peak load - Beam B27-6. 220 
Fig. 4-182: Distribution of longitudinal, transverse, and shear strains at failure load Beam B27-6 221

Fig. 4-183: Principal strain field at peak and failure load - Beam B27-6

Fig. 4-184: Development of principal tensile strains along the critical inclined crack Beam B27-6 223

Fig. 4-185: Load versus deflection and reinforcement strain relationships - Beam B27-7

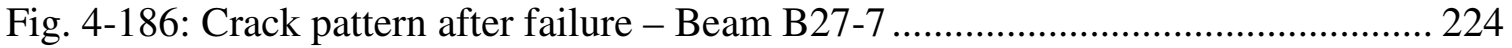

Fig. 4-187: Numbering of markers, elements, and strain gauges - Beam B27-7 ......... 225

Fig. 4-188: Distribution of longitudinal, transverse, and shear strains at $90.3 \mathrm{k}-$ Beam B27-7. 225

Fig. 4-189: Development of principal tensile strains along the critical inclined crack (close-up at the peak load) - Beam B27-7. 226

Fig. 4-190: Crack pattern prior to failure - Beam B27-8 …..................................... 228

Fig. 4-191: Crack pattern after failure - Beam B27-8 …............................................ 228

Fig. 4-192: Load versus deflection and reinforcement strain relationships - Beam B27-8 228

Fig. 4-193: Numbering of markers, elements, and strain gauges - Beam B27-8 .......... 229

Fig. 4-194: Distribution of longitudinal, transverse, and shear strains at $128 \mathrm{k}-$ Beam B27-8. 229

Fig. 4-195: Transverse and shear strains averaged over the beam depth - Beam B27-8230 Fig. 4-196: Principal strain field at 128 kips - Beam B27-8 230

Fig. 4-197: Development of principal tensile strains along the critical inclined crack Beam B27-8 231

Fig. 5-1: Reaction (shear) forces in test beams 232

Fig. 5-2: Determination of the number of inclined cracks, average inclined crack spacing, and angle and length of the critical crack (Series B18) 239 Fig. 5-3: Determination of the number of inclined cracks, average inclined crack spacing, and angle and length of the critical crack (Series B27) ..... 240

Fig. 5-4: Relationship between inclined crack spacing and either shear stress or normalized shear stress 
Fig. 5-5: Relationship between inclined crack spacing and longitudinal reinforcement ratio, and between inclined crack spacing and fiber reinforcing index

Fig. 5-6: Relationship between average inclined crack angle versus shear stress for beams with fibers 244

Fig. 5-7: Effect of fiber volume fraction on shear stress and normalized shear stress ... 247 Fig. 5-8: Effect of longitudinal reinforcement ratio on shear stress and normalized shear stress. 248

Fig. 5-9: Shear strength prediction of SFRC beams tested in this research using previously proposed expressions 251

Fig. 5-10: Proposed model to predict shear strength of SFRC beams 254 Fig. 5-11: Normal compressive and shear stress relationship used in this study (Bresler and Pister, 1958) 255

Fig. 5-12: Comparison of two methods to obtain a uniform shear stress 258 Fig. 5-13: Derivation of uniform tensile stress versus crack width relationship from fourpoint bending tests 260

Fig. 5-14: Average tensile stress versus crack width relationship 262 Fig. 5-15: Assumed contribution from different shear resisting mechanisms at different crack widths 267

Fig. 5-16: Predicted shear strengths of test beams using the proposed model 267 Fig. 5-17: Comparison of experimentally obtained SFRC average tensile stress and recommended tensile stress.

Fig. 5-18: Shear strength prediction for SFRC beams tested by this and other research groups. 


\section{LIST OF TABLES}

Table 2-1: Pullout test results pertinent to hooked steel fibers embedded in a cementbased matrix (from Naaman and Najm, 1991) .............................................................. 9

Table 2-2: Test results pertinent to hooked steel fibers embedded in a concrete matrix

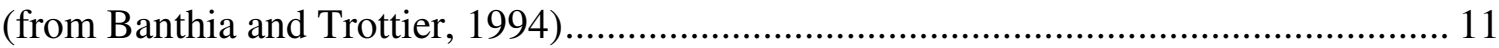

Table 2-3: Previous researches on SFRC beams without stirrup reinforcement ............. 32

Table 2-4: Research programs that dealt with practical beam depths ............................. 33

Table 3-1: Design properties of beams in Series B18 and B27 ................................... 50

Table 3-2: Calculation of the required beam length ................................................. 52

Table 3-3: Properties of hooked steel fibers as reported by the manufacturer ................ 54

Table 3-4: Calculation of beam shear and flexural strengths ..................................... 56

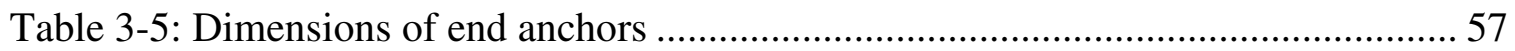

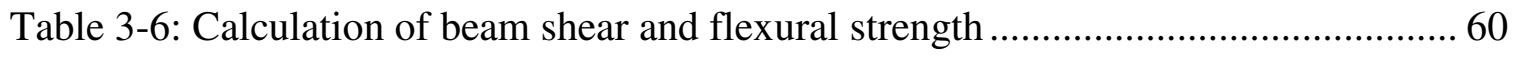

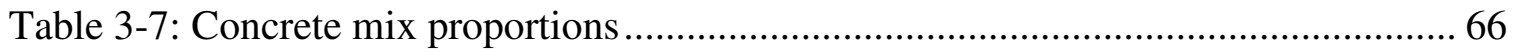

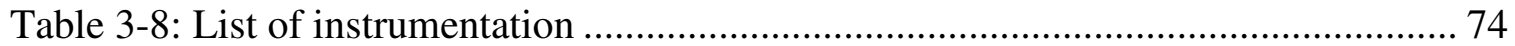

Table 3-9: Properties of reinforcing bars …........................................................... 75

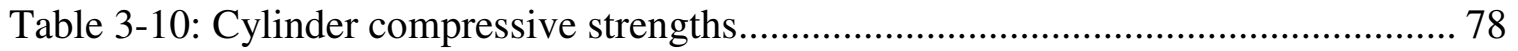

Table 3-11: Information of specimens at a deflection of 0.12 inches.............................. 81

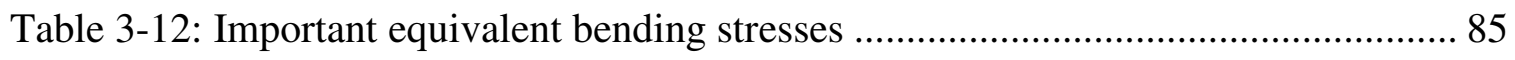

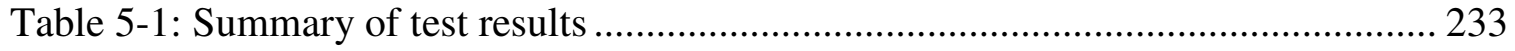

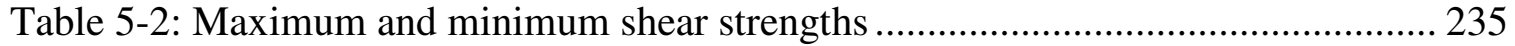

Table 5-3: Number of inclined cracks, crack spacing, and crack angles ...................... 242

Table 5-4: Ratio of shear stresses for SFRC beams with different types of fibers ......... 245

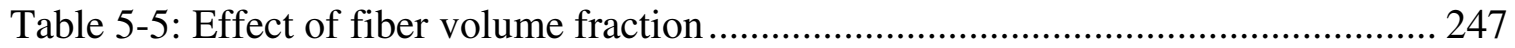

Table 5-6: Effect of longitudinal reinforcement ratio............................................... 248

Table 5-7: Prediction of beam shear strength .......................................................... 266 
Table 5-8: Shear strength prediction for SFRC beams tested by previous research groups

273 


\begin{abstract}
This research aims to study: (1) the behavior and ultimate shear strength of steel fiber reinforced concrete (SFRC) beams; (2) the possibility of using steel fibers as minimum shear reinforcement in reinforced concrete (RC) beams; and (3) the effectiveness of steel fibers as a means to reduce shear size effect in RC beams. A total of 28 simply-supported beams with a shear span-to-depth ratio of 3.5 were subjected to monotonically-increased, concentrated load. Target concrete compressive strength for all beams was $6000 \mathrm{psi}$. The studied parameters included fiber type, fiber volume fraction (between 0.75 and 1.5\%), longitudinal tension reinforcement ratio, and beam depth (18 in. or 27 in.). Three types of steel fibers were considered, all with hooks at their ends. Two of the fibers evaluated were 1.2 inch long, with aspect ratio of either 55 or 80 . The third type of fiber investigated was 2.36 inch long, with an aspect ratio of 80 . The $30 \mathrm{~mm}$ long fiber with an aspect ratio of 80 was made out of a high-strength (330 ksi) wire, while the other two fiber types were made out of a regular strength (160 ksi) wire. Various longitudinal reinforcement ratios (approximately 1.6, 2.0 and 2.7\%) were evaluated to investigate the behavior of SFRC beams failing in shear either prior to or after flexural yielding.

Test results showed that the use of hooked steel fibers in a volume fraction greater than or equal to $0.75 \%$ led to a substantial increase in shear strength and significantly reduced the size effect for shear strength of beams with depths of up to 27 in. The results also indicated that hook steel fibers can be used as minimum shear reinforcement in $\mathrm{RC}$ beams constructed with normal-strength concrete and within the depth range considered.

A method to predict the shear strength of SFRC beams based on a Pister and Bresler concrete failure criterion and on the average tensile strength of the SFRC obtained from a standard ASTM four-point bending test was proposed. The method
\end{abstract}


proved to provide reasonable predictions of shear strengths for the SFRC beams tested in this experimental program, as well as in previous research reported in the literature. An average tensile strength for the SFRC as a function of fiber volume fraction and aspect ratio was also recommended for the purpose of shear design of SFRC beams. 


\section{CHAPTER 1}

\section{INTRODUCTION}

\subsection{BACKGROUND AND MOTIVATION}

The problem of shear failure and the traditional solution. When subjected to a combination of moment and shear force, a reinforced concrete (RC) beam with either little or no transverse reinforcement can fail prematurely in shear before reaching its full flexural strength. This type of shear failure is sudden in nature and usually catastrophic because it does not give ample warning to inhabitants. One well-known example is the shear failure of beams in the U.S. Air Force Warehouse (Anderson, 1957), as shown in Fig. 1-1.

To prevent shear failures, beams are traditionally reinforced with stirrups. In general, the use of stirrups is expensive because of the labor cost associated with reinforcement installation. Also, casting concrete in beams with closely-spaced stirrups could be difficult and might lead to voids and associated poor bond between concrete and reinforcing bars. An alternative solution to stirrup reinforcement is the use of randomly oriented steel fibers, which have been shown to increase shear resistance (for example, Kwak et al., 2000). The use of deformed steel fibers in place of minimum stirrup reinforcement is currently allowed in ACI Code Section 11.4.6 (ACI Committee 318, 2008). The benefits of using steel fiber reinforcement for shear resistance, however, have not been fully exploited yet, primarily due to lack of understanding of the role which steel fibers play on the shear behavior of beams with and without stirrup reinforcement. The development of steel fibers, their application in the concrete industry, and a proposal for their use will be discussed next. 


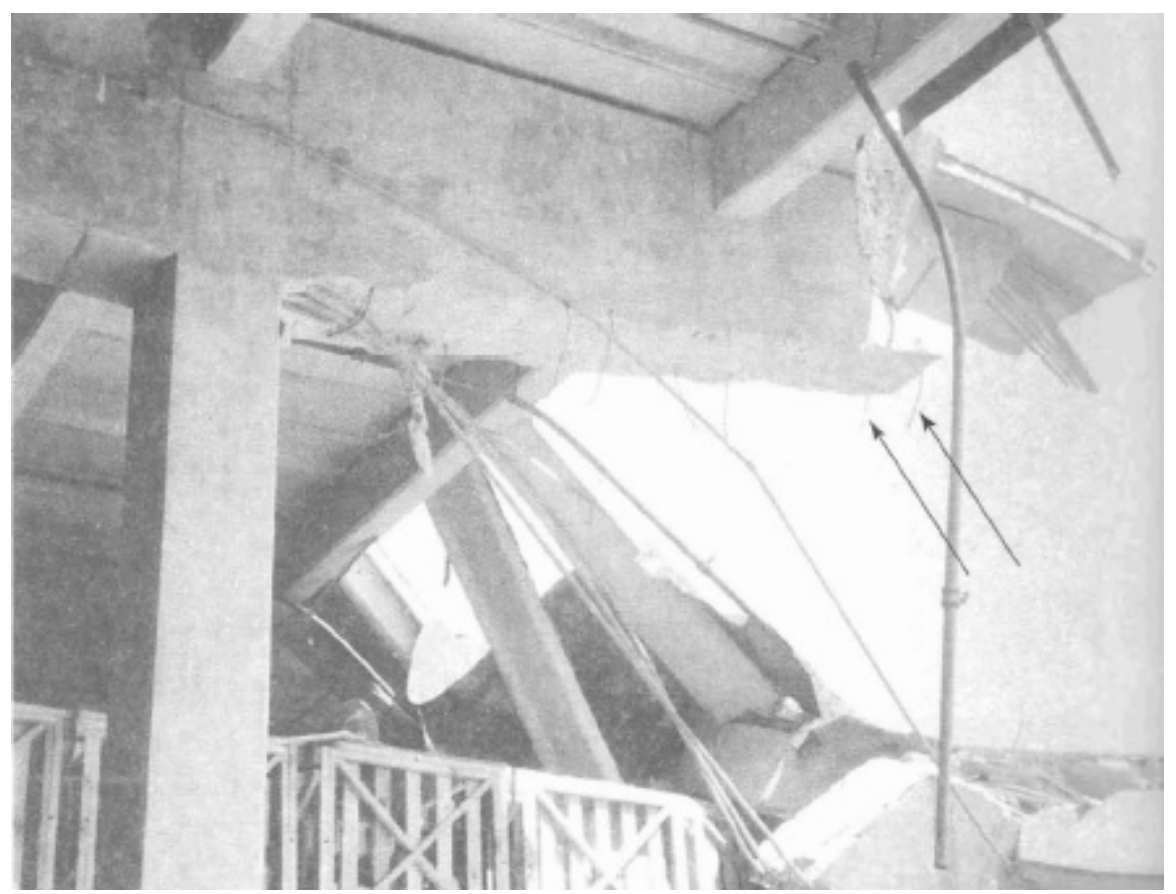

Fig. 1-1: Shear failure of reinforced concrete beams in the U.S. Air Force Warehouse (from Wight and MacGregor, 2009)

Development of fiber-reinforced concrete (FRC). According to Naaman (1985), even though the concept of using fibers to reinforce concrete is not new, the application of FRC in the concrete industry did not flourish until the early 1960s. Fibers are made from various materials (steel, glass, carbon, or synthetic material) and with different geometrical characteristics (length, diameter, longitudinal shape, cross-sectional shape, and surface roughness). Among the various types of fibers, steel fibers are most widely used in the concrete industry. Steel fibers are short (typically from 0.5 to 2.5 inches) and generally deformed to enhance bond with the concrete (Fig. 1-2). Available commercial steel fibers have a tensile strength of up to approximately $300 \mathrm{ksi}$.
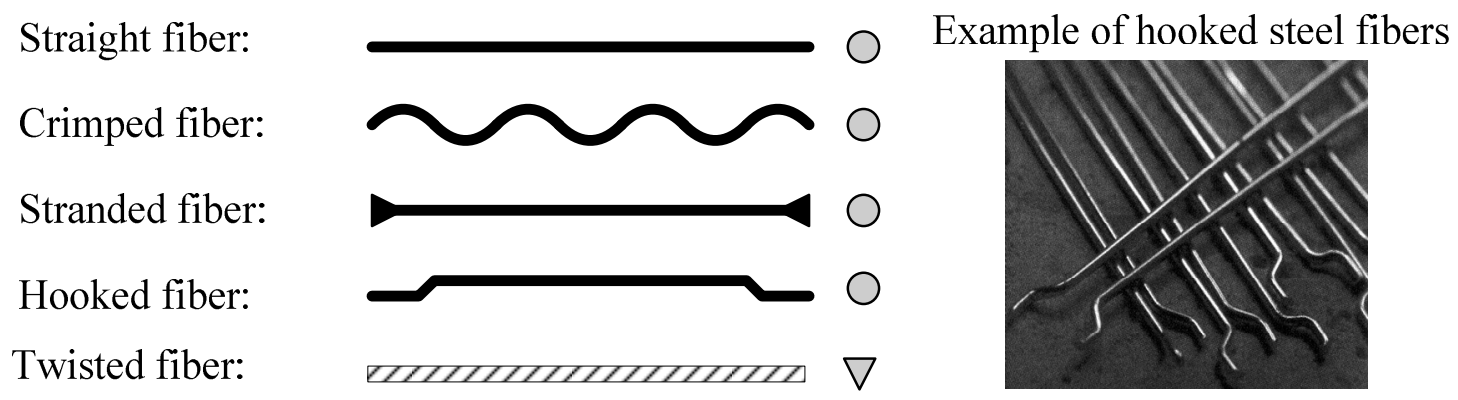

Fig. 1-2: Different types of steel fibers 
Applications of steel fiber-reinforced concrete (SFRC). When added to concrete, steel fibers significantly improve its post-cracking tensile resistance and toughness (Hannant, 1978). SFRC has been used extensively in construction of industrial floors, bridge deck overlays, airport runways, highway pavements, tunnel linings, spillways, dams, slope stabilizations, and many precast products. Recent examples include the floor slab for the Chrysler Jefferson North Assembly Plant (Robinson et al., 1991), the Barr Lake Dam (Mass, 1997), and the Gotthard Base Tunnel (Kronenberg, 2006). Nevertheless, the use of SFRC in buildings has been very limited, even though steel fibers have been shown to enhance the flexural (Hannant, 1978), and shear (for example, Batson and Jenkins, 1972) behavior of concrete members. The limited use of SFRC in building structures is primarily due to the lack of design provisions in building codes.

Shear design provisions for SFRC beams. The section entitled "Shear in Beams" of the report "Design Considerations for Steel Fiber Reinforced Concrete" (ACI Committee 544, 1999) has no design equations for SFRC beams in shear. Only test results are reported and an equation to predict shear strength is given. There are two potential reasons for the lack of design equations. This is probably because most tested SFRC beams reported by the committee were small (from 6 to 12 inches) and contained obsolete types of fibers, such as straight steel fibers. In general, small scale beams exhibit higher shear strength than large beams do. Even though the shear size effect is believed to be less important for SFRC beams than regular concrete beams, experimental verification is needed. Beams using early-developed fibers, such as chopped straight wire, have exhibited much lower shear strength than those reinforced with modern, commercially available deformed fibers (Kwak et al., 2002). Another potential reason for the lack of shear design provisions is the fact that the mechanisms for shear resistance in RC beams are not fully understood. When steel fibers are added to RC beams, these mechanisms become even more complicated.

Motivation for the research. The need for more experimental data for large SFRC beams failing in shear and the little knowledge on the shear behavior of SFRC 
beams are the primarily motivations for this research. In particular, this research was aimed at evaluating the shear resisting mechanism in SFRC beams and the ability of various steel fibers to serve as replacement of minimum stirrup-type shear reinforcement.

\subsection{OBJECTIVES AND SCOPE OF THE RESEARCH}

Prior to listing the objectives and scope of this research, a discussion of the shear problem of SFRC beams is necessary.

\section{Shear behavior of simply-supported SFRC beams under a monotonically-} increasing concentrated load. When subjected to a monotonically-increased, concentrated load near midspan, a simply supported SFRC beam often fails due to either moment (flexural failure), shear action (shear failure), or a combination of both. While a flexural failure usually occurs for slender beams with a low amount of longitudinal reinforcement, a shear failure may occur in shorter beams with a moderate to high amount of longitudinal reinforcement. This failure may occur prior to or after flexural yielding. An SFRC beam experiencing a shear failure usually goes through four phases of cracking: (1) flexural (vertical) cracks appear in the maximum moment region, (2) shear (inclined) cracks occur in the mid-depth region along with the spreading of flexural cracks toward the supports and the propagation and bending of pre-existing flexural cracks toward to the compression region (flexural cracks may or may not turn into flexural-shear cracks), (3) the shear and flexural cracks widen, and finally, (4) the beam fails with the sudden opening of the critical inclined crack. An SFRC beam resists shear forces through several mechanisms: shear force across the compression region, aggregate interlock, tension in the fibers, and the dowel action of longitudinal reinforcing bars.

The shear-resisting mechanisms in an SFRC beam are inter-related and depend on several factors. In additional to determining the relative moment-shear demand, the shear span-to-effective depth ratio affects the lines of forces within the beam. Slender beams are likely to resist less shear force than those with low shear span-to-effective depth ratio, due to the arch action. Similarly, together with aggregate size, the properties 
and amount of fibers, matrix properties, and fiber-matrix interaction will affect how much force could be transferred along and across the inclined shear cracks at a given crack width. The concrete compressive strength and the amount and strength of longitudinal reinforcement certainly affect the shear strength of the compression zone and the dowel action, as well as the crack distribution and width along the beam span. It should be noted that the dowel action is also affected by the SFRC tensile strength.

Studies on shear behavior of SFRC beams. Experimental research on the shear behavior of SFRC beams has been conducted for the past three decades. Most of these test programs have investigated key parameters known to affect shear behavior, including shear span-to-effective depth ratio, longitudinal reinforcement ratio, fiber volume fraction, and concrete compressive strength. However, there are still parameters that have not been extensively investigated. For example, the majority of test beams have been of a small depth. Therefore, the conclusion that size effect is negligible in SFRC beams (for example, Kwak et al., 2002) has not been fully validated by experimental data. Also, while many test programs investigated the use of either straight or crimped fibers, hooked steel fibers are more common nowadays. The limited test data on the shear behavior of SFRC beams with hooked fibers further supports the need for additional experiments, particularly on beams with depth greater than 12 inches. The interaction between fibers and stirrup reinforcement is little known and thus, this topic also requires investigation.

With regard to analytical research on the shear behavior of SFRC beams, no significant advances have been made beyond the existing theories for RC members. Most of the analytical work has relied on regression analysis of experimental data to derive expressions to predict the shear strength of SFRC beams. Thus, the equations derived might only be applicable to beams similar to those in which the expressions were based.

Objectives of the research. The primary objectives of this research were to: (1) study the behavior and ultimate shear strength of SFRC beams without stirrup reinforcement, (2) investigate the possibility of using steel fibers as minimum shear reinforcement in RC beams, (3) evaluate the effectiveness of steel fibers as a means to 
reduce the effect of depth on shear strength of RC beams, and (4) develop a mechanicsbased analytical model to predict the shear strength of SFRC beams.

Scope of the research. This investigation was limited to: (1) simply-supported beams, (2) hooked steel fibers, and (3) concentrated monotonic loading.

It has been found (for example, Rodriguez et al., 1959) that expressions developed from tests of simply-supported beams give a conservative prediction of the shear strength of continuous RC beams. However, issues related to behavior and design of transverse and tensile reinforcement at points of inflection in a continuous beam are worthy of a separate investigation.

This research is also limited to concrete reinforced with hooked steel fibers because they currently are the most popular fibers. The results of this investigation, however, should be generally applicable to SFRC beams constructed with other deformed steel fibers that exhibit bond stress versus slip response similar to that of the hooked steel fibers used in this investigation.

The beams in this study were subjected to monotonically-increasing loading. Other loading schemes such as cyclic, reversed cyclic and dynamic loading, which are substantially different from monotonic loading, were not investigated. The loading was also restricted to a single concentrated load. It is known that loads on beams are frequently uniform. However, research on RC beams, see for example Bernaert and Siess (1956) and Brown et al. (2006), has shown that RC beams can sustain higher shear induced by uniform loads than by concentrated loads. Therefore, it can be argued that the results obtained from this study can be conservatively applied to the design of reinforced SFRC beams subjected to uniform loads.

\subsection{ORGANIZATION OF THE THESIS}

This thesis has six chapters. Chapter 2 starts with a review of bond properties of steel fibers in a regular-strength concrete matrix. The compressive, tensile, flexural, and shear strength of SFRC, as well as its strength under multiaxial stresses, are also 
discussed. The second part of Chapter 2 includes a discussion of the failure mechanisms in RC and SFRC beams, either with or without stirrup reinforcement. The remaining of this chapter is dedicated to reporting test results of SFRC beams from previous investigations.

The experimental program, including the choice of studied parameters and the design, construction, and testing of SFRC beam specimens, is discussed in Chapter 3. Tests to obtain mechanical properties of the materials used in the beam specimens are also discussed in this chapter.

The shear behavior of the tested beam specimens is discussed in Chapter 4. Test results are reported and analyzed in that chapter. Analyses include the investigation of the strain field throughout the tests and its relationship with the strength and failure mode of the test beams.

In Chapter 5, the effect of the studied parameters on beam behavior was investigated. A mechanics-based model to predict the shear strength of SFRC beams without shear reinforcement is also proposed. Finally, conclusions from this research, along with major findings and design recommendations, are featured in Chapter 6. 


\section{CHAPTER 2}

\section{LITERATURE REVIEW}

\subsection{MECHANICAL PROPERTIES OF SFRC}

\subsubsection{Types of steel fibers and bond between steel fibers and concrete}

General discussion. Steel fibers and concrete form a composite, known as steel fiber-reinforced concrete (SFRC), which has an improved post-cracking behavior compared to plain concrete. The performance of SFRC greatly depends on bond characteristics between steel fibers and concrete, fiber content, and distribution of fibers in the concrete matrix.

The primary role of steel fibers is to bridge cracks due to tension in the concrete. Depending on bond strength, the fibers can either fracture or pull out of the concrete as cracks open. The latter form of failure is more favorable because SFRC will be more ductile and absorb a greater amount of energy. The development of different types of steel fibers has been driven by the desire to improve bond characteristics between steel fibers and concrete.

Early versions of steel fibers developed in the early 1960s were straight fibers. The primary source of bond for these fibers comes from the friction between the fibers and concrete. Therefore, fibers with a higher surface area to volume ratio have higher bond strengths. Thus, fibers with rectangular sections are more efficient than fibers with circular sections and for the same fiber length, fibers with smaller diameters (higher aspect ratio) are more efficient.

Bond strength between steel fibers and concrete can be greatly improved by introducing deformations in fibers. The common types of deformed steel fibers are 
crimped fibers with a sinusoidal shape along its length, stranded fibers with enlarged ends, hooked fibers with deformed ends, and twisted fibers (Fig. 1-2).

When being pulled out, the ends of a hooked steel fiber must bend significantly and yield before the fiber can be pulled out of the concrete. This process allows SFRC with hooked steel fibers to absorb a great amount of energy prior to a complete failure. In this sense, a hooked steel fiber with a higher yield strength will produce a stronger composite.

It should be noted that changing fiber cross section, increasing fiber aspect ratio, or introducing mechanical anchorage do not always improve the fiber matrix bond strength because these changes may result in an SFRC mix with inadequate workability and fiber distribution (ACI Committee 544, 1988).

Bond test results. From the pullout tests of fibers embedded in a cement-based matrix, Naaman and Najm (1991) concluded that an increase in matrix strength resulted in an increase in bond strength and a faster debonding. Pulling out a hooked steel fiber required a work that was four times greater than that required for a smooth steel fiber. The slip at peak load when pulling out a hooked steel fiber was up to two times greater than that for a smooth steel fiber. Test results pertinent to hooked steel fibers are listed in Table 2-1. It can be noted that the slips at peak loads were less than 0.04 inches $(1 \mathrm{~mm})$ and the average bond stress varied from 640 to 1110 psi, depending on the matrix strength.

Table 2-1: Pullout test results pertinent to hooked steel fibers embedded in a cementbased matrix (from Naaman and Najm, 1991)

\begin{tabular}{cccccc}
\hline $\begin{array}{c}\text { Diameter } \\
\text { (in.) }\end{array}$ & $\begin{array}{c}\text { Embedment } \\
\text { length } \\
\text { (in.) }\end{array}$ & $\begin{array}{c}\text { Matrix } \\
\text { strength } \\
(\mathrm{psi})\end{array}$ & $\begin{array}{c}P_{\text {peak }} \\
\text { (lb) }\end{array}$ & $\begin{array}{c}\Delta_{\text {peak }} \\
\text { (in.) }\end{array}$ & $\begin{array}{c}\text { Average bond } \\
\text { stress at peak load } \\
\text { (psi) }\end{array}$ \\
\hline & & 4850 & 59 & 0.031 & 640 \\
0.0295 & 1 & 7400 & 80 & 0.035 & 860 \\
$(0.75 \mathrm{~mm})$ & $(25.4 \mathrm{~mm})$ & 8650 & 103 & 0.029 & 1110 \\
\hline
\end{tabular}


Fig. 2-1 shows the effect of matrix strength and embedment length. Doubling the embedment length of the fibers did not significantly affect the load-slip curves because the hooked ends contributed most of the effective bond strength of the fibers.
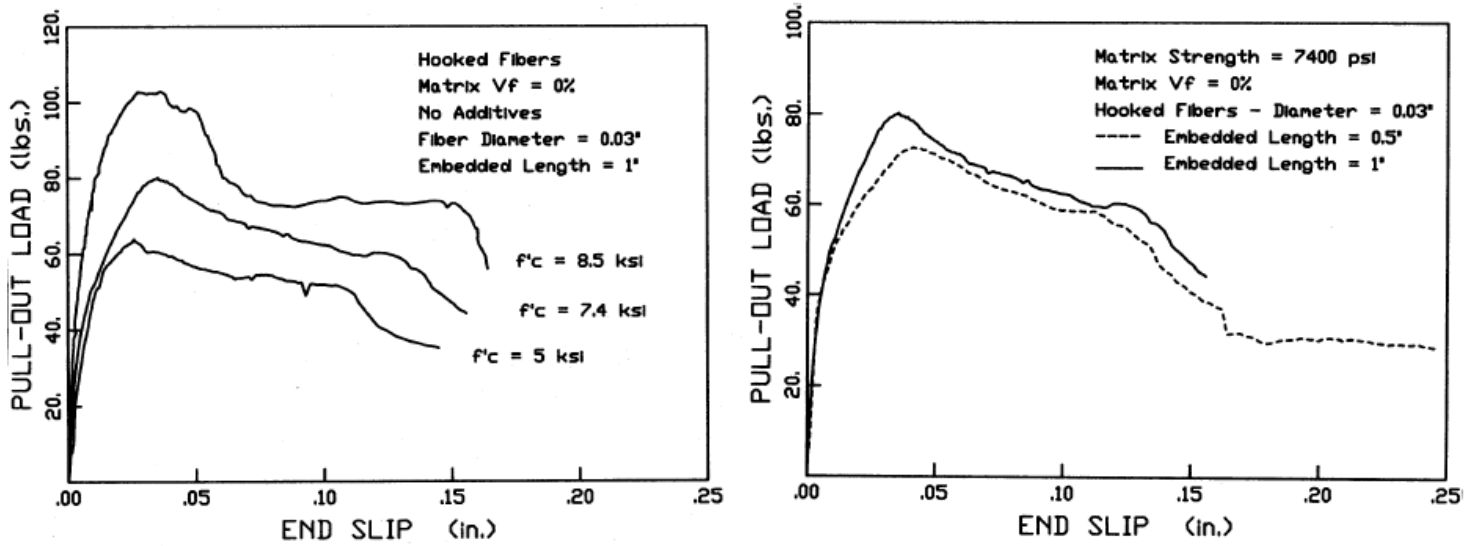

Fig. 2-1: Effect of matrix strength and fiber embedment length on pullout behavior (from Naaman and Najm, 1991)

Bond tests for a concrete-based matrix and commercially available fibers are rare. Banthia and Trottier (1994) investigated the pullout behavior of hooked, crimped, and stranded steel fibers embedded in concrete matrices with different strengths. Aggregates used in the concrete matrix had a maximum size of $3 / 8$ inches $(10 \mathrm{~mm})$. The fibers were inclined from the loading direction at an angle that varied from 0 to 90 degrees. Only test results for hooked steel fibers with 0-degree inclination are reported in Table 2-2. The conclusion that bond strength increased with respect to an increase in matrix strength, as found from the test results of Naaman and Najm (1991), still held. However, compared with the results for a cement-based matrix, an increase in the concrete matrix strength resulted in only a modest increase in the average bond stress at peak load. In addition, the slips at peak load were greater when a concrete matrix was used. 
Table 2-2: Test results pertinent to hooked steel fibers embedded in a concrete matrix (from Banthia and Trottier, 1994)

\begin{tabular}{|c|c|c|c|c|c|}
\hline $\begin{array}{l}\text { Diameter } \\
\text { (in.) }\end{array}$ & $\begin{array}{l}\text { Embedded } \\
\text { length } \\
\text { (in.) }\end{array}$ & $\begin{array}{l}\text { Matrix } \\
\text { strength } \\
\text { (psi) }\end{array}$ & $\begin{array}{l}P_{\text {peak }} \\
(\mathrm{lb})\end{array}$ & $\begin{array}{l}\Delta_{\text {peak }} \\
\text { (in.) }\end{array}$ & $\begin{array}{c}\text { Average bond } \\
\text { stress at peak load } \\
(\mathrm{psi})\end{array}$ \\
\hline \multirow{3}{*}{$\begin{array}{c}0.0295 \\
(0.75 \mathrm{~mm})\end{array}$} & \multirow{3}{*}{$\begin{array}{c}1.18 \\
(30 \mathrm{~mm})\end{array}$} & 5800 & 61 & 0.061 & 560 \\
\hline & & 7540 & 65 & 0.039 & 590 \\
\hline & & 12330 & 67 & 0.047 & 610 \\
\hline
\end{tabular}

Fig. 2-2 shows the effect of fiber inclination angle with respect to the loading direction for normal-, mid-, and high-strength concrete (5.8, 7.5, and $12.3 \mathrm{ksi}$, respectively). For normal- and mid-strength concrete, a change in the angle from 0 to 15 degrees did not significantly affect the load-slip curve. When the inclination angle increased beyond 30 degrees, the effect of fiber inclination was more pronounced up to a slip of $0.14 \mathrm{in}$. (3.5 $\mathrm{mm})$. 
(a) Normal-strength concrete

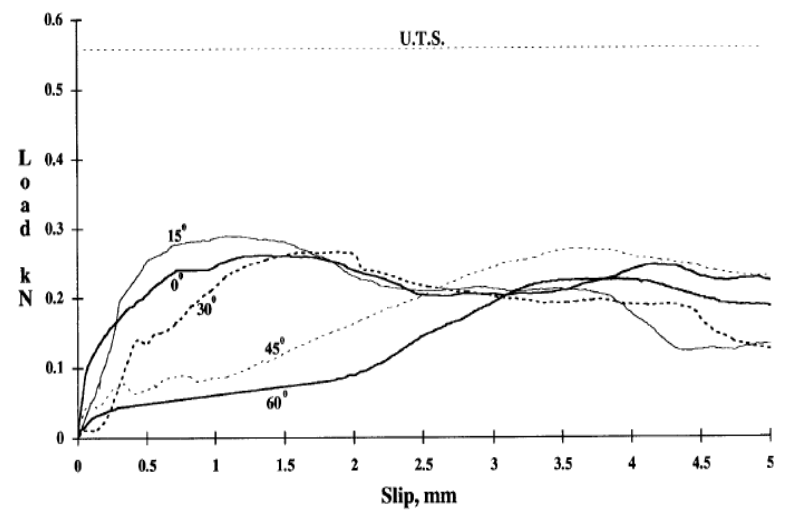

(b) Mid-strength concrete

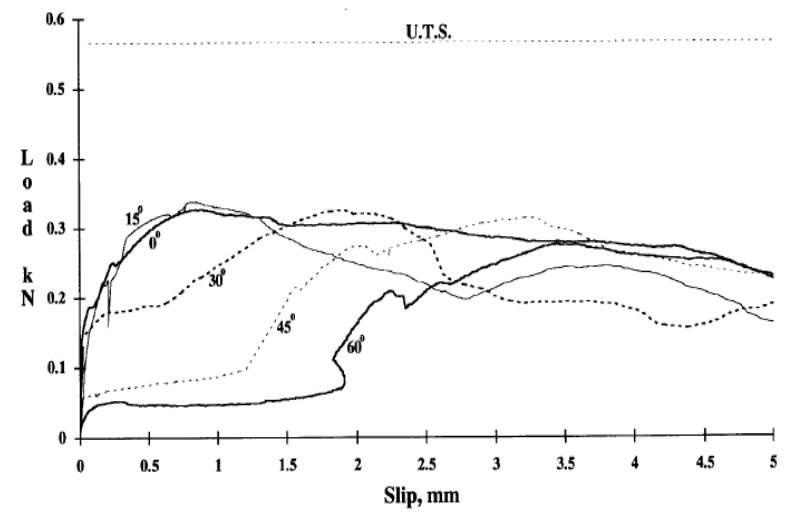

(c) High-strength concrete

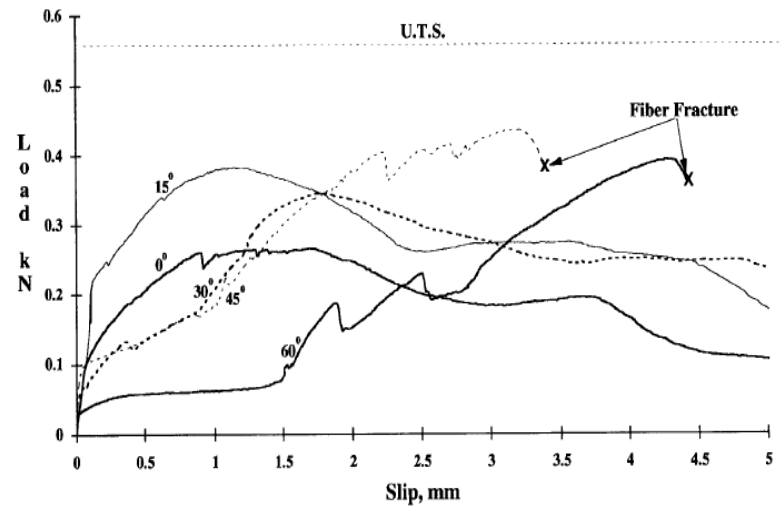

Fig. 2-2: Effect of fiber inclination for hooked steel fiber in normal-, mid-, and highstrength concrete (from Banthia and Trottier, 1994) 


\subsubsection{SFRC direct tensile strength}

General discussion. The tensile properties of SFRC are difficult to experimentally assess. To the author's knowledge, there has been no agreement upon a standard test method to evaluate the direct tensile behavior of SFRC. The problem arises primarily from the need for specimens that have a sufficiently-large cross section such that a fiber distribution similar to that in real structural members is obtained. A tension specimen of that size often poses difficulty in the design of fixtures to grip the ends of the specimen. Therefore, direct tensile test results are usually significantly scattered. The strain is also difficult to interpret because after cracking, average strain is due primarily to local crack opening. Therefore, the selection of gauge length also affects the measured average strain. In recent direct tensile test results, researchers tend to report the deformation in terms of crack width or extension, particularly for strain-softening materials.

Test results. Due to the reasons mentioned above, direct tensile test results for SFRC are rare. Shah (1978) performed tests with straight, hooked, and stranded steel fibers and cement-based matrices. Test results are reported in ACI Committee 544 and shown in Fig. 2-3.

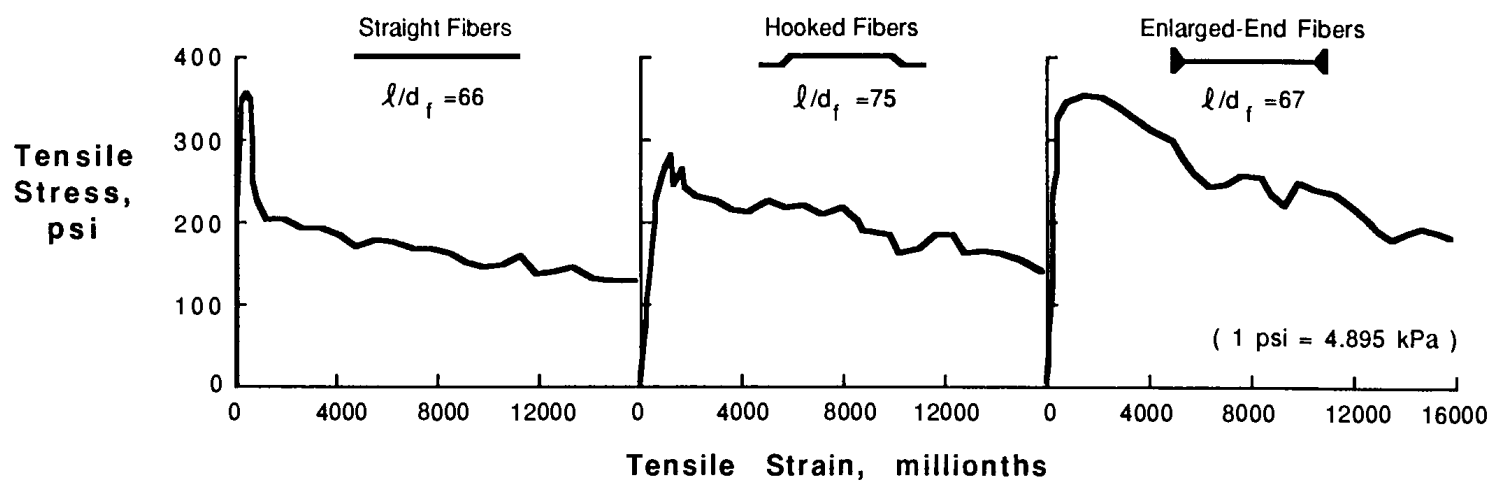

Fig. 2-3: Direct tensile stress-strain curves for different types of SFRCs (from ACI

Committee 544, 1988)

Using a dog-bone specimen with a gauge length of 7.87 inches $(200 \mathrm{~mm})$ and a width of 2.76 inches $(70 \mathrm{~mm})$, Lim et al. (1987) evaluated the performance of SFRCs 
with straight and hooked steel fibers. The matrix used was concrete with a maximum aggregate size of 3/8 inches $(10 \mathrm{~mm})$. Fig. 2-4 shows the load-extension relationship for the specimens containing hooked steel fibers with a diameter of 0.02 inches $(0.5 \mathrm{~mm})$. Unfortunately, Lim et al. did not report the thickness of the specimens and thus, the tensile strength of the specimens is not known. Nevertheless, the test results suggested that with the same fiber length, an increase in fiber content (from 0.5 to either 1.0 or $1.5 \%$ ) led to an almost proportional increase in post-cracking strength (from 1.35 kips (6 $\mathrm{kN})$ to $2.7 \mathrm{kips}(12 \mathrm{kN})$ and $4.05 \mathrm{kips}(18 \mathrm{kN})$, respectively). Similarly, the test results also showed that for the same fiber content of $1 \%$ by volume, an increase in fiber length from 1.18 inches $(30 \mathrm{~mm})$ to 1.97 inches $(50 \mathrm{~mm})$ led to an increase in post-cracking strength from $2.7 \mathrm{kips}(12 \mathrm{kN})$ to $3.83 \mathrm{kips}(17 \mathrm{kN})$.
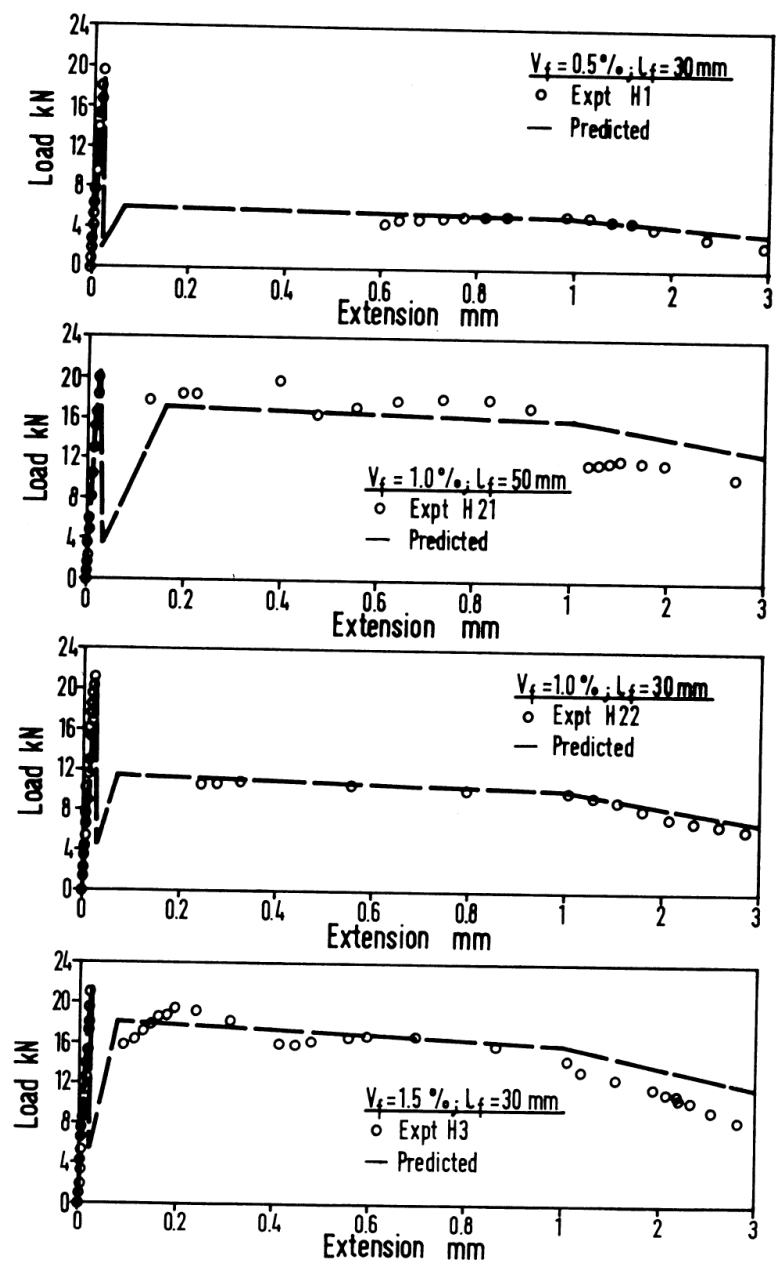

Fig. 2-4: Load-extension relationship for different SFRC mixes (from Lim et al., 1987) 
Noghabai (2000) tested notched concrete cylinder specimens (2.76 inches (70 $\mathrm{mm})$ in diameter and 3.35 inches $(85 \mathrm{~mm})$ in height, Fig. 2-5a) with hooked steel fibers and aggregate size from 0.315 inches $(8 \mathrm{~mm})$ to 0.63 inches $(16 \mathrm{~mm})$. The crack width was measured with a gauge length of 1.18 inches $(30 \mathrm{~mm})$. The use of high strength concrete (HSC) seems to increase both the first crack and the post-cracking tensile strength of SFRC (Fig. 2-5b).

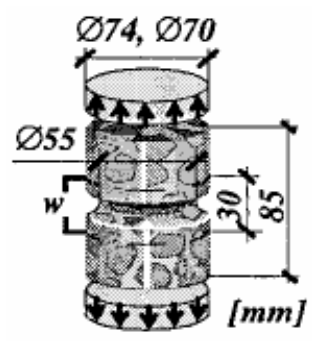

(a): Test set-up

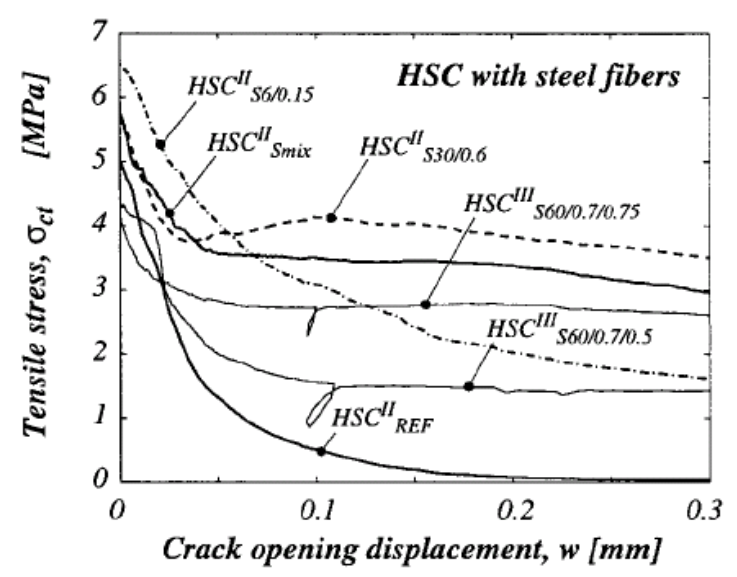

(b) Tensile stress-crack opening relationship

Fig. 2-5: Tensile stress-crack opening relationship for different SFRC mixes (from

Noghabai, 2000)

First-crack tensile strength and elastic modulus of SFRC with fibers aligned in one, two, or three dimensions. The following review, treated in many textbooks (for example, Hannant, 1978), is important to facilitate the discussion in the following chapters. The law of mixtures leads to the following expressions for the composite tensile strength at first crack, $\sigma_{c c}$, and stiffness, $E_{c}$ :

$$
\begin{gathered}
\sigma_{c c}=\sigma_{f} V_{f}+\sigma_{m u}\left(1-V_{f}\right) \\
E_{c}=E_{f} V_{f}+E_{m}\left(1-V_{f}\right) .
\end{gathered}
$$

where $\sigma_{m u}, E_{m}, E_{f}$, and $V_{f}$ are, respectively, the matrix tensile strength at first crack, matrix modulus, fiber modulus, and fiber volume fraction. Because the fiber volume fractions in concrete are generally small (less than $2 \%$ ), it turns out that the increase in composite strength and stiffness is not significant. For example, with a concrete modulus 
of elasticity of 4,420 ksi (concrete strength of $6 \mathrm{ksi}$ ), a fiber modulus of elasticity of $29,000 \mathrm{ksi}$, and a fiber volume fraction of $0.75 \%$, the first-crack tensile strength and modulus of elasticity of the composite increase less than 5\%.

When the fibers align randomly in 2 or 3 dimensions, a fiber length factor, $\eta_{1}$,

and a fiber orientation factor, $\eta_{2}$, can be multiplied to the fiber terms (the first terms in the right hand sides of Eq. 2-1 and 2-2), and thus, the first-crack tensile strength and elastic modulus can be calculated as follows:

$$
\begin{gathered}
\sigma_{c c}=\eta_{1} \eta_{2} \sigma_{f} V_{f}+\sigma_{m u}\left(1-V_{f}\right) \\
E_{c}=\eta_{1} \eta_{2} E_{f} V_{f}+E_{m}\left(1-V_{f}\right)
\end{gathered}
$$

In essence, the fiber length factor takes into consideration the ability to develop the bond along the fibers. Allen (1972) proposed a fiber length factor as follows:

$$
\eta_{1}=\left\{\begin{array}{l}
\frac{L_{f}}{2 L_{c}} \text { if } L_{f} \leq L_{c} \\
1-\frac{L_{c}}{2 L_{f}} \text { if } L_{f}>L_{c}
\end{array}\right.
$$

where $L_{f}$ is the fiber length and $L_{c}$ is two times the length of the fiber embedment required to induce fiber fracture as opposed to fiber pullout.

The fiber orientation factor takes into account the fact that fibers, which are inclined at a certain angle compared with the direction of loading, may not be as effective in resisting the applied load. As reported by Hannant, Cox (1952) estimated a fiber orientation factor of $1 / 3$ and $1 / 6$ for fibers randomly distributed in 2 and 3 dimensions, respectively. Krenchel (1976) proposed similar values for the fiber orientation factor, 3/8 and $1 / 5$ for the cases of 2 and 3 dimensions, respectively.

\section{Post-cracking tensile strength of SFRC with fibers aligned in two or three}

dimensions. After the matrix cracks, the fibers are debonded and pulled out. The postcracking tensile strength can be therefore calculated on the basis of the average bond strength, the length over which the bond acts, and the number of fibers across a unit area (Naaman and Reinhardt, 1995): 


$$
\sigma_{p c}=\left(\lambda_{1} L_{f} \cdot \pi D_{f} \cdot \lambda_{2} \tau\right) \cdot\left(\lambda_{3} \frac{V_{f}}{\pi D_{f}^{2}}\right)=\lambda_{1} \lambda_{2} \lambda_{3} \tau V_{f} \frac{L_{f}}{D_{f}}
$$

It should be noted that $\lambda_{1}$ and $\lambda_{2}$ are, respectively, the fiber length and orientation factors for a post-cracking state, while $\lambda_{3}$ is the group factor associated with the number of fibers crossing a unit area. For example, with a mean fiber embedded length of $L_{f} / 4$ (Naaman, 1972 and Aveston et al., 1974), i.e. $\lambda_{1}$ equal to 0.25 , and the number of fibers across a unit area of $\frac{2 V_{f}}{\pi D_{f}^{2}}$ for the case of three dimensions (Aveston et al., 1974), i.e. $\lambda_{3}$ equal to 2, and a post-cracking fiber orientation factor of 1.2 (Naaman and Reinhardt, 1995), the peak post-cracking strength of SFRC can be estimated to be equal to $0.6 \tau V_{f} L_{f} / D_{f}$

Critical fiber volume fraction. The critical fiber volume fraction is defined to be the one above which the composite can carry additional stress after the matrix cracks ( $\sigma_{p c} \geq \sigma_{c c}$, see Fig. 2-6). In essence, when the matrix first cracks at a stress, $\sigma_{m u}$, and strain, $\varepsilon_{m u}$, the number of fibers must be sufficient to carry all the composite stress, $\sigma_{c c}$. If the maximum failure stress of the fibers embedded in the matrix is $\sigma_{f u}$, the critical fiber volume fraction for the case of fibers aligned in the loading direction and a fiber fracture mode takes the following form:

$$
V_{f}(\text { critical })=\frac{1}{1+\frac{\sigma_{f u}}{\sigma_{m u}}-\frac{\varepsilon_{m u} E_{f}}{\sigma_{m u}}}
$$

For example, with a concrete compressive strength of 6,000 psi (concrete cracking strength $\sigma_{m u}$ varies from $3 \sqrt{f_{c}^{\prime}}(230 \mathrm{psi})$ to $\left.4 \sqrt{f_{c}^{\prime}}(310 \mathrm{psi})\right)$, a first-crack strain of $0.01 \%$, a maximum failure stress of the fibers, $\sigma_{f u}$, of $160 \mathrm{ksi}$ (assuming fiber fracture), and a modulus of elasticity of the fibers, $E_{f}$, of $29,000 \mathrm{ksi}$, the critical fiber volume fraction varies from 0.15 to $0.25 \%$. 
It should be emphasized that this example is calculated for the case of fibers aligned in the loading direction and for a fiber fracture mode. In typical concrete construction, fiber distribution is random in three dimensions and the normal fiber failure mode is debonding and pullout. Thus, the critical fiber volume would be substantially greater than that obtained from Eq. 2-7. Naaman and Reinhardt (1995) derived an expression for the critical fiber volume fraction, taking into account the randomness of fiber distribution and the bond between fibers and matrix, $\tau$, as follows:

$$
V_{f}(\text { critical })=\frac{1}{1+\frac{\tau}{\sigma_{m u}} \frac{L_{f}}{D_{f}}\left(\lambda_{1} \lambda_{2} \lambda_{3}-\alpha_{1} \alpha_{2}\right)}
$$

where $\alpha_{1}$ and $\alpha_{2}$ are the fiber length and fiber orientation factors for the uncracked composite (similar to $\eta_{1}$ and $\eta_{2}$ ). Naaman and Reinhardt predicted a critical fiber volume fraction of $2 \%$ for SFRC using hooked steel fibers with an aspect ratio of 100 .

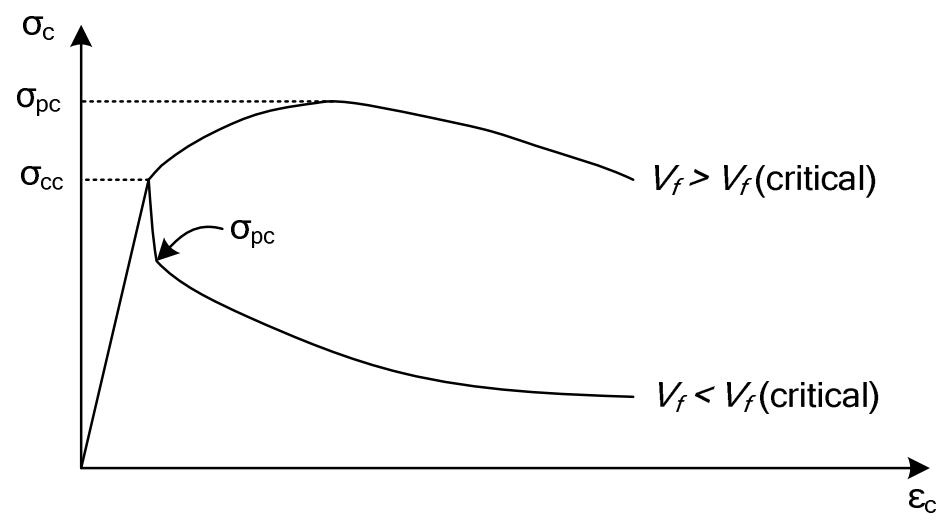

Fig. 2-6: Different stress-strain curves for brittle matrix composites 


\subsubsection{SFRC flexural tensile strength}

General discussion. ACI Committee 544 (1988) commented that two strength values should be reported from a flexural test. These are the first-crack flexural strength and the peak post-cracking flexural strength, which can be calculated using the assumption of a linear stress distribution. The first-crack flexural strength, if expressed in terms of the square root of the concrete compressive strength, is often referred to as the modulus of rupture, a quantity reported in the literature. Another important property that should be reported is the toughness, which is defined as the area underneath the loaddeflection curve from a bending test.

Test results. Shah and Rangan (1971) found that the first-crack flexural strength increased by less than $100 \%$ when concrete was reinforced with $1 \%$ by volume of straight fibers with a rectangular cross section. Wafa and Ashour (1992) found an increase of $67 \%$ in first-crack flexural strength when a $1.5 \%$ volume fraction of fibers with an aspect ratio of 80 was added to high strength concrete (13,500 psi). Song and Hwang (2004) reported that the increase in first-crack flexural strength was up to $127 \%$ when $2 \%$ (by volume) of hooked steel fibers with an aspect ratio of 64 were added to high strength concrete. Thomas and Ramaswamy (2007) found that adding hooked steel fibers with an aspect ratio of 60 in amounts ranging from 0.5 to $1.5 \%$ by volume to concrete with strength ranging from 5000 to 12,300 psi increased the first-crack flexural strength up to $40 \%$.

Ramakrishnan et al. (1980) found that hooked steel fibers were superior to straight fibers in terms of providing a higher ultimate flexural strength and flexural toughness. As seen in Fig. 2-7a, the use of hooked steel fibers in an amount greater than or equal to 80 pounds per cubic yard (approximately $0.6 \%$ by volume) led to a postcracking strength nearly equal to or greater than the first-crack strength. The superiority of hooked steel fibers compared with straight steel fibers was also confirmed by Soroushian and Bayasi (1991), as seen in Fig. 2-7b.

Khaloo and Kim (1996) found that adding the same type of fibers with the same fiber volume fraction resulted in a higher increase in modulus of rupture for normal- 
strength concrete $(4.5 \mathrm{ksi})$ than for mid- and high-strength concrete $(7.3$, and $12 \mathrm{ksi}$, respectively).

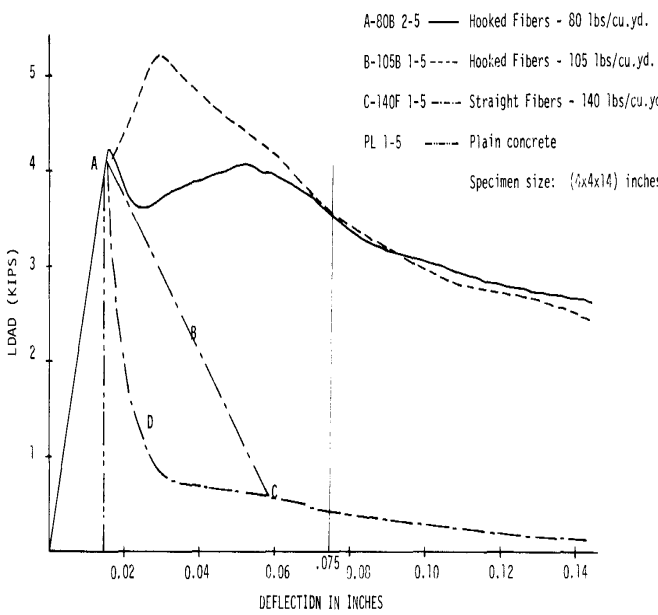

(a) from Ramakrishnan et al., 1980

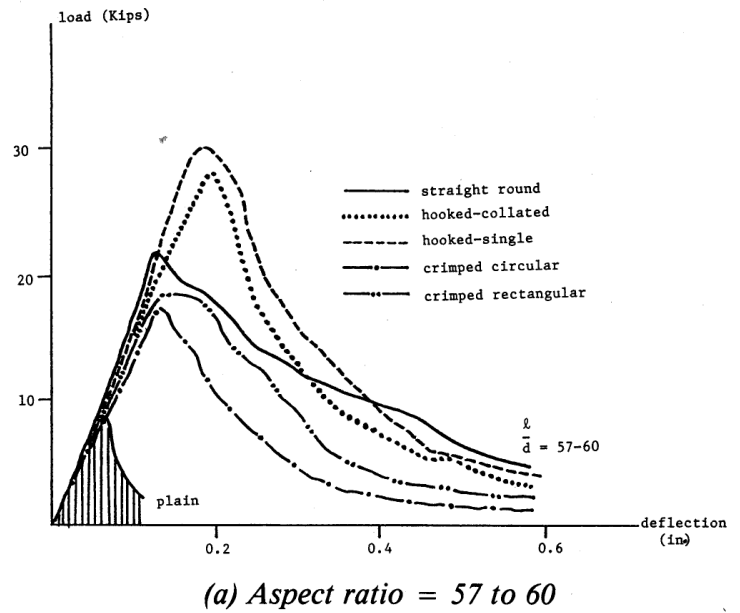

(b) from Soroushian and Bayasi, 1991

Fig. 2-7: Effect of hooked and straight steel fibers on flexural performance of concrete 


\subsubsection{SFRC compressive strength}

Test results. When added to concrete, steel fibers do not significantly increase the peak compressive strength (Ramakrishnan et al, 1980; Soroushian and Bayasi, 1991; Khaloo and Kim, 1996; and Thomas and Ramaswamy, 2007). Thomas and Ramaswamy (2007) reported that the increase in peak strength was less than $10 \%$ when fibers were added in volume fractions of up to $1.5 \%$. Khaloo and Kim (1996) reported a higher increase in compressive strength (37\%) for a fiber content of $1.5 \%$ by volume. With the presence of steel fibers, the strain at peak stress also increased slightly. Thomas and Ramaswamy (2007) reported that the increase in strain at peak stress was less than $30 \%$.

The key role of steel fibers is to reduce the rate of strength loss after the peak stress. Soroushian and Bayasi (1991) and Ramakrishnan et al. (1980) found that hooked and crimped steel fibers are more effective in enhancing the compressive post-peak behavior than straight steel fibers. The difference in the post-peak behavior of concrete with hooked and crimped steel fibers was found to be insignificant. Fig. 2-8 shows examples of compressive stress-strain curves with different types of fibers and fiber aspect ratios.

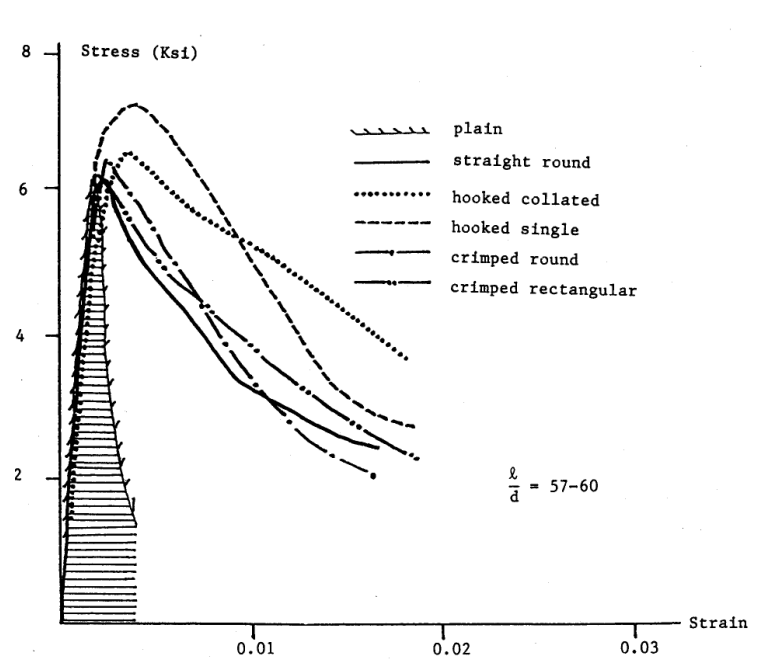

(a) Aspect ratio $=57$ to 60

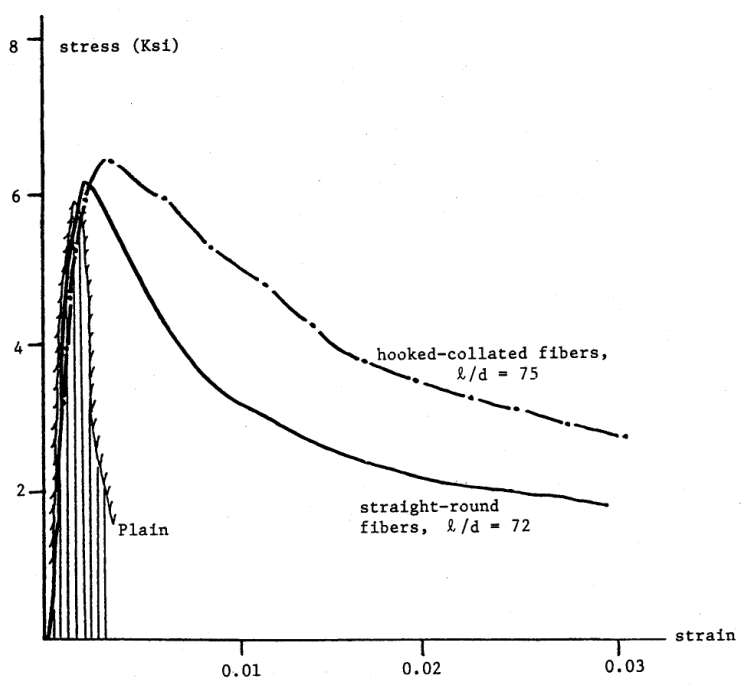

(b) Aspect ratio $=72$ to 75

Fig. 2-8: Examples of compressive stress-strain relationships with different fiber types and fiber aspect ratios (from Soroushian and Bayasi, 1991) 
Modeling of compressive stress-strain relationship. Fanella and Naaman (1985) proposed a normalized compressive stress versus strain model for SFRC that reflected the higher strain at peak stress and the modified unloading branch, as shown in Fig. 2-9. The constants were determined from the results of their experimental program. The increase in the peak strain was found to linearly increase with the fiber reinforcing index, a product of fiber volume fraction, and fiber aspect ratio. The researchers also found that an increase in fiber volume fraction and fiber aspect ratio led to a less steep descending branch.

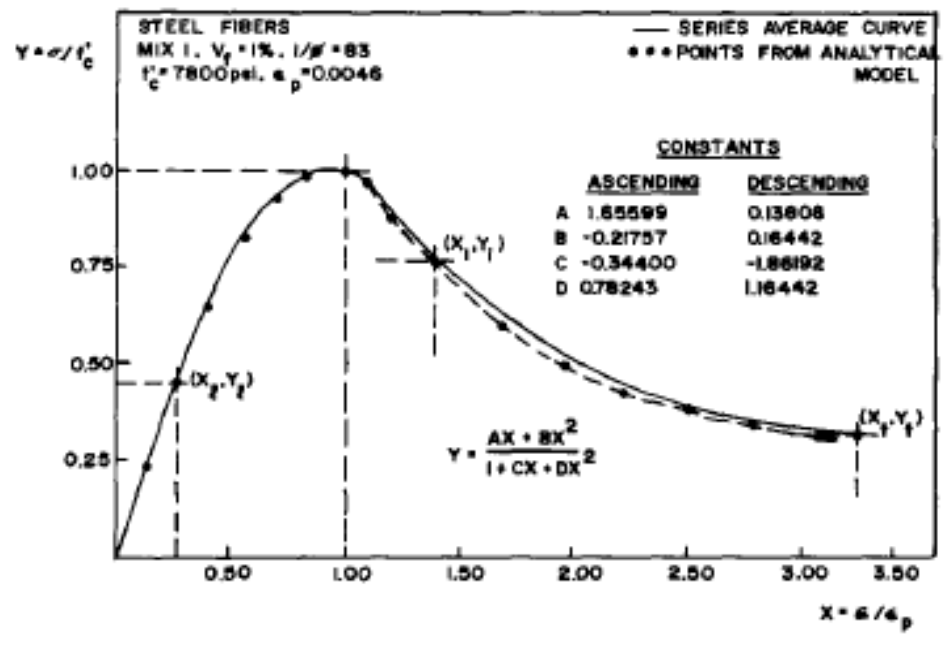

Fig. 2-9: Model of SFRC compressive stress-strain relationship (from Fanella and Naaman, 1985) 


\subsubsection{SFRC direct shear strength}

Valle and Büyüköztürk (1993) and Khaloo and Kim (1997) tested push-off concrete-based specimens reinforced with steel fibers (Fig. 2-10a). From the test results, the direct shear strength was found to vary from 10 to $15.6 \sqrt{f_{c}^{\prime}}$ (psi) for normal strength concrete. Based on the work on shear transfer in reinforced concrete by Hsu et al. (1987), Valle and Büyüköztürk (1993) derived a tri-linear shear transfer model for SFRC reinforced with steel bars as shown by Curve (b) in Fig. 2-10b, as a principal tensile stress-strain relationship.

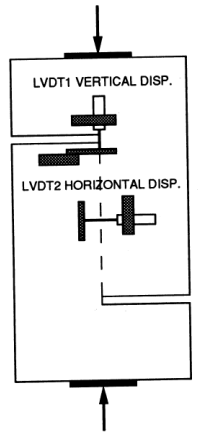

(a) Test setup

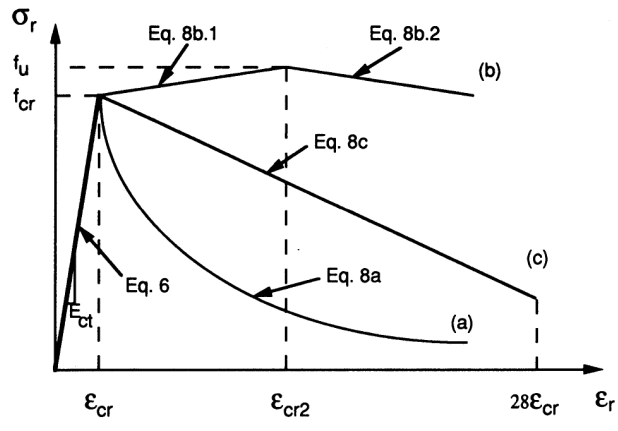

(b) Principal tensile stress-strain model

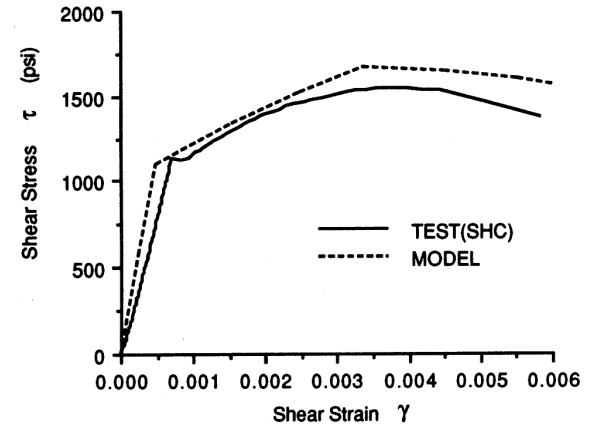

(c) Validation of the model

Fig. 2-10: Work of Valle and Büyüköztürk, 1993

Valle and Büyüköztürk assumed a cracking strength $f_{c r}=7.5 \sqrt{f_{c}^{\prime}}$ (psi) for normal strength concrete. The initial modulus, $E_{c t}$, adopts a form from the law of mixtures with modification factors $C, \eta_{\ell}$, and $\eta_{o}$ to take into account the biaxial stress effect, fiber length effect, and fiber orientation effect, respectively:

$$
E_{c t}=C\left(E_{m t} V_{m t}+E_{f} V_{f} \eta_{\ell} \eta_{o}\right)
$$

The second and third branches in Fig. 2-10b are described by the following equations:

$$
\begin{aligned}
& \sigma_{r}=f_{c r}+E_{f} V_{f} \eta_{\ell} \eta_{o}\left(\varepsilon_{r}-\varepsilon_{c r}\right) \\
& \sigma_{r}=f_{u}-E_{f} V_{f} \eta_{\ell} \eta_{o}\left(\varepsilon_{r}-\varepsilon_{c r 2}\right) .
\end{aligned}
$$

The strain at peak tensile strain takes the following form:

$$
\varepsilon_{c r 2}=\varepsilon_{c r}+\frac{\sigma_{s f u}}{E_{s f}} .
$$


where $\sigma_{s f u}$ and $E_{s f}$ are the ultimate steel fiber strength and modulus of elasticity, respectively. Fig. 2-10c shows the validation of the model.

\subsubsection{Bond between reinforcing bars and SFRC}

Experimental studies on this particular topic arrived at the following conclusions:

(1) Adding steel fibers to concrete improved significantly the bond strength between reinforcing bars and concrete, particularly if a splitting bond failure, rather than a pullout bond failure, occurred (Ezeldin and Balaguru, 1989 and Harajli et al., 1995). Ezeldin and Balaguru found that reinforcing bars with a large diameter greater than or equal to that of \#5 bars experienced splitting bond failure, while a pullout bond failure was observed for \#3 bars.

(2) Adding steel fibers to concrete also increased the bond slip at peak bond stress and enhanced the post-peak ductility (Ezeldin and Balaguru, 1989, Harajli et al., 1995, and Hota and Naaman, 1997).

(3) An increase in either fiber volume fraction or concrete compressive strength generally increased the bond strength between reinforcing bars and concrete (Ezeldin and Balaguru, 1989, Harajli et al., 1995, and Hota and Naaman, 1997). 


\subsection{SHEAR FAILURE MECHANISM AND TEST RESULTS OF SFRC BEAMS}

\subsubsection{Stress distribution and failure of plain concrete beams}

Consider a simply-supported, rectangular plain concrete beam (no stirrups and no longitudinal reinforcement) subjected to a concentrated load at midspan as shown in Fig. 2-11. Any section of the beam is subjected to moment and shear that cause normal stress and shear stress, respectively. From beam theory, the normal stress and shear stress, for $x \leq a$, are determined as follows:

$$
\begin{aligned}
& f(x, y)=-\frac{M y}{I}=-\frac{12 V x y}{b h^{3}} \\
& v(y)=\frac{V Q}{I b}=\frac{6 V}{b h^{3}}\left(\frac{h^{2}}{4}-y^{2}\right) .
\end{aligned}
$$

The distribution of normal and shear stresses is shown in Fig. 2-11. It can be seen that at the top or bottom surfaces, the beam is subjected to a pure compression or tension stress state. At a section located at a distance $x$ from the left support, the maximum tensile normal stress occurs at the bottom of the beam surface and is equal to:

$$
f_{\max }(x, y=-h / 2)=\frac{6 V x}{b h^{2}} .
$$

This maximum tensile stress causes vertical flexural cracks in the beam. For plain concrete beams, complete failure will immediately follow when a flexural crack occurs.

At the mid-depth level, the beam is subjected to a maximum shear stress, which is determined as follows:

$$
v_{\max }(y=0)=\frac{3 V}{2 b h}
$$

Since this is a pure shear stress state, the corresponding principal tension and compression stress have the same magnitude as that of the shear stress. The principal tension stress, often referred to as diagonal tension, is the cause of diagonal cracking at the mid-depth level of the beam. For plain concrete beams, however, flexural cracking will likely precede diagonal cracking and hence lead to immediate collapse of the beam. 


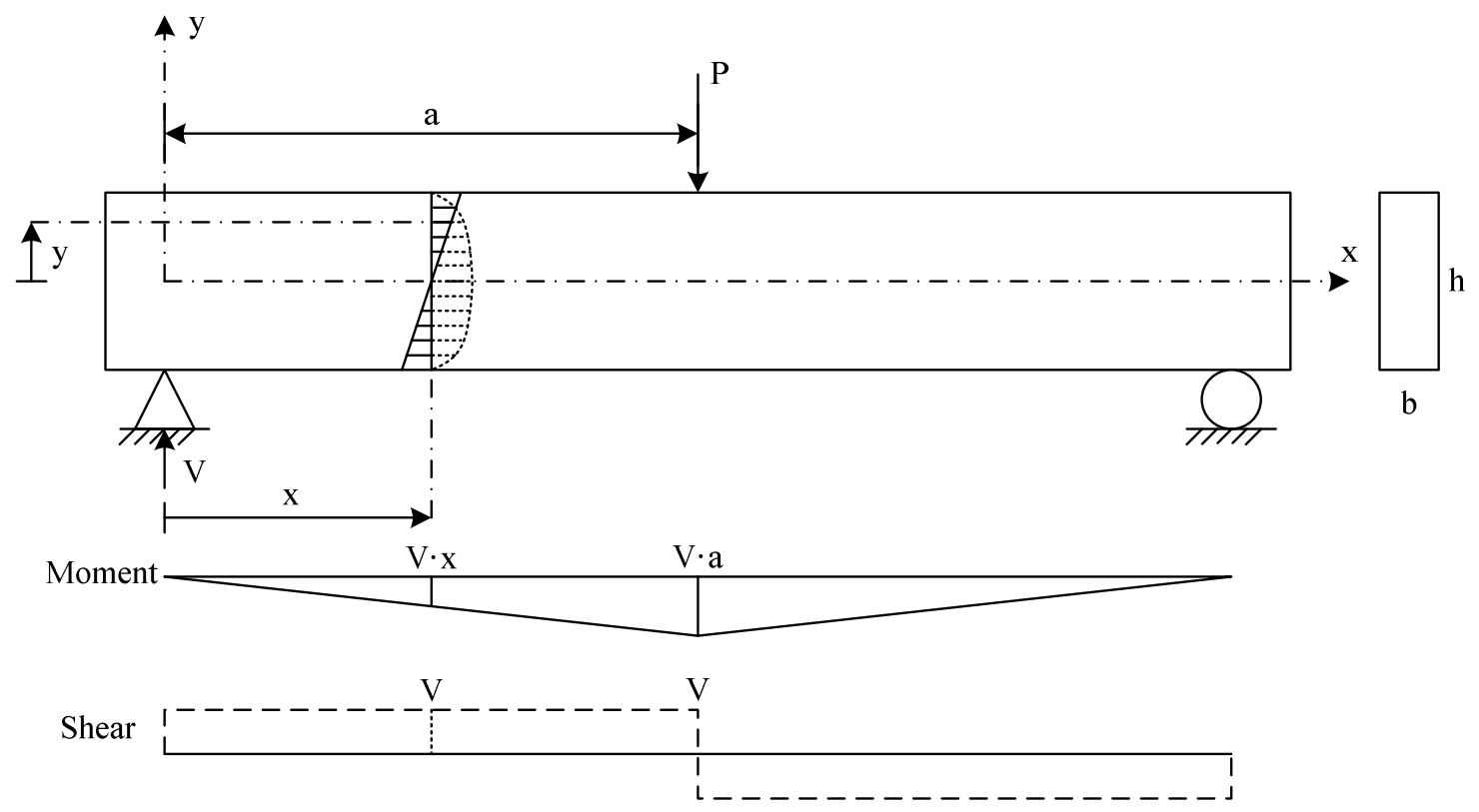

Fig. 2-11: Plain concrete beam subjected to a concentrated load

\subsubsection{Failure of RC beams without stirrup reinforcement}

When longitudinal reinforcement is added to the beam shown in Fig. 2-11, the beam flexural strength increases significantly. The beam will exhibit more flexural cracks due to the ability of the longitudinal reinforcement to bridge cracks and transfer the stress to the concrete. Unlike plain concrete beams, RC beams without shear reinforcement may fail due to shear action. Therefore, the shear span, which dictates the relative demand between moment and shear force, becomes an important factor affecting crack propagation, failure mechanism, and strength of the RC beam. Bond between concrete and reinforcement and the anchorage of reinforcement at the supports are also critical factors that affect the behavior of RC beams.

In general, an $\mathrm{RC}$ beam can fail due to either moment or shear action. If the beam is slender, the beam is likely to fail in flexure due to crushing of the compression region near the point of application of the concentrated load. Prior to crushing, the longitudinal reinforcement may or may not experience yielding, depending on the amount of reinforcement. If the reinforcement yields extensively, the beam will exhibit a ductile 
behavior and provide ample warning before failure occurs. If the amount of reinforcement is too small, on the other hand, the reinforcement may fracture before the concrete crushes.

For short or slender beams with a high tensile reinforcement ratio, the beam may fail due to the formation of diagonal cracks. These cracks may start at the beam midheight or from the top of flexural cracks. There are two possible scenarios after the formation of diagonal cracks. In the first scenario, the beam collapses immediately, caused by a widely-opened diagonal crack that splits the beam into two pieces. Before collapse, this diagonal crack is often not clearly evident. In the second scenario, the beam sustains additional loads after diagonal cracking before a failure occurs. The commonly observed failure mode for such a beam is a widely-opened diagonal crack, together with crushing of the compression region near the point of loading. These two scenarios represent shear failures. Fig. 2-12a shows an example of shear failure of an RC beam without stirrup reinforcement due to diagonal tension.

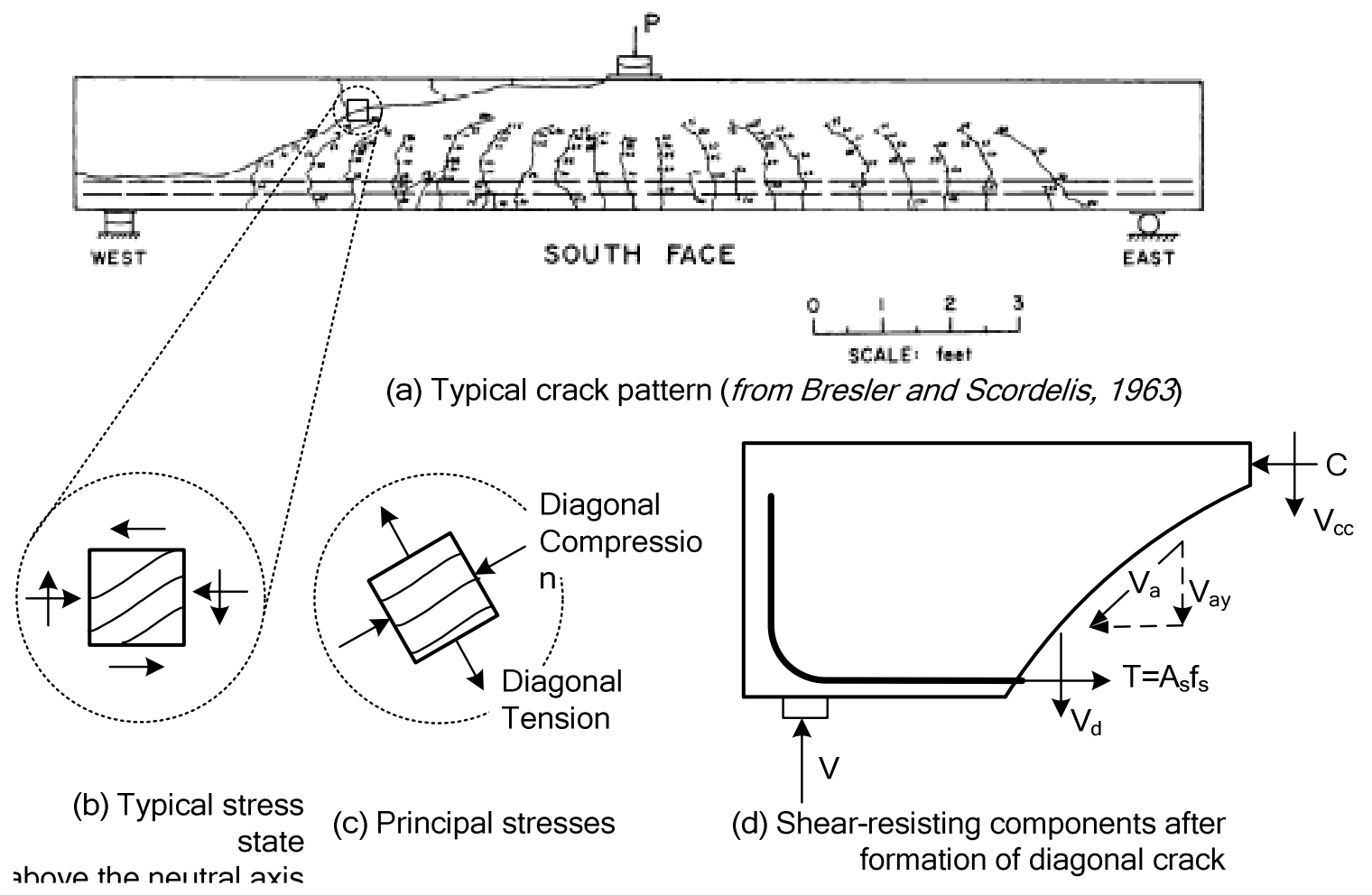

Fig. 2-12: Behavior of RC beam without stirrup reinforcement 
A widely-accepted explanation for diagonal cracking is diagonal tension. ACIASCE Committee 326 (1962) reported that this concept was first introduced by Ritter (1899). Diagonal tension, together with diagonal compression, occurs when shear is present (see Fig. 2-12b and Fig. 2-12c). When diagonal tension reaches the concrete tensile strength, diagonal cracks will form.

A diagonally-cracked beam may carry shear force through dowel action of the tensile reinforcement $\left(V_{d}\right)$, aggregate interlock $\left(V_{a y}\right)$, and shear carried in the compression region $\left(V_{c c}\right)$, as shown in Fig. 2-12d. Tensile reinforcement carrying shear as a dowel, a mechanism referred to as dowel action, may split the lower portion of the beam from the upper portion over a short length. Aggregate interlock comes from the fact that the crack surface is not smooth, allowing the two protruding portions of the beam to bear against each other and hence, resist shear (sliding). Shear resistance from aggregate interlock decreases as the width of the diagonal crack increases. The ability of the compression region to resist shear depends on the degree of penetration of the diagonal crack. Obviously, there is an interaction of the three shear-resisting components. If any of them fails, a redistribution of internal stresses occurs until all of them fail. Determination of a shear failure mechanism remains a challenging task. In experimental studies, researchers often describe different shear failure mechanisms based on crack patterns. Primary beam failure modes, defined by ASCE-ACI Committee 426 (1973), are shown in Fig. 2-13.

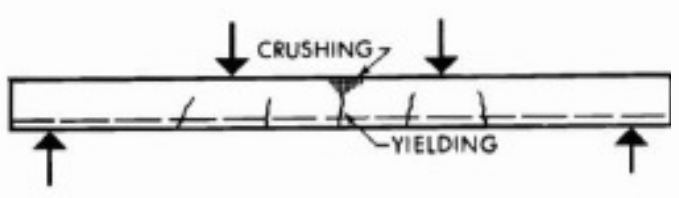

(a) Flexural failure

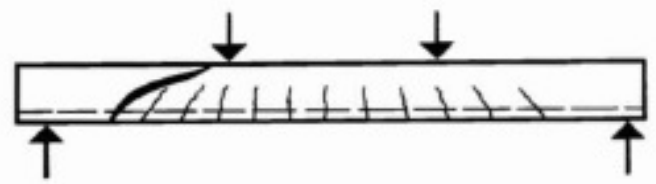

(c) Diagonal tension failure

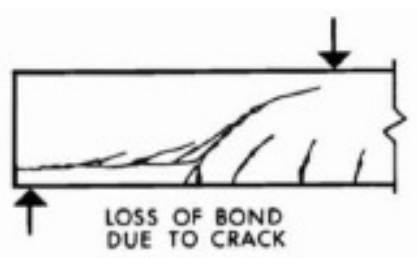

(b) Shear tension failure

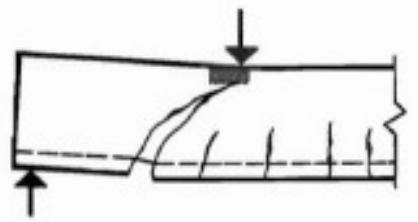

(d) Shear compression failure

Fig. 2-13: Beam failure modes (from ACI-ASCE Committee 426, 1973) 


\subsubsection{Roles of stirrup reinforcement in RC beams}

In an RC beam, stirrup reinforcement is only effective after the formation of diagonal cracks, as shown experimentally by Richart (1927) and Moretto (1945). The main role of the stirrup reinforcement is to carry the redistributed shear stresses, mainly through tension, after the formation of diagonal cracks. This tension is transferred back to the concrete, which may lead to the formation of additional diagonal cracks. Stirrup reinforcement also slows down the propagation of diagonal cracks, which helps the compression region sustain shear force. It is also effective in preventing a premature splitting failure along the tensile reinforcement. In addition, stirrup reinforcement controls crack opening, and hence, helps aggregate interlock resist shear. After the formation of diagonal cracks, there is an interaction among the four main contributors to shear resistance: tensile reinforcement through dowel action, concrete compression region, aggregate interlock, and stirrup reinforcement. Fig. 2-14 schematically shows these components and their relative contributions to beam shear strength at increasing levels of shear force.

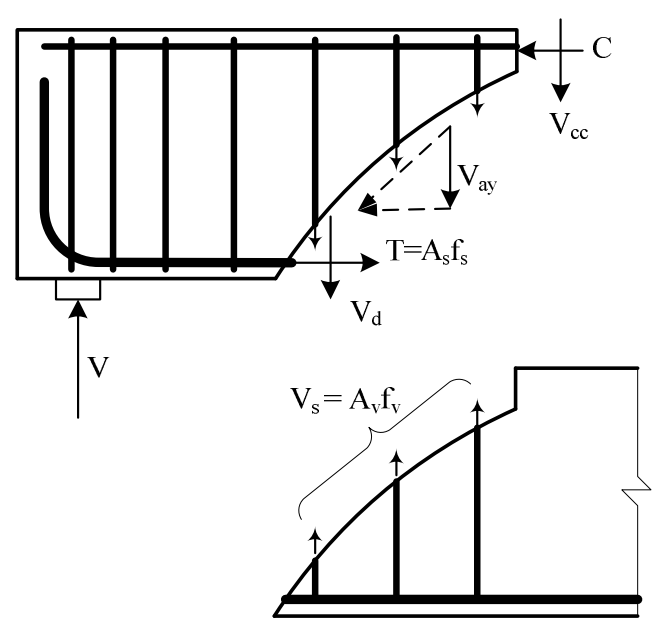

(a) Shear-resisting components after formation of diagonal crack

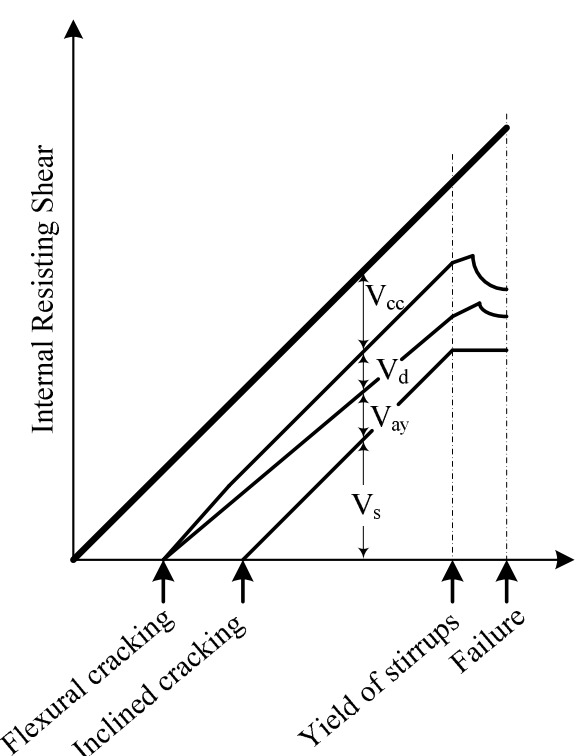

(b) Relative contribution from shearresisting components (from ACI-ASCE Committee 426, 1973)

Fig. 2-14: Shear resistance in RC beams with stirrup reinforcement 


\subsubsection{Failure of SFRC beams without stirrup reinforcement}

In a longitudinally-reinforced SFRC beam without stirrup reinforcement, steel fibers play a role similar to that of the stirrup reinforcement in an $\mathrm{RC}$ beam. For example, steel fibers carry redistributed tensile stress and delay the propagation and opening of diagonal cracks. They also prevent premature concrete splitting along the tensile reinforcement, just as stirrup reinforcement does. Steel fibers are effective in controlling crack width and fostering the formation of multiple diagonal cracks. This role is even more evident in beams made of strain-hardening, high-performance fiber reinforced concrete.

The shear strength analysis of SFRC beams without shear reinforcement presents several challenges. First, the distribution of fibers in the concrete is somewhat uncertain. The most important issue related to fiber reinforcement is the proper distribution of fibers to develop uniform mechanical properties. Second, the opening of wide diagonal cracks in SFRC beams is the result of fiber pullout instead of shear reinforcement yielding, as in the case of an RC beam. The pullout of fibers and bond between fibers and concrete is a complicated problem.

In general, the shear behavior of a simply-supported SFRC beam subjected to a concentrated and monotonic load is affected by the following parameters: (1) cross section shape, (2) beam size (particularly depth), (3) beam slenderness, (4) tensile reinforcement ratio, (5) SFRC compressive strength, (6) aggregate size, and (7) SFRC tensile strength and ductility.

If stirrup reinforcement is added to a reinforced SFRC beam, the problem is even more complicated. An interesting question is whether the shear strength of stirrup reinforcement, assuming yielding, can simply be superimposed to the contribution from the fiber reinforced concrete, as is typically done in shear design of RC beams. The addition of steel fibers to an RC beam with stirrup reinforcement should not change the role of stirrup reinforcement. However, the extent to which fibers contribute to resist shear could change due to the presence of stirrup reinforcement. 


\subsubsection{Tests of SFRC beams without stirrup reinforcement}

Listed in Table 2-3 are parameters investigated by previous research groups that studied the shear behavior of reinforced SFRC beams without stirrup reinforcement. The notations used in the table are defined at the end of the table.

Cross section. It can be seen that most research groups focused on rectangular sections. Only Swamy and Bahia (1985) and Rosenbusch and Teutsch (2002c) compared the shear behavior of rectangular beams with that of T-beams. The T-beams in the work of Swamy and Bahia had a flange thickness equal to one fifth of the beam depth and a flange width equal to three times the web width. Their test results showed a 30\% increase in ultimate shear strength compared to rectangular beams. Rosenbusch and Teutsch showed that a change in flange width (from 20 to 30, and to 40 inches) did not lead to a significant change in load versus deflection response. Their beam with a flange thickness of 9 inches showed a $54 \%$ increase in ultimate strength when compared to the rectangular beam of the same height (20 inches). The beams with a flange thickness of either 4 inches or 6 inches behaved similarly to the rectangular beam with the same height. 
Table 2-3: Previous researches on SFRC beams without stirrup reinforcement

\begin{tabular}{|c|c|c|c|c|c|c|c|c|}
\hline Research group & Type of sections & $\begin{array}{c}d \\
\text { (in.) }\end{array}$ & $a / d$ & $\begin{array}{c}\rho \\
(\%)\end{array}$ & $f_{c}^{\prime}(\mathrm{ksi})$ & Type of fibers & $L_{f} / D_{f}$ & $V_{f}(\%)$ \\
\hline Batson et al. (1972) & $\mathrm{R}$ & 5 & $1.2-5^{*}$ & $3^{*}$ & & $\mathrm{~S}, \mathrm{C}^{*}$ & - & $0.22-2.66^{*}$ \\
\hline Williamson and Knab (1975) & $\mathrm{R}$ & 18 & 4.7 & 2.54 & $4.11-4.66$ & - & - & 1.5 \\
\hline Swamy and Bahia (1985) & $\mathrm{R}, \mathrm{T}^{*}$ & 8.3 & 4.5 & $1.95-4^{*}$ & $5.1-6.24$ & $\mathrm{C}$ & 100 & $0.4-1.2^{*}$ \\
\hline Mansur et al. (1986) & $\mathrm{R}$ & 7.8 & $2-4.4^{*}$ & $0.79-2 *$ & $2.4-3.9^{*}$ & $\mathrm{H}$ & 60 & $0.5-1^{*}$ \\
\hline Murty and Venkatacharyulu (1987) & $\mathrm{R}$ & 7.1 & $2,3^{*}$ & 1.25 & $2.6-3.8$ & $\mathrm{~S}$ & $50,100^{*}$ & $0.5,1,1.5^{*}$ \\
\hline Lim et al. (1987) & $\mathrm{R}$ & 8.7 & $1.5,2.5,3.5^{*}$ & $1.1,2.2 *$ & 4.93 & $\mathrm{H}$ & 60 & $0.5,1^{*}$ \\
\hline Narayanan and Darwish (1987) & $\mathrm{R}$ & 5.1 & $2,2.5,3^{*}$ & $2,3.69,5.7 * 2$ & $4.3-9.2 *$ & $\mathrm{~S}$ & $100,133^{*}$ & $0.25-3^{*}$ \\
\hline Narayanan and Darwish (1988) & $\mathrm{R}$ & 13.6 & $0.5-0.8^{*}$ & 3.55 & $4.4-7.9^{*}$ & S & 100 & $0.25-1.25^{*}$ \\
\hline Li et al. (1992) & $\mathrm{R}$ & $\begin{array}{ll}4 \\
3\end{array}$ & $1-3^{*}$ & $1.1,2.2,3.3^{*}$ & $3.3-8.3$ & $\mathrm{H}$ & 60,100 & $1,2^{*}$ \\
\hline Ashour et al. (1992) & $\mathrm{R}$ & 8.5 & $2,4,6^{*}$ & $0.374-4.58$ & $13.4-14.7$ & $\mathrm{H}$ & 75 & $0.5,1,1.5^{*}$ \\
\hline Schantz (1993) & $\mathrm{R}$ & 21.5 & 2.8 & 1.84 & $6.1-7.1$ & C & 80 & $0.5,1,1.5^{*}$ \\
\hline Swamy et al. (1993) & I & 10.4 & $2,3.43,4.91^{*}$ & $1.55,2.76,4.31^{*}$ & $4.7-5.9$ & $\mathrm{C}$ & 100 & 1 \\
\hline Tan et al. (1993) & I & 13.4 & $1.5,2,2.5^{*}$ & 3.44 & $4.8-5.2$ & $\mathrm{H}$ & 60 & $0.5,0.71 *$ \\
\hline Shin et al. (1994) & $\mathrm{R}$ & 6.9 & $2,3,4.5,6^{*}$ & $3.59,7.18^{*}$ & 11.6 & S & 100 & $0.5,1^{*}$ \\
\hline Adebar et al. (1997) & $\mathrm{R}$ & 21.9 & 1.64 & 1.64 & $5.9-8.7$ & $\mathrm{H}$ & 60,100 & $0.4-1.5^{*}$ \\
\hline Casanova and Rossi (1999) & $\mathrm{R}$ & 8.9 & 2.89 & $2.21-3.57$ & 13.1 & $\mathrm{H}$ & 60 & \\
\hline Noghabai (2000) & $\mathrm{R}$ & $9.8,27.6^{*}$ & $2.8,2.9,3,3.3$ & $2.9-4.5$ & & $\mathrm{~S}, \mathrm{H}^{*}$ & $40,86^{*}$ & $0.5,0.75,1^{*}$ \\
\hline Kwak et al. (2002) & $\mathrm{R}$ & 8.4 & $2,3,4^{*}$ & 1.48 & $4.5-10^{*}$ & $\mathrm{H}$ & 63 & $0.5,0.75^{*}$ \\
\hline Rosenbusch and Teutsch (2002a) & $\mathrm{R}$ & 10.2 & 3.46 & 3.56 & $6.4-7$ & $\mathrm{H}$ & 68 & $0.25,0.51,0.76^{*}$ \\
\hline Rosenbusch and Teutsch (2002b) & $\mathrm{R}$ & 10.2 & $1.5,2.5,4^{*}$ & $1.15-3.56$ & $5.6-6$ & H & 68 & $0.25,0.76^{*}$ \\
\hline Rosenbusch and Teutsch (2002c) & $\mathrm{R}, \mathrm{T}^{*}$ & $11.8,18.1,21.3^{*}$ & 3.5 & $2.4-2.83$ & $4.7-5.6$ & $\mathrm{H}$ & 68 & \\
\hline Cho and Kim (2003) & $\mathrm{R}$ & 6.6 & 1.43 & $2,2.82 *$ & $9.8-13^{*}$ & $\mathrm{H}$ & 60 & $0.5,1,1.5,2^{*}$ \\
\hline Cucchiara et al. (2004) & $\mathrm{R}$ & 8.6 & $2,2.8^{*}$ & 1.9 & $5.9-6.3$ & $\mathrm{H}$ & 60 & $1,2 *$ \\
\hline Kim et al. (2004) & $\mathrm{R}$ & 8.7 & $1.8,2.5,3.5,4^{*}$ & $0.75-4.14 *$ & $17.5-19.4$ & $\mathrm{H}$ & - & $1,2 *$ \\
\hline Han et al. (2004) & $\mathrm{R}$ & $12.4,15.6$ & $4,6,8^{*}$ & $2,3.5,4,5^{*}$ & $16.7-17.6$ & $\mathrm{H}$ & - & $1,2,3^{*}$ \\
\hline Entire Range & $\mathrm{R}, \mathrm{T}, \mathrm{I}$ & $4-27.6$ & $0.5-8$ & $0.37-7.18$ & $2.4 \div 19.4$ & $\mathrm{~S}, \mathrm{C}, \mathrm{H}$ & $10-133$ & $0.22-3^{*}$ \\
\hline Remarks & $\begin{array}{l}90 \% \text { with } \\
\text { rectangular sections }\end{array}$ & $\begin{array}{l}85 \% \text { with } \\
d<11 \text { in. }\end{array}$ & $\begin{array}{l}\text { mostly with } \\
\text { a/d }>2.5\end{array}$ & $\begin{array}{l}3 \% \text { with } \\
\rho<1 \%\end{array}$ & $\begin{array}{l}60 \% \text { with } \\
f_{c}^{\prime} \leq 6 \mathrm{ksi}\end{array}$ & $\begin{array}{l}35 \% \text { with } \\
\text { hooked fibers }\end{array}$ & & \\
\hline
\end{tabular}

Notes: (1) An asterisk indicates the studied parameters.

(2) R, T, and I: rectangular, T-, and I- section, respectively; $d$ : section effective depth; $a$ : shear span; $\rho$ : tensile reinforcement ratio; $f_{c}^{\prime}:$ SFRC compressive strength

$\mathrm{S}, \mathrm{C}$, and $\mathrm{H}$ : straight, crimped, and hooked fibers, respectively; $L_{f}$ : fiber length; $D_{f}$ : fiber diameter; $V_{f}$ : fiber volume fraction. 
Effective depth. Approximately $85 \%$ of beams tested found in the literature review had an effective depth less than 11 inches (see Table 2-3). Only few research programs dealt with more realistic beam depths, as listed in Table 2-4.

Table 2-4: Research programs that dealt with practical beam depths

\begin{tabular}{|c|c|c|}
\hline Research Group & Number of beams & Effective depth (inches) \\
\hline Williamson and Knab (1975) & 3 & 18 \\
Schanzt (1993) & 4 & 21.5 \\
Adebar et al. (1997) & 8 & 21.9 \\
Noghabai (2000) & 9 & 16.1 \\
& 3 & 22.4 \\
Rosenbusch and Teutsch (2002c) & 9 & 18.1 \\
\hline
\end{tabular}

Therefore, there is a need for more data for large-size beams, especially those with an effective depth larger than 20 inches. Additionally, testing beams of a larger size, along with those of smaller sizes, would provide further experimental validation on the belief that the shear "size effect" is not significant in SFRC beams. In the study of Noghabai (2000), an increase in beam effective depth from 16.1 to 22.4 inches, while everything else was kept the same, resulted in a $15 \%$ decrease in the average shear stress at failure (see Beams 7, 8 type C and Beam 4, type D).

Shear span-to-effective depth ratio. The effect of shear span-to-effective depth ratio on the shear behavior of SFRC beams has been extensively studied by various researchers (Table 2-3). A general conclusion is that beams with a smaller span-to-depth ratio can resist more shear. This is due to arch action, which is basically the direct transfer of the load to the support through a compressive strut. To distinguish between short and slender beams, Batson et al. (1972) proposed a critical value of shear span-toeffective depth ratio of 3 for SFRC beams, similar to 2.5 for RC beams, as proposed by Zsutty (1968). Shown in Fig. 2-15 is the effect of shear span-to-effective depth ratio from results reported by Mansur et al. (1986), Ashour et al. (1993), Swamy et al. (1993), Shin et al. (1994), and Kwak et al. (2002). In the figure, $f_{y f}$ and $f_{u f}$ are the fiber yield and ultimate tensile strengths, respectively. It can be seen that the beam shear strength increases rapidly when the shear span-to-effective depth ratio is less than 2.0. 

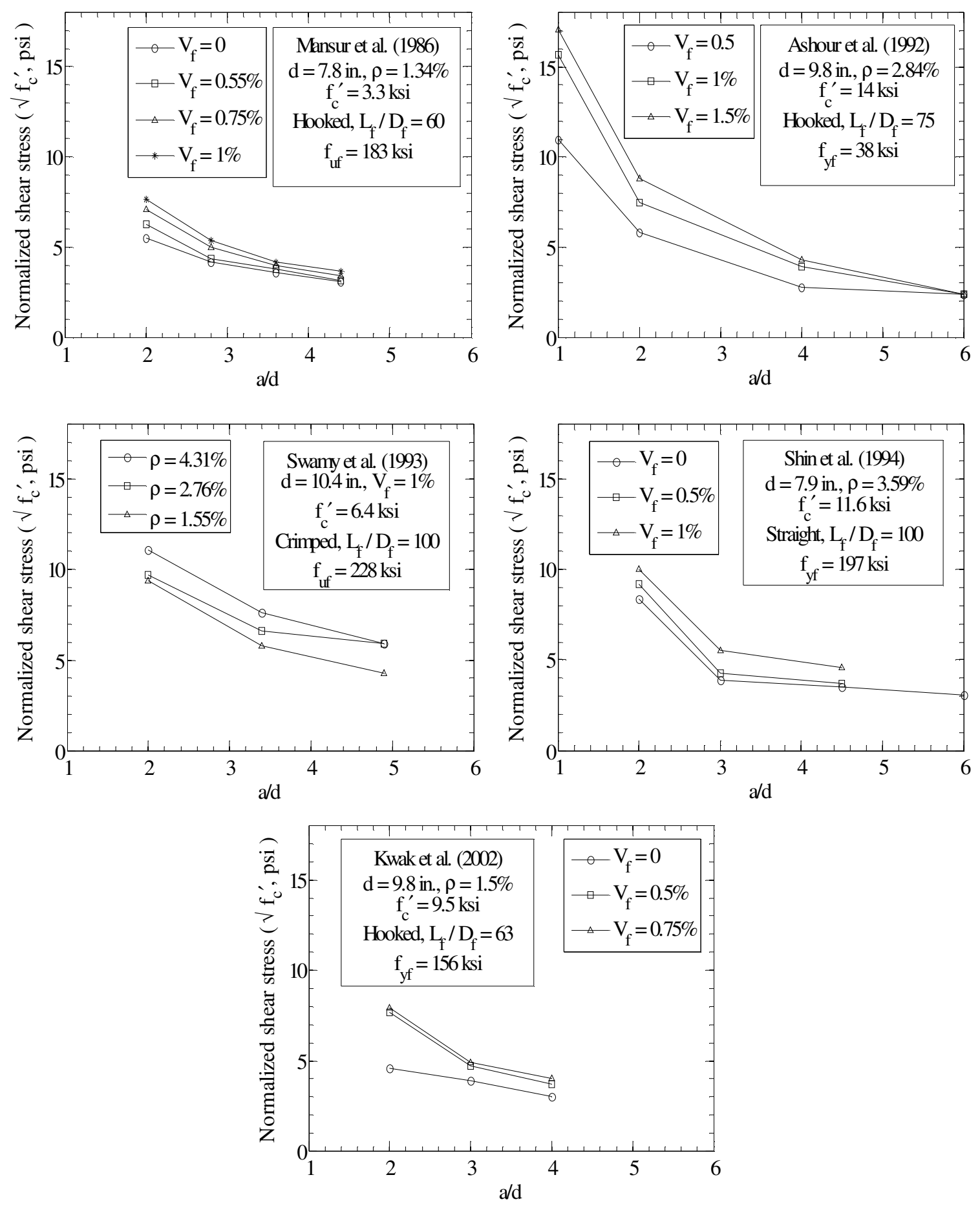

Fig. 2-15: Effect of shear span-to-effective depth ratio on SFRC beam strength from previous investigations

Longitudinal reinforcement ratio. The effect of longitudinal tensile reinforcement ratio on beam shear strength has also been extensively investigated. It is 
generally concluded that a higher ratio of tensile reinforcement results in a higher shear stress at failure because of increased dowel action and a deeper compression zone (Ashour et al., 1992 and Swamy et al., 1993).

SFRC compressive strength. The effect of SFRC compressive strength on the shear strength of reinforced SFRC beams was evaluated in at least five studies, as seen in Table 2-3. Generally, an increase in SFRC compressive strength leads to an increase in beam shear strength. For example, when the concrete strength was doubled while everything else was kept the same, the shear strength increased $20 \%$ for slender beams (Kwak et al., 2002).

Aggregate size. With regard to the effect of aggregate size, it is commonly believed that beams made of concrete containing greater aggregate sizes have higher shear resistance due to increased aggregate interlock. Therefore, most researchers choose small aggregate sizes, very often $3 / 8$ inches, to be on the safe side. However, in reinforced SFRC beams, this might not necessarily be conservative because smaller aggregate sizes tend to cause less disturbance to the bond between fibers and the concrete matrix and thus, may lead to an increase in shear strength. To date, the effect of aggregate size on the shear strength of reinforced SFRC beam has not been adequately studied.

Fiber volume fraction. The effect of using steel fibers in the concrete is typically studied by varying fiber volume fraction and the type of fiber, which includes changing the fiber aspect ratio, fiber length, and fiber strength. The effect of fiber volume fraction has been intensively investigated, as indicated in Table 2-3. However, the improvement in shear strength due to the increase in volume fraction depends heavily on other factors. Fig. 2-15 shows some examples of such increases. 


\subsection{PREDICTION OF SHEAR STRENGTH OF SFRC BEAMS}

Because the shear failure of SFRC beams is complicated, most of the predictive methods rely on a regression analysis. Factors considered to affect shear strength are decided based on a rational evaluation of the shear failure mechanism. The primary controlling factors are the shear span-to-effective depth ratio, longitudinal reinforcement ratio, and other factors that affect tensile strength of fiber reinforced concrete, which include fiber aspect ratio, fiber geometry, fiber content, fiber tensile strength, and concrete matrix properties.

Sharma (1986). Sharma proposed an empirical expression to predict ultimate shear strength based on the splitting tensile strength, $f_{c t}$, and shear span-to-effective depth ratio, $a / d$.

$$
v_{u}=\left(k f_{c t}\right)\left(\frac{d}{a}\right)^{1 / 4}
$$

where $k=2 / 3$. The product, $k f_{c t}$, could be understood as the direct tensile strength because Sharma followed the work of Wright (1955), who found out that the direct tensile stress is in the order of $2 / 3$ of the splitting tensile strength. The expression is therefore not related to contribution of fibers. Moreover, the role of reinforcement is also not introduced as pointed out by Kwak et al. (2002). In the absence of $f_{c t}$, Sharma used the following relation recommended by the European Concrete Committee (Comite EuroInternational du Béton, 1993):

$$
f_{c t}=9.5 \sqrt{f_{c}^{\prime}} \quad(\mathrm{psi})
$$

The expression was validated against 41 beams and showed reasonable estimates.

Narayanan and Darwish (1987). These researchers considered the shear contribution of fiber reinforced concrete through the splitting tensile strength, $f_{c t}$, dowel action (as a function of longitudinal reinforcement ratio, $\rho$ ), shear span-to-effective depth ratio, $a / d$, and "fiber pullout forces along the inclined crack" through a term $v_{b}$, as follows: 


$$
v_{u}=e A^{\prime} f_{c t}+e B^{\prime} \rho \frac{d}{a}+v_{b}(\mathrm{MPa}) .
$$

The coefficient $e$ considers the arch/beam action effect and is approximately 1 for slender beams $(a / d>2.8)$ and is $2.8 d / a$ for shorter beams $(a / d \leq 2.8)$. Based on regression analysis, the researchers proposed the following formula for computing $f_{c t}$ from cube compressive strength, $f_{\text {cuf }}$, and fiber factor, $F$ :

$$
f_{c t}=\frac{f_{c u f}}{20-\sqrt{F}}+0.7+\sqrt{F}(\mathrm{MPa}),
$$

where

$$
F=V_{f} \frac{L_{f}}{D_{f}} \beta .
$$

The bond factor $\beta$, adopted from the work of Narayanan and Kareem-Palanjian (1984), is approximately $0.5,0.75$, and 1 for round, crimped, and indented fibers, respectively. $A^{\prime}$ and $B^{\prime}$ were determined based on a regression analysis of 91 tests, which led to $A^{\prime}=0.24$ and $B^{\prime}=80 \mathrm{MPa}$. The $v_{b}$ term is based on the bond stress of all fibers crossing a 45-degree diagonal. The fiber bond stress, $\tau$, more precisely "average fiber matrix interfacial bond stress", was assumed to exist along $1 / 4$ of the fiber length. The number of fibers over a unit area, $n_{w}$, was adopted from the work of Romualdi et al. (1964) as follows:

$$
n_{w}=\frac{1.64 V_{f}}{\pi D_{f}^{2}}
$$

The number of fibers is counted on a diagonal crack that has a vertical projection equal to the distance between the center of reinforcement to the lower tip of the compression region. With all those definitions, the authors derived the formula for $v_{b}$ as follows:

$$
v_{b}=0.41 \tau V_{f} \frac{L_{f}}{D_{f}}
$$

To account for the effect of fiber geometry on bond, they added the bond factor $\beta$ to Eq. (2-23): 


$$
v_{b}=0.41 \tau V_{f} \frac{L_{f}}{D_{f}} \beta=0.41 \tau F .
$$

The bond stress is equal to 4.15 MPa, as proposed by Swamy et al. (1974).

In this model, the contribution of shear across the compression region was not specified. The authors also neglected the contribution of aggregate interlock. Regardless of this, their formula was reported to be conservatively acceptable.

Ashour, Hasanain, and Wafa (1992). These researchers modified one of the ACI Committee 318 equations on shear by applying factors determined from a regression analysis to the parameters $\sqrt{f_{c}^{\prime}}$ and $\rho \frac{d}{a}$ :

$$
v_{u}=0.7 \frac{d}{a} \sqrt{f_{c}^{\prime}}+7 \frac{d}{a} F+17.2 \rho \frac{d}{a} \quad(\mathrm{MPa}) .
$$

They also modified the equation proposed by Zsutty (1968) by introducing the fiber factor $F$ as follows:

$$
\begin{aligned}
v_{u} & =\left(2.11 \sqrt[3]{f_{c}{ }^{\prime}}+7 F\right)\left(\rho \frac{d}{a}\right)^{0.333}(\mathrm{MPa}) \quad \text { for } a / d>2.5 \\
v_{u}=\left[\left(2.11 \sqrt[3]{f_{c}{ }^{\prime}}+7 F\right)\left(\rho \frac{d}{a}\right)^{0.333}\right]\left(2.5 \frac{d}{a}\right)+v_{b}\left(2.5-\frac{a}{d}\right) \quad(\mathrm{MPa}) & \text { for } a / d<2.5
\end{aligned}
$$

In the latter formula, these researchers took into account the supplementary "shear strength of fiber" along the shear crack, $v_{b}$. The coefficient $\left(2.5-\frac{a}{d}\right)$ indicates that the last term only applies to short beams. The two modified approaches were reported to give good estimations of shear strength of their tested beams, except for those with low reinforcement ratios $(0.37 \%)$.

The usage of these formulae should be restrained to beams that have similar parameters $\left(a / d, \rho\right.$, and $\left.f_{c}^{\prime}\right)$ because they were only validated against the accompanying experiments. 
Mansur, Ong, and Paramasivam (1986). These researchers used the equation for shear strength at diagonal cracking for an RC beam without stirrups proposed by ACIASCE Committee 426 (1973) in order to account for the contribution of concrete. The contribution of steel fibers was based on the post-cracking tensile strength, $\sigma_{p c}$, obtained from direct tensile tests of dog-bone specimens. In the absence of test data, they suggested using $\sigma_{p c}$ as proposed by Swamy and Al-Taan (1981). This stress was assumed to be uniformly distributed over the critical diagonal crack. They assumed that the horizontal projection of this critical crack was equal to the beam effective depth. Therefore, the total shear strength has the following form:

$$
v_{c}=0.167 \sqrt{f_{c}^{\prime}}+17.2 \frac{\rho V d}{M}+\sigma_{p c} \quad(\mathrm{MPa})
$$

In this equation, the critical $M / V$ ratio is defined as:

$$
\begin{aligned}
& \frac{M}{V}=\frac{M_{\max }}{V}-\frac{a}{2} \text { for } a / d \leq 2 \\
& \frac{M}{V}=\frac{M_{\max }}{V}-d \text { for } a / d>2
\end{aligned}
$$

The equation was reported to give a good prediction of the shear strength for their beams. It overestimated the shear strength of beams with larger $a / d$ values and underestimated the strength of those with smaller $a / d$ values.

In general, the equation is attractive because the formulation is based on simple reasoning. The direct tensile test also gives the most fundamental tensile strength of fiber reinforced concrete. Unfortunately, research in this area is still immature.

Al-Ta'an and Al-Feel (1990). Al-Ta' an and Al-Feel proposed a method based on a combination of shear-resisting mechanism. The contribution of the compression region, aggregate interlock, and dowel action has the following form, which is adopted from the work of Zsutty (1971) and Placas and Regan (1971):

$$
\begin{aligned}
& v_{c}=\left(10 \rho f_{c}^{\prime} \frac{d}{a}\right)^{1 / 3} \text { for } a / d>2.5 \\
& v_{c}=\left(160 \rho f_{c}^{\prime}\right)^{1 / 3}\left(\frac{d}{a}\right)^{4 / 3} \text { for } a / d<2.5
\end{aligned}
$$


The contribution of fiber is taken into account by considering a post-cracking tensile stress along the diagonal crack, similar to what Narayanan \& Darwish (1987) and Mansur et al. (1986). However, they excluded the depth of the compression region from the crack height. The depth of the compression region is calculated by equating the external moment to the nominal moment, as follows:

$$
\begin{aligned}
& M_{u}=M_{n} \\
& M_{u}=V_{u} a \\
& M_{n}=0.85 f_{c}^{\prime}(\beta c) b\left(d-\frac{\beta c}{2}\right)+A_{s}^{\prime} f_{y}\left(d-d^{\prime}\right) .
\end{aligned}
$$

The number of fibers crossing a unit area follows the proposal of Aveston et al. (1974):

$$
n_{w}=\frac{2 V_{f}}{\pi D_{f}^{2}}
$$

This leads to a post-cracking strength $\sigma_{p c}$ as follows:

$$
\sigma_{p c}=0.5 \tau F
$$

If the depth of the compression region is $c$, the vertical component of the pullout force is:

$$
V_{f p}=\sigma_{p c} b(h-c) .
$$

The method was found to give an "acceptable results" compared to experimental data.

Khuntia, Stojadinovic and Goel (1999). These researchers proposed an expression that takes into account the fiber factor, which has the form of Eq. 2-21. The contribution of the compression region, aggregate interlock, and dowel action are lumped into a single lower bound term, as stipulated by ACI Committee 318, i.e.:

$$
v_{c}=0.167 \sqrt{f_{c}^{\prime}}(\mathrm{MPa})
$$

The contribution of fibers is based on the post-cracking tensile strength, which is taken equivalent to $0.41 \tau F$. By assuming a bond stress of $\tau=0.68 \sqrt{f_{c}^{\prime}}$ and a vertical projection of the diagonal crack equal to $0.9 d$, the shear contribution of the fibers becomes:

$$
v_{f r}=0.41 \times 0.68 \sqrt{f_{c}^{\prime}} \times F \times 0.9=0.25 F \sqrt{f_{c}^{\prime}}(\mathrm{MPa})
$$


By multiplying the arch action factor $\alpha$, equal to $2.5 \mathrm{~d} / a$, to the term $v_{c}$, the total shear strength becomes:

$$
v_{u}=(0.167 \alpha+0.25 F) \sqrt{f_{c}^{\prime}}(\mathrm{MPa})
$$

The expression was validated against tested beams with a wide range of variables and was shown to be conservative. 


\subsection{SHEAR THEORY OF RC BEAMS}

The distribution of shear stress on the cross section of an RC beam subjected to shear is uncertain. However, it is generally accepted that shear strength in beams is provided by reinforcement through dowel action, aggregate interlock, and the concrete compression region. Of these components, dowel action is considered to be minor compared to the other two, particularly in beams with a low flexural reinforcement ratio. Over the years, two schools of thought have clearly emerged. The first school emphasizes the importance of aggregate interlock, while the second assigns a dominant role to the concrete compression region. Researchers in the first group include Mitchell and Collins (1974), Nielsen (1984), Vecchio and Collins (1986), Marti (1986), and Berlabi and Hsu (1995). Those from the second group, including Zwoyer and Siess (1954), Bresler and Pister (1958), Kani (1964), and Frosch (2003), assume that shear resistance comes primarily from the shear stresses in the concrete compression region. The next section summarizes important concepts from each school of thought.

\subsubsection{Shear strength models based on the contribution of the compression region}

Work of Bresler and Pister (1958). Bresler and Pister (1958) used the concept of average shear stress and average normal stress in the compression region to calculate the ultimate shear strength of RC beams. The average shear stress $\bar{\tau}$ and average normal stress $\overline{\boldsymbol{\sigma}}$ are defined as follows:

$$
\begin{gathered}
\bar{\tau}=\frac{V_{u}}{A_{e}} \\
\bar{\sigma}=\frac{C}{A_{e}}
\end{gathered}
$$

where $V_{u}$ is the ultimate shear strength of the section; $C$ is the compressive force acting on the compression region; and $A_{e}$ is the effective area of the compression region resisting shear, defined by $(k b) d$ (see Fig. 2-16). 


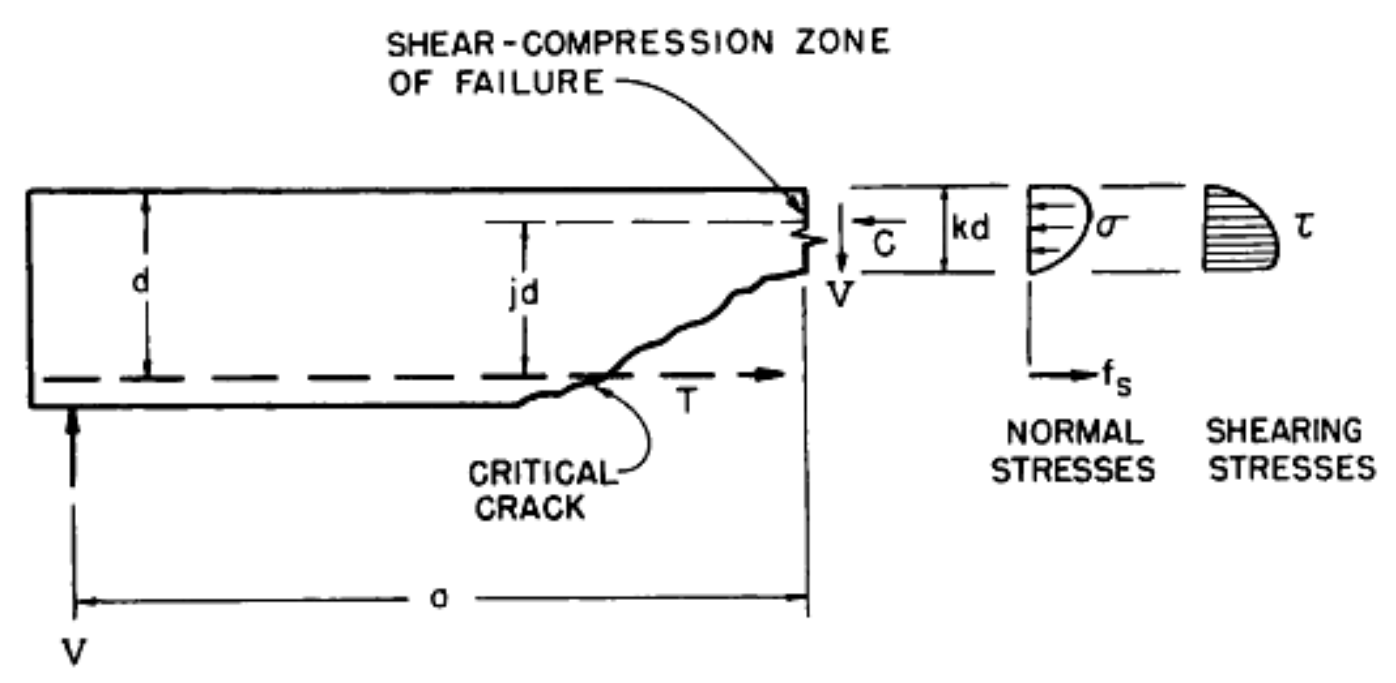

Fig. 2-16: Stress distribution in RC beams (from Bresler and Pister, 1958)

By equilibrium, the compressive force $C$ equals the tensile force $T$ in the reinforcement, which is assumed to yield at ultimate strength. A compressive stress at failure, based on the work of Hognestad et al. (1955), was assumed as follows:

$$
\bar{\sigma}=\frac{3900+0.35 f_{c}^{\prime}}{3200+f_{c}^{\prime}} f_{c}^{\prime}
$$

The height of the compression region can be calculated as:

$$
k d=\frac{\rho f_{y}}{\bar{\sigma}} d
$$

where $\rho$ is the tensile reinforcement ratio. A failure criterion that relates the average shear and compression stresses allows the calculation of the corresponding shear stress at failure, as follows:

$$
\bar{\tau}=0.1 f_{c}^{\prime}\left[0.62+7.86\left(\frac{\bar{\sigma}}{f_{c}^{\prime}}\right)-8.46\left(\frac{\bar{\sigma}}{f_{c}^{\prime}}\right)^{2}\right]^{1 / 2}
$$

The ultimate shear strength can then be calculated as follows:

$$
V_{u}=\bar{\tau} A_{e}=\bar{\tau} b(k d)
$$

Work of Tureyen and Frosch (2003). Tureyen and Frosch (2003) proposed a method for estimating the ultimate shear strength of RC beams. They assumed that failure 
of the concrete in the compression zone occurs when the principal tensile stress reaches the concrete tensile strength. Considering a concrete element in the compression region subjected to shear and normal stress, the relationship between shear stress $\tau$, normal stress $\sigma$, and the principal tensile stress, which is equal to the concrete tensile strength $f_{t}$, has the following form:

$$
\tau=\sqrt{f_{t}^{2}+f_{t} \sigma}
$$

By considering a free body of the compression zone above a crack, they assumed, in addition to the compression that the uncracked concrete is subjected to, bending. This assumption leads to a parabolic shear stress distribution with the maximum value at the centroid of the compression region, as shown in Fig. 2-17. This maximum shear stress $\tau_{\max }$ can be calculated as follows:

$$
\tau_{\max }=\frac{3 V}{2 b_{w} c}
$$

where $b_{w}$ is the beam web width.

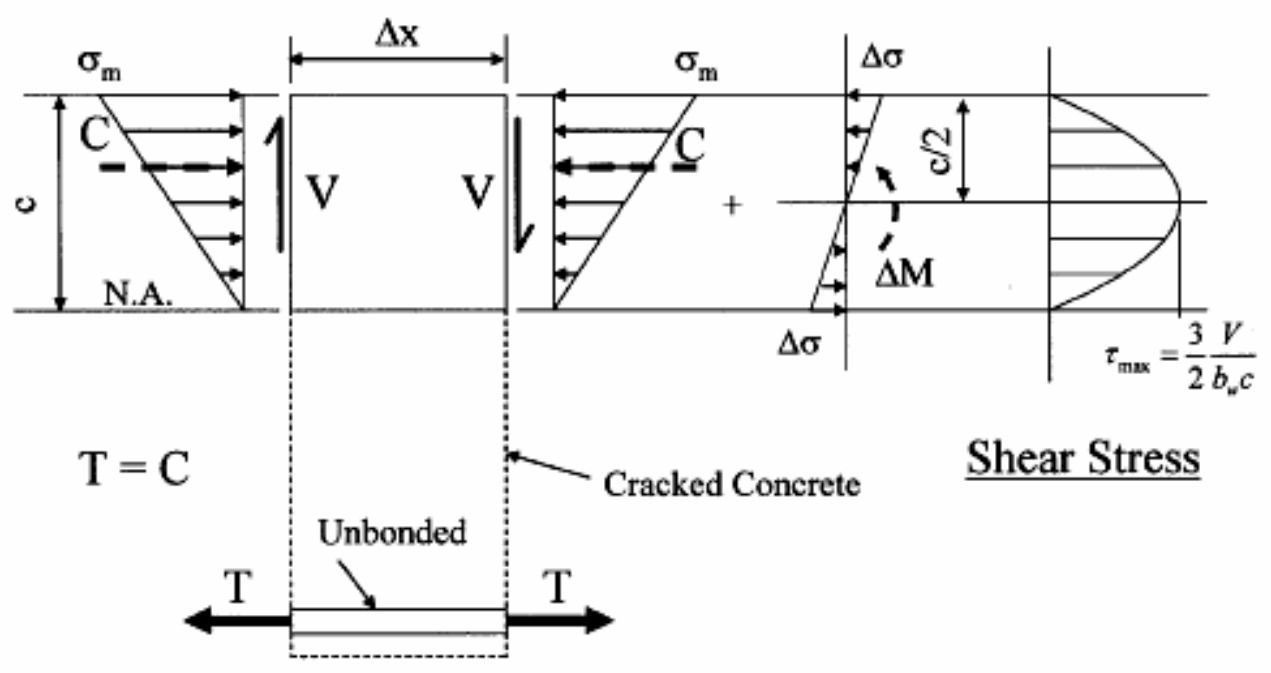

Fig. 2-17: Free body diagram at a crack (from Tureyen and Frosch, 2003)

By assuming a linear distribution of compressive stress with the zero value at the neutral axis and the maximum value $\sigma_{m}$ at the top fiber, as seen in Fig. 2-17, the maximum shear stress and hence, the shear force can be calculated as: 


$$
V=\frac{2}{3} b_{w} c \sqrt{f_{t}^{2}+f_{t} \frac{\sigma_{m}}{2}}
$$

For conservatism, they recommended the use of $\sigma_{m}$ corresponding to the cracking moment of the section. This method is reported to give conservative shear strength predictions for RC beams.

\subsubsection{Shear strength models based on the contribution of the aggregate interlock}

Work of Vecchio and Collins (1986). Vecchio and Collins (1986) introduced the Modified Compression Field Theory for cracked concrete. It is based on the concept of average stress and strain in cracked concrete. The contribution from steel reinforcement in resisting shear through dowel action is neglected. In developing the theory, it is assumed that perfect bond exists between reinforcement and concrete. Average stress versus strain relationships for concrete in compression and tension were proposed based on the results of reinforced concrete panel tests. It was found that concrete subjected to transverse tension strain is "softer and weaker" in the direction of principal compressive stress than concrete in a cylinder test. The principal compressive stress versus strain relationship (Fig. 2-18) depends on the two principal strains, as well as on other constants obtained from a cylinder test, as follows:

$$
f_{c 2}=f_{c 2 \max }\left[2\left(\frac{\varepsilon_{2}}{\varepsilon_{c}^{\prime}}\right)-\left(\frac{\varepsilon_{2}}{\varepsilon_{c}^{\prime}}\right)^{2}\right]
$$

where:

$$
f_{c 2 \max }=\frac{f_{c}^{\prime}}{0.8-0.34 \varepsilon_{1} / \varepsilon_{c}^{\prime}} \leq f_{c}^{\prime}
$$

and $f_{c 2}$ is the principal compressive stress; $\varepsilon_{1}$ and $\varepsilon_{2}$ are, respectively, the principal tensile and compressive strains; and $f_{c}^{\prime}$ and $\varepsilon_{c}^{\prime}$ are, respectively, the peak compressive stress and strain at peak stress obtained from a cylinder test. Meanwhile, the principal tensile stress versus strain relationship (Fig. 2-18d) was assumed to depend on only one strain parameter, as follows: 


$$
\begin{array}{ll}
f_{c 1}=E_{c} \varepsilon_{1} & \text { for } \varepsilon_{1} \leq \varepsilon_{c r} \\
f_{c 1}=\frac{f_{c r}}{1+\sqrt{200 \varepsilon_{1}}} & \text { for } \varepsilon_{1}>\varepsilon_{c r}
\end{array}
$$

where $f_{c 1}$ is the principal tensile stress; $E_{c}$ is the concrete modulus of elasticity; and $f_{c r}$ and $\varepsilon_{c r}$ are, respectively, the concrete stress and strain at cracking.

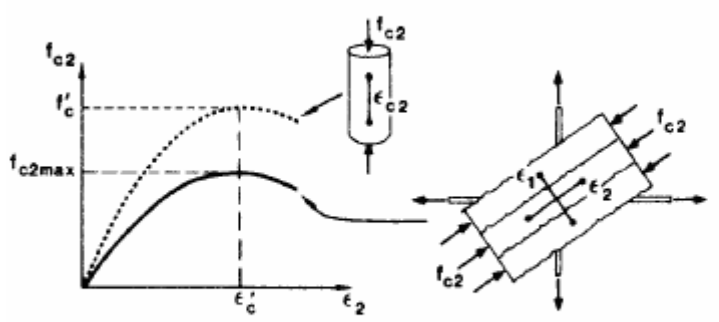

(a) Stress-Strain Relationship for Cracked Concrete in Compression

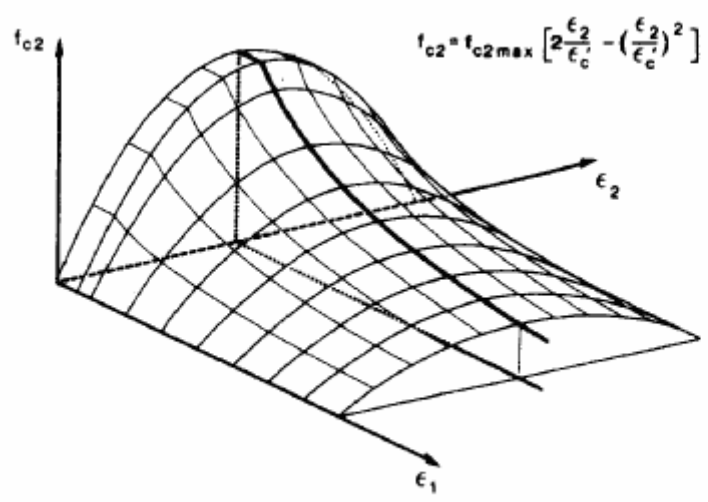

(c)

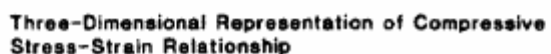

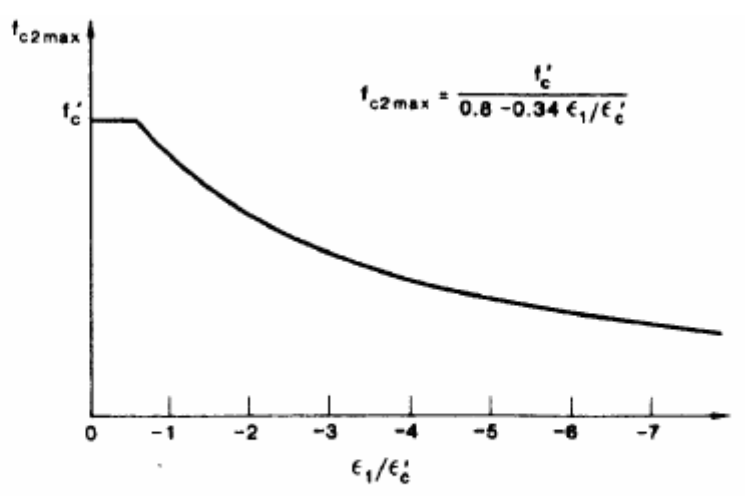

(b)

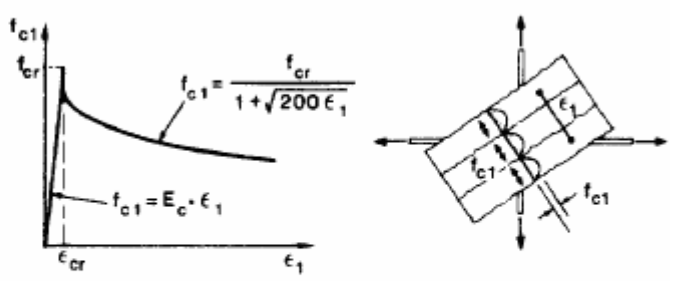

(d) Average Stress-Strain Relationship for Cracked Concrete in Tension

Fig. 2-18: Stress versus strain relationships for reinforced concrete in the Modified Compression Field Theory (from Vecchio and Collins, 1986)

In the Modified Compression Field Theory, it is assumed that the direction of principal stress coincides with that of principal strain. In addition, the local variation of stress inbetween and at the cracks is recognized. Following the work of Walraven (1981), the shear stress, $v_{c i}$, at a crack is related to the crack width, aggregate size, and compressive stress transmitted across the crack, as follows: 


$$
v_{c i}=\frac{2.16 \sqrt{f_{c}^{\prime}}}{0.3+\frac{24 w}{a+0.63}}
$$

where $w$ is the crack width (inches) and $a$ is the aggregate size (inches). The crack width is calculated based on the principal tensile strain and crack spacing. The relationship between the average stress and strain is then obtained through an iterative procedure.

The Modified Compression Field Theory has been implemented in some design codes, for example Canadian Concrete Code (Technical Committee on Reinforced Concrete Design, 1994) and AASHTO Code (LRFD Bridge Specifications and Commentary, 1998), and finite element codes. Obviously, this theory cannot be directly applied to predict the response of SFRC beams because the concrete stress versus strain relationships were developed from tests of panels made of regular reinforced concrete. Thus, new stress versus strain relationships for SFRC must be developed if this theory is to be applied to the shear design of SFRC beams

Work of Vecchio (2000 and 2001). The Disturbed Stress Field Theory introduced by Vecchio (2000 and 2001) is an improved version of the Modified Compression Field Theory. The theory recognizes that elemental stress fields are disturbed due to the formation of cracks, while still using the concept of average stresses and strains. It also accounts for the fact that the direction of principal stress is often different from that of principal strain in cracked reinforced concrete. It explicitly considers slip along cracks, which creates an "apparent" shear strain in additional to the actual shear strain in the concrete. The relationship between apparent shear strain due to crack slip $\gamma_{s}$ and (total) shear strain $\gamma_{x y}$ is as follows:

$$
\gamma_{s}=\gamma_{x y} \cos 2 \theta_{\sigma}+\left(\varepsilon_{y}-\varepsilon_{x}\right) \sin 2 \theta_{\sigma}
$$

where $\theta_{\sigma}$ is the principal tensile stress angle, assumed to be equal to that for the principal concrete strain. If the actual concrete strain field is $\left[\varepsilon_{c}\right]^{T}=\left\{\begin{array}{lll}\varepsilon_{c x} & \varepsilon_{c y} & \gamma_{c x y}\end{array}\right\}$, the principal stress angle can be calculated as follows: 


$$
\theta_{\sigma}=\frac{1}{2} \tan ^{-1}\left[\frac{\gamma_{c x y}}{\varepsilon_{c x}-\varepsilon_{c y}}\right]
$$

Meanwhile, $\varepsilon_{x}$ and $\varepsilon_{y}$ are normal strain components of the total strain field $[\varepsilon]^{T}=\left\{\begin{array}{lll}\varepsilon_{x} & \varepsilon_{y} & \gamma_{x y}\end{array}\right\}$. Their relation to the total principal strain $\theta_{\varepsilon}$ has the following form:

$$
\theta_{\varepsilon}=\frac{1}{2} \tan ^{-1}\left[\frac{\gamma_{x y}}{\varepsilon_{x}-\varepsilon_{y}}\right]
$$

Changes to the concrete stress versus strain relationships and shear stresses along cracks were also made. The theory is reported to give more accurate results than the Modified Compression Field Theory. Similar to the Modified Compression Field Theory, the application to reinforced SFRC beams requires a modification of the concrete constitutive relationships to account for the presence of fibers. 


\section{CHAPTER 3}

\section{EXPERIMENTAL PROGRAM}

\subsection{INTRODUCTION}

As mentioned in Chapters 1 and 2, although many experimental programs were conducted to test SFRC beams without web reinforcement over the last forty years, very few dealt with large-scale beams and deformed, commercially available steel fibers. The experimental program of this research aims to provide a further understanding of shear behavior of large-scale beams reinforced with steel fibers.

The experimental program strived to answer the following questions:

(1) What are the shear failure mechanisms, ultimate shear strength, and ductility of SFRC beams?

(2) How do these results change if the beam depth, fiber content, fiber type, and longitudinal reinforcement ratio vary?

(3) What are the distribution and magnitude of strains in SFRC beams prior to shear failure? What are the implications of these results?

(4) Can steel fibers be used in place of the minimum shear reinforcement required by ACI Committee 318 for RC beams?

The experimental program involved the design, construction, and testing of simply-supported beam specimens subjected to a monotonically-increased, concentrated load. A total of 28 beams were tested. In addition, tests to obtain mechanical properties of materials - cylinder tests, four-point bending tests, direct tensile tests for SFRC dog-bone specimens and steel bars - were also conducted. Subsequent sections provide details of the experimental program. 


\subsection{DESIGN OF BEAM SPECIMENS}

The experimental program consisted of two series of beams: Series B18 and Series B27. There were 8 pairs of beams for Series B18 and 4 pairs plus 4 single beams for Series B27. This made a total of 28 beams tested in the program. Beams from each pair were nominally "identical" to reduce the uncertainty of the shear data. Table 3-1 lists the properties of beams in Series B18 and B27. In general, the fixed parameters for each series were beam size, shear span-to-effective depth ratio, and concrete compressive strength, while the varied parameters were fiber type, fiber content, and longitudinal reinforcement ratio. Details of the parameters listed in Table 3-1 are discussed in the following sections.

Table 3-1: Design properties of beams in Series B18 and B27

\begin{tabular}{|c|c|c|c|c|c|c|c|c|}
\hline Beam $(*)$ & $\begin{array}{l}d \\
\text { (in) }\end{array}$ & $a / d$ & $\begin{array}{c}\rho \\
(\%)\end{array}$ & $\begin{array}{l}\text { Fiber } \\
\text { type }\end{array}$ & $\begin{array}{l}V_{f} \\
(\%)\end{array}$ & $\begin{array}{c}\text { Targeted } \\
f_{c}^{\prime} \\
\text { (psi) }\end{array}$ & $\begin{array}{l}\text { Concrete } \\
\text { mix ID }\end{array}$ & $\begin{array}{c}\text { Measured } \\
f_{c}^{\prime} \\
(\mathrm{psi})\end{array}$ \\
\hline B18-0a, b & 15 & 3.43 & 2.67 & No fiber & 0 & 6000 & - & 6210 \\
\hline B18-1a, b & 15 & 3.43 & 1.96 & ZP305 & 0.75 & 6000 & LAB 1 & 6500 \\
\hline B18-2a, b & 15 & 3.50 & 1.96 & ZP305 & 1.00 & 6000 & - & 5530 \\
\hline B18-2c, d & 15 & 3.50 & 2.67 & ZP305 & 1.00 & 6000 & - & 5530 \\
\hline B18-3a, b & 15 & 3.43 & 2.67 & ZP305 & 1.50 & 6000 & - & 4500 \\
\hline B $18-3 c, d$ & 15 & 3.43 & 2.67 & ZP305 & 1.50 & 6000 & LAB 1 & 6520 \\
\hline B18-5a, b & 15 & 3.43 & 2.67 & $\mathrm{RC} 80 / 60 \mathrm{BN}$ & 1.00 & 6000 & LAB 1 & 7140 \\
\hline B18-7a, b & 15 & 3.43 & 1.96 & RC80/30BP & 0.75 & 6000 & LAB 1 & 6290 \\
\hline B27-1a, b & 24 & 3.50 & 2.06 & ZP305 & 0.75 & 6000 & SUP1 & 7370 \\
\hline B27-2a, b & 24 & 3.50 & 2.06 & RC80/60BN & 0.75 & 6000 & SUP2 & 4170 \\
\hline B27-3a, b & 24 & 3.50 & 1.56 & ZP305 & 0.75 & 6000 & SUP2 & 6140 \\
\hline B27-4a, b & 24 & 3.50 & 1.56 & RC80/60BN & 0.75 & 6000 & SUP2 & 4290 \\
\hline B27-5 & 24 & 3.50 & 2.06 & ZP305 & 1.50 & 6000 & SUP3 & 6450 \\
\hline B27-6 & 24 & 3.50 & 2.06 & $\mathrm{RC} 80 / 60 \mathrm{BN}$ & 1.50 & 6000 & SUP3 & 6210 \\
\hline B27-7 & 24 & 3.50 & 1.56 & No fiber & 0 & 6000 & SUP3 & 5370 \\
\hline B27-8 $(\dagger)$ & 24 & 3.50 & 1.56 & No fiber & $0(\dagger)$ & 6000 & SUP3 & 5370 \\
\hline $\begin{array}{l}(*) \text { : All bea } \\
\text { had dimens } \\
(\dagger) \text { : This be }\end{array}$ & s in $\mathrm{S}$ & ies B18 & ad dim & $\begin{array}{l}\text { nsions of } b \times h \\
\text { in. } \times 170 \mathrm{in} .\end{array}$ & $\ell=6 \mathrm{il}$ & 18 in $\times 96$ & ; all beams & eries B27 \\
\hline
\end{tabular}




\subsubsection{Fixed parameters}

Shear span-to-effective depth ratio. All beams in this test program had a shear span-to-effective depth ratio, $a / d$, of approximately 3.5 to minimize the effect of arch action. It is noted that for beams with an $a / d$ ratio less than approximately 2.5 , a direct concrete strut from the loading point to the support is formed, leading to an increase in beam strength compared with slender beams, which are less likely to have this effect. If the beams are too slender, a flexural failure mode will be likely. Due to these reasons, an a/d ratio of 3.5 was selected.

Beam size. Each beam in Series B18 was 6 inches in width by 18 inches in depth by 96 inches in length. Each beam in Series B27 was 8 inches in width by 27 inches in depth by 170 inches in length. From the review of tested beams found in research papers (see Chapter 2), it was decided that testing beams with a depth of at least 18 inches was necessary. Beams with a depth of 27 inches represented an increase of $50 \%$ in depth compared to Series B18 and should be useful for evaluating the effect of beam size on shear strength of SFRC beams. The width of the beams was chosen on the basis of concrete cover of 1 inches and steel bar arrangement.

The length of beams was designed such that the critical (left) shear span-toeffective depth ratio $(a / d)$ was 3.5 and that no anchorage failures would occur (see Fig. 3-1). The shorter shear span was reinforced with stirrups to force a shear failure in the longer shear span.

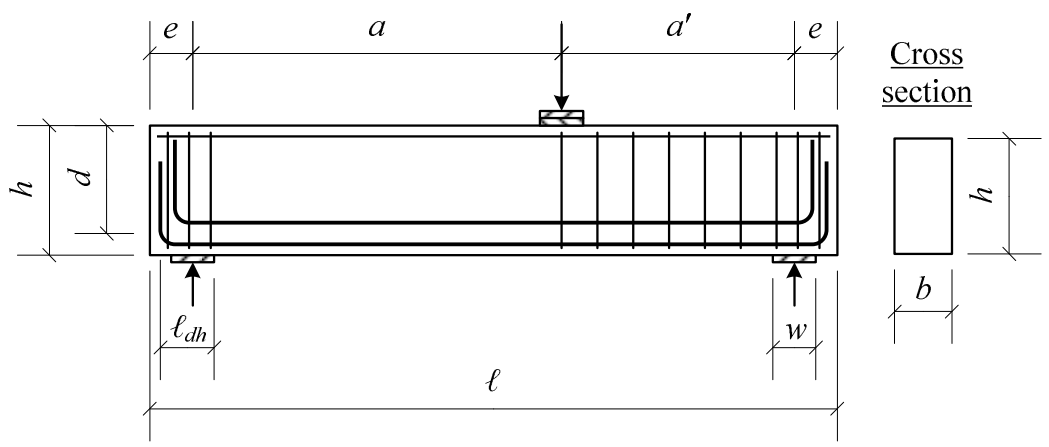

Fig. 3-1: Basic dimensions, load, and boundary conditions for a test beam 
Table 3-2 shows the calculation of required beam length for Series B18 and B27. In Series B18, two types of anchorage methods were used: mechanical anchorage (Lenton terminators) and $90^{\circ}$ hooks. The dimension $e$ was first determined based on the anchorage for the Lenton terminators. The selected length of beams B18 was then 96 inches. The first four beams in Series B18, which were constructed with Lenton terminators, were B18-2a, b, c, \& d. The subsequent beams in Series B18, constructed with $90^{\circ}$-hooked anchorage, also had a length of 96 inches to make use of the same formwork. It turned out that the available space for developing the $\# 790^{\circ}$-hooked bars was 8 inches, which was 3 inches less than the required development length for \#7 $90^{\circ}$ hooked bars enclosed with stirrups in concrete with a compressive strength of 6000 psi. Regardless of the deficiency in development length, the anchorage using $90^{\circ}$-hooked bars worked adequately, as will be shown in Chapter 4. In Series B27, 90-hooked bars were used for all beams. The required development length for $\# 890^{\circ}$-hooked bars enclosed by stirrups in concrete with a compressive strength of 6000 psi is 13 inches, less than the available space (17 inches). As in the beams in Series B18, the anchorage worked adequately.

Table 3-2: Calculation of the required beam length

\begin{tabular}{cccc}
\hline Dimension & $\begin{array}{c}\text { Series B18 } \\
\text { (anchored by } \\
\text { Lenton terminators })\end{array}$ & $\begin{array}{c}\text { Series B18 } \\
\text { (anchored by } \\
\left.90^{\circ} \text { hooks }\right)\end{array}$ & Series B27 \\
\hline$d=$ & 15 in. & 15 in. & 24 in. \\
$a / d=$ & 3.5 & 3.5 & 3.5 \\
$a \approx 3.5 d=$ & 52.5 in. & 51.5 in. & 84 in. \\
$a^{\prime}=2 a / 3=$ & 35.0 in. & 35.0 in. & 56 in. \\
$e=$ & 4 in. & 6 in. & 15 in. \\
$\ell=2 e+a+a^{\prime}=$ & 95.5 in. (select $\underline{96}$ in.) & $\underline{96}$ in. & $\underline{170}$ in. \\
$w=$ & 6 in. & 6 in. & 6 in. \\
$\ell_{d h}($ available $)=w / 2+e-1$ & 6 in. & 8 in. & 17 in. \\
\hline
\end{tabular}

Concrete compressive strength. As seen from Table 3-1, the design compressive strength was fixed at 6000 psi. However, the measured strength varied in the range of $\pm 30 \%$ of the design strength. This was mainly due to: (1) different mixes were 
used, (2) the concrete was either supplied by a local ready-mix supplier or mixed at the Structural Engineering Laboratory of the University of Michigan, (3) all beams were not cast at the same time - typically, each pair was cast at a time, and (4) all beams were not tested at the same age. Details of concrete mixtures will be provided in Section 3.4. 


\subsubsection{Varied parameters}

\subsubsection{Selection of fiber types}

Fiber types. For each series, two main types of fibers were used - Dramix ZP305 and RC80/60BN fibers. The exception was pair B18-7 in Series B18, in which Dramix RC80/30BP fibers were used. These fibers were manufactured by Bekaert Cooperation and are likely the most popular type of fibers. All of these fibers are hooked at their ends and collated into bundles by dissolvable glue, as shown in Fig. 3-2.

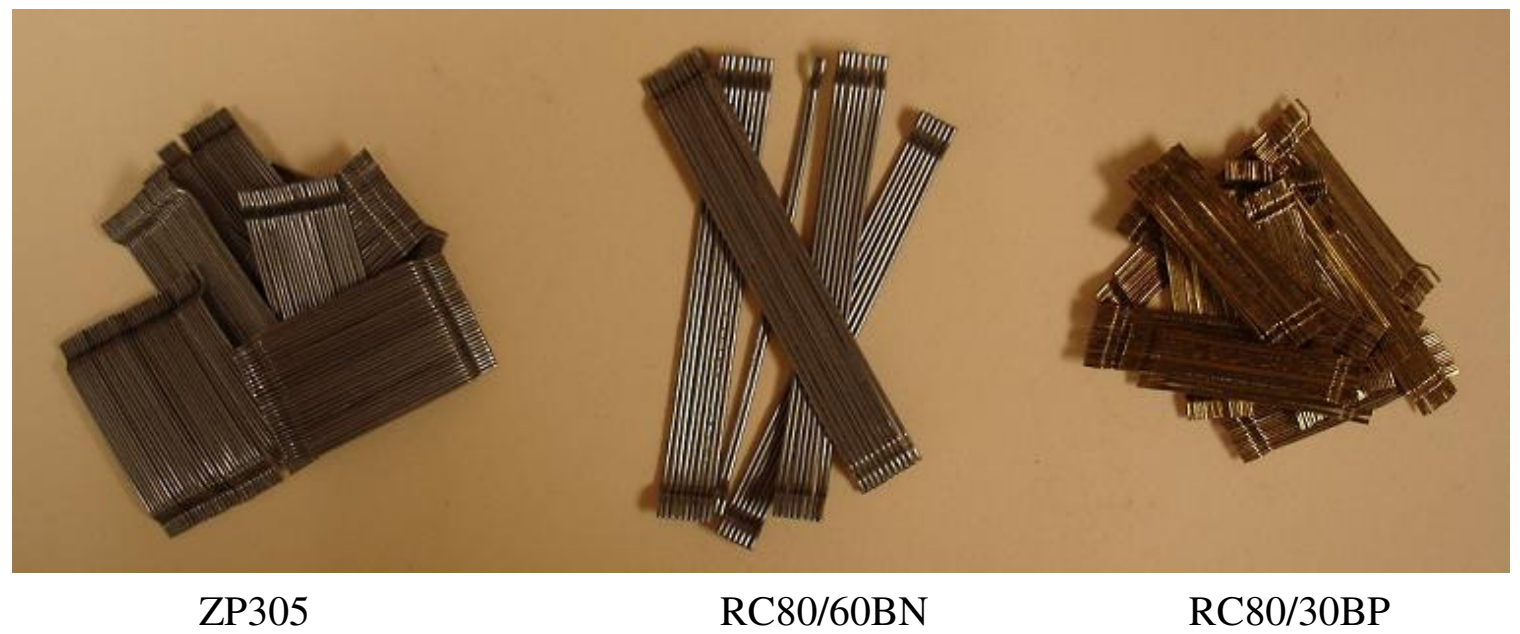

Fig. 3-2: Dramix hooked steel fibers used in this study

Fiber properties are listed in Table 3-3. Dramix ZP305 and RC80/60BN fibers, made of normal strength wire with a tensile strength of approximately $160 \mathrm{ksi}$, are often used in conventional fiber-reinforced concrete (FRC). Dramix RC80/30BP fibers, with a higher strength (330 ksi), are often employed in high-strength concrete. Dramix ZP305 fibers have a smaller aspect ratio (55) than that of RC80/60BN and RC80/30BP fibers (80).

Table 3-3: Properties of hooked steel fibers as reported by the manufacturer

\begin{tabular}{ccccc}
\hline Fiber & Length & Diameter & Aspect ratio & Strength \\
\hline ZP305 & 1.18 in. $(30 \mathrm{~mm})$ & 0.022 in. $(0.55 \mathrm{~mm})$ & 55 & $160 \mathrm{ksi}(1100 \mathrm{MPa})$ \\
RC80/60BN & 2.36 in. $(60 \mathrm{~mm})$ & 0.030 in. $(0.75 \mathrm{~mm})$ & 80 & $152 \mathrm{ksi}(1050 \mathrm{MPa})$ \\
RC80/30BP & 1.18 in. $(30 \mathrm{~mm})$ & 0.015 in. $(0.38 \mathrm{~mm})$ & 80 & $330 \mathrm{ksi}(2300 \mathrm{MPa})$ \\
\hline
\end{tabular}




\subsubsection{Selection of fiber volume fraction and reinforcement ratio for Series B18}

Selection of fiber volume fraction. A total of eight pairs of beams were tested for Series B18. The first pair of beams (B18-0a \& b), with no fibers, served as control specimens. The next five pairs (B18-1a \& b, B18-2a \& b, B18-2c \& d, B18-3a \& b, and B18-3c \& d) were reinforced with Dramix ZP305 fibers in volume fractions of either $0.75,1.0$, or $1.5 \%$. Beams B18-5a \& b contained Dramix RC80/60BN fibers in a $1 \%$ volume fraction, while Beams 18-7a \& b were reinforced with Dramix RC80/30BP fibers in a $0.75 \%$ volume fraction.

Selection of longitudinal bars. For each fiber volume fraction, the longitudinal reinforcement ratio was selected such that the beam would fail due to the action of shear. In some instances, a shear failure prior to flexural yielding was likely, while in other cases, the reinforcement ratio was selected such as to develop some flexural yielding prior to the shear failure. The next section will elaborate on the selection of the reinforcement. It turned out that a reinforcement ratio of $1.96 \%$ was selected for beams with a fiber volume fraction of $0.75 \%$ (Beams B18-1a \& b and B18-7a \& b). For beams with a fiber volume fraction of $1.0 \%$ (Beams B18-2c \& $d$ and B18-5a \& b) or 1.5\% (Beams B18-3a, b, c \& d), a reinforcement ratio of $2.67 \%$ was selected. It should be noted that the beam pair B18-3c \& d was the repetition of pair B18-3a \& b. This was because the observed shear strengths of the two beams B18-3a and B18-3b differed significantly from each other. It was therefore decided that a duplication of the pair B18$3 \mathrm{a} \& \mathrm{~b}$ was necessary. Beams B18-2a \& b had a fiber volume fraction of $1.0 \%$ and a longitudinal reinforcement ratio of $1.96 \%$ to evaluate the effect of fiber content (when compared with Beams B18-1a \& b) and reinforcement ratio (when compared with B18$2 c \& d)$.

As mentioned above, the longitudinal reinforcement was selected such that a shear failure would occur. From the proposal of Parra-Montesinos (2006), a shear strength of $3.5 \sqrt{f_{c}^{\prime}} b d$ was assumed for beams with a fiber volume fraction of $0.75 \%$ or $1.0 \%$, regardless of the type of fibers. Similarly, for beams with a fiber volume fraction 
of $1.5 \%$, a higher shear strength of $4.5 \sqrt{f_{c}^{\prime}}$ bd was arbitrarily assumed. Table 3-4 shows the calculation of the beam shear strength based on those assumptions. For example, the load required to reach the shear strength of Beams B18-1a \& b is:

$$
P_{s}=\frac{a+a^{\prime}}{a^{\prime}} V_{c}=\frac{5}{2} V_{c}=\frac{5}{2}(3.5 \sqrt{6000} \text { psi } \times 6 \text { in } \times 15 \text { in. })=61,000 \mathrm{lbs}=61 \mathrm{kips} .
$$

Table 3-4: Calculation of beam shear and flexural strengths

\begin{tabular}{cccccccccccc}
\hline Beam & \begin{tabular}{c}
$v_{u}$ \\
\cline { 2 - 10 }
\end{tabular} & $\begin{array}{c}f_{c}^{\prime} \\
f_{c}^{\prime}\end{array}$ & $\begin{array}{c}b \\
\text { (in.) }\end{array}$ & $\begin{array}{c}d \\
\text { (in.) }\end{array}$ & $\begin{array}{c}P_{s} \\
\text { (in.) }\end{array}$ & $\begin{array}{c}A_{s} \\
\text { (k) }\end{array}$ & $\begin{array}{c}a_{c} \\
\text { (in.) }\end{array}$ & $\begin{array}{c}a \\
\text { (in.) }\end{array}$ & $\begin{array}{c}M_{n} \\
\text { (k-in) }\end{array}$ & $\begin{array}{c}P_{m} \\
\text { (k) }\end{array}$ & $P_{s}<P_{m}$ \\
\hline B18-0a, b & 2.00 & 6000 & 6 & 15 & 34.9 & 2.4 & 4.71 & 51.5 & 1820 & 88.4 & OK \\
B18-1a, b & 3.50 & 6000 & 6 & 15 & 61.0 & 1.76 & 3.45 & 51.5 & 1400 & 68.0 & OK \\
B18-2a, b & 3.50 & 6000 & 6 & 15 & 61.0 & 1.76 & 3.45 & 52.5 & 1400 & 66.8 & OK \\
B18-2c, d & 3.50 & 6000 & 6 & 15 & 61.0 & 2.4 & 4.71 & 52.5 & 1820 & 86.7 & OK \\
B18-3a, b & 4.50 & 6000 & 6 & 15 & 78.4 & 2.4 & 4.71 & 51.5 & 1820 & 88.4 & OK \\
B18-3c, d & 4.50 & 6000 & 6 & 15 & 78.4 & 2.4 & 4.71 & 51.5 & 1820 & 88.4 & OK \\
B18-5a, b & 3.50 & 6000 & 6 & 15 & 61.0 & 2.4 & 4.71 & 51.5 & 1820 & 88.4 & OK \\
B18-7a, b & 3.50 & 6000 & 6 & 15 & 61.0 & 1.76 & 3.45 & 51.5 & 1400 & 68.0 & OK \\
\hline
\end{tabular}

The calculation of beam flexural strength followed the traditional method used for RC beams. For example, the nominal moment capacity for Beams B18-1a \& b reinforced with 4 \#6 bars was calculated, as follows:

$$
\begin{aligned}
& a_{c}=\frac{A_{s} f_{y}}{0.85 f_{c}^{\prime} b}=\frac{\left(4 \times 0.44 \mathrm{in}^{2}\right) \times 60 \mathrm{ksi}}{0.85 \times 6 \mathrm{ksi} \times 6 \mathrm{in} .}=3.45 \mathrm{in} . \\
& M_{n}=A_{s} f_{y}\left(d-a_{c} / 2\right)=(4 \times 0.44) \times 60 \mathrm{ksi} \times(15-3.45 / 2)=1400 \mathrm{k}-\mathrm{in} .
\end{aligned}
$$

The load required to reach the flexural strength is then:

$$
P_{m}=\frac{5 M_{n}}{2 a}=\frac{5 \times 1400 \mathrm{ksi}}{2 \times 51.5 \mathrm{in} .}=68 \mathrm{kips}
$$

It can be seen from Table 3-4 that all beams in Series B18 had a load $P_{m}<P_{s}$. It is worth noting that the beam shear strength may be higher than the assumed value and possibly higher than the beam flexural strength (even when actual materials properties are considered). In that case, the longitudinal reinforcement may yield prior to the occurrence of a shear failure. This was considered to be acceptable because the use of higher reinforcement ratios may lead to an unrealistically high shear strength. Also, the 
calculation of beam flexural strength did not taken into account the probable yield strength of the reinforcement and the contribution of the steel fibers to the flexural strength of the beam.

Selection of stirrups for the shorter spans. The stirrups in the right shear span were selected such that a shear failure would not occur in that span. With \#3 stirrups spaced at 5 in., the calculated shear capacity of the beam is:

$$
\begin{aligned}
V_{n}(\text { right span }) & =V_{c}+V_{s}=3.5 \sqrt{f_{c}^{\prime}} b d+A_{v} f_{y} \frac{d}{s} \\
& =3.5 \sqrt{6000} \text { psi } \times 6 \text { in. } \times 15 \text { in. }+0.22 \text { in. }^{2} \times 60000 \mathrm{psi} \times \frac{15 \mathrm{in} .}{5 \mathrm{in} .} \\
& =23400+39600=63000 \mathrm{lbs}=63 \mathrm{kips}
\end{aligned}
$$

The corresponding load is:

$$
P=\frac{a+a^{\prime}}{a} V_{n}=\frac{5}{3} V_{n}(\text { right span })=105 \text { kips } .
$$

This strength is greater than any of the predicted strengths listed in Table 3-4. Therefore, a shear failure in the shorter span was not expected.

Table 3-5: Dimensions of end anchors

\begin{tabular}{cccc}
\hline Bar & $\begin{array}{c}\text { Bar diameter } \\
\text { (in.) }\end{array}$ & $\begin{array}{c}\text { End anchor diameter } \\
\text { (in.) }\end{array}$ & $\begin{array}{c}\text { End anchor body length } \\
\text { (in.) }\end{array}$ \\
\hline$\# 6$ & $3 / 4$ & $1-7 / 8$ & $1-1 / 8$ \\
$\# 7$ & $7 / 8$ & 2 & $1-1 / 4$ \\
\hline
\end{tabular}

Details of end anchorage. Fig. 3-3 shows the reinforcement details of all beams in Series B18. As seen in Fig. 3-3, two types of anchorage were employed: oversized end anchors (Lenton terminators) and $90^{\circ}$ hooks. Table 3-5 provides details of the end anchors used.

Selection of steel plates. Steel plates were used at the supports and load point to distribute the loads such that bearing stresses remained below acceptable limits. Under 
the steel plate at the load point, a layer of non-shrink grout was also added to ensure that the load was uniformly applied over the loading area. The size of the steel plates under the loading point and at the supports was selected on the basis of a permissible bearing stress of $0.85 f_{c}^{\prime}$ such that no concrete crushing would occur. For a 6 in. by 6 in. steel plate, the permissible bearing load is $184 \mathrm{kips}$, over twice as much as the maximum beam strength predicted in Table 3-4 (88.4 kips). 
(a) Reinforcement details for B18-1a, b; B18-7a, b

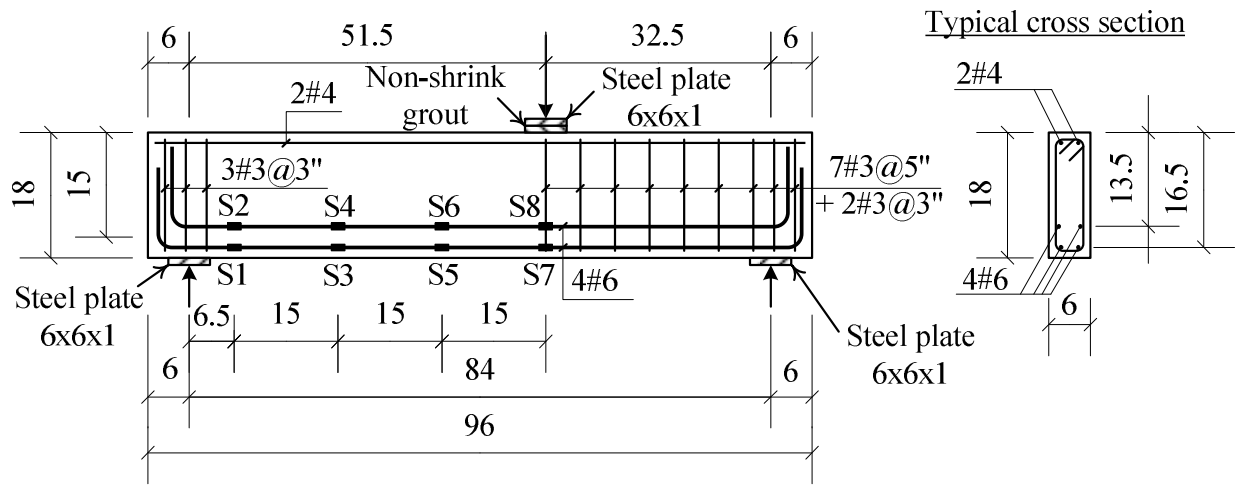

(b) Reinforcement details for B18-0a, b; B18-3a, b, c, d; B18-5a, b

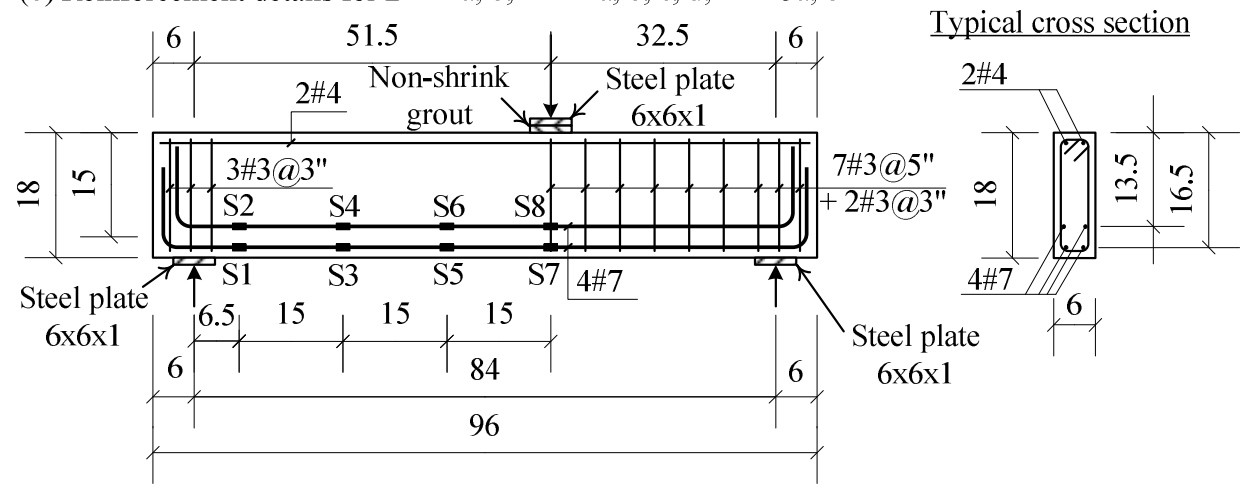

(c) Reinforcement details for B18-2a, b

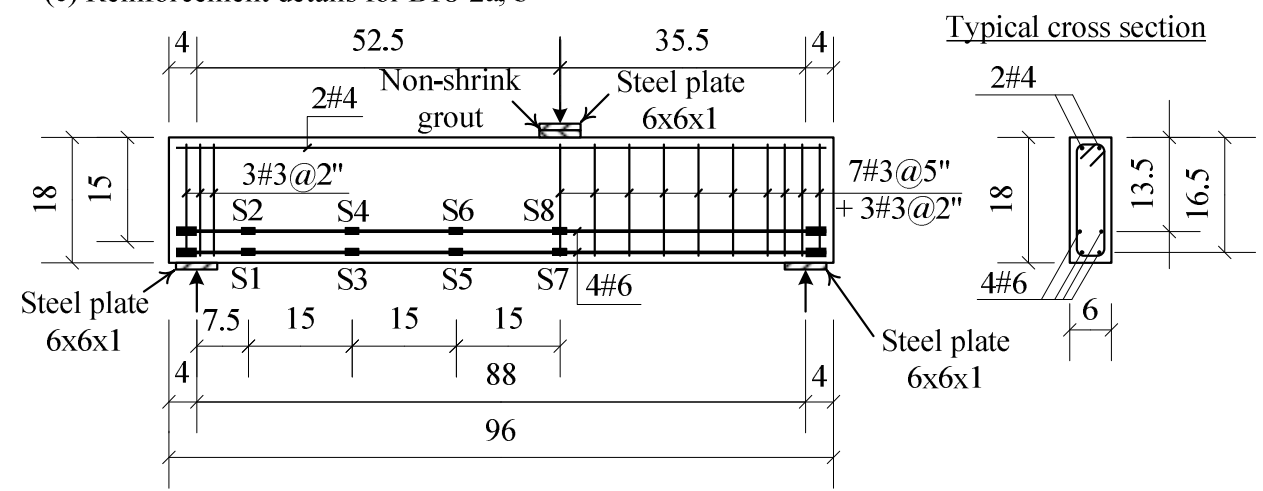

(d) Reinforcement details for B18-2c, d

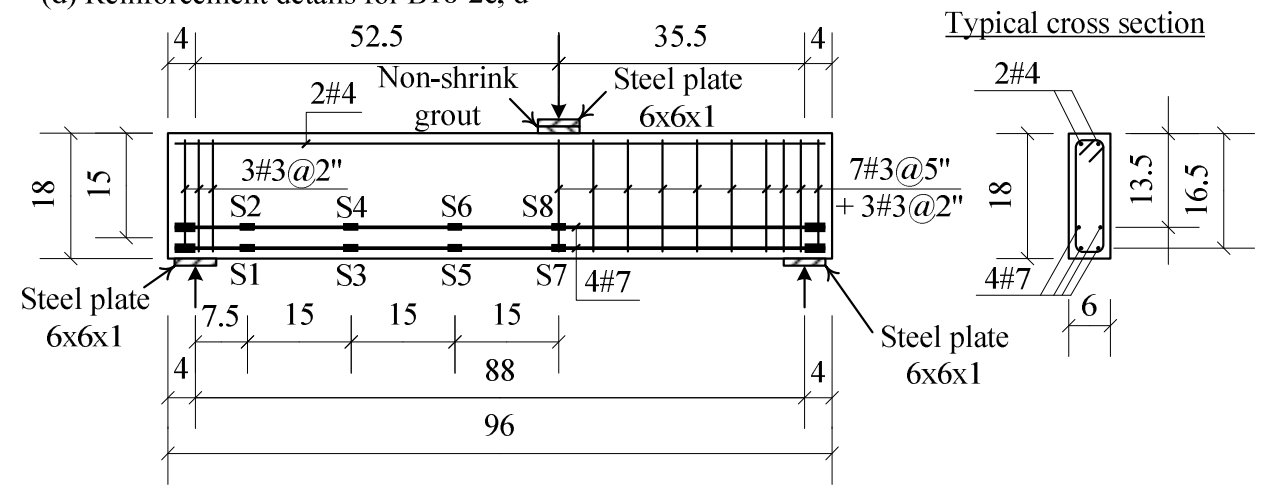

Fig. 3-3: Reinforcement details and strain gauge placement for beams in Series B18 


\subsubsection{Selection of fiber volume fraction and reinforcement ratio for Series B27}

Selection of fiber volume fraction. For Series B27, four pairs and four single beams were tested. In the four pairs of beams, either ZP305 or RC80/60BN fibers in a volume fraction of $0.75 \%$ were used.

Beams B27-1a \& b and B27-2a \& b were reinforced with Dramix fibers ZP305 and $\mathrm{RC} 80 / 60 \mathrm{BN}$, respectively, in a fiber volume fraction of $0.75 \%$. These beams had a reinforcement ratio of $2.06 \%$, sufficiently close to $1.96 \%$ such that a comparison between beams in Series B18 and B27 could be made. Similar to Series B18, a reinforcement ratio of $2.06 \%$ was chosen such that a shear failure was to be expected. The calculated flexural strengths and shear strengths for Beams B27-1a \& b and B27-2a \& b are shown in Table 3-6. Without considering the effect of beam depth, the same shear strength of $3.5 \sqrt{f_{c}^{\prime}} b d$ and $4.5 \sqrt{f_{c}^{\prime}} b d$ was assumed for beams with a fiber volume fraction of 0.75 and $1.5 \%$, respectively. It can be seen from the table that Beams B27-1a \& b and B27-2a \& b were expected to fail in shear prior to flexural yielding

Table 3-6: Calculation of beam shear and flexural strength

\begin{tabular}{cccccccccccc}
\hline Beam & \begin{tabular}{c}
$v_{u}$ \\
\cline { 2 - 10 }
\end{tabular} & $\begin{array}{c}f_{c}^{\prime} \\
\text { (in.) }\end{array}$ & $\begin{array}{c}b \\
\text { (in.) }\end{array}$ & $\begin{array}{c}d \\
\text { (in.) }\end{array}$ & $\begin{array}{c}P_{s} \\
\text { (k) }\end{array}$ & $\begin{array}{c}A_{s} \\
\left.\text { (in. }{ }^{2}\right)\end{array}$ & $\begin{array}{c}a_{c} \\
\text { (in.) }\end{array}$ & $\begin{array}{c}a \\
\text { (in.) }\end{array}$ & $\begin{array}{c}M_{n} \\
(\mathrm{k}-\mathrm{in})\end{array}$ & $\begin{array}{c}P_{m} \\
\text { (k) }\end{array}$ & $P_{s}<P_{m}$ \\
\hline B27-1a, b & 3.50 & 6000 & 8 & 24 & 130 & 3.95 & 5.81 & 84 & 5000 & 149 & OK \\
B27-2a, b & 3.50 & 6000 & 8 & 24 & 130 & 3.95 & 5.81 & 84 & 5000 & 149 & OK \\
B27-3a, b & 3.50 & 6000 & 8 & 24 & 130 & 3 & 4.41 & 84 & 3920 & 117 & NO \\
B27-4a, b & 3.50 & 6000 & 8 & 24 & 130 & 3 & 4.41 & 84 & 3920 & 117 & NO \\
B27-5 & 4.50 & 6000 & 8 & 24 & 167 & 3.95 & 5.81 & 84 & 5000 & 149 & NO \\
B27-6 & 4.50 & 6000 & 8 & 24 & 167 & 3.95 & 5.81 & 84 & 5000 & 149 & NO \\
B27-7 & 2.00 & 6000 & 8 & 24 & 74 & 3 & 4.41 & 84 & 3920 & 117 & OK \\
\hline
\end{tabular}

Beams B27-3a \& b and B27-4a \& b were also reinforced with Dramix fibers ZP305 and RC80/60BN, respectively, in a fiber volume fraction of $0.75 \%$. However, these beams contained a lower amount of longitudinal reinforcement (1.56\%) to evaluate the behavior of SFRC beams exhibiting flexural yielding prior to shear failure. Therefore, the predicted flexural strength (117 kips) is lower than the predicted shear strength (130 kips), as shown in Table 3-6. 
Single beams B27-5 and B27-6 were also reinforced with Dramix fibers ZP305 and $\mathrm{RC} 80 / 60 \mathrm{BN}$, respectively, in a fiber volume fraction of $1.5 \%$. It was decided that for a fiber volume fraction of $1.5 \%$, single beams, instead of pairs of beams, would be tested because test results for the pairs of SFRC beams were sufficiently close - the variance was less than 5\%. A reinforcement ratio of $2.06 \%$ was selected for the purpose of comparison with Beams B27-1a \& b and B27-2a \& b, which have a lower fiber volume fraction $(0.75 \%)$. For beams B27-5 and B27-6, the calculated flexural strength was lower than the shear strength (see Table 3-6).

Similar to Beams B18-0a \& b in Series B18, Beam B27-7 served as control specimen for other beams in Series B27. In addition, Beam B27-8 was constructed with normal-strength concrete (no fibers) and transverse reinforcement conforming to the minimum requirement in ACI Code Section 11.4.6 (ACI Committee 318, 2008). This beam was tested to evaluate the potential of hooked steel fibers as a replacement for minimum stirrup-type shear reinforcement. Indented \#D4 bars, with an area of 0.04 in. $^{2}$ and a $0.2 \%$ offset yield strength of $90 \mathrm{ksi}$, were provided at a spacing of $12 \mathrm{in}$., half of the effective depth $d$, which is the maximum allowable stirrup spacing permitted by ACI Committee 318 for the expected shear demand. The resulting shear reinforcement area was then $0.0067 \mathrm{in}{ }^{2} / \mathrm{in}$. The required shear reinforcement was calculated on the basis of a concrete compressive strength of 6000 psi and a stirrup yield strength of $90 \mathrm{ksi}$, as follows:

$$
\frac{A_{v}}{s}=\frac{0.75 \sqrt{f_{c}^{\prime}} b_{w}}{f_{y}}=\frac{0.75 \sqrt{6000} \mathrm{psi} \times 8 \mathrm{in} .}{90000 \mathrm{psi}}=0.0052 \mathrm{in} .^{2} / \mathrm{in} .
$$

It should be noted that the provided shear reinforcement was $30 \%$ greater than the minimum required amount. Due to the lack of smaller-size bars and the limit on the maximum stirrup spacing regulated by ACI Committee 318, it was impossible to design a beam with reinforcement closer to the minimum shear reinforcement.

Selection of steel plates. Similar to Series B18, the size of steel bearing plates was selected to prevent a bearing failure. The concrete bearing capacity of the 8 in. by 8 in. steel plate was $326 \mathrm{kips}$, nearly two times greater than the maximum predicted beam strength as shown in Table 3-6 (167 kips). With this predicted beam strength, the 
maximum load the right support would bear is $100 \mathrm{kips}$, less than the bearing capacity of a 6 in. by 6 in. steel plate (184 kips).

Selection of stirrups for the shorter spans. With \#3 stirrups spaced at 4 in., the calculated shear capacity of the beam and corresponding applied load were:

$$
\begin{aligned}
& V_{n}(\text { right span })=V_{c}+V_{s}=3.5 \sqrt{f_{c}^{\prime}} b d+A_{v} f_{y} \frac{d}{s} \\
&=3.5 \sqrt{6000} \mathrm{psi} \times 8 \mathrm{in} . \times 24 \mathrm{in} .+0.22 \mathrm{in} .^{2} \times 60000 \mathrm{psi} \times \frac{24 \mathrm{in} .}{4 \mathrm{in} .} \\
&=52000+79200 \mathrm{lbs}=131 \mathrm{kips} \\
& P_{n}=\frac{5}{3} V_{n}(\text { right span })=218 \mathrm{kips}
\end{aligned}
$$

This is greater than any of the predicted beam strengths listed in Table 3-6. The final design of beams in Series B27 is provided in Fig. 3-4. 
(a) Reinforcement details for B27-1a, b; B27-2a, b; B27-5; B27-6

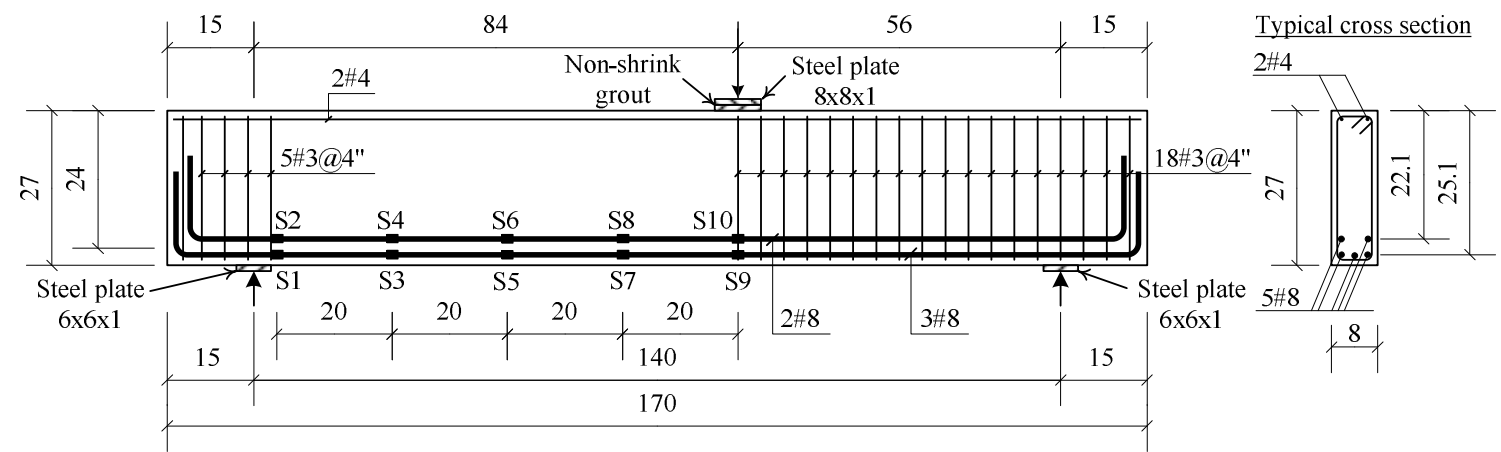

(b) Reinforcement details for B27-3a, b; B27-4a,b; B27-7

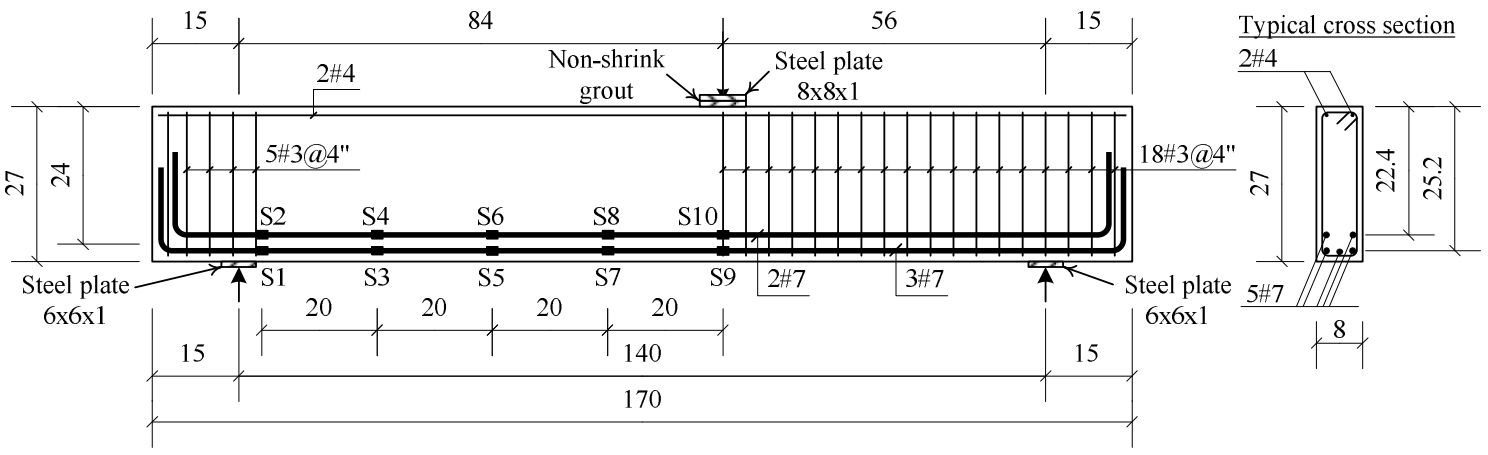

(c) Reinforcement details for B27-8

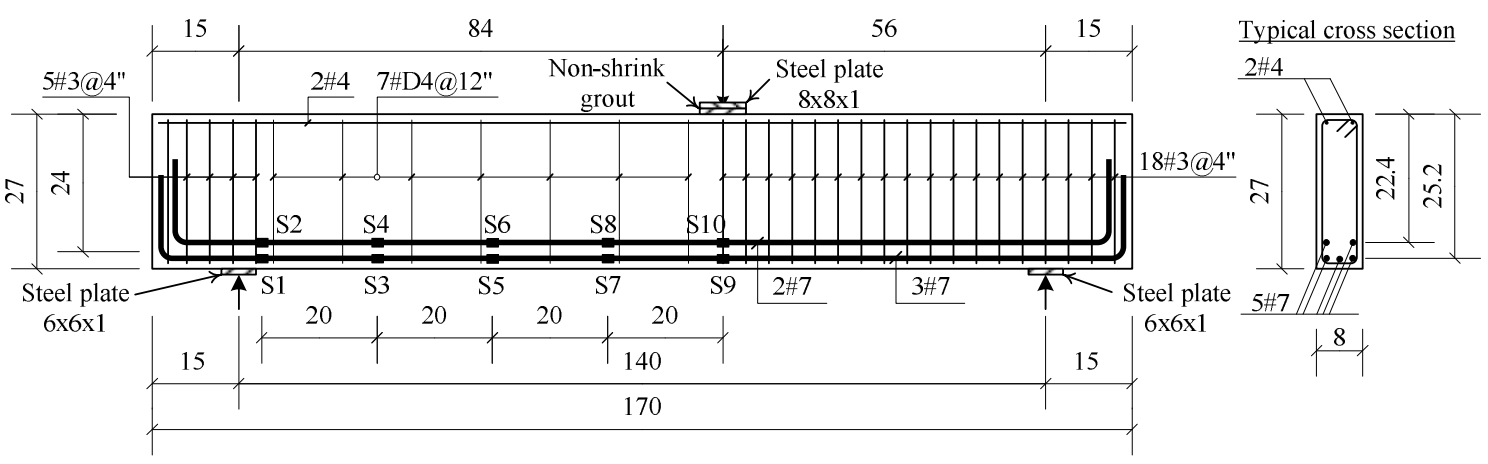

Fig. 3-4: Reinforcement details and strain gauge placement for beams in Series B27 


\subsection{FABRICATION OF REINFORCEMENT CAGE}

Strain gauges. For each beam, foil-type strain gauges were installed onto bottom reinforcing bars. For each beam in Series B18, except for Beams B18-0a \& b, eight strain gauges labeled S1 to S8 were placed at a spacing of 15 inches, starting from the loading point position (see Fig. 3-3). Similarly, ten strain gauges labeled S1 to S10 were spaced at 20 inches for beams in Series B27 (see Fig. 3-4). Each strain gauge was glued to a degreased flat area through cyanoacrylate glue and protected by three coating layers, namely polyurethane, nitrile coating, and a rubber pad sealed by electric liquid tape.

Reinforcement cage and formwork. Reinforcement cages were constructed at the University of Michigan Structural Engineering Laboratory. Formwork was oiled and caulked prior to placement of the reinforcement cage. A concrete cover of one inch for all reinforcement was attained by placing one-inch-high reinforcing bar chairs between reinforcement cages and formwork. Fig. 3-5a shows an example of a reinforcement cage ready for placing in the formwork. It should be noted that hooks were installed at the beam tops for the purpose of lifting and moving the beam. Fig. 3-5b shows an example of the laboratory site ready for casting. 


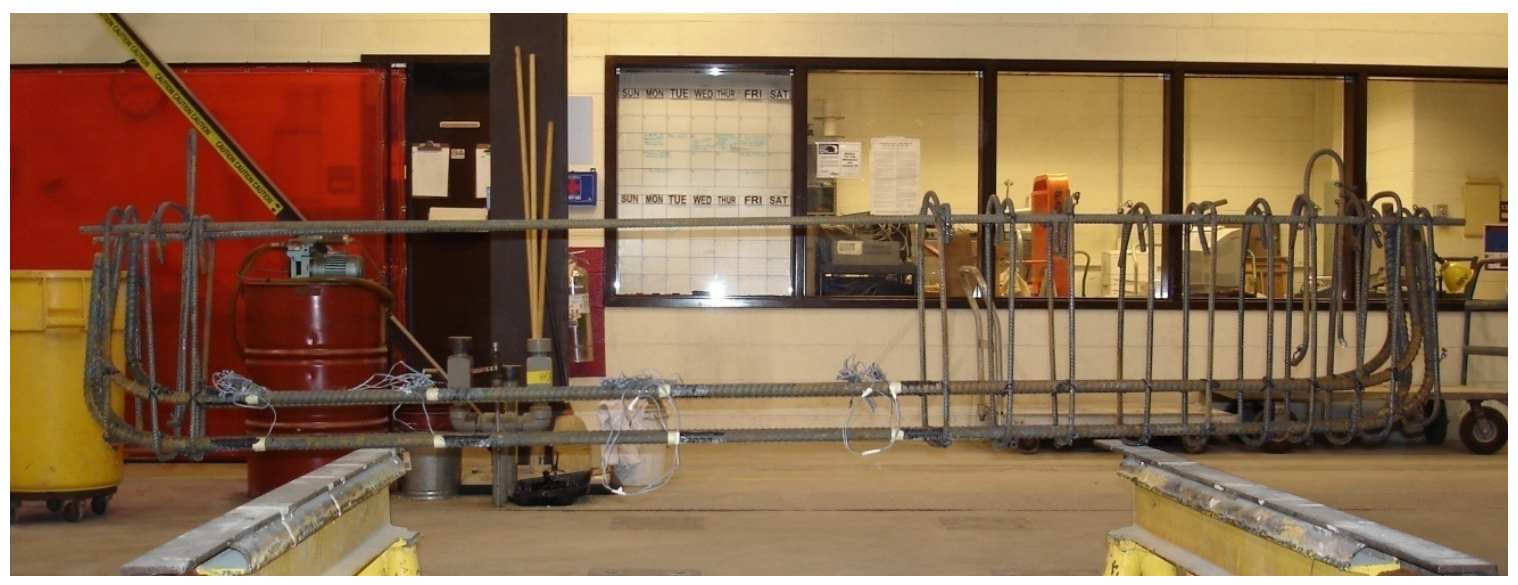

(a) Reinforcing cage ready for placement

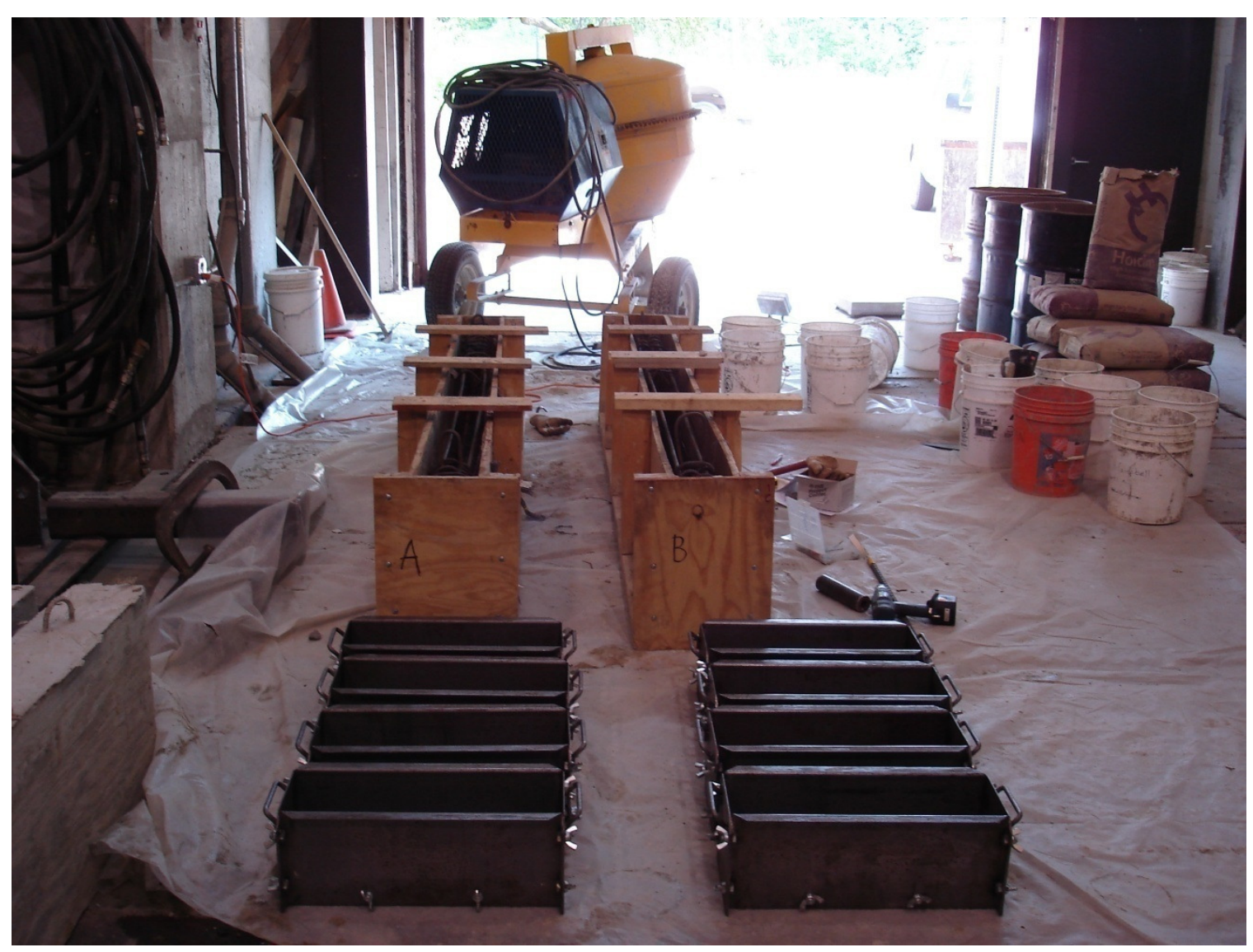

(b) Laboratory site ready for casting

Fig. 3-5: Reinforcing cage ready for placement and laboratory site ready for casting 


\subsection{PROPORTIONING AND MIXING OF SFRC - CASTING AND CURING OF BEAM SPECIMENS}

Proportioning SFRC. Concrete mix IDs for all beams are listed in Table 3-1. In the table, concrete mixes prefixed by LAB or SUP refer to concrete mixed at The University of Michigan Structural Engineering Laboratory or provided by a local concrete supplier, respectively. Detailed concrete mix proportions are provided in Table 3-7.

Table 3-7: Concrete mix proportions

\begin{tabular}{ccccc}
\hline \multirow{2}{*}{ Mix ID } & \multicolumn{4}{c}{ Proportion by weight } \\
\cline { 2 - 5 } & Concrete $(*)$ & Water & Aggregate $(\dagger)$ & Sand $(\ddagger)$ \\
\hline LAB1 & 1 & 0.48 & 1.45 & 1.55 \\
SUP1 & 1 & 0.48 & 2.49 & 2.00 \\
SUP2 & 1 & 0.39 & 3.27 & 2.94 \\
SUP3 & NA & NA & NA & NA \\
\hline
\end{tabular}

(*): High early strength cement type III was used for Mix LAB1, while cement type I was used for mixes SUP1, SUP2, and SUP3.

$(\dagger)$ : Crushed limestone Grade 26A with a maximum aggregate size of 3/8 in., conforming to MDOT "Standard Specification for Construction", was used for all mixes.

(†): Natural sand Grade 2NS, conforming to MDOT “Standard Specification for Construction”, was used for all mixes.

Mixing SFRC. For concrete mixed at The University of Michigan Structural Engineering Laboratory, cement and sand were mixed until a uniform color was seen throughout the mix. Coarse aggregates were then thrown in the mixing drum and mixed until they blended well with the cement and sand. Water was next gradually poured into the mixing drum and mixed until reaching a uniform consistency. Similar to the inclusion of water, steel fibers were added to the rotating drum in a gradual manner until they were well-dispersed in the fresh concrete. In several cases, a high-range water reducer was added to obtain the desired workability.

For concrete provided by a local concrete supplier, all required steel fibers were dumped into the truck mixing drum and mixed until a uniform distribution of the fibers 
was attained. Water was often added for better workability. According to the local concrete supplier, the amount of materials in each batch was less accurate than that mixed at the University of Michigan laboratory because the materials were dropped onto the scale from a certain height. This affected the accuracy of the scale reading.

Casting beam specimens. Concrete was poured and compacted layer by layer. Each layer had a thickness of approximately 5 inches. Even though good workability was obtained for all mixes, fiber congestion was observed in the regions with clear spacing between reinforcing bars substantially less than the fiber length. Fiber congestion was more pronounced for concrete mixed with longer fibers RC80/60BN (2.36 inches). Due to this problem, three beams had voids (Fig. 3-6). The beams were subsequently repaired by pouring high strength grout into the voids. The beams were positioned such that the voids would retain the fresh grout liquid before it hardened.

Curing beam specimens. After casting, all beam specimens were moist-cured and covered with plastic sheets. Beams were demolded at the age of seven days and aircured in the laboratory until being tested. 


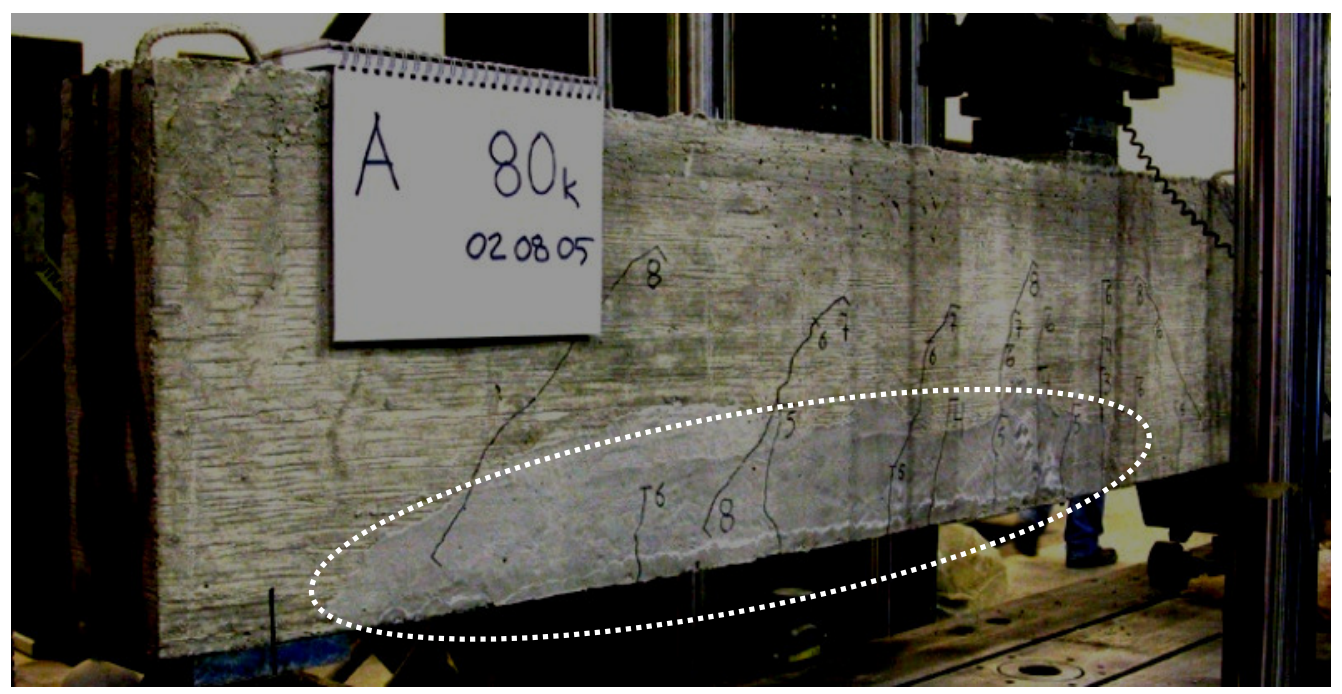

(a) Beam B18-2b - using ZP305 fibers (1.18 inches long)

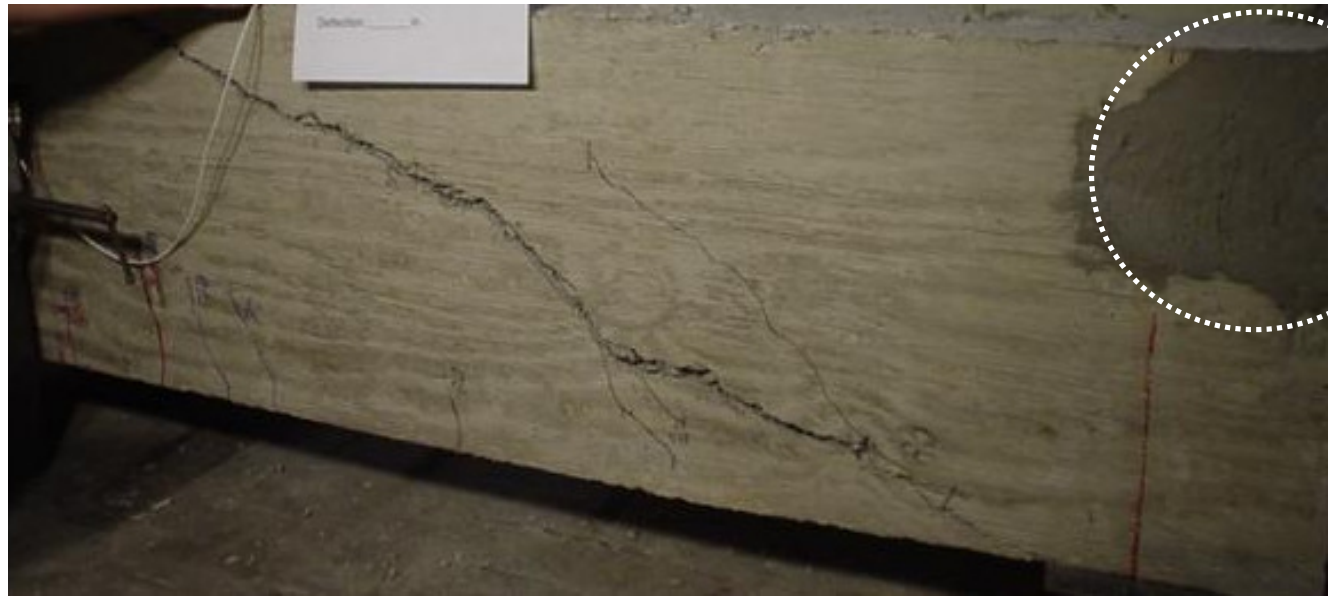

(b) Beam B18-3a - using ZP305 fibers (1.18 inches long)

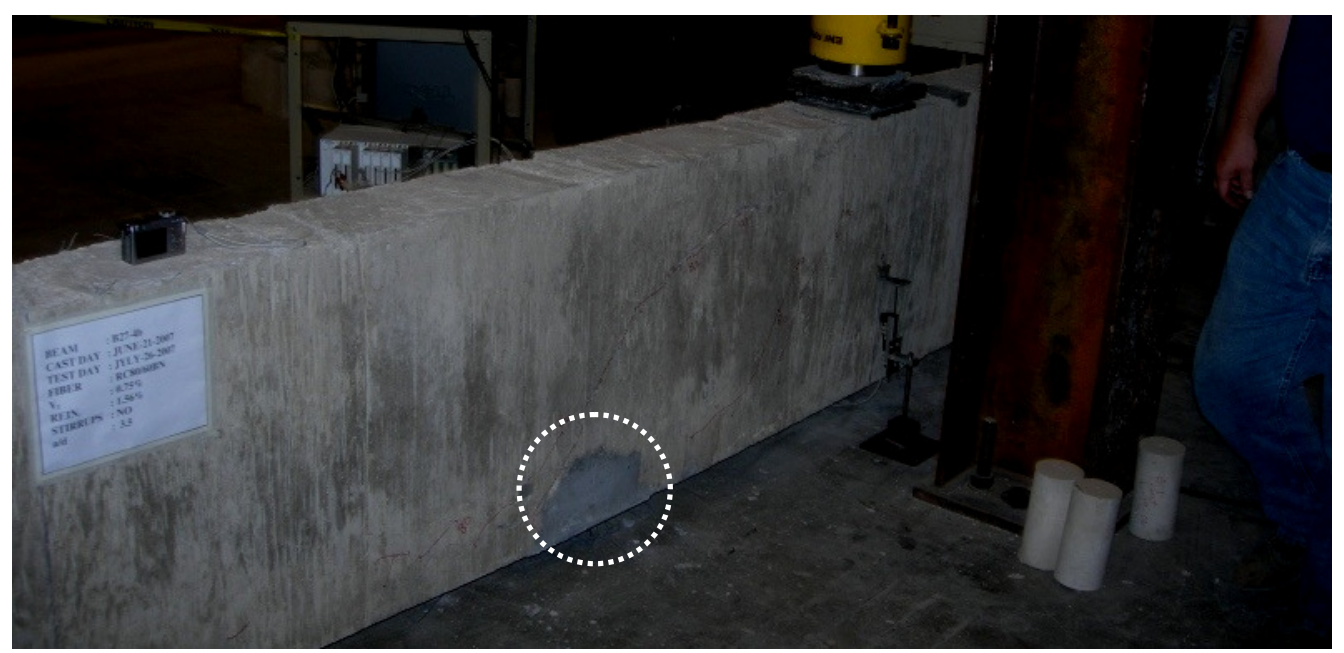

(c) Beam B27-4b - using RC80/60BN fibers (2.36 inches long)

Fig. 3-6: Beams with voids that required repair 


\subsection{INSTRUMENTATION AND TESING}

Potentiometers. In addition to strain gauges, described in Section 3.3, two linear potentiometers were installed under the loading point of each beam to measure its deflection (see Fig. 3-10, Fig. 3-11, and Fig. 3-12). It should be noted that the potentiometer tip was in contact with an aluminum angle glued to the beam with an epoxy resin.

Markers. To record the exact position of various points of interest, an active infrared optical position tracking system - OptoTRAK Certus - was used. Fig. 3-7 illustrates the key devices and their connections for the system. In this system, a light emitting diode (marker) emits infrared light at a certain frequency (marker frequency) controlled by a strober. If the infrared light is detected by the high resolution sensors, the three dimensional position of the marker is then calculated through triangulation. The marker frequency can be controlled by a computer program (NDI First Principle) through a system unit controller and a strober. Maximum sampling rate depends on the number of markers used and their programmed frequency. An OptoTRAK Certus system allows a maximum number of 512 markers (OptoTRAK Certus User Guide, 2005).

In order to be accurately detected, the marker has to be in the characteristic volume of the OptoTRAK system. Fig. 3-8 shows the characteristic volume of the OptoTRAK Certus close-focus system used in this experimental program. If the marker is placed beyond the characteristic volume (in the z-direction), the accuracy is unknown. In general, the nearer the markers are to the position sensors, the more accurate is the reading of their coordinates. Any marker within the characteristic volume and on a plane whose distance to the sensors is $2.25 \mathrm{~m}$ has an accuracy of 0.00394 inches $(0.1 \mathrm{~mm})$ for its $\mathrm{x}$ - or $\mathrm{y}$ - coordinates and 0.00591 inches $(0.15 \mathrm{~mm})$ for its $\mathrm{z}$ - coordinate (OptoTRAK Certus User Guide, 2005).

To measure the strain field on the face of the test beams, a grid of markers was attached onto the tested span using thermoplastic adhesives (hot-melt glue). Fig. 3-9 shows the arrangement of markers on beam Series B18 and B27. It should be noted that

markers were installed for only Beams B18-1a \& b and B18-7a \& b in Series B18 
because the OptoTRAK system was only available for the later phase of the research program.

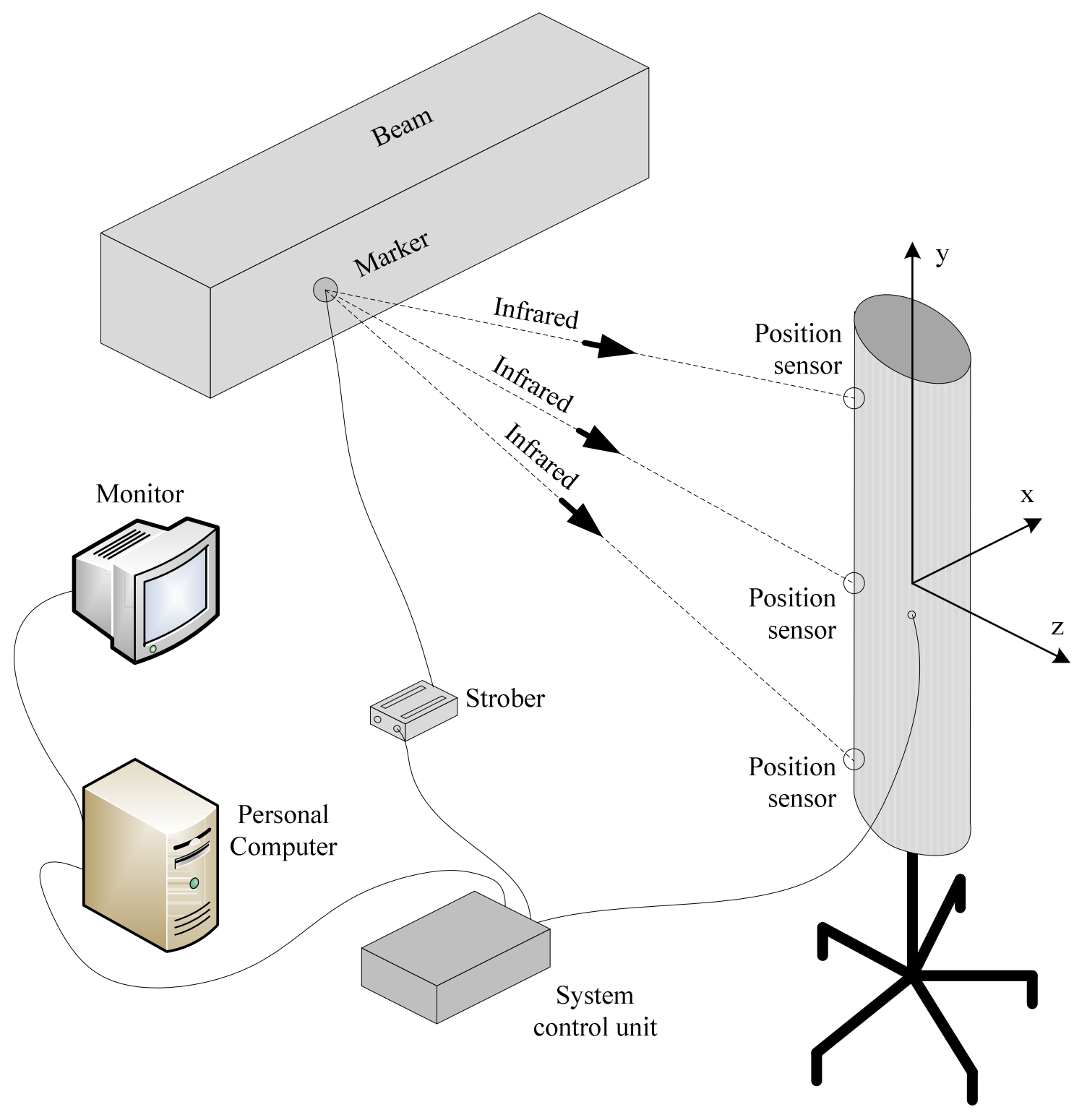

Fig. 3-7: Devices in an active infrared optical tracking system 


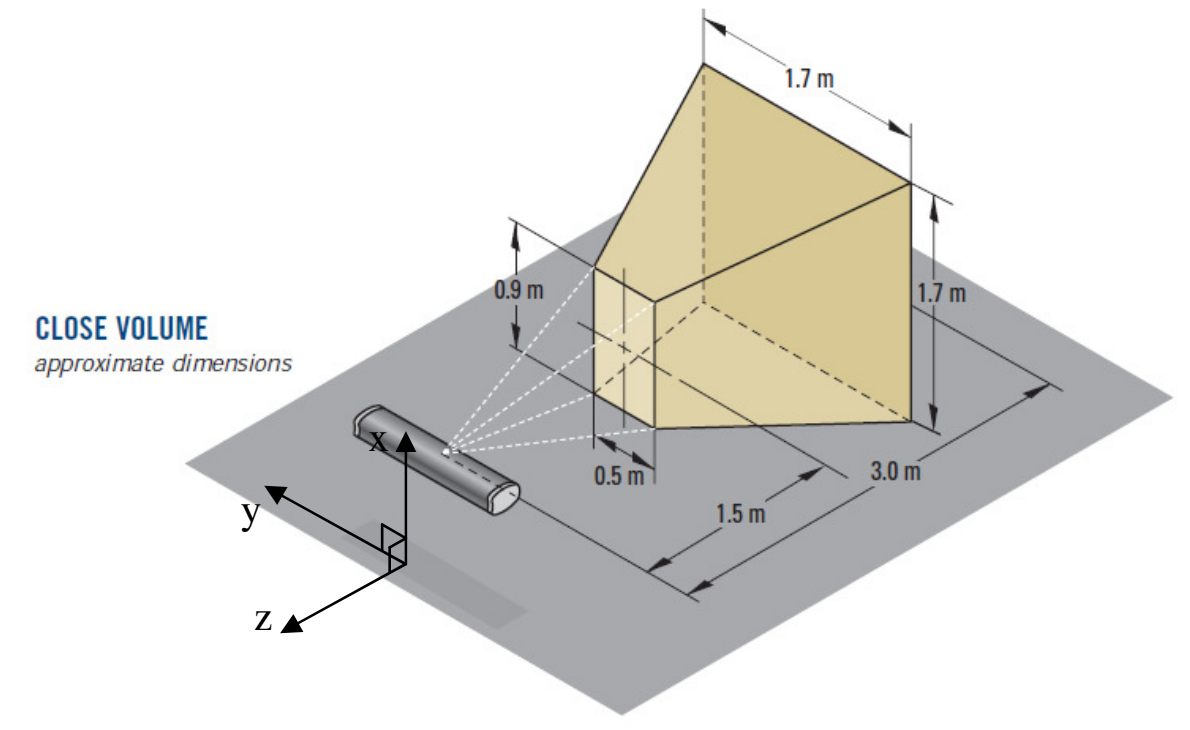

Fig. 3-8: Characteristic volume of close-focus OptoTRAK Certus sensors (from OptoTRAK Certus User Guide, 2005)

(a): Arrangement of markers on beams B18-1a, b; and B18-7a, b

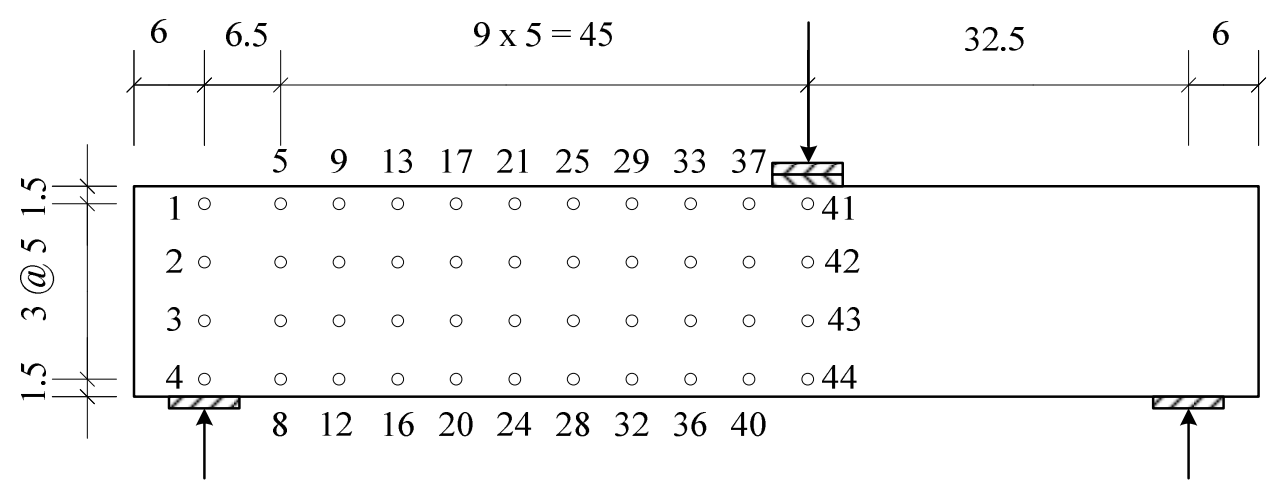

(b): Arrangement of markers on all beams in Series B27

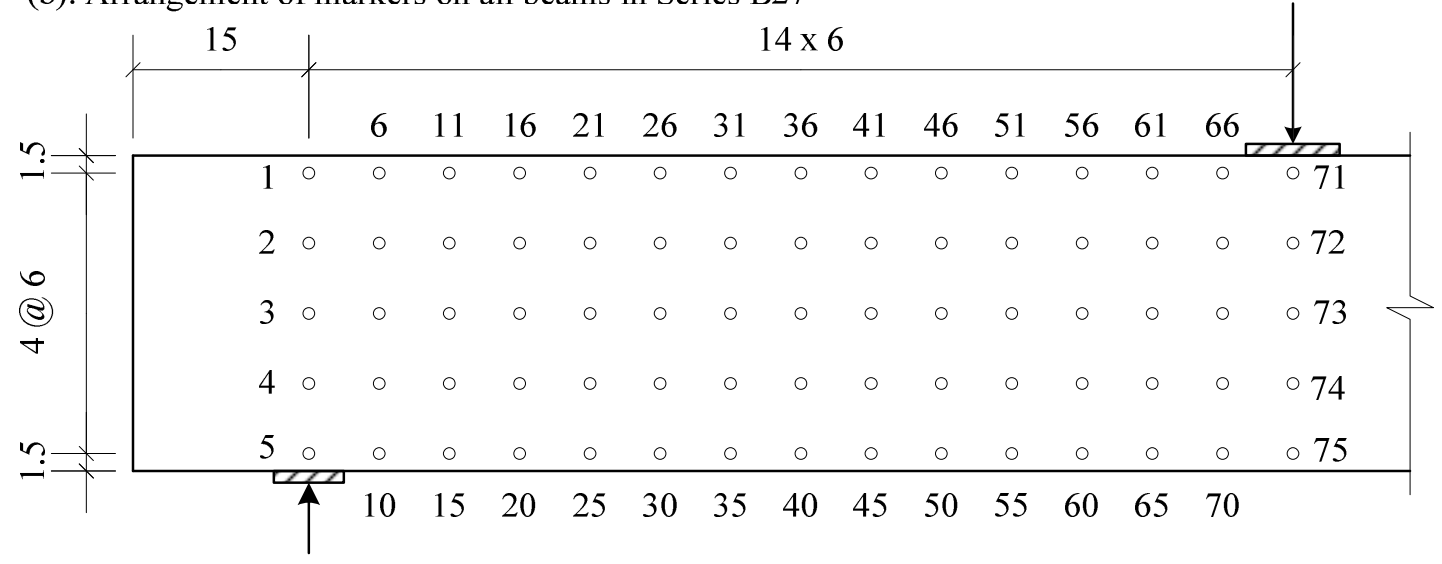

Fig. 3-9: Typical arrangement of markers on beams 
Loading devices. Test setups were selected based on the predicted strength of the beams. Three different test setups - INS115, ACT235, and CYL500 - were used, as shown in Fig. 3-10, Fig. 3-11, and Fig. 3-12, respectively. INS115 is an Instron servohydraulic closed-loop testing machine with a 115-kip loading capacity. In this system, the force and motion of the machine hydraulic actuator are measured, respectively, by the machine load cell and linear variable differential transformer (LVDT) integrated in the machine. ACT235 is a hydraulic actuator with a 235-kip loading capacity connected to a reaction frame. Its force is measured by an external 500-kip load cell placed between the beam and the actuator (see Fig. 3-11). CYL500 is an ENERPAC single-acting high tonnage hydraulic cylinder with a 500-kip loading capacity and 5.91-inch stroke. This hydraulic cylinder is pressurized by an ENERPAC submerged electric pump with an oil flow rate at $20 \mathrm{in.} . / \mathrm{min}$ at a rated pressure of 1000 to $10,000 \mathrm{psi}$ (Hydraulic Power for all Industrial Applications, Enerpac, 2001). Similar to the case of ACT235, a 500-kip load cell was used to measure the applied load. It should be repeated that in all cases, the beam deflection was measured by means of linear potentiometers. Because multiple instrumentations were used, Table 3-8 lists the instrumentation used in each beam.

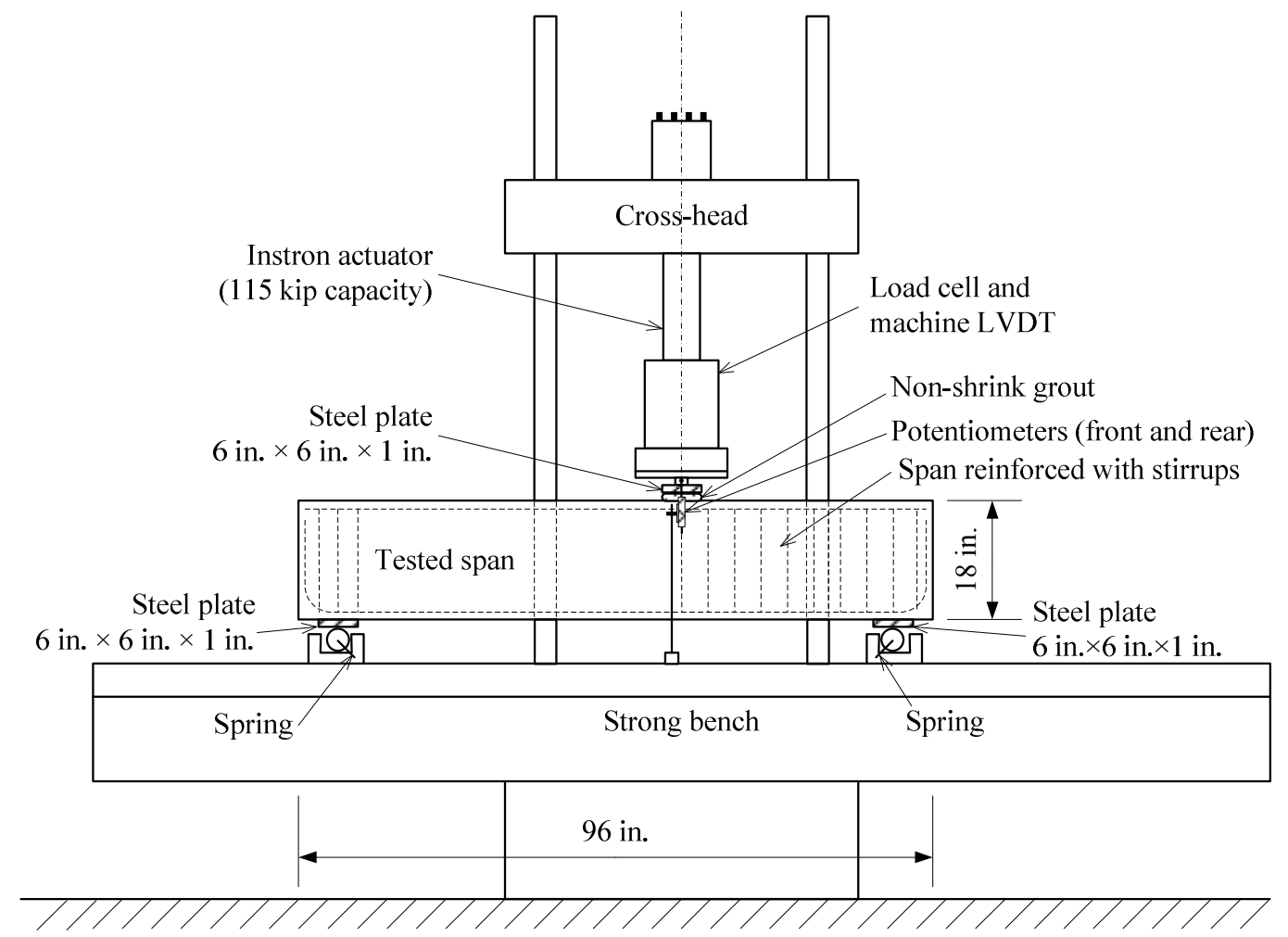

Fig. 3-10: Test setup for beams in Series B18 with 115-kip Instron machine (INS115) 


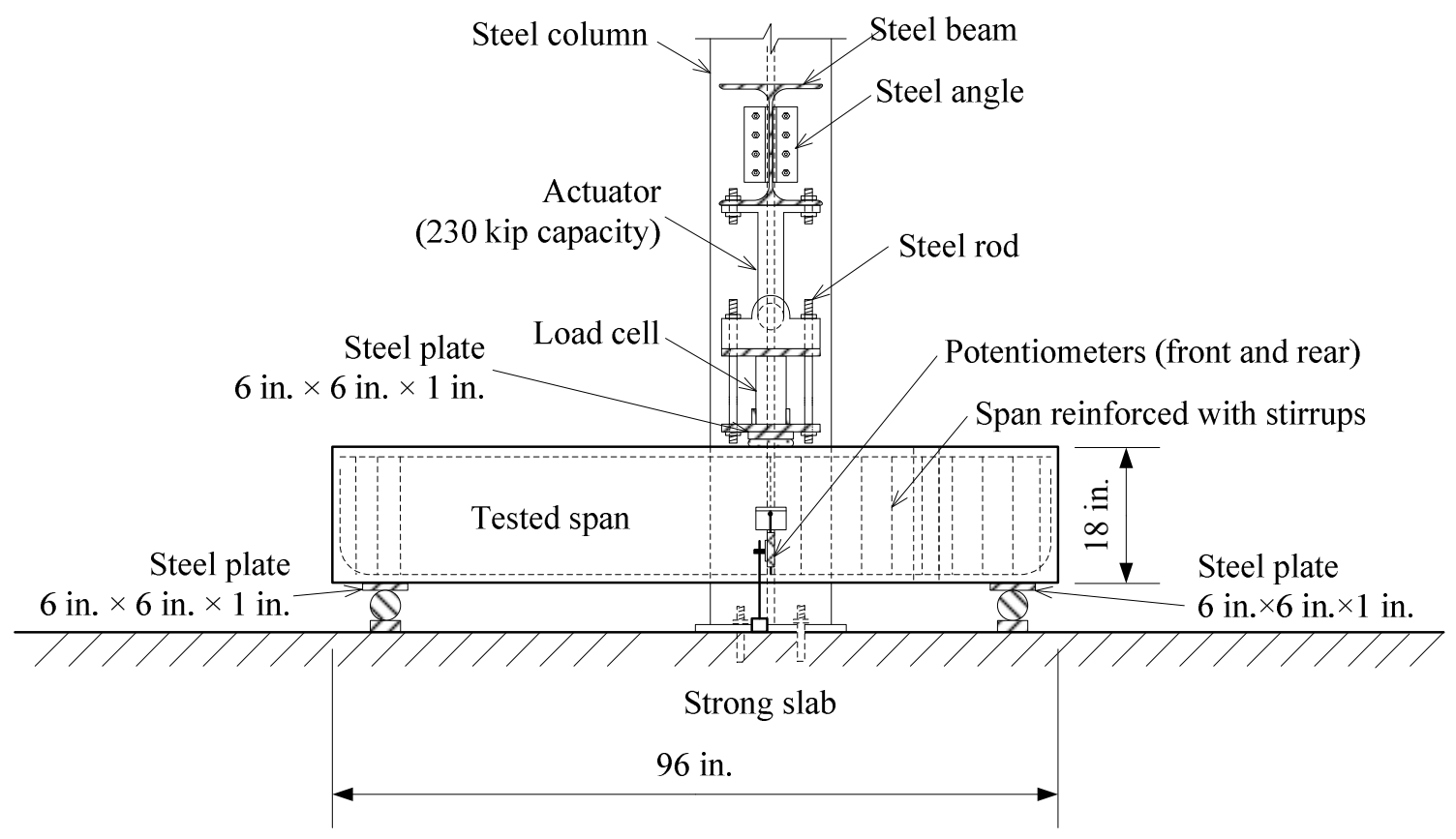

Fig. 3-11: Test set-up for beams in Series B18 with 235-kip hydraulic actuator (ACT235)

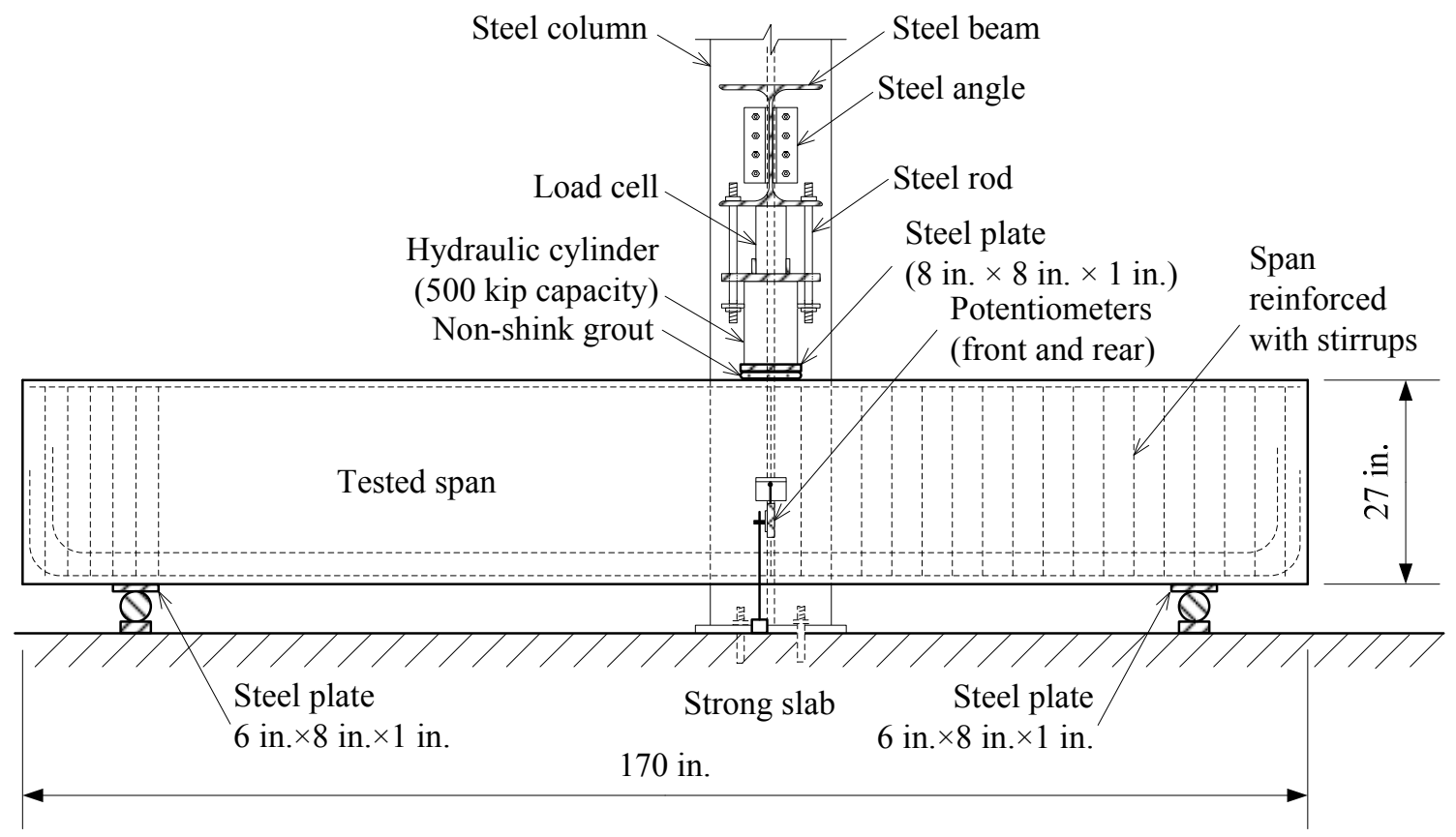

Fig. 3-12: Test setup for beams in Series B27 with 500-kip hydraulic cylinder (CYL500) 
Table 3-8: List of instrumentation

\begin{tabular}{ccccc}
\hline Beam & $\begin{array}{c}\text { Strain gauge } \\
(\mathrm{SG})\end{array}$ & Potentiometer (P) & Marker (M) & Loading devices \\
\hline B18-0a, b & NA & NA & NA & INS115 \\
B18-1a, b & 8 SG & 2 P & $44 \mathrm{M}$ & INS115 \\
B18-2a, b & 8 SG & NA & NA & INS115 \\
B18-2c, d & 8 SG & NA & NA & INS115 \\
B18-3a, b & 8 SG & 2 P & NA & ACT235 \\
B18-3c, d & 8 SG & 2 P & NA & ACT235 \\
B18-5a, b & 8 SG & 2 P & NA & ACT235 \\
B18-7a, b & 8 SG & 2 P & $44 \mathrm{M}$ & INS115 \\
B27-1a, b & $10 \mathrm{SG}$ & 2 P & $75 \mathrm{M}$ & CYL500 \\
B27-2a, b & $10 \mathrm{SG}$ & $2 \mathrm{P}$ & $75 \mathrm{M}$ & CYL500 \\
B27-3a, b & $10 \mathrm{SG}$ & $2 \mathrm{P}$ & $75 \mathrm{M}$ & CYL500 \\
B27-4a, b & $10 \mathrm{SG}$ & $2 \mathrm{P}$ & $75 \mathrm{M}$ & CYL500 \\
B27-5 & $10 \mathrm{SG}$ & $2 \mathrm{P}$ & $75 \mathrm{M}$ & CYL500 \\
B27-6 & $10 \mathrm{SG}$ & $2 \mathrm{P}$ & $75 \mathrm{M}$ & CYL500 \\
B27-7 & $10 \mathrm{SG}$ & $2 \mathrm{P}$ & $75 \mathrm{M}$ & CYL500 \\
B27-8 & $10 \mathrm{SG}$ & $2 \mathrm{P}$ & $75 \mathrm{M}$ & CYL500 \\
\hline
\end{tabular}




\subsection{MATERIAL TESTING AND PROPERTIES}

\subsubsection{Reinforcing bars}

Testing of reinforcing bars. Tensile stress-strain relationships for the reinforcing bars used in the research were obtained by direct tensile testing following ASTM A370. For each reinforcing bar diameter, three 24-inch long coupons were tested using a hydraulic testing machine. In this system, strain was measured by an MTS extensometer.

Test results. Fig. 3-13 shows the test results and Table 3-9 lists the yield strength $\left(f_{y}\right)$ and ultimate strength $\left(f_{s u}\right)$, as well as strains at the beginning and at the end of the yield plateau ( $\varepsilon_{y}$ and $\varepsilon_{s h}$, respectively). For the \#D4 and \#3 bars, which did not have a clear yield plateau, the yield strength was determined based on the intersection between the stress-strain curve and a $0.2 \%$-offset line parallel to the ascending linear branch.

Table 3-9: Properties of reinforcing bars

\begin{tabular}{ccccccc}
\hline Bar & Area (in. $\left.{ }^{2}\right)$ & $\varepsilon_{y}$ & $f(\mathrm{ksi})$ & $\varepsilon_{z}(\mathrm{ksi})$ & $\varepsilon_{s h}$ & $f_{s u}(\mathrm{ksi})$ \\
\hline \# D4 & 0.04 & - & 91 & 28,000 & - & 96 \\
$\# 3$ & 0.11 & - & 60 & 29,000 & - & 84 \\
$\# 4$ & 0.20 & 0.00239 & 67 & 28,000 & 0.0080 & 100 \\
$\# 6$ & 0.44 & 0.00257 & 72 & 28,000 & 0.0090 & 109 \\
$\# 7$ & 0.60 & 0.00232 & 65 & 28,000 & 0.0080 & 98 \\
$\# 8$ & 0.79 & 0.00236 & 66 & 28,000 & 0.0080 & 100 \\
\hline
\end{tabular}



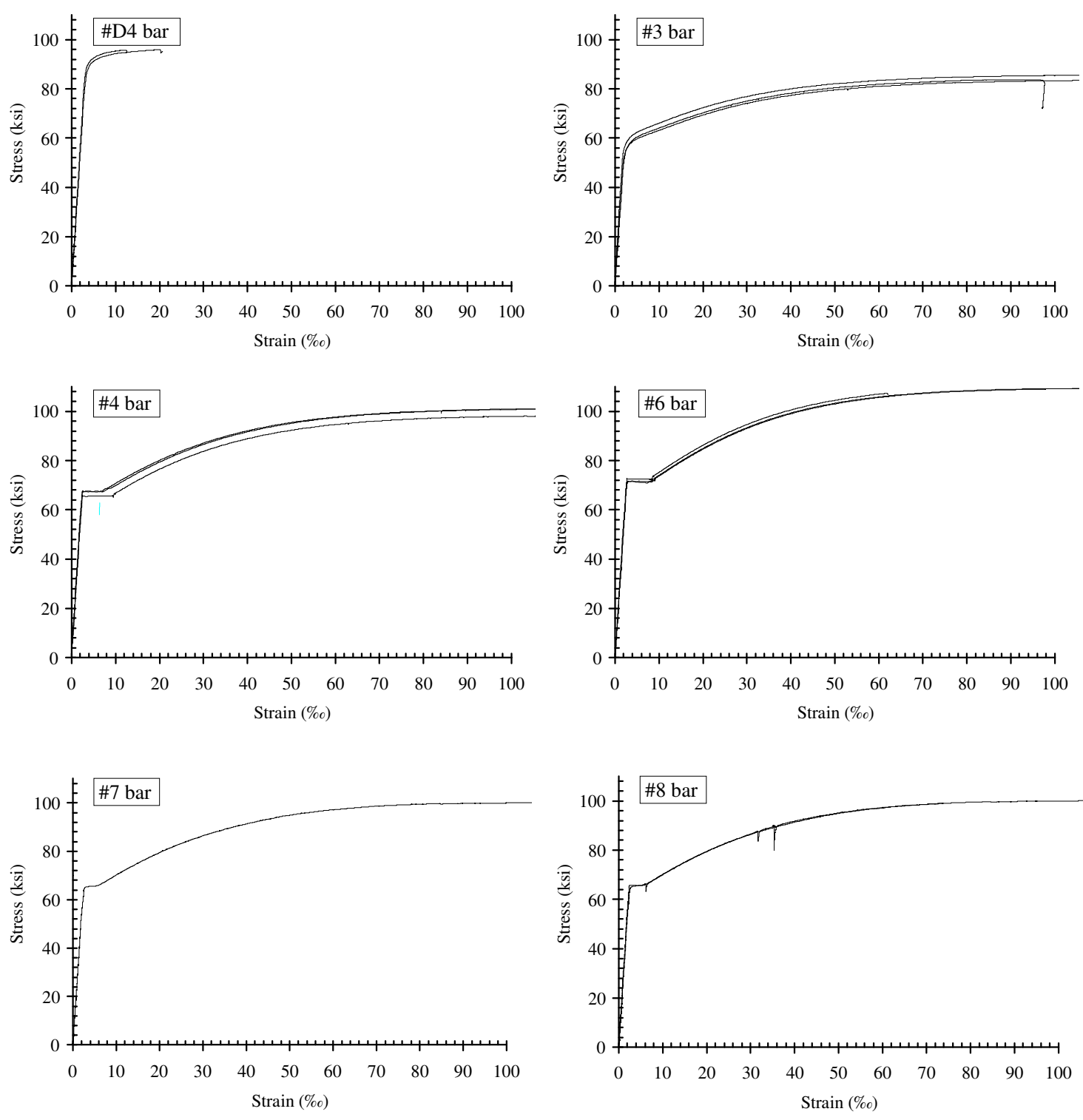

Fig. 3-13: Stress versus strain relationships of reinforcing bars 


\subsubsection{SFRC compressive strength}

Casting, curing, capping, and testing of cylindrical concrete specimens. For each pair of beam specimens or single beam specimen, at least three cylindrical specimens with a size of 4 inches in diameter and 8 inches high were cast. Given the size of the fibers used, a cylinder 6 inches in diameter and 12 inches in high would have been required to ensure a fiber distribution similar to that in the beam specimens. However, because the main intention of the cylinder tests was to determine compressive strength which is not significantly affected by the presence of fibers, the use of a smaller cylinder size was believed to be adequate. Each set of cylinders was sampled following ASTM C172 (1999), compacted, and cured following ASTM C31/C31M. The specimens were covered with a lid or a plastic sheet and left in the laboratory for one day. They were demolded the next day and immersed in a water tank for curing until being tested. The cylinders were pulled out of the tank approximately two days prior to their test date. To ensure that the cylinders were uniformly and axially loaded, the ends of the cylinders were capped with a sulfur compound such that the two capped planes at the two ends were parallel to each other. The capping process followed ASTM C617/C617M (2003). The cylinders were tested using an Instron hydraulic testing machine and following ASTM C39/C39M (2003), which stipulates a loading rate of approximately 0.05 inches per minute.

Test results. Table 3-10 reports the concrete compressive strength for cylinders corresponding to all the test beams. 
Table 3-10: Cylinder compressive strengths

\begin{tabular}{|c|c|c|c|c|c|c|c|c|c|}
\hline \multirow{2}{*}{ Beam } & \multicolumn{7}{|c|}{$f_{c}^{\prime}(\mathrm{psi})$} & \multirow[b]{2}{*}{ Mean } & \multirow{2}{*}{$\begin{array}{l}\text { Coefficient } \\
\text { of variation }\end{array}$} \\
\hline & $\mathrm{C \# 1}$ & $\mathrm{C \# 2}$ & $\mathrm{C \# 3}$ & $\mathrm{C \# 4}$ & $\mathrm{C \# 5}$ & $\mathrm{C \# 6}$ & $\mathrm{C \# 7}$ & & \\
\hline B18-0a, b & 5860 & 6,080 & 6,700 & - & - & - & - & 6210 & 0.070 \\
\hline B18-1a, b & 7490 & 6250 & 6620 & 5640 & - & - & - & 6500 & 0.119 \\
\hline B18-2a, b & 5900 & 5660 & 5690 & 4750 & 5700 & 5490 & - & 5530 & 0.073 \\
\hline B18-2c, d & 5900 & 5660 & 5690 & 4750 & 5700 & 5490 & - & 5530 & 0.073 \\
\hline B18-3a, b & 4740 & 4310 & 4460 & - & - & - & - & 4500 & 0.048 \\
\hline $\mathrm{B} 18-3 \mathrm{c}, \mathrm{d}$ & 7690 & 5290 & 6590 & - & - & - & - & 6520 & 0.184 \\
\hline B18-5a, b & 7640 & 6800 & 7950 & 7210 & 7580 & 6920 & 5910 & 7140 & 0.095 \\
\hline B18-7a, b & 6350 & 6080 & 7090 & 5830 & 6080 & - & - & 6290 & 0.077 \\
\hline B27-1a, b & 7610 & 7580 & 6930 & - & - & - & - & 7370 & 0.052 \\
\hline B27-2a, b & 3820 & 4480 & 4340 & 4040 & - & - & - & 4170 & 0.071 \\
\hline B27-3a, b & 6180 & 6370 & 5880 & - & - & - & - & 6140 & 0.040 \\
\hline B27-4a, b & 4310 & 4270 & 4230 & 4340 & - & - & - & 4290 & 0.011 \\
\hline B27-5 & 6430 & 6150 & 6780 & - & - & - & - & 6450 & 0.049 \\
\hline B27-6 & 6330 & 6250 & 6060 & 6370 & 6320 & 5920 & - & 6210 & 0.029 \\
\hline B27-7 & 5120 & 5160 & 5320 & 5560 & 5680 & - & - & 5370 & 4.7 \\
\hline B27-8 & 5120 & 5160 & 5320 & 5560 & 5680 & - & - & 5370 & 4.7 \\
\hline
\end{tabular}




\subsubsection{SFRC flexural strength}

Casting, curing, and testing of beam specimens. Similar to cylinder tests, at least three beam specimens were sampled for each pair of beams (or single beam). The beam specimens had dimensions of 6 inches $\times 6$ inches $\times 20$ inches. Each beam specimen was sampled following ASTM C172, compacted and cured following ASTM C31/C31M. Each specimen was covered with a plastic sheet for one day in the laboratory before being demolded and cured in a water tank. The beam specimens were tested at the age of 28 days following ASTM C1609 (2006). The four-point bending test setup is shown in Fig. 3-14. The beams were loaded at a rate of 0.005 inches per second. The beam midspan deflection was measured by a pair of linear potentiometers attached to a frame such that a net midspan deflection would be measured, according to ASTM C1609. The test was terminated at a deflection of 0.12 inches, equivalent to $1 / 150$ of the span length (18 inches).
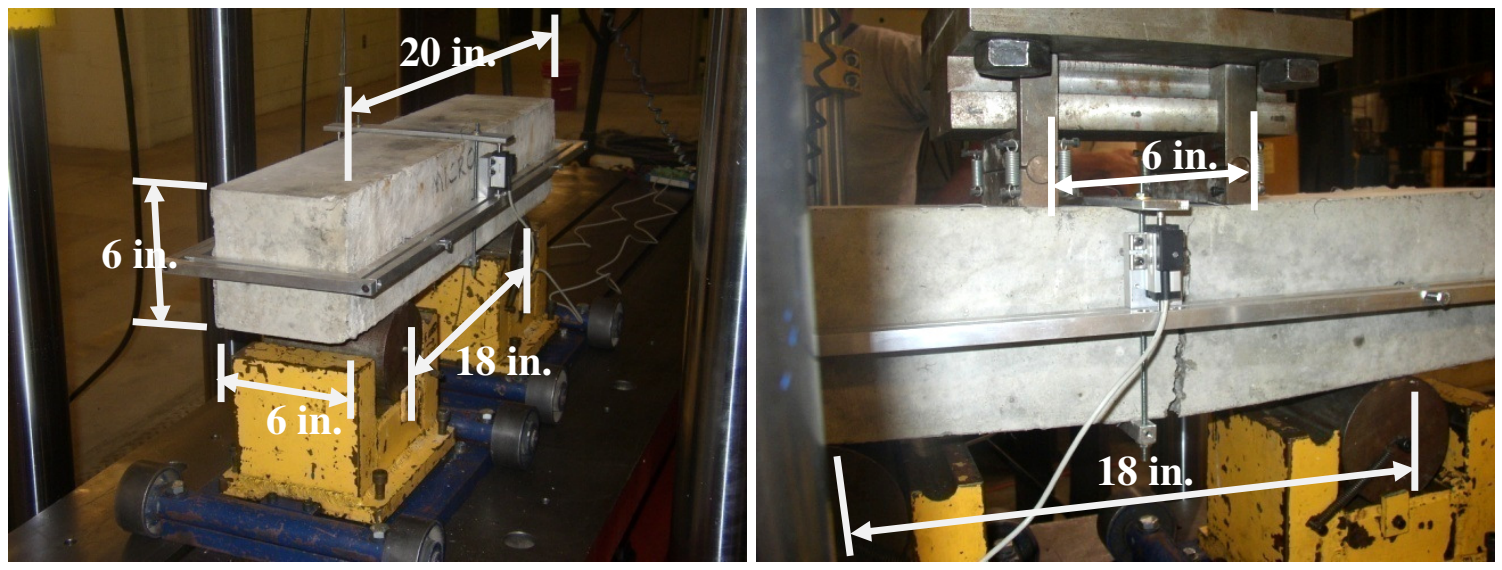

Fig. 3-14: Test setup for four-point bending tests

Location of flexural cracks and depth of the compression region. When the net midspan deflection of the beam reached 0.12 inches (1/150 of the span length), the test was stopped. The compression region depth, $c$, crack width at the bottom face, $w$, and the largest distance from the crack location to a support, $a$, were measured. Table 3-11 lists the information. The distribution of these parameters is shown in Fig. 3-15. As seen in in the figure, the compression region depth ranged from 4 to $17 \%$ of the specimen 
depth, with an average of 0.53 inches. The average crack width was 0.2 inches. It should be noted that the measurement of crack width is subject to errors because some concrete spalling at the tip of the crack can increase the measured crack width. The crack location fell well within the range from 6 inches to 12 inches - the region of constant and maximum moment. This means that the specimens failed due to flexure, without any shear influence.
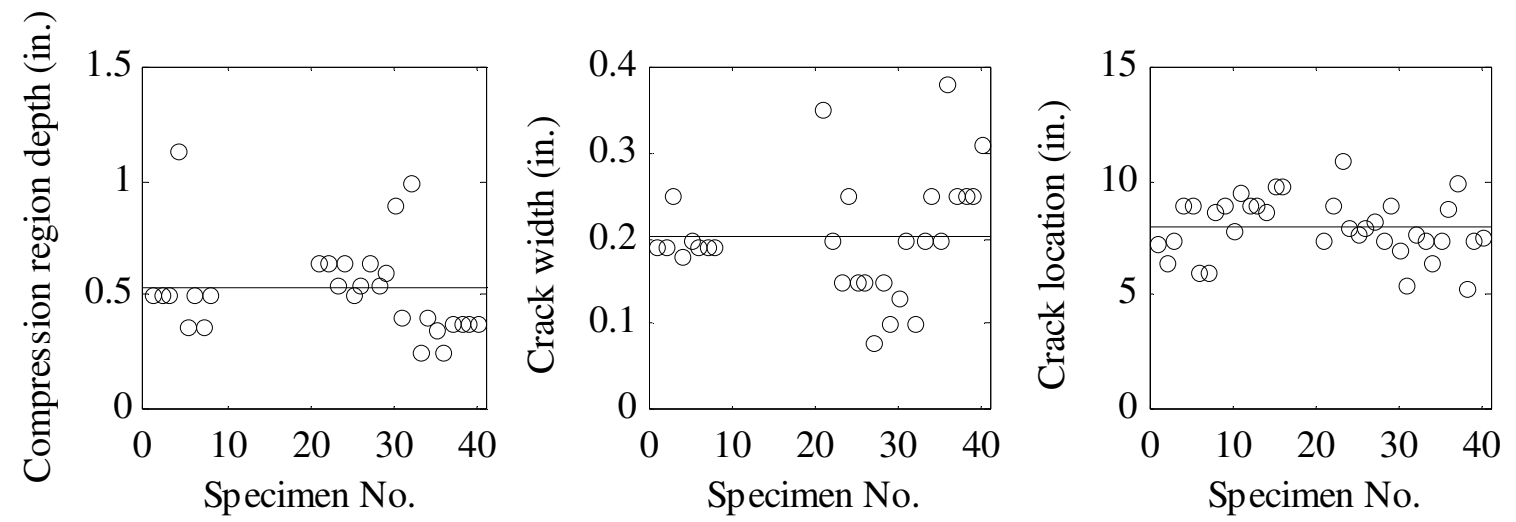

Fig. 3-15: Distribution of compression region depth, crack width, and crack location at a 0.12 in. (1/150 of span length) midspan deflection 
Table 3-11: Information of specimens at a deflection of 0.12 inches

\begin{tabular}{|c|c|c|c|c|c|c|}
\hline Beam & $\begin{array}{c}\text { Specimen } \\
\text { No. }\end{array}$ & $\begin{array}{l}\text { Fiber } \\
\text { type }\end{array}$ & $\begin{array}{l}\mathrm{V}_{\mathrm{f}} \\
(\%)\end{array}$ & $\begin{array}{c}\mathrm{c} \\
\text { (in.) }\end{array}$ & $\begin{array}{l}\mathrm{w} \\
\text { (in.) }\end{array}$ & $\begin{array}{l}\mathrm{a} \\
\text { (in.) }\end{array}$ \\
\hline B18-1 & 1 & ZP305 & 0.75 & 0.50 & 0.19 & 10.8 \\
\hline B18-1 & 2 & ZP305 & 0.75 & 0.50 & 0.19 & 11.5 \\
\hline B18-1 & 3 & ZP305 & 0.75 & 0.50 & 0.25 & 10.5 \\
\hline B18-1 & 4 & ZP305 & 0.75 & 1.13 & 0.18 & 9.0 \\
\hline B18-1 & 5.1 & ZP305 & 0.75 & 0.36 & 0.20 & 9.0 \\
\hline B18-1 & 5.2 & ZP305 & 0.75 & 0.50 & 0.19 & 12.0 \\
\hline B18-1 & 5.3 & ZP305 & 0.75 & 0.36 & 0.19 & 12.0 \\
\hline B18-1 & 5.4 & ZP305 & 0.75 & 0.50 & 0.19 & 9.3 \\
\hline B27-1 & 1 & ZP305 & 0.75 & - & - & 9.0 \\
\hline B27-1 & 2 & ZP305 & 0.75 & - & - & 10.1 \\
\hline B27-1 & 3 & ZP305 & 0.75 & - & - & 9.0 \\
\hline B27-1 & 4 & ZP305 & 0.75 & - & - & 9.0 \\
\hline B27-3 & 1 & ZP305 & 0.75 & - & - & 9.0 \\
\hline B27-3 & 2 & ZP305 & 0.75 & - & - & 9.3 \\
\hline B27-3 & 3 & ZP305 & 0.75 & - & - & 9.0 \\
\hline B27-3 & 4 & ZP305 & 0.75 & - & - & 9.0 \\
\hline B27-2 & 1 & RC80/60BN & 0.75 & & & \\
\hline B27-2 & 2 & RC80/60BN & 0.75 & - & - & - \\
\hline B27-2 & 3 & RC80/60BN & 0.75 & - & - & - \\
\hline B27-2 & 4 & $\mathrm{RC} 80 / 60 \mathrm{BN}$ & 0.75 & - & - & - \\
\hline B27-4 & 1 & RC80/60BN & 0.75 & 0.65 & 0.35 & 10.5 \\
\hline B27-4 & 2 & RC80/60BN & 0.75 & 0.65 & 0.20 & 9.0 \\
\hline B27-4 & 3 & RC80/60BN & 0.75 & 0.55 & 0.15 & 9.0 \\
\hline B27-4 & 4 & $\mathrm{RC} 80 / 60 \mathrm{BN}$ & 0.75 & 0.65 & 0.25 & 10.0 \\
\hline B18-7 & 1 & RC80/30BP & 0.75 & 0.50 & 0.15 & 10.3 \\
\hline B18-7 & 2 & RC80/30BP & 0.75 & 0.55 & 0.15 & 10.0 \\
\hline B18-7 & 3 & RC80/30BP & 0.75 & 0.65 & 0.08 & 9.8 \\
\hline B18-7 & 4 & RC80/30BP & 0.75 & 0.55 & 0.15 & 10.5 \\
\hline B18-7 & 5.1 & RC80/30BP & 0.75 & 0.60 & 0.10 & 9.0 \\
\hline B18-7 & 5.2 & RC80/30BP & 0.75 & 0.90 & 0.13 & 11.0 \\
\hline B18-7 & 5.3 & $\mathrm{RC} 80 / 30 \mathrm{BP}$ & 0.75 & 0.40 & 0.20 & 12.5 \\
\hline B18-7 & 5.4 & RC80/30BP & 0.75 & 1.00 & 0.10 & 10.3 \\
\hline B27-5 & 1 & ZP305 & 1.50 & 0.25 & 0.38 & 9.1 \\
\hline B27-5 & 2 & ZP305 & 1.50 & 0.38 & 0.25 & 9.0 \\
\hline B27-6 & 1 & RC80/60BN & 1.50 & 0.38 & 0.25 & 12.6 \\
\hline B27-6 & 2 & RC80/60BN & 1.50 & 0.38 & 0.25 & 10.5 \\
\hline B27-6 & 3 & RC80/60BN & 1.50 & 0.38 & 0.31 & 10.4 \\
\hline
\end{tabular}


Equivalent bending stress versus deflection relationship. The load recorded for each specimen was converted to an equivalent bending stress by assuming a linear stress distribution. The maximum stress within a beam occurs at the extreme top and bottom beam fibers over the constant moment region. The conversion is as follows:

$$
\sigma=\frac{6 M}{b h^{2}}=\frac{6\left(\frac{P}{2} \times 6 \mathrm{in} .\right)}{6 \mathrm{in} . \times(6 \mathrm{in} .)^{2}}=\frac{P}{12}(\text { psi if } P \text { is in pounds) }
$$

The maximum equivalent bending stress versus midspan deflection relationships for all specimens where these data were available are plotted in Fig. 3-16. A summary of key results is presented in Table 3-12.
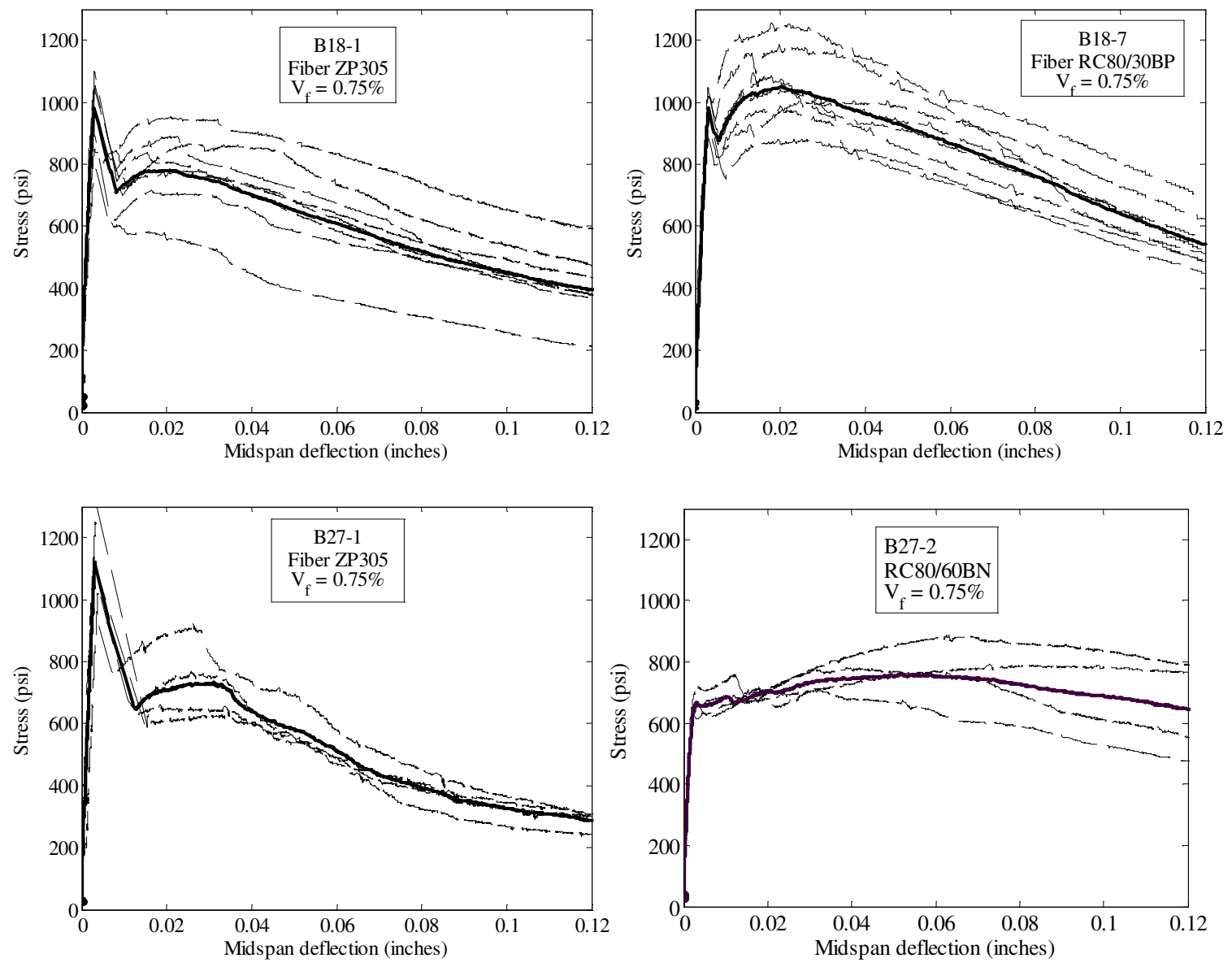

Fig. 3-16: Equivalent bending stress versus midspan deflection relationship from fourpoint bending tests 

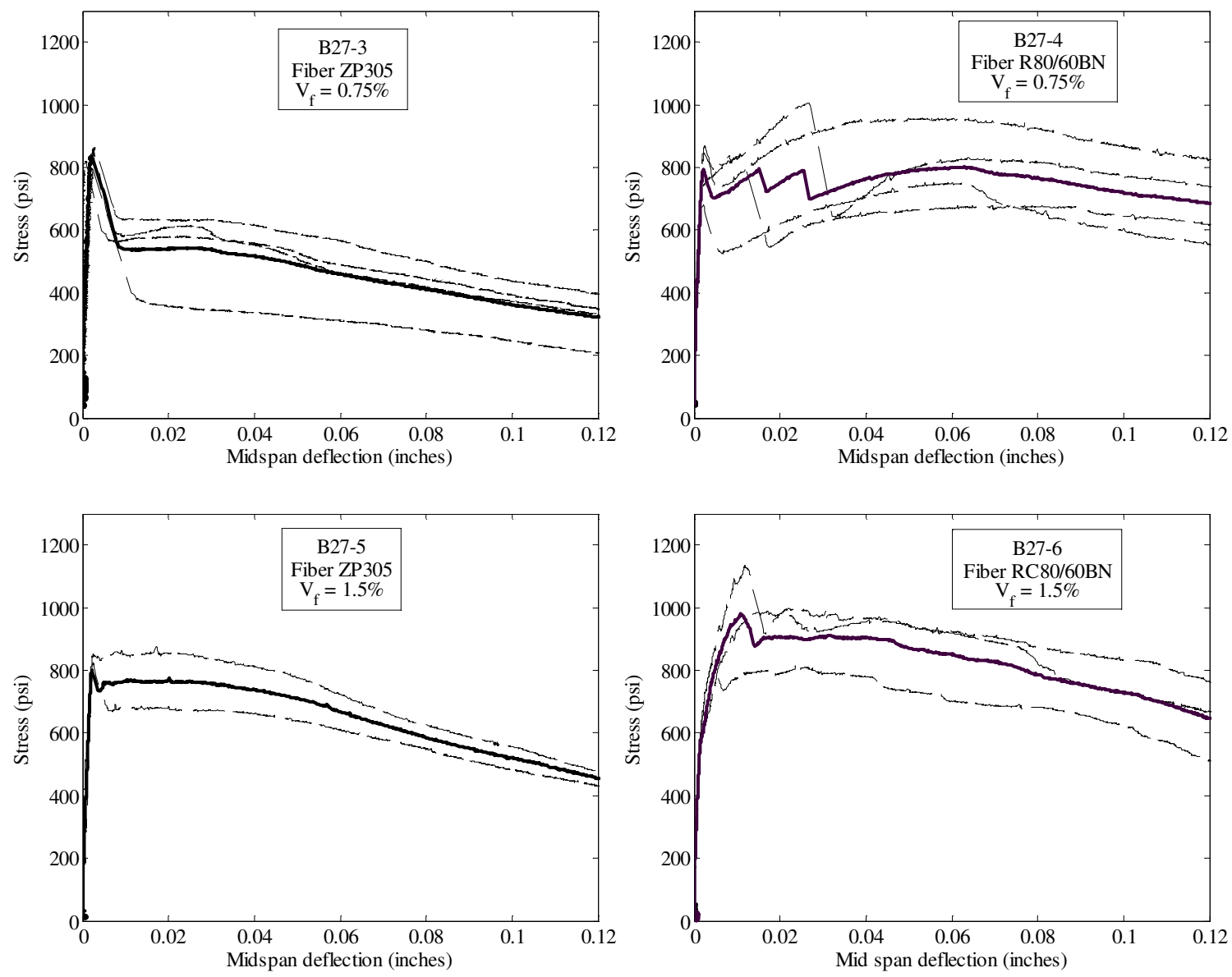

Fig. 3-16: Equivalent bending stress versus midspan deflection relationship from fourpoint bending tests (continued)

In Fig. 3-16, the light dashed curves show the measured test results from different specimens, while the bold continuous curve represents the average response. The averaging procedure is conceptually shown in Fig. 3-17. The average curve is generated by first calculating a deflection that is the mean of all deflections at first-crack stress $\left(\delta_{c}\right)$. The average stress corresponding to an intermediate point of a deflection $\delta_{i}$ between $\delta_{c}$ and the maximum deflection $\delta_{12}$ was then calculated by interpolating between the two nearest points on each curve. 


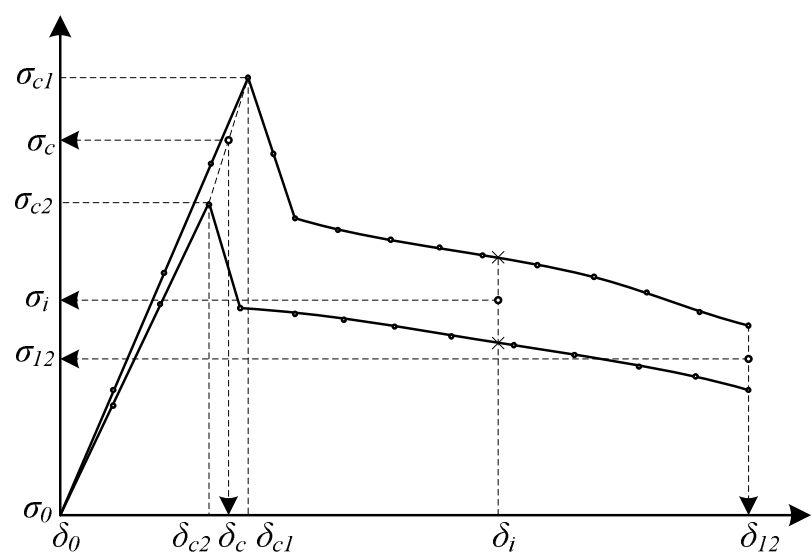

Fig. 3-17: Curve averaging procedure

From Fig. 3-16, it can be seen that the equivalent bending stress versus midspan deflection relationship is approximately linear up to first structural cracking, typically followed by a sudden drop in strength. After this point, the beam may behave in two different ways. In the first case, the beam gains some strength prior to steadily dropping its strength again, as schematically shown by Curve (1) in Fig. 3-18. In the second case, the strength drops continuously without any hardening, as shown by Curve (2) in Fig. 3-18. Important points of the stress-deflection relationship are also identified in Fig. 3-18, namely, first-crack stress $\left(\sigma_{c}\right)$, post-cracking peak stress $\left(\sigma_{p c}\right)$, stress at the deflection of 0.06 inches $\left(\sigma_{6}\right)$, and stress at the deflection of 0.12 inches $\left(\sigma_{12}\right)$ (end of the curve). If a specimen does not exhibit deflection hardening after the initial stress drop, the stress corresponding to the first point after cracking is considered to be the post-cracking peak stress $\left(\sigma_{p c}\right)$. These important stresses are listed in Table 3-12.

It can be seen from Fig. 3-16 that most specimens exhibited a deflection hardening after the initial stress drop, except for the specimens associated with Beams B27-3, B27-5, and B27-6, which showed a minor hardening. Specimens containing ZP305 and RC80/30BP fibers (1.18 inches in length) in a $0.75 \%$ volume fraction exhibited a significant drop in strength after first cracking. This was not observed in the specimens reinforced with RC80/60BN fibers (60 mm in length). 
Table 3-12: Important equivalent bending stresses

\begin{tabular}{|c|c|c|c|c|c|c|c|c|}
\hline Beam & $\begin{array}{l}\text { Spe. } \\
\text { No. }\end{array}$ & Fiber & $\begin{array}{c}\sigma_{c} \\
\text { (psi) }\end{array}$ & $\begin{array}{c}\sigma_{p c} \\
(\mathrm{psi})\end{array}$ & $\begin{array}{c}\sigma_{\sigma} \\
(\mathrm{psi})\end{array}$ & $\begin{array}{c}\sigma_{12} \\
(\mathrm{psi})\end{array}$ & $\begin{array}{c}\delta_{p c} \\
\text { (in.) }\end{array}$ & $\begin{array}{c}\text { Pass } \\
\text { ACI Code } \\
\text { Requirement }\end{array}$ \\
\hline B18-1 & 1 & ZP305 & 1055 & 785 & 638 & 437 & 0.0206 & $\mathrm{~N}$ \\
\hline B18-1 & 2 & ZP305 & 1100 & 838 & 585 & 378 & 0.0154 & $\mathrm{~N}$ \\
\hline B18-1 & 3 & ZP305 & 1011 & 782 & 626 & 384 & 0.0191 & $\mathrm{~N}$ \\
\hline B18-1 & 4 & ZP305 & 1015 & 953 & 831 & 591 & 0.0153 & $\mathrm{~N}$ \\
\hline B18-1 & 5.1 & ZP305 & 978 & 892 & 676 & 382 & 0.0225 & $\mathrm{~N}$ \\
\hline B18-1 & 5.2 & ZP305 & 898 & 585 & 362 & 214 & 0.0101 & $\mathrm{~N}$ \\
\hline B18-1 & 5.3 & ZP305 & 837 & 867 & 753 & 477 & 0.0250 & $\mathrm{~N}$ \\
\hline B18-1 & 5.4 & ZP305 & 787 & 718 & 546 & 371 & 0.0165 & $\mathrm{~N}$ \\
\hline B27-1 & 1 & ZP305 & 1003 & 922 & 580 & 310 & 0.0261 & $\mathrm{~N}$ \\
\hline B27-1 & 2 & ZP305 & 1300 & 767 & 470 & 242 & 0.0257 & $\mathrm{~N}$ \\
\hline B27-1 & 3 & ZP305 & 1138 & 629 & 492 & 303 & 0.0329 & $\mathrm{~N}$ \\
\hline B27-1 & 4 & ZP305 & 1035 & 661 & 489 & 301 & 0.0184 & $\mathrm{~N}$ \\
\hline B27-3 & 1 & ZP305 & 864 & 614 & 490 & 347 & 0.0253 & $\mathrm{~N}$ \\
\hline B27-3 & 2 & ZP305 & 798 & 399 & 311 & 208 & 0.0114 & $\mathrm{~N}$ \\
\hline B27-3 & 3 & ZP305 & 862 & 647 & 566 & 396 & 0.0077 & $\mathrm{~N}$ \\
\hline B27-3 & 4 & ZP305 & 822 & 599 & 462 & 329 & 0.0063 & $\mathrm{~N}$ \\
\hline B27-2 & 1 & RC80/60BN & 627 & 791 & 767 & 761 & 0.0729 & $\mathrm{Y}$ \\
\hline B27-2 & 2 & RC80/60BN & 664 & 707 & 637 & 475 & 0.0310 & $\mathrm{~N}$ \\
\hline B27-2 & 3 & RC80/60BN & 721 & 781 & 757 & 554 & 0.0398 & Y \\
\hline B27-2 & 4 & RC80/60BN & 660 & 895 & 876 & 792 & 0.0613 & Y \\
\hline B27-4 & 1 & RC80/60BN & 869 & 1008 & 819 & 738 & 0.0265 & $\mathrm{Y}$ \\
\hline B27-4 & 2 & RC80/60BN & 847 & 964 & 952 & 823 & 0.0482 & $\mathrm{Y}$ \\
\hline B27-4 & 3 & RC80/60BN & 683 & 755 & 749 & 554 & 0.0625 & Y \\
\hline B27-4 & 4 & RC80/60BN & 774 & 843 & 669 & 616 & 0.0105 & $\mathrm{~N}$ \\
\hline B18-7 & 1 & RC80/30BP & 968 & 886 & 748 & 488 & 0.0272 & $\mathrm{~N}$ \\
\hline B18-7 & 2 & RC80/30BP & 988 & 1085 & 789 & 512 & 0.0179 & $\mathrm{~N}$ \\
\hline B18-7 & 3 & RC80/30BP & 984 & 1061 & 856 & 561 & 0.0184 & $\mathrm{~N}$ \\
\hline B18-7 & 4 & $\mathrm{RC} 80 / 30 \mathrm{BP}$ & 946 & 1009 & 892 & 486 & 0.0245 & $\mathrm{~N}$ \\
\hline B18-7 & 5.1 & $\mathrm{RC} 80 / 30 \mathrm{BP}$ & 1048 & 1257 & 1008 & 695 & 0.0181 & $\mathrm{~N}$ \\
\hline B18-7 & 5.2 & RC80/30BP & 1018 & 1098 & 943 & 622 & 0.0136 & $\mathrm{~N}$ \\
\hline B18-7 & 5.3 & $\mathrm{RC} 80 / 30 \mathrm{BP}$ & 1026 & 1188 & 974 & 523 & 0.0197 & $\mathrm{~N}$ \\
\hline B18-7 & 5.4 & RC80/30BP & 884 & 989 & 737 & 447 & 0.0200 & $\mathrm{~N}$ \\
\hline B18-2 & 1 & ZP305 & 1010 & 843 & 547 & 321 & 0.0139 & $\mathrm{~N}$ \\
\hline B18-2 & 2 & ZP305 & 961 & 794 & 569 & 362 & 0.0154 & $\mathrm{~N}$ \\
\hline B18-2 & 3 & ZP305 & 867 & 727 & 611 & 404 & 0.0248 & $\mathrm{~N}$ \\
\hline B27-5 & 1 & ZP305 & 826 & 690 & 611 & 431 & 0.0075 & $\mathrm{~N}$ \\
\hline B27-5 & 2 & ZP305 & 851 & 877 & 722 & 476 & 0.0172 & $\mathrm{~N}$ \\
\hline B27-6 & 1 & RC80/60BN & 821 & 811 & 702 & 512 & 0.0261 & $\mathrm{~N}$ \\
\hline B27-6 & 2 & RC80/60BN & 1133 & 999 & 923 & 764 & 0.0222 & $\mathrm{~N}$ \\
\hline B27-6 & 3 & $\mathrm{RC} 80 / 60 \mathrm{BN}$ & 606 & 988 & 918 & 673 & 0.0171 & Y \\
\hline
\end{tabular}




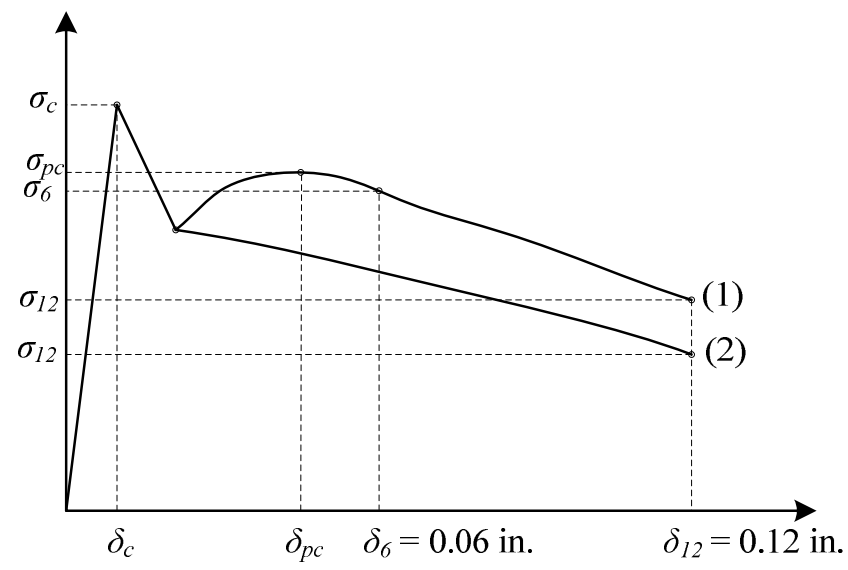

Fig. 3-18: Two different equivalent bending stress versus deflection responses and important points on the curves

Characteristics of first-crack equivalent bending stress. Fig. 3-19 shows the relationship between the fiber reinforcing index $\left(V_{f} L_{f} / D_{f}\right)$ and the equivalent bending stresses at key points. It should be mentioned that fiber reinforcing index is considered by many researchers to be the quantity that better correlates with the tensile performance of SFRC (for example, Hannant, 1978).

As can be seen from Fig. 3-19, there is no clear correlation between the firstcracking stress and the fiber factor. This confirms a well-established fact that fibers only work efficiently once the concrete matrix has cracked. An increase in the fiber reinforcing index leads to an increase in post-cracking stresses $\sigma_{p c}, \sigma_{6}$, and $\sigma_{12}$.

Did the specimens pass the ACI Code requirement for use of SFRC as shear reinforcement? ACI Committee 318 requires that the equivalent bending stress at the deflection of $1 / 300$ and $1 / 150$ of the span length (0.06 inches and 0.12 inches, respectively) be greater than $90 \%$ and $75 \%$ of the first-cracking stress, respectively. As listed in Table 3-12, most the specimens did not pass this requirement, except for those with RC80/60BN fibers. However, in terms of improving shear strength of RC beams, all three types of fibers performed similarly, as will be shown in Chapter 4. 

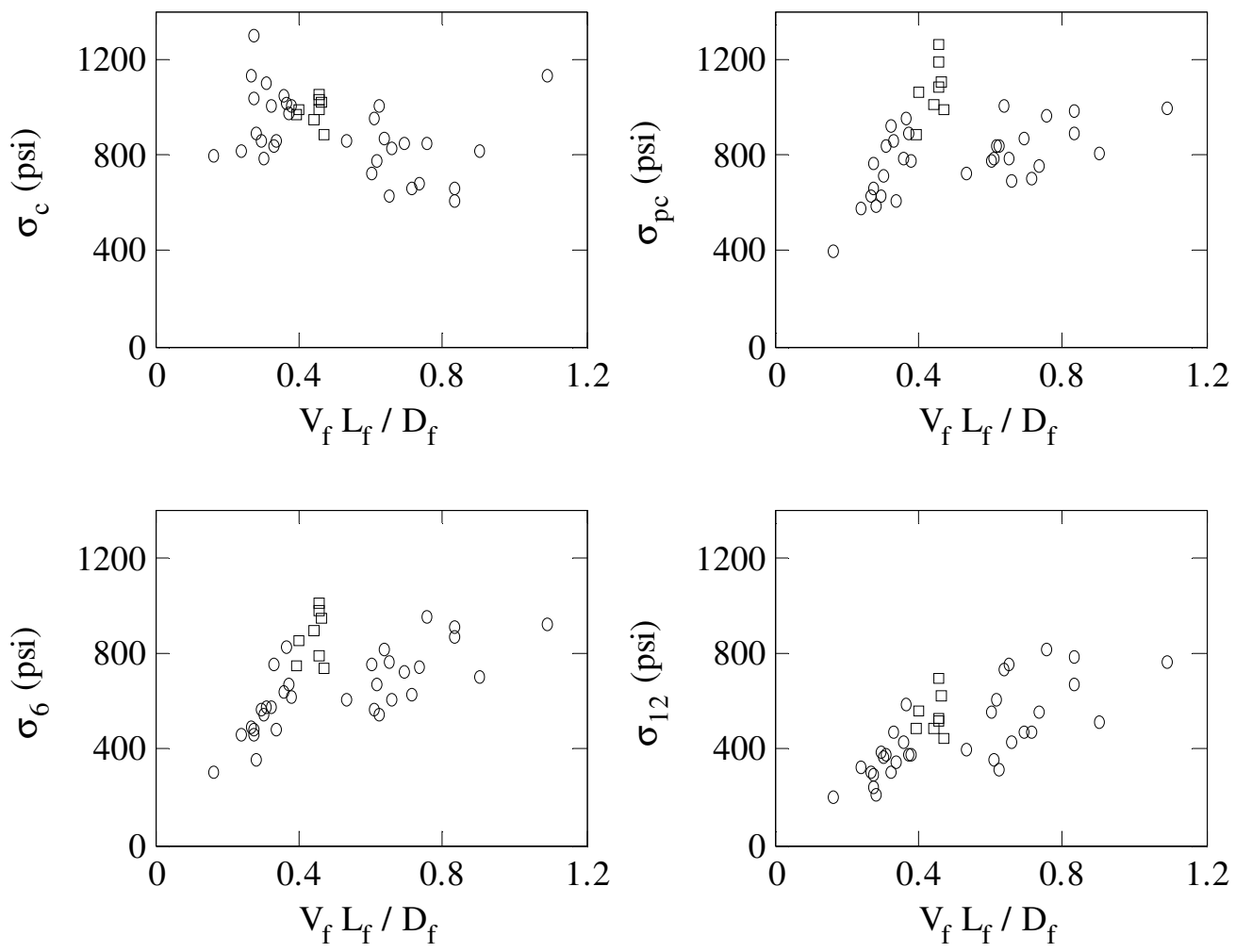

Fig. 3-19: Different equivalent bending stresses versus fiber reinforcing index relationships 


\subsubsection{SFRC direct tensile strength}

Casting and testing of dog bone specimens. When each pair of beams was constructed, at least three dog-bone specimens were cast using dog bone-shaped molds. Their dimensions are shown in Fig. 3-20a. The setup for a typical dog-bone test is shown in Fig. 3-20b. The dog-bone specimens were loaded in tension by an MTS testing machine. The average strain within a gauge length of 8 inches was measured by a pair of OptoTRAK markers attached to the specimen, as shown in Fig. 3-20b.

Test results. The stress versus strain relationships for dog-bone specimens corresponding to Beams B18-1, B18-7, B27-2, B27-3, and B27-4 are shown in Fig. 3-21a through e. It can be seen that the relationships are not consistent and vary from specimen to specimen for the same material, except for the dog-bone specimens associated with Beams B18-7. The main reason is that the size of the dog-bone specimens was not sufficiently large to ensure a random distribution of fibers. Fig. 3-21f shows examples of cracked dog-bone specimens for Beam B27-4. The number of fibers varied from as low as 7 fibers to 19 fibers. The shape of the stress versus strain relationships also differed substantially. For instance, dog-bone specimens for Beams B18-1 and B18-7 cracked at low stresses (approximately $150 \mathrm{psi}$ ) and then gained additional strength with a significant change in stiffness. On the contrary, those for Beams B27-2, B27-3, and B274 had a significantly higher first-cracking stress (from 450 to 600 psi). In these specimens, a rapid strength loss occurred after cracking, followed by a gradual strength decay. Again, these differences should be attributed to the size of the dog-bone specimens, which did not allow a uniform distribution of fibers. 


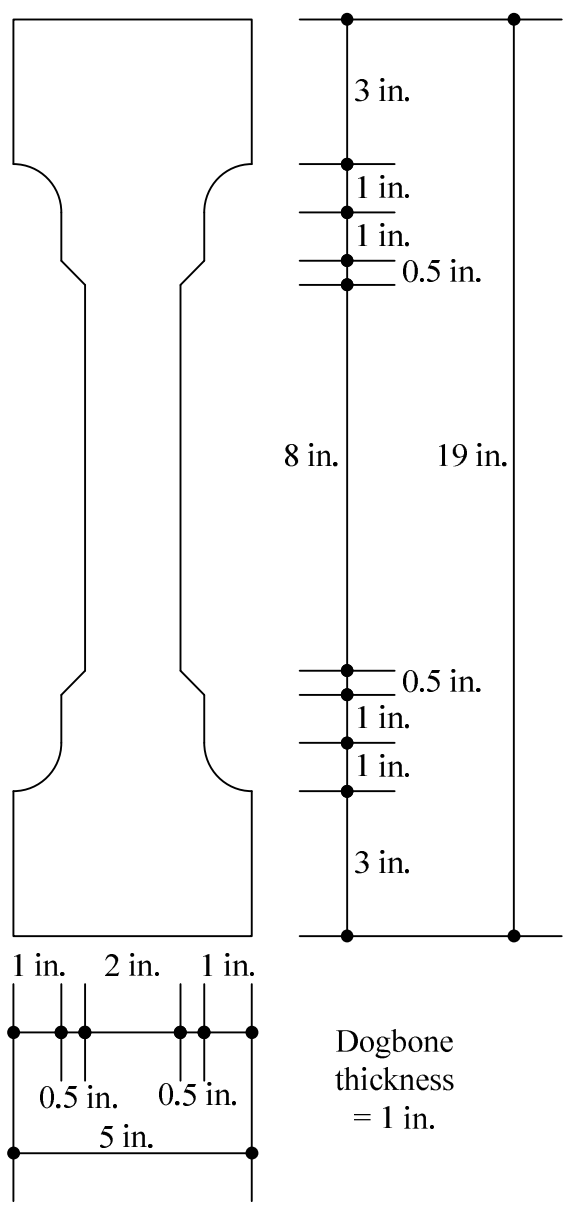

(a) Dimensions

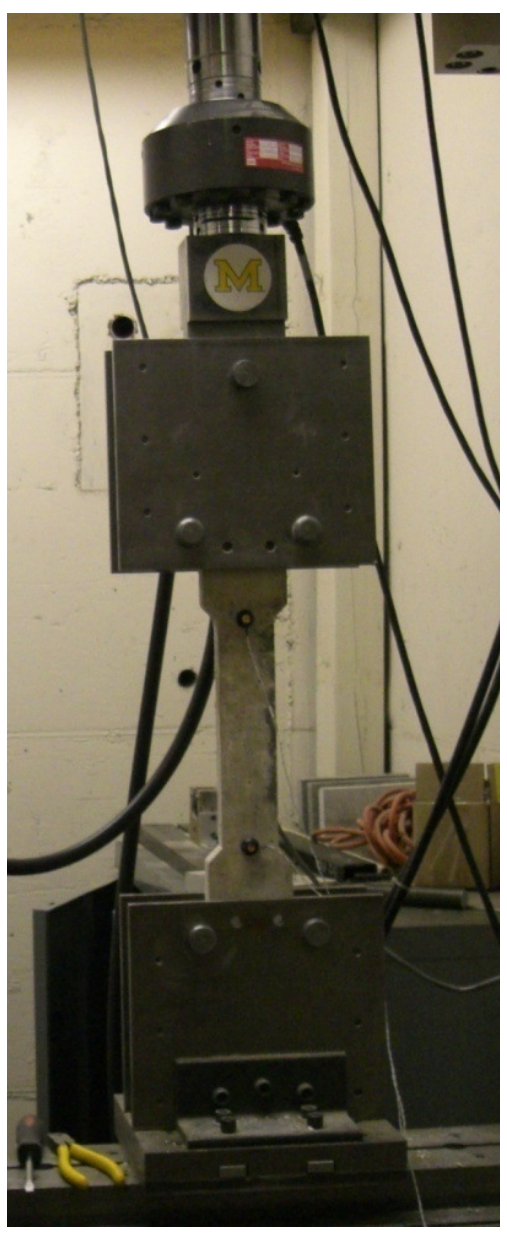

(b) Test setup

Fig. 3-20: Direct tensile tests of dog-bone specimens 


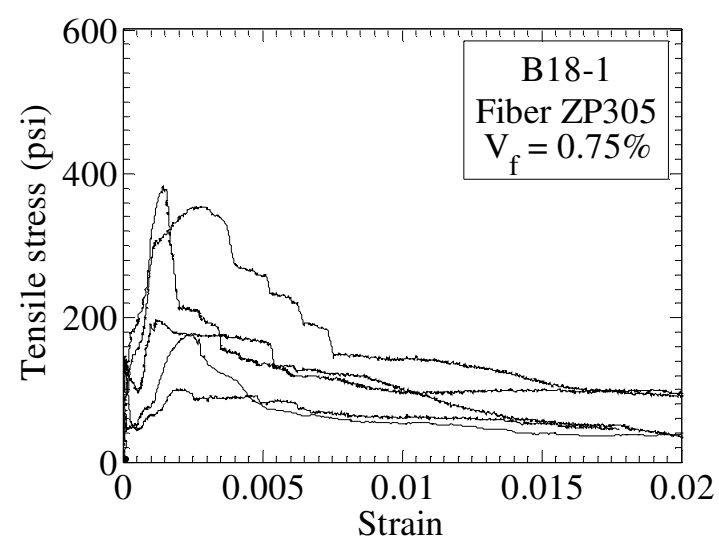

(a)

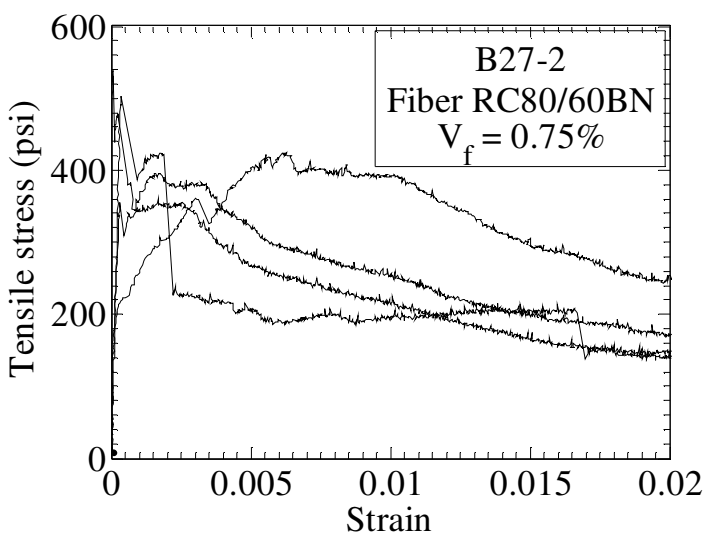

(c)

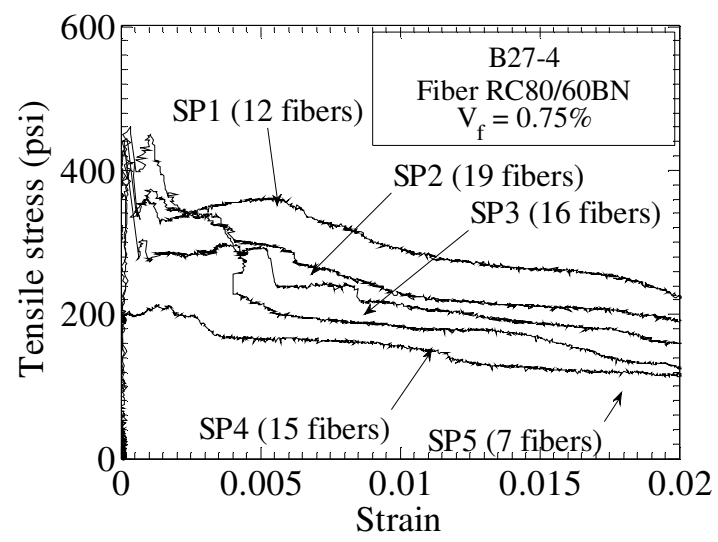

(e)

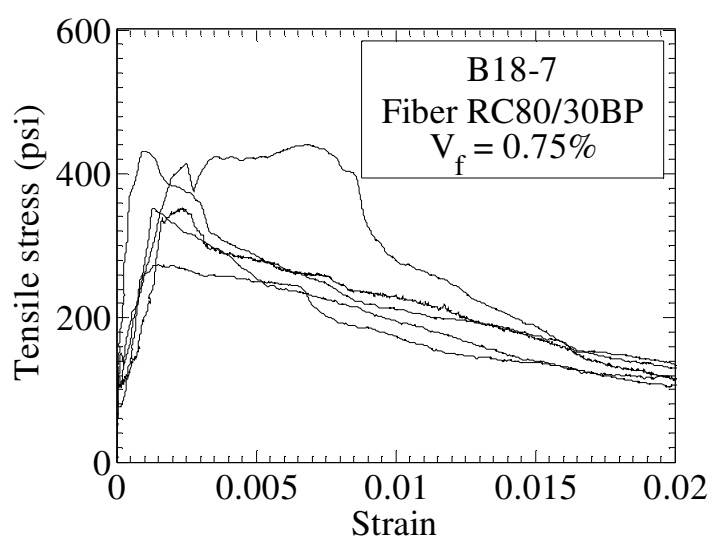

(b)

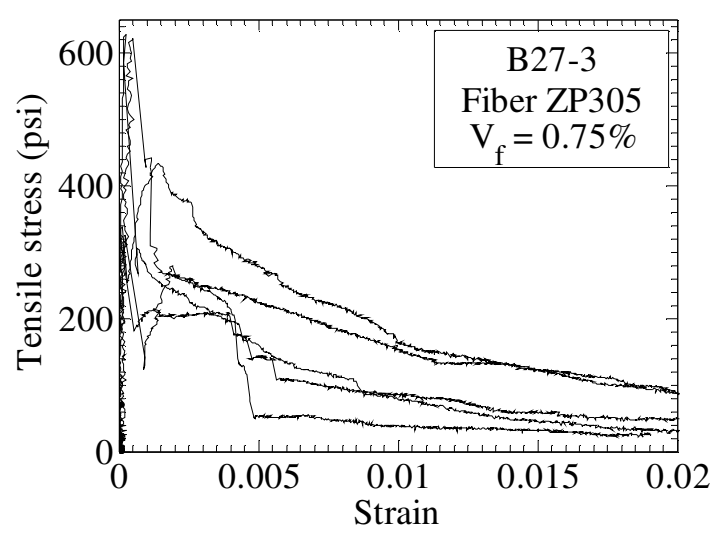

(d)

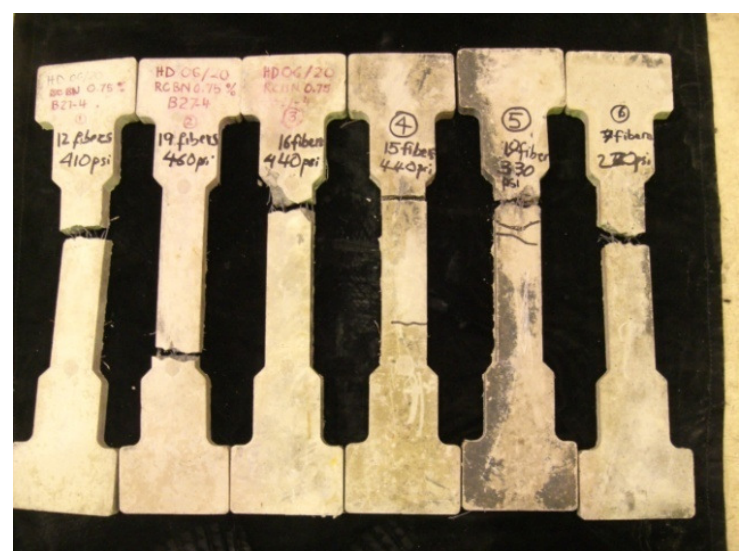

(f)

Fig. 3-21: Results of dog-bone direct tensile tests 


\section{CHAPTER 4}

\section{RESULTS OF}

\section{THE EXPERIMENTAL PROGRAM}

\subsection{INTRODUCTION}

This chapter provides a detailed analysis of the behavior of all tested beams. Each beam will be analyzed separately to fully understand its behavior, including its load versus deflection relationship, crack pattern, reinforcement strains, and distribution and magnitude of concrete strains in the critical shear span. A comparison between the beam test results will also be made to analyze the effect of the studied parameters on beam behavior.

The concrete average strain data were obtained from an infrared-based tracking system (OptoTRAK) that measured the location of selected points on the surface of the beam. A discussion of how these data were processed is given in the following section.

\subsection{PROCESS OF OPTOTRAK DATA AND CALCULATION OF AVERAGE CONCRETE STRAINS}

\subsubsection{Transformation of coordinates}

The OptoTRAK Certus system described in Section 3.5 has the default origin $O$ at the middle position sensor (see Fig. 3-8 and Fig. 4-1). For this testing program, the 
plane $X O Y$ was not parallel to the beam vertical plane to which the markers were attached. Therefore, the coordinates relative to the OptoTRAK OXYZ coordinate system could not be used to compute the location of the markers within the beam vertical plane. Consequently, the coordinates of each marker relative to the OptoTRAK OXYZ coordinate system were transformed to a reference coordinate system whose $x y$ plane coincided with the beam vertical plane. The $x y z$ coordinate system with its origin at $B$, shown in Fig. 4-1, is an example of a useful reference coordinate system.

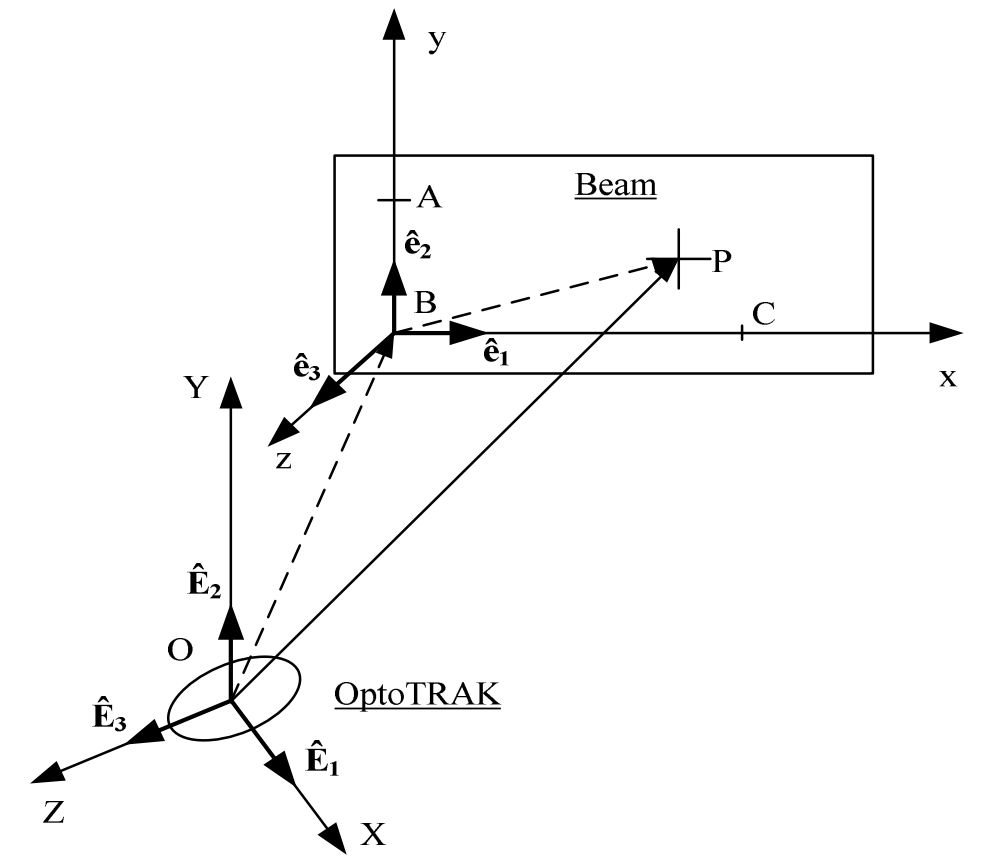

Fig. 4-1: Transformation of coordinates of markers

Let $\left\langle\hat{E}_{1}, \hat{E}_{2}, \hat{E}_{3}\right\rangle$ and $\left\langle\hat{e}_{1}, \hat{e}_{2}, \hat{e}_{3}\right\rangle$ be the bases of the coordinate system $O X Y Z$ and Bxyz, respectively (see Fig. 4-1), and $\left\langle X_{P}, Y_{P}, Z_{P}\right\rangle$ and $\left\langle x_{P}, y_{P}, z_{P}\right\rangle$ be the coordinates of point $P$ in the two coordinate systems $O X Y Z$ and $B x y z$, respectively. The transformation of marker coordinates from $O X Y Z$ to Bxyz follows the rule of coordinate transformation discussed in many textbooks of continuum mechanics (for example, Continuum Mechanics for Engineers by George E. Mase and G. Thomas Mase, 1999), and is given as follows: 


$$
\left\{\begin{array}{l}
x_{P} \\
y_{P} \\
z_{P}
\end{array}\right\}=\left[\begin{array}{ccc}
\hat{e}_{1} \cdot \hat{E}_{1} & \hat{e}_{1} \cdot \hat{E}_{2} & \hat{e}_{1} \cdot \hat{E}_{3} \\
\hat{e}_{2} \cdot \hat{E}_{1} & \hat{e}_{2} \cdot \hat{E}_{2} & \hat{e}_{2} \cdot \hat{E}_{3} \\
\hat{e}_{3} \cdot \hat{E}_{1} & \hat{e}_{3} \cdot \hat{E}_{2} & \hat{e}_{3} \cdot \hat{E}_{3}
\end{array}\right]\left(\left\{\begin{array}{c}
X_{P} \\
Y_{P} \\
Z_{P}
\end{array}\right\}-\left\{\begin{array}{c}
X_{B} \\
Y_{B} \\
Z_{B}
\end{array}\right\}\right)
$$

In this equation, the base $\left\langle\hat{E}_{1}, \hat{E}_{2}, \hat{E}_{3}\right\rangle$ takes the unit value as follows:

$$
\hat{E}_{1}=(1,0,0) ; \hat{E}_{2}=(0,1,0) ; \hat{E}_{3}=(0,0,1) .
$$

The base $\left\langle\hat{e}_{1}, \hat{e}_{2}, \hat{e}_{3}\right\rangle$ must be determined to represent the beam vertical plane. Three markers $A, B$, and $C$ must be chosen (see Fig. 4-1). The first unit vector can be determined as follows:

$$
\hat{e}_{1}=\frac{\overrightarrow{B C}}{|\overrightarrow{B C}|} .
$$

Since $A B$ and $B C$ may not be perfectly perpendicular to each other, the third unit vector has to be calculated such that it is orthogonal to the $A B C$ plane. A cross product is used to satisfy this requirement:

$$
\hat{e}_{3}=\frac{\hat{e}_{1} \times \overrightarrow{B A}}{\left|\hat{e}_{1} \times \overrightarrow{B A}\right|} .
$$

Another cross product is then used to back calculate the second unit vector based on the known unit vectors $\hat{e}_{1}$ and $\hat{e}_{3}$ :

$$
\hat{e}_{2}=\hat{e}_{3} \times \hat{e}_{1} .
$$

Note that the coordinates of the base $\left\langle\hat{e}_{1}, \hat{e}_{2}, \hat{e}_{3}\right\rangle$ and origin $B$ must remain constant throughout the loading process. Therefore, they were often selected to correspond to the loading frame with the load equal to zero (frame 1), while the coordinates of point $P$ in the OptoTRAK system, and hence in the beam system, varied along with the change of loading frames. This can be expressed as:

$$
\left\{\begin{array}{l}
x_{P} \\
y_{P} \\
z_{P}
\end{array}\right\}_{\text {frame } i}=\left[\begin{array}{lll}
\hat{e}_{1} \cdot \hat{E}_{1} & \hat{e}_{1} \cdot \hat{E}_{2} & \hat{e}_{1} \cdot \hat{E}_{3} \\
\hat{e}_{2} \cdot \hat{E}_{1} & \hat{e}_{2} \cdot \hat{E}_{2} & \hat{e}_{2} \cdot \hat{E}_{3} \\
\hat{e}_{3} \cdot \hat{E}_{1} & \hat{e}_{3} \cdot \hat{E}_{2} & \hat{e}_{3} \cdot \hat{E}_{3}
\end{array}\right]_{\text {frame } 1}\left(\left\{\begin{array}{l}
X_{P} \\
Y_{P} \\
Z_{P}
\end{array}\right\}_{\text {frame } i} \quad-\left\{\begin{array}{l}
X_{B} \\
Y_{B} \\
Z_{B}
\end{array}\right\}\right)
$$




\subsubsection{Displacements of markers}

Once the coordinates of markers in the beam coordinate system are specified, the displacements $\left\langle u_{P}, v_{P}, w_{P}\right\rangle$ of marker $P$ are computed as follows:

$$
\left\{\begin{array}{l}
u_{P} \\
v_{P} \\
w_{P}
\end{array}\right\}_{\text {frame } i}=\left\{\begin{array}{l}
x_{P} \\
y_{P} \\
z_{P}
\end{array}\right\}_{\text {frame } i}-\left\{\begin{array}{l}
x_{P} \\
y_{P} \\
z_{P}
\end{array}\right\}_{\text {frame 1 }} \quad \text { with } i=1 \ldots n
$$

where $n$ is the number of data samples collected. 


\subsubsection{Calculation of concrete strains}

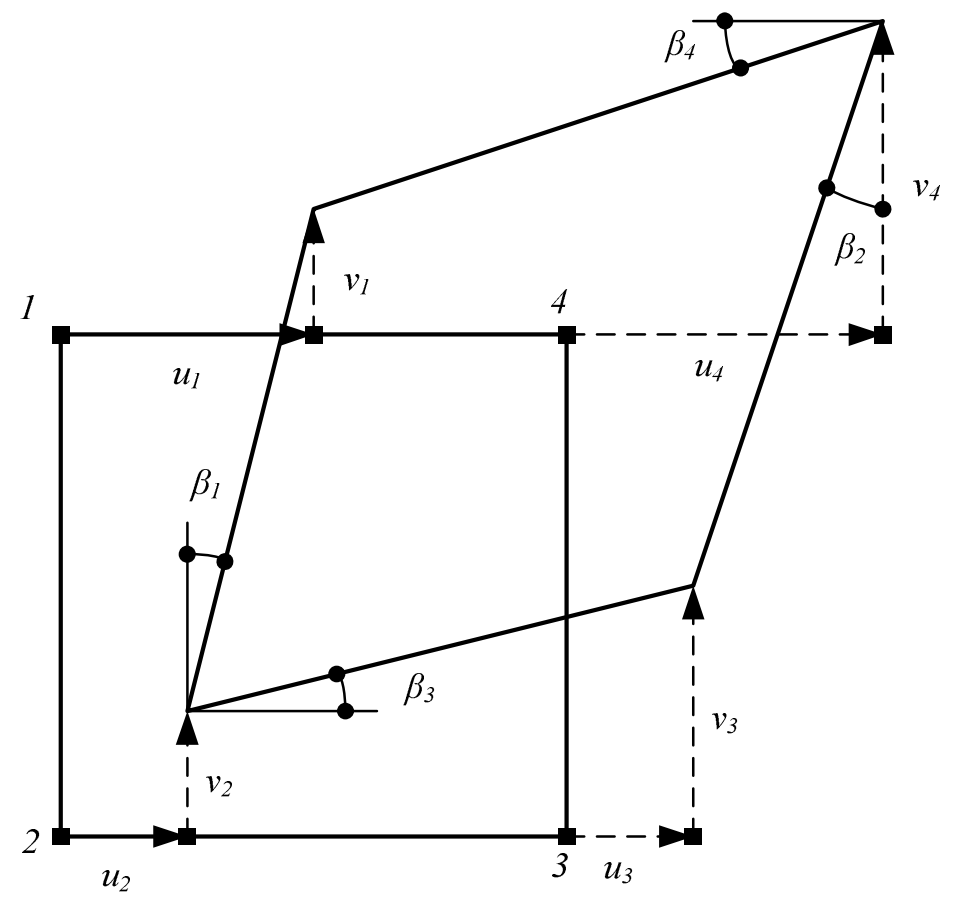

Fig. 4-2: Deformation of a quadrilateral element

Consider a plane quadrilateral element deformed as shown in Fig. 4-2. If the plane coordinates and displacements of all four nodes 1, 2, 3, and 4 are known, the plane strains $\varepsilon_{x}, \varepsilon_{y}$ and $\gamma_{x y}$ of the element can be calculated as follows:

$$
\begin{aligned}
& \left(\varepsilon_{x}\right)_{\text {frame i }}=\frac{1}{2}\left(\frac{\left(u_{3}\right)_{\text {frame i }}-\left(u_{2}\right)_{\text {frame i }}}{\left(\Delta x_{23}\right)_{\text {frame 1 }}}+\frac{\left(u_{4}\right)_{\text {frame i }}-\left(u_{1}\right)_{\text {frame i }}}{\left(\Delta x_{14}\right)_{\text {frame 1 }}}\right) \\
& \left(\varepsilon_{y}\right)_{\text {frame i }}=\frac{1}{2}\left(\frac{\left(v_{1}\right)_{\text {frame i }}-\left(v_{2}\right)_{\text {frame i }}}{\left(\Delta y_{21}\right)_{\text {frame 1 }}}+\frac{\left(v_{4}\right)_{\text {frame i }}-\left(v_{3}\right)_{\text {frame i }}}{\left(\Delta y_{34}\right)_{\text {frame 1 }}}\right) \\
& \left(\gamma_{x y}\right)_{\text {frame i }}=\frac{1}{2}\left[\left(\beta_{1}\right)_{\text {frame i }}+\left(\beta_{2}\right)_{\text {frame i }}\right]+\frac{1}{2}\left[\left(\beta_{3}\right)_{\text {frame i }}+\left(\beta_{4}\right)_{\text {frame i }}\right] \\
& =\frac{1}{2}\left[\frac{\left(u_{1}\right)_{\text {frame i }}-\left(u_{2}\right)_{\text {frame i }}}{\left(\Delta y_{21}\right)_{\text {frame } 1}}+\frac{\left(u_{4}\right)_{\text {frame i }}-\left(u_{3}\right)_{\text {frame i }}}{\left(\Delta y_{34}\right)_{\text {frame } 1}}\right] \\
& +\frac{1}{2}\left[\frac{\left(v_{3}\right)_{\text {frame i }}-\left(v_{2}\right)_{\text {frame i }}}{\left(\Delta x_{23}\right)_{\text {frame } 1}}+\frac{\left(v_{4}\right)_{\text {frame i }}-\left(v_{1}\right)_{\text {frame i i }}}{\left(\Delta x_{14}\right)_{\text {frame 1 }}}\right]
\end{aligned}
$$


where:

$$
\begin{aligned}
& \left(u_{P}\right)_{\text {frame i }}=\left(x_{P}\right)_{\text {frame i }}-\left(x_{P}\right)_{\text {frame 1 }} ; P=1 \ldots 4 \\
& \left(v_{P}\right)_{\text {frame i }}=\left(y_{P}\right)_{\text {frame i }}-\left(y_{P}\right)_{\text {frame 1 }} ; P=1 \ldots 4 \\
& \left(\Delta x_{P Q}\right)_{\text {frame 1 }}=\left|\left(x_{Q}\right)_{\text {frame 1 }}-\left(x_{P}\right)_{\text {frame 1 }}\right| ; P Q=23 \text { or } 14 \\
& \left(\Delta y_{P Q}\right)_{\text {frame 1 }}=\left|\left(y_{Q}\right)_{\text {frame 1 }}-\left(y_{P}\right)_{\text {frame 1 }}\right| ; P Q=21 \text { or } 34
\end{aligned}
$$

\subsubsection{Calculation of principal strains and principal directions}

Once $\varepsilon_{x}, \varepsilon_{y}$ and $\gamma_{x y}$ are known, the principal strains and directions are computed as follows:

$$
\begin{aligned}
& \varepsilon_{1}=\frac{1}{2}\left(\varepsilon_{x}+\varepsilon_{y}\right)+\frac{1}{2} \sqrt{\left(\varepsilon_{x}-\varepsilon_{y}\right)^{2}+\gamma_{x y}{ }^{2}} \\
& \varepsilon_{2}=\frac{1}{2}\left(\varepsilon_{x}+\varepsilon_{y}\right)-\frac{1}{2} \sqrt{\left(\varepsilon_{x}-\varepsilon_{y}\right)^{2}+\gamma_{x y}{ }^{2}} \\
& \theta=\frac{1}{2} \tan ^{-1}\left(\frac{\gamma_{x y}}{\varepsilon_{x}-\varepsilon_{y}}\right)
\end{aligned}
$$




\subsection{BEHAVIOR OF BEAMS IN SERIES B18}

In the following sections, the behavior of the beams with a depth of 18 inches is discussed in detail. While some of the beam pairs are discussed in a single section, particularly when their behavior was nearly identical, other pairs are discussed in greater detail in separate sections. It should be noted that for consistency, some of the beam photos were flipped horizontally, such that the failure shear span would always correspond to the left span. This led to the numbers adjacent to the cracks, which represent the load level at which the crack was first observed, be also flipped.

\subsubsection{Beams B18-0a \& b}

Fig. 4-3 through Fig. 4-5 show the load versus deflection relationships and crack patterns of Beams B18-0a \& b. Flexural cracks first developed from the bottom surface of the beam in the region below the loading point, where the maximum moment occurred. As the applied load increased, flexural cracks spread out toward the support. When the load was sufficient to impose a diagonal tension greater than the concrete tensile strength, an inclined crack occurred, immediately followed by failure of the beam.

Beam B18-0a, for instance, developed the first flexural cracks and the first inclined crack at the applied load of approximately 30 kips and 36 kips, respectively. The peak applied load was 37.7 kips, only $4.7 \%$ greater than that at the first observed inclined crack. Similarly, the load in Beam B18-0b at first flexural cracking, first inclined crack, and failure was 24,35 , and, 36.5 kips, respectively. 


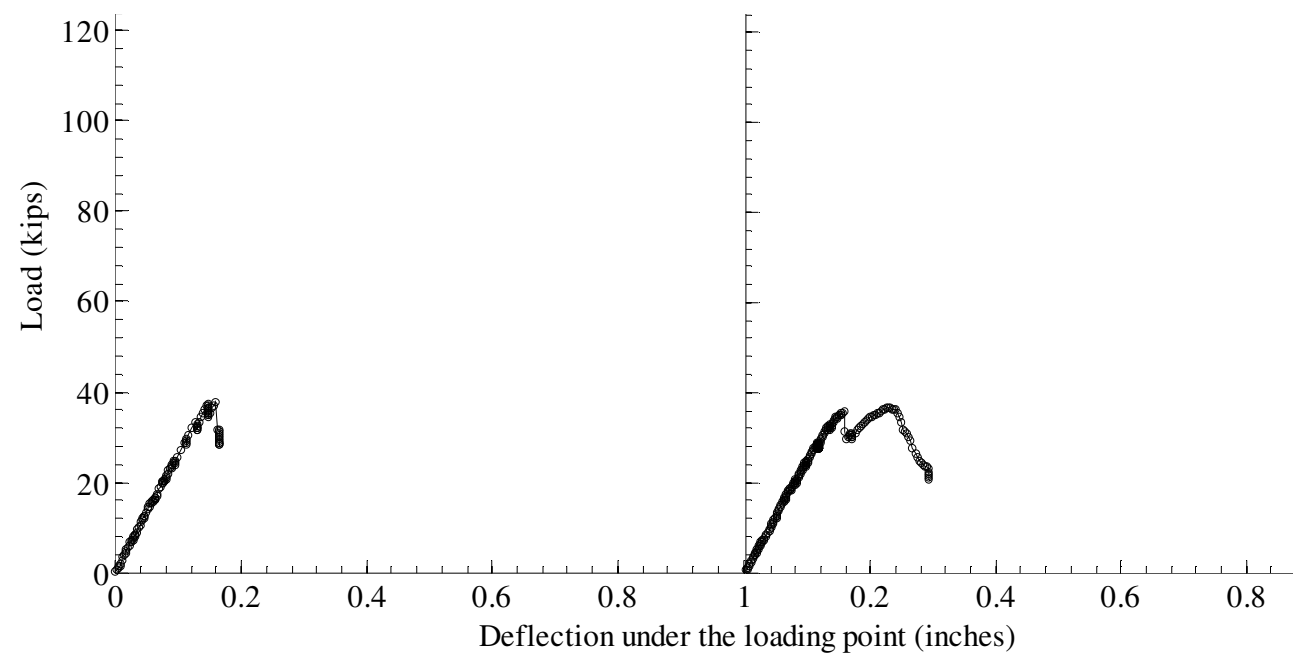

Fig. 4-3: Load versus deflection relationships - Beams B18-0a \& b

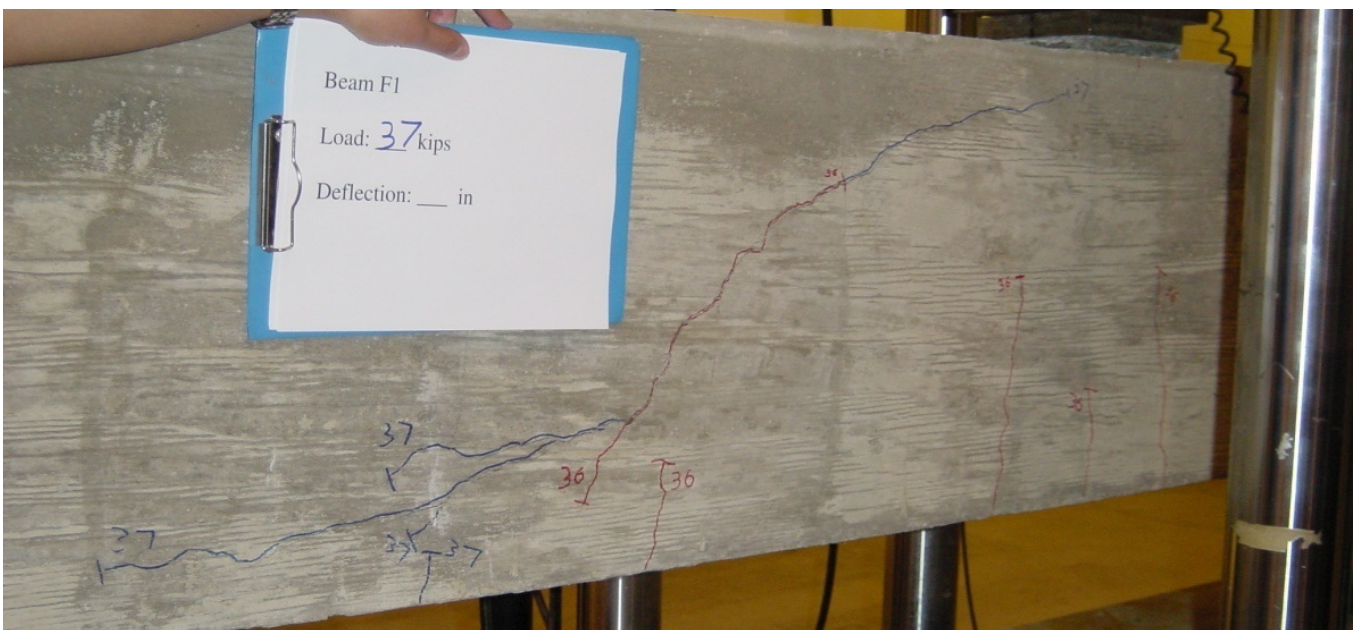

Fig. 4-4: Crack pattern after failure - Beam B18-0a

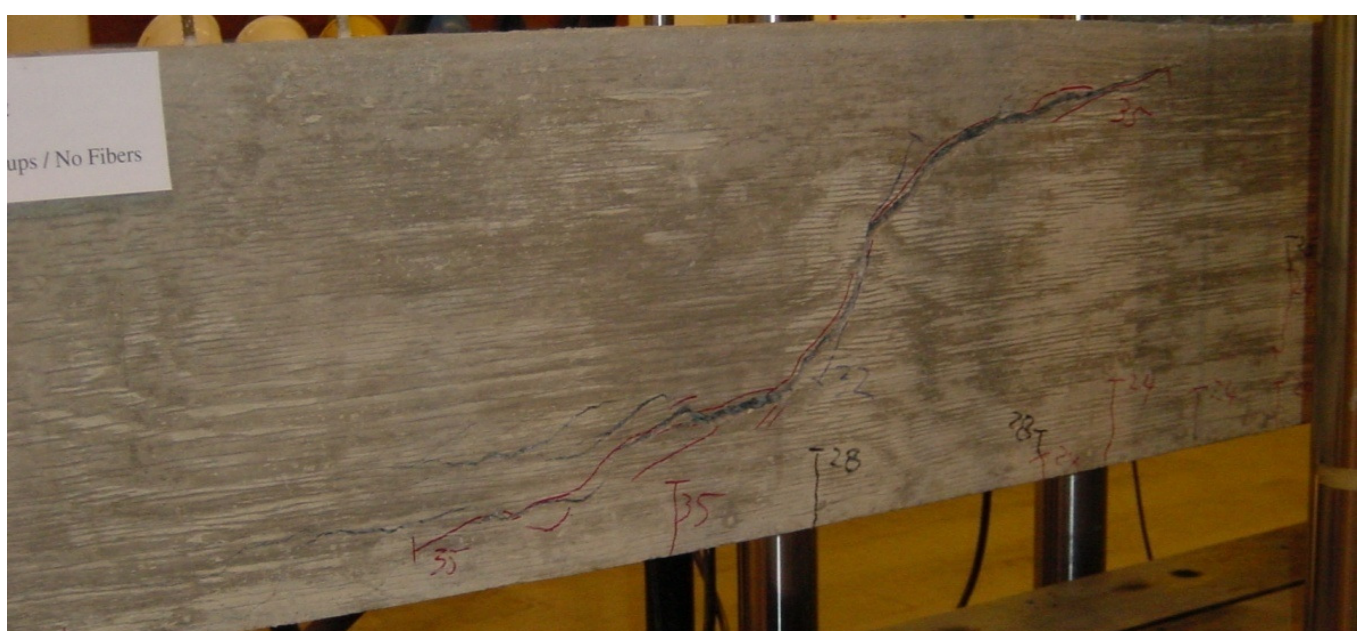

Fig. 4-5: Crack pattern after failure - Beam B18-0b 


\subsubsection{Beam B18-1b}

Beam B18-1b contained a $0.75 \%$ volume fraction of ZP305 fibers, which have a diameter of 0.0022 inches and a length of 1.18 inches.

Load versus deflection relationship, crack pattern, and failure mode. Fig. 4-6a shows the load versus deflection relationship of Beam B18-1b. At 60 kips, the first diagonal cracking was observed. Beyond this load, the beam stiffness decreased gradually until reaching the peak load of 92.8 kips. Beyond the peak load, the beam strength dropped to 74.8 kips ( $81 \%$ of the maximum load), prior to a sudden shear failure.

It can seen from Fig. 4-7 and Fig. 4-8 that Beam B18-1b exhibited first flexural and inclined cracks at 40 and 60 kips, respectively (numbers on beam indicate load). Additional cracks developed over the shear span before a wider crack opening was observed on the third inclined crack from the support (Fig. 4-7) and a sudden failure occurred. At the lower end of the critical inclined crack, cracking propagated along the top layer of reinforcing bars (Fig. 4-8), which could be attributed to the mobilization of a dowel action mechanism in combination with a certain degree of bond failure at the reinforcement level. This indicated a partial shear-tension failure, as described by ACIASCE Committee 426 (1973). The failure mode of Beam B18-1b can then reasonably be defined as a combination of a diagonal-tension and a shear-tension failure.

Reinforcement strains. Fig. 4-6b shows the load versus reinforcement strain relationship for Beam B18-1b (see Fig. 4-9 for the positions of the strain gauges). In this figure, the open markers indicate the point at the peak applied load.

It can be seen that the strains at S1 and S2 were of the same order up to the load at first diagonal cracking (60 kips). The strains at these locations were also very small during this loading stage. Beyond the first diagonal cracking load, these strains started to increase due to numerous flexural and inclined cracks that appeared in the vicinity of the gauges. On the other hand, the strains measured by strain gauges S3 and S4 were different prior to the load of 90 kips, the S3 strains being slightly larger. A similar difference was observed in the pairs of strain gauges S5 \& S6 and S7 \& S8, and can be 
explained by the fact that strain gauges S3, S5, and S7 (on the bottom layer of reinforcement) were farther away from the beam neutral axis than strain gauges S4, S6, and S8, respectively. The difference in the straining rate of S1 and S2 was negligible because the moment demand at $\mathrm{S} 1$ and $\mathrm{S} 2$ was very small. The jump in the reinforcement strains at S1 and S2 when the load increased above 60 kips was due to the formation of a diagonal crack near the support (see Fig. 4-7).

At the peak load, the reinforcement strains at S1 through S4, S6, and S8 were still in the linear range of the reinforcement stress-strain curve, while the reinforcement at S5 and S7 had already yielded.

Fig. 4-10 shows the strains along the reinforcing bars at various applied loads. It can be seen that the strains for both the upper and lower layers of reinforcing bars were nearly linearly distributed along the beam axis before yielding of reinforcement.

Average bond stress. In order to evaluate development of bond between SFRC and reinforcing bars, the average bond stress between two adjacent strain gauge locations $i$ and $j$ was calculated, as follows:

$$
\tau_{i j}=\frac{\left(f_{s j}-f_{s i}\right) \pi \frac{d_{b}^{2}}{4}}{\pi d_{b} \ell_{i j}}=\frac{\left(f_{s j}-f_{s i}\right) d_{b}}{4 \ell_{i j}}
$$

where $d_{b}$ and $\ell_{i j}$ are the bar diameter and the distance between the two strain gauges, respectively. The terms $f_{s j}$ and $f_{s i}$ are the longitudinal reinforcement stresses at the location closer to the loading point and support, respectively. Each stress value is calculated based on the steel stress versus strain model shown in Fig. 4-11. The reinforcement stress was calculated as follows:

$$
f_{s}=\left\{\begin{array}{l}
E_{s} \varepsilon_{s} \text { for } \varepsilon_{s} \leq \varepsilon_{y} \\
f_{y} \text { for } \varepsilon_{y}<\varepsilon_{s} \leq \varepsilon_{s h} \\
f_{y}+\left(f_{s u}-f_{y}\right)\left(2 \xi-\xi^{2}\right) \text { for } \varepsilon_{s h}<\varepsilon_{s} \leq \varepsilon_{s m} \\
f_{s u} \text { for } \varepsilon_{s}>\varepsilon_{s m}
\end{array}\right.
$$


where:

$$
\xi=\frac{\varepsilon_{s}-\varepsilon_{s h}}{\varepsilon_{s m}-\varepsilon_{s h}}
$$

The values $f_{y}, \varepsilon_{y}, E_{s}, \varepsilon_{s h}, f_{s u}$, and $\varepsilon_{s m}$ were obtained from direct tensile tests for reinforcing bars, as reported in Section 3.6.1. In most cases, the bond stress had a positive value because the stress at the location closer to the loading point was generally greater than that at the adjacent strain gauge location closer to the support.

The average bond stresses along the reinforcing bars at various applied loads are shown in Fig. 4-12 and Fig. 4-13. It can be seen that the maximum bond that was developed, was approximately 360 psi, significantly less than the bond developed in bond tests, which was greater than approximately 1000 psi (see Section 2.1.6). This low bond stress is not an indication of strength, but a consequence of the bond stress demand. For the middle region of the beam, between S5 \& S6 and S7 \& S8, lower bond stresses were developed throughout the test, which indicated an almost constant stress in the tension reinforcement over this region of the beam. This result corresponds to an assumed truss model with compression struts propagating away from the load point toward the bottom of the beam at points closer to the supports (Fig. 4-14). For such a truss, the tension in the lower cord would be constant between the points A and B.

Distribution of longitudinal concrete strains. Fig. 4-15 shows the vertical distribution of longitudinal concrete strains $\varepsilon_{x}$ at certain applied loads. It can be seen that an approximately linear distribution of longitudinal strains was measured at all sections until the peak applied load of 92.8 kips was reached. However, the derived zero strain points did not seem to represent neutral axis locations. This is probably due to the fact that the local cracks that appeared within a certain concrete element made the longitudinal strain of that element larger than that of the adjacent ones. For instance, at 92.8 kips, an average tensile strain of 0.00066 was measured in Element 22 (Fig. 4-9) on the top row because the critical inclined shear crack had reached that element at this load.

The localized effect due to the cracks also affected the horizontal distribution of longitudinal strains along the beam, which normally follows that of the moment prior to yielding. It can be seen in Fig. 4-15 that the longitudinal strain along the bottom row did 
not necessarily increased linearly from the support to midspan. Therefore, an approximation is necessary to "bridge" this localized effect.

Fig. 4-16 compares measured concrete strains at the level of the lower reinforcing bars, obtained using the lowest row of OptoTRAK markers, and the measured reinforcement strains for those bars. It can be seen that while the reinforcement strains showed a relatively smooth trend, the concrete strains fluctuated widely. However, a linear approximation of the concrete strains is roughly parallel to the reinforcement strains, except for the segment connecting the two strain gauges closest to the loading point at the peak load where yielding of the reinforcement occurred. Fig. 4-17 shows the linear trend of concrete strains at different applied loads. It can be seen that the concrete strains increased at a uniform rate along the beam up to the load of 80 kips. For the peak load of $92.8 \mathrm{kips}$, the slope of concrete strain distribution increased due to significant cracking, which reduced the beam stiffness.

The maximum tensile strain at the peak load was approximately 0.0042 , between the two markers M36 and M40 (see Fig. 4-9). Similarly, the maximum compressive strain at peak load was approximately 0.0011, between M13 and M17, and between M29 and M33. It should be noted that these maximum concrete strain values should be lower than the true maximum value because the markers were not located at the lowest or highest levels of the beam. However, it is likely that the maximum compressive strain did not reach 0.003 .

Distribution of transverse concrete strains. Fig. 4-18 shows the vertical distribution of transverse (vertical) concrete strains $\varepsilon_{y}$. As can be seen, the transverse strains were practically zero up first diagonal cracking at the load of 60 kips. At the peak load of $92.8 \mathrm{kips}$, the transverse strains were only significant in the parallelogram region formed by the dashed lines shown in Fig. 4-18. Fig. 4-19 shows the component strains for top, middle, and bottom row elements within the parallelogram. It can be seen that no significant transverse strains developed until reaching an applied load of approximately 60 kips. The more "exact" load was approximately 50, 60, and 70 kips for the middle, bottom, and top rows, respectively. This coincides with the fact that the diagonal cracks first occurred in the middle region, propagated towards the bottom reinforcement level, 
and then toward the top compression region. With respect to the magnitude, the transverse strain reached a value of 0.0065 (Element 12) at the peak load.

Distribution of concrete shear strains. The distribution of concrete shear strains was similar to that for transverse strains in the sense that higher shear strains mostly concentrated in the parallelogram region shown in Fig. 4-18 (see Fig. 4-20). Unlike transverse strains, shear strains started developing at relatively low loads (see Fig. 4-19 and Fig. 4-20). Fig. 4-20 also shows that the beam exhibited shear deformation throughout the full depth of a beam section with more shear deformation near the bottom of the beam at higher load levels.

In order to better compare the transverse and shear strains over a cross section, these strains were averaged over an element size of 5 inches in width by 15 inches in depth. Fig. 4-21 shows the distribution of these averaged transverse and shear strains along the beam axis. It can be seen that the average transverse strain was very small near the support and the load point, and reached a peak value near the middle section of the shear span. Shear strains followed a similar trend, but with more fluctuations. It is also noted that the differences in shear strains along the beam axis were more dramatic when the load was larger because of the localized effect due to the opening of a wide diagonal crack.

Distribution of principal strains. Fig. 4-22 shows the crack pattern, principal strains $\left(\varepsilon_{1}\right.$ and $\left.\varepsilon_{2}\right)$, and their directions at first diagonal cracking and peak load. The state of strain for each element was calculated from the displacements of its nodes (markers), following the strain calculation discussed in Section 4.2.3. In order to facilitate the discussion, the critical shear span is divided into 3 regions: the triangular region above the reaction point (Region $\mathrm{R} 1$ ), the triangular region below the loading point (Region R2), and the middle parallelogram (Region R3).

Principal tensile strains were significantly larger in Region 3, which is consistent with the abundant cracks that formed in this region. The direction of principal tensile strains in Region 2 was nearly parallel to the beam longitudinal axis, showing that longitudinal strains dominated. It should be noted that tension-compression strain states 
were observed for elements in the top and middle row of elements in Region 2, while tension-tension strain states were observed for elements in the bottom row and to the right of the critical diagonal crack.

The directions of principal tensile strains did not remain constant (Fig. 4-23), when the load was increased from 60 to 92.8 kips. Rather, the directions of principal tensile strains rotated clockwise, as shown by solid and dashed lines within each element in Fig. 4-23. It is noted that a larger scale factor was used to display the strains at 60 kips. The directions of principal tensile strain at $60 \mathrm{kips}$, first diagonal cracking, were approximately perpendicular to the diagonal cracks (see Fig. 4-22a). However, this was not true for those at peak load (see Fig. 4-22b). The average change in the direction of the principal strains from diagonal cracking to the peak load over the shear span was $17^{\circ}$.

Development of the shear failure mechanism. In order to understand how the failure propagated along the critical diagonal crack, an analysis of strains in the concrete elements surrounding the critical diagonal crack (Fig. 4-22) for loads greater than 82 kips was conducted. Fig. 4-24 shows the strain growth over time for these elements. From the load of 82 to $92.8 \mathrm{kips}$, the rate of increase for principal tensile strains, $\varepsilon_{1}$, was higher for E12 and E15 than for any other elements. In terms of magnitude, the principal tensile strain decreased from Element 15 to 12, 17, 14, 16, 19, 22 and 25. These observations for the rate of increase in principal tensile strains and magnitude held true as the load decreased from 92.8 to 92 kips, except for a sudden increase in the strain magnitude of Element E12 near the peak load. Below the load of 92 kips in the post-peak response, the principal tensile strain rate and magnitude for all elements increased rapidly, except for Element 25, for which the principal tensile strain rate remained relatively constant up to a load slightly below 80 kips. It should be noted that the principal compressive strain $\varepsilon_{2}$ of the top elements $(\mathrm{E} 19,22$, and 25) during these load stages was insignificant, less than 0.0005 . 


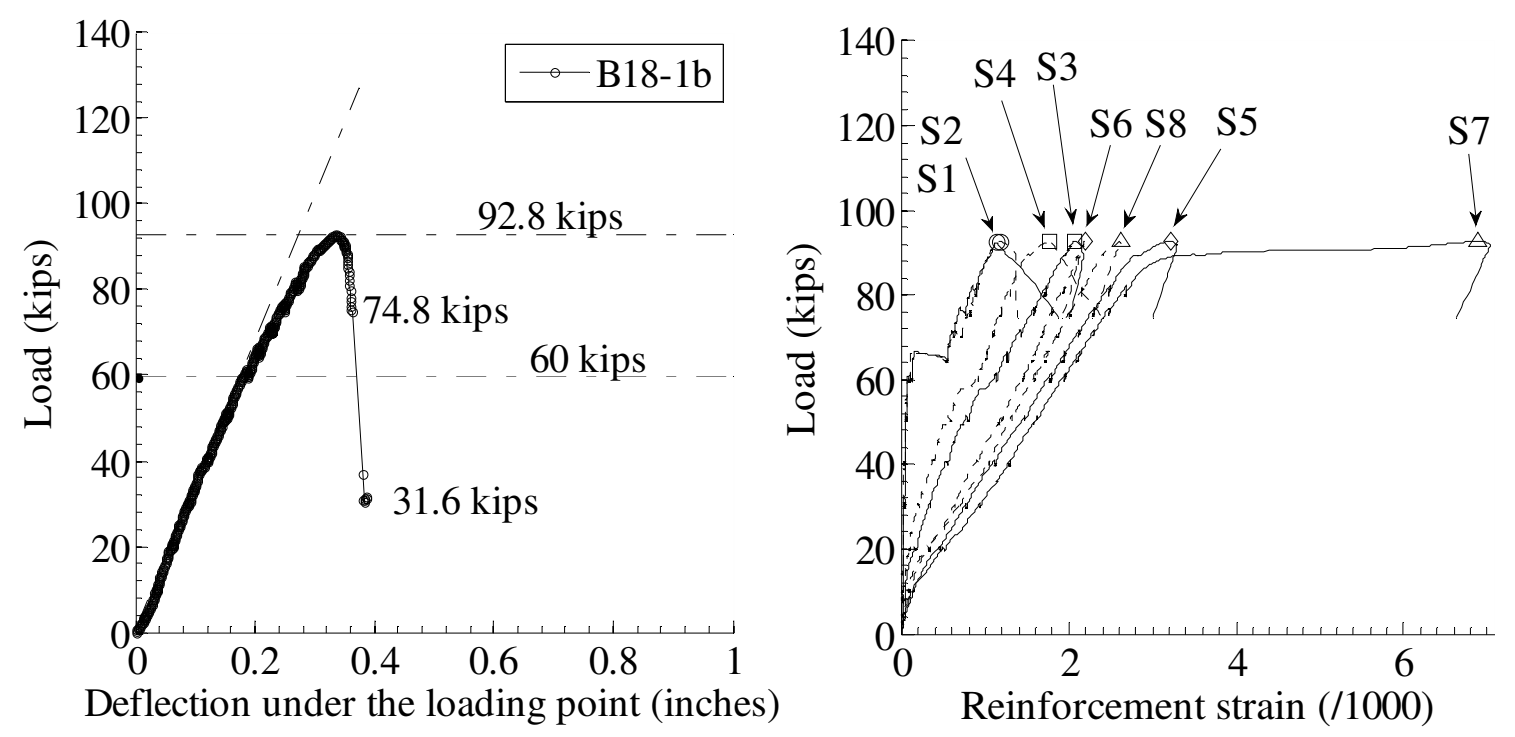

Fig. 4-6: Load versus deflection and reinforcement strain relationships - Beam B18-1b

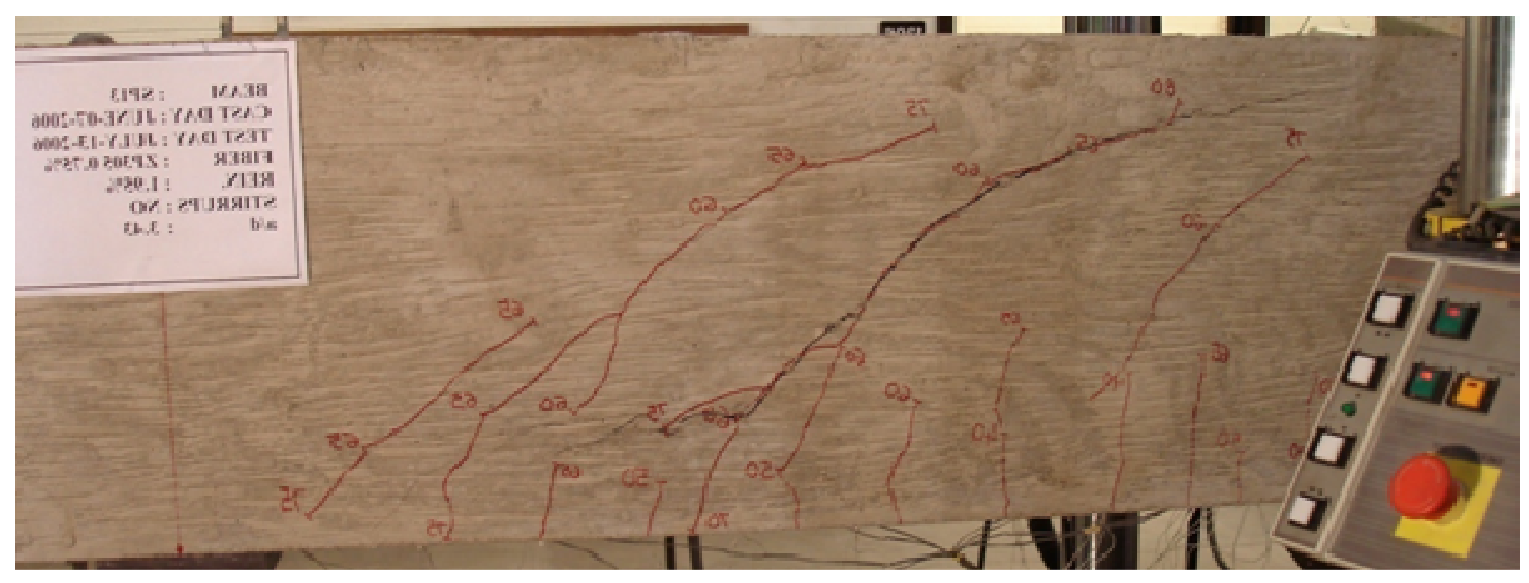

Fig. 4-7: Crack pattern prior to failure - Beam B18-1b

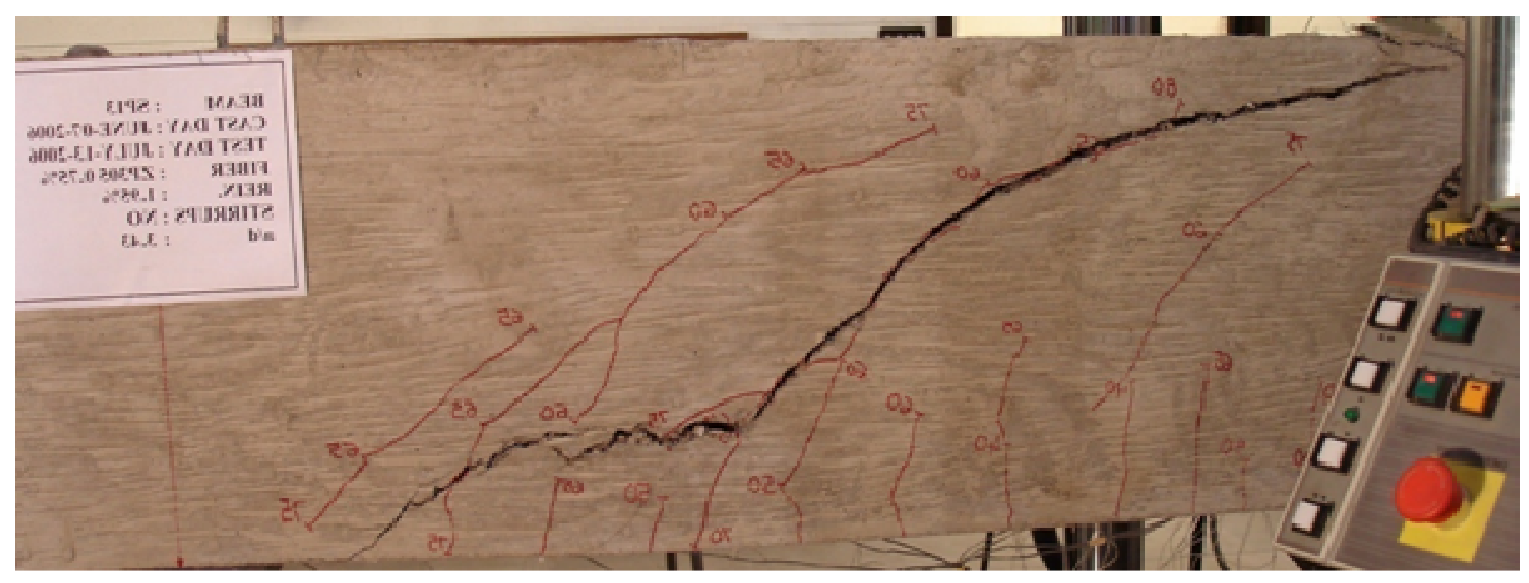

Fig. 4-8: Crack pattern after failure - Beam B18-1b 


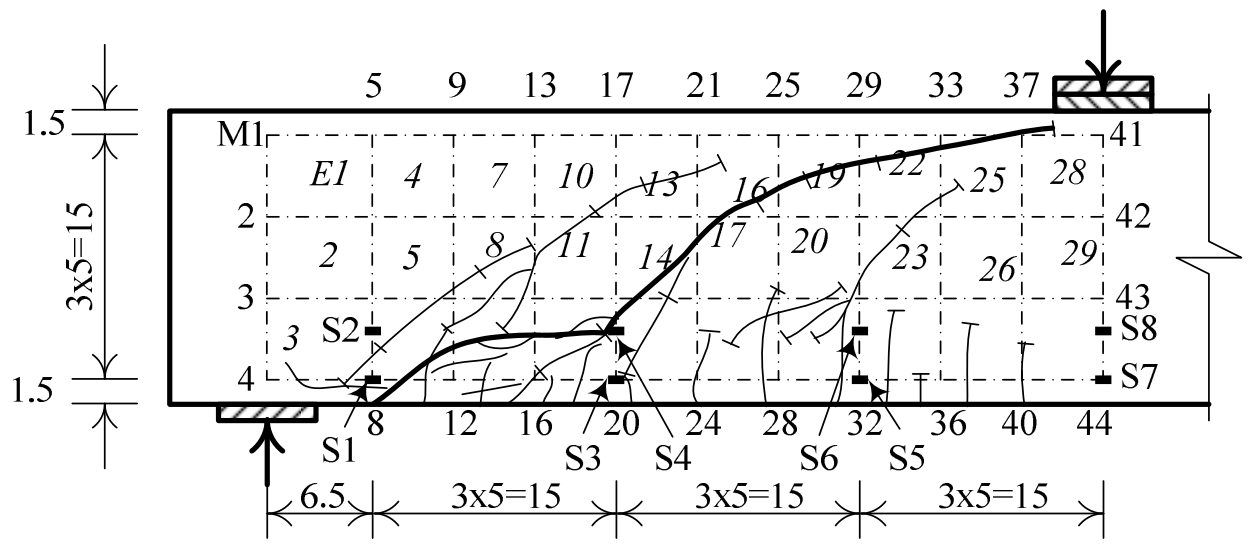

Fig. 4-9: Numbering of markers, elements, and strain gauges - Beam B18-1b

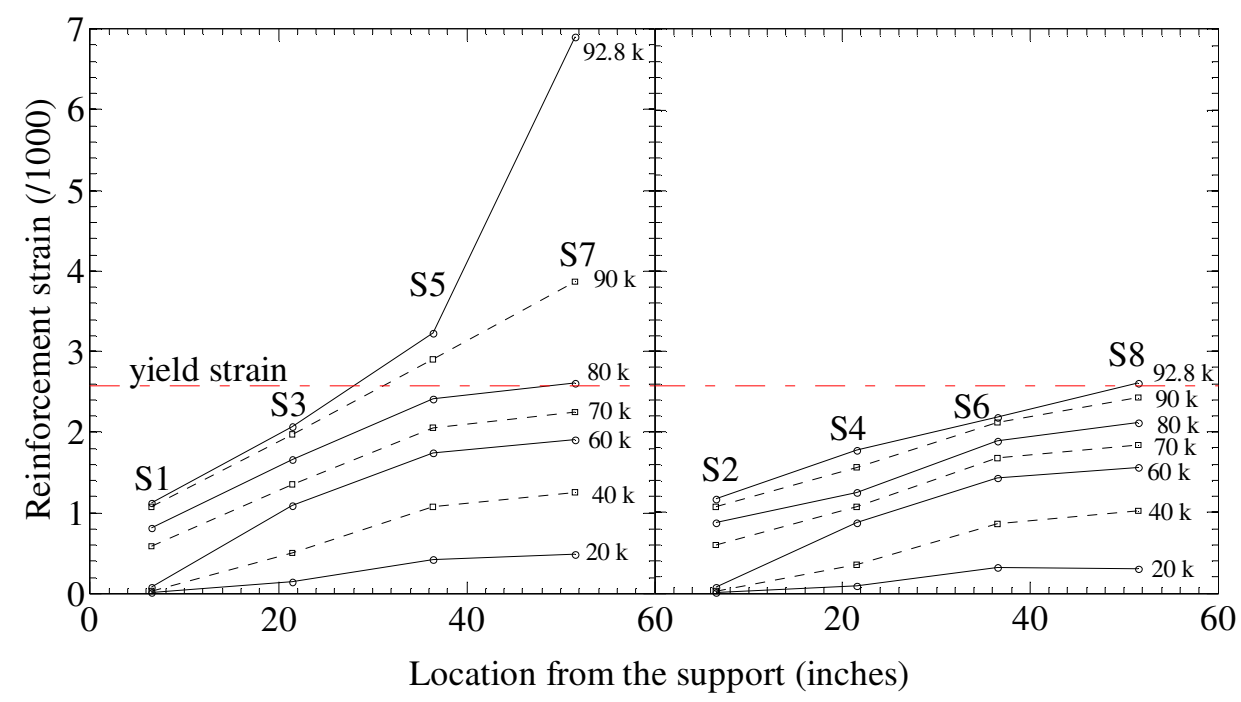

Fig. 4-10: Reinforcement strains along the beam axis - Beam B18-1b

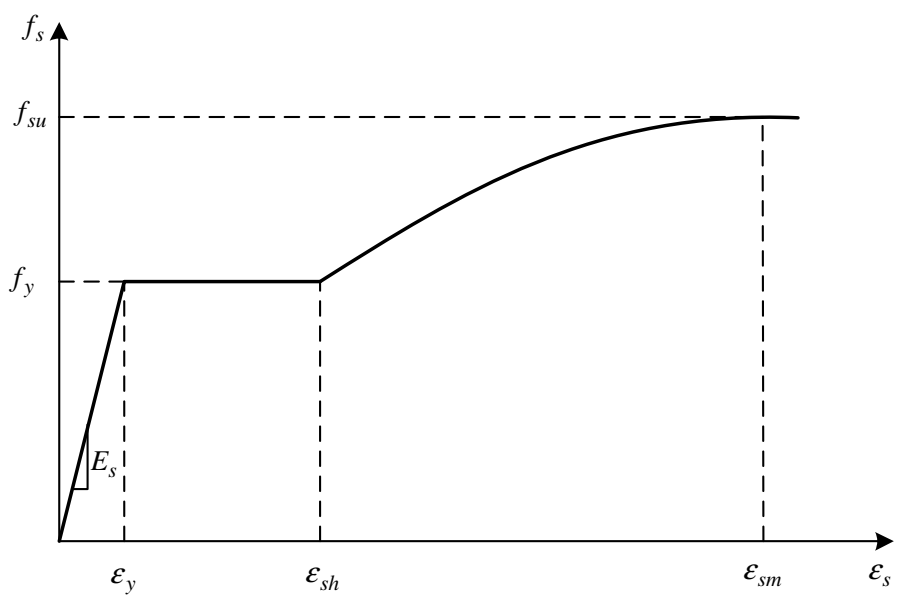

Fig. 4-11: Model of direct tensile stress versus strain relationship for reinforcing bars 


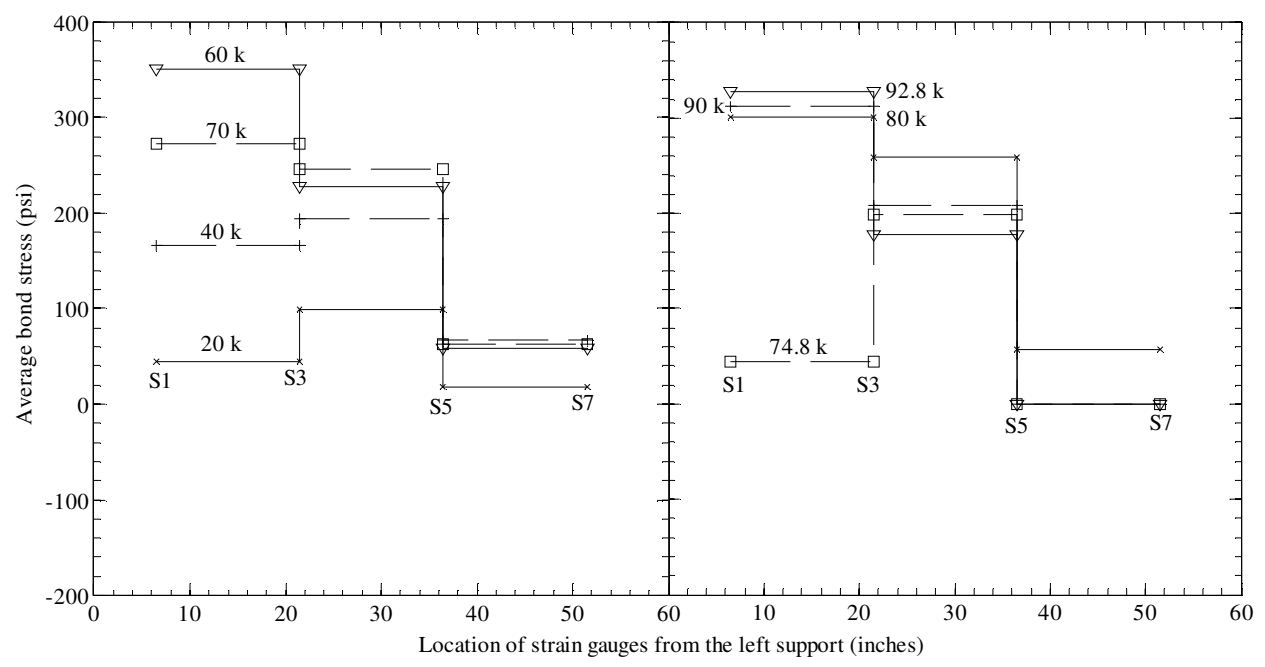

Fig. 4-12: Bond along the lower reinforcing bars at various applied loads - Beam B18-1b

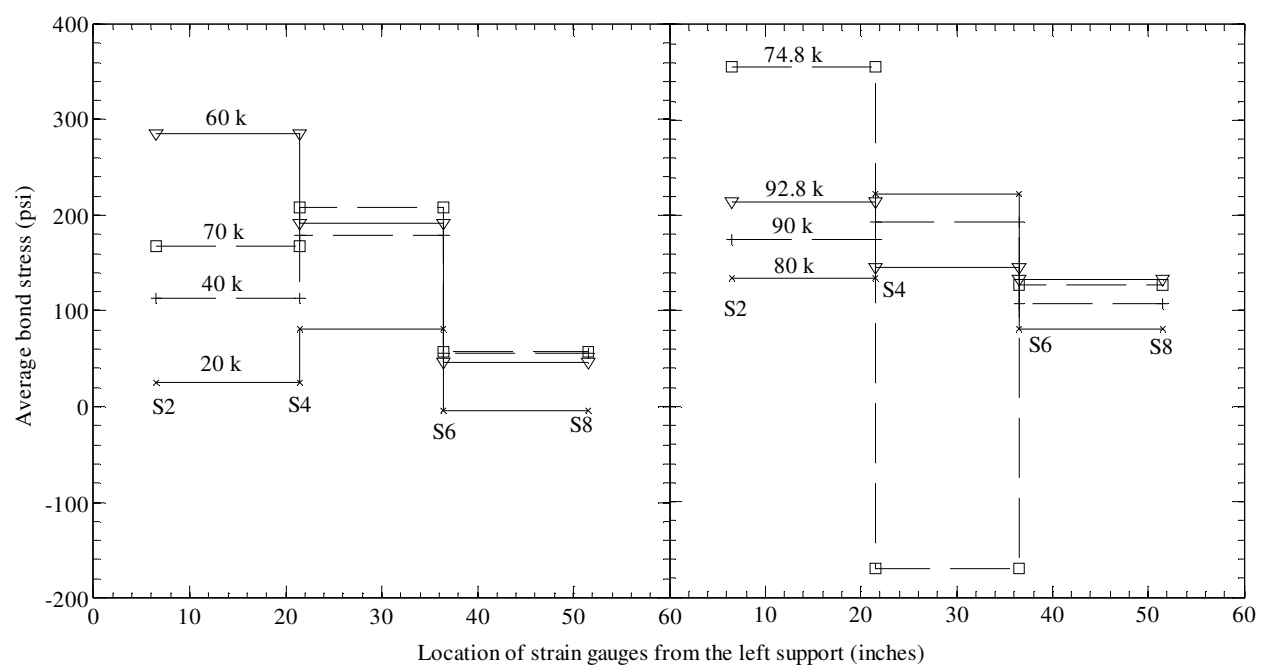

Fig. 4-13: Bond along the upper reinforcing bars at various applied loads - Beam B18-1b

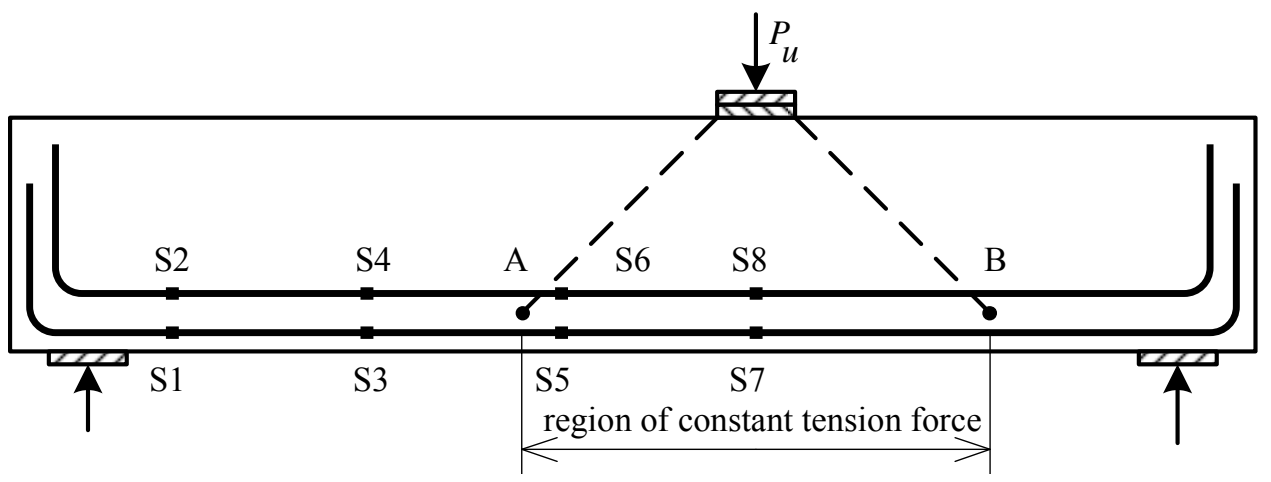

Fig. 4-14: Truss model 
(a)

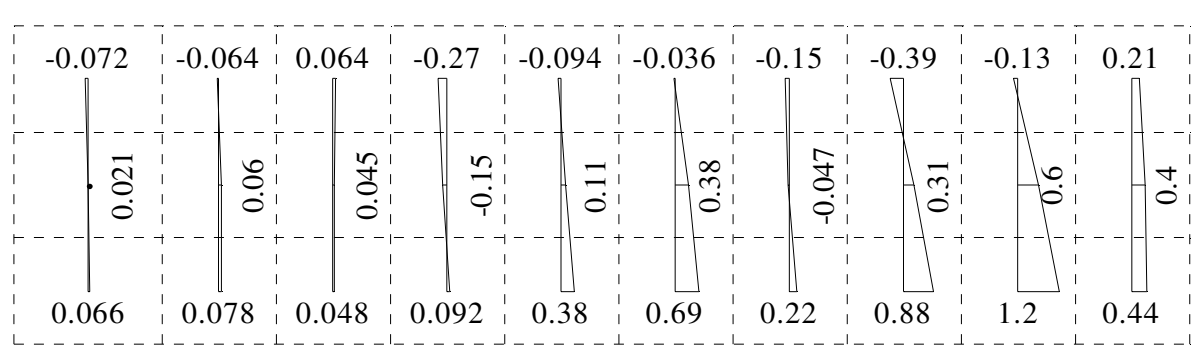

4 Distribution of longitudinal strain (/1000) at 40 kips

(b)

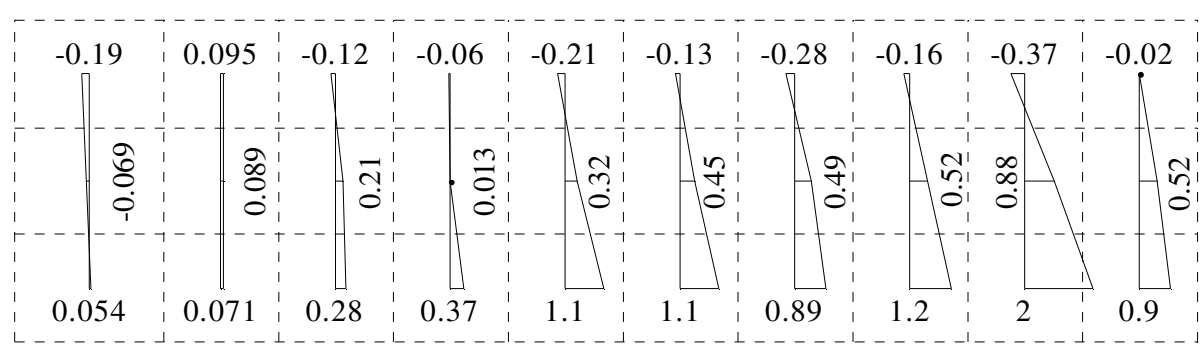

Distribution of longitudinal strain (/1000) at 60 kips

(c)

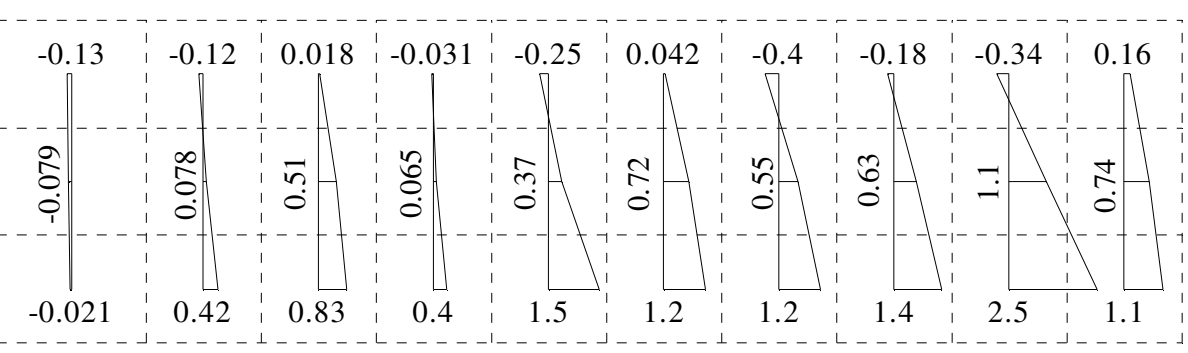

Distribution of longitudinal strain (/1000) at 70 kips

(d)

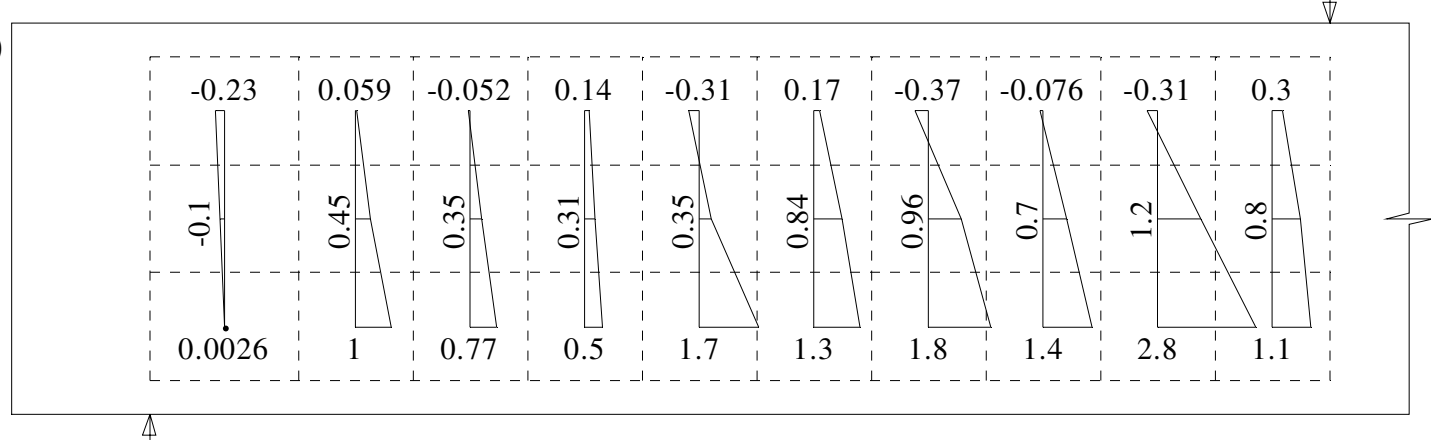

Distribution of longitudinal strain (/1000) at 80 kips

Fig. 4-15: Distribution of longitudinal strains at various applied loads - Beam B18-1b 
(e)

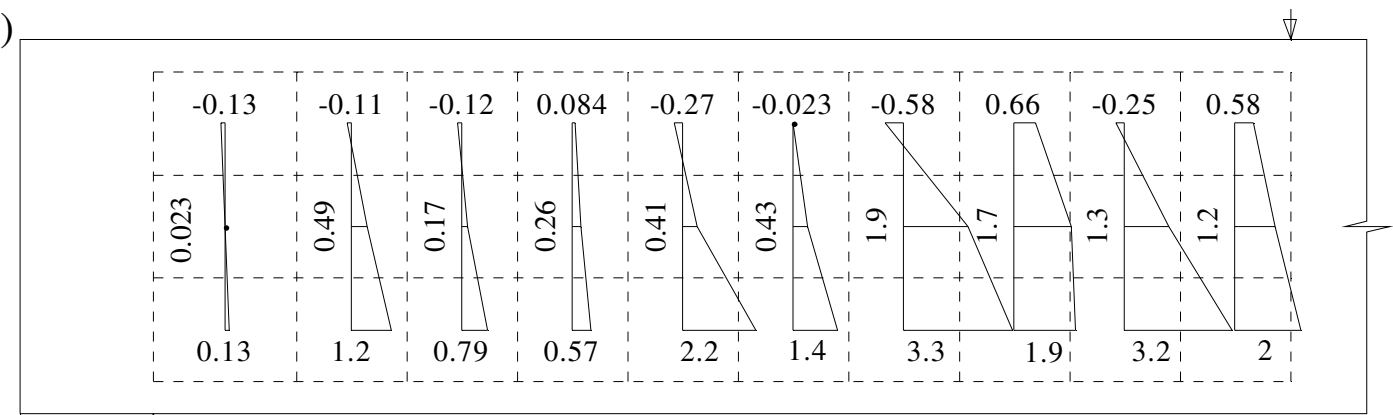

Distribution of longitudinal strain (/1000) at 92.8 kips

Fig. 4-15: Distribution of longitudinal strains at various applied loads - Beam B18-1b (continued)

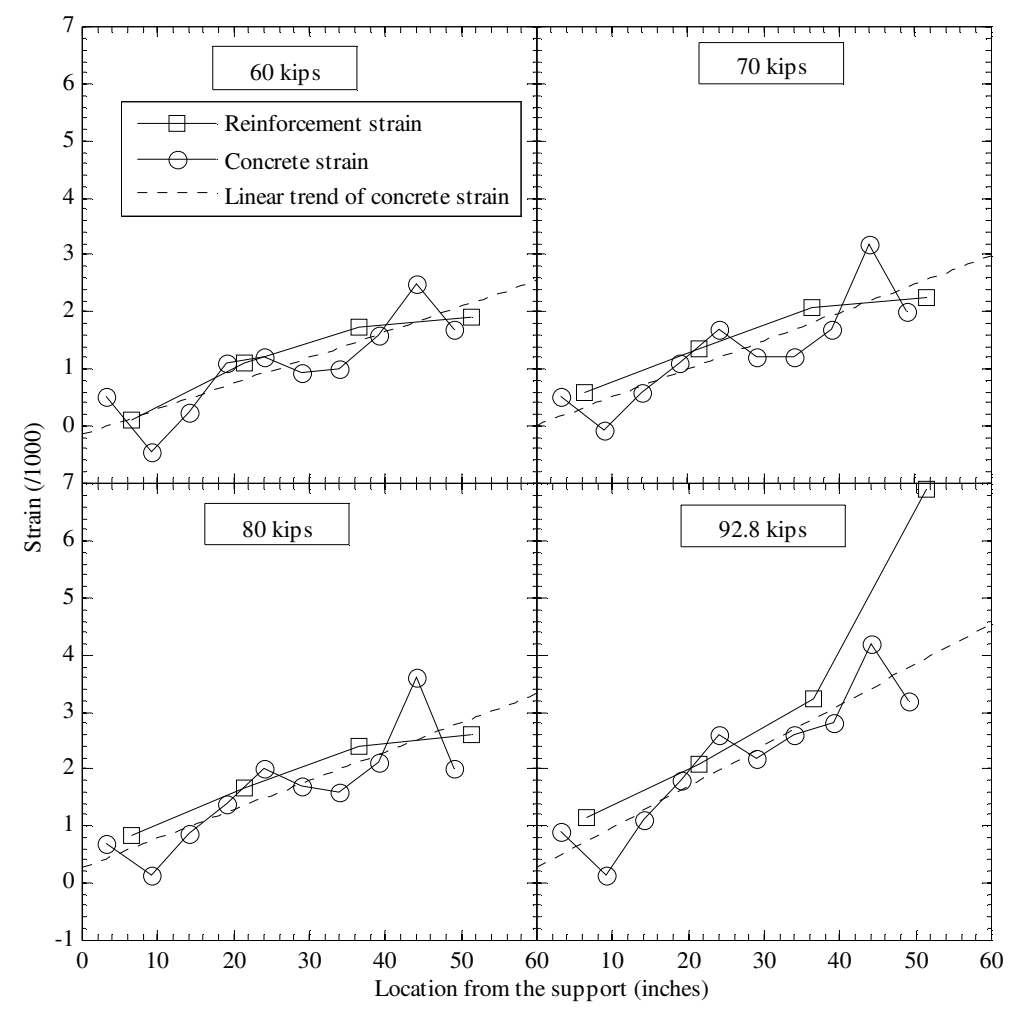

Fig. 4-16: Comparison of reinforcement and average concrete strains at the lower level of reinforcing bars - Beam B18-1b 

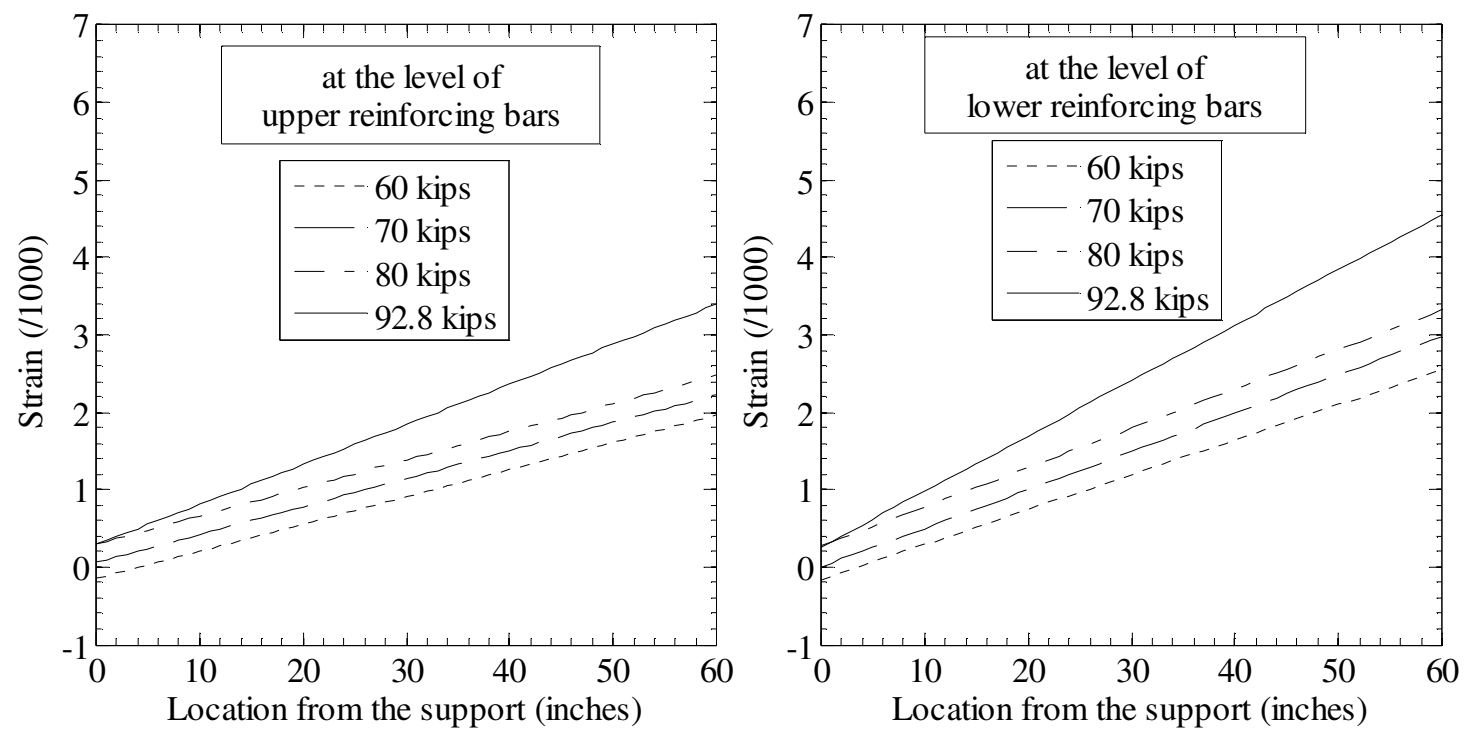

Fig. 4-17: Distribution of average concrete strains - Beam B18-1b

(a)

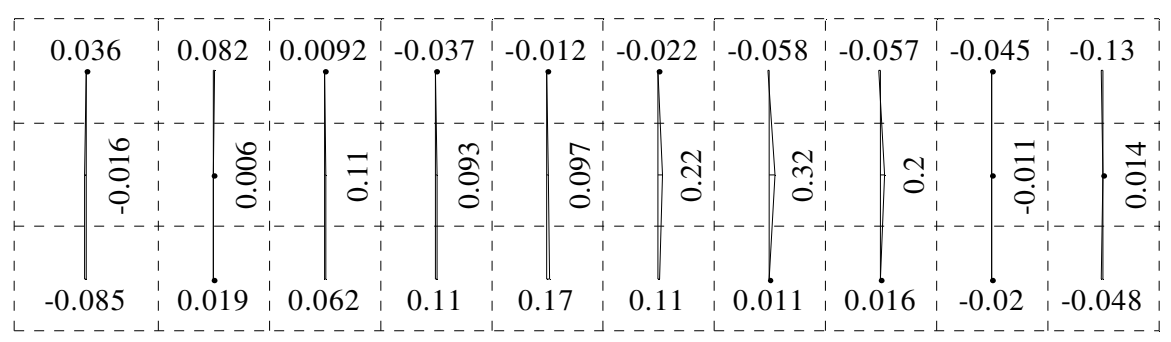

4 Distribution of transverse strain (/1000) at $60 \mathrm{kips}$

(b)

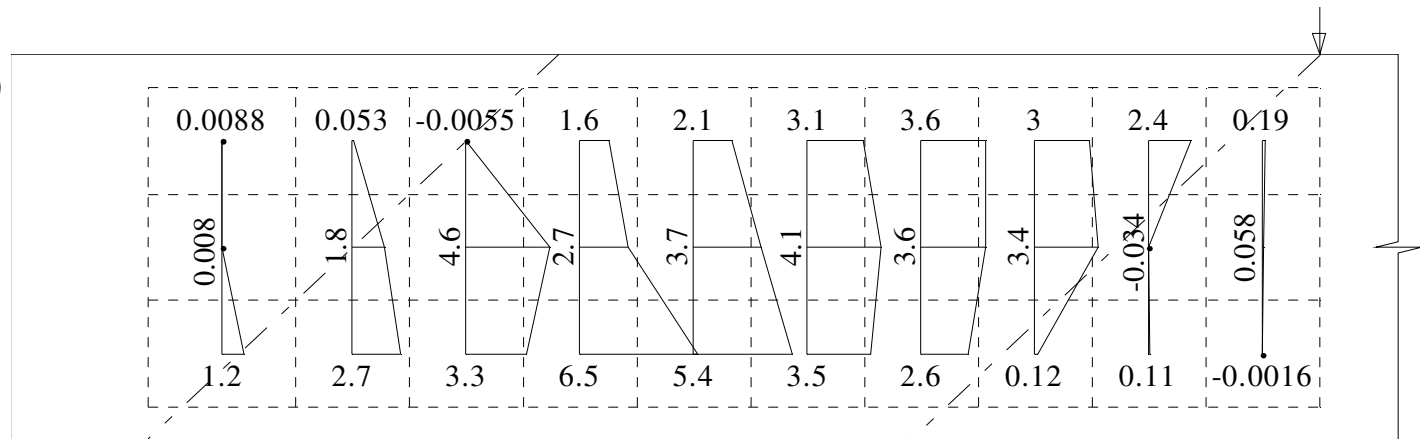

Distribution of transverse strain (/1000) at 92.8 kips

Fig. 4-18: Distribution of transverse strains at various applied loads - Beam B18-1b 


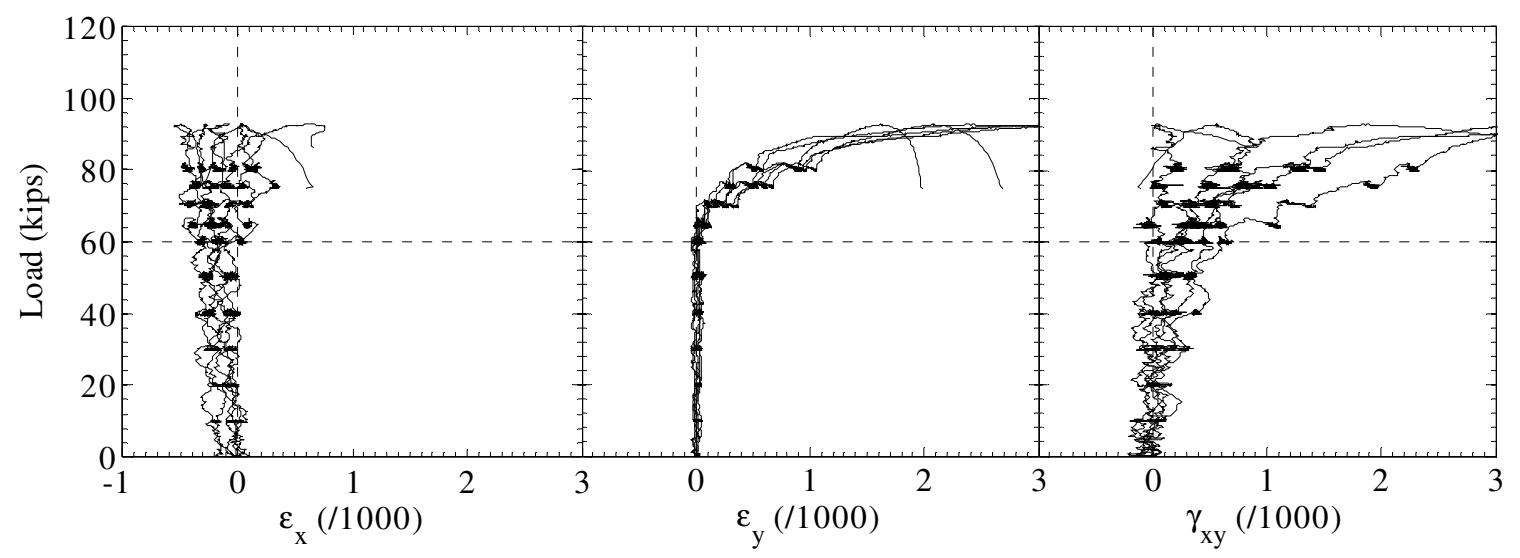

(a) Top row

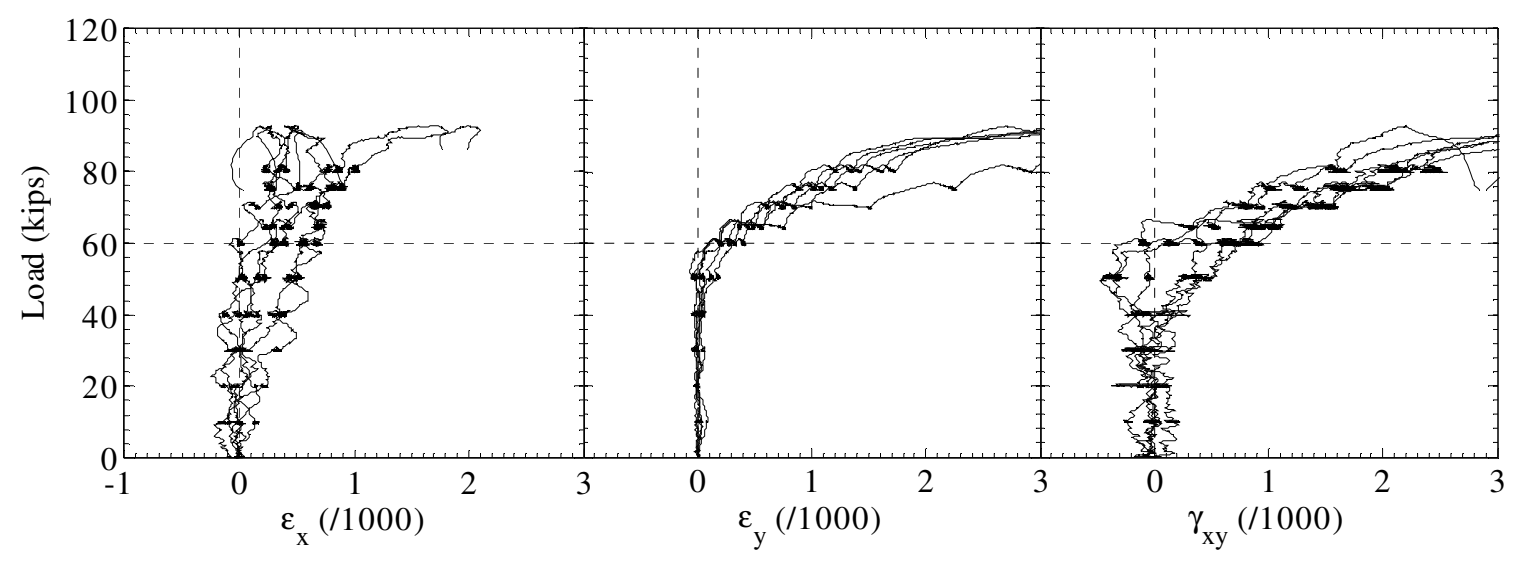

(b) Middle row

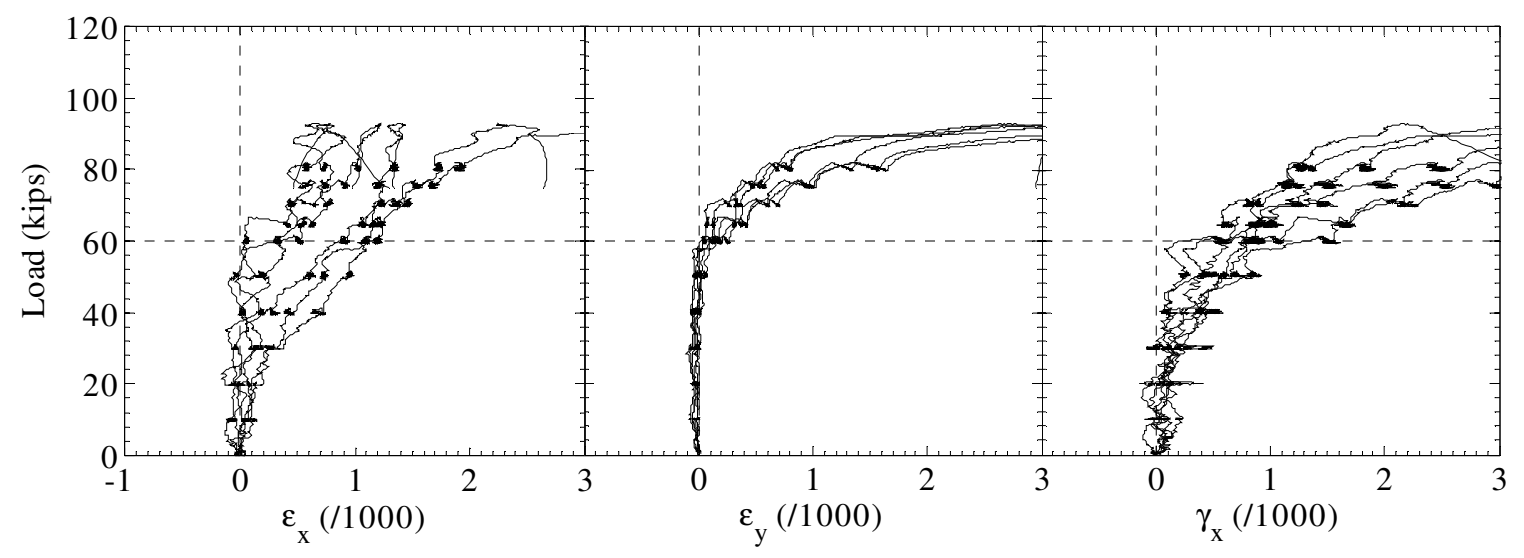

(c) Bottom row

Fig. 4-19: Concrete strains in the parallelogram region shown in Fig. 4-18 - Beam B18$1 b$ 
(a)

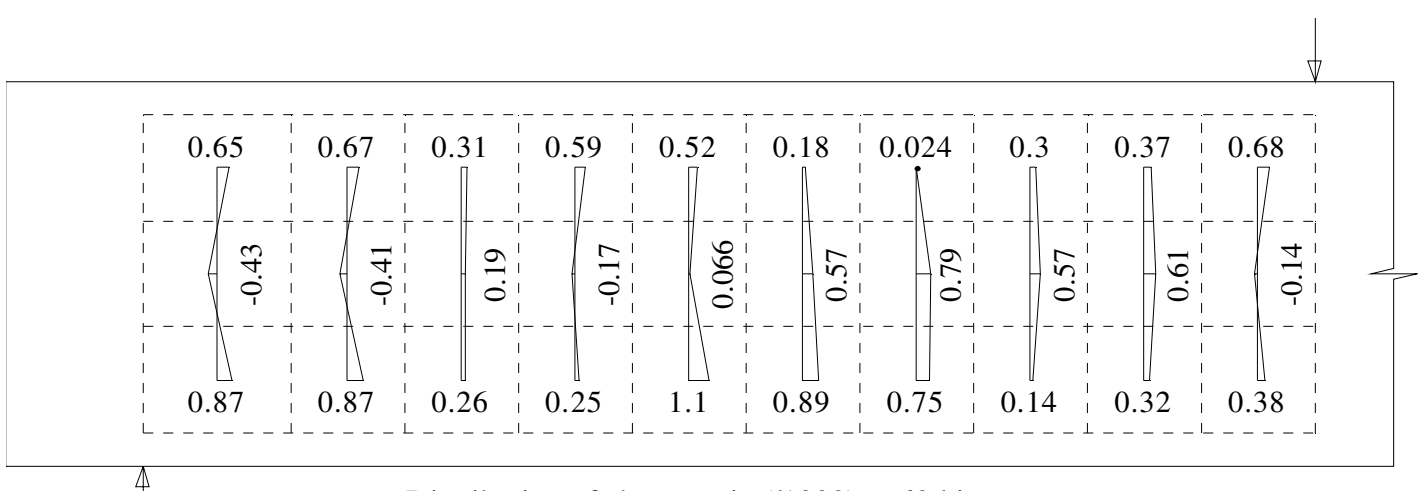

Distribution of shear strain (/1000) at 60 kips

(b)

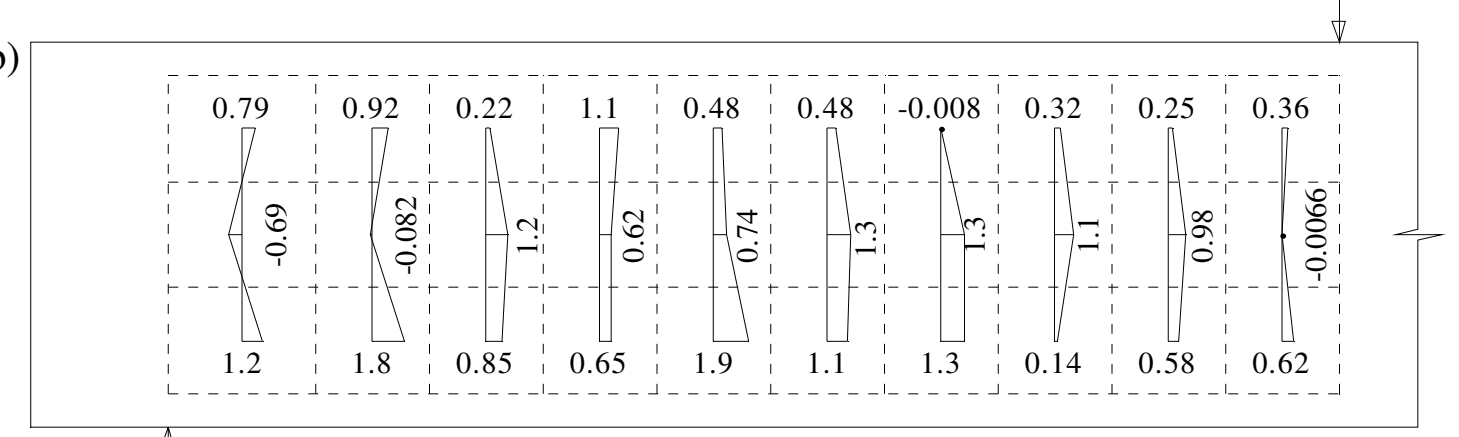

Distribution of shear strain (/1000) at $70 \mathrm{kips}$

(c)

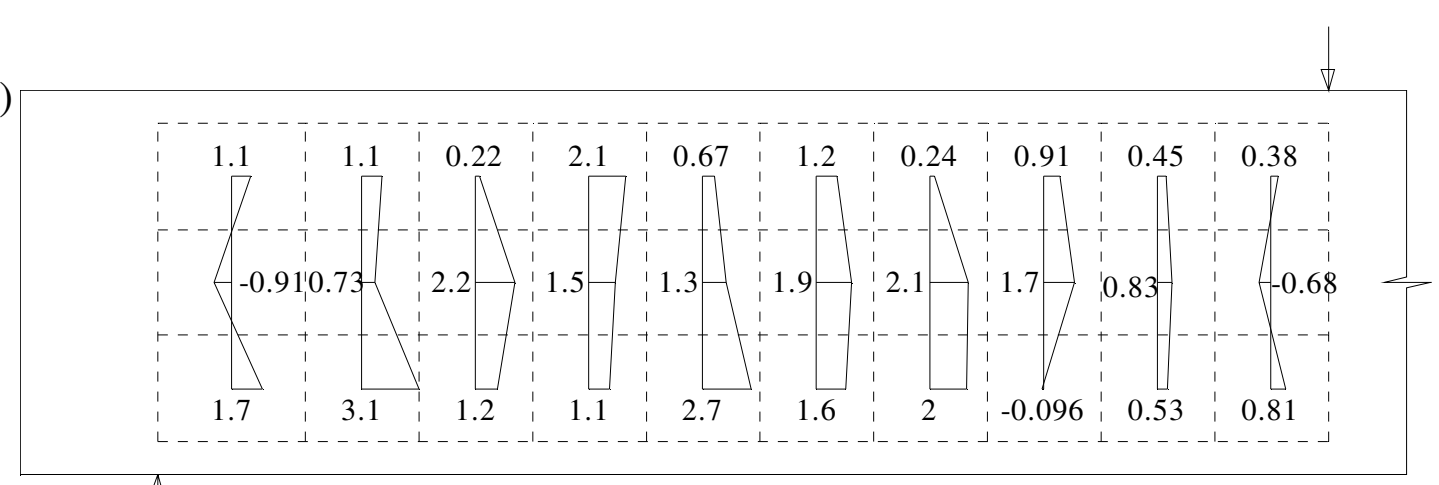

Distribution of shear strain (/1000) at 80 kips

(d)

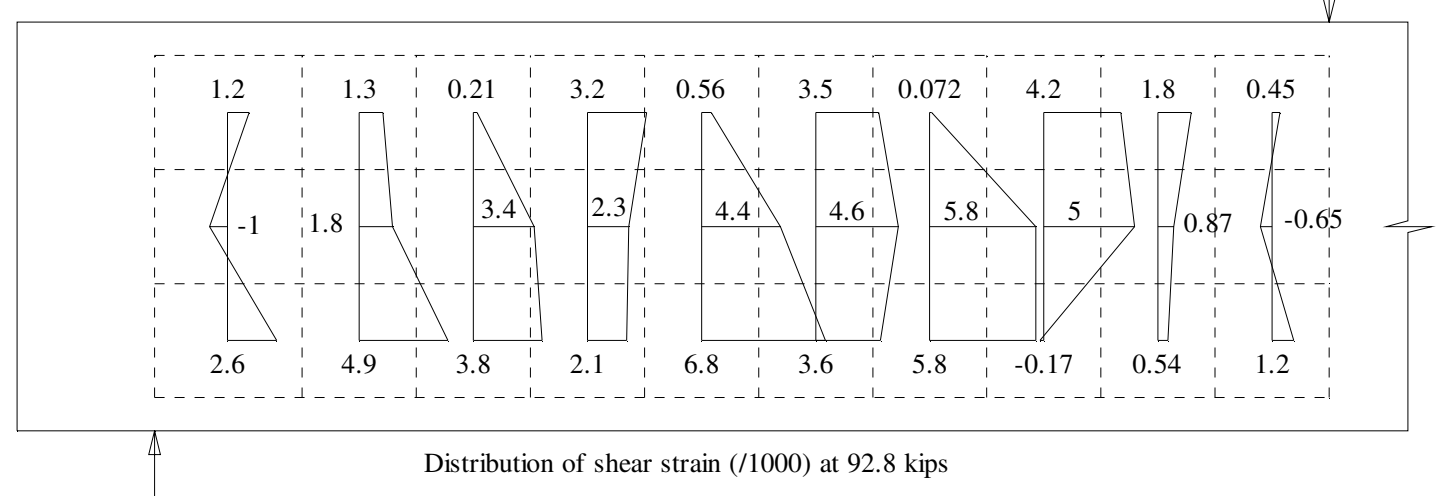

Fig. 4-20: Distribution of shear strains at various applied loads - Beam B18-1b 

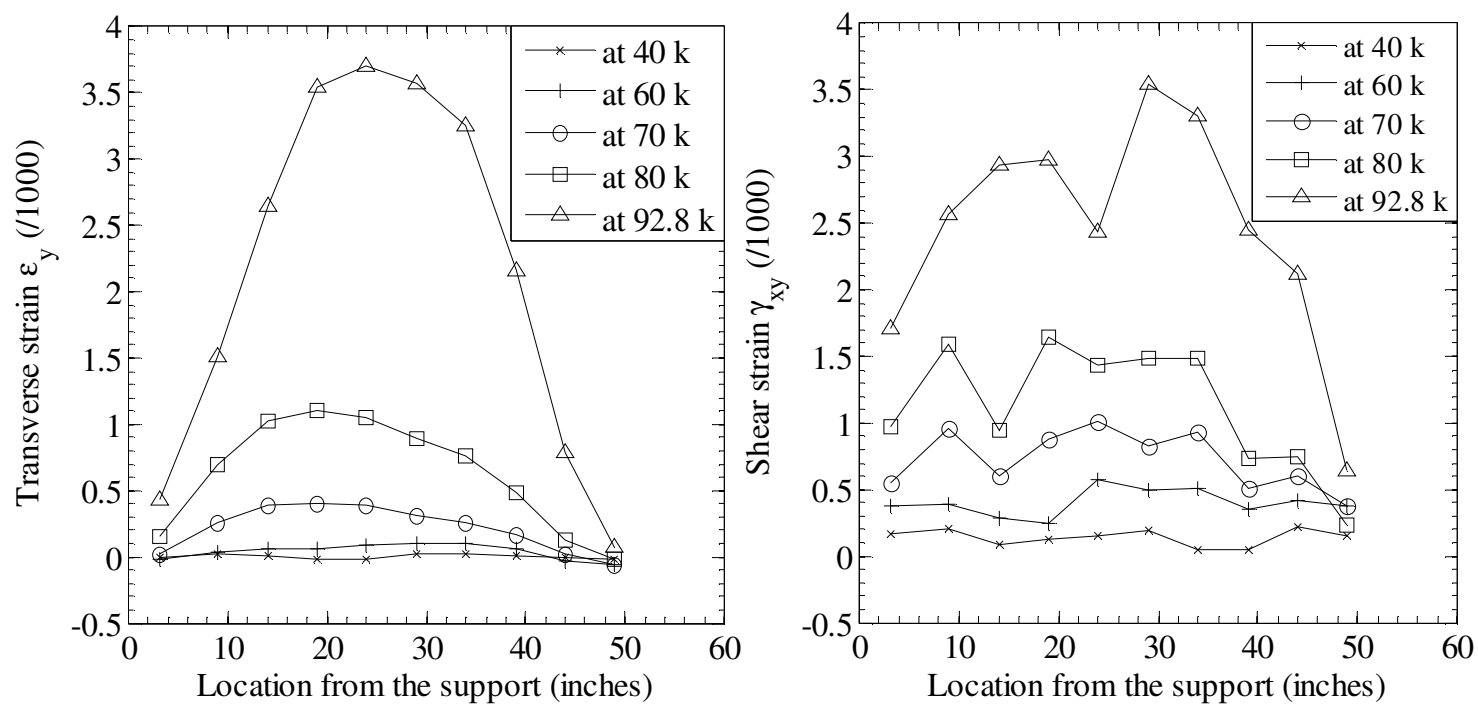

Fig. 4-21: Distribution of transverse and shear strains averaged over the beam depth Beam B18-1b
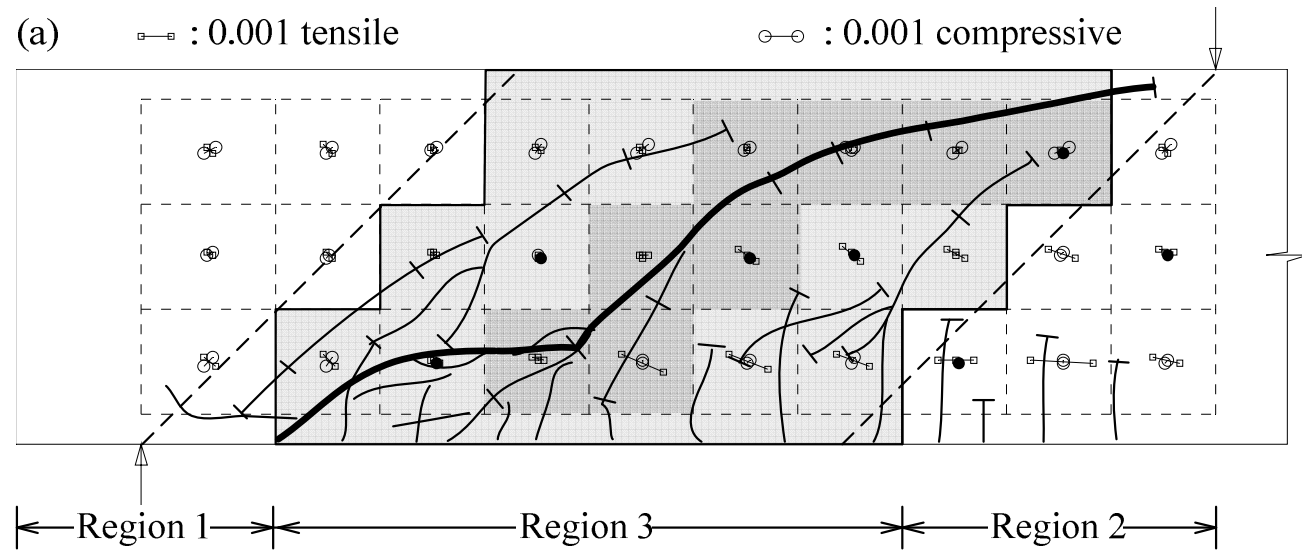

Region $2 \longrightarrow$

(b) $\square: 0.001$ tensile $\odot: 0.001$ compressive

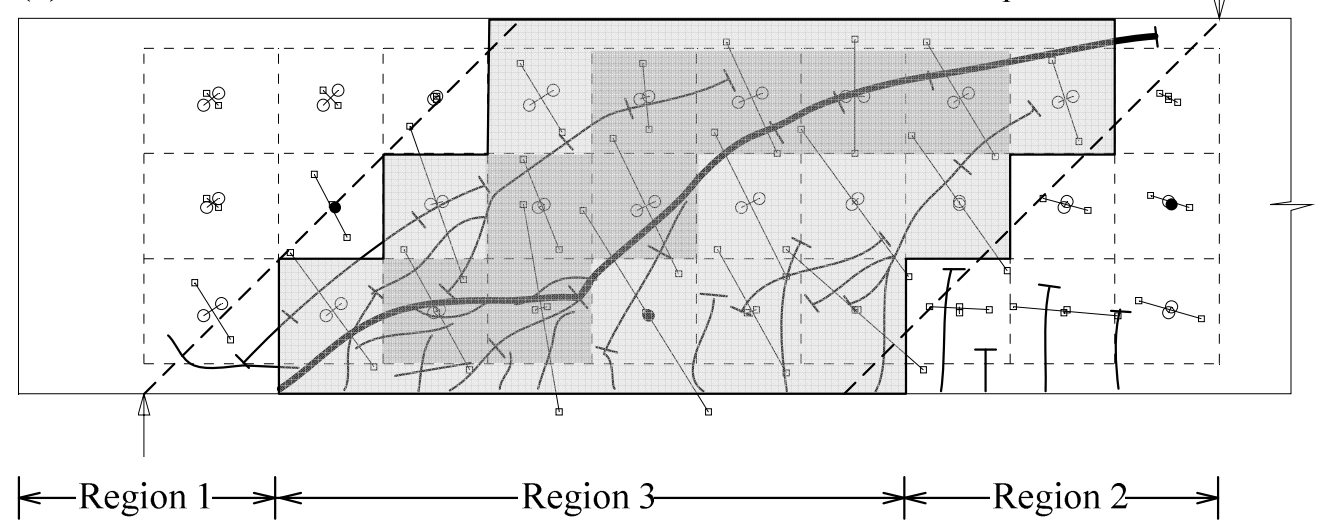

Fig. 4-22: Principal strain field at first diagonal cracking (a) and peak load (b) - Beam B18-1b 


\begin{tabular}{|c|c|c|c|c|c|c|c|c|c|}
\hline $6^{\circ .88^{\circ}}$ & $5^{\circ}$ & $3^{\circ}$ & $12^{\circ}$ & $28^{\frac{8}{0^{\circ}}}$ & $6^{\circ}$ & 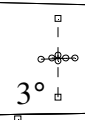 & $5^{\circ}$ & $7^{\circ}$ & $16^{8.86}$ \\
\hline $3^{\circ}$ & $24^{\circ}$ & $40^{\circ}$ & $12^{\circ}$ & 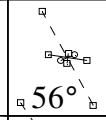 & $30^{\circ}$ & & $25^{\circ}$ & $1^{\circ}$ & $7^{\circ}$ \\
\hline $16^{\circ}$ & $10^{\circ}$ & $37^{\circ}$ & $58^{\circ}$ & $33^{\circ}$ & $40^{\circ}$ & $22^{\circ}$ & $0^{\circ}$ & $0^{\circ}$ & $4^{\circ}$ \\
\hline
\end{tabular}

Fig. 4-23: Rotation of principal angles from first diagonal cracking (continuous lines) to maximum load (dashed lines) - Beam B18-1b

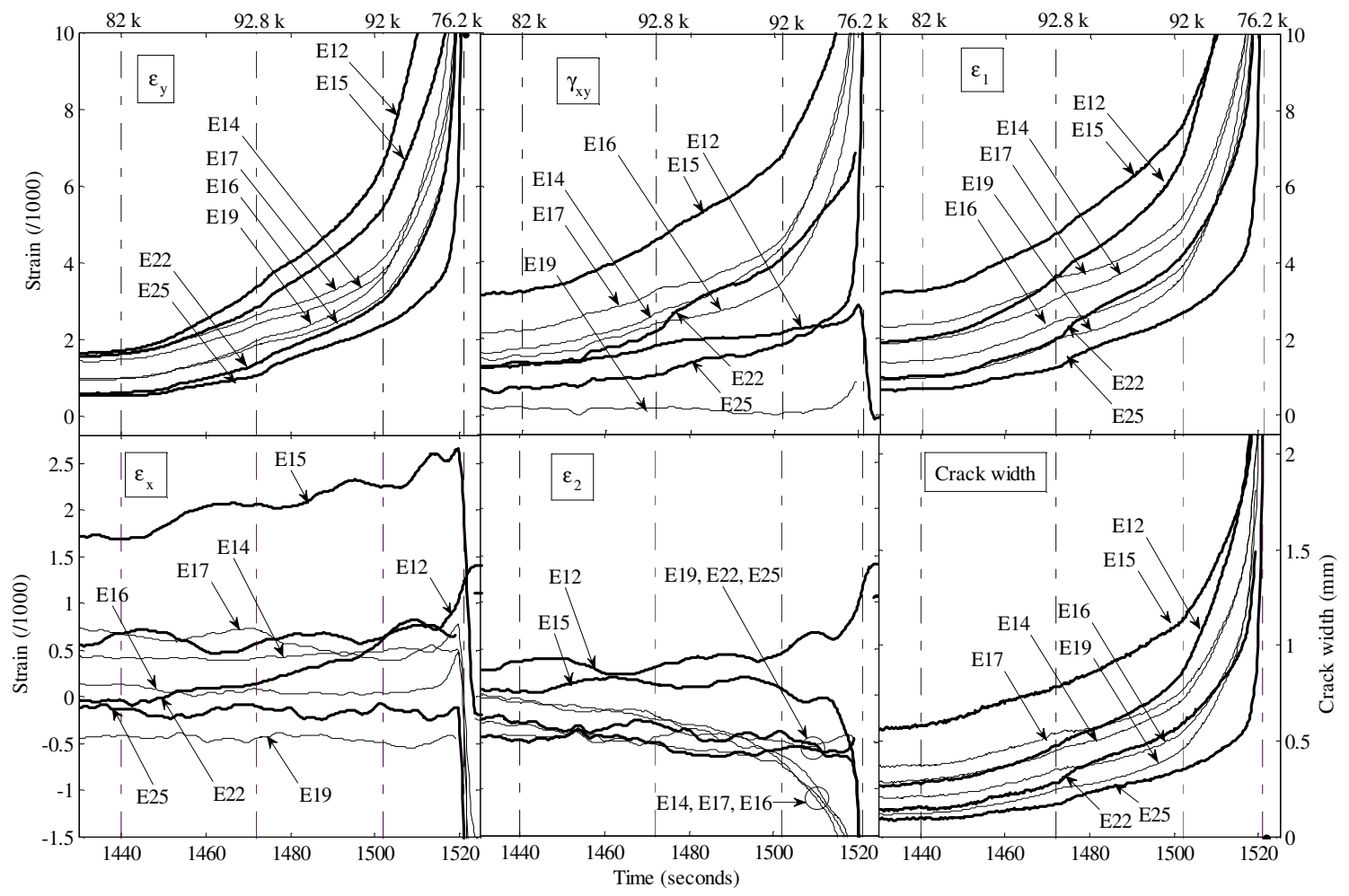

Fig. 4-24: Development of strains along the critical diagonal crack around the peak load Beam B18-1b 


\subsubsection{Beam B18-1a}

Load versus deflection relationship, crack pattern, and failure mode. Beam B18-1a had "identical" properties to Beam B18-1b. Fig. 4-25 shows the load versus deflection relationship and crack patterns of Beam B18-1a. Similar to Beam B18-1b, Beam B18-1a exhibited first diagonal cracking at a load of approximately 60 kips (Fig. 4-26) and reached the ultimate strength of 99.2 kips after significant flexural and diagonal cracks developed throughout the shear span. A sign of partial shear-tension failure was also observed (Fig. 4-27). Compared with Beam B18-1b, Beam B18-1a had a slightly higher strength, which is believed to be the result of a better redistribution of internal stresses due to additional diagonal cracks (five in total).

Reinforcement strain and average bond stress. Similar to Beam B18-1b, the following observations were made with regard to reinforcement strains and average bond stress:

(1) The straining rate was somewhat different for strain gauges S3, S5, and S7 on the lower layer of reinforcement compared to strain gauges S4, S6, and S8 on the upper layer (Fig. 4-29 and Fig. 4-30).

(2) The reinforcement strains at S1 through S4 were still in the linear range of the reinforcement stress-strain curve while the reinforcement at S5 through S8 yielded. Notably, the strains at S5 and S7 approached the strain-hardening region.

(3) The strains were linearly distributed along the beam axis up to the load at first diagonal cracking (60 kips) for both the upper and lower layers of reinforcing bars (Fig. 4-30).

(4) Bond stresses were largest near the support, ranging between 300 and 500 psi at peak load, while being negligible in the trapezoidal region under the loading point.

Analysis of the strain field. The distribution of longitudinal, transverse, and shear strains in Beam B18-1a was very similar to that of Beam B18-1b. The following observations were made: 
(1) Using the average strain per block, as discussed in Section 4.2.3, the vertical distribution of longitudinal strains was almost linear up to the peak load of $99.2 \mathrm{kips}$ (Fig. 4-33a).

(2) Using only the displacements of two adjacent markers, the longitudinal strain distribution over the beam depth was irregular with maximum compressive and tensile concrete strains of 0.0015 and 0.0076 , respectively (Fig. 4-33b).

(3) The slope of the approximated linear distribution of concrete strain at the reinforcement level (obtained as shown in Fig. 4-34) over the shear span increased significantly at the peak load (Fig. 4-35).

(4) Transverse and shear strains measured over the beam depth were larger in the middle of the shear span (Fig. 4-33 and Fig. 4-36).

(5) Transverse and shear strains developed only after first diagonal cracking, while longitudinal strains at the beam bottom steadily increased with increasing load (Fig. 4-37).

(6) Large principal tensile strains (Fig. 4-38) concentrated in the parallelogram region defined in Fig. 4-33c.

Distribution of principal strains and failure mechanism. Fig. 4-38 shows the principal strain distribution, while Fig. 4-39 shows the development of principal tensile strains along the critical inclined cracks versus time. It can be seen that the strain of the bottom element E18 (see Fig. 4-28 for the numeration of elements) was larger than that of other elements up to an applied load of approximately 90 kips, at which load the principal strain of Element E20 started to exceed that of Element E18. At the maximum load, the principal tensile strain was largest at Element E20 and lower in the bottom elements E18 and E15 and even lower in the top elements E22 and E25. This indicated that the beam failure was triggered by the crack opening in the beam mid-depth region, propagating towards the tension reinforcement and then the compression zone region. 


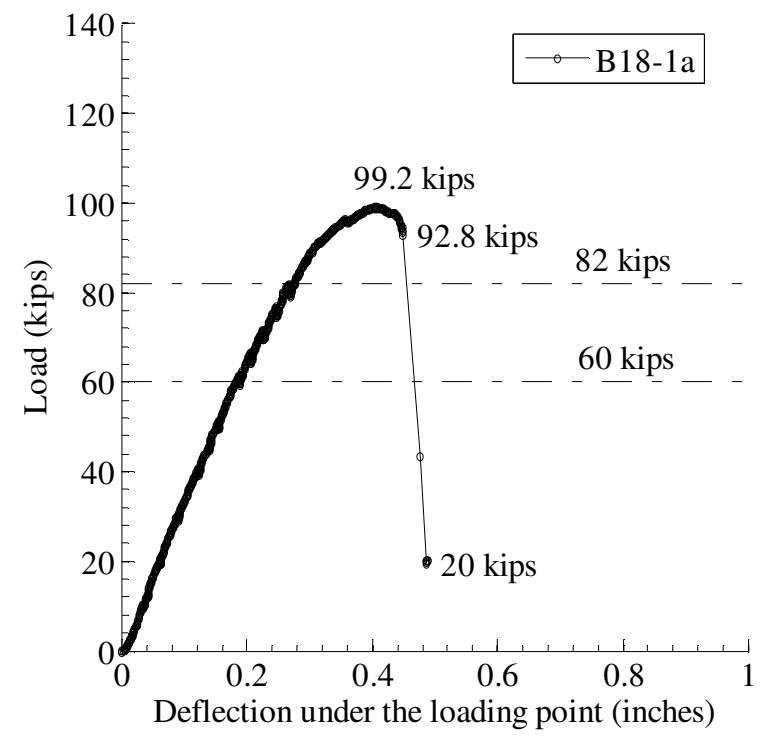

Fig. 4-25: Load versus deflection relationship - Beam B18-1a

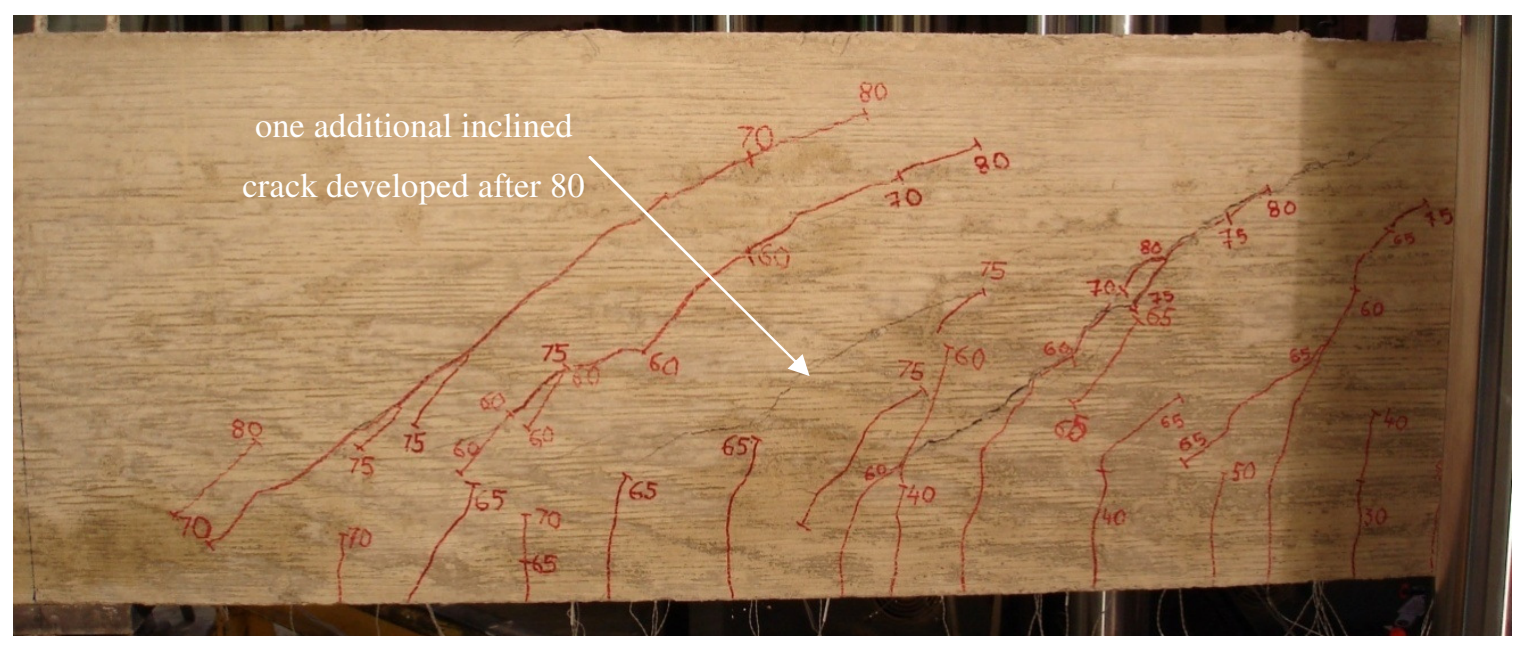

Fig. 4-26: Crack pattern prior to failure - Beam B18-1a

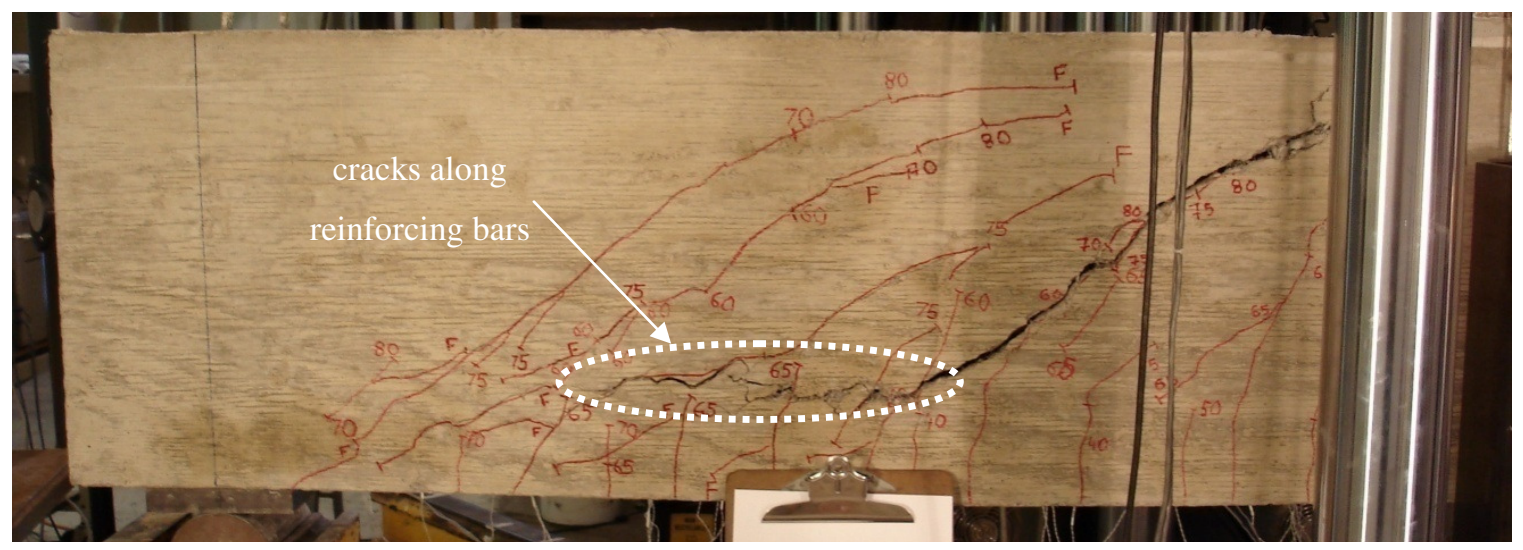

Fig. 4-27: Crack pattern after failure - Beam B18-1a 


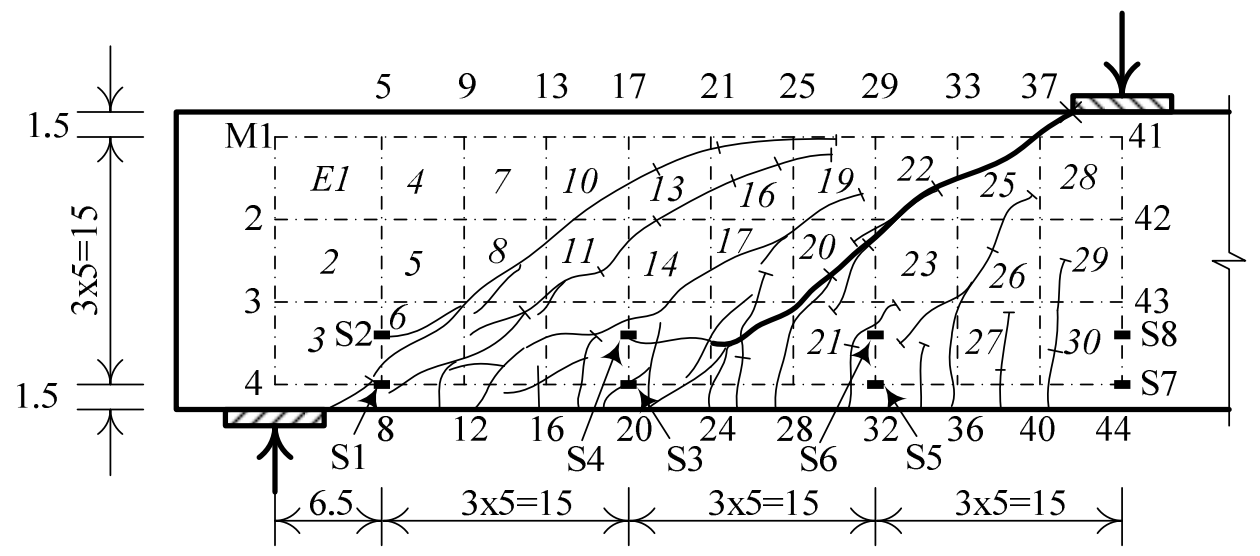

Fig. 4-28: Numeration of markers, elements, and strain gauges - Beam B18-1a

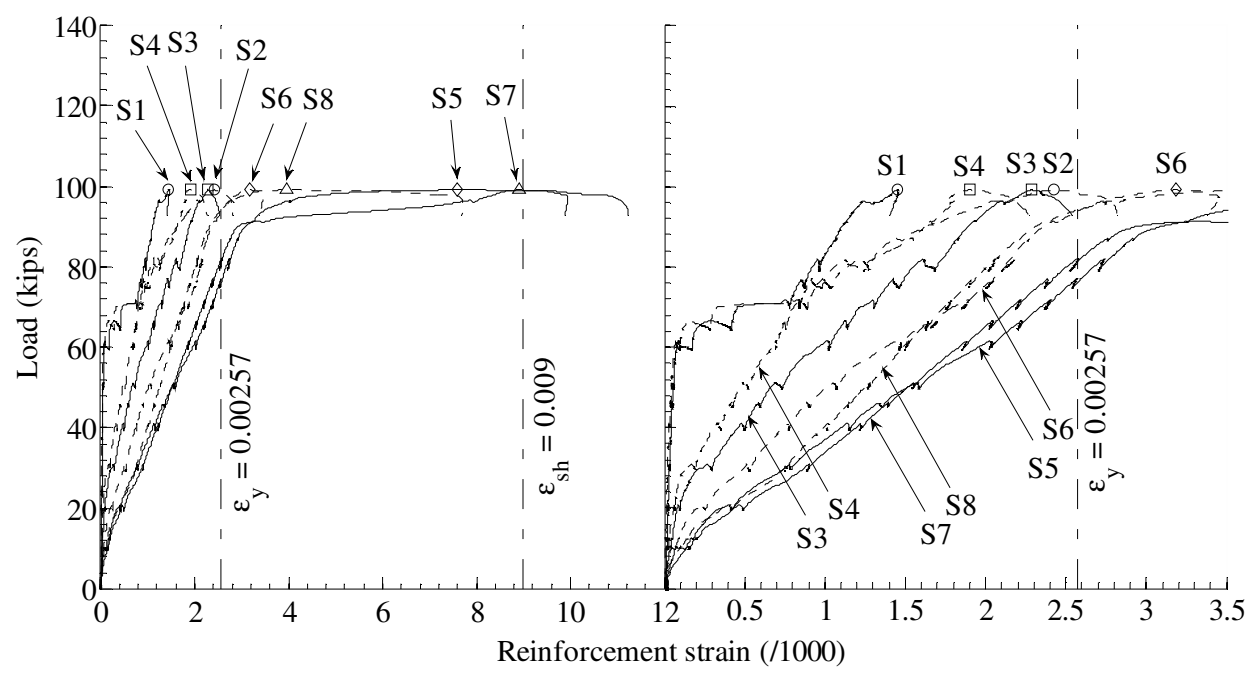

Fig. 4-29: Load versus reinforcement strain relationships - Beam B18-1a

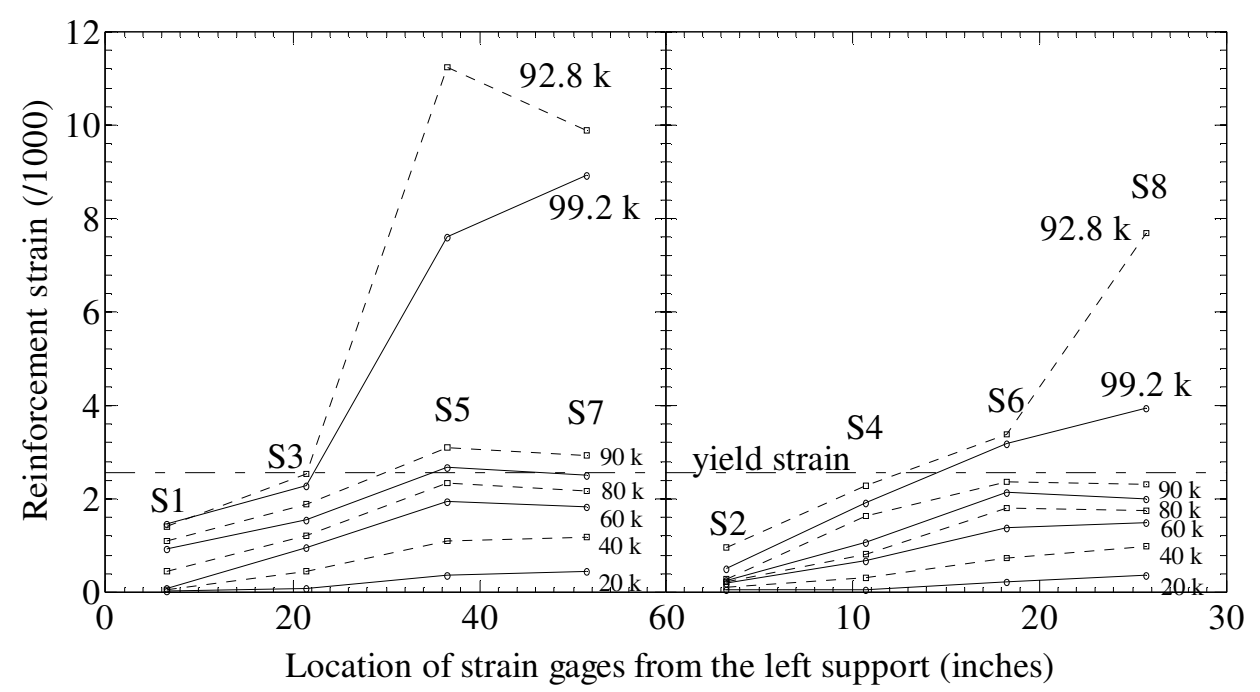

Fig. 4-30: Reinforcement strains along the beam axis - Beam B18-1a 


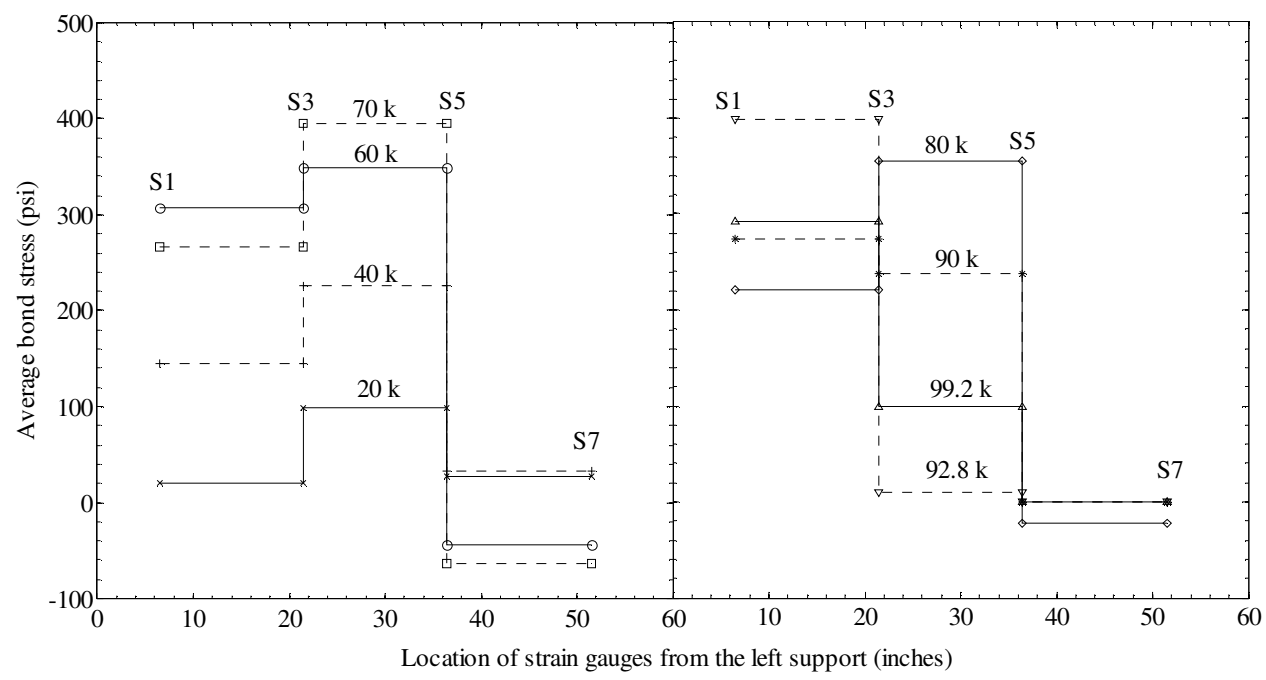

Fig. 4-31: Average bond stress in the lower tension reinforcement - Beam B18-1a

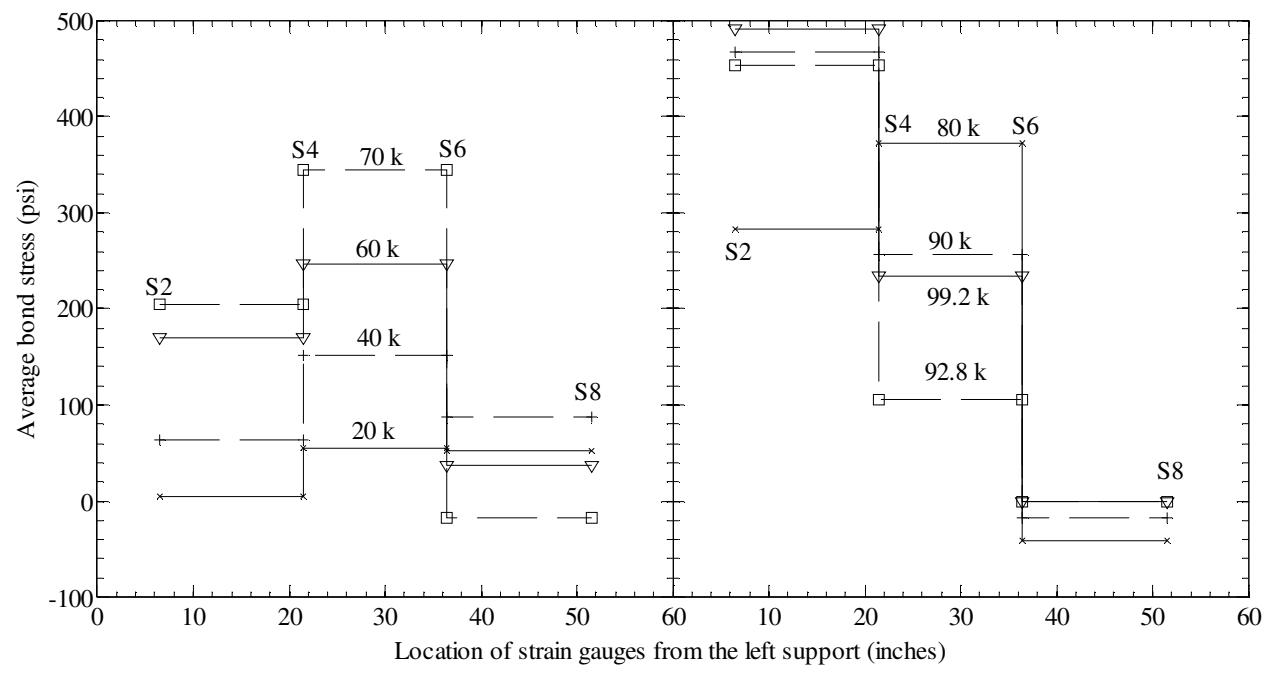

Fig. 4-32: Average bond stress in the upper tension reinforcement - Beam B18-1a 
(a)

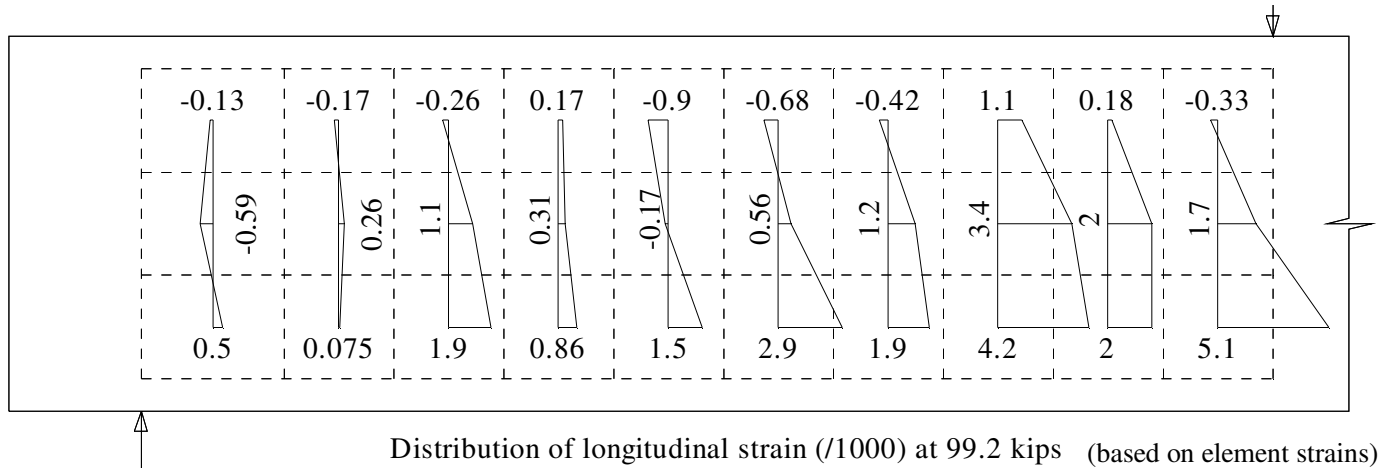

(b)

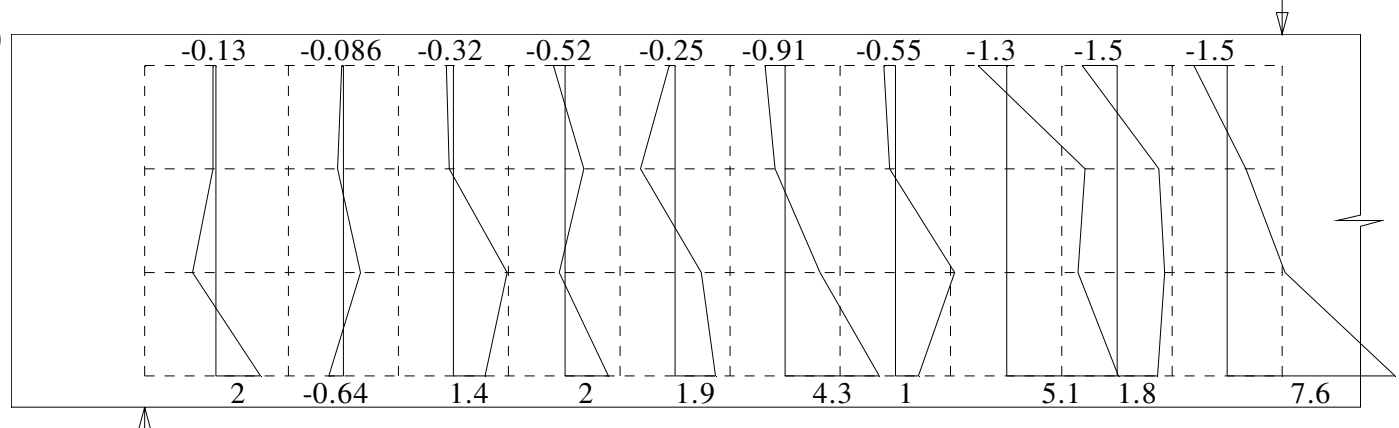

Distribution of longitudinal strains (/1000) at 99.2 kips (based on adjacent markers)

(c)

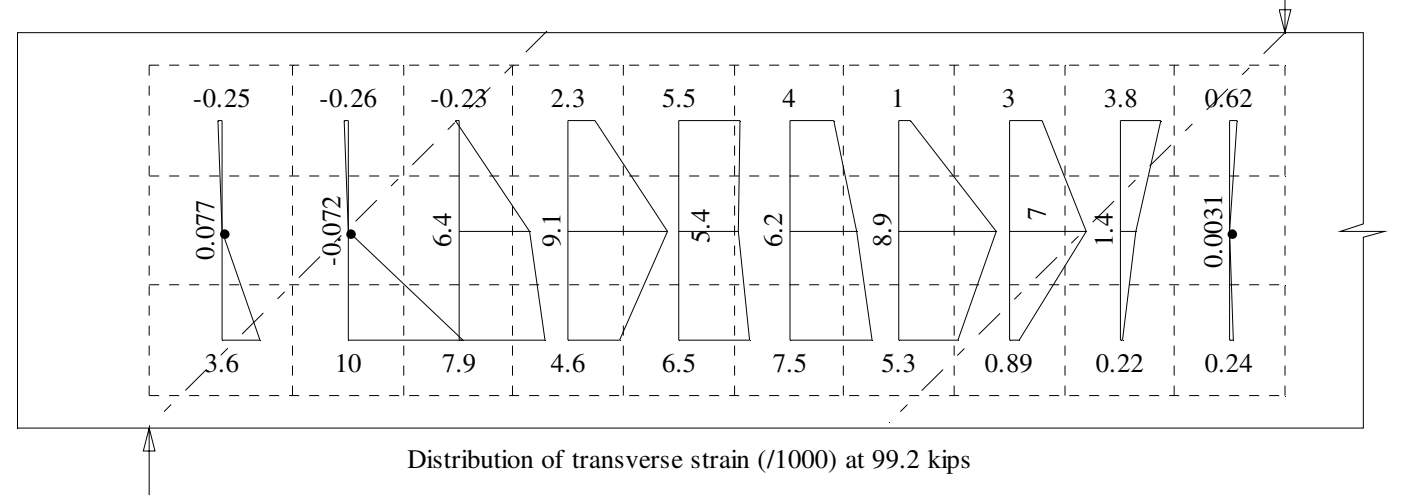

(d)

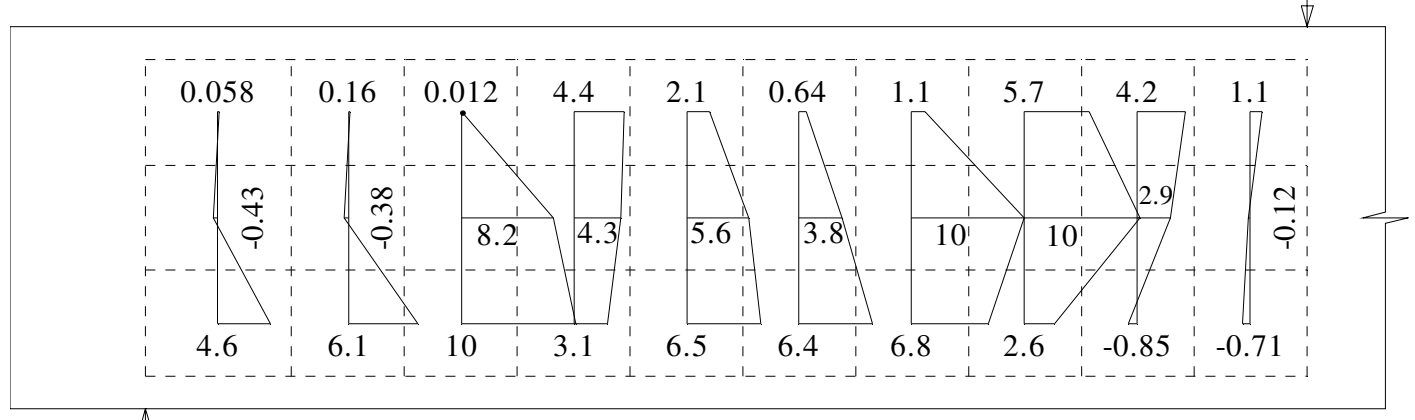

Distribution of shear strain (/1000) at 99.2 kips

Fig. 4-33: Distribution of longitudinal, transverse, and shear strains at peak load - Beam

B18-1a 


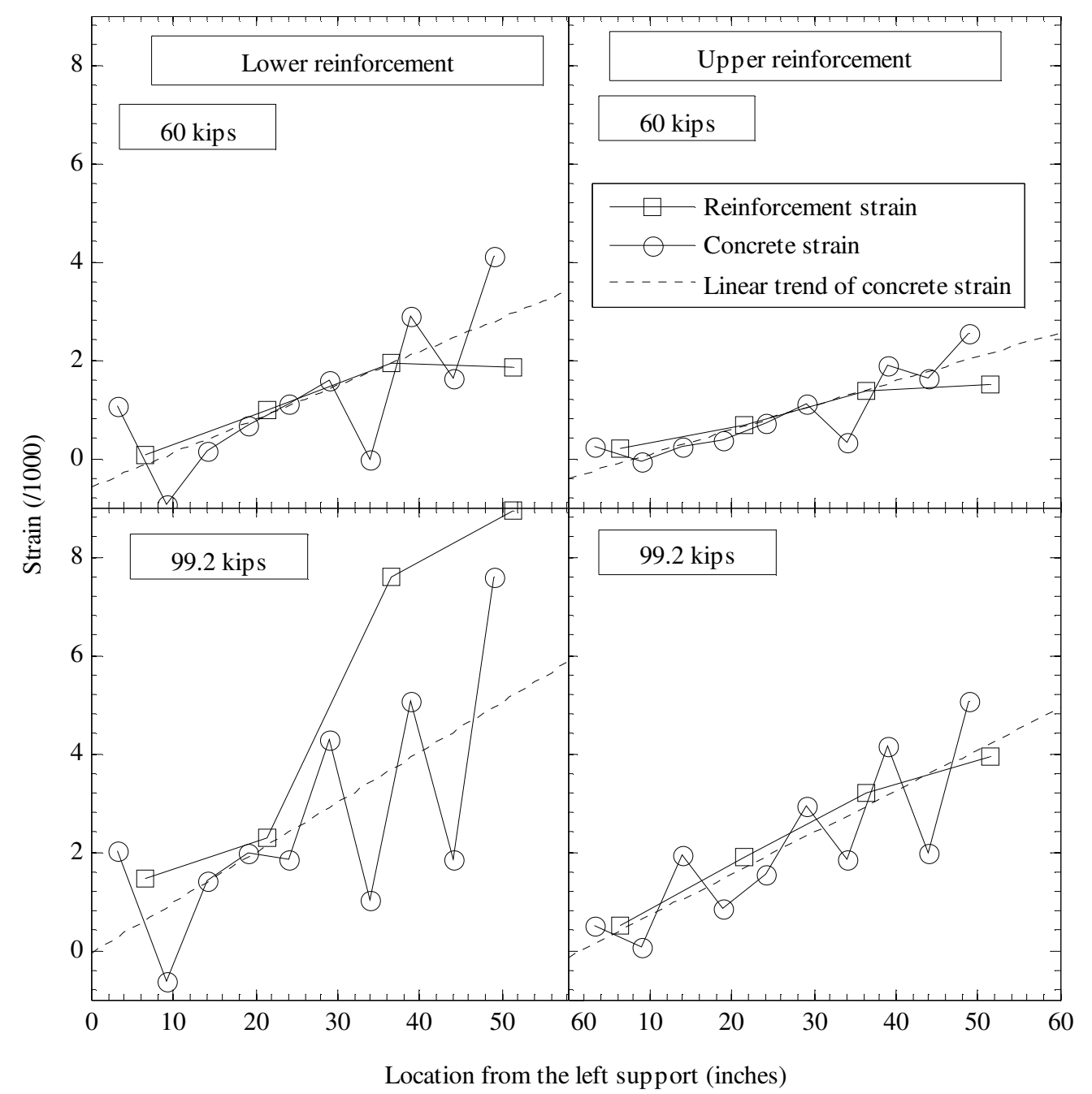

Fig. 4-34: Comparison of reinforcement and average concrete strain at the level of lower and upper reinforcing bars - Beam B18-1a
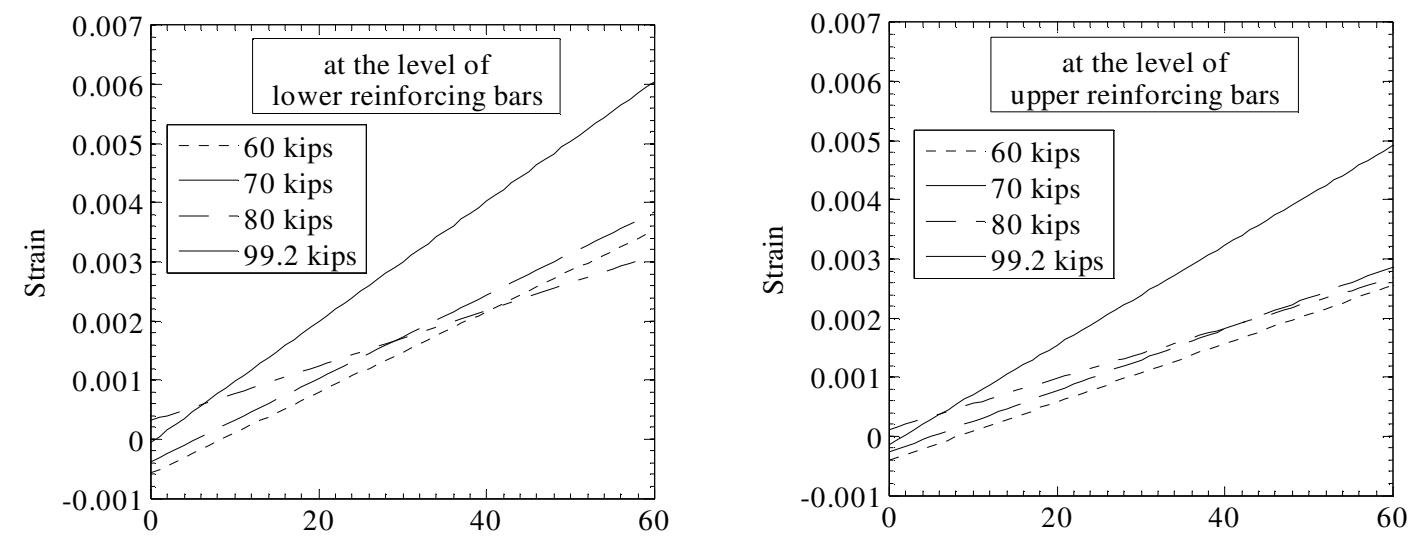

Location of measured strain from the left support (inches) Location of measured strain from the left support (inches)

Fig. 4-35: Distribution of average concrete strain at various applied loads - Beam B18-1a 


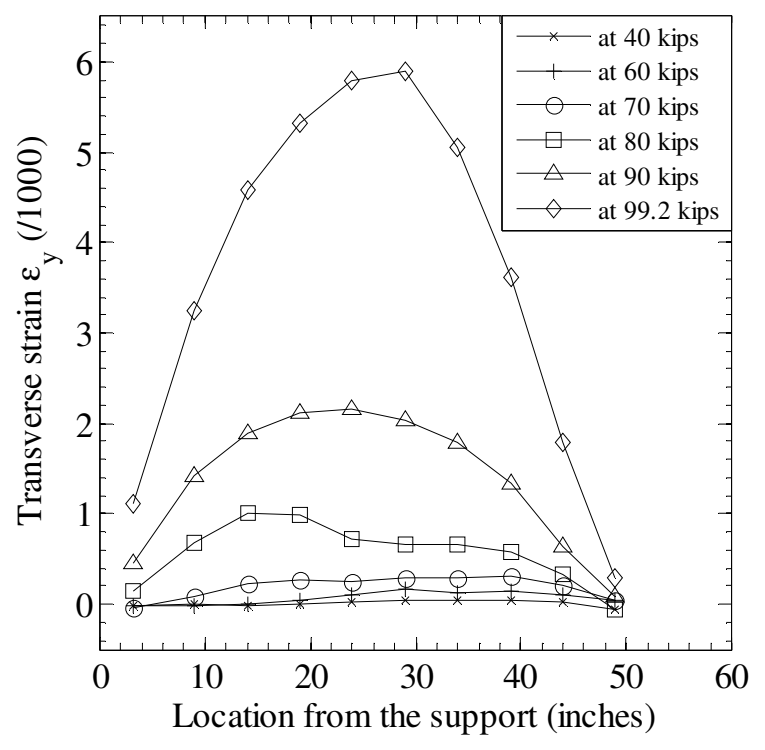

(a)

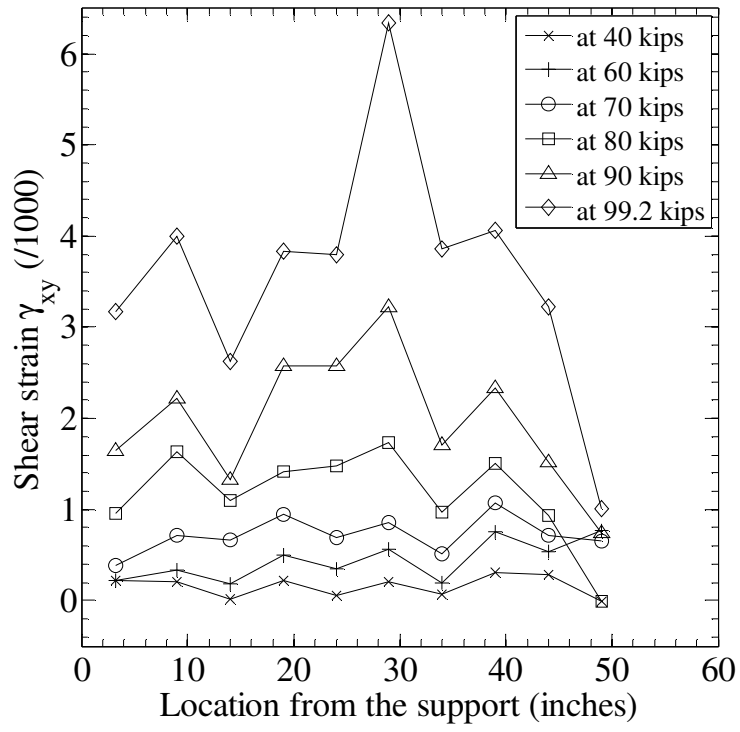

(b)

Fig. 4-36: Distribution of transverse and shear strains averaged over the beam depth -

\section{Beam B18-1a}

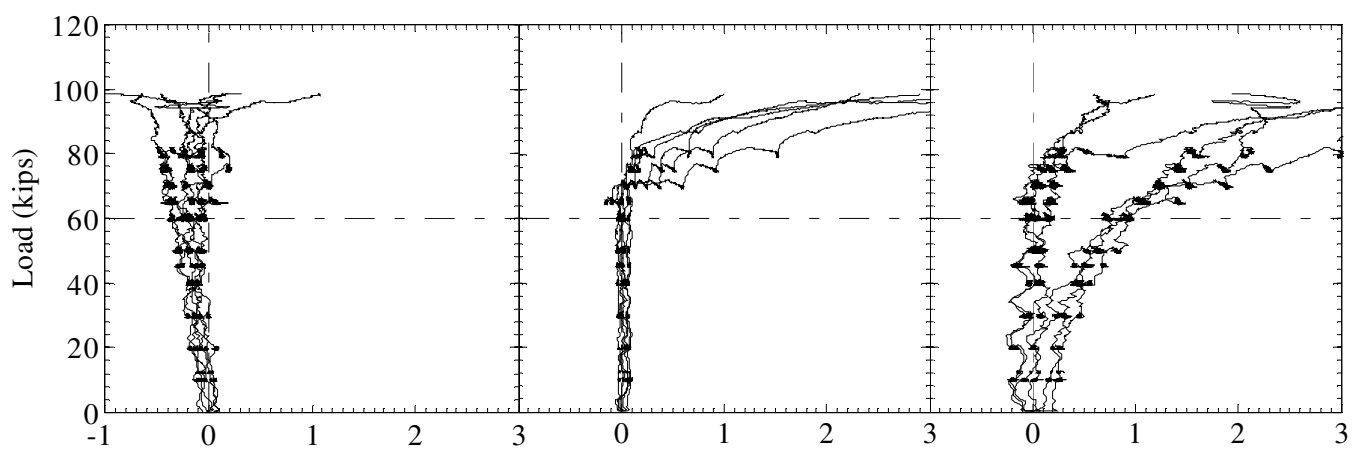

(a) Top row

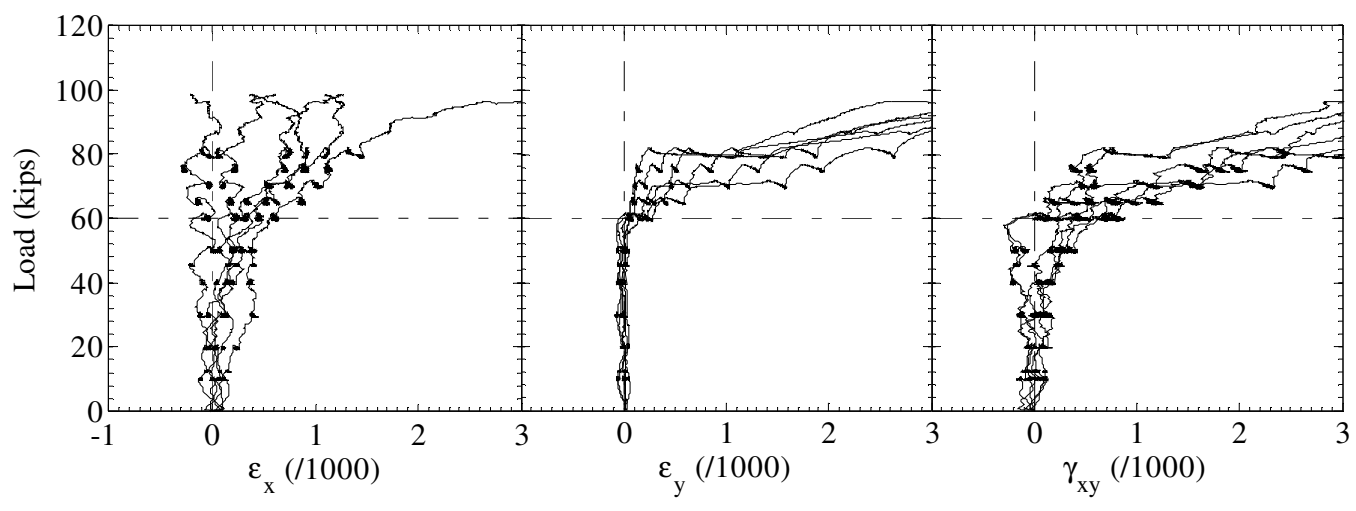

(b) Middle row

Fig. 4-37: Load versus concrete strain relationships in the parallelogram region shown in Fig. 4-33c - Beam B18-1a 


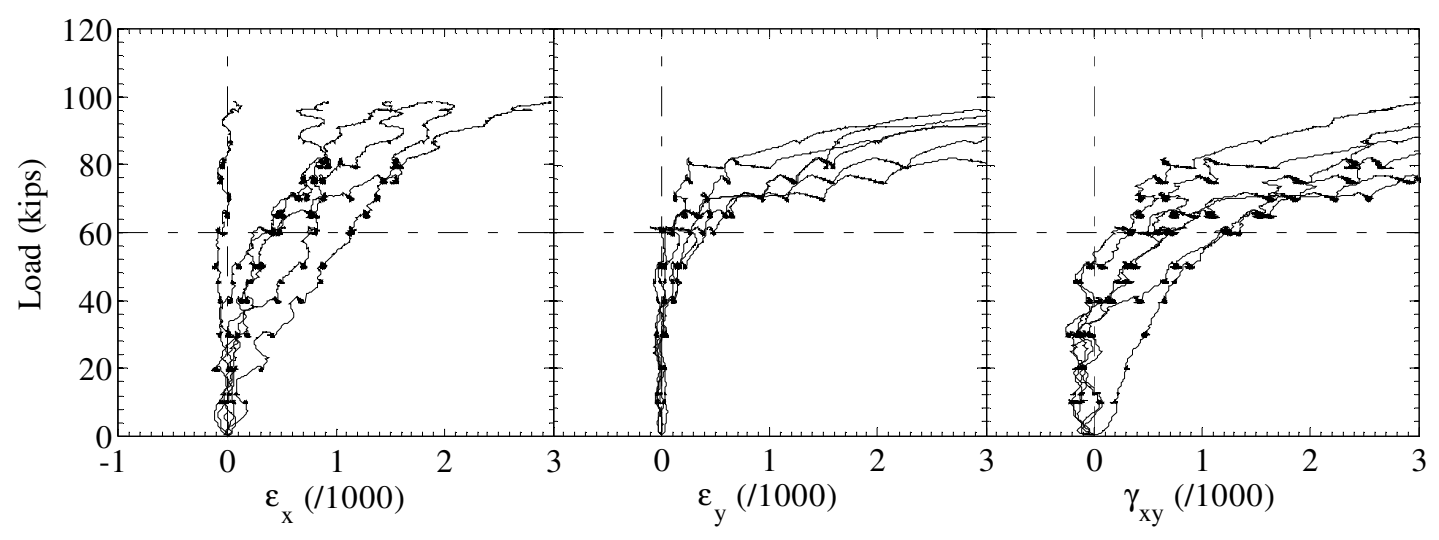

(c) Bottom row

Fig. 4-37: Load versus concrete strain relationships in the parallelogram region shown in Fig. 4-33c - Beam B18-1a (continued)

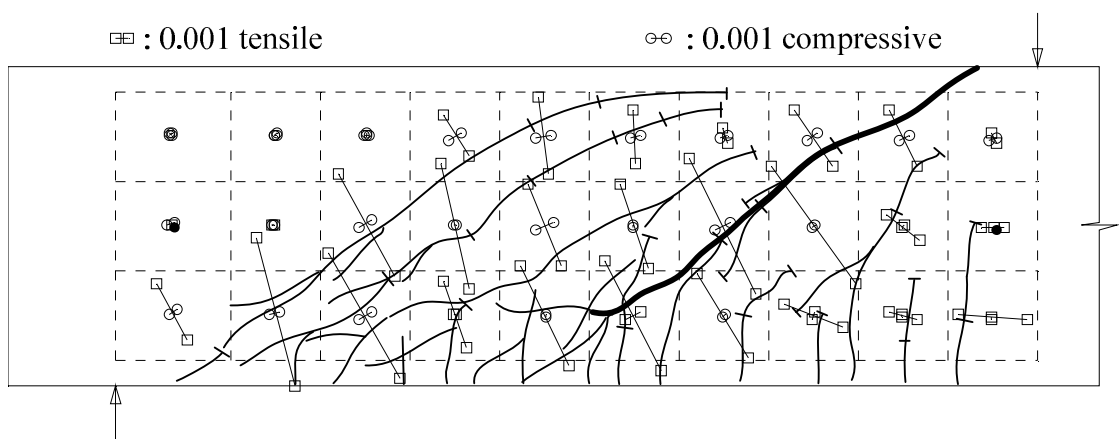

Fig. 4-38: Principal strain field at 99.2 kips - Beam B8-1a

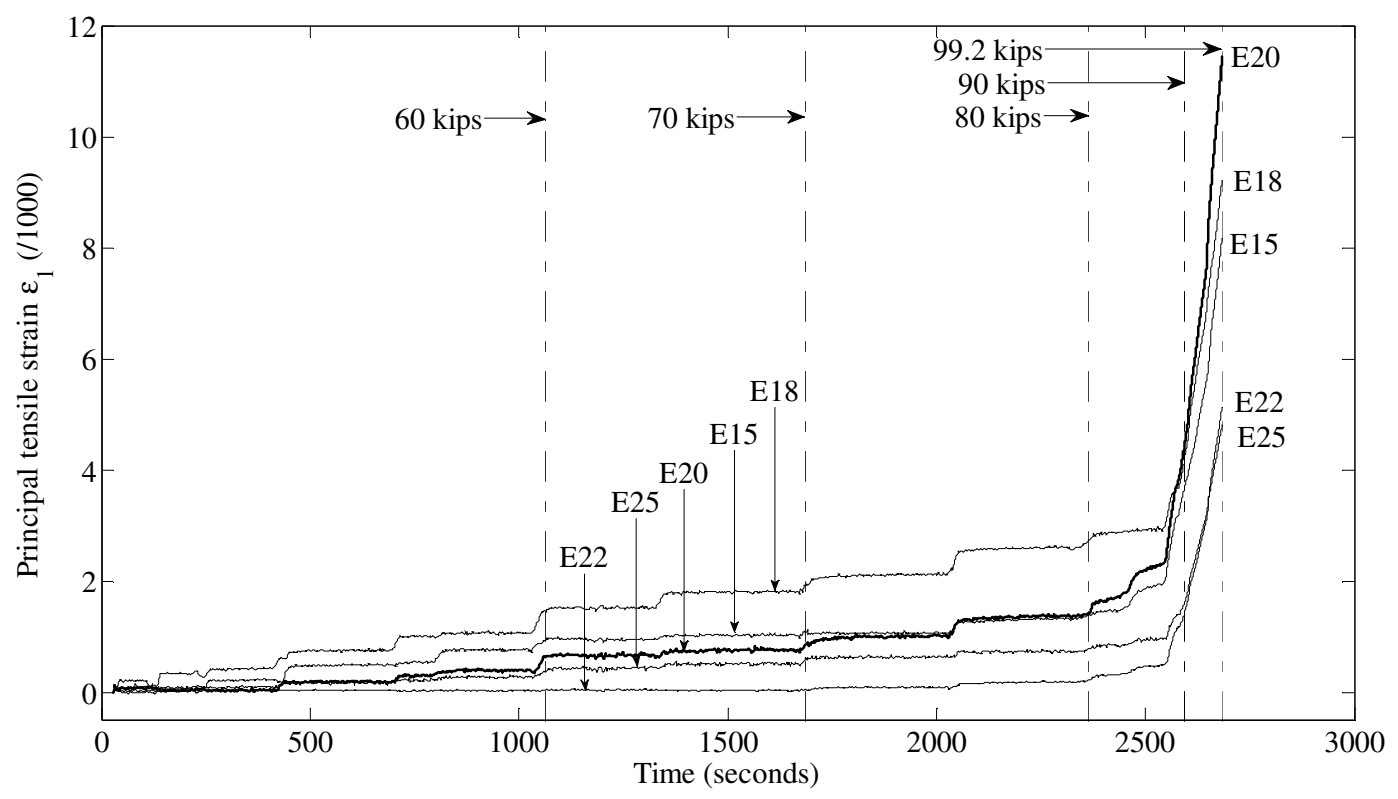

Fig. 4-39: Development of principal tensile strain versus time - Beam B18-1a 


\subsubsection{Beam B18-2a}

Beam B18-2a (and B18-2b) had similar properties to Beams B18-1a \& b, except that Beam B18-2a had a higher fiber volume fraction (1\%) compared with Beams B18-1a $\& \mathrm{~b}(0.75 \%)$. The fibers used in this beam were Dramix ZP305, which have a diameter of 0.022 inches and a length of 1.18 inches (an aspect ratio of 55). In addition, Beam B18-2a (and B18-2b) had a longitudinal reinforcement ratio of $1.96 \%$. Mechanical anchorages were used to anchor the reinforcing bars into the supports.

\section{Load versus displacement relationship, crack pattern, and failure mode.} Beam B18-2a had a similar crack pattern (Fig. 4-40) to Beam B18-1b (Fig. 4-7). The numbers next to the cracks, except " 85 ", in Fig. 4-40 show the applied loads in multiples of 10 kips. Flexural and inclined cracks were first observed at 40 and 60 kips, respectively. The beam was able to exhibit several inclined cracks prior to a complete failure. The beam attained its full strength at 98.2 kips (Fig. 4-42a). A short "yield" plateau was observed on the load-deflection curve. With significant splitting along the upper layer of reinforcing bars (Fig. 4-41), the failure mode of the beam can be reasonably considered as a combination of shear-tension and diagonal tension.

Reinforcement strain and average bond stress. Fig. 4-42b shows the load versus reinforcement strain relationship for Beam B18-2a (see Fig. 3-3 for the location of strain gauges). It should be mentioned that the strain gauge S2 had been damaged before the beam was tested. The rate of reinforcement straining at the same longitudinal location was similar (compare S3 \& S4, S5 \& S6, and S7 \& S8). The load versus reinforcement strain relationship was practically linear for strain gauges S1 through S6. The reinforcement at locations S7 and S8 started to yield at a load of approximately 90 kips and was eventually strained beyond the yielding plateau.

The strain distribution along the beam axis was linear up to a load of 80 kips for both the upper and lower reinforcing bars (Fig. 4-43). From the load of 80 to 98.2 kips, the strain under the loading point (S7 and S8) increased significantly. The bond stress developed in the upper bars was larger than in the lower bars (Fig. 4-44). This could be 
due to the concrete struts that applied compressive forces on the reinforcing bars, and hence increased the friction stress between concrete and reinforcing bars. The bond stress near the loading point was smaller than that near the support.

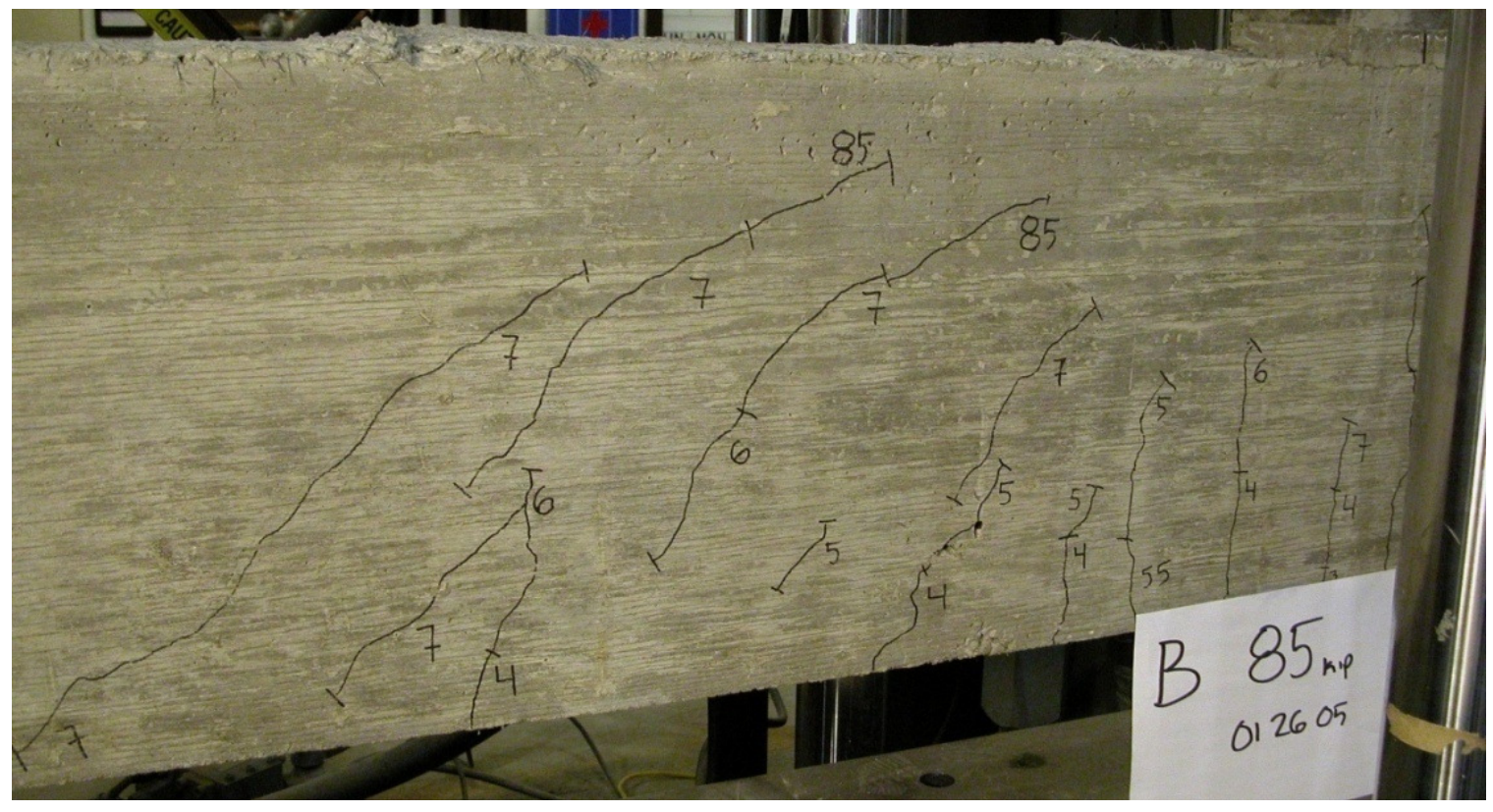

Fig. 4-40: Crack pattern prior to failure - Beam B18-2a

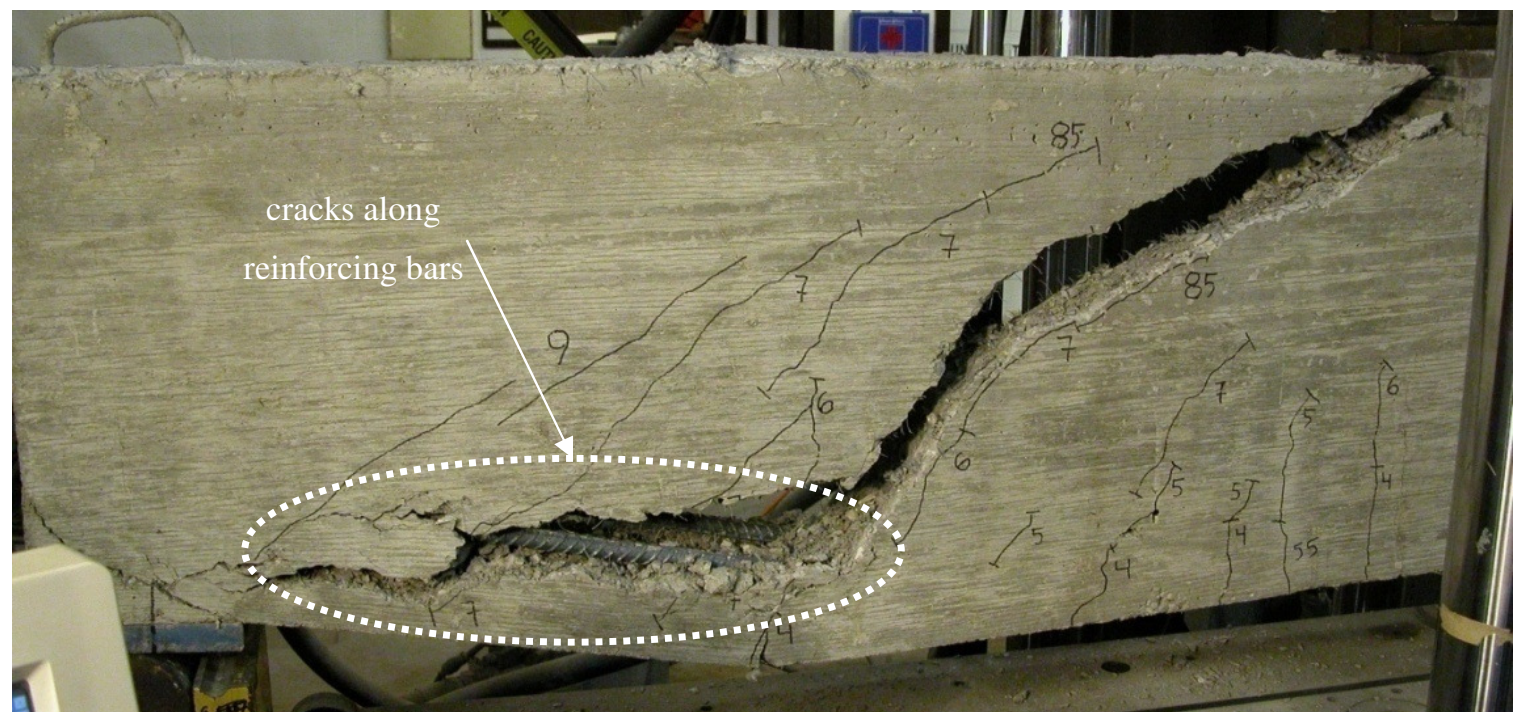

Fig. 4-41: Crack pattern after failure - Beam B18-2a 
(a)

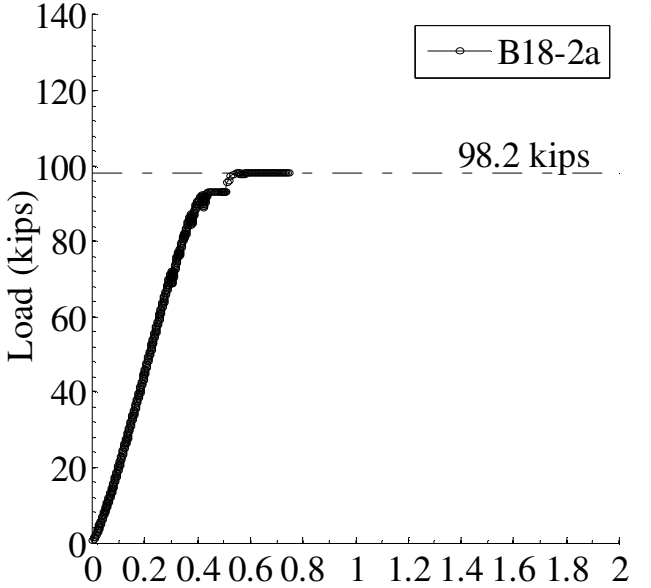

Deflection under the loading point (in.) (b) 140

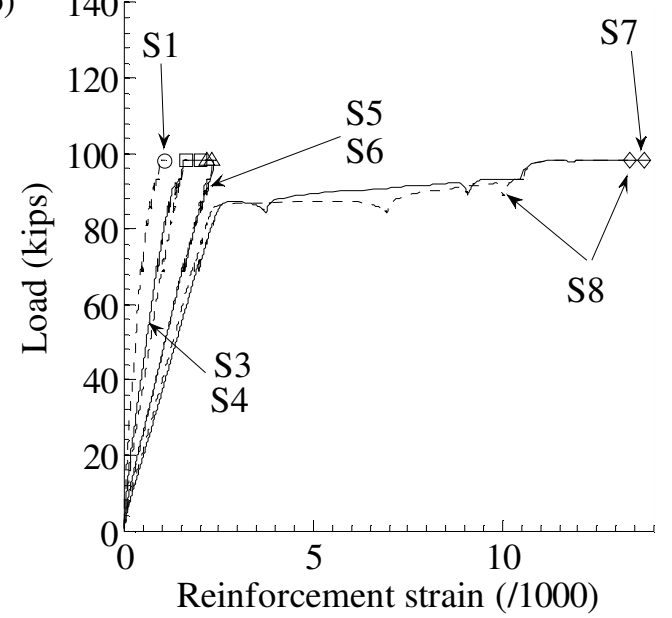

Fig. 4-42: Load versus deflection and reinforcement strain relationships - Beam B18-2a

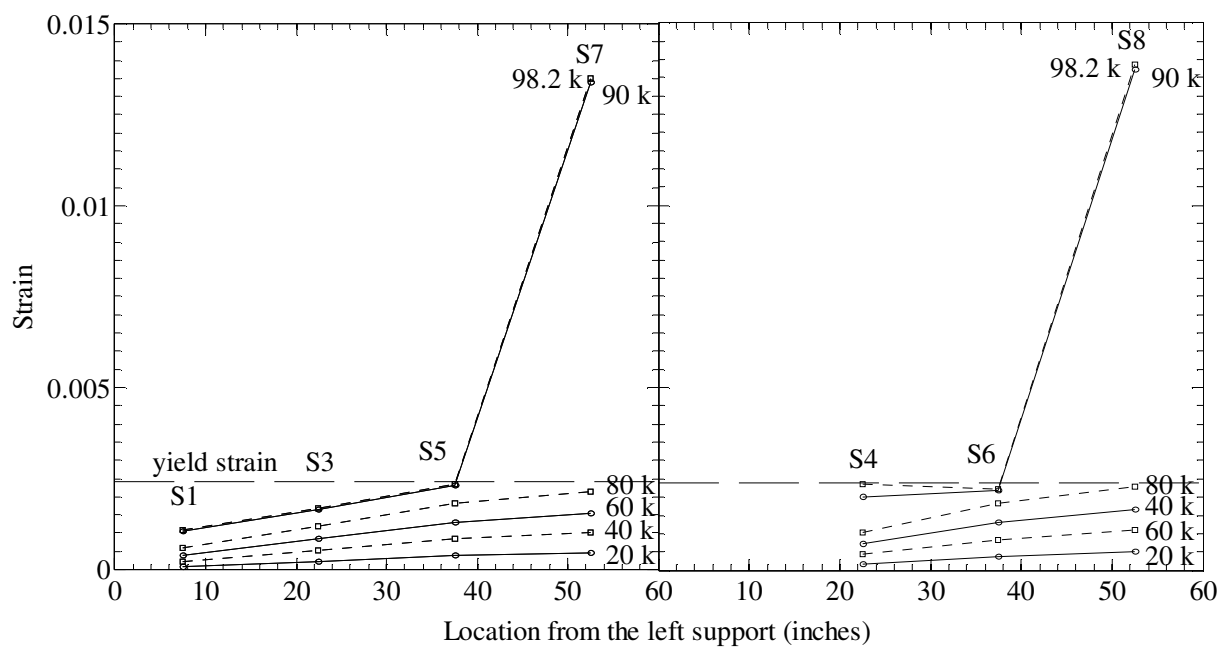

Fig. 4-43: Reinforcement strains along the beam axis - Beam B18-2a

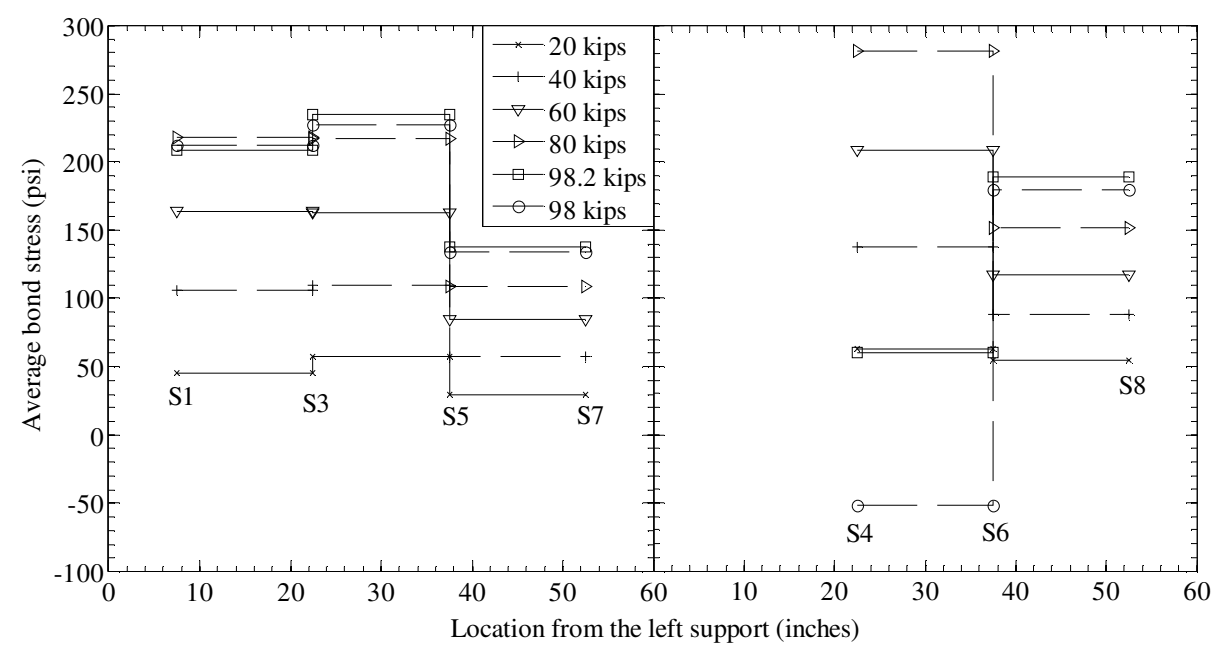

Fig. 4-44: Average bond stress along the beam axis - Beam B18-2a 


\subsubsection{Beam B18-2b}

\section{Load versus displacement relationship, crack pattern, and failure mode.}

Beam B18-2b had similar properties to Beam B18-2a. Although Beam B18-2b had a large void along the longitudinal reinforcement level, which was subsequently repaired by grouting, its performance was comparable to that of Beam B18-2a. Several inclined cracks were observed (Fig. 4-45). The beam ultimate strength was 100 kips (Fig. 4-46a), slightly higher than that of Beam B18-2a (98.2 kips), which had no visible flaws. Compared with Beam B18-2a, the "yield plateau" of Beam B18-2b was longer.

Reinforcement strain and average bond stress. Similar to Beam B18-2a, the reinforcement strains at the same longitudinal position were comparable prior to a load of 90 kips (compare S1 and S2 in Fig. 4-46b). The reinforcement strains along the beam axis were also linearly distributed up to a load of 80 kips (Fig. 4-47). Compared with Beam B18-2a, the reinforcement at S5 and S6 was more severely strained at peak load, while higher inelastic strains were measured at S7 and S8. Only the strains at S1 through S4 were linear up to the peak load.

The observed maximum bond stress was 480 psi (Fig. 4-48), which was higher than that in Beam B18-2a (280 psi). However, a more uniform bond stress was observed in Beam B18-2a.

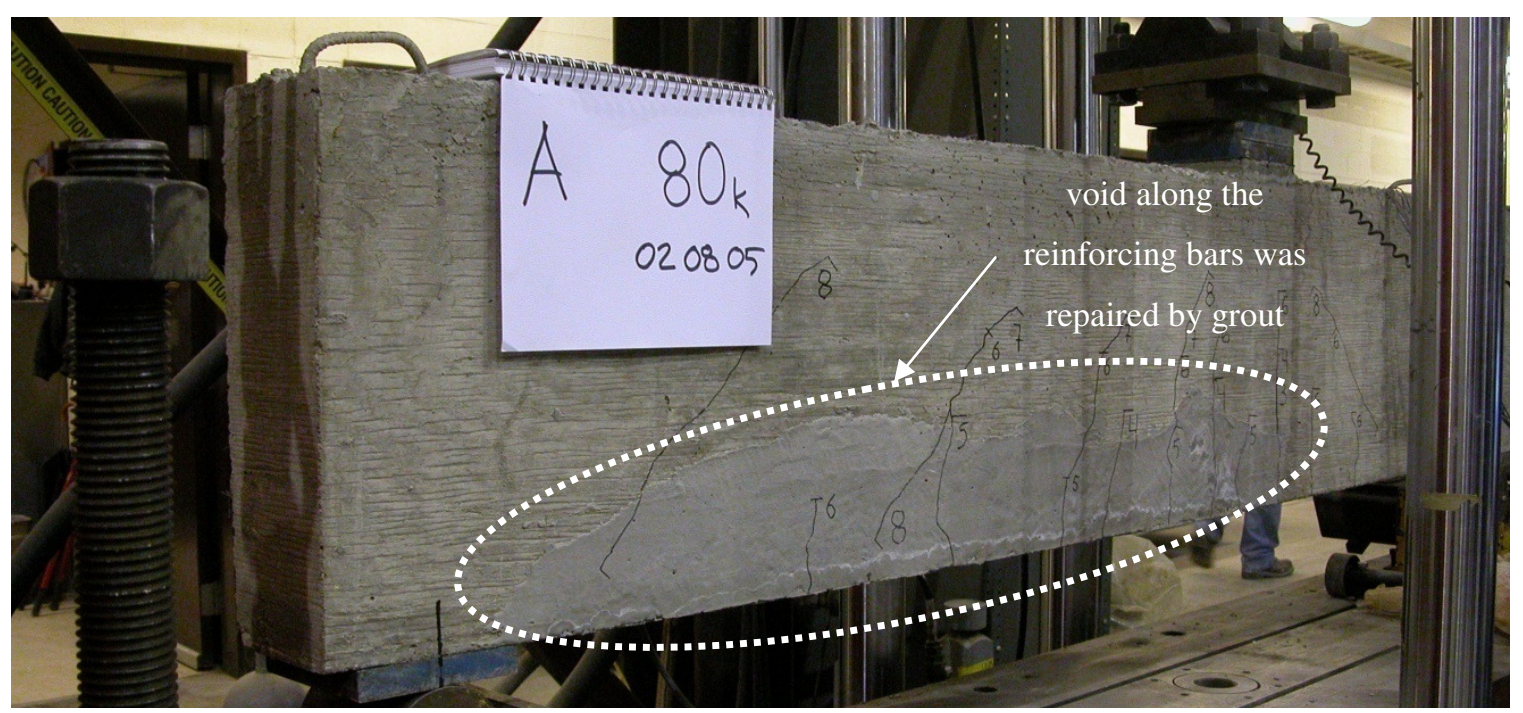

Fig. 4-45: Crack pattern prior to failure - Beam B18-2b 
(a)

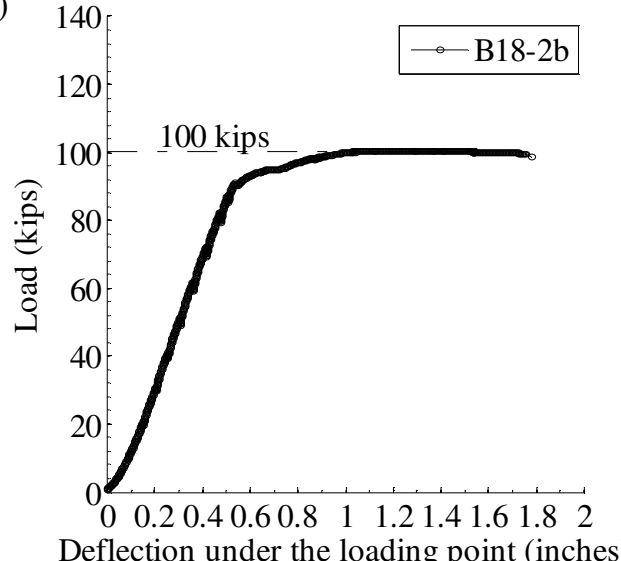

(b)

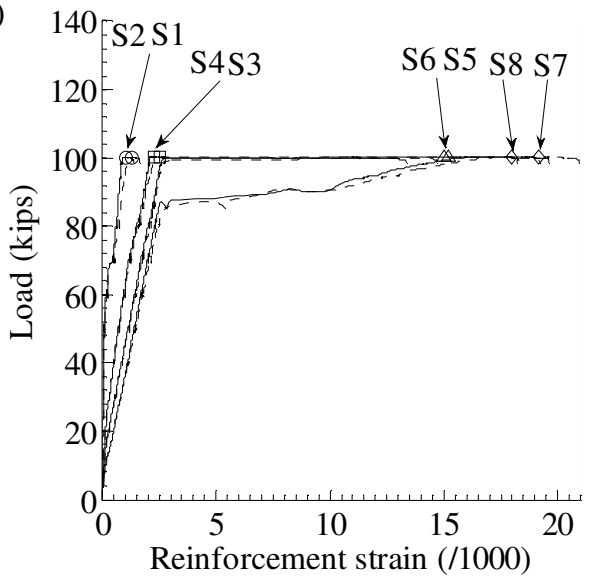

Fig. 4-46: Load versus deflection and reinforcement strain relationships - Beam B18-2b

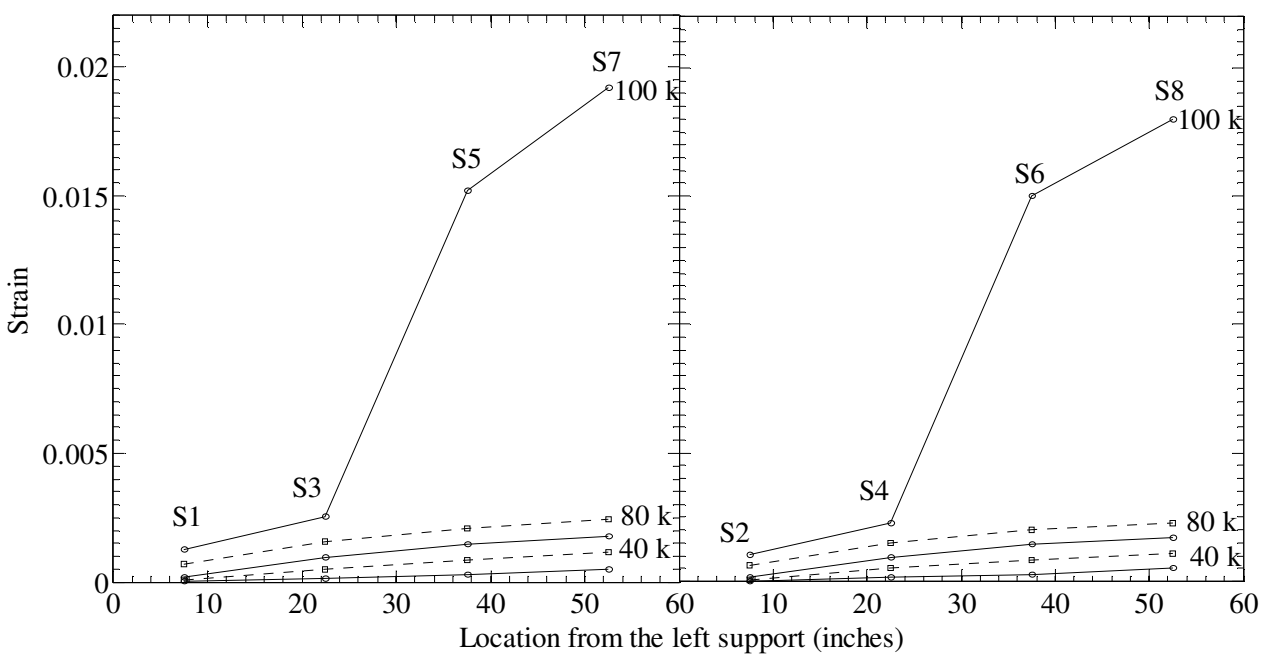

Fig. 4-47: Reinforcement strains along the beam axis - Beam B18-2b

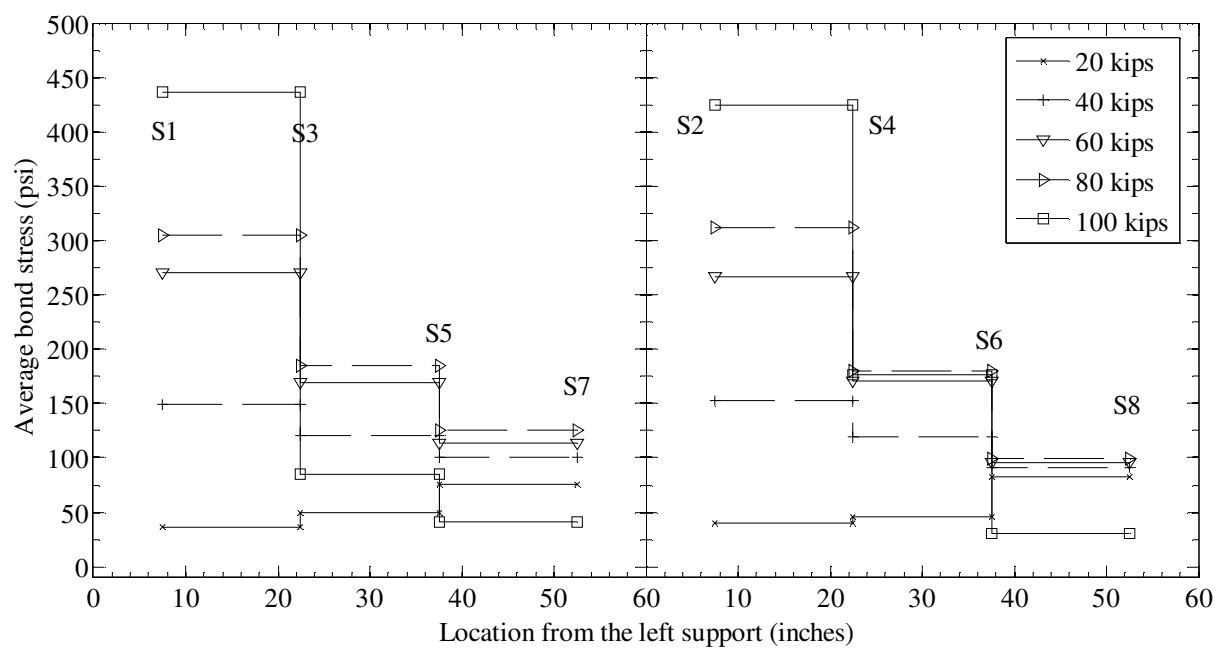

Fig. 4-48: Average bond stress along the beam axis - Beam B18-2b 


\subsubsection{Beams B18-2c \& d}

Beams B18-2c \& d were similar to Beams B18-2a \& b, except that they contained a higher amount of longitudinal reinforcement (2.67\% compared with $1.96 \%)$. These beam also used mechanical anchors (Lenton terminators) for anchorage of longitudinal bars. The fibers and volume fraction were Dramix ZP305 and 0.75\%, respectively.

Beam B18-2c. Fig. 4-49a shows the load versus deflection relationship for Beam B18-2c. The beam failed shortly after reaching the peak load of 113 kips, higher than the strengths of Beams B18-2a \& b, which were 98.2 and 100 kips, respectively. This was expected because Beam B18-2c had a higher longitudinal reinforcement ratio. This high amount of longitudinal reinforcement also led to lower reinforcement strains (Fig. 4-49b). Except for locations S5 and S7, the reinforcement at other strain gauge locations was not strained beyond the yield point. The distribution of longitudinal reinforcement strains was close to linear over the shear span for all loading stages (Fig. 4-50). The distribution of bond stresses in the shear span (Fig. 4-51) was similar to that observed in the previous test specimens, with larger bond demand near the support.

Beam B18-2d. Compared with Beam B18-2c, Beam B18-2d exhibited a much lower strength of 82.5 kips (Fig. 4-52a). With the increase in longitudinal reinforcement, the strength of Beams B18-2c \& d was expected to be higher than that of Beams B18-2a $\&$ b. Therefore, this low strength of Beam B18-2d is not representative for the pair B182c \& d. The significant increase in reinforcement strains at S1 and S4 from 60 kips to the peak load of 82.5 kips (Fig. 4-53) suggests a significant degradation in bond near the support, which is believed to have triggered the shear failure of Beam B18-2d. This can be seen in Fig. 4-54, in which the average bond stress near the support decreased for loads greater than 60 kips. 
(a)

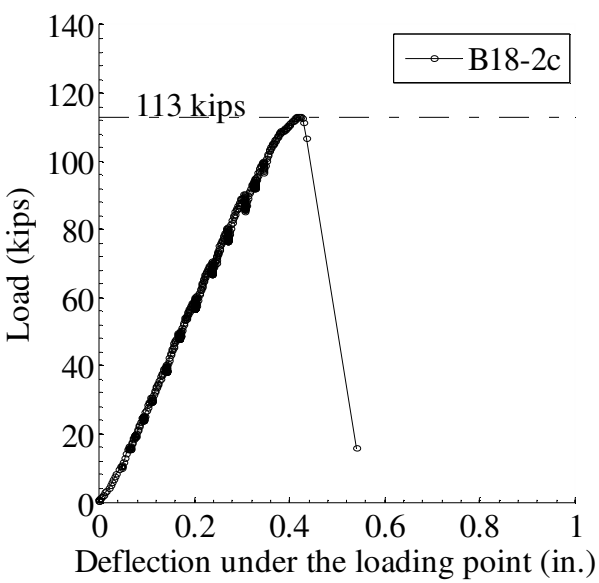

(b)

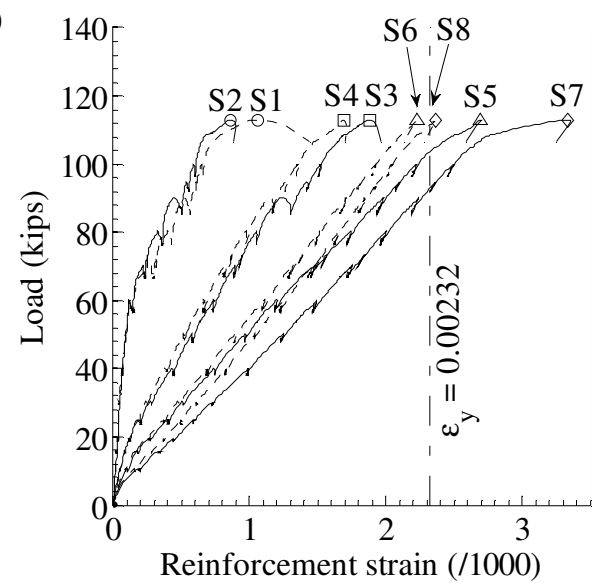

Fig. 4-49: Load versus deflection and reinforcement strain relationships - Beam B18-2c

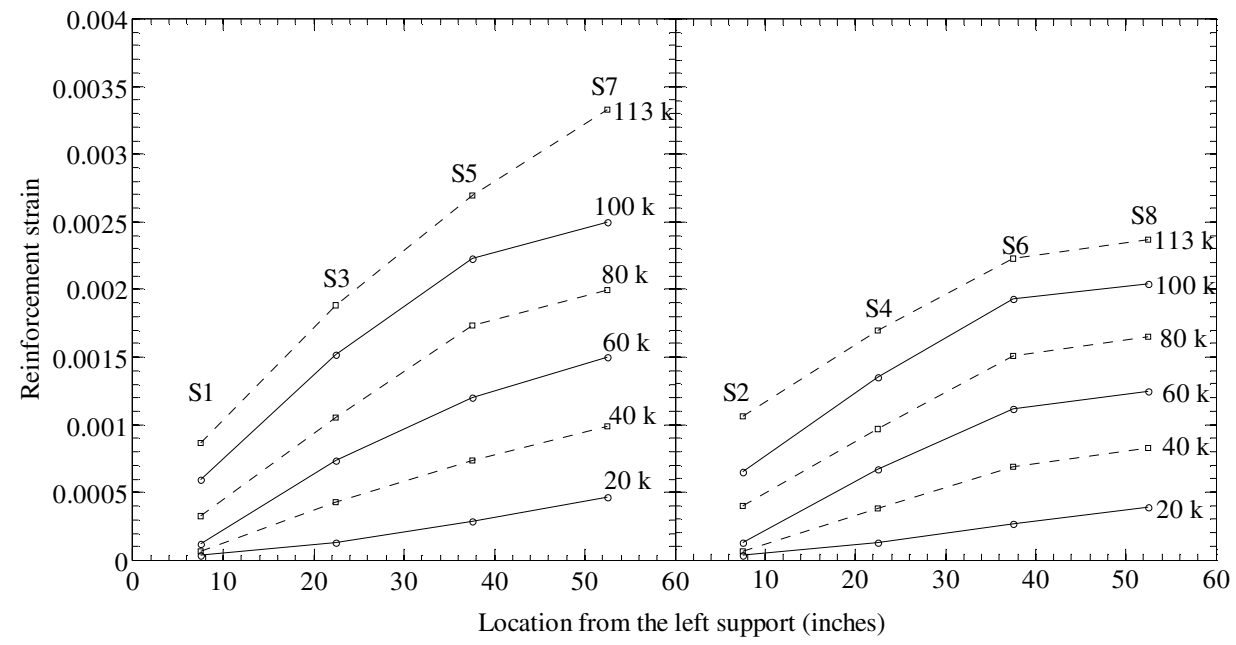

Fig. 4-50: Reinforcement strains along the beam axis - Beam B18-2c

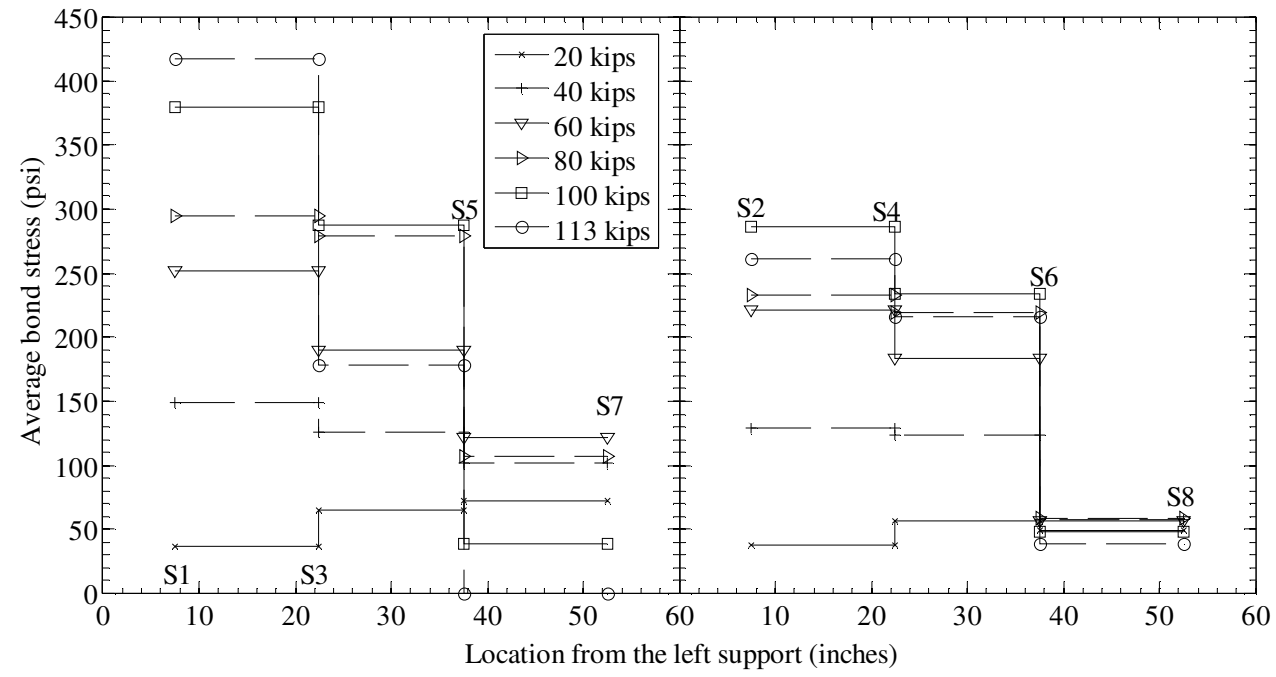

Fig. 4-51: Average bond stress along the beam axis - Beam B18-2c 
(a)

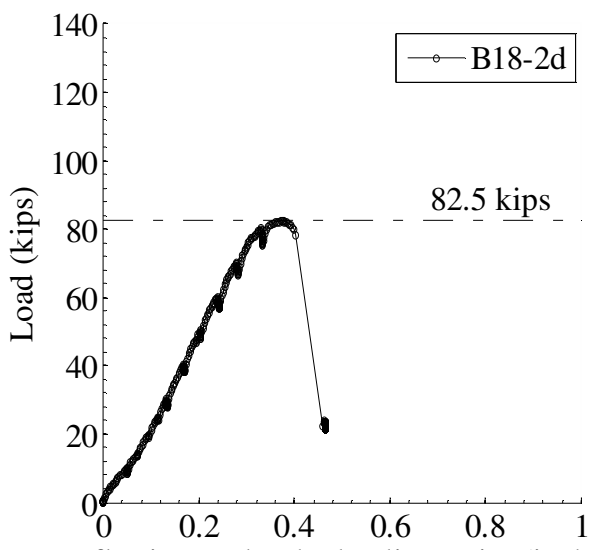

Deflection under the loading point (inches) (b)

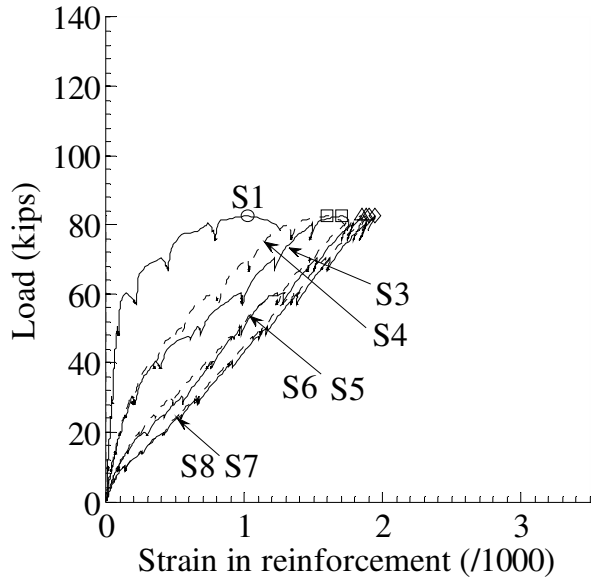

Fig. 4-52: Load versus deflection and reinforcement strain relationships - Beam B18-2d

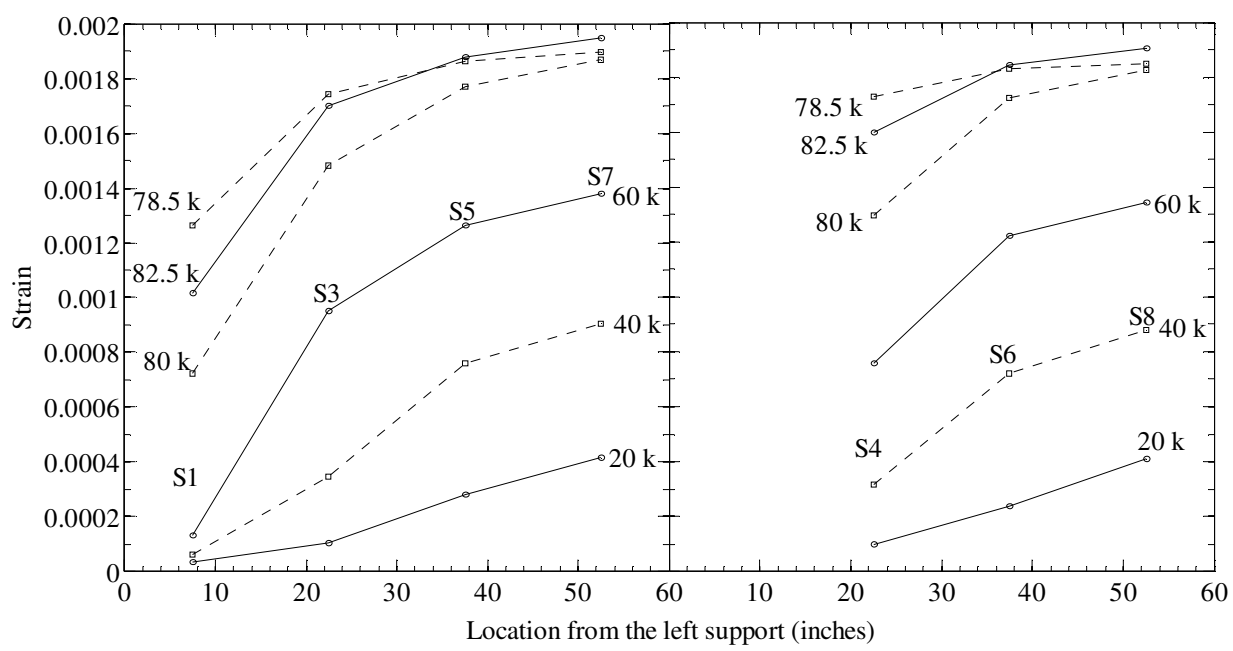

Fig. 4-53: Reinforcement strains along the beam axis - Beam B18-2d

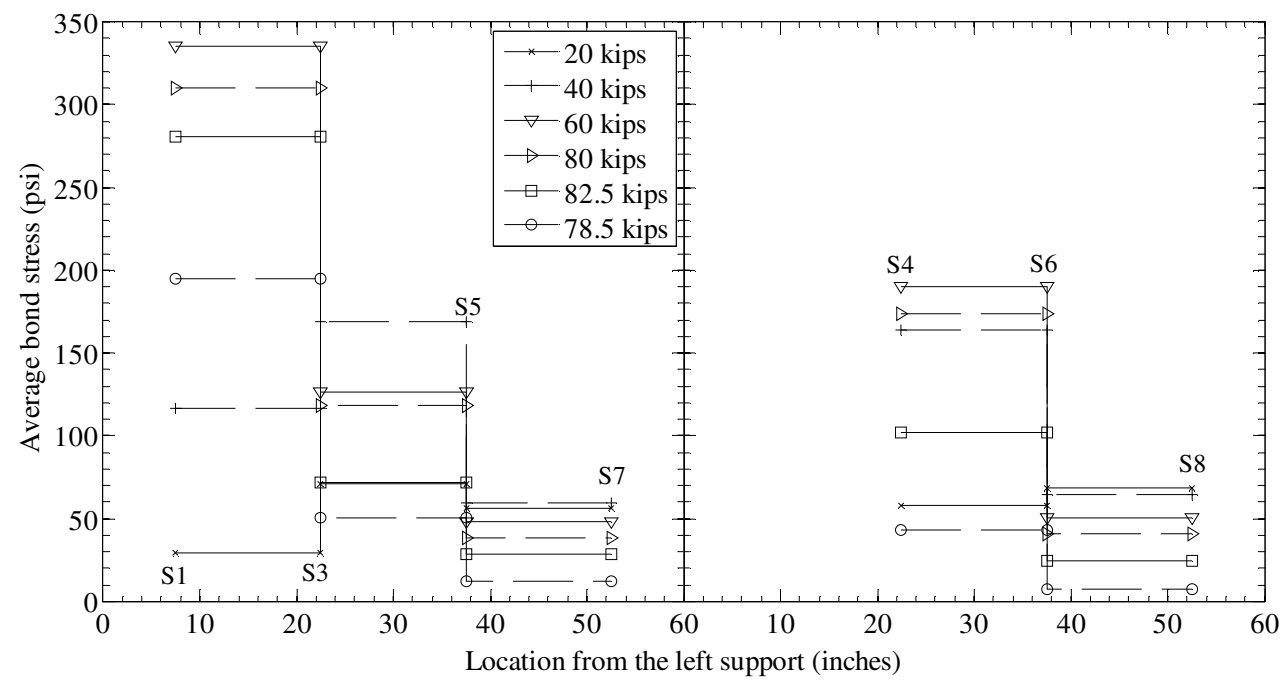

Fig. 4-54: Average bond stress along the beam axis - Beam B18-2d 


\subsubsection{Beam B18-3a}

Beam B18-3a was similar to Beams B18-2c \& d, except that it had a higher fiber volume fraction (1.5\% compared with $1 \%$ ). It should be repeated that Beam B18-3a had a longitudinal reinforcement ratio of $2.67 \%$ and contained ZP305 fibers. In addition, $90^{\circ}$ hooks, instead of Lenton terminators, were used for the purpose of bar anchorage in Beam B18-3a.

Despite the increase in longitudinal reinforcement ratio (2.67\% versus $1.96 \%)$ and fiber volume fraction (1.5\% versus 1\%), Beam B18-3a exhibited a much lower strength of 86.4 kips (Fig. 4-55a), compared with that of Beams B18-2a \& b (98.2 \& 100 kips, respectively).

Although there was no visible sign of defects along the reinforcing bars to explain for the low bond at later stages of the test, a large void was observed above the support (Fig. 4-56). It was also possible that the fibers stacked at the reinforcement levels, leading to poor concrete consolidation. It should be mentioned that the length of fibers used in this beam was 1.18 inches, while the clear bar spacing was 1.5 inches.

The weak bond was confirmed by a large increase in strains at S1 and S2 for loads greater than 70 kips (Fig. 4-55b and Fig. 4-57). At the end of the test, the strains near the support were close to those at the maximum moment region. It should be noted that this phenomenon was seen in Beam B18-2d (see Fig. 4-52b and Fig. 4-53), which also had a lower strength than its counterpart (Beam B18-2c). From Fig. 4-58, it is clear that the bond was nearly completely lost near the support when the beam was loaded beyond 60 kips. 
(a)

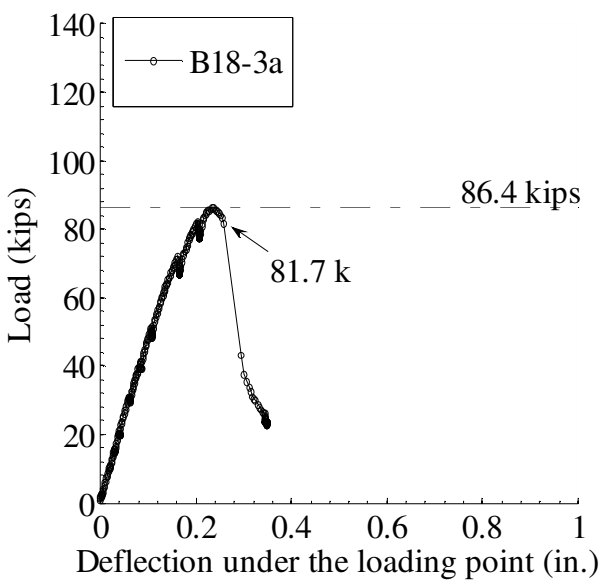

(b)

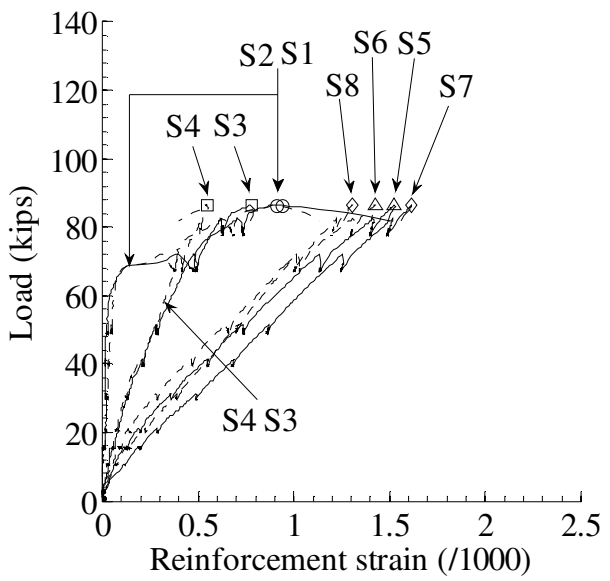

Fig. 4-55: Load versus deflection and reinforcement strain relationships - Beam B18-3a

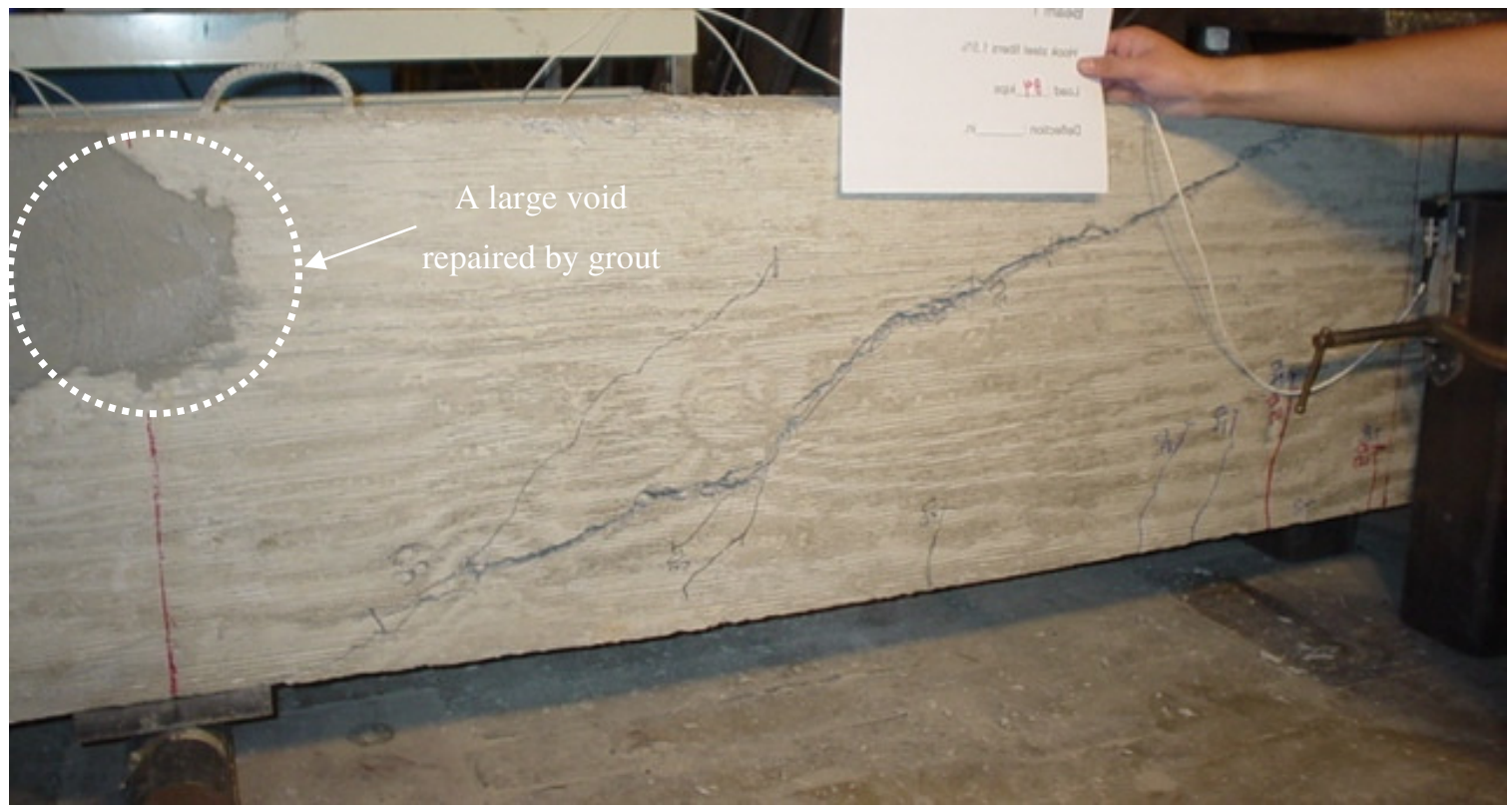

Fig. 4-56: Crack pattern after failure - Beam B18-3a 


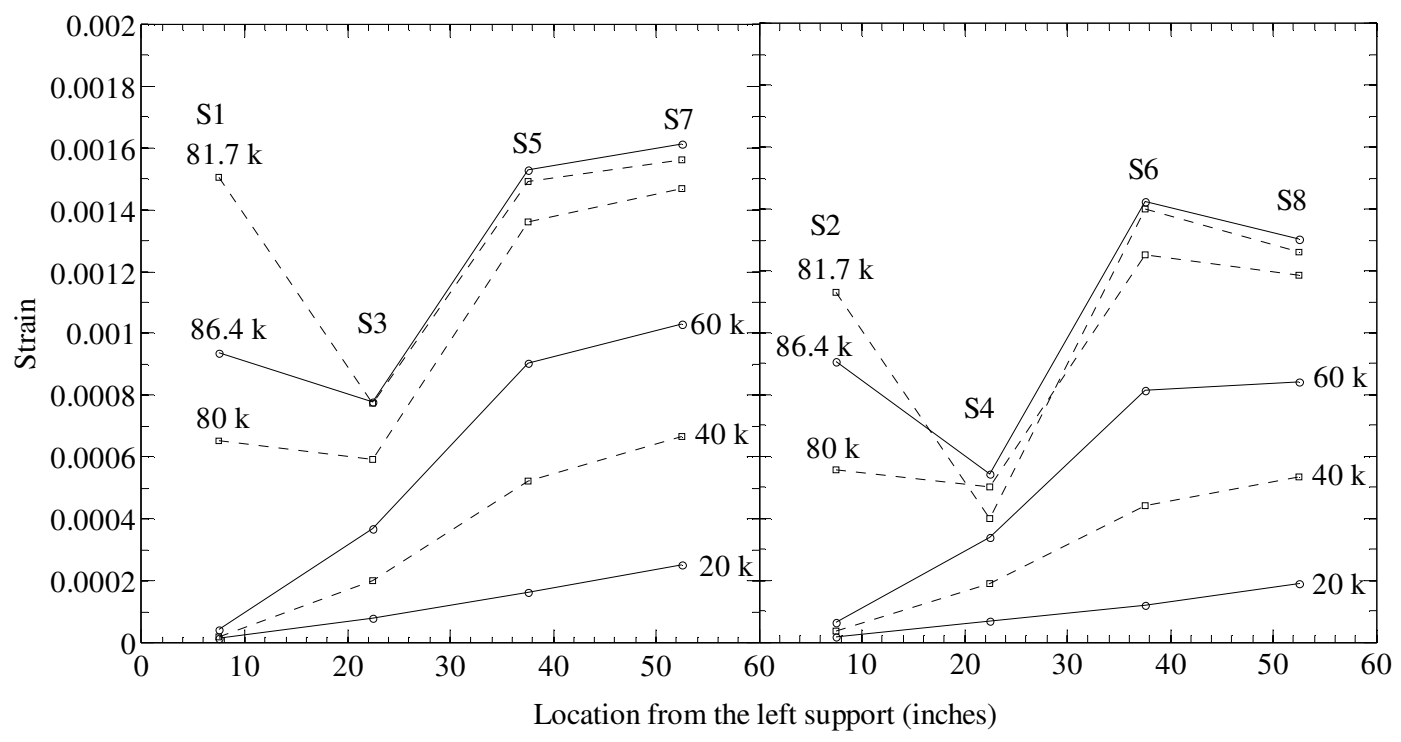

Fig. 4-57: Reinforcement strains along the beam axis - Beam B18-3a

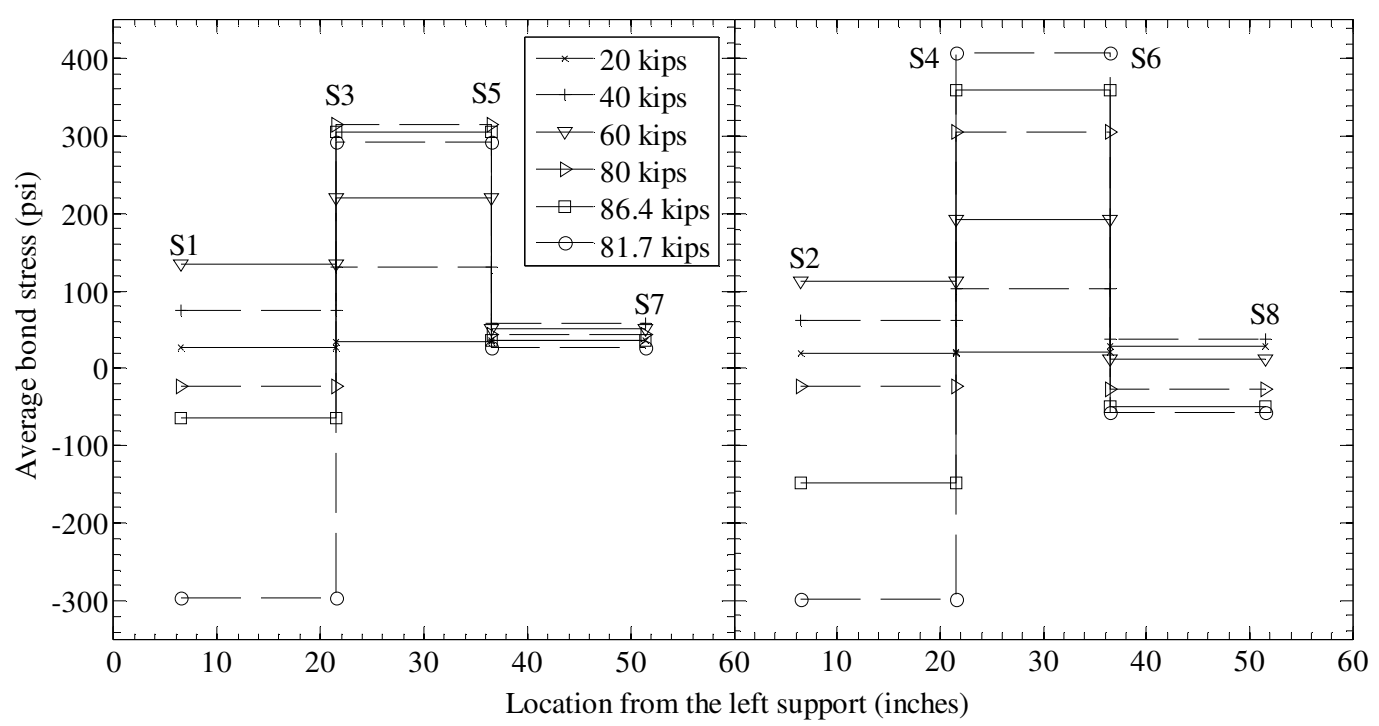

Fig. 4-58: Average bond stress along the beam axis - Beam B18-3a 


\subsubsection{Beam B18-3b}

Load versus displacement relationship, crack pattern, and failure mode. Compared with its counterpart (Beam B18-3a), which had a strength of 86.4 kips, Beam B18-3b had a significantly higher strength of 114 kips (Fig. 4-59). Beam B18-3b exhibited its first diagonal crack at 70 kips (Fig. 4-60), compared with 60 kips in beams that contained 0.75 or $1 \%$ fibers by volume. It is assumed that the higher fiber volume fraction of Beam B18-3b led to a modest increase in strength at first diagonal cracking. Also, only three inclined cracks developed (Fig. 4-60), less than the number of cracks seen in Beams B18-1a \& b with the same fiber type but half in volume.

The inclined cracks, except for the critical one, did not penetrate as close to the beam top as those in Beams B18-1a \& b. This can be explained by two facts. First, Beam B18-3b had a higher longitudinal reinforcement ratio (2.67\%) compared with that of Beams B18-1a \& b (1.96\%). This higher amount of reinforcement would result in a larger compression zone that would stop the propagation of the diagonal cracks. Second, with a $1.5 \%$ fiber volume fraction, the concrete is more resistant to crack opening, preventing the inclined cracks from penetrating all the way to the neutral axis.

With the severe splitting along the upper flexural reinforcing bars and some crushing near the loading point, the failure mode of the beam can be considered to be a combination of shear-tension and shear-compression (Fig. 4-60).

Reinforcement strain and average bond stress. None of the reinforcing bars in the beam were strained beyond the yield point (Fig. 4-59b), which was 0.0023 for the No. 7 bars used in this beam. Similar reinforcement strains at the same applied load were observed for S1 \& S2 throughout the test, but not for S3 \& S4, S5 \& S6, and S7 \& S8. The strain distribution along the shear span of Beam B18-3b was marked by a drop in strain magnitude at S5 \& S6 (Fig. 4-61). This was probably due to a loss of bond from S3 to S5 and from S4 to S6 (Fig. 4-62). However, this seems to indicate that while a loss of bond occurred from S1 \& S2 to S3 \& S4, respectively, there was still good bond between S3 \& S4 and the loading point 
(a)

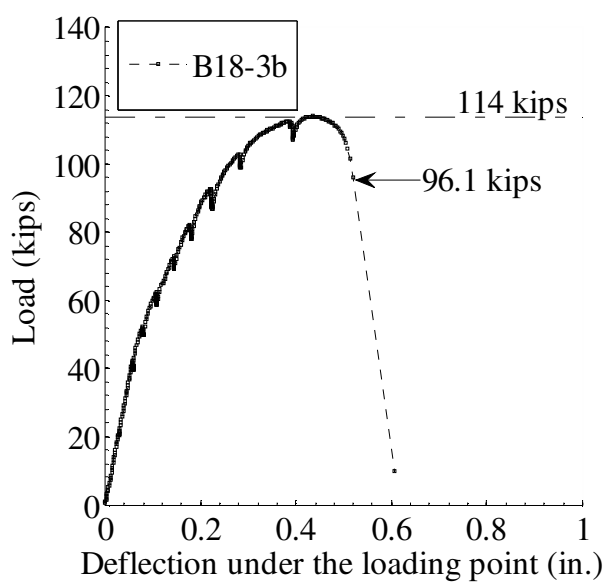

(b)

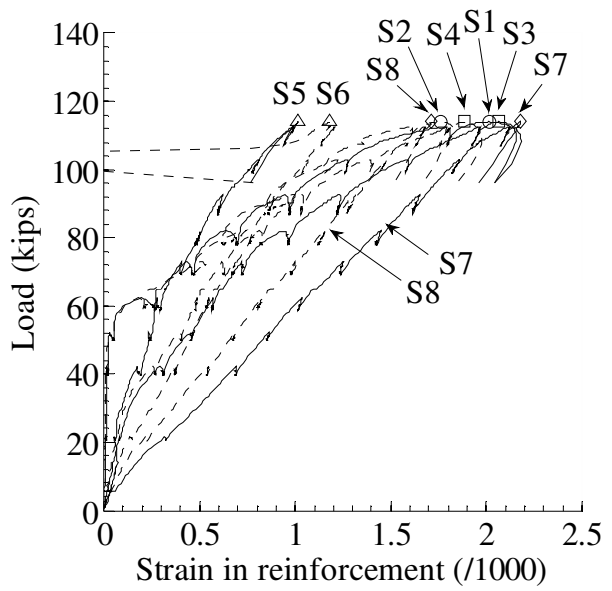

Fig. 4-59: Load versus deflection and reinforcement strain relationships - Beam B18-3b

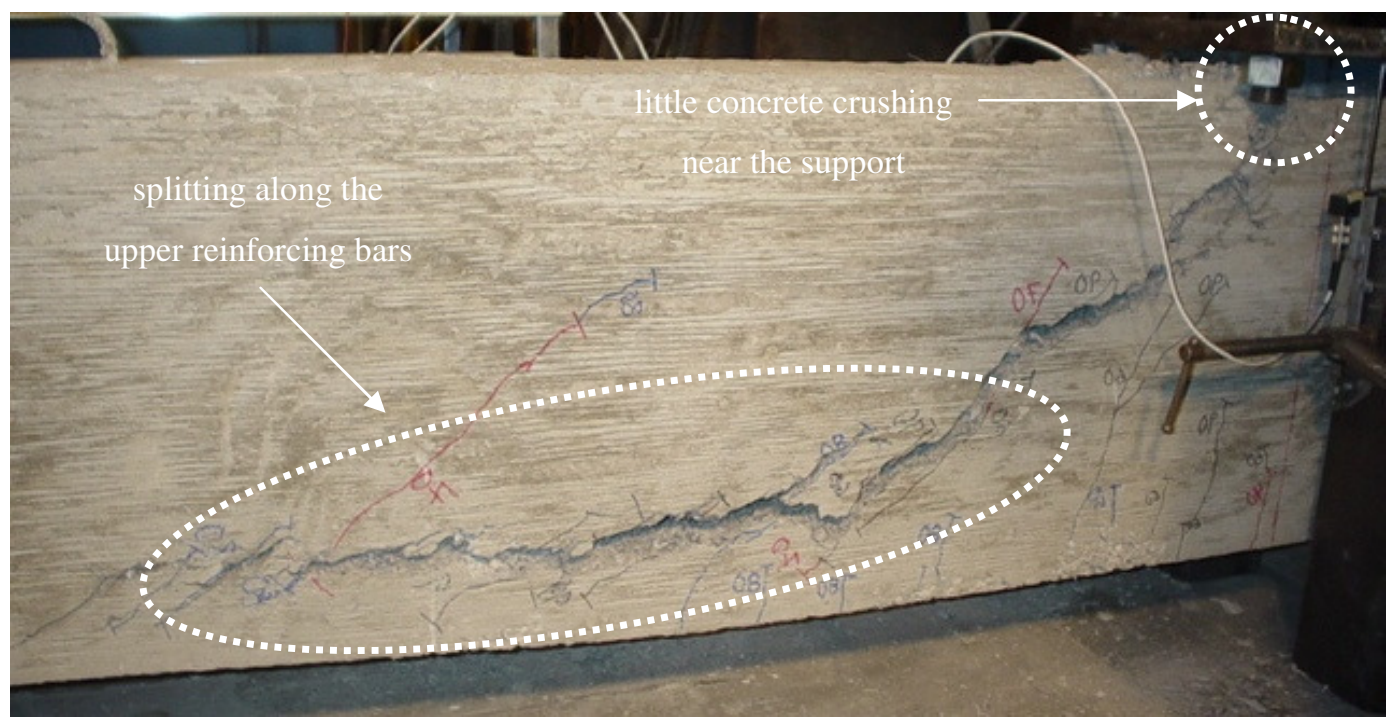

Fig. 4-60: Crack pattern after failure - Beam B18-3b

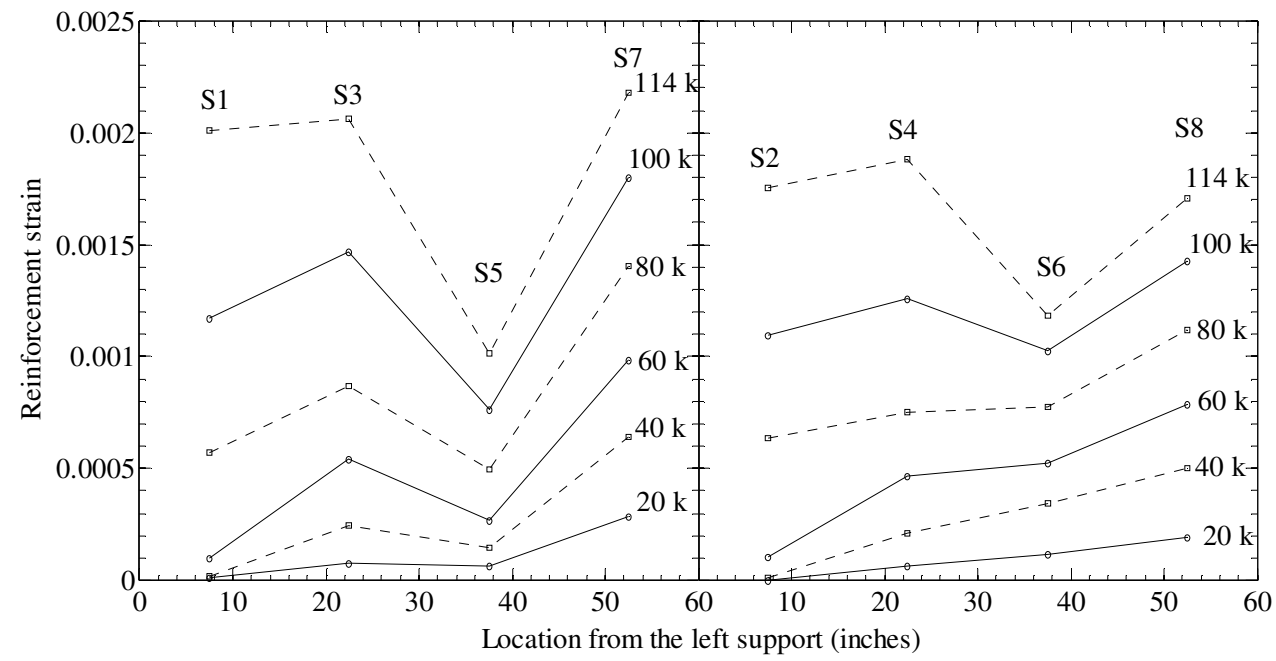

Fig. 4-61: Reinforcement strains along the beam axis - Beam B18-3b 


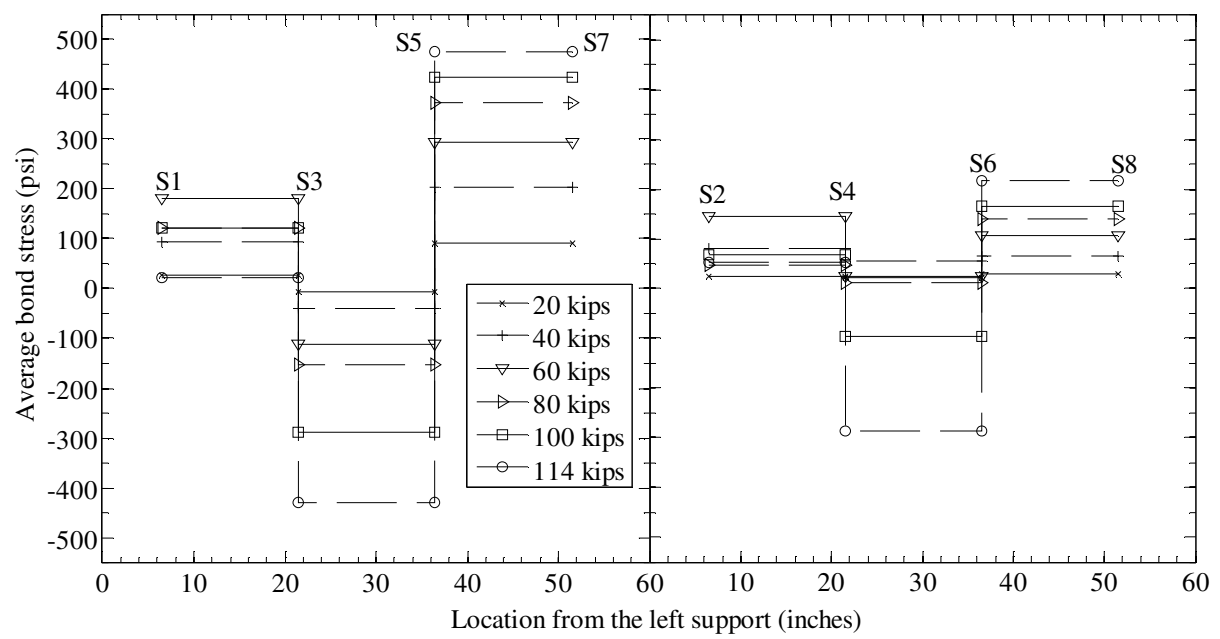

Fig. 4-62: Average bond stress along the beam axis - Beam B18-3b

\subsubsection{Beams B18-3c \& d}

Load versus deflection relationship, crack pattern, and failure mode. Due to the significant difference $(32 \%)$ in the ultimate strengths of Beams B18-3a \& b, it was decided that a similar pair would be constructed and tested. It turned out that the beam strengths of Beams B18-3c \& d were 111 (Fig. 4-63a) and 110 (Fig. 4-67a) kips, respectively. With similar ultimate strengths, Beams B18-3b, c, and d can be assumed to have defined the expected strength for this class of beams. Nevertheless, these ultimate strengths were not significantly higher than that of Beam B18-2c, which had a lower fiber volume fraction.

Similar to Beam B18-3b, only a few inclined cracking were observed (Fig. 4-64 and Fig. 4-68). These inclined cracks, except for the critical ones, stayed below the middepth level of the beam. With splitting cracks having developed along the upper layer of reinforcing bars (Fig. 4-64 and Fig. 4-68), the failure mode of Beams B18-3c \& d can be considered as a combination of shear-tension and diagonal tension.

Reinforcement strain and average bond stress. For both beams, the reinforcement strains were in the linear range of the stress-strain curve of No. 7 bars (Fig. 4-63b and Fig. 4-67b). A significant jump in the reinforcement strains at S1 and S2 near 
the peak load was observed (Fig. 4-65 and Fig. 4-69). This led to a drop in bond stress from $\mathrm{S} 1$ to $\mathrm{S} 3$ and from S2 to S4 (Fig. 4-66). It should be noted in Fig. 4-66 that the bond was very small from S5 to S7 and from S6 to S8, as observed in several previous beams.

(a)

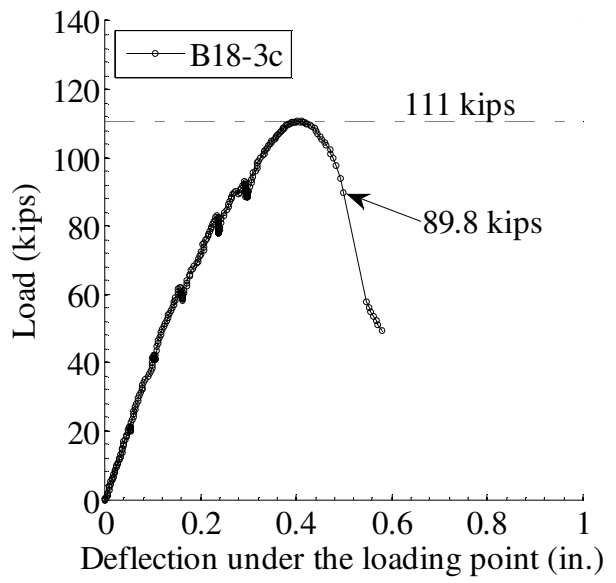

(b)

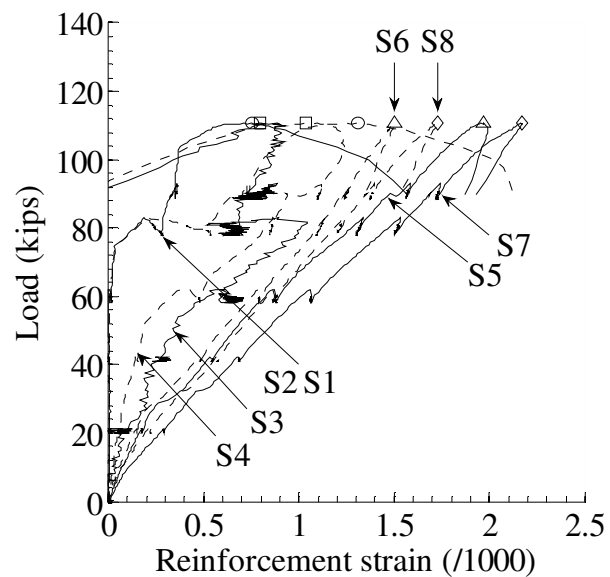

Fig. 4-63: Load versus deflection and reinforcement strain relationships - Beam B18-3c

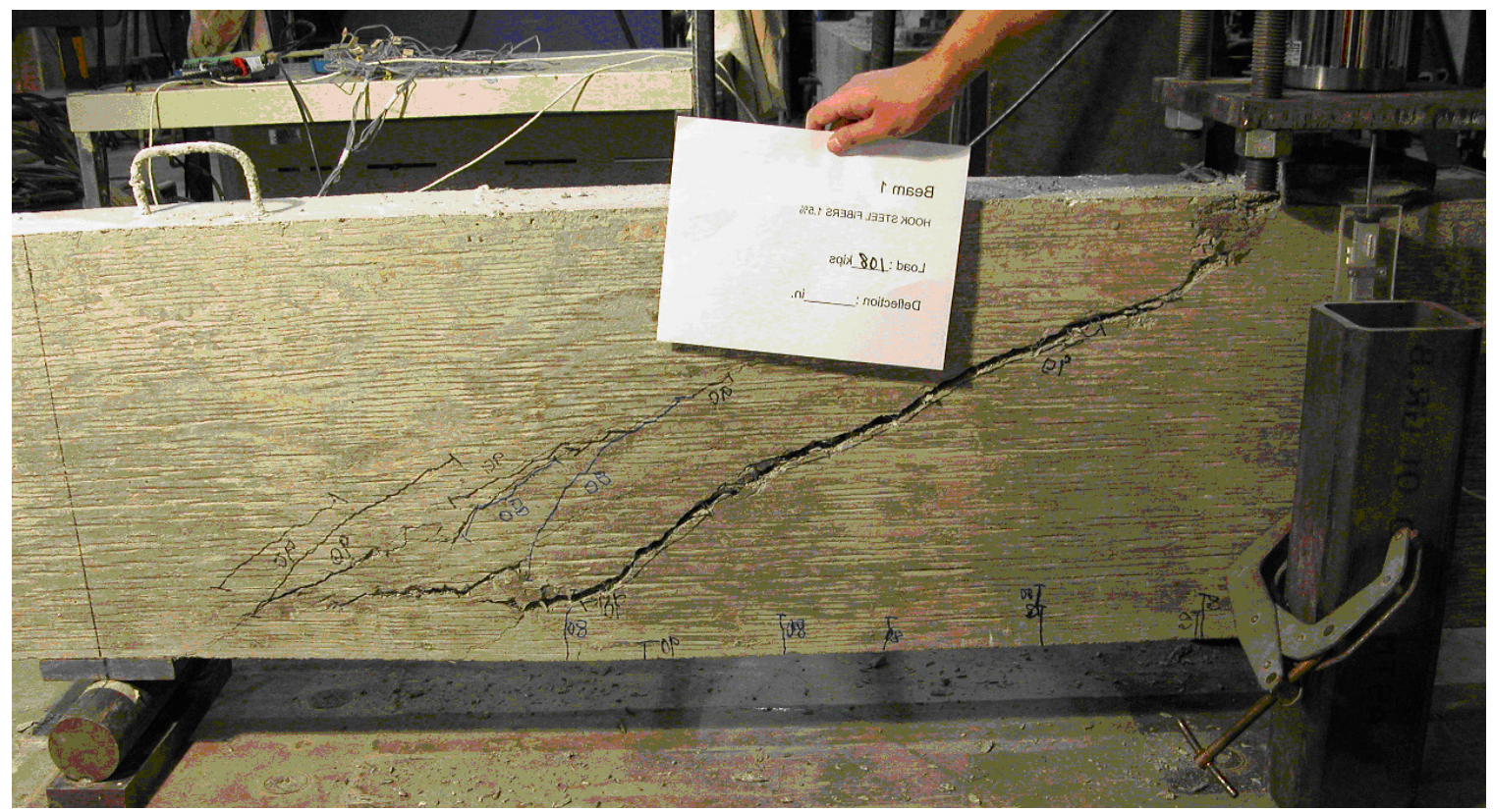

Fig. 4-64: Crack pattern after failure - Beam B18-3c 


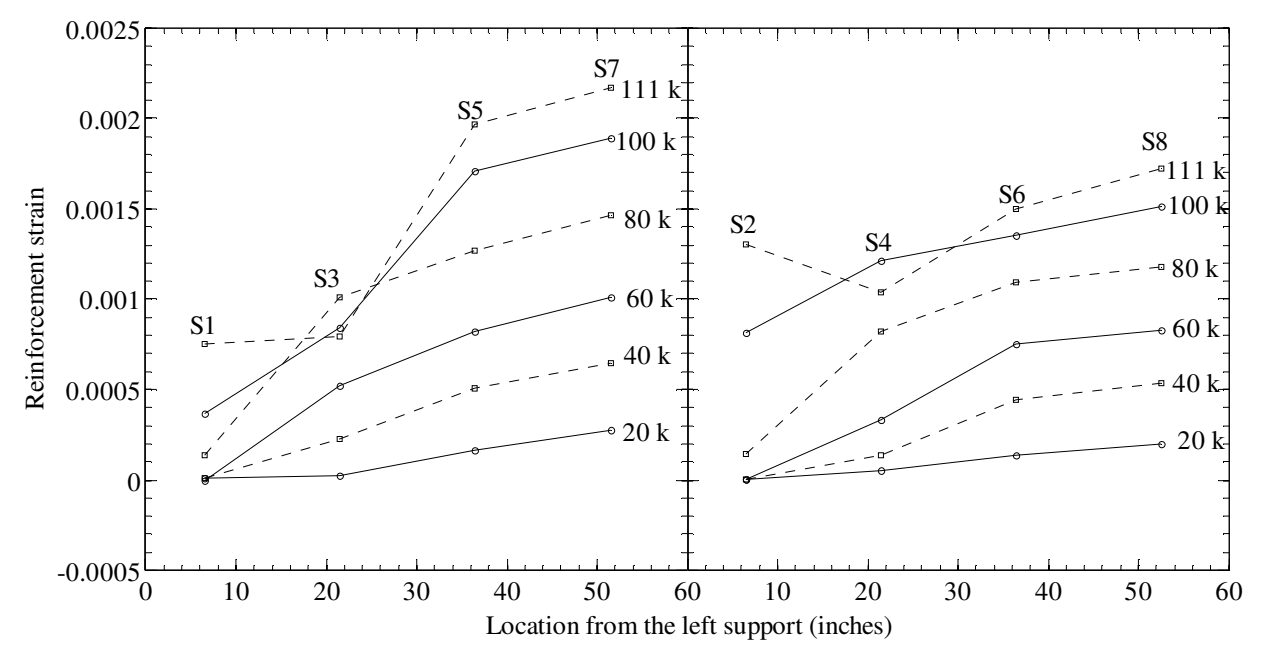

Fig. 4-65: Reinforcement strains along the beam axis - Beam B18-3c

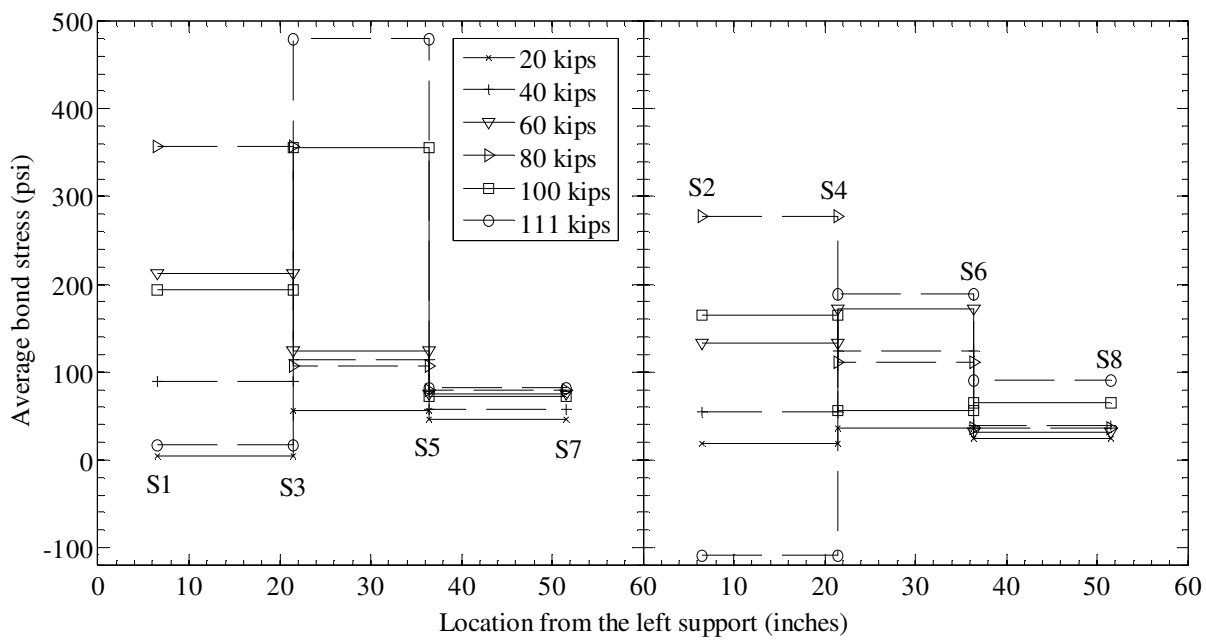

Fig. 4-66: Average bond stress along the beam axis - Beam B18-3c

(a)

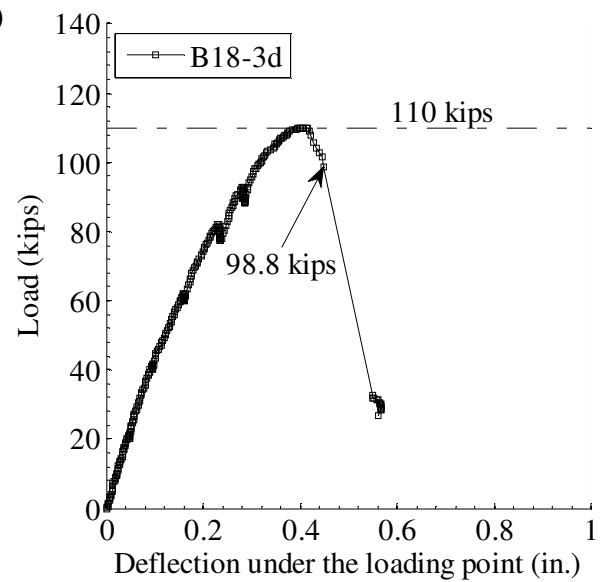

(b)

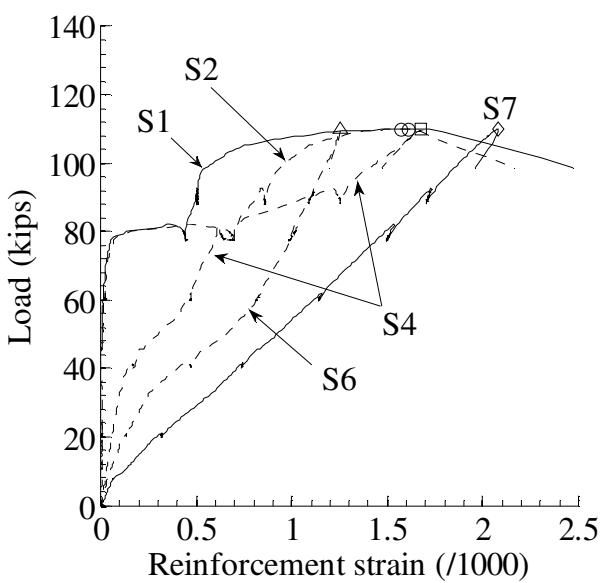

Fig. 4-67: Load versus deflection and reinforcement strain relationships - Beam B18-3d 


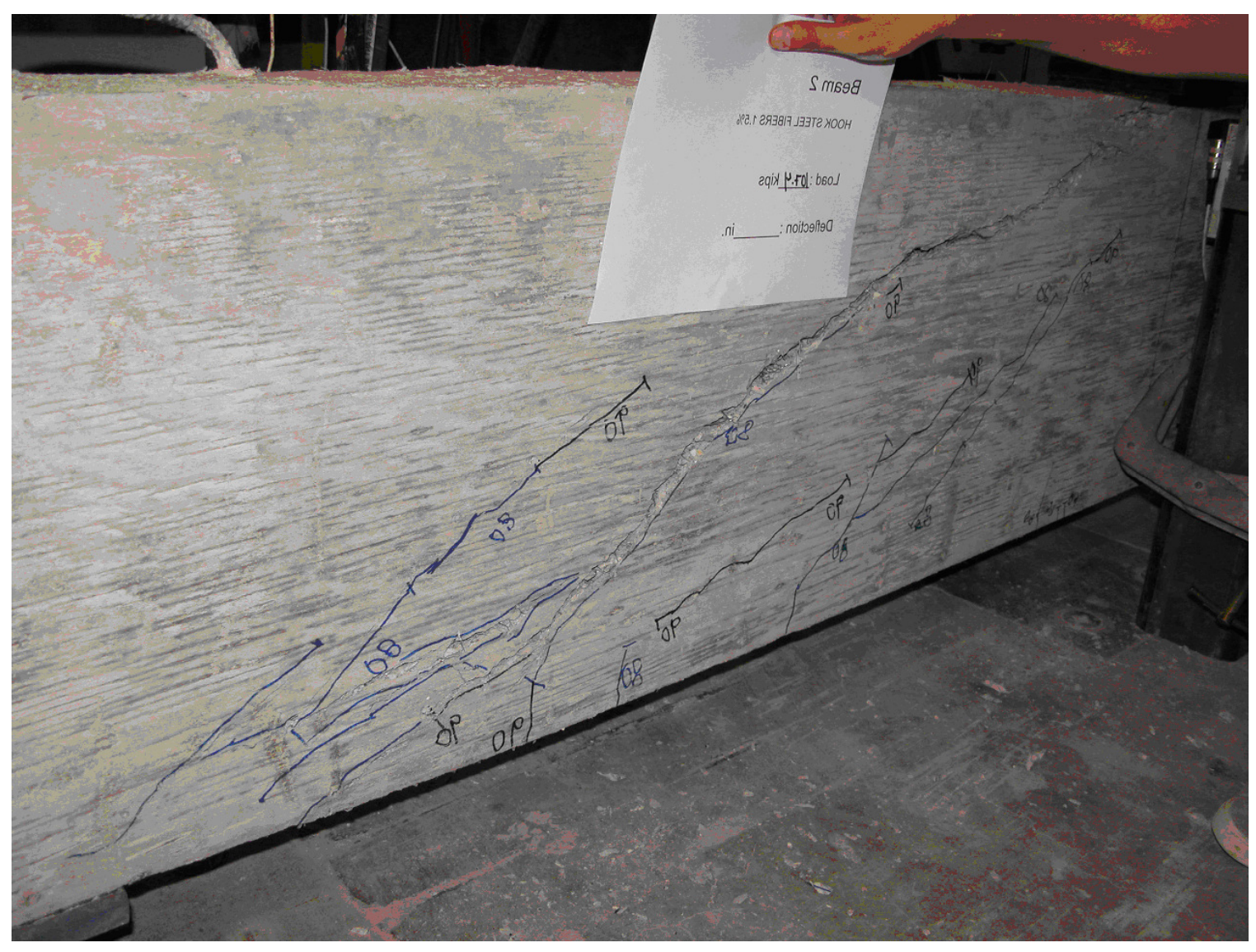

Fig. 4-68: Crack pattern after failure - Beam B18-3d

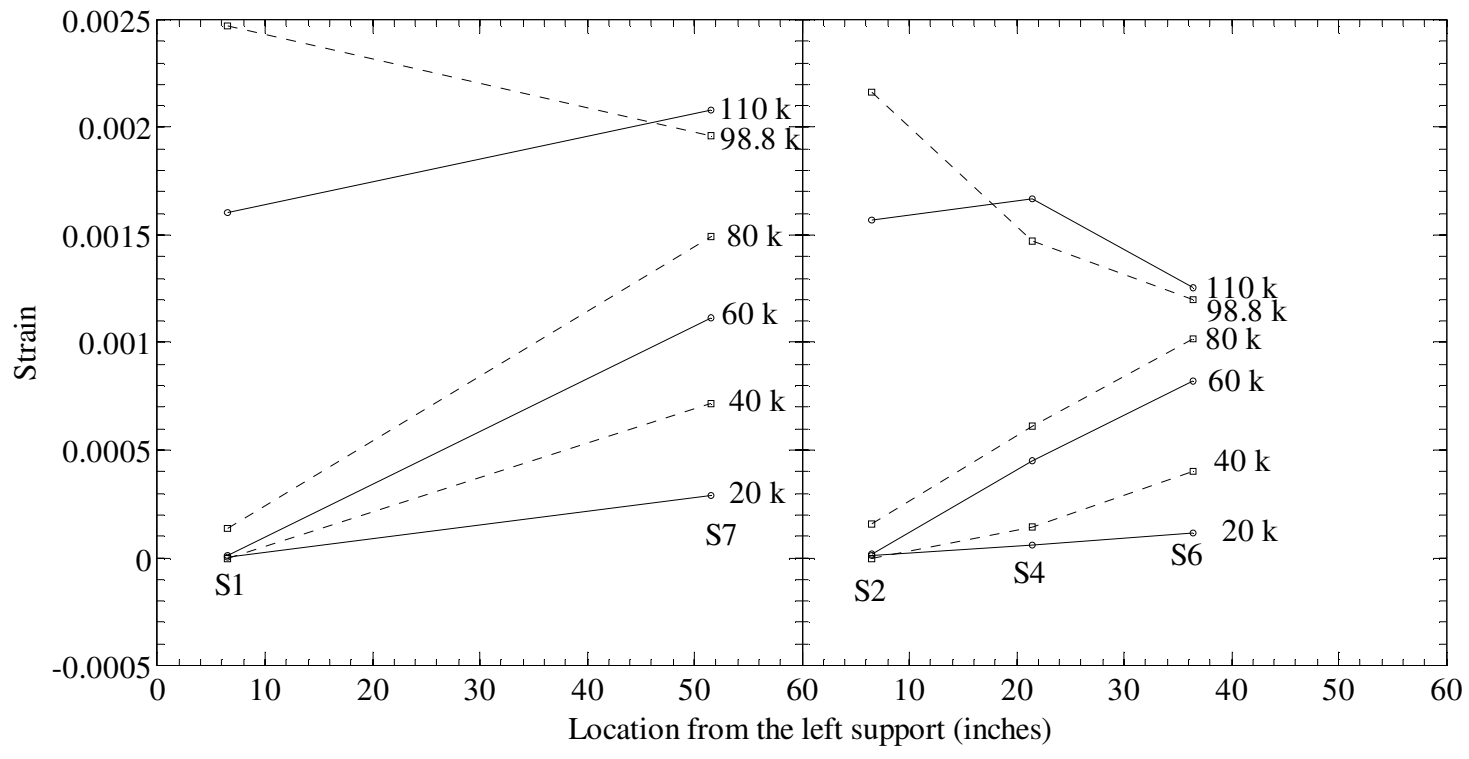

Fig. 4-69: Reinforcement strains along the beam axis - Beam B18-3d 


\subsubsection{Beams B18-5a \& b}

Beams B18-5a \& b contained the same fiber volume fraction (1\%) as Beams B18-2c \& d, but Beams B18-5a \& b contained Dramix fibers RC80/60BN with a larger diameter (0.030 versus 0.022 inches) and a longer length (2.36 inches versus 1.18 inches), compared to ZP305 fibers. It should be mentioned that the reinforcement ratio of Beams B18-5a \& b was $2.67 \%$. In addition, the anchorage used in these beams was $90^{\circ}$ hooks, instead of Lenton terminators, which were used in Beams B18-2c \& d.

Load versus deflection relationship, crack pattern, and failure mode. The ultimate strength of Beams B18-5a \& b was 100 (Fig. 4-70a) and 127 kips (Fig. 4-74a), respectively. Based on the strength of Beam B18-2c (113 kips), it was difficult to decide whether the strength of Beam B18-5a or B18-5b was more representative.

The first diagonal crack in Beam B18-5a appeared at a load of approximately 80 kips and three inclined cracks developed prior to failure (Fig. 4-71). The beam failed shortly after reaching the peak load. Significant crack opening at the mid-depth and narrower openings near the reinforcement and compression region of the beam are a strong indication of a pure diagonal tension failure. This failure mode caused the concrete block above the crack to displace up and to the right (Fig. 4-71).

While Beam B18-5b exhibited its first diagonal crack at the same load (80 kips) as Beam B18-5a, it showed four inclined cracks before failure (Fig. 4-75). This beam also had a different failure mode. Simultaneous wide opening was observed at two critical inclined cracks. If the beam had failed along the first crack from the support, the failure mode would have been a pure diagonal tension failure. However, several concrete compressive struts that intersected at the loading point seem to have created a significant compressive stress demand in the compression region, leading to concrete crushing near the loading point (Fig. 4-75). Together with this crushing, the splitting cracks along the reinforcement after failure suggested that the beam failed due to a combination of shearcompression and shear-tension. It is possible that the better cracking distribution in Beam B18-5b led to the higher shear strength of this beam compared to Beam B18-5a. 
Reinforcement strain and average bond stress. Strain gauges S6 and S8 in Beam B18-5a and S6 in Beam B18-5b failed prior to the beam tests. Similar to the previous beams with a high amount of longitudinal reinforcement, the reinforcing bars were not strained beyond the yield point (Fig. 4-70b and Fig. 4-74b). In general, a nearly linear distribution of reinforcement strains along the shear span was seen up to the peak load (Fig. 4-72 and Fig. 4-76). In Beam B18-5a, however, there was a jump in reinforcement strain near the support (S1 and S2) as the load increased from 60 to 80 kips due to the crossing of a diagonal crack at this location (Fig. 4-72). A similar situation occurred in Beam B18-5b, particularly at S2, but at a higher load. In general, bond stresses were larger near the support (Fig. 4-73 and Fig. 4-77). In both beams, a decrease in bond stress on the top reinforcement layer, near the support, occurred when the load approached the peak.

(a)

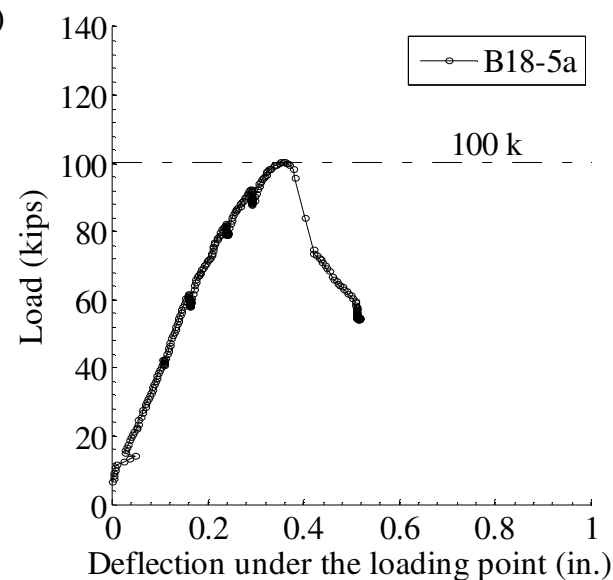

(b)

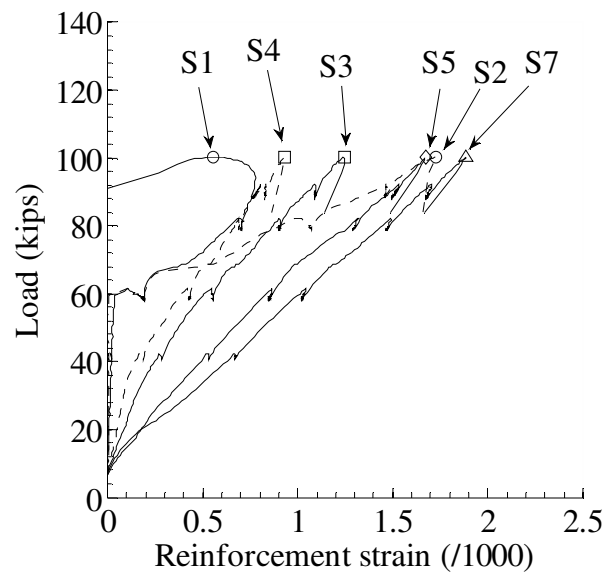

Fig. 4-70: Load versus deflection and reinforcement strain relationships - Beam B18-5a

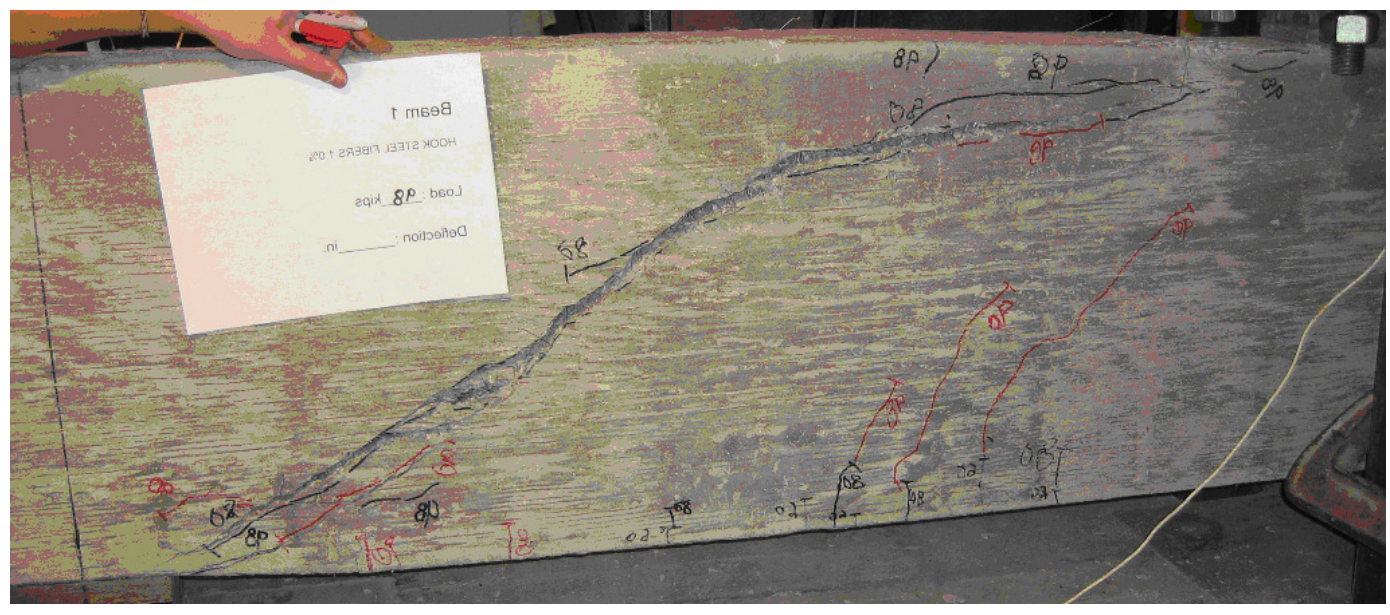

Fig. 4-71: Crack pattern after failure - Beam B18-5a 


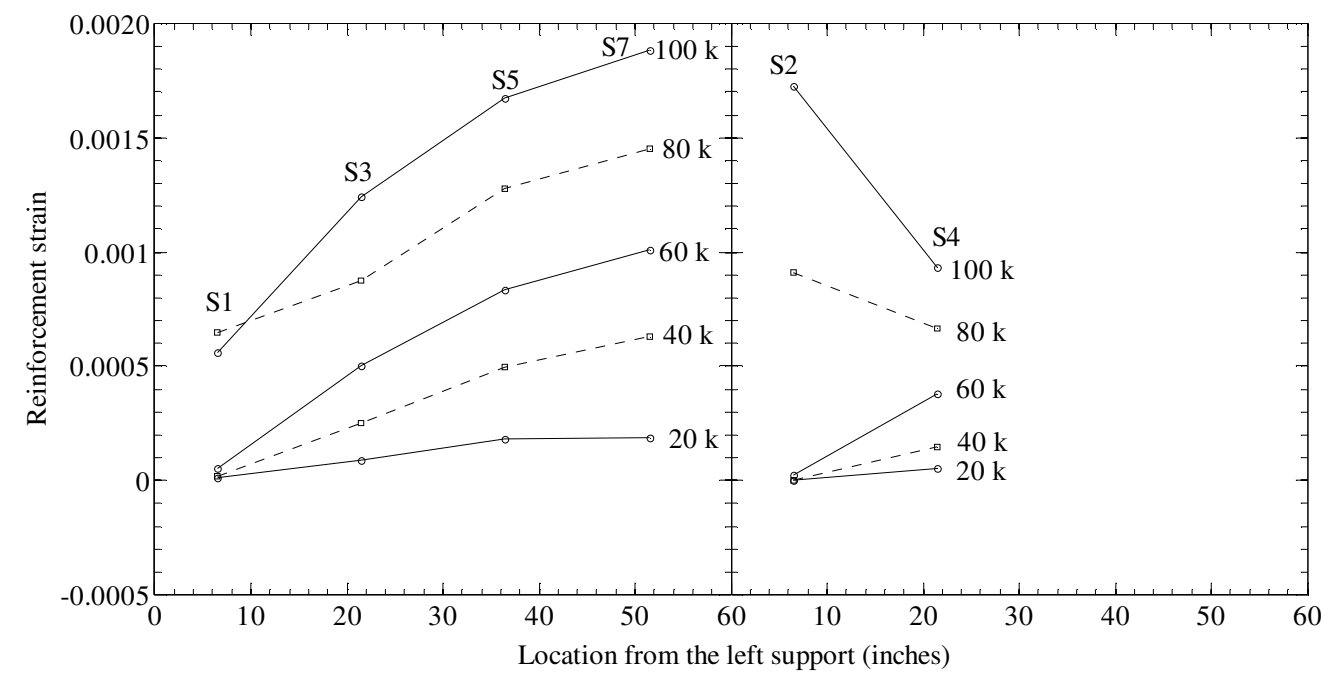

Fig. 4-72: Reinforcement strains along the beam axis - Beam B18-5a

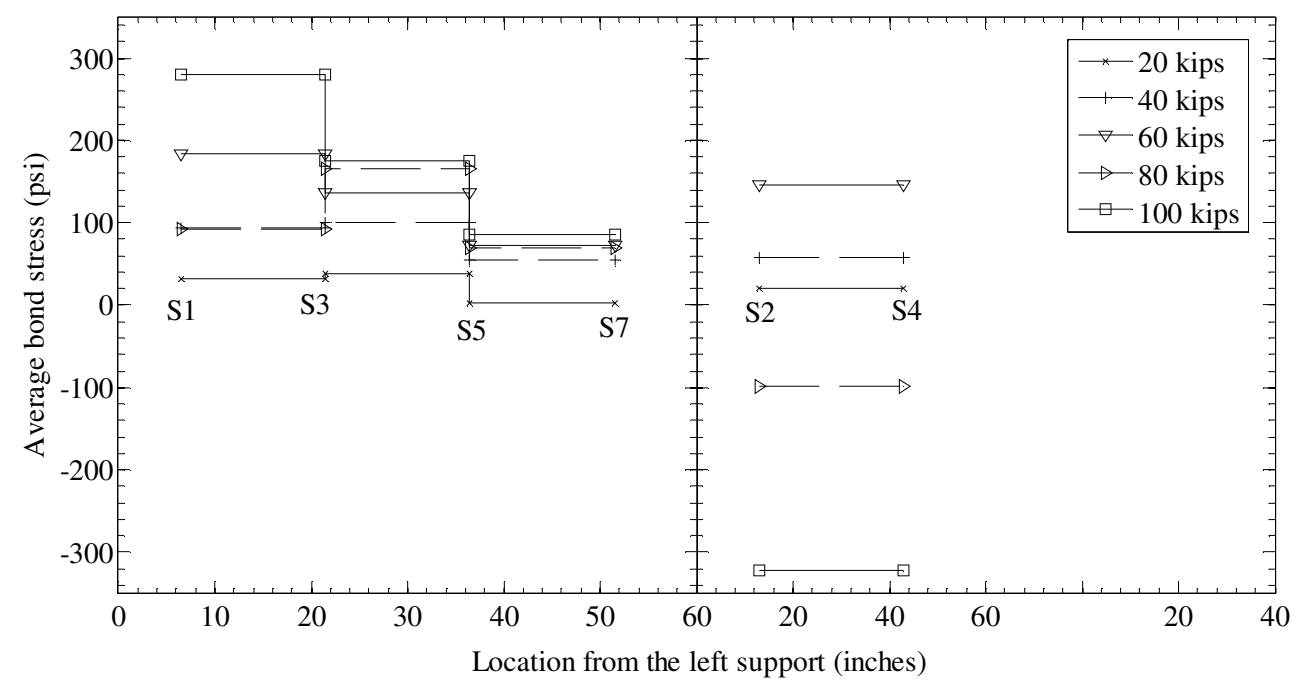

Fig. 4-73: Average bond stress along the beam axis - Beam B18-5a

(a)

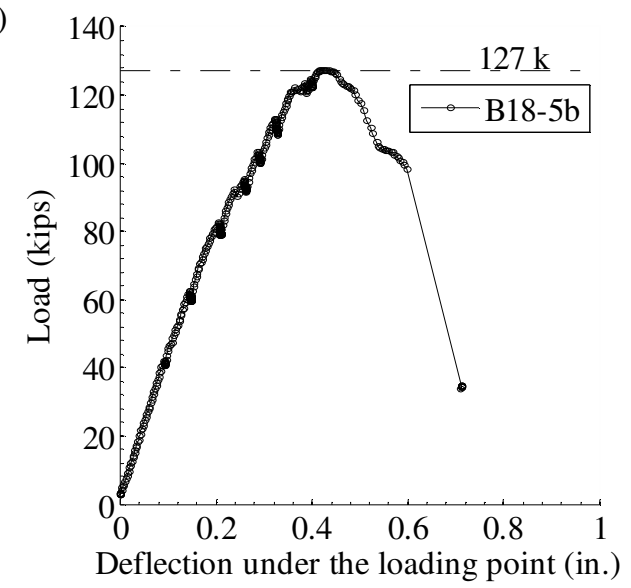

(b)

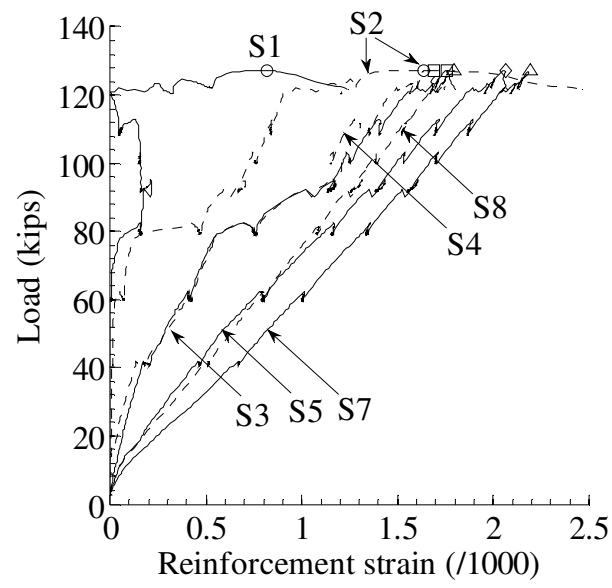

Fig. 4-74: Load versus deflection and reinforcement strain relationships - Beam B18-5b 


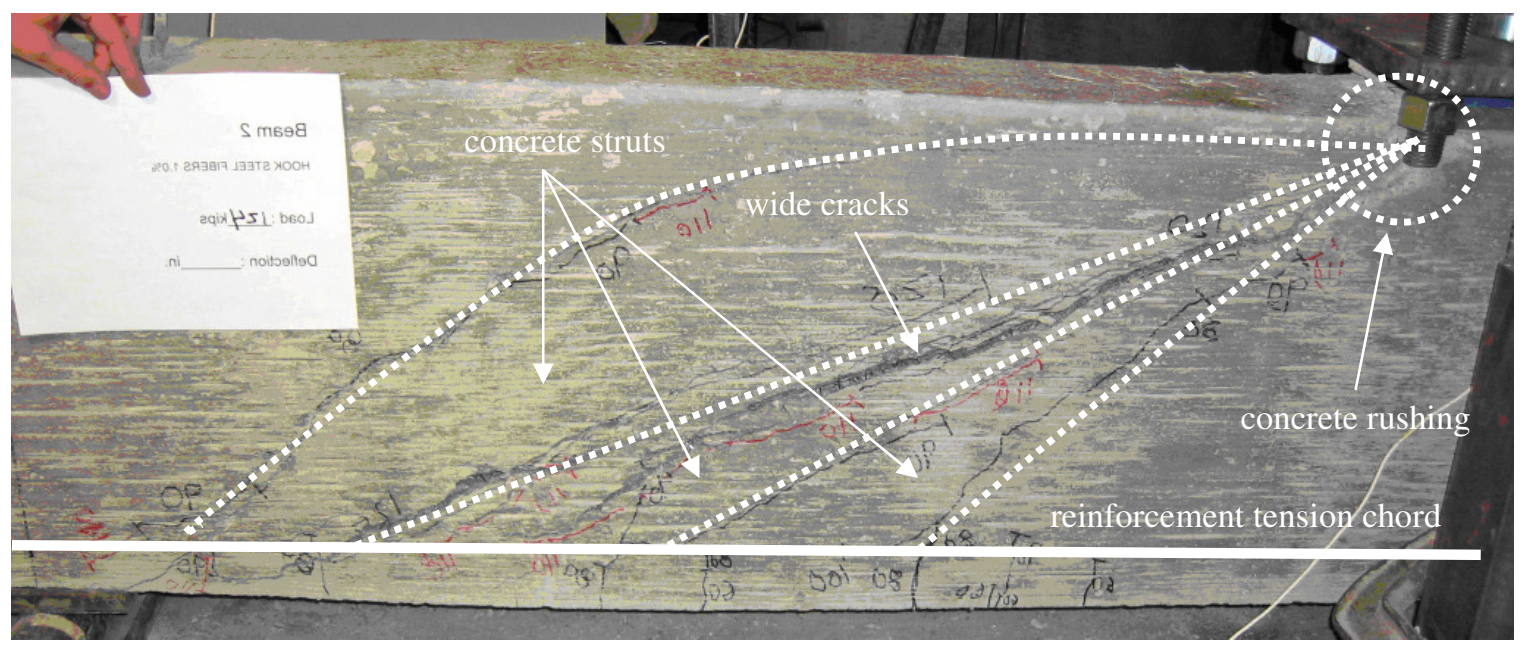

Fig. 4-75: Crack pattern after failure - Beam B18-5b

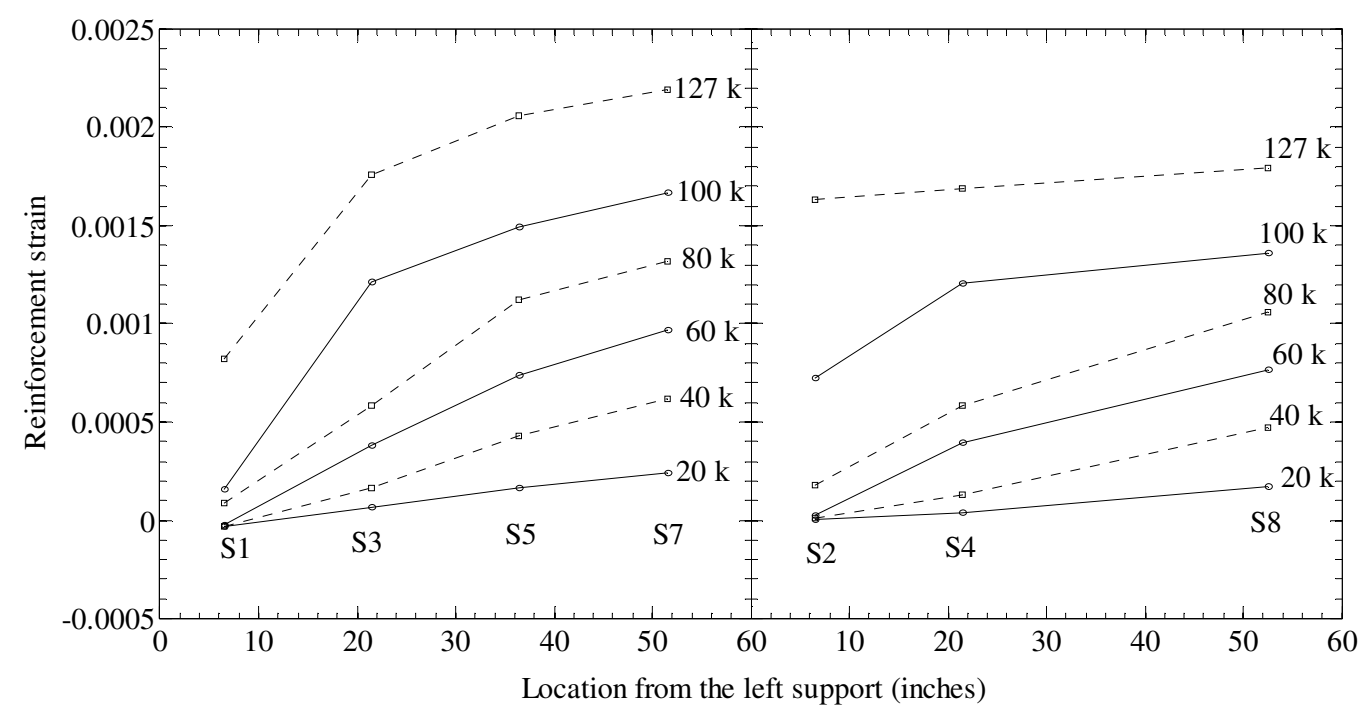

Fig. 4-76: Reinforcement strains along the beam axis - Beam B18-5b

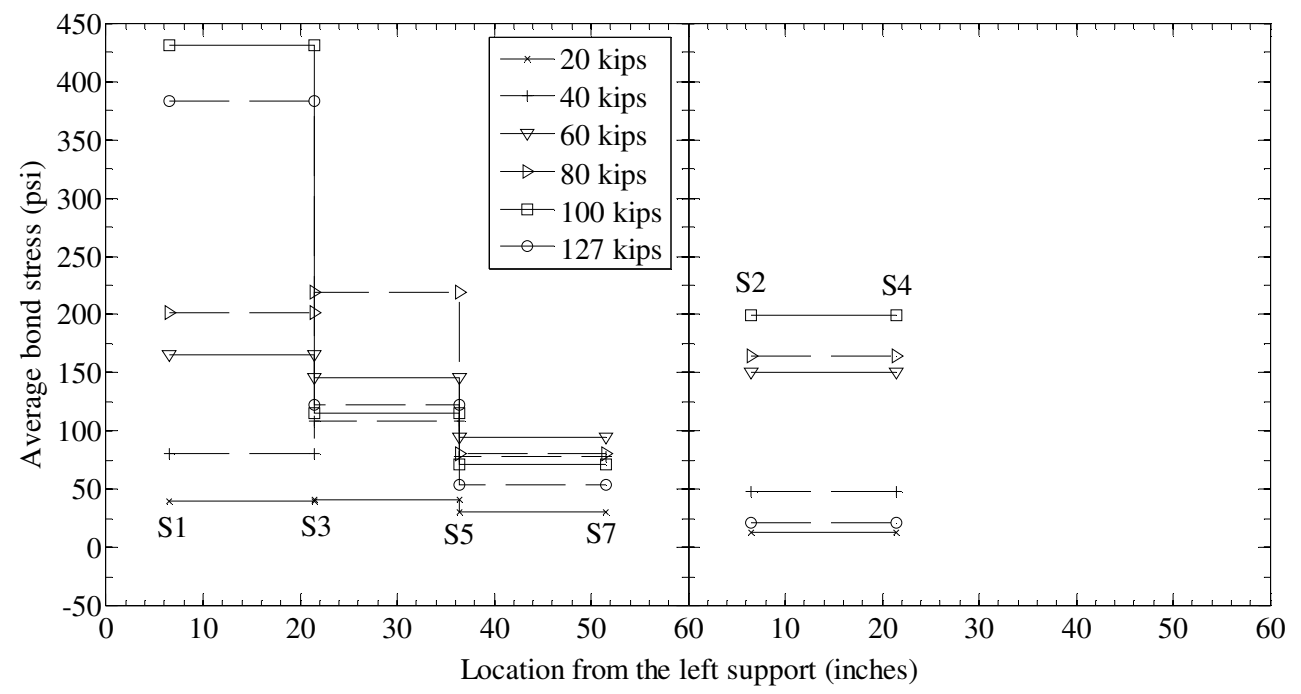

Fig. 4-77: Average bond stress along the beam axis - Beam B18-5b 


\subsubsection{Beam B18-7a}

Beam B18-7a had the same fiber volume fraction $(0.75 \%)$ and longitudinal reinforcement ratio $(1.96 \%)$ as Beams B18-1a \& b. While Beams B18-1a \& b contained ZP305 fibers, Beam B18-7a contained RC80/30BP fibers. It should be mentioned that these two types of fibers have the same length (1.18 inches). However, RC80/30BP fibers have a smaller diameter ( 0.015 inches compared to 0.022 inches), and hence a greater aspect ratio (80 versus 60). Also, RC80/30BP fibers have a higher tensile strength (330 ksi compared with $150 \mathrm{ksi}$ ).

\section{Load versus deflection relationship, crack pattern, and failure mode.}

Flexural cracks developed first at 30 kips and subsequently bent towards the loading point to become flexural-shear cracks at approximately 60 kips (Fig. 4-78). Compared with Beams B18-1a \& b, Beam B18-7a exhibited a widely distributed crack pattern. As many as seven inclined cracks were seen to pass the beam mid-depth level. Also, the inclined cracks in this beam penetrated high into the beam prior to failure. The beam reached its ultimate strength at 112 kips (Fig. 4-79), higher than those of Beams B18-1a $\& b$ (99.2 and 92.8, respectively). It should be noted that the beam was able to maintain a strength similar to its ultimate strength while deflecting an additional 0.2 inches. It is worth mentioning that several data points corresponding to bad OptoTRAK data during this yielding phase were deleted from Fig. 4-79. With a crack along the reinforcing bars (Fig. 4-78), the beam can be assumed to have failed due to a combination of diagonal tension and shear tension.

The enhanced performance of Beam B18-7a is attributable to the use of RC80/30BP fibers with their superior tensile strength and higher aspect ratio, which led to better bond characteristics and pullout behavior (Section 2.1.1). Bending test results (Section 3.6.3) also confirmed that SFRC containing RC80/30BP fibers, with the same fiber volume fraction, exhibited a higher equivalent bending stress than that with ZP305 fibers at the same deflection demand. 
Reinforcement strain and average bond stress. Similar observations made for previous beams also hold for this beam. At the same load, reinforcement strains near the support (S1 and S2) were similar up to the load of 60 kips, while those at S3 and S4 were different (Fig. 4-81). This was also seen for S5 \& S6 and S7 \& S8. Throughout the test, the reinforcement strains at S1 through S4 were still in the linear range, while strain gauges S5 through S8 were strained beyond the yield point. Specifically, S5, S7, and S8 were strained into the strain hardening region. Strain gauge S7 was strained beyond the yield point at the load of 75 kips. It should be noted that strain gauge S4 malfunctioned after the load of 95 kips. An approximately linear distribution of reinforcement strains along the shear span was seen up to a load of 80 kips (Fig. 4-82). Similar to previous beams, low bond stresses were observed for the region below the loading point (Fig. 4-83).

Compared with Beams B18-1a \& b, the reinforcing bars of Beam B18-7a were more highly strained. In conclusion, the use of RC80/30BP fibers allowed the beam to maintain its strength and show significant ductility. This ductility was attained through a favorable crack pattern and better fiber performance, as discussed above.

Analysis of concrete strain field. It is interesting to note that the vertical distribution of the longitudinal strains showed a high degree of linearity even at the peak load (Fig. 4-84a). The maximum compressive and tensile strains were 0.0034 between markers M37 \& M41 and 0.014 between M36 \& M40, respectively (Fig. 4-84b, see Fig. 4-80 for the numeration of markers). The maximum measured transverse and shear strains were 0.0077 and 0.01, respectively (Fig. 4-84c \& d).

The distribution of transverse strains, measured over the beam depth, along the shear span was similar to previous beams, with its peak at the middle of the shear span and some degree of shear distortion and transverse strain near the support (Fig. 4-85).

Fig. 4-86 shows the principal tensile strain field at the peak load. Significant principal tensile strains were observed not only along the critical crack, but also along other flexural-shear cracks to the right of the critical one. None of the elements were compressed beyond a strain of 0.003 . 
Throughout the test, the principal tensile strain of Element E15 at the lower end of the critical crack was dominant (Fig. 4-87a). At approximately 68 kips, the tensile strain of Element E17 at the mid-depth suddenly increased, but was still smaller than that of E15. The principal tensile strain of Element E12 was also smaller than that of E15 and E17, and then surpassed that of E17 after the load of 100 kips, as a longitudinal crack opened along top reinforcement layer.

Prior to reaching the peak load, the principal tensile strains for all element suddenly increased at the load of 105 kips (Fig. 4-87b). This can be considered the critical strain state beyond which the beam started to fail. The maximum principal tensile strain and crack width at E15 were 0.008 and 0.046 inches $(1.2 \mathrm{~mm})$, respectively.

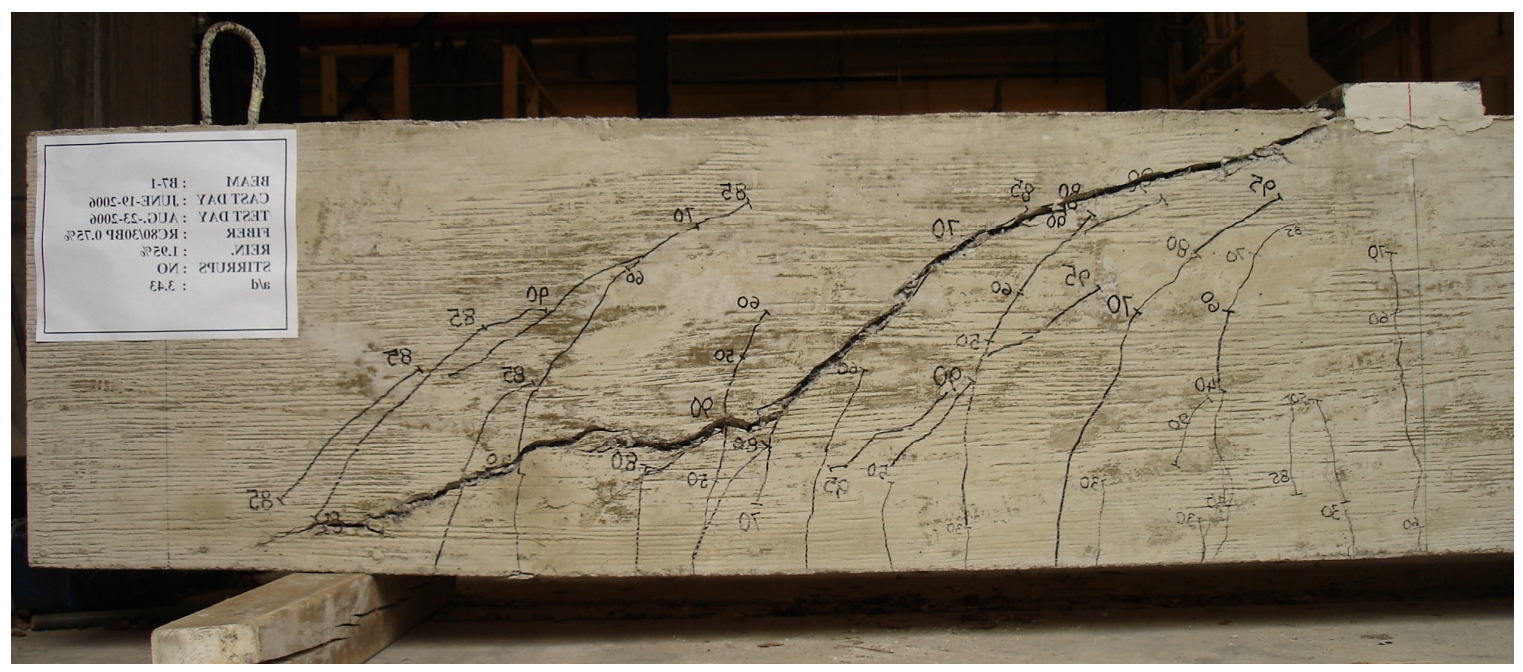

Fig. 4-78: Crack pattern after failure - Beam B18-7a

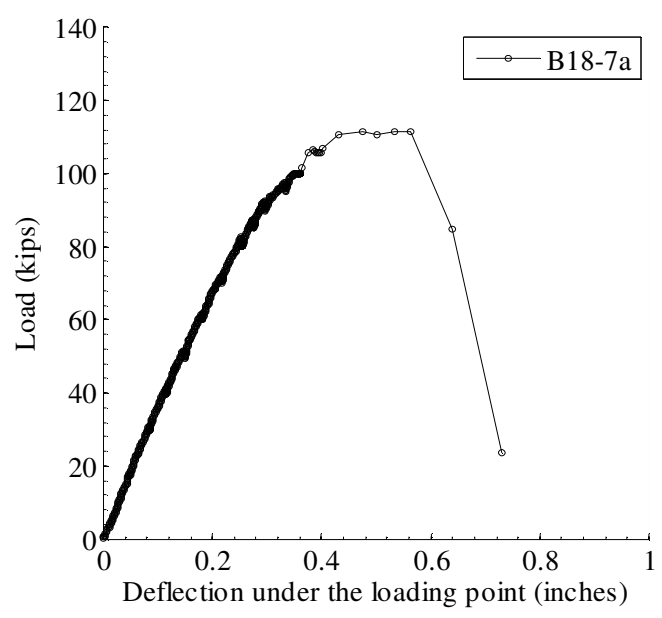

Fig. 4-79: Load versus deflection relationship - Beam B18-7a 


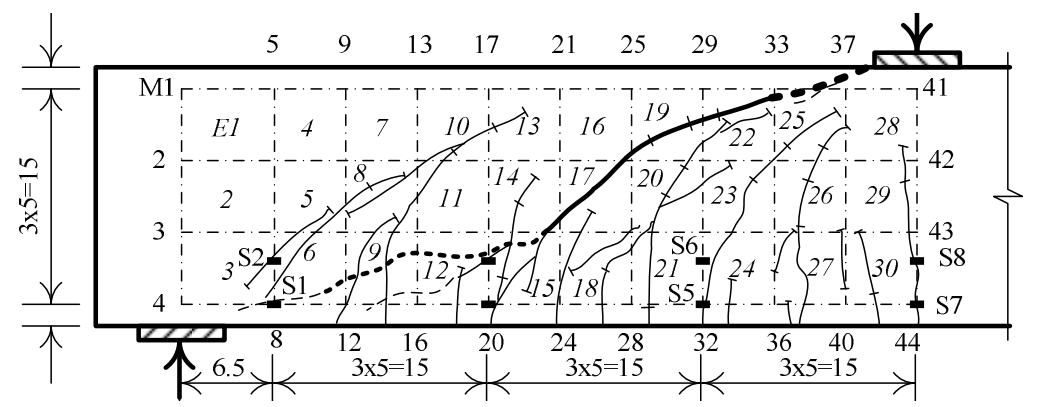

Fig. 4-80: Numbering of markers, elements, and strain gauges - Beam B18-7a

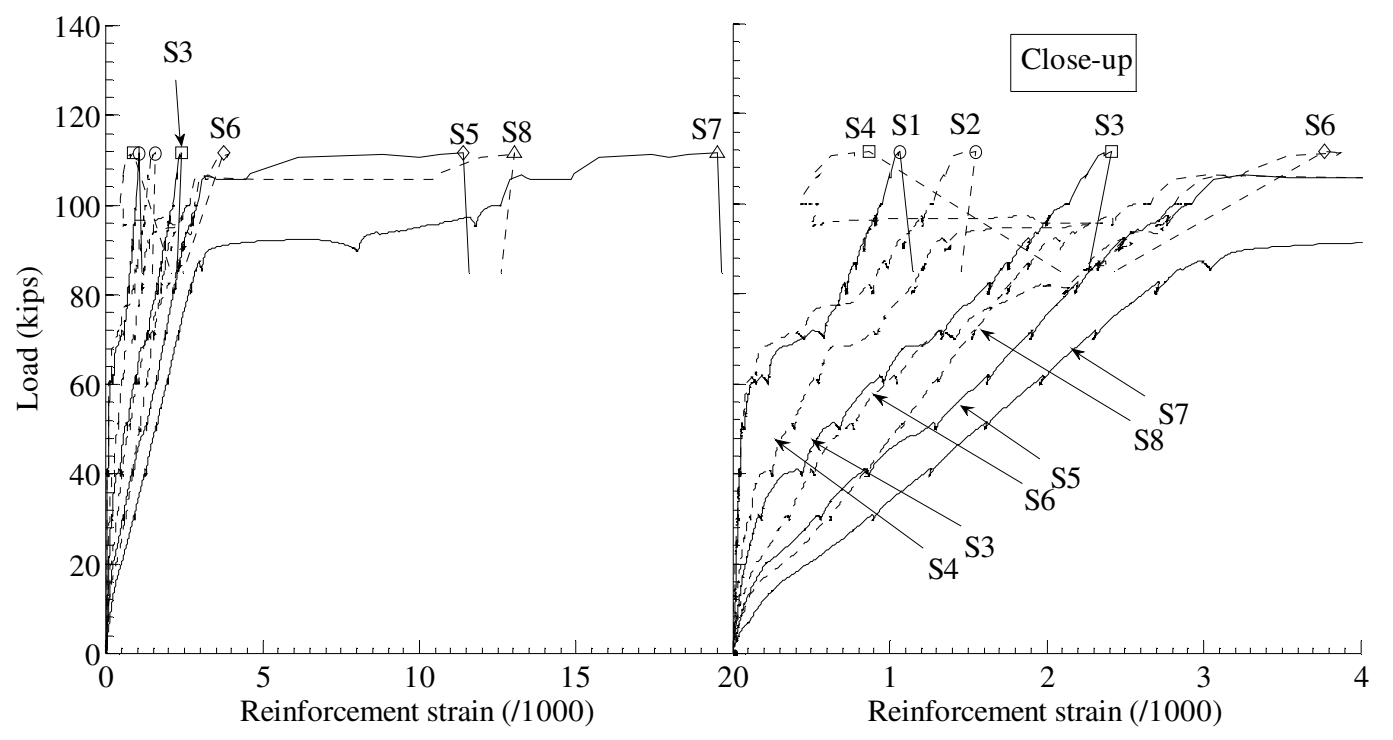

Fig. 4-81: Load versus reinforcement strain relationships - Beam B18-7a

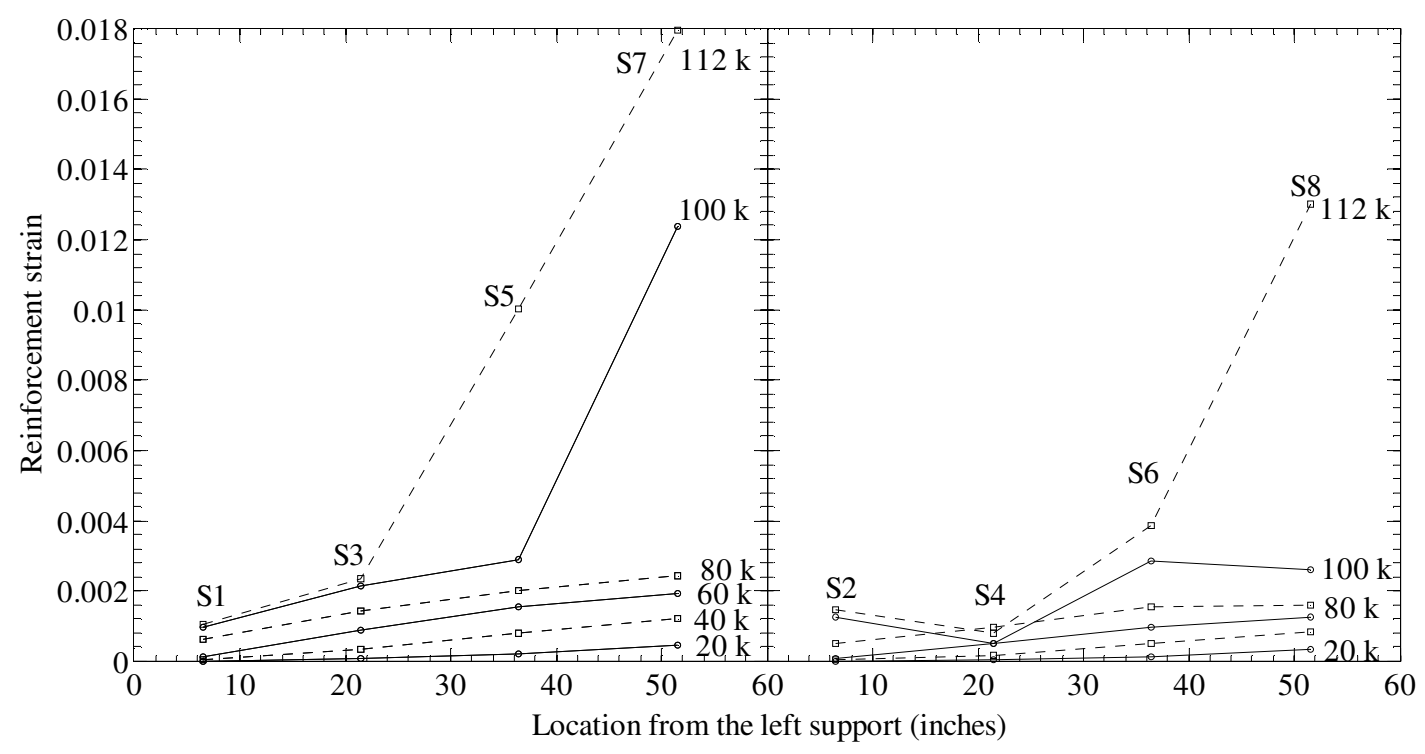

Fig. 4-82: Reinforcement strains along the beam axis - Beam B18-7a 


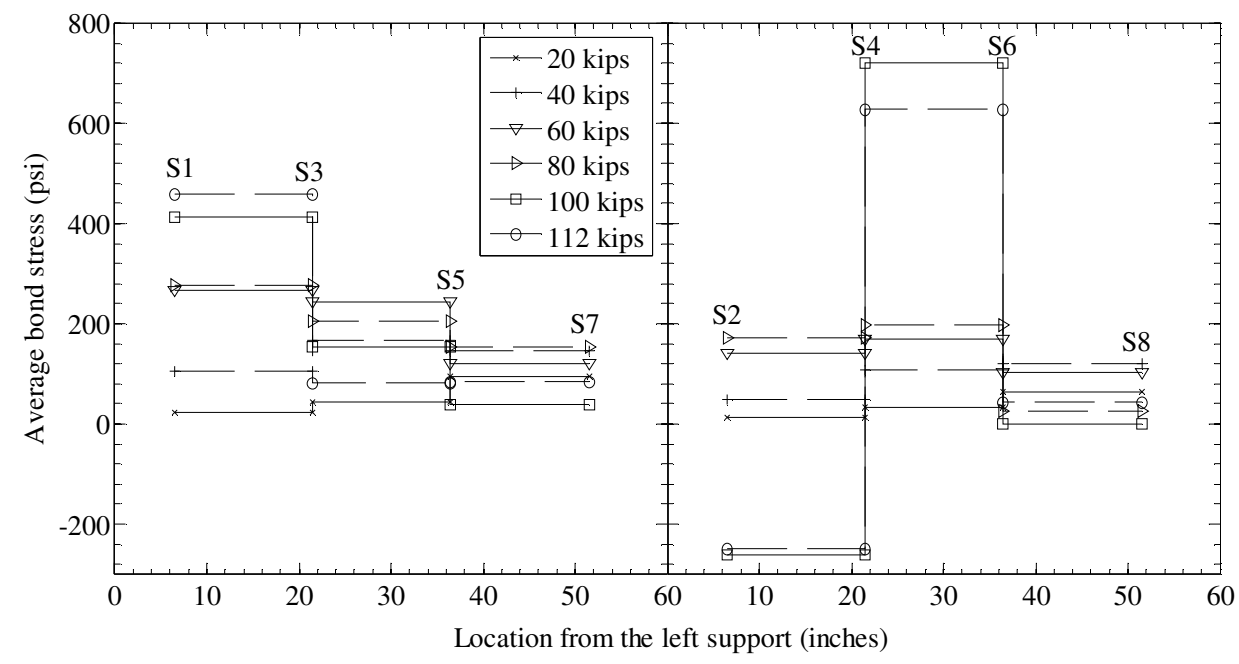

Fig. 4-83: Average bond stress along the beam axis - Beam B18-7a

(a)

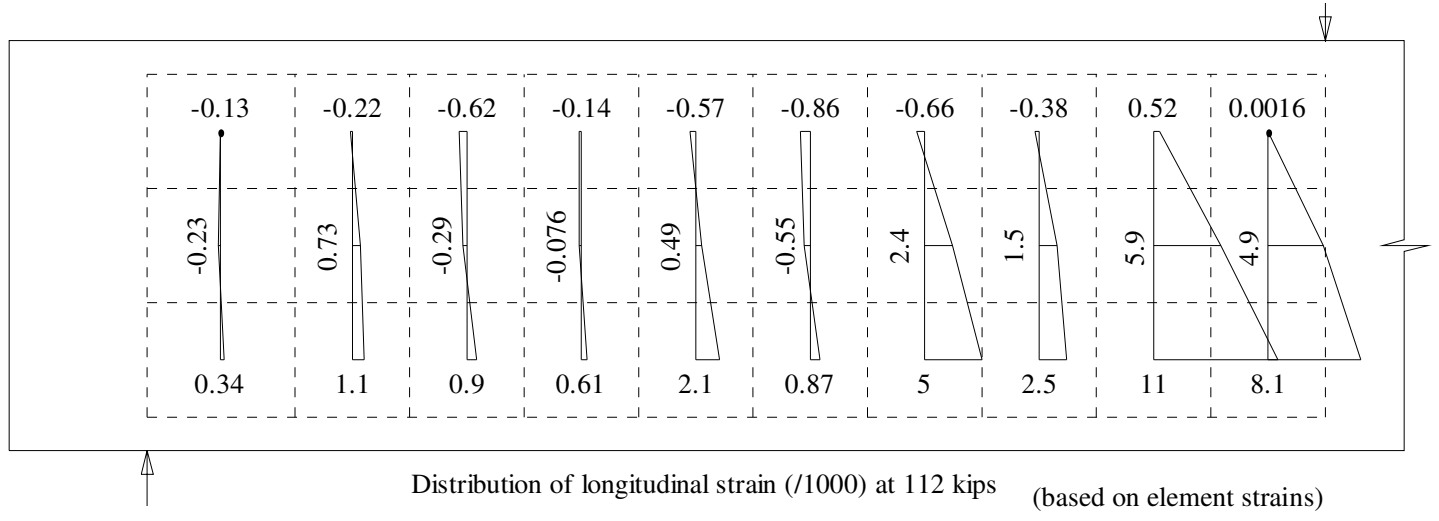

(b)

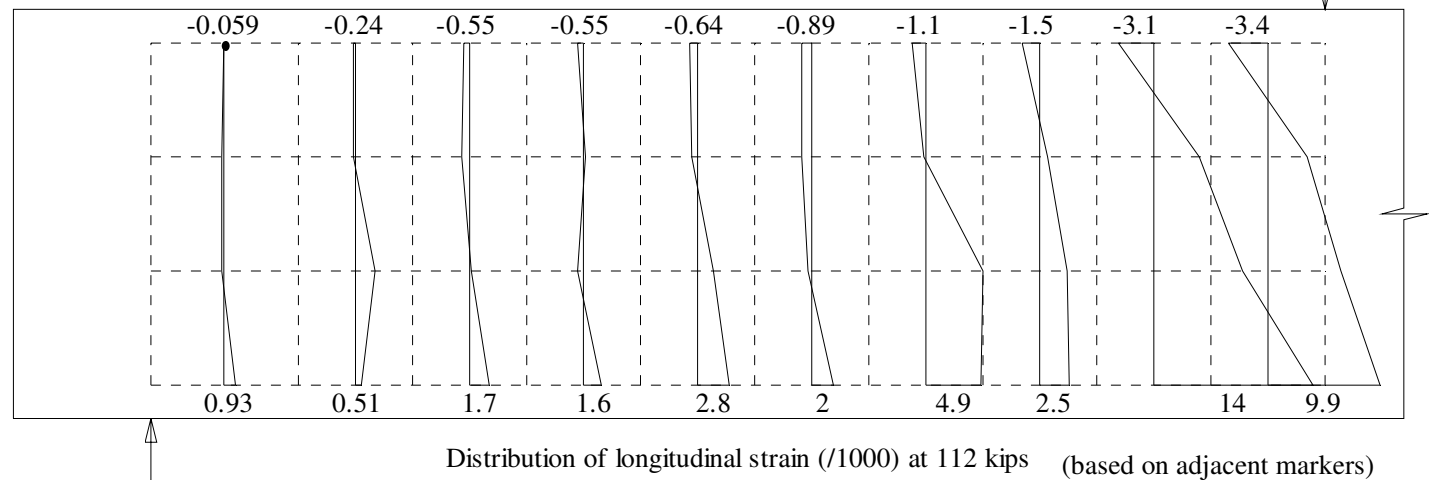

Fig. 4-84: Distribution of longitudinal, transverse, and shear strains - Beam B18-7a 
(c)

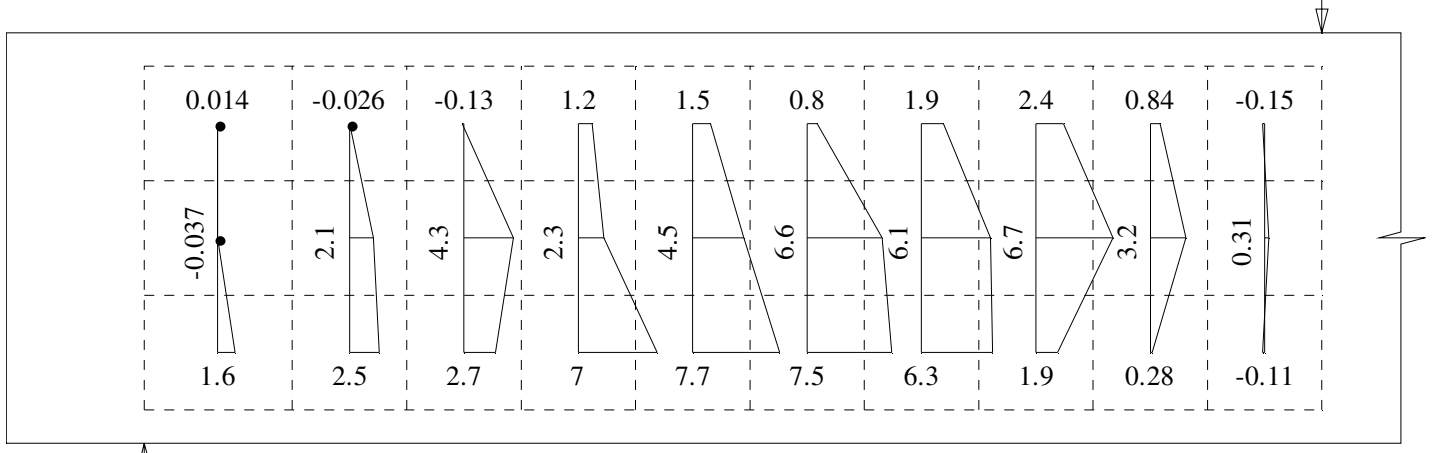

Distribution of transverse strain (/1000) at $112 \mathrm{kips}$

(d)

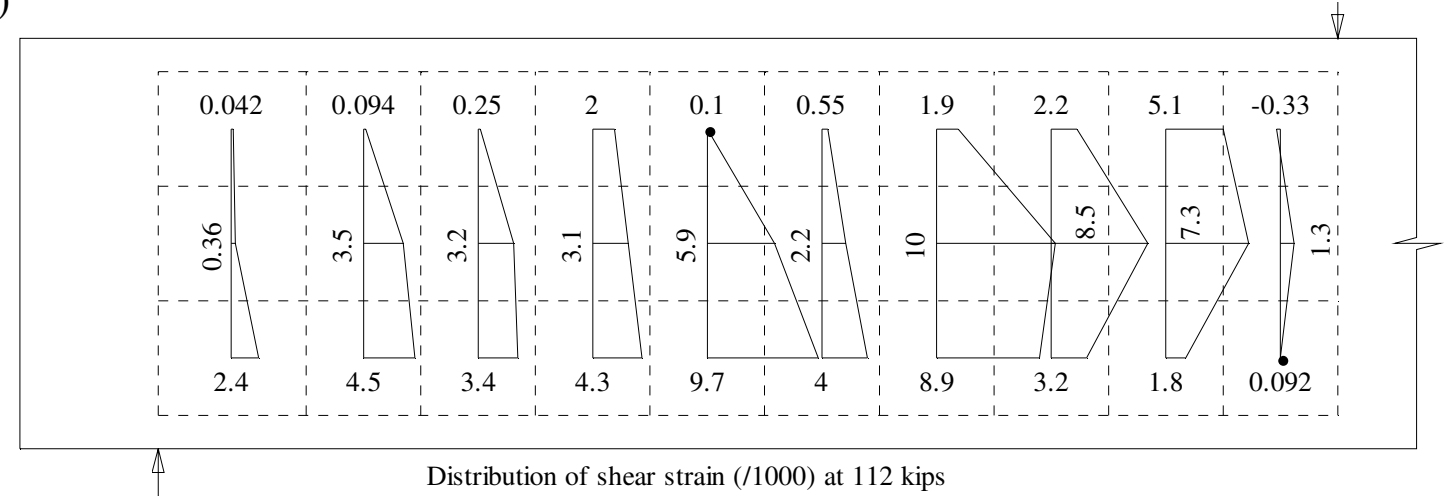

Fig. 4-84: Distribution of longitudinal, transverse, and shear strains - Beam B18-7a (continued)

(a)

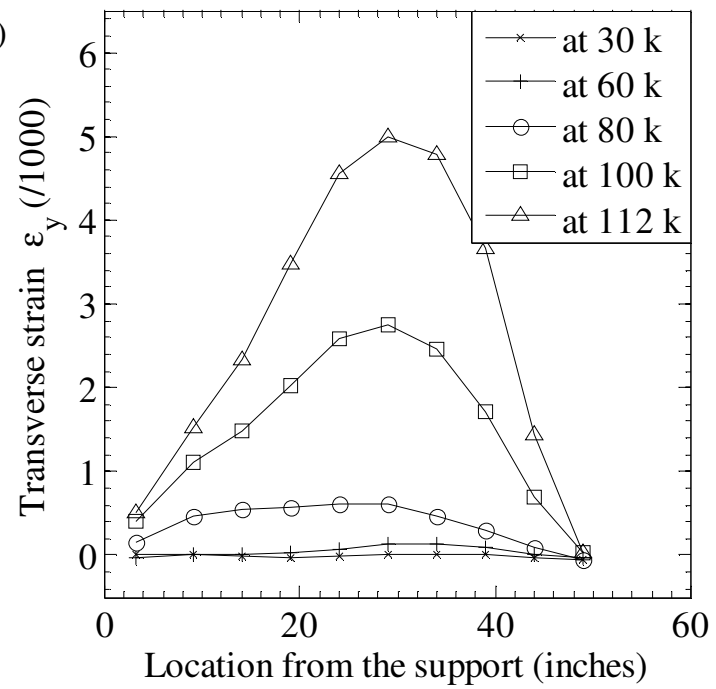

(b)

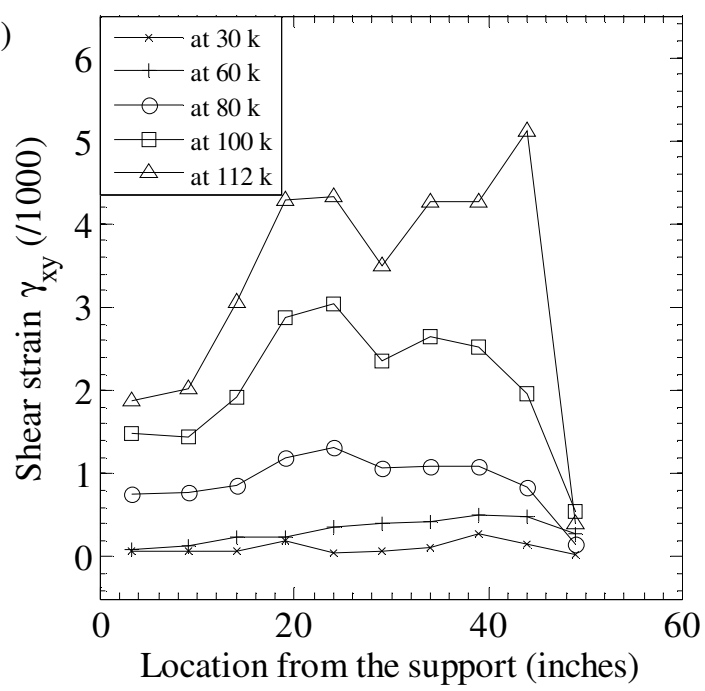

Fig. 4-85: Distribution of transverse and shear strains averaged over the beam depth Beam B18-7a 


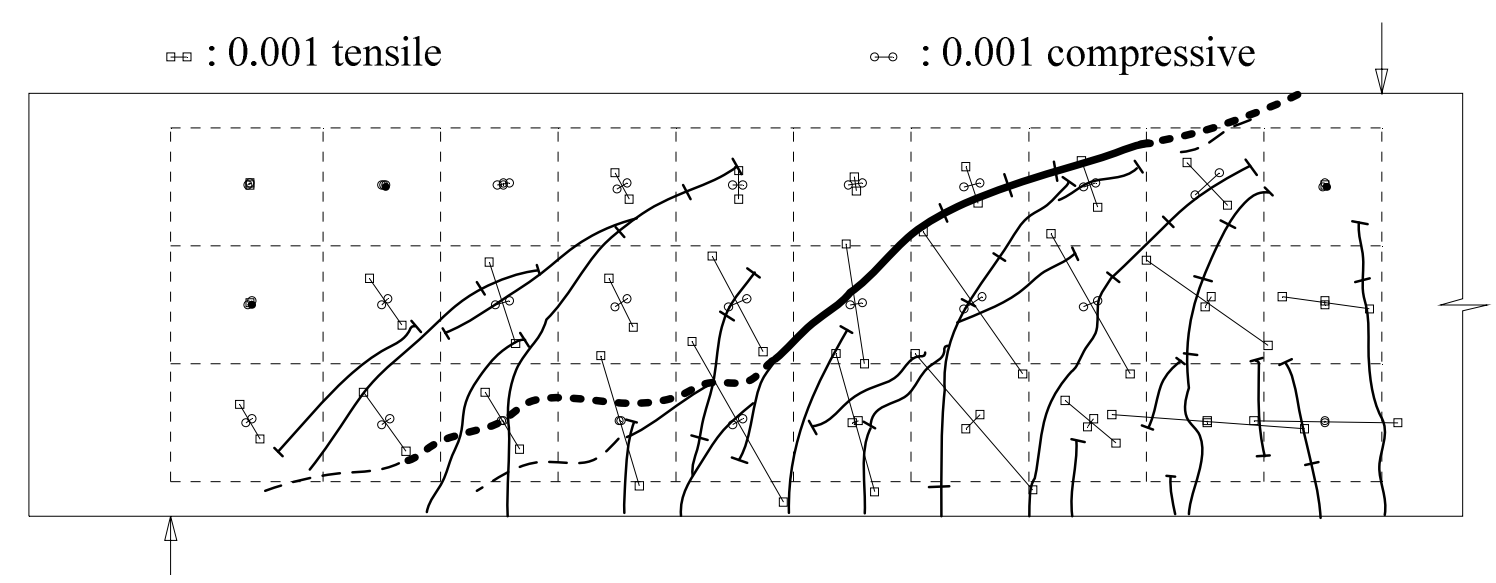

Fig. 4-86: Principal strain field at 112 kips - Beam B18-7a

(a)

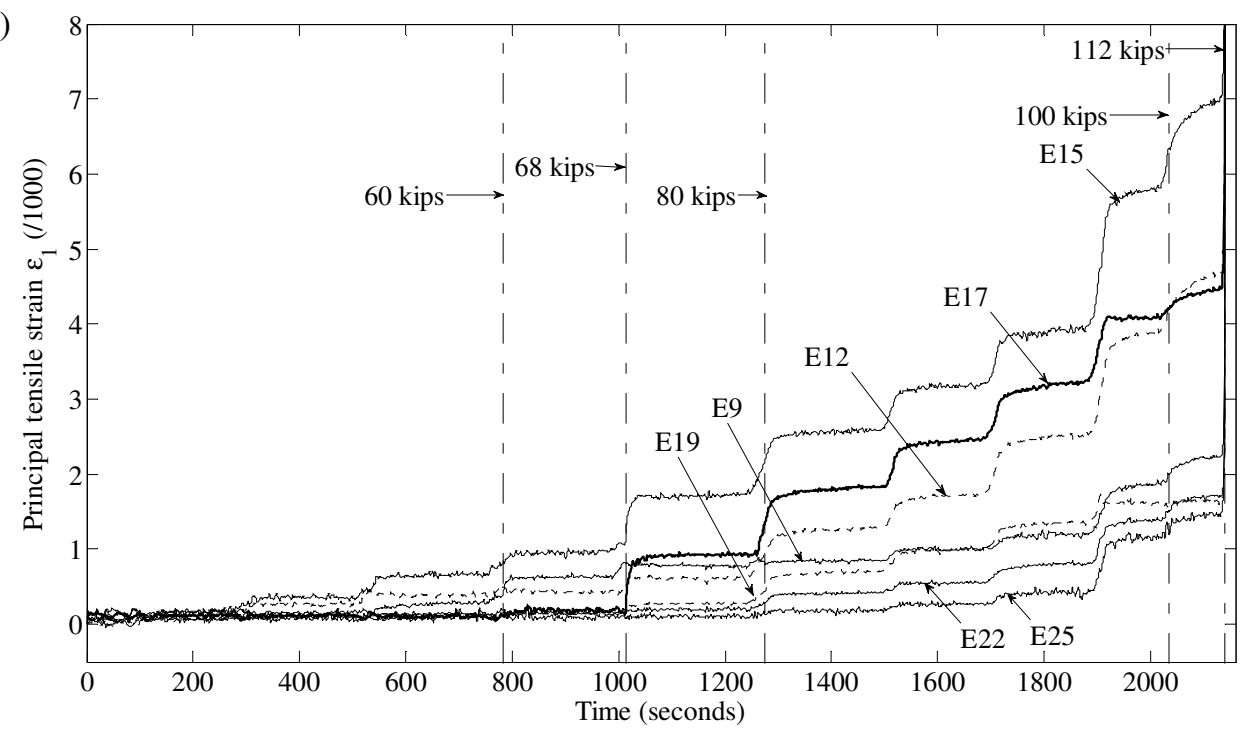

(b)

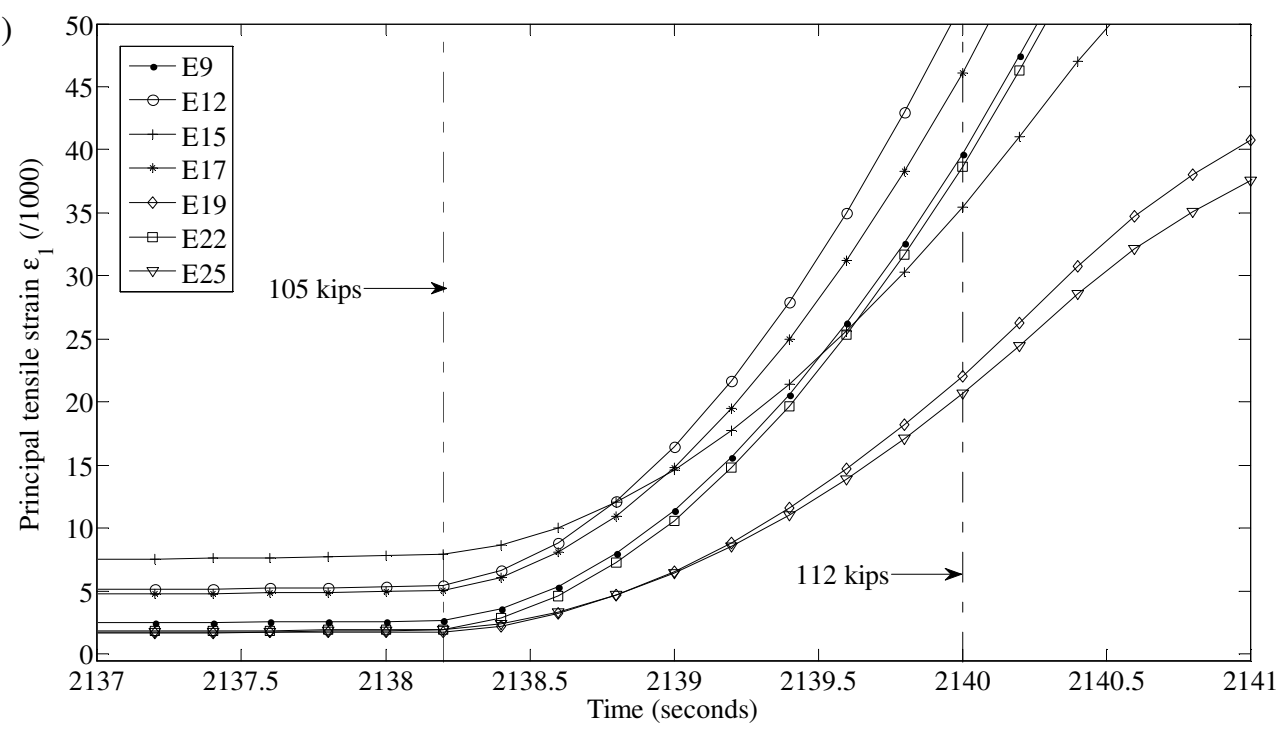

Fig. 4-87: Development of principal tensile strain versus time - Beam B18-7a 


\subsubsection{Beam B18-7b}

Beam B18-7b had the same design as Beam B18-7a and its behavior was very similar to that of Beam B18-7a. Beam B18-7b also exhibited five inclined cracks that passed the beam mid-depth level (Fig. 4-88a) and approached the compression region (Fig. 4-88b) when the applied load got near the beam ultimate strength. The beam was able to exhibit a "yield" plateau and attained its ultimate strength at 110 kips (Fig. 4-89a), very close to that of Beam B18-7a. The failure mode of Beam 18-7b can also be considered to be a combination of diagonal tension and shear-tension.

The reinforcing bars were strained well beyond the yield strain at locations S5, S6, and S7 (Fig. 4-89b). Linear distribution of reinforcement strains along the shear span was observed up to the load of 80 kips (Fig. 4-90). Bond stresses were larger near the support (from S1 to S3 and S2 to S4, Fig. 4-91) and smaller close to the loading point (from S5 to S7, Fig. 4-91). Notably, the bond stress from S1 to S3 and from S2 to S4 increased continuously up to the peak load.

Maximum concrete compressive and tensile longitudinal strains at the peak load were 0.004 and 0.013, respectively (Fig. 4-92a). The observed maximum transverse and shear strains were 0.01 (Fig. 4-92b and c). The transverse and shear strains averaged over the beam depth are shown in Fig. 4-94. The transverse strain demand was a little smaller than that for Beam B18-7a (Fig. 4-85).

Large principal tensile strains were not as widely distributed throughout the shear span as those in Beam B18-7a. Principal compression strains in the elements were below 0.003 (Fig. 4-93). The development of principal tensile strains with time (Fig. 4-95a) suggests that the beam failure was initiated by diagonal tension, with the largest

principal tensile strains at the mid-depth in Element E17 and at the reinforcement-level in Element E15. At 108 kips after the peak load, the principal strains of all elements significantly increased, signifying the final failure of the beam. 

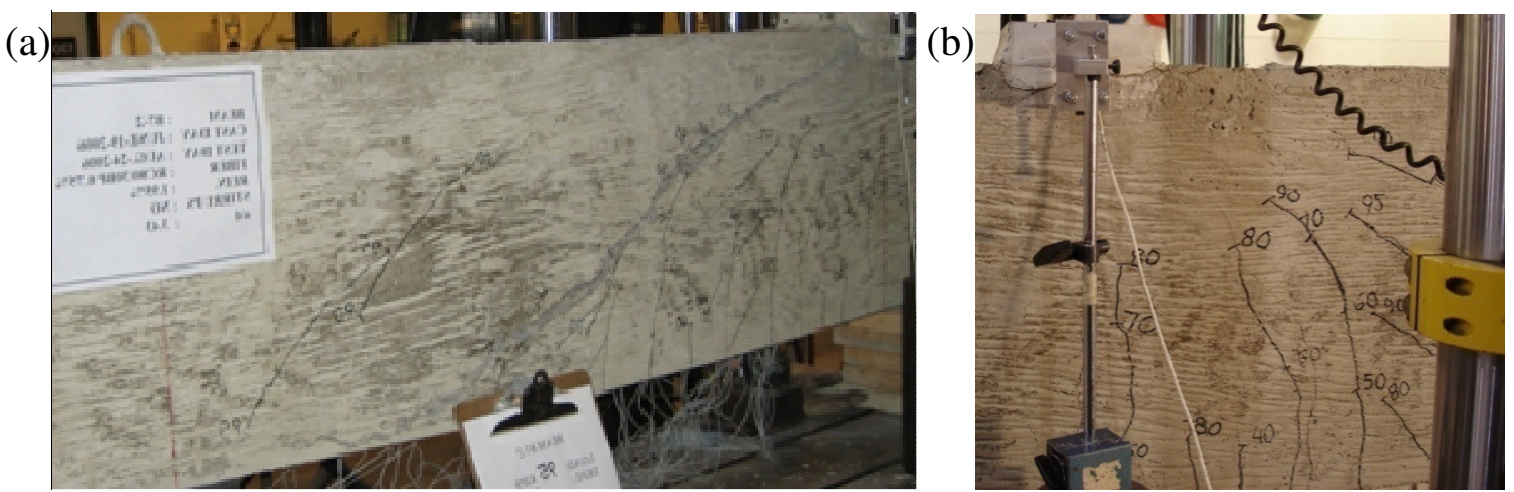

Fig. 4-88: Crack pattern after failure - Beam B18-7b

(a)

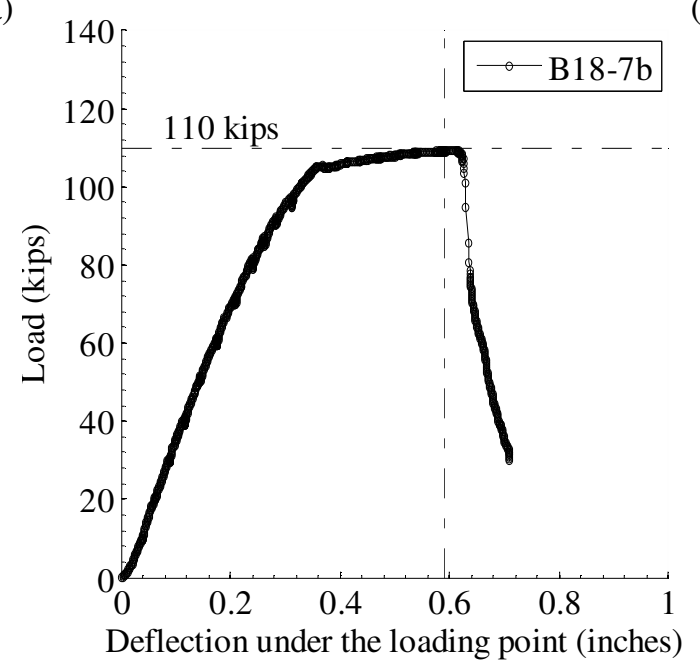

(b)

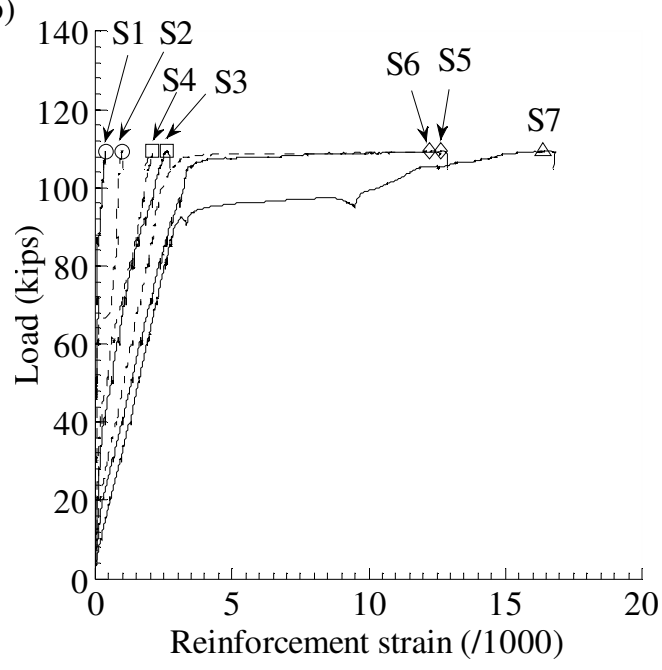

Fig. 4-89: Load versus deflection and reinforcement strain relationships - Beams B18-7b

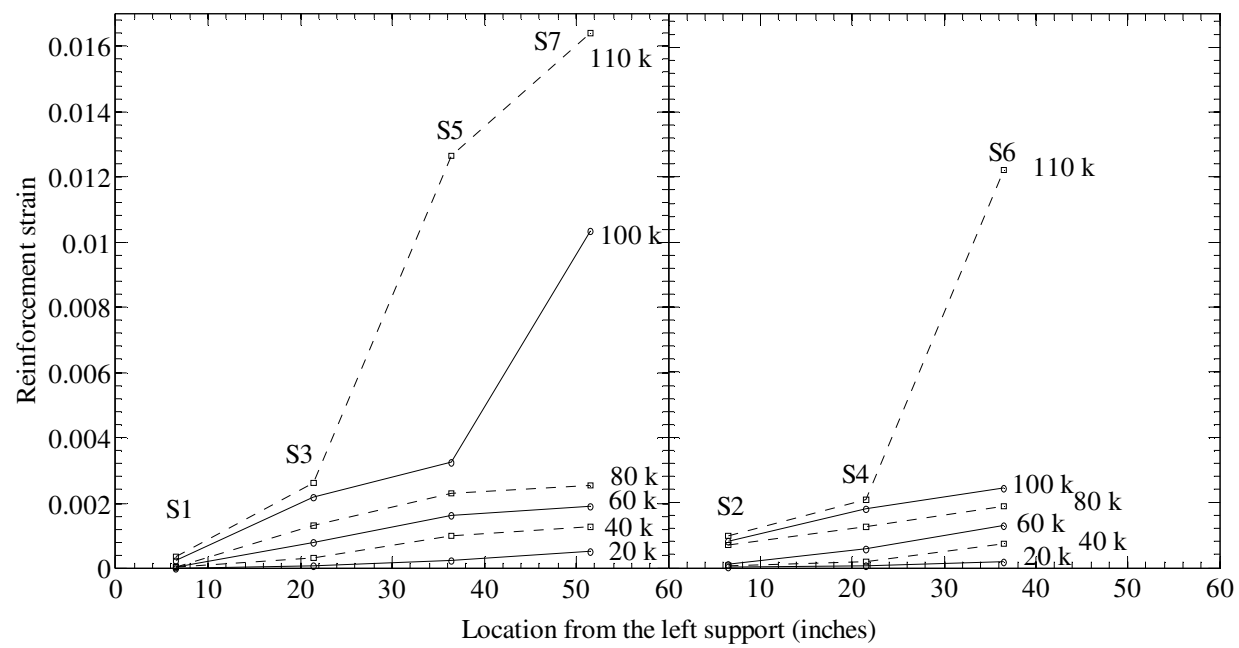

Fig. 4-90: Reinforcement strains along the beam axis - Beam B18-7b 


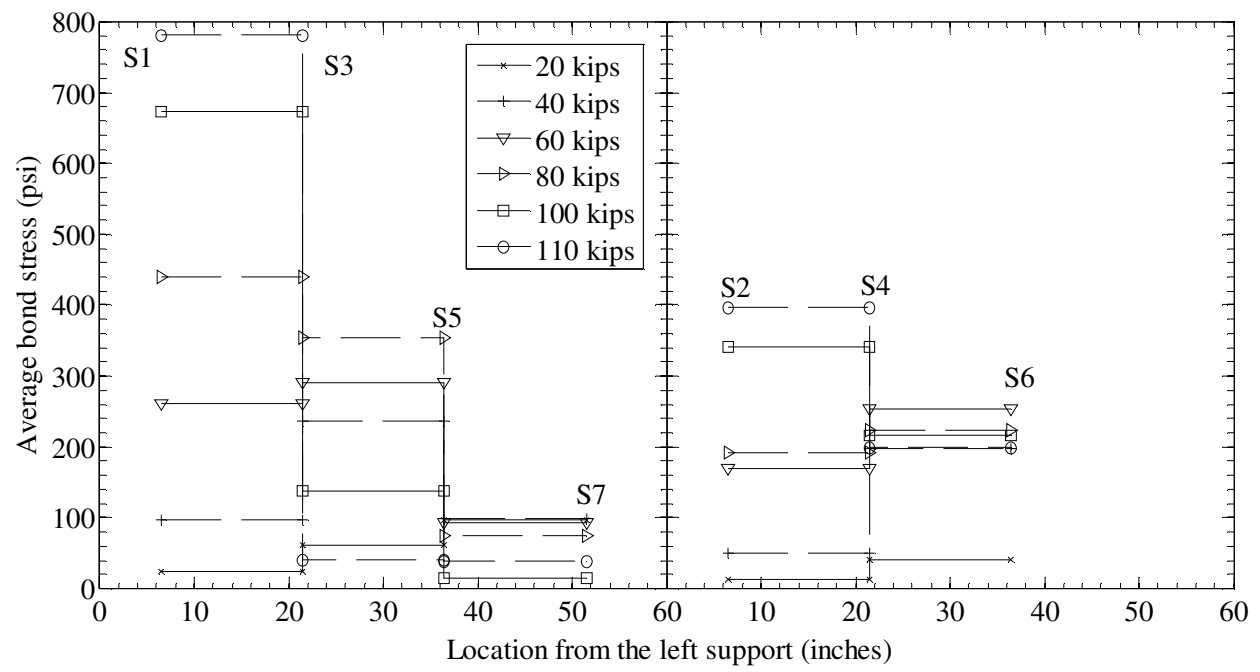

Fig. 4-91: Average bond stress along the beam axis - Beam B18-7b

(a)

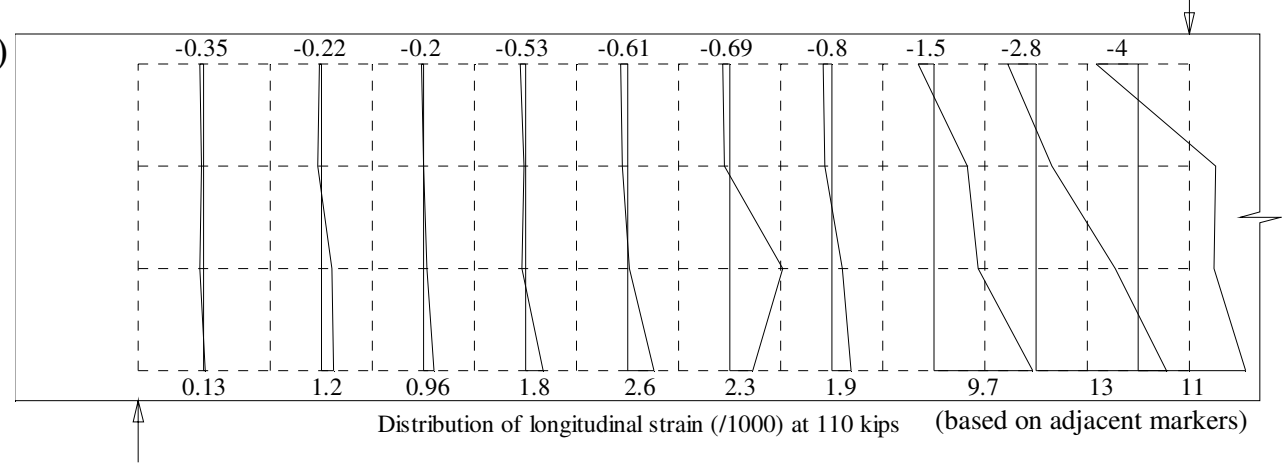

(b)

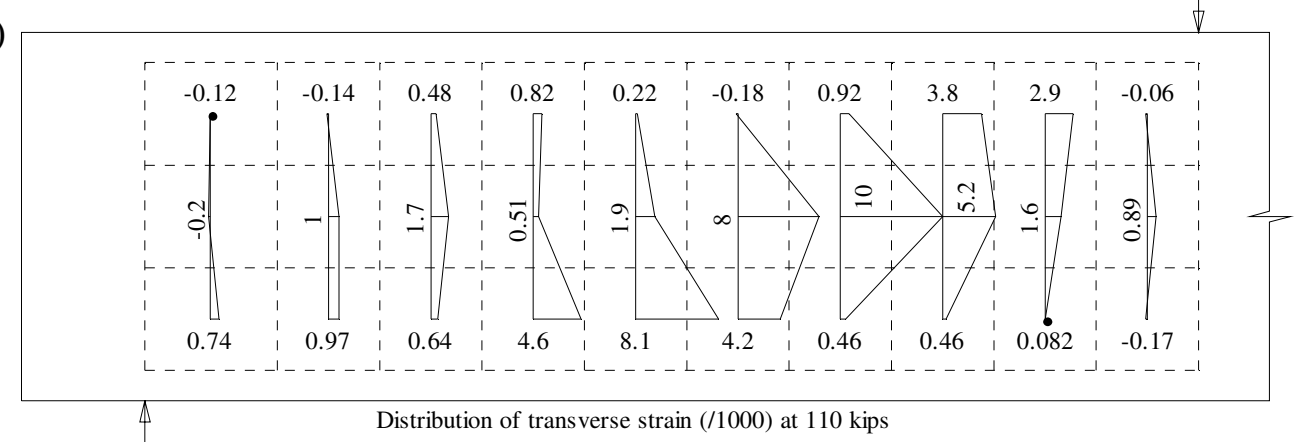

(c)

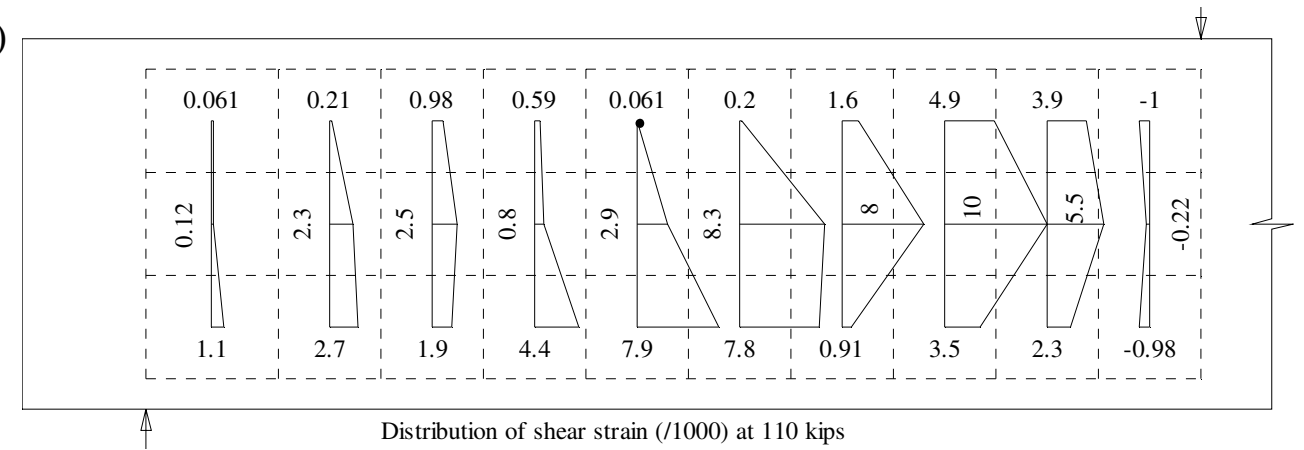

Fig. 4-92: Distribution of longitudinal, transverse, and shear strains - Beam B18-7b 

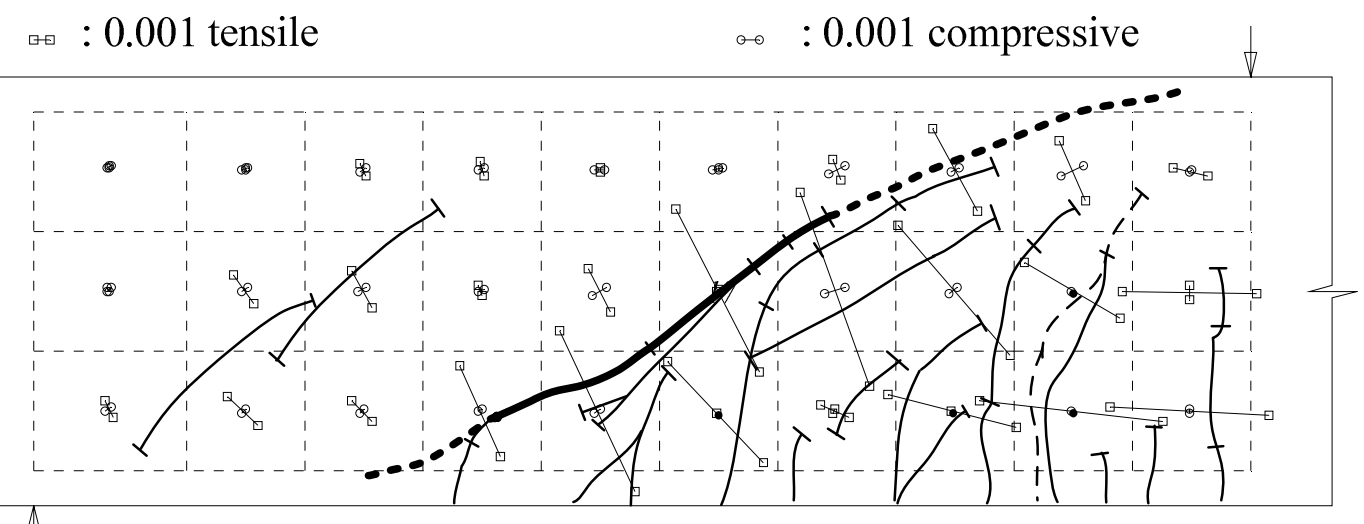

Fig. 4-93: Principal strain field at 110 kips - Beam B18-7b

(a)

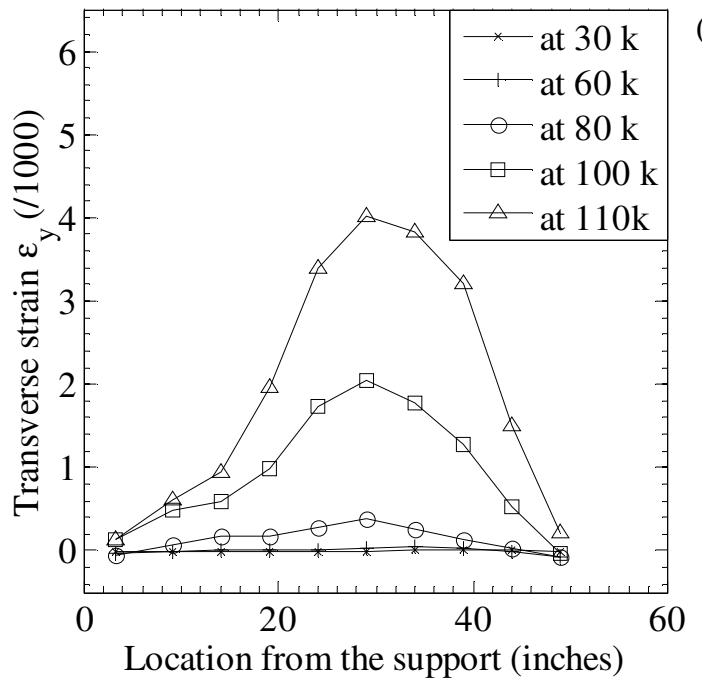

(b)

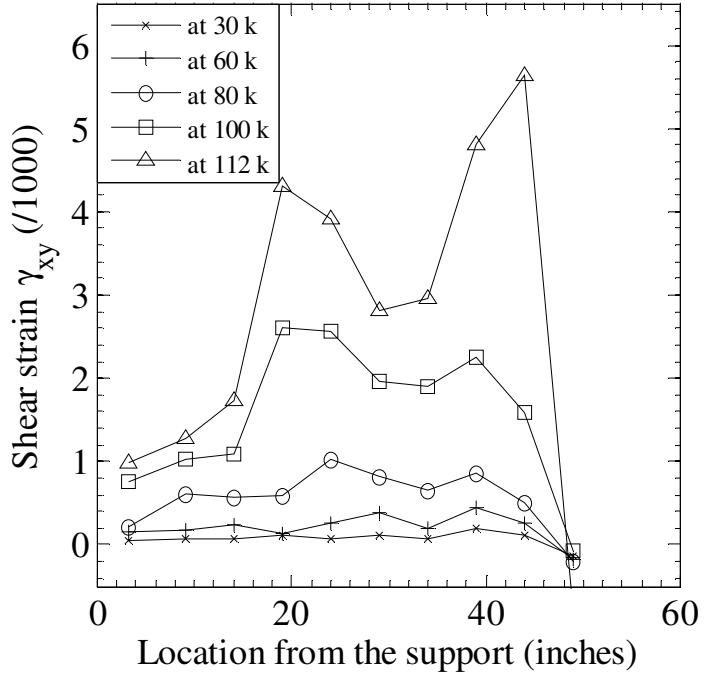

Fig. 4-94: Distribution of transverse and shear strains averaged over the beam depth Beam B18-7b 
(a)

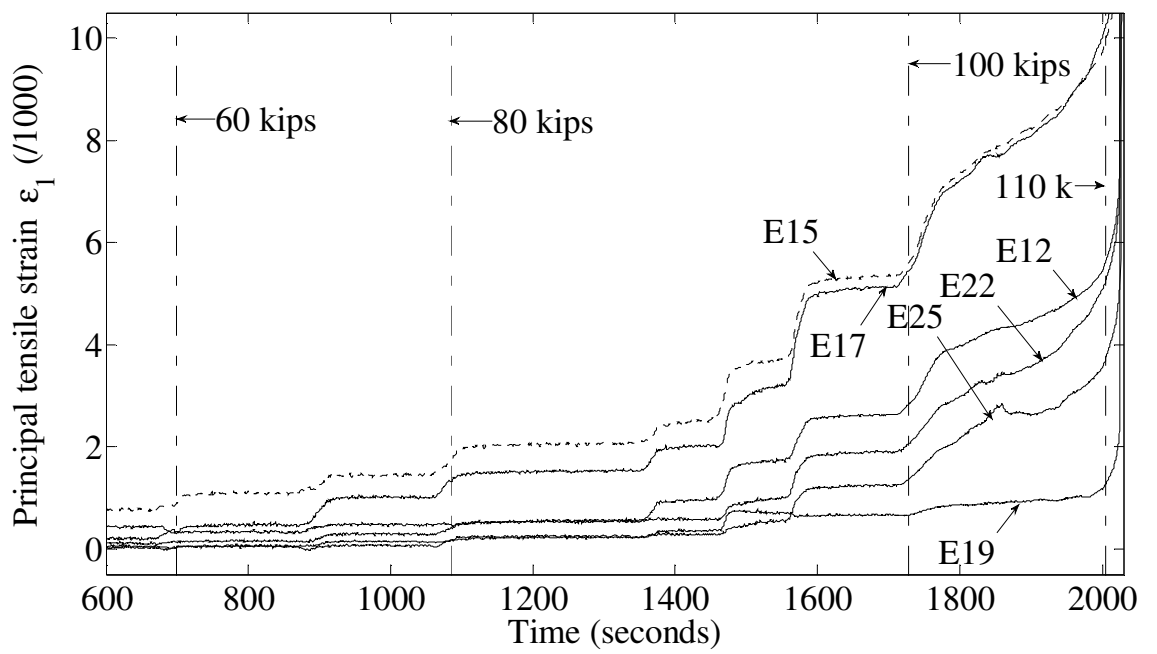

(b)

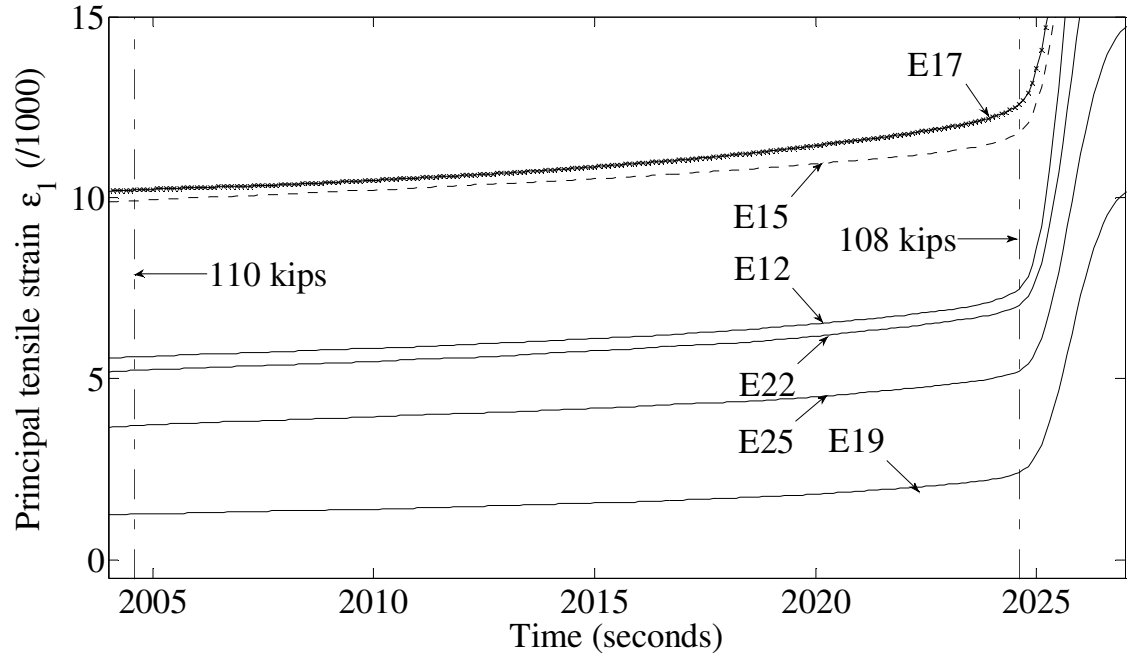

Fig. 4-95: Development of principal tensile strain versus time - Beam B18-7b 


\subsection{BEHAVIOR OF BEAMS IN SERIES B27}

Similar to Section 4.3, this section discusses the behavior of all beams in Series B27.

\subsubsection{Beam B27-1a}

Beam B27-1a had similar properties to Beams B18-1a \& b, i.e. it contained a $0.75 \%$ volume fraction of ZP305 fibers with a length of 1.18 inches and a diameter of 0.022 inches (corresponding to an aspect ratio of 55), and had a longitudinal reinforcement ratio of $2.06 \%$. Beam B27-1a had a depth of 27 inches, $50 \%$ greater than that of Beams B18-1a \& b.

\section{Load versus deflection relationship, crack pattern, and failure mode.} Unlike Beams B18-1a \& b, Beam B27-1a exhibited inclined cracks only in the region adjacent to the loading point (Fig. 4-96). The applied load at first diagonal cracking was approximately 130 kips, at which point the beam stiffness decreased slightly (Fig. 4-97). The beam reached the maximum load of 204 kips and failed shortly after that. The wide opening of the critical diagonal crack, which progressed from the bottom to the top of the crack, and the splitting along the longitudinal reinforcing bars (Fig. 4-96) indicated that the beam failed due to a combination of diagonal-tension and shear-tension. The small concrete break-out (Fig. 4-96) was likely due to the downward movement of the portion of the beam to the right of the diagonal crack that pulled down the left piece through the top reinforcing bars.

Reinforcement strain and average bond stress. Fig. 4-97b shows the load versus reinforcement strain relationship (see Fig. 4-98 for strain gauges locations). It should be mentioned that strain gauges S1, S4, and S9 failed prior to beam testing. Therefore, the average strain at a given section was calculated based on the assumption that the reinforcement strains of the lower and upper reinforcing bars were offset $\pm 5 \%$ 
from the average value. Fig. 4-99a and b show the average reinforcement strain and bond stress along the bars at different load levels.

It can be seen from Fig. 4-97b that the reinforcement was not strained beyond the measured yield strain (0.0024) for the \#8 longitudinal bars used in this beam. At the peak load, the strain distribution along the shear span was still approximately linear (Fig. 4-99a). Fig. 4-99b shows no sign of bond deterioration along the reinforcing bars. However, from the load of 120 kips to the peak load, the bond stress between S3-S4 and S7-S8 remained stationary while that between S1-S2 and S3-S4 increased significantly, up to 470 psi.

Concrete strain field. The maximum compressive strain was 0.0024 (Fig. 4-100a), between markers M31 and M37 (see Fig. 4-98 for the location of markers). Similar to the beams in Series B18, the linear trend of measured concrete strains at the centroid level of reinforcement was very similar to the distribution of measured reinforcement strains (Fig. 4-101). The fluctuation of the concrete strain was more significant near the loading point due to the local flexural cracks that were more pronounced in that region. There was a small increase in the linear slope of concrete strains with increasing loads.

It can be seen from Fig. 4-100b and c that the distribution of transverse strains followed closely that of shear strains. Most of the transverse and shear strains focused from the mid-depth level to the bottom surface of the beam. The magnitude of transverse strains was relatively small, reaching a maximum value of 0.0028 (Fig. 4-100b) at Element E8 (see Fig. 4-98 for the numbering of elements). Similarly, the maximum shear strain was 0.0049 radians, also at E8 (Fig. 4-100c). The transverse and shear strains averaged over the beam depth (Fig. 4-102) were approximately one-half of the local maximum transverse and shear demands.

Fig. 4-103 shows the principal strain field at the peak load superimposed on top of the crack pattern. No elements had a principal compressive strain greater than 0.0035 . Along the critical crack, Element E4 had the largest tensile strain. The beam basically failed due to the wide opening of the critical diagonal crack near Element E4. This wide opening first propagated towards the reinforcement level, and then toward the 
compression region. Fig. 4-104a further confirmed this observation with the largest principal tensile strain measured in Element E4. There is a significant jump in the principal tensile strain of all elements along the critical crack at a load of 203 kips after the peak load (Fig. 4-104b). This load can be considered as the failure load, which led to the beam splitting apart, as seen in Fig. 4-96. It is noted that the principal tensile strain of Element E4 at the failure load was 0.0031 (corresponding to a crack width of $0.98 \mathrm{~mm}$ ), which was not significantly different from that at the peak load $(0.0028$, corresponding to a crack width of $0.9 \mathrm{~mm})$.

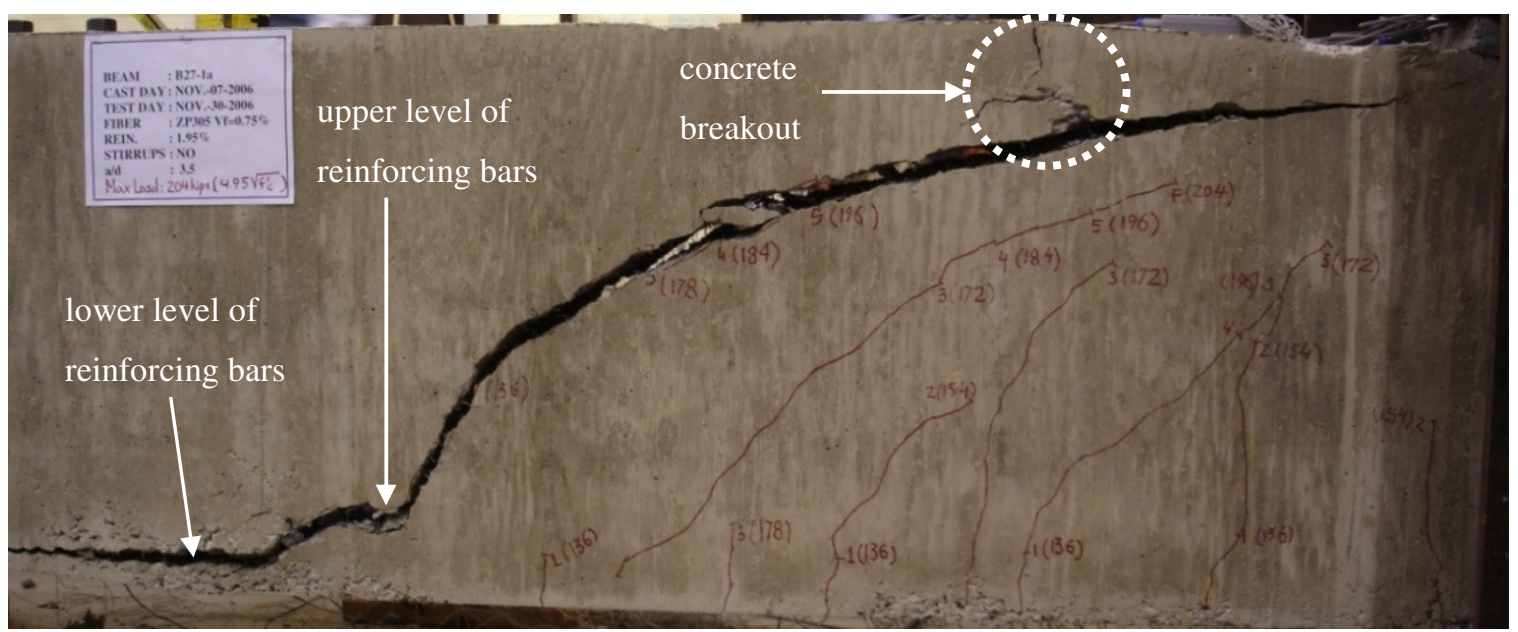

Fig. 4-96: Crack pattern after failure - Beam B27-1a
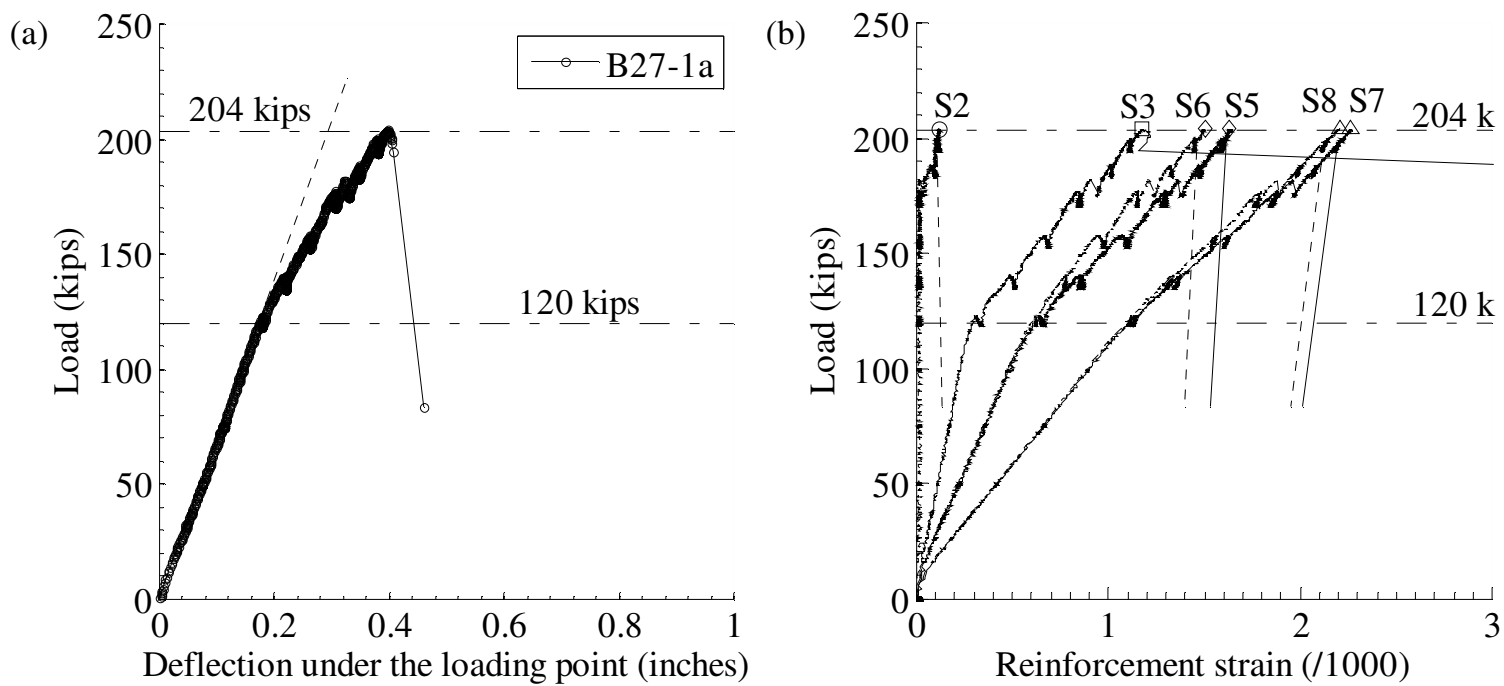

Fig. 4-97: Load versus deflection and reinforcement strain relationships - Beam B27-1a 


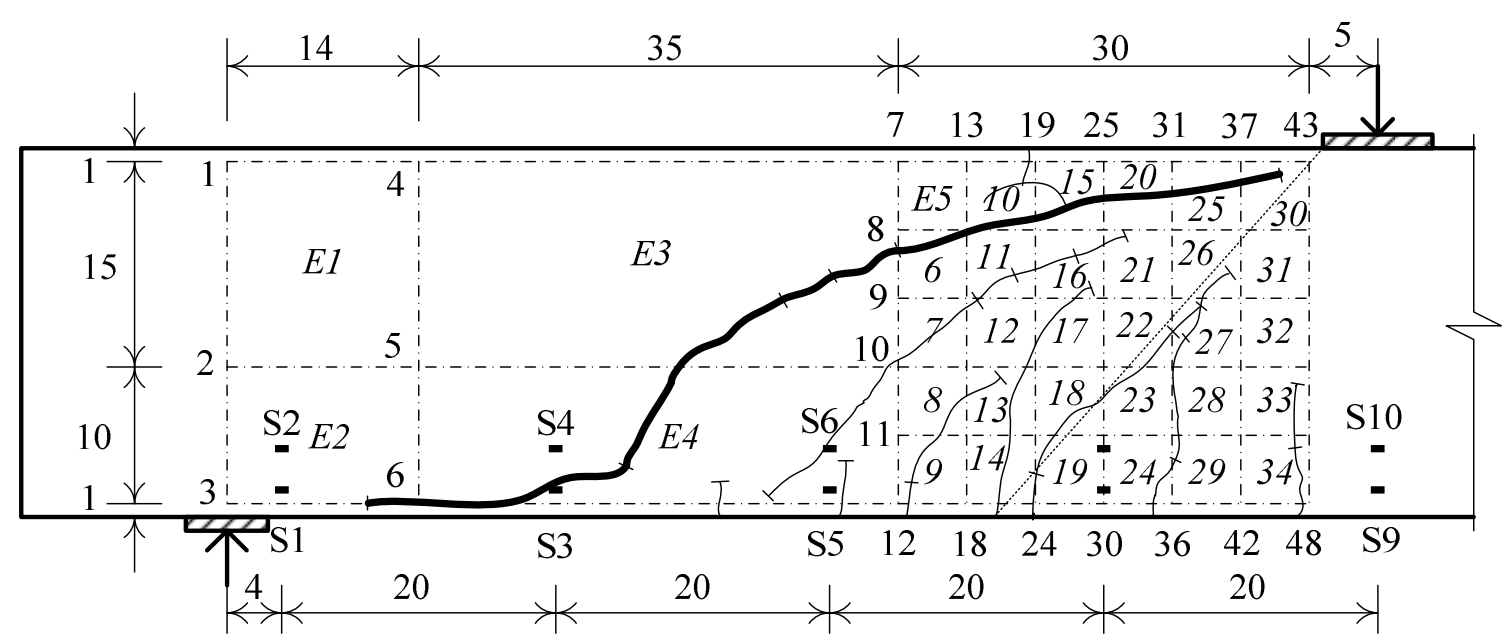

Fig. 4-98: Numbering of markers, elements, and strain gauges - Beam B27-1a

(a)

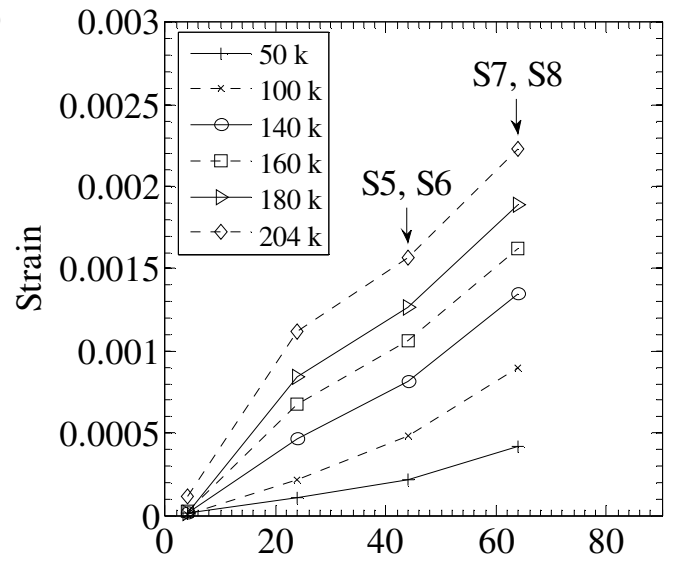

(b)

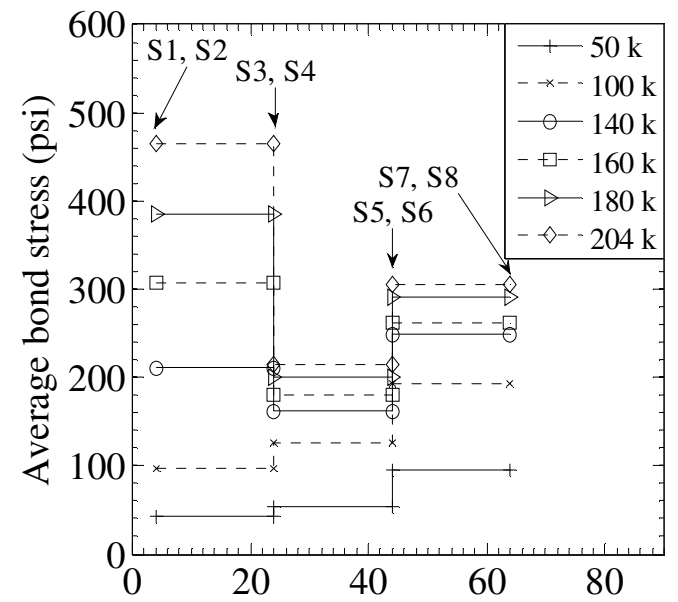

Location from the left support (inches)

Fig. 4-99: Reinforcement strains and average bond stress along the beam axis - Beam B27-1a 
(a)

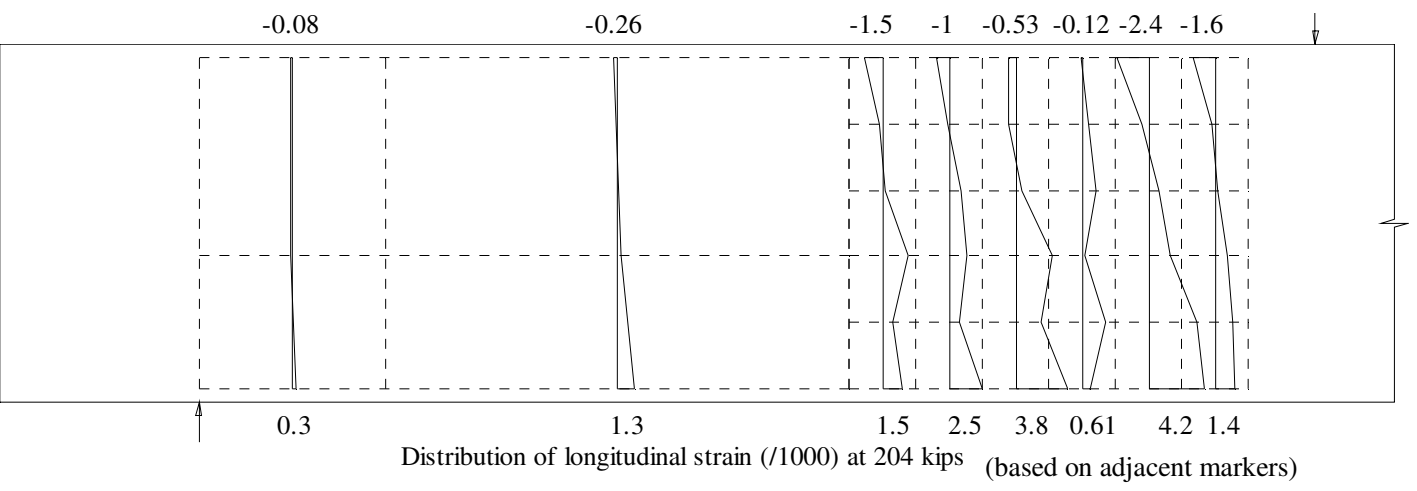

(b)

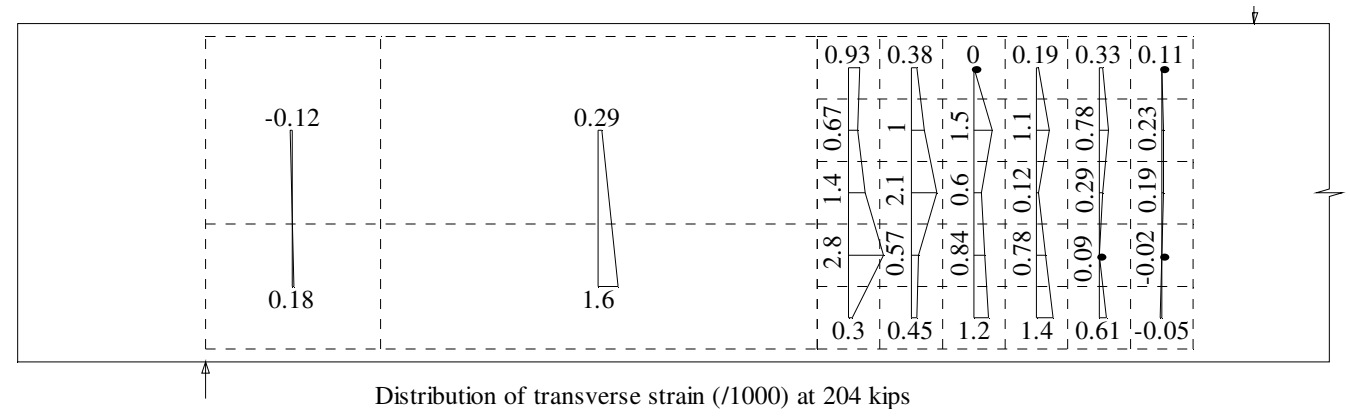

(c)

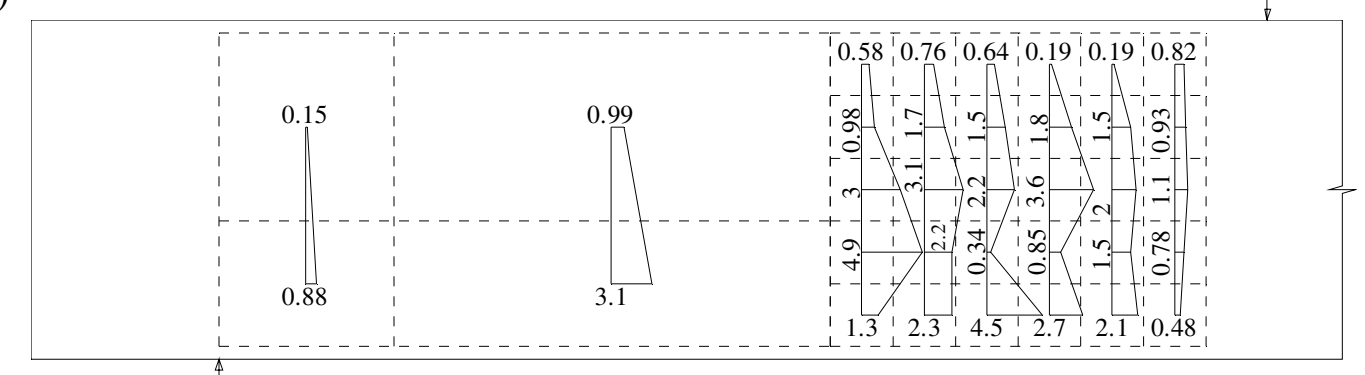

Distribution of shear strain (/1000) at 204 kips

Fig. 4-100: Distribution of longitudinal, transverse, and shear strains at 204 kips - Beam B27-1a 


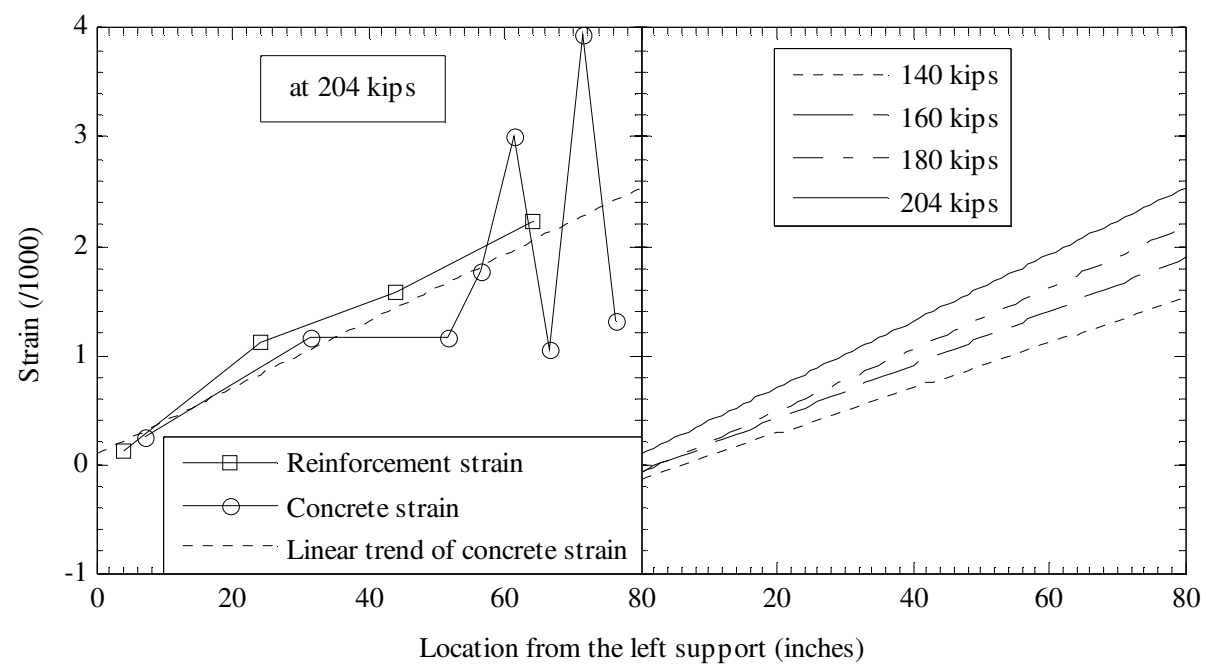

Fig. 4-101: Comparison of reinforcement and concrete strains - Beam B27-1a

(a)

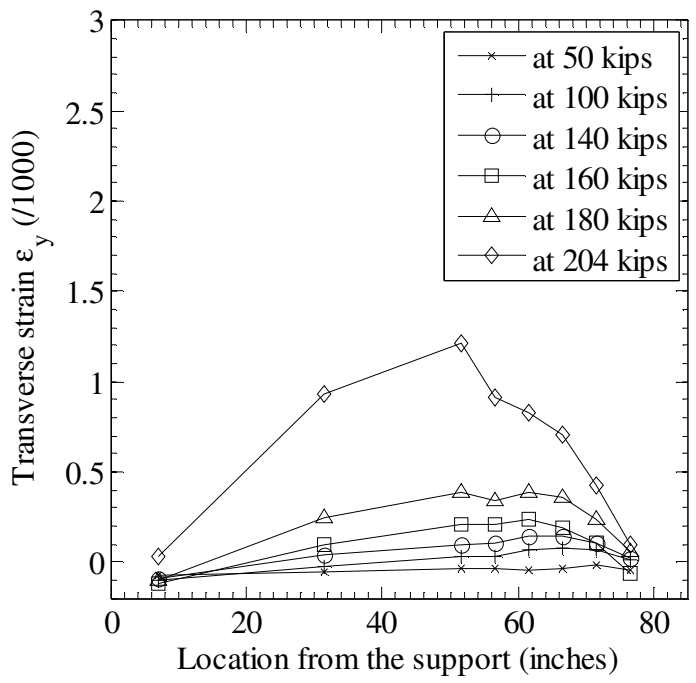

(b)

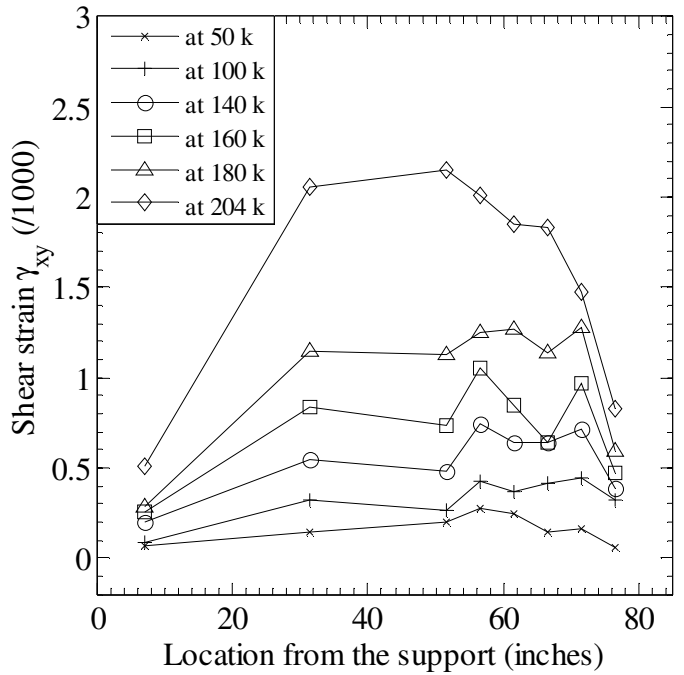

Fig. 4-102: Distribution of transverse and shear strains averaged over the beam depth Beam B27-1a

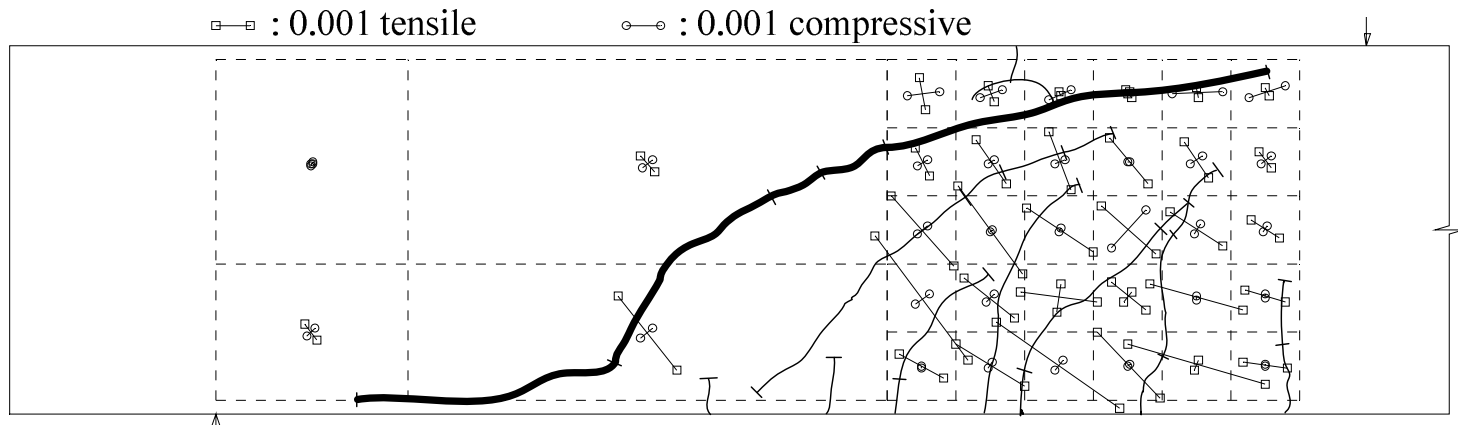

Principal strain field

Fig. 4-103: Principal strain field at 204 kips - Beam B27-1a 
(a)

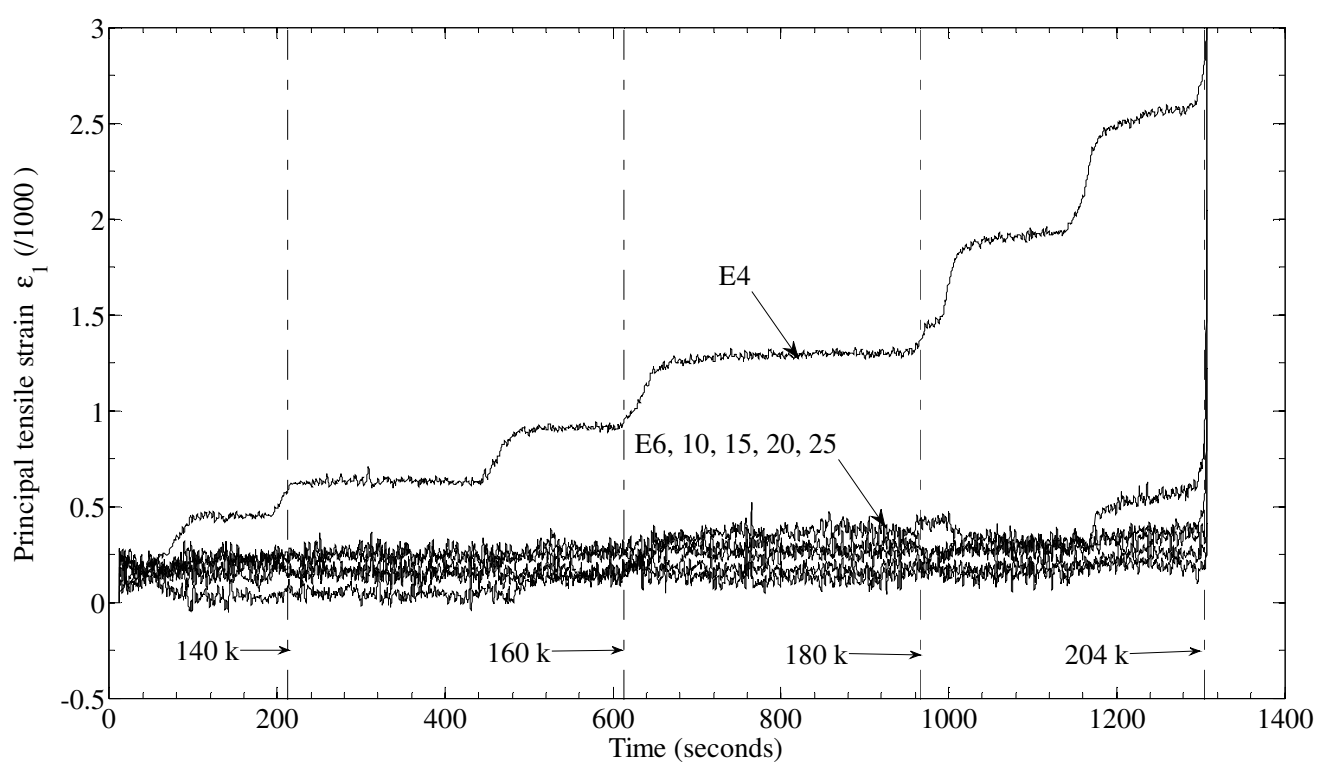

(b)

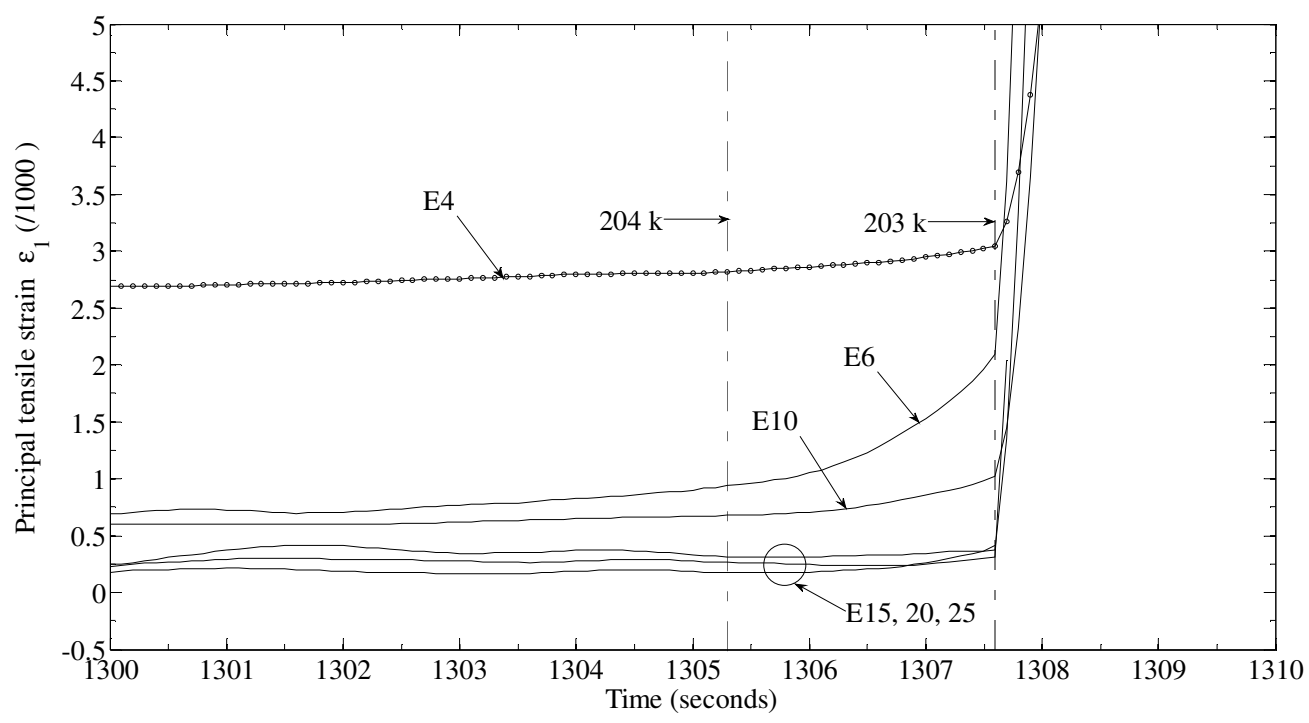

Fig. 4-104: Development of principal tensile strains along the critical inclined crack Beam B27-1a 


\subsubsection{Beam B27-1b}

Load versus deflection relationship, crack pattern, and failure mode. Beam B27-1b had similar properties to Beam B27-1a. Therefore, its crack pattern was similar to that in Beam B27-1a. The first diagonal crack was observed at 160 kips (Fig. 4-105), at which load no significant stiffness reduction was observed (Fig. 4-106a). Flexural-shear cracks were spread throughout the shear span before the beam reached its ultimate strength of 188 kips. It is noted that this strength was comparable to that of Beam B27-1a.

The beam failed due to diagonal tension along the critical crack connecting the loading point and the support. It is noted that the crack did not propagate all the way to the loading point (Fig. 4-105). The measured crack opening along the critical crack was largest at the mid-depth and smaller in both the compression and tension regions.

Reinforcement strain and average bond stress. Strain gauges $\mathrm{S} 1,4,6,8,9$, and 10 failed before beam testing. Strains at other locations are shown in Fig. 4-106b. Therefore, average strain values, similar to those discussed for Beam B27-1a are shown in Fig. 4-107. The strain distribution along the beam span was linear up to the load of 160 kips. At the peak load, due to the formation of the critical crack in the vicinity of strain gauges S1 and S2 (Fig. 4-108), the increase in reinforcement strain was more rapid in this region. It should be noted that the bond in the region from S3 (S4) to S7 (S8) quickly increased with an increase in load, but remained stationary after the load of 140 kips, while the bond in the region from S1 (S2) to S3 (S4) increased steadily throughout the test up to a load of $160 \mathrm{kips}$, after which a drop in bond stress occurred. Overall, the peak average bond demand was low, 280 psi, compared to 470 psi for Beam B27-1a. This was probably due to a more even distribution of flexural cracks throughout the shear span.

Concrete strain field. Fig. 4-109 shows the distribution of longitudinal, transverse, and shear strains at peak load. The maximum longitudinal tensile strain was of a similar magnitude to that of Beam B27-1a. However, the maximum compressive strain was much larger, reaching a value of 0.0043 at Element E33, where the critical crack stopped (see Fig. 4-108 for the numbering of elements). 
The maximum shear strain was of a similar magnitude (0.0052) to that of Beam B27-1a (0.0049). However, the maximum transverse strain was more than twice as large as that in Beam B27-1a (0.0059 compared with 0.0028). Fig. 4-110 shows that the average transverse strain was significantly higher near the support, while the shear strain was spread evenly throughout the shear span.

Error! Reference source not found. shows the principal strain field at peak load (188 kips). Element E33 was compressed with a principal compressive strain of up to 0.0035 , but no sign of concrete crushing was observed.

Fig. 4-112 confirms that the principal tensile strains at Elements E7, 10, and 14 at the beam mid-depth level were significantly larger than others prior to the peak load of 188 kips. The opening of the diagonal crack was therefore initiated in the mid-depth region and propagated towards the reinforcement and compression region, as discussed previously. Right after the peak load the strains in all elements jumped significantly at the load of 187 kips (Fig. 4-112b), which can be considered to be the failure load. The maximum crack width at Element E10 was $0.92 \mathrm{~mm}$, which was comparable to that in Beam B27-1a.

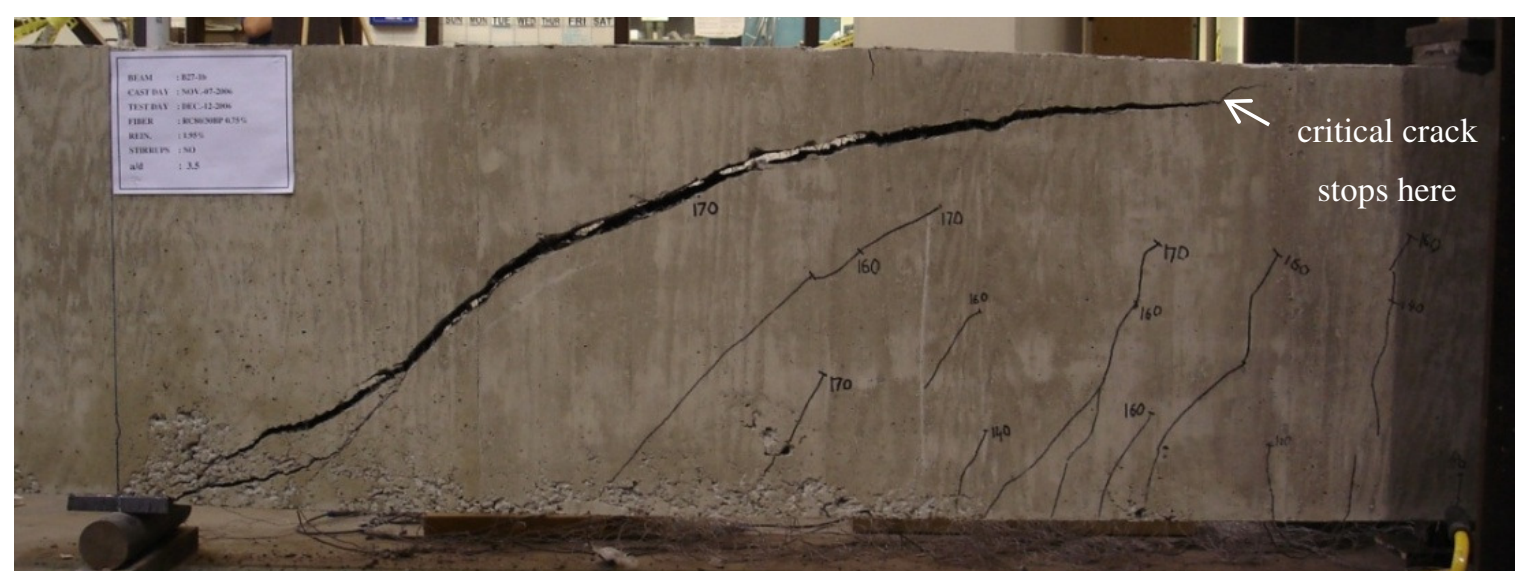

Fig. 4-105: Crack pattern after failure - Beam B27-1b 
(a)

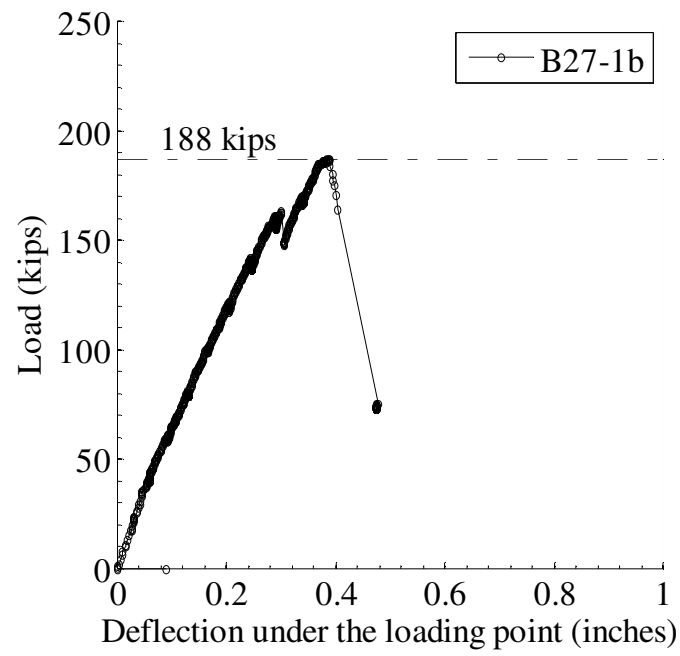

(b)

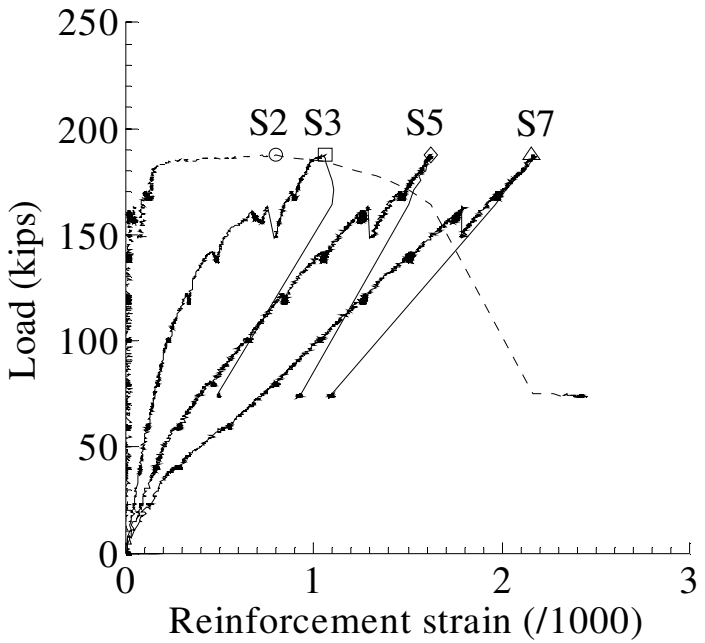

Fig. 4-106: Load versus deflection and reinforcement strain relationships - Beam B27-1b

(a)

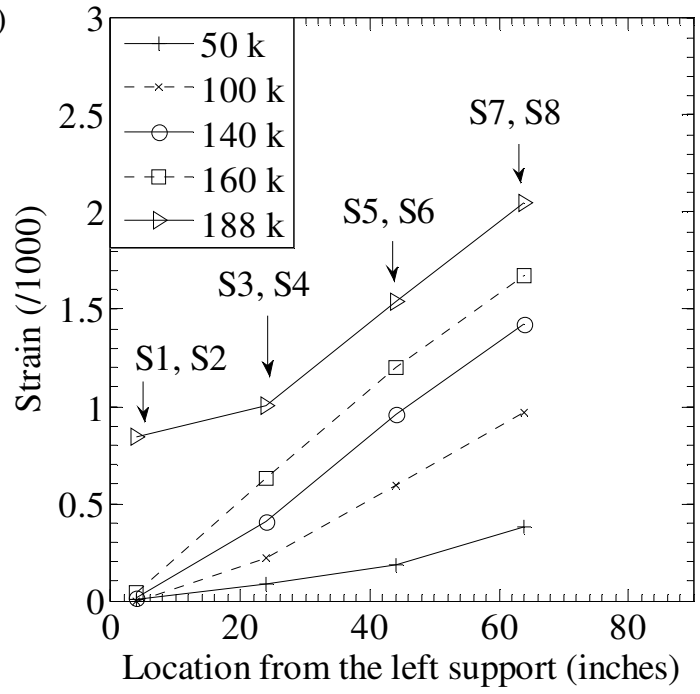

(b)

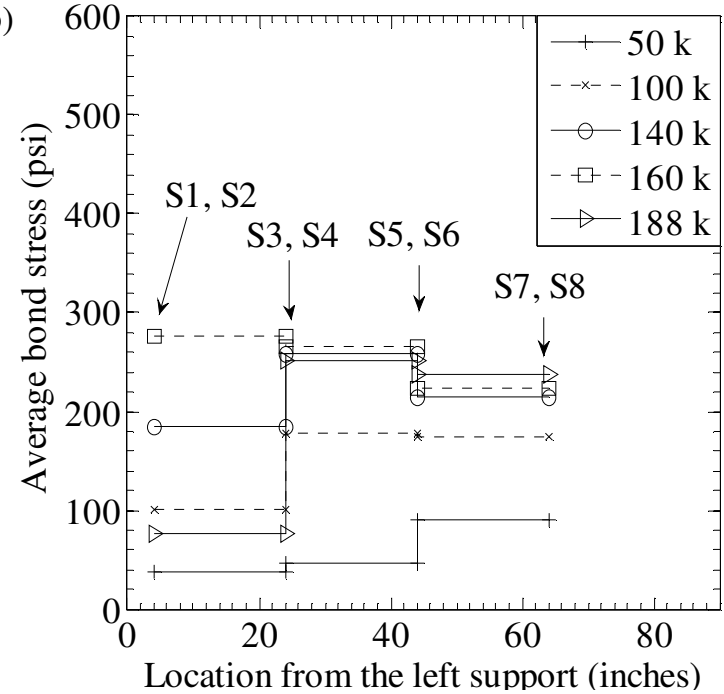

Fig. 4-107: Reinforcement strains and average bond stress along the beam axis - Beam B27-1b

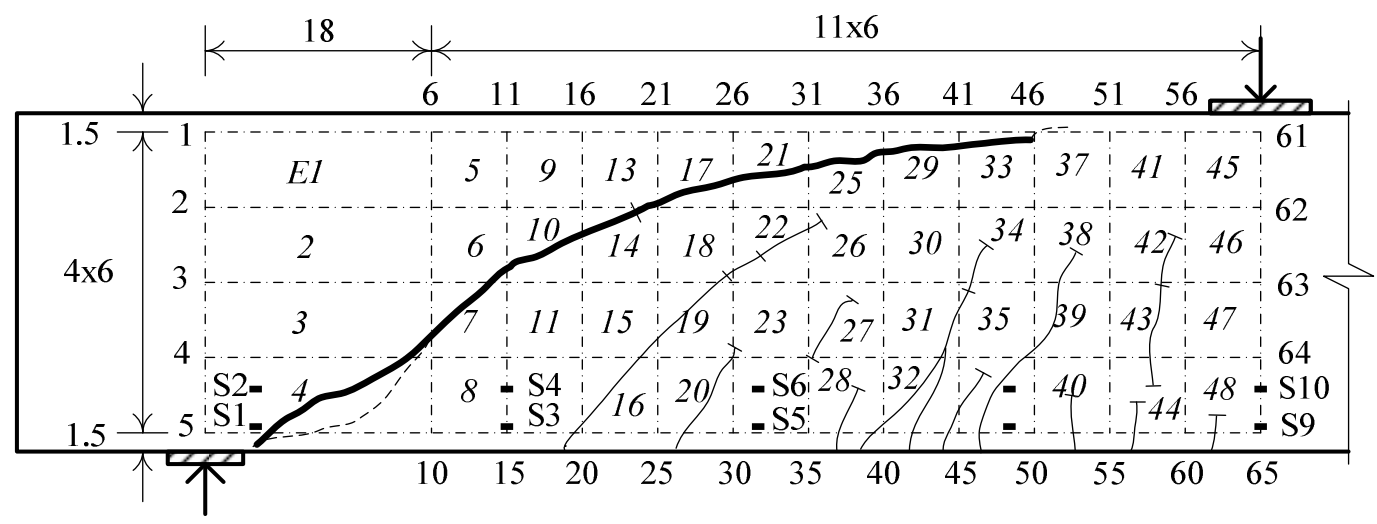

Fig. 4-108: Numbering of markers, elements, and strain gauges - Beam B27-1b 
(a)

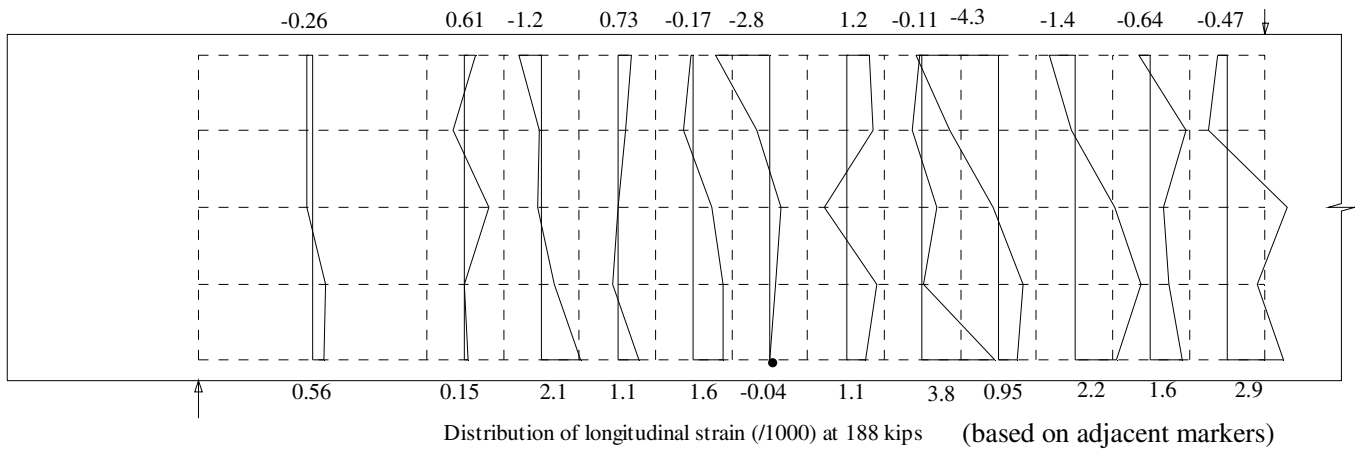

(b)

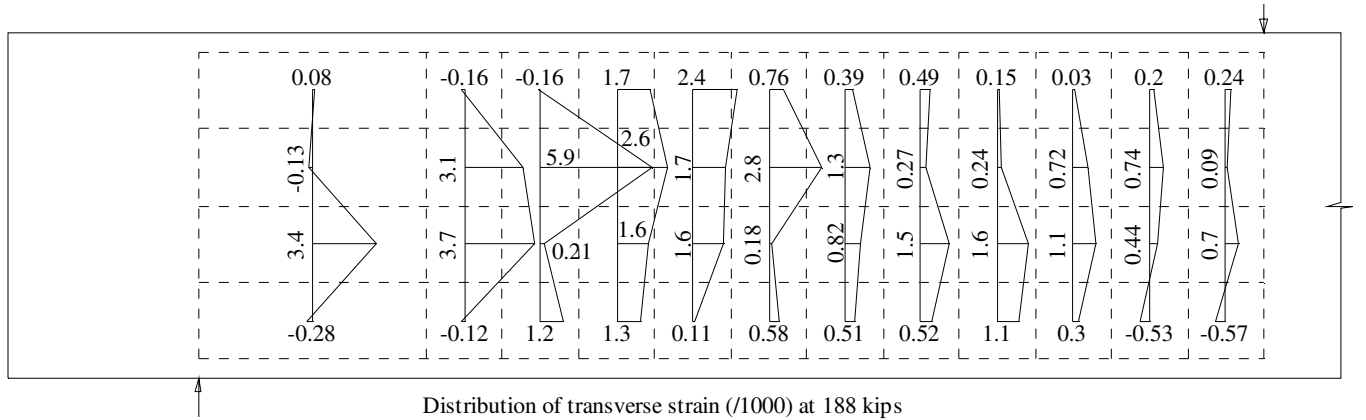

(c)

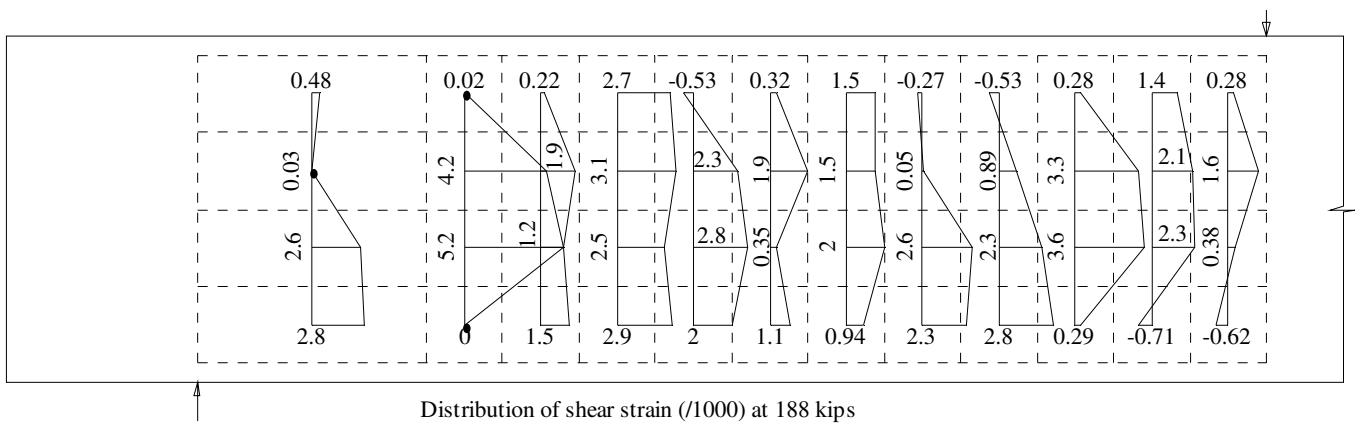

Fig. 4-109: Distribution of longitudinal, transverse, and shear strains at $188 \mathrm{k}-$ Beam B27-1b 
(a)

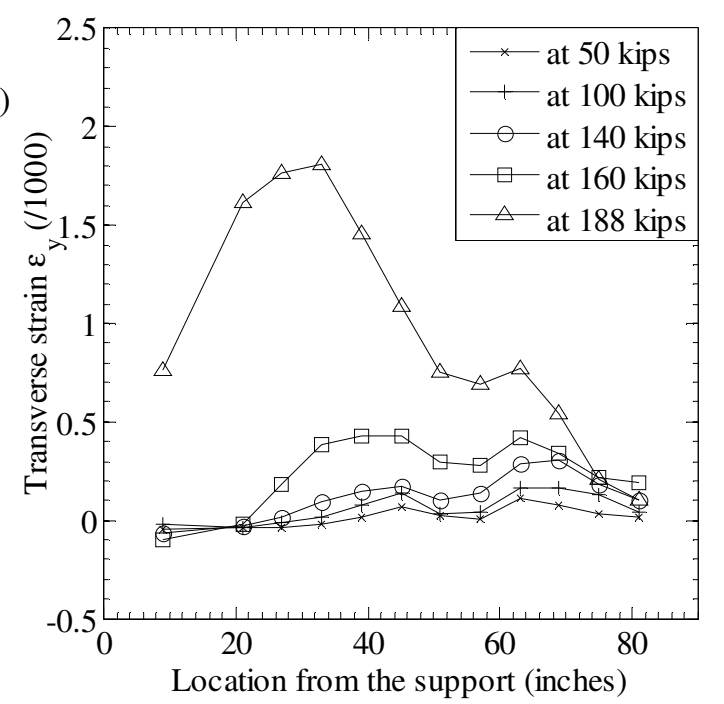

(b)

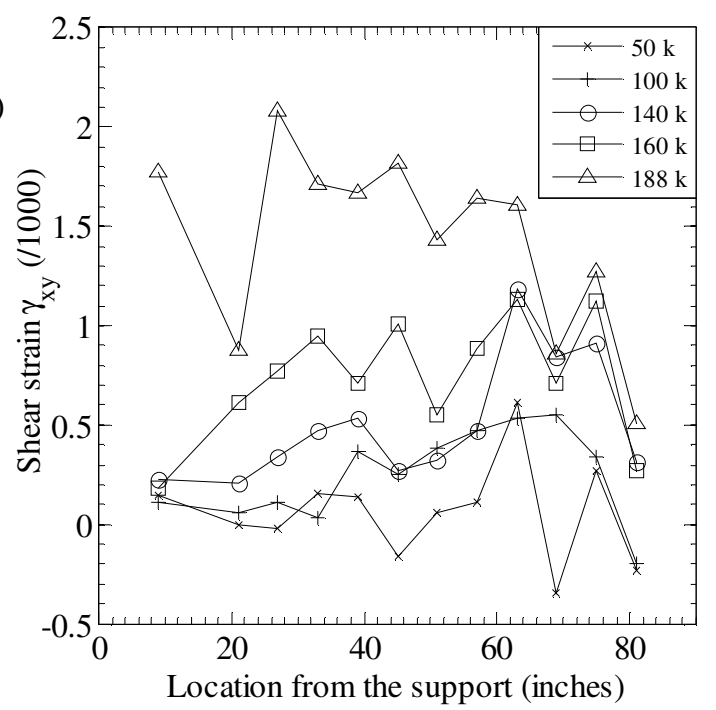

Fig. 4-110: Distribution of transverse and shear strains averaged over the beam depth Beam B27-1b

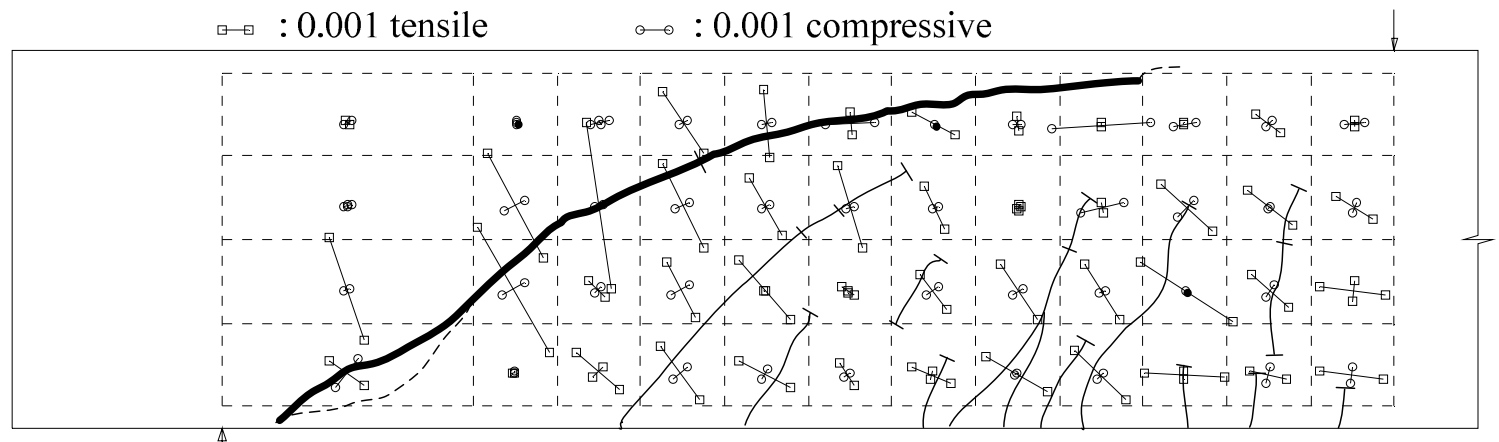

Principal strain field

Fig. 4-111: Principal strain field at 188 kips - Beam B27-1b 
(a)

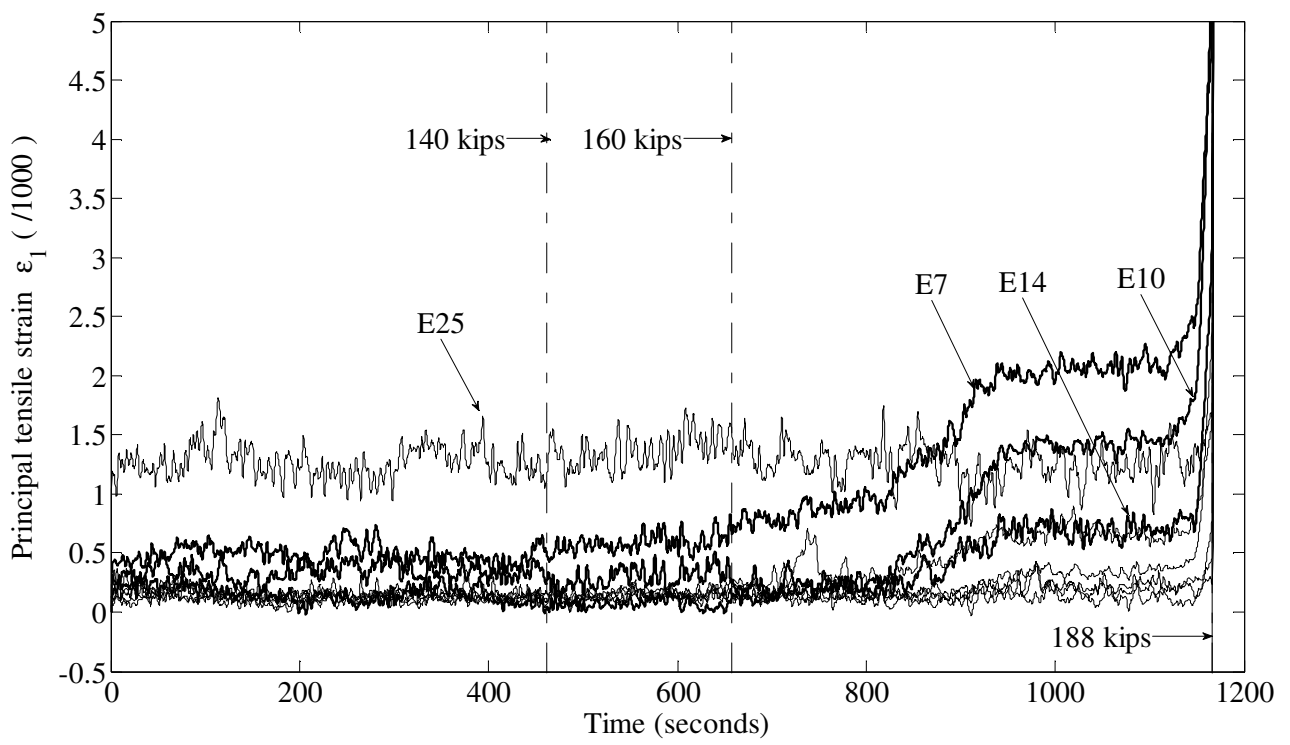

(b)

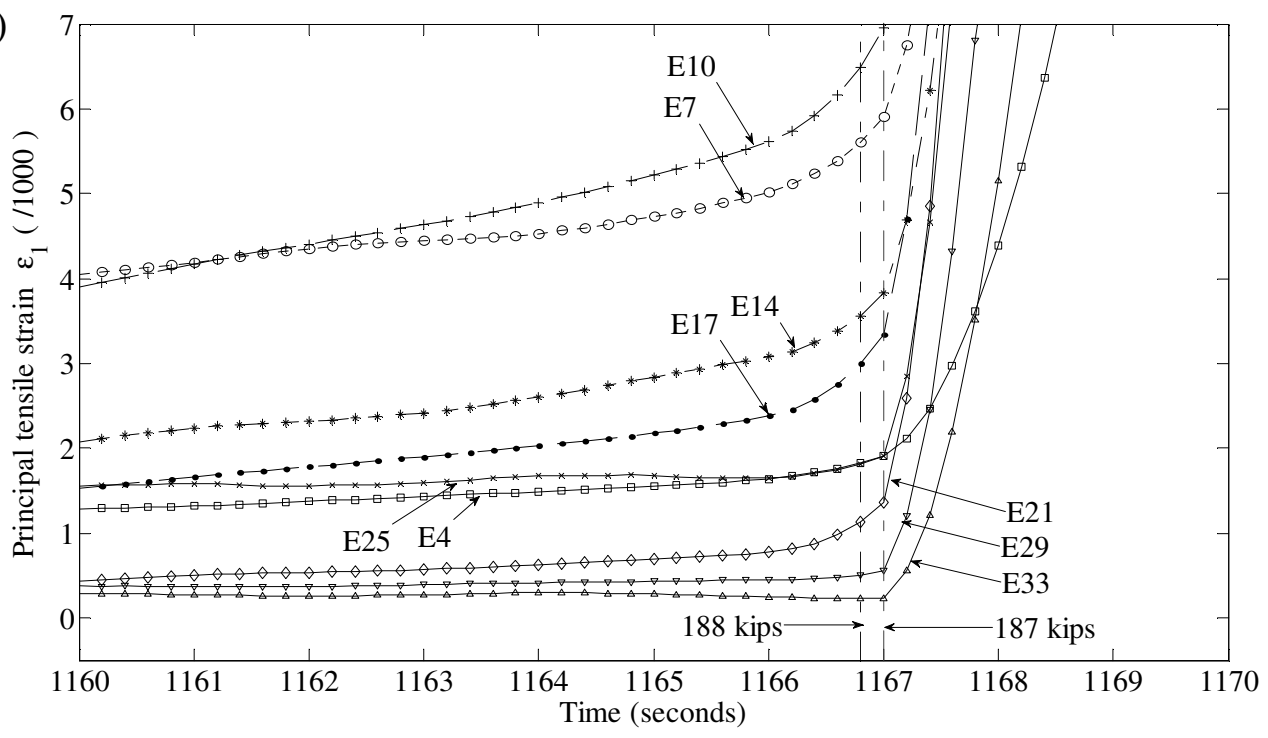

Fig. 4-112: Development of principal tensile strains along the critical inclined crack Beam B27-1b 


\subsubsection{Beam B27-2a}

Beam B27-2a had the same fiber volume fraction $(0.75 \%)$ and longitudinal reinforcement ratio $(2.06 \%)$ as Beams B27-1a \& b, but it contained longer and larger diameter fibers $(\mathrm{RC} 80 / 60 \mathrm{BN})$ with a fiber length of 2.36 inches and a diameter of 0.03 inches, corresponding to a fiber aspect ratio of 80 .

Load versus deflection relationship, crack pattern, and failure mode. The first inclined cracks of this beam occurred at a load of 120 kips (Fig. 4-113), smaller than that in Beams B27-1a \& b. Also, the load versus deflection curve (Fig. 4-114a) had a smooth and steady reduction in stiffness from zero up to the peak load. Nevertheless, the beam was able to maintain the strength of 196 kips for a deflection of approximately 0.2 inches prior to a complete failure. However, there was no indication of reinforcement yielding at the strain gauge locations (Fig. 4-114b) and there were no significant flexural cracks under the loading point. It is likely that local bar yielding occurred in between strain gauge location. With wide cracks along longitudinal reinforcement and some concrete crushing at the top of the critical inclined crack, Beam B27-2a failed due to a combination of shear-tension and shear-compression.

Reinforcement strain and average bond stress. Fig. 4-116 shows the distribution of reinforcement strains along the beam axis and Fig. 4-117 shows the bond stress distribution along the beam. As in other beams, a nearly linear strain distribution was observed throughout the test. The average bond stress did not increase for the regions from S3 (S4) to S7 (S8) from a load of 140 kips to the peak load, while that from S1 (S2) to S3 (S4) kept increasing with load, up to a value of 470 psi at the peak load (Fig. 4-117).

Concrete strain field. It can be seen from Fig. 4-118a that small longitudinal strains occurred near the end of the beam. Near the top of the critical inclined crack, the longitudinal compression strain was large, up to 0.0057, between markers M61 and M66, while the longitudinal tension strain near the bottom of that crack was not significant, 
only 0.0035 between markers M40 and M45 (see Fig. 4-115 for location of markers and elements). This level of compression was large enough to cause crushing, as stated before. The longitudinal strains fluctuated significantly around a linear trend, but their trend was still parallel to the linear trend for the measured reinforcement strains (Fig. 4-120), as observed in other beams.

With only minor cracking near the support, the transverse and shear strains (Fig. 4-118b and c) were only non-negligible for the bottom elements in this region. However, significant transverse and shear strains were measured on the region associated with the critical diagonal crack. The maximum transverse strain of 0.0085 occurred at Element E24 (Fig. 4-118b). The peak shear strain was also 0.0085 radians at E28 (Fig. 4-118c). Similar to Beams B27-1a \& b, the peak transverse and shear strains, averaged over the beam depth, were approximately one-half of the corresponding peak local strain (Error! Reference source not found.).

Fig. 4-121 shows the direction of principal strains at peak load. It can be seen that significant principal tensile strains concentrated on elements along the critical diagonal crack. Element E49 was subjected to a principal compressive strain of 0.0035 . Fig. 4-122 shows the development of the principal tensile strains at the elements along the critical inclined crack. It can be seen that the principal tensile strain for Elements E32, E36, and E39 were largest throughout the test. This indicated that the critical crack was opened from the level of reinforcement to the compression region. This held true even at the peak load with the exception that the principal tensile strain of Element E49 exceeded that of Element E39.

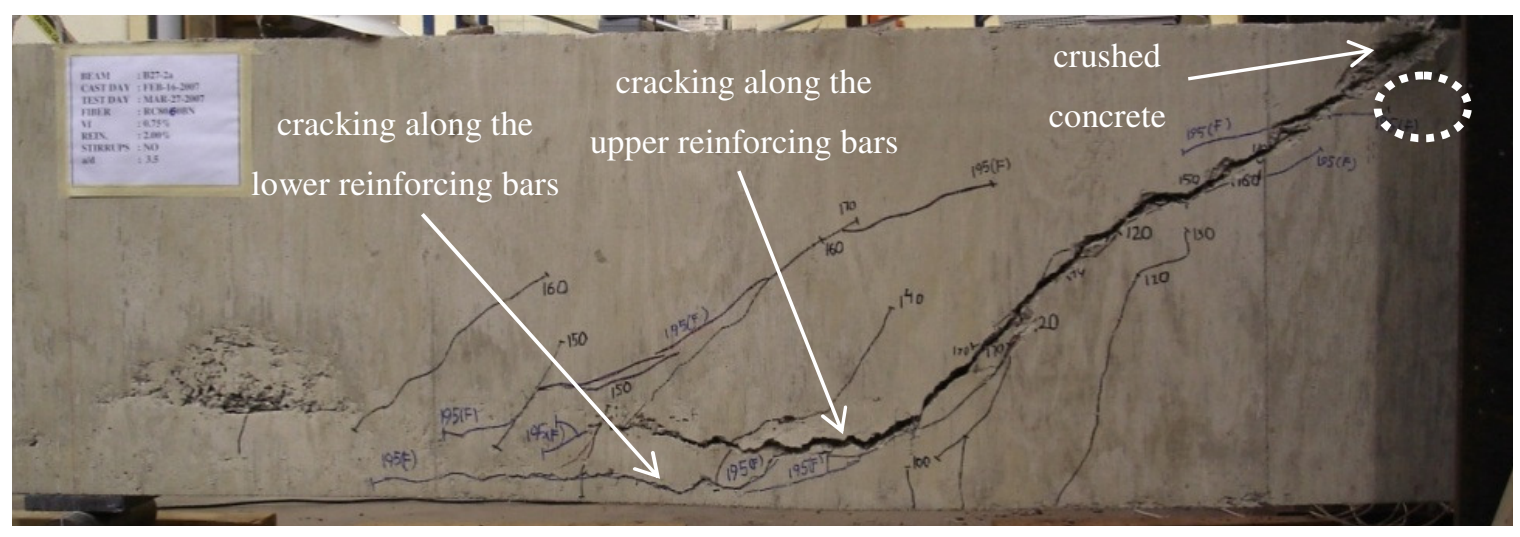

Fig. 4-113: Crack pattern after failure - Beam B27-2a 
(a)

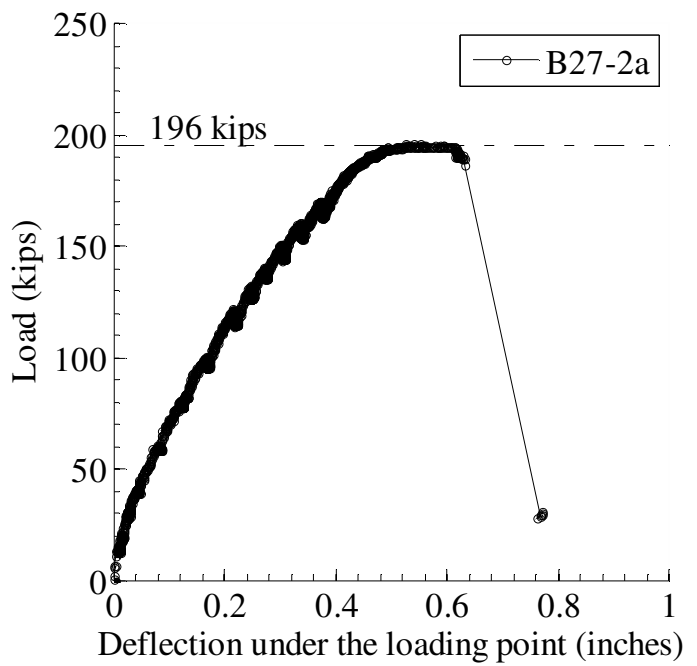

(b)

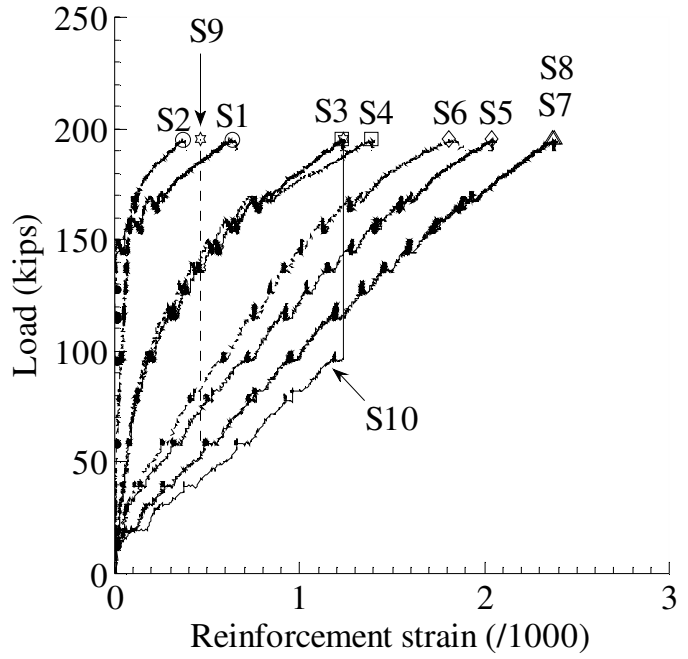

Fig. 4-114: Load versus deflection and reinforcement strain relationships - Beam B27-2a

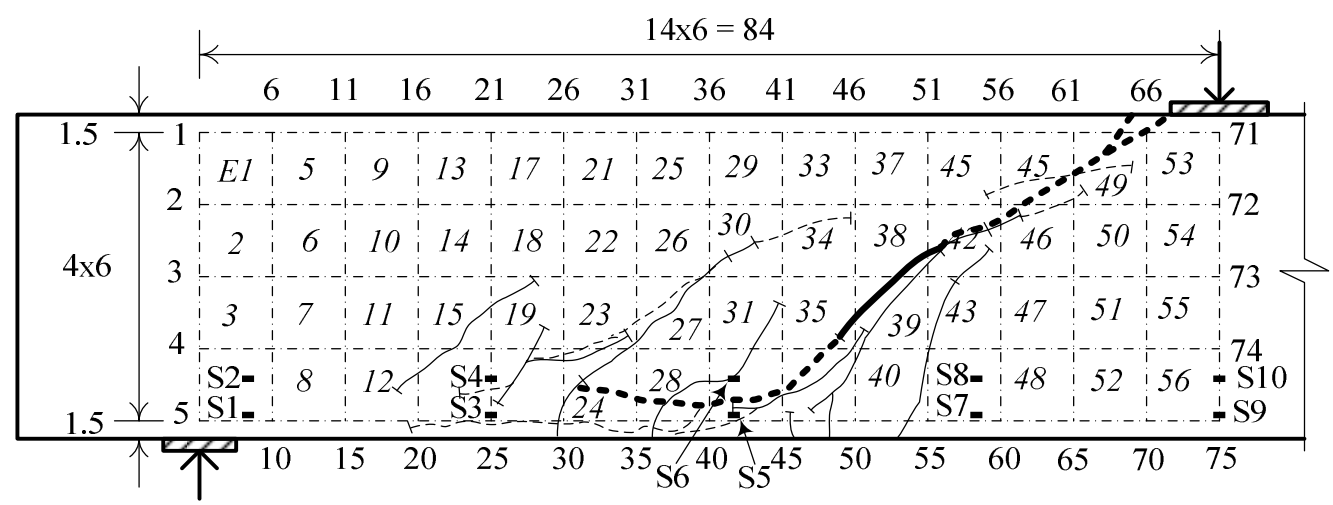

Fig. 4-115: Numbering of markers, elements, and strain gauges - Beam B27-2a 


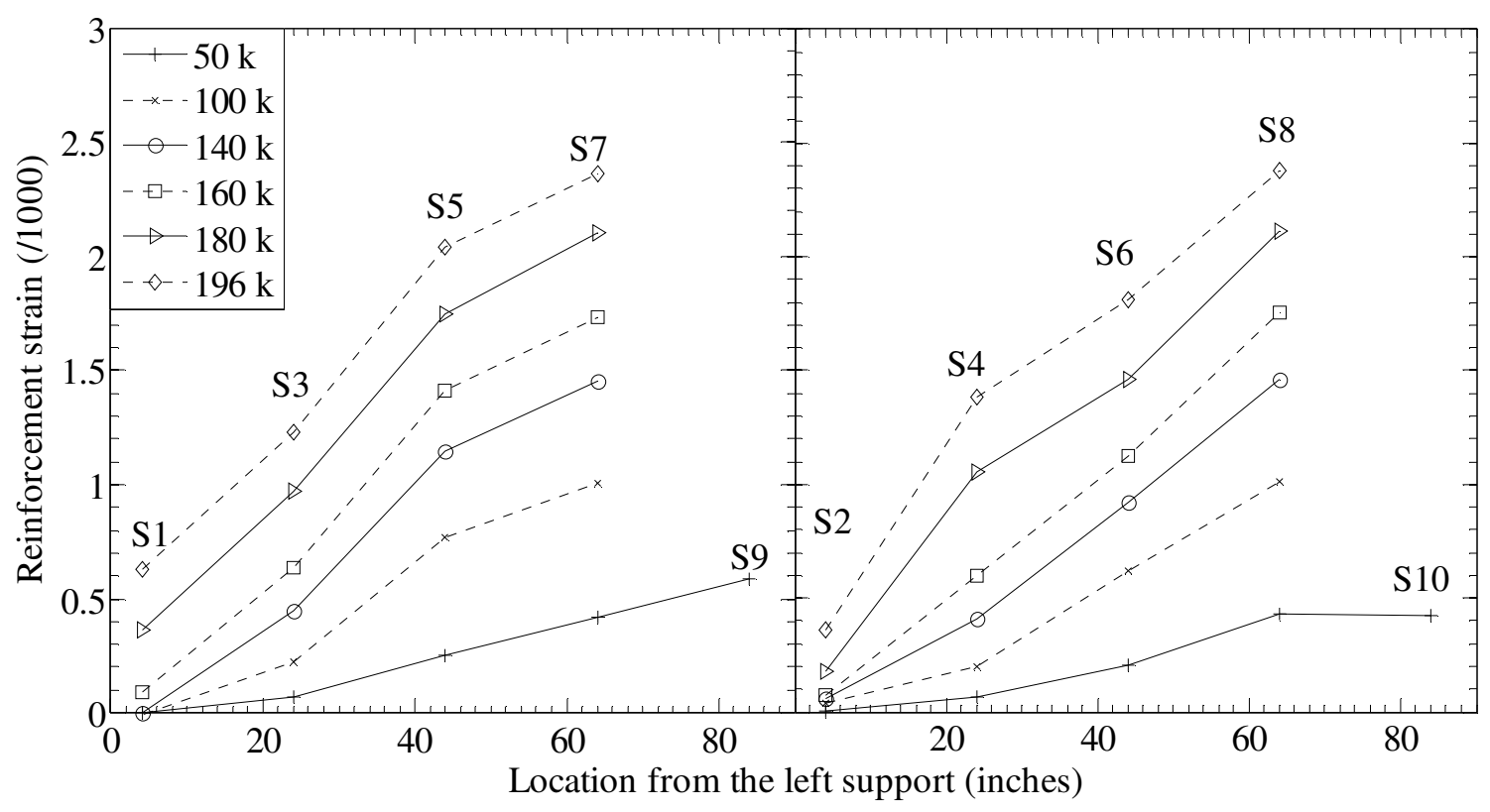

Fig. 4-116: Reinforcement strains along the beam axis - Beam B27-2a

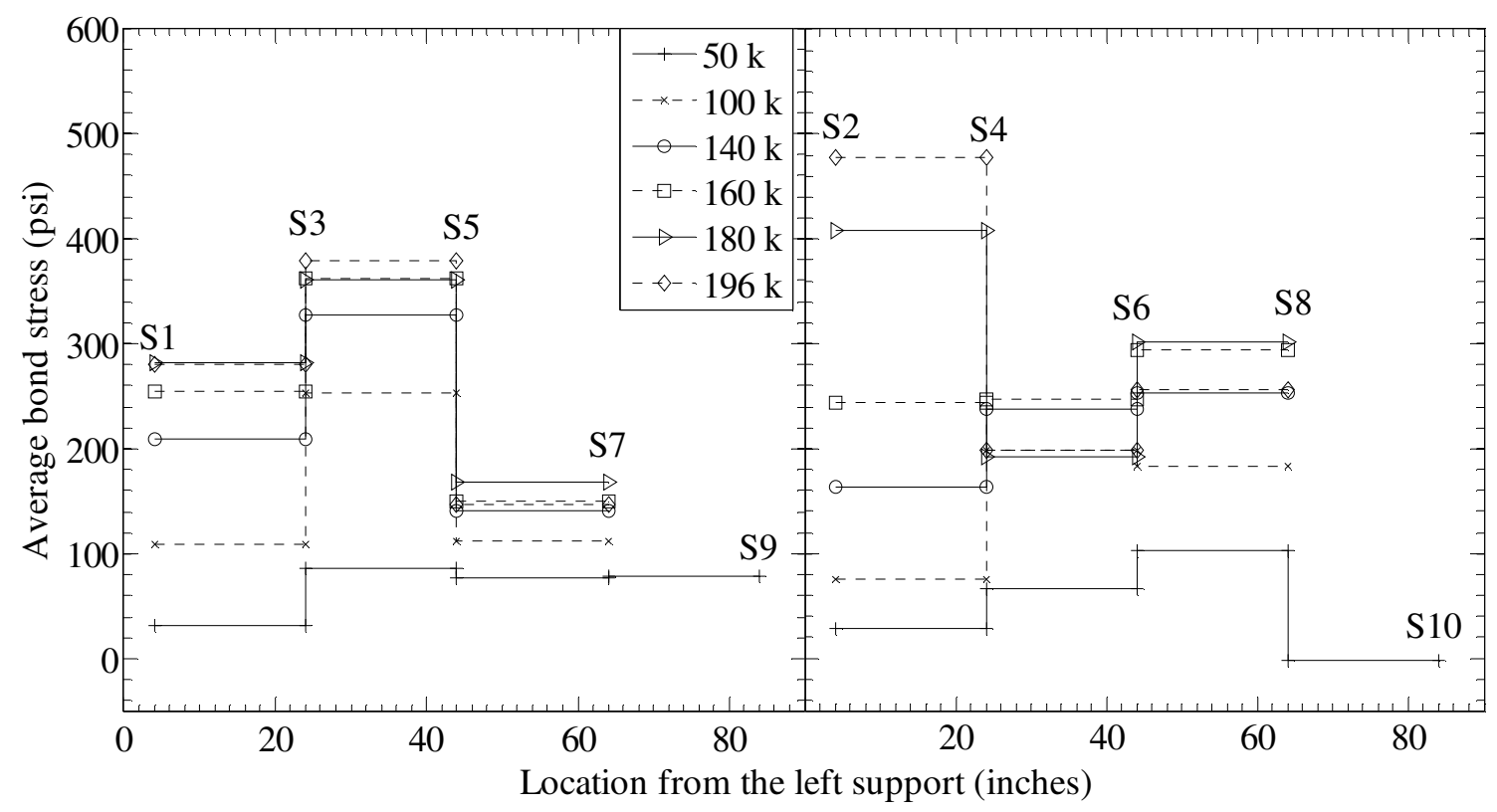

Fig. 4-117: Average bond stress along the beam axis - Beam B27-2a 
(a)

$\begin{array}{llllllllllllll}0.1 & -0.29 & 0.16 & -0.64 & 0.72 & -0.093 & -1.9 & -0.96 & -1.1 & -2.1 & 0.8 & -1.1 & -5.7 & -1.3\end{array}$

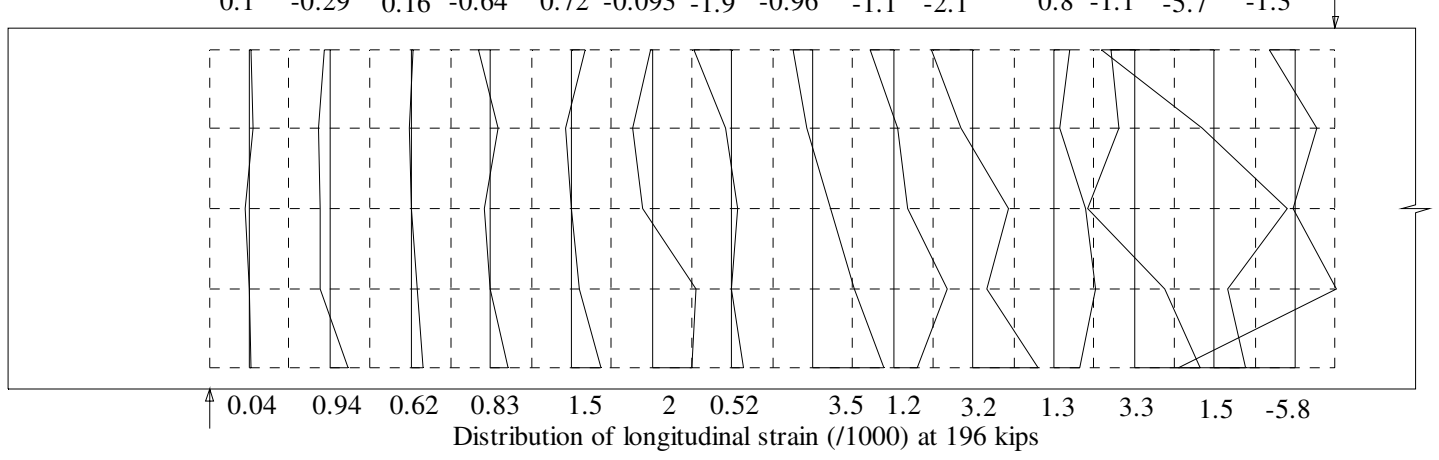

(b)

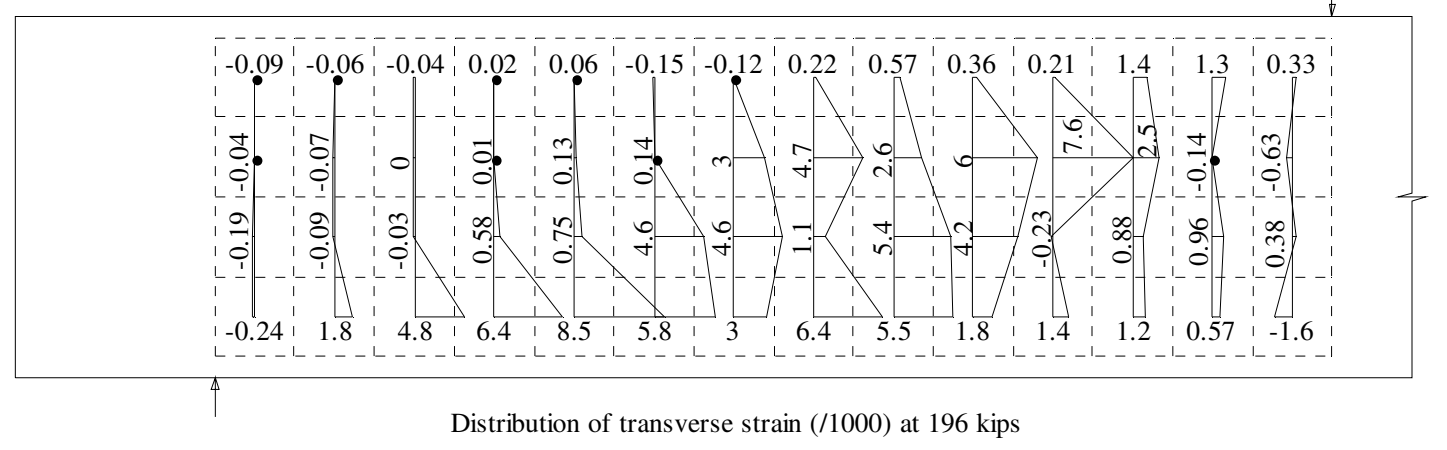

(c)

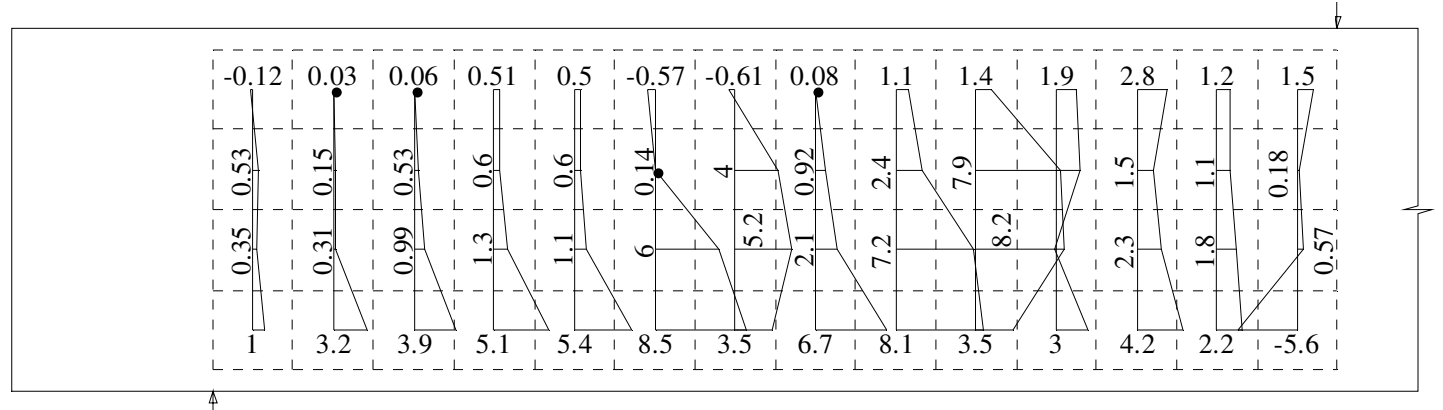

Distribution of shear strain (/1000) at 196 kips

Fig. 4-118: Distribution of longitudinal, transverse, and shear strains at $196 \mathrm{k}-$ Beam B27-2a 

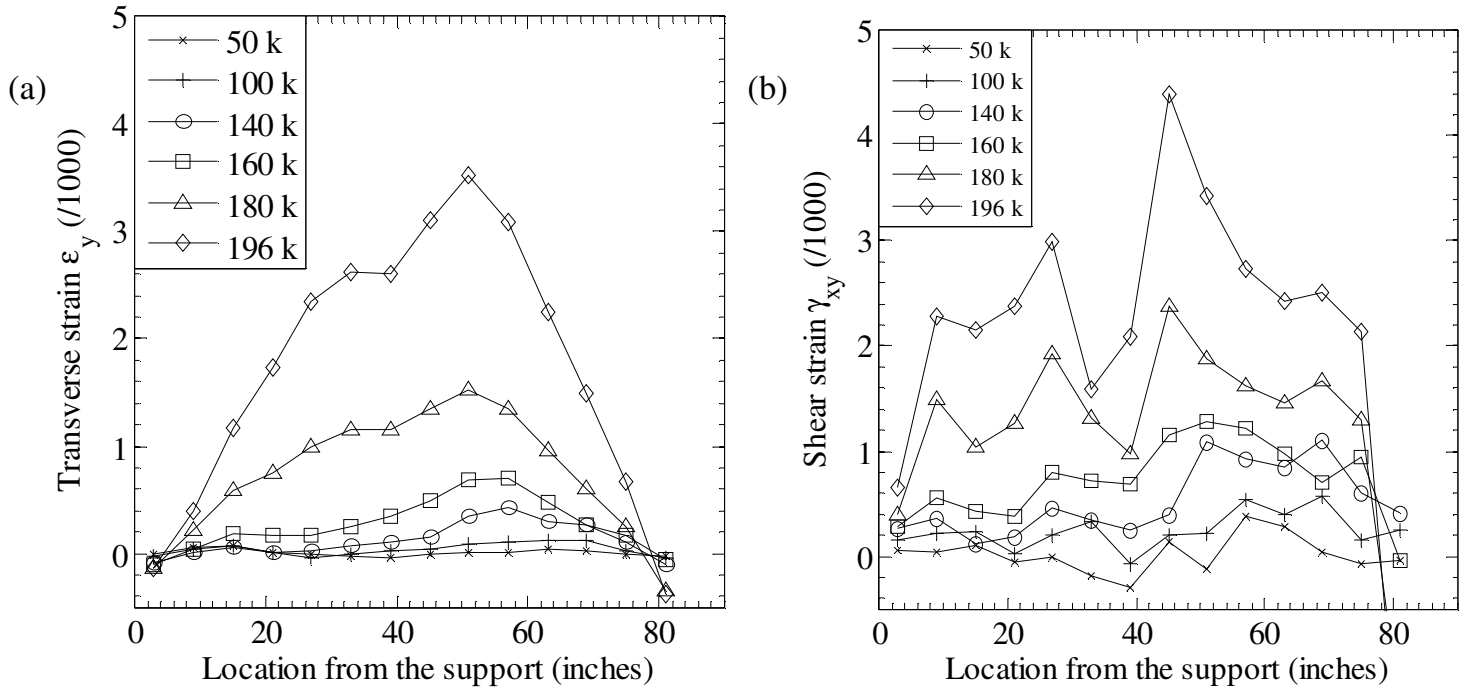

Fig. 4-119: Distribution of transverse and shear strains averaged over the beam depth -

Beam B27-2a

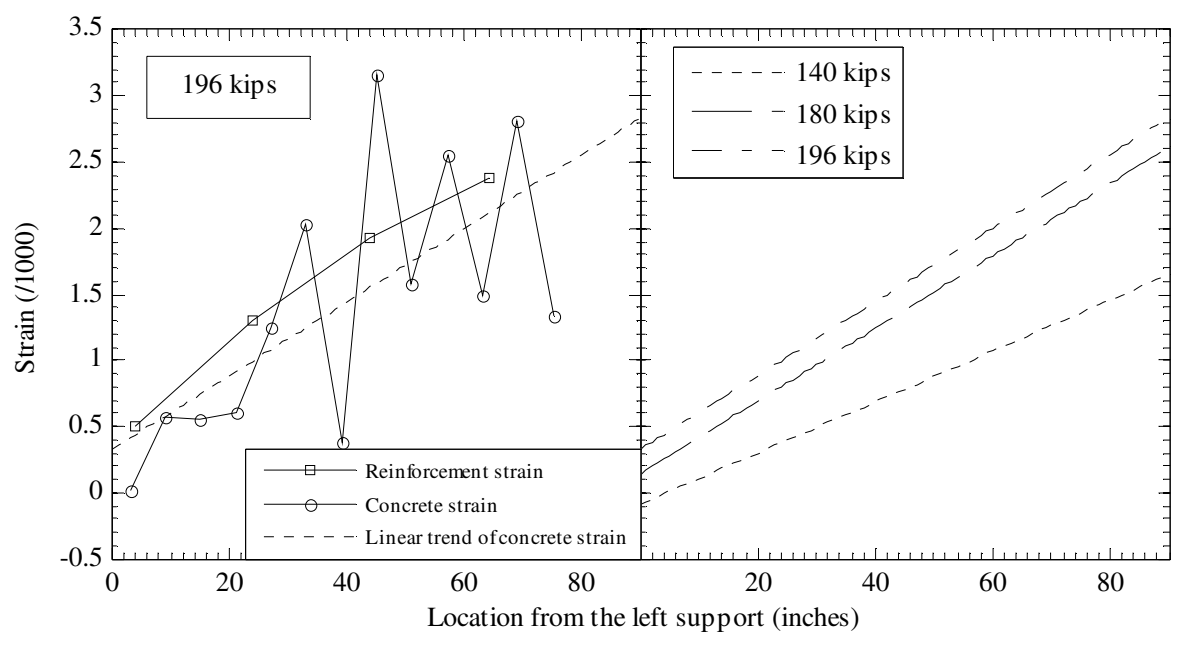

Fig. 4-120: Comparison of reinforcement and concrete strains - Beam B27-2a

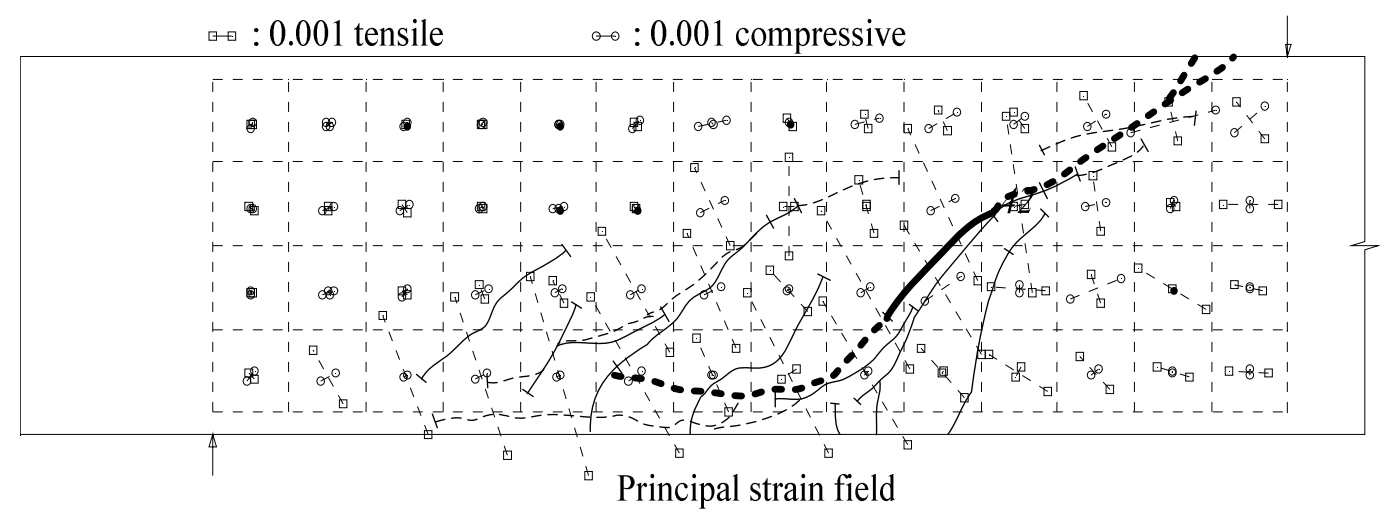

Fig. 4-121: Principal strain field at 196 kips - Beam B27-2a 
(a)

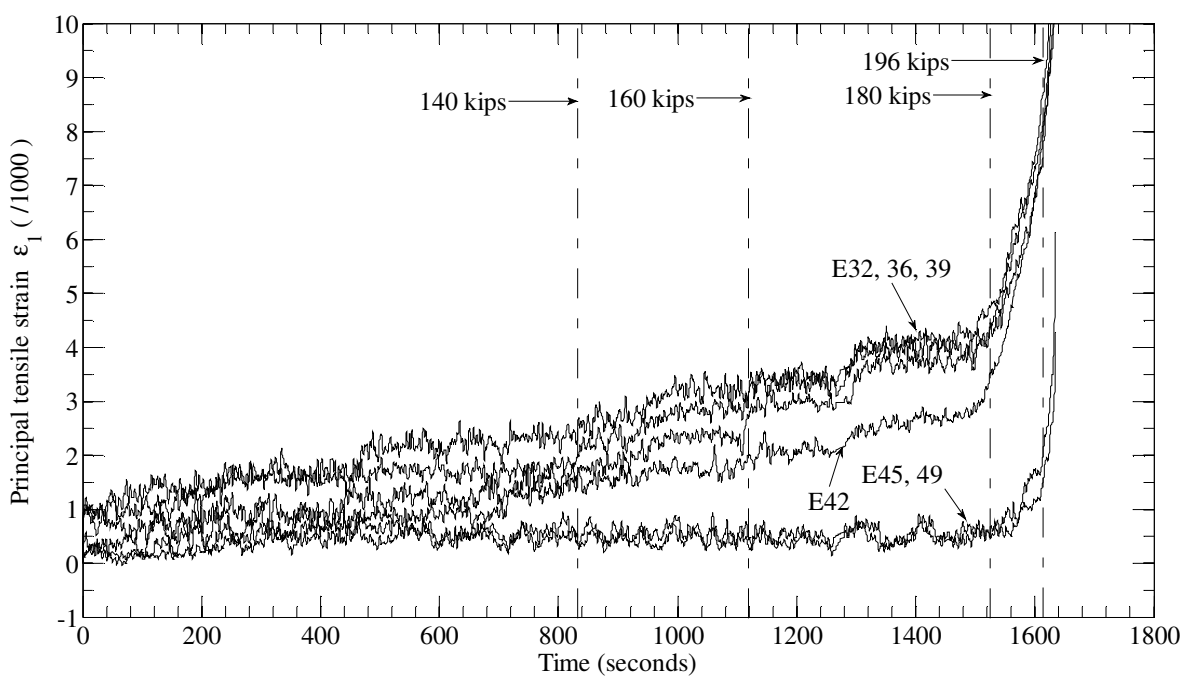

(b)

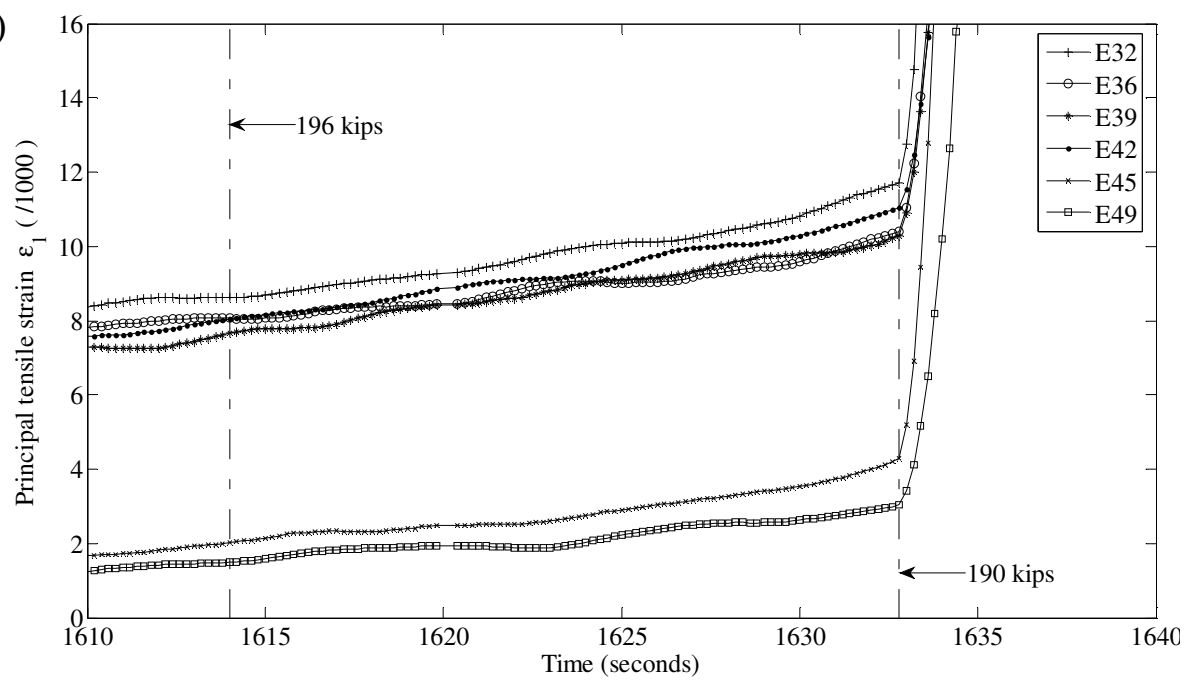

(c)

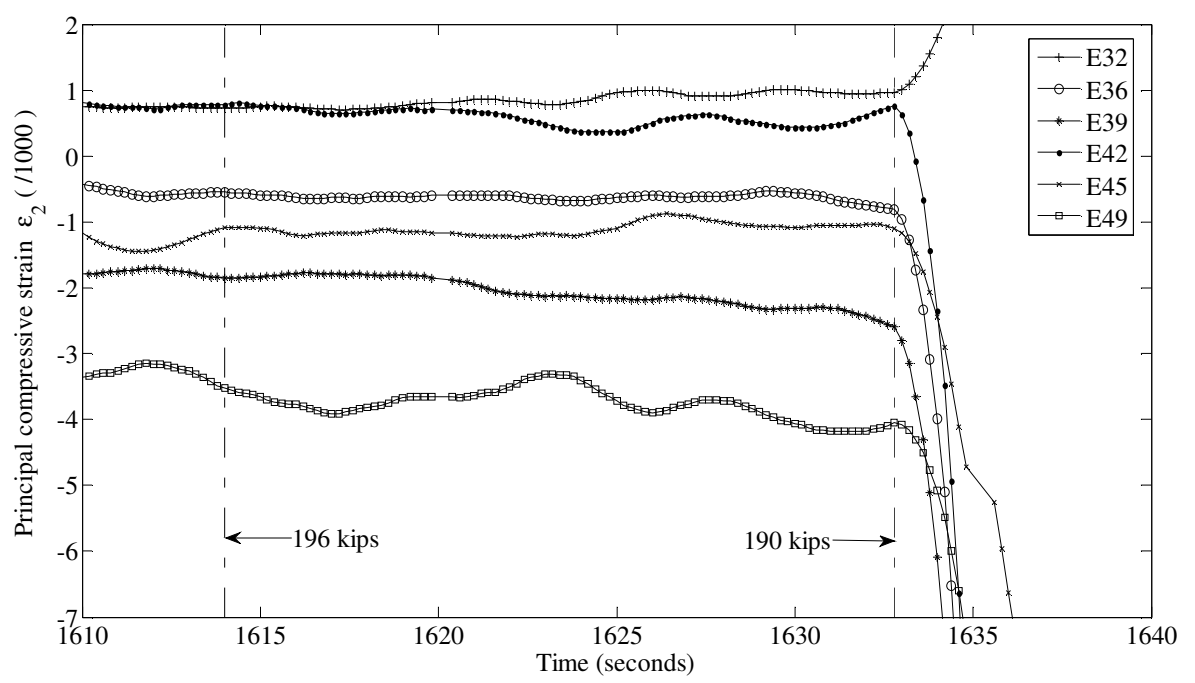

Fig. 4-122: Development of principal tensile and compressive strains along the critical inclined crack - Beam B27-2a 


\subsubsection{Beam B27-2b}

Load versus deflection relationship, crack pattern, and failure mode. Beam B27-2b had identical properties to Beam B27-1a. The behavior of Beam B27-2b was similar to that of Beam B27-2a in the following aspects:

(a) The load-deflection curve was nonlinear from the beginning (see Fig. 4-123a) and showed a steady decrease in stiffness after the first inclined crack, which appeared at 100 kips (Fig. 4-124).

(b) The flexural-shear cracks spread throughout the shear span.

In spite of these similarities, the failure mode was totally different. Beam B27$2 \mathrm{~b}$ failed due to the opening of a diagonal crack that connected the loading point and the support (Fig. 4-125). Fig. 4-124 shows the initial formation of the critical inclined crack, which opened up at the beam mid-depth and propagated toward both the tension reinforcement and the compression region. It was clear from Fig. 4-124 that the crack width was larger near the mid-depth region than it was at both the reinforcement and compression regions.

Compared to Beam B27-2a, Beam B27-2b did not have a "yielding" plateau. Rather, the beam failed quickly after reaching the peak load of 192 kips. The reason was probably due to the different failure mechanism. While a shear-compression (Beam B272a) failure with a steep crack angle tends to allow more rotation of the two pieces about the upper crack tip, a shallow diagonal crack (Beam B27-2b) tends to split through the compression region rapidly without allowing much rotation.

Reinforcement strain and average bond stress. The propagation of the critical crack towards the support caused the strain at S2 to rapidly increase (Fig. 4-123b and Fig. 4-126) from a load of approximately 140 kips. Similar to Beam B27-2a, average bond stress was largest near the support (from S1 to S3).

Concrete strain field. As mentioned above, the splitting of the compression region due to diagonal tension resulted in a less severe compressive strain demand in the compression region (Fig. 4-128a). The maximum compression strain of 0.0043 between 
markers M66 and M71 (see Fig. 4-129 for the numbering of markers) was mainly due to the flexural demand. The maximum tension strain was 0.0065 between markers M19 and M24, where the critical diagonal crack extended into the tension region. Meanwhile, the transverse and shear strains were large, especially for elements along the critical crack. The maximum transverse and shear strains were 0.025 (Element 26, Fig. 4-128b) and 0.018 radians (Element 16, Fig. 4-128c), respectively. Observations similar to those for other beams were also made for Beam B27-2b with respect to the correlation between concrete and reinforcement strains (Fig. 4-130) and to the distribution of average transverse and shear strains measured over the beam depth (Fig. 4-131).

Fig. 4-132 shows the principal strain field at peak load. As expected, the principal tensile strain was significantly larger along the critical diagonal crack. Fig. 4-133a shows the development of principal tensile strains along the critical crack with time. When the applied load reached 140 kips, significant strains developed for middepth elements (E19, 22, and 26), which then propagated to the reinforcement (E15, 12, and 8) and compression region (E37, 41, and 45). This figure also shows that the crack growth toward the reinforcement level was faster than that toward the compression region. This was obvious because the reinforcement in tension tended to accelerate the opening while the compression region did the opposite. The crack propagation clearly defined the beam failure as pure diagonal tension. An expansion of the time scale at the peak load (Fig. 4-133b) shows that there was a significant change in the straining rate at a load of 192 kips, which can be considered as the critical failure load. 
(a)

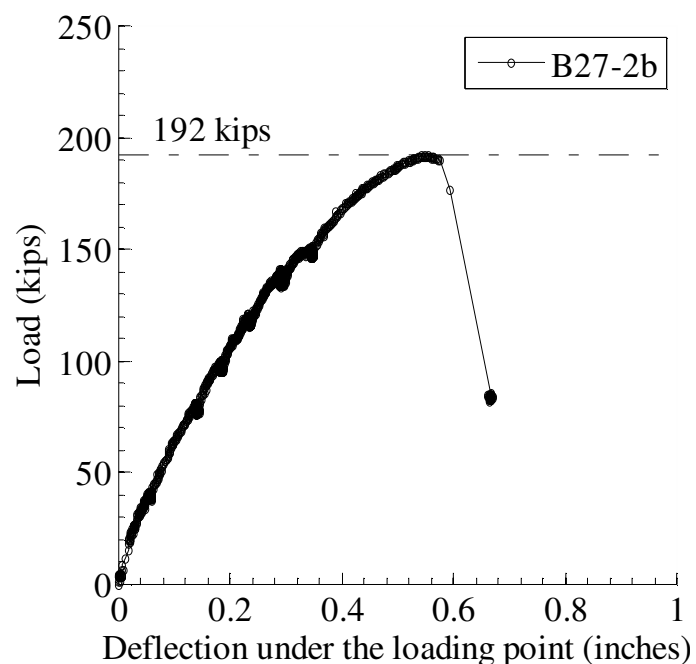

(b)

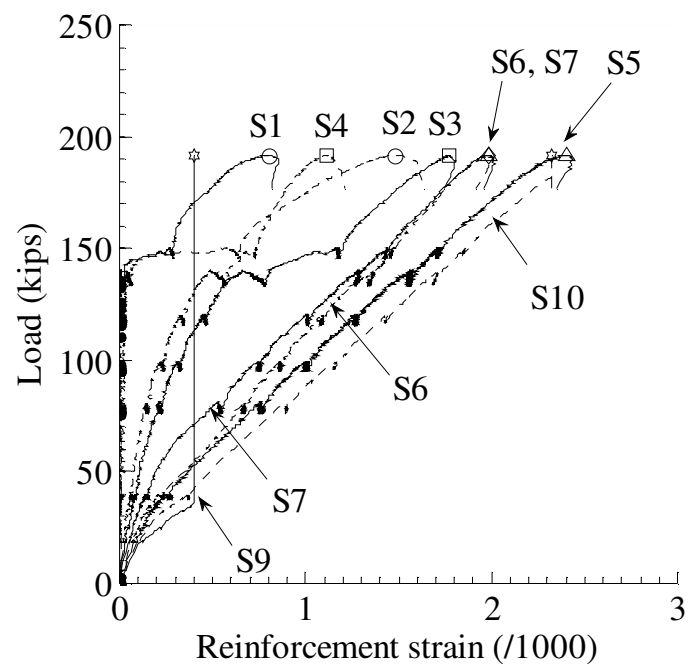

Fig. 4-123: Load versus deflection and reinforcement strain relationships - Beam B27-2b

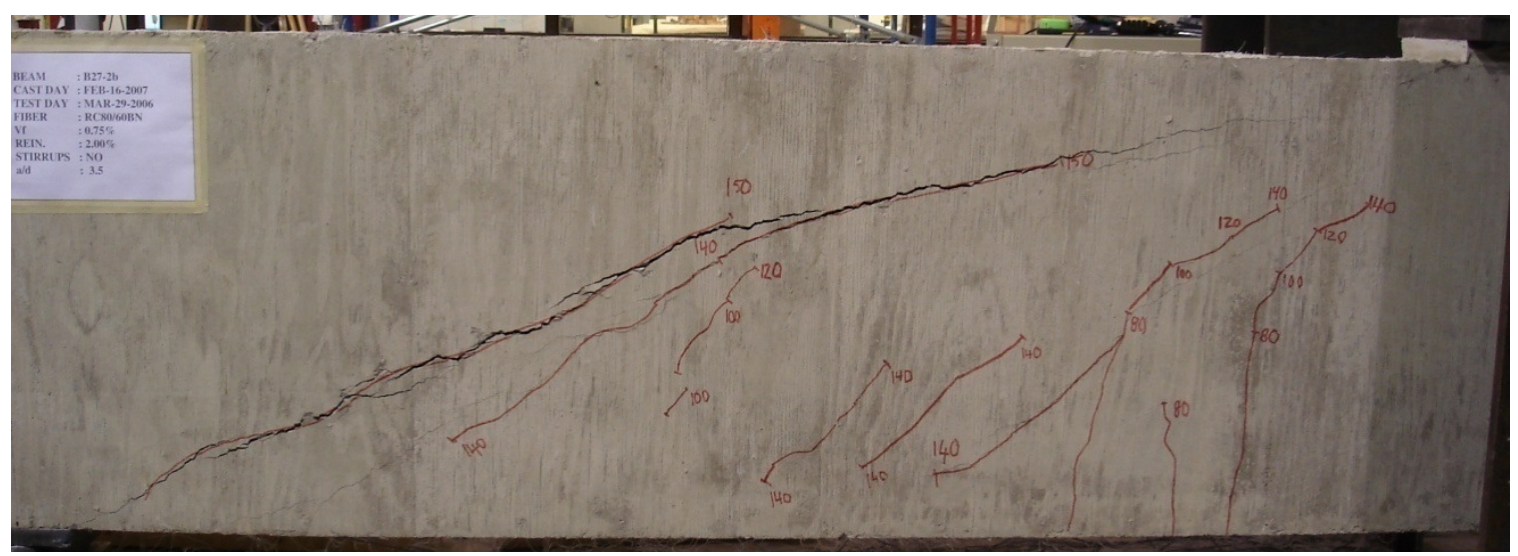

Fig. 4-124: Crack pattern prior to failure - Beam B27-2b

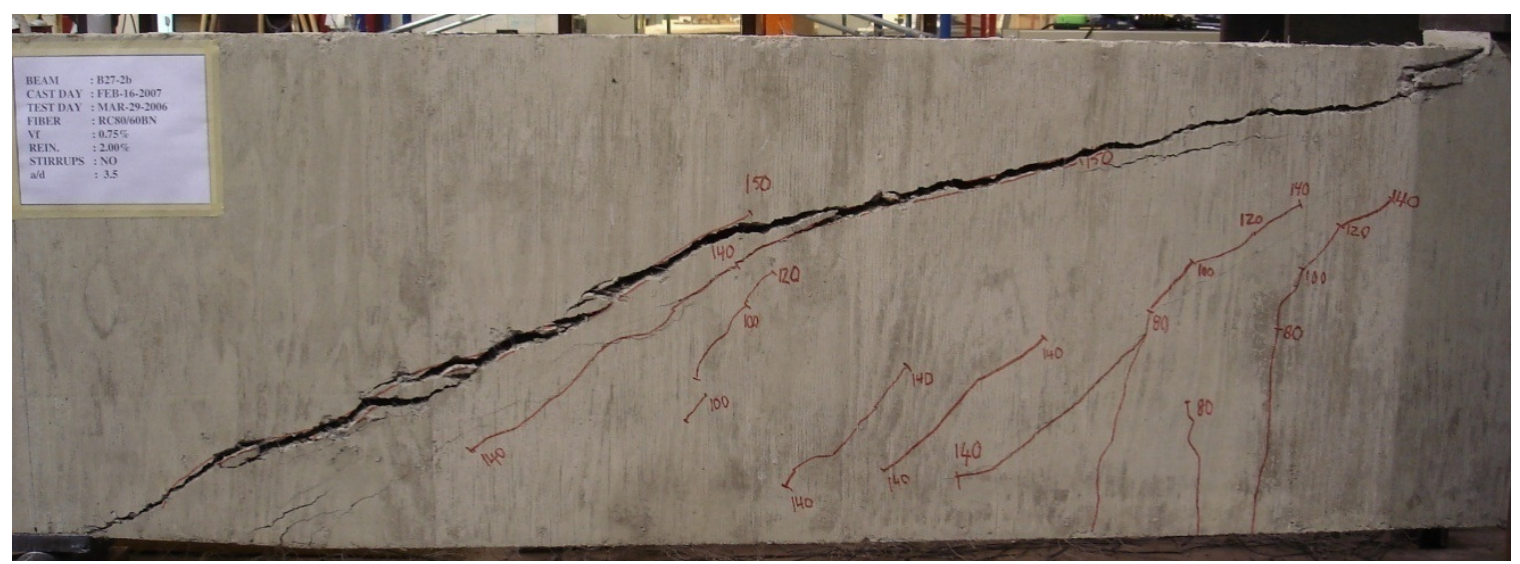

Fig. 4-125: Crack pattern after failure - Beam B27-2b 


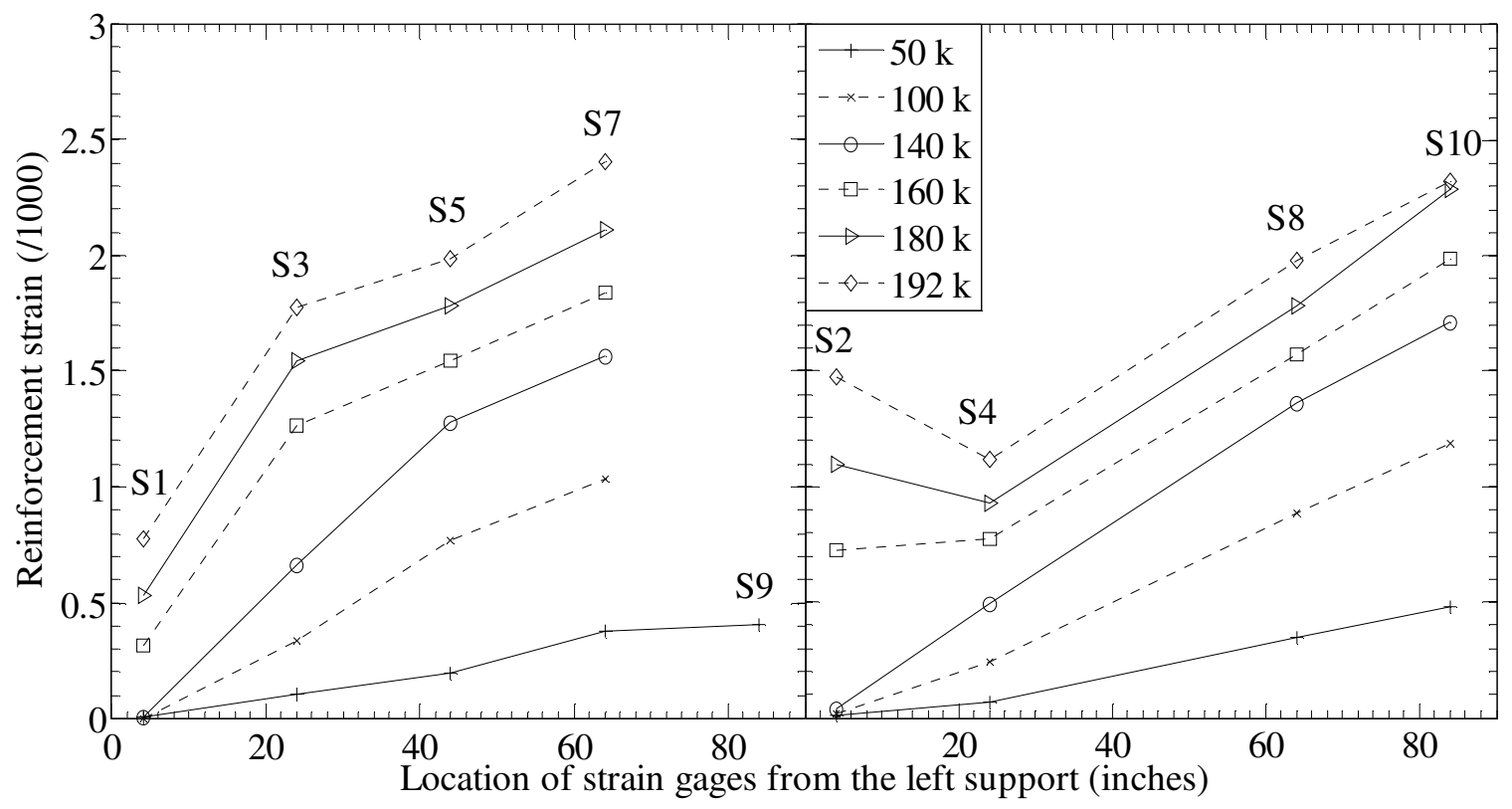

Fig. 4-126: Reinforcement strains along the beam axis - Beam B27-2b

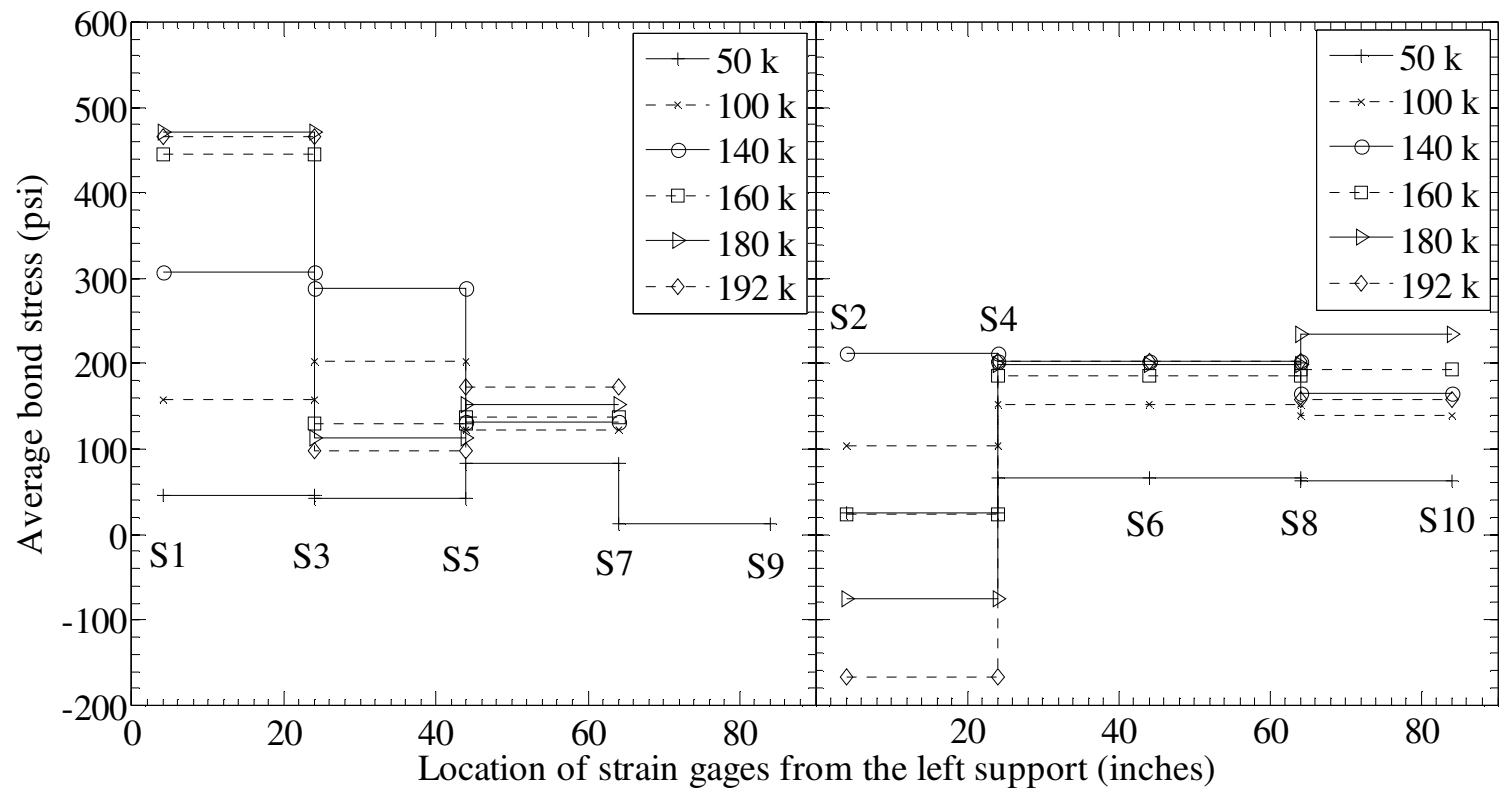

Fig. 4-127: Average bond stress along the beam axis - Beam B27-2b 
(a)

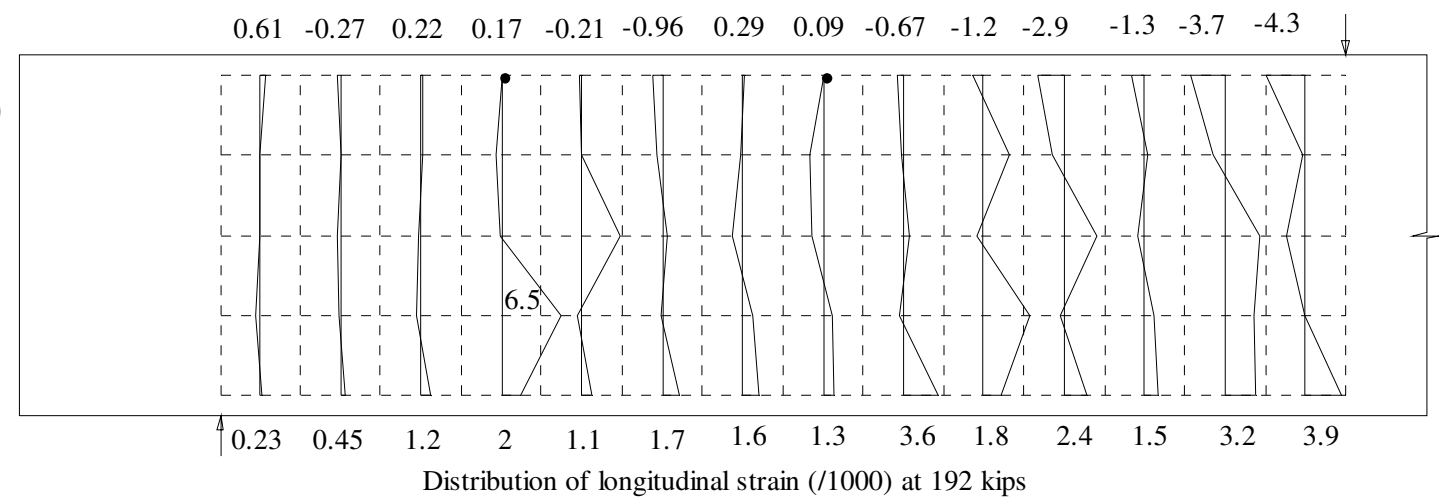

(b)

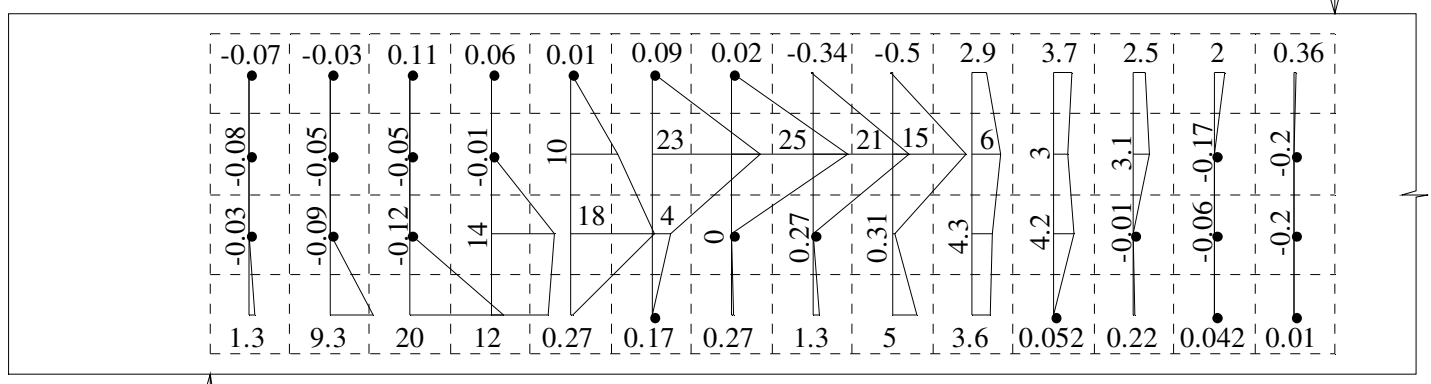

Distribution of transverse strain (/1000) at 192 kips

(c)

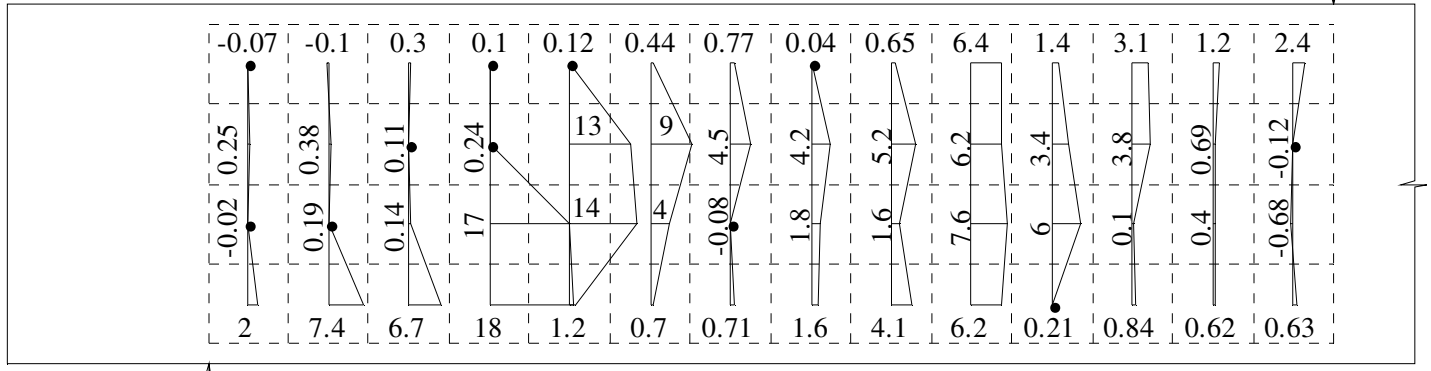

Distribution of shear strain (/1000) at 192 kips

Fig. 4-128: Distribution of longitudinal, transverse, and shear strains at $192 \mathrm{k}-$ Beam

$$
\text { B27-2b }
$$

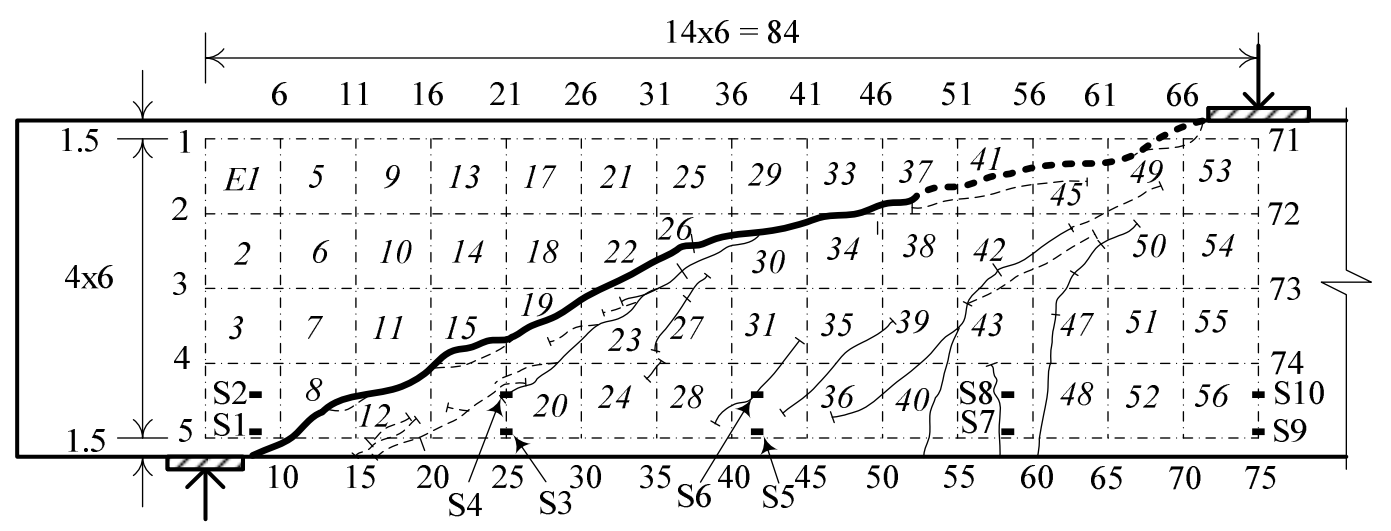

Fig. 4-129: Numbering of markers, elements, and strain gauges - Beam B27-2b 


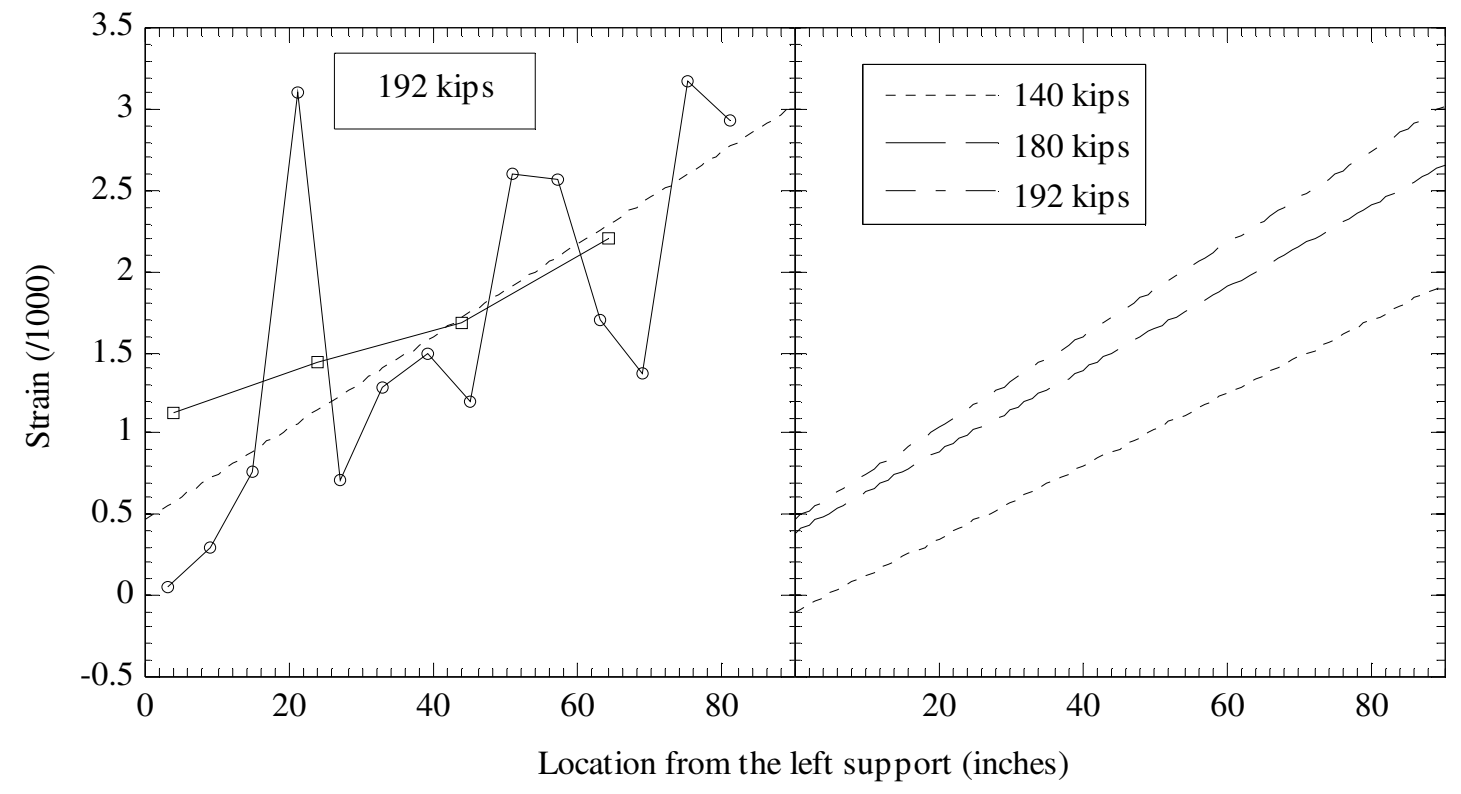

Fig. 4-130: Comparison of reinforcement and concrete strains - Beam B27-2b

(a)

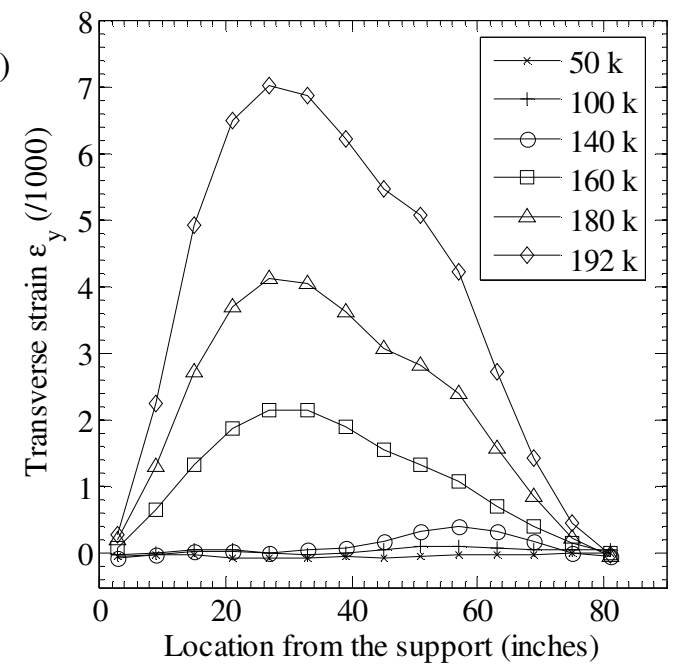

(b)

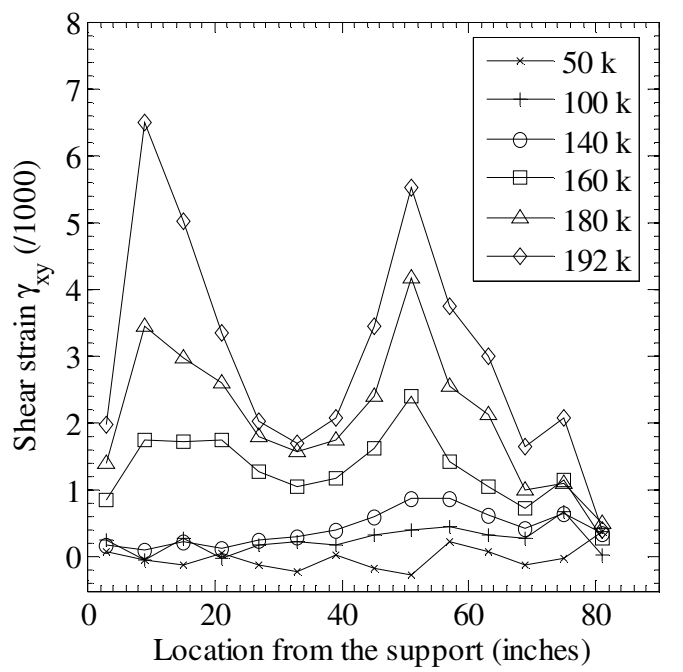

Fig. 4-131: Distribution of transverse and shear strains averaged over the beam depth -

Beam B27-2b 


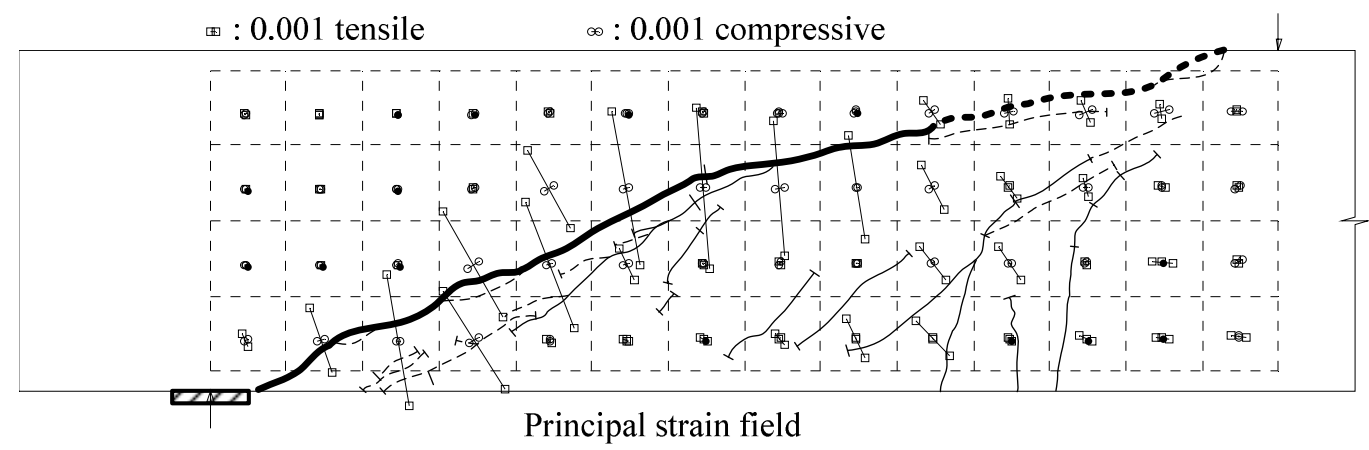

Fig. 4-132: Principal strain field at 192 kips - Beam B27-2b

(a)

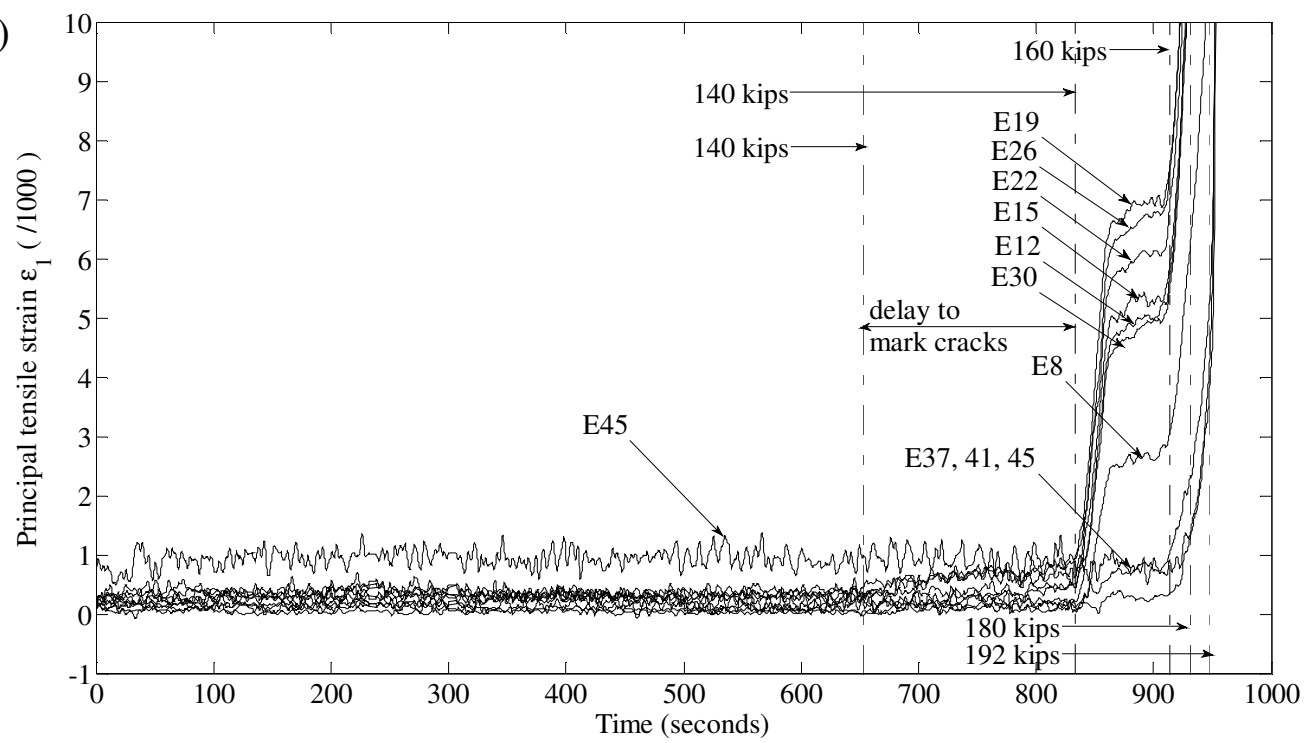

(b)

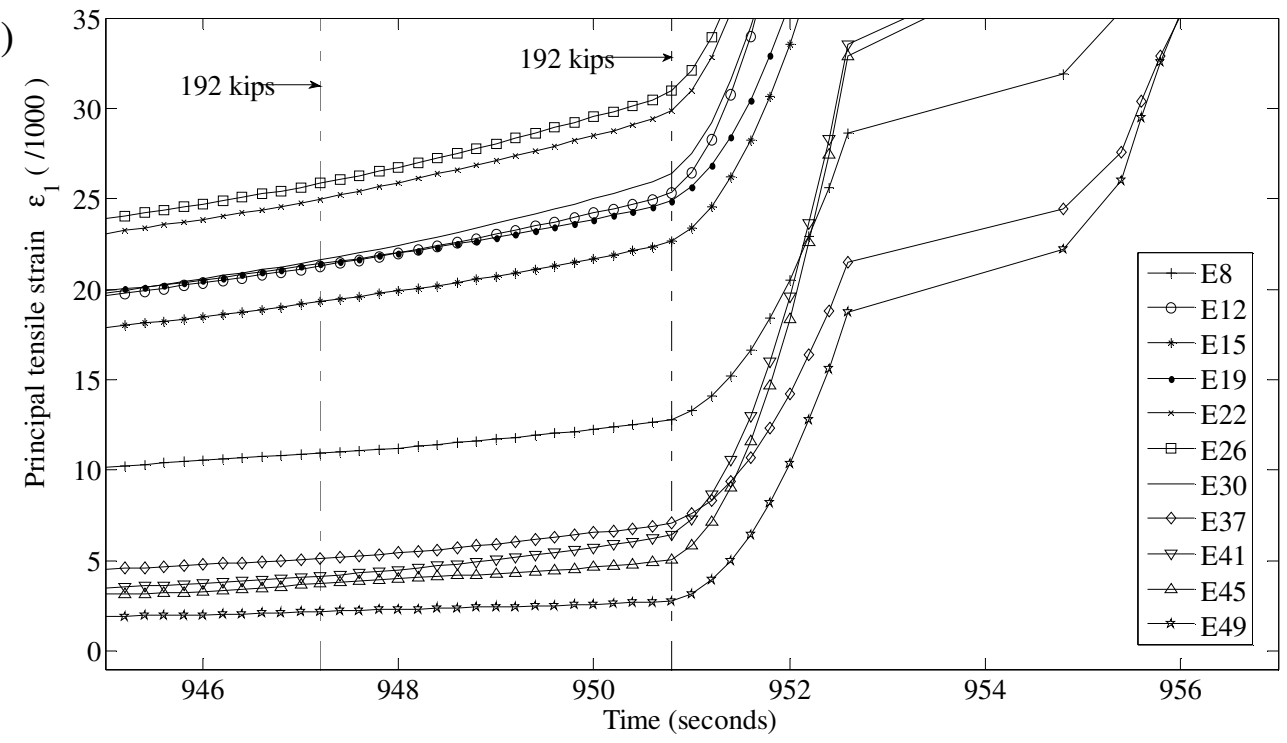

Fig. 4-133: Development of principal tensile strains along the critical inclined cracks Beam B27-2b 


\subsubsection{Beam B27-3a}

Compared with Beams B27-1a \& b, Beam B27-3a had similar properties, except that it had a lower reinforcement ratio (1.56\% compared with $2.06 \%)$. This means that Beam B27-3a contained a $0.75 \%$ volume fraction of ZP305 fibers, which have a length of 1.18 inches and a diameter of 0.022 inches (corresponding to an aspect ratio of 55).

\section{Load versus deflection relationship, crack pattern, and failure mode.} Compared with Beams B27-1a \& b, which exhibited first inclined cracks at 140 and 160 kips, respectively, the first inclined crack of Beam B27-3a was detected at a smaller load, 100 kips (Fig. 4-134). Nevertheless, the load versus deflection stiffness did not reduce at first diagonal cracking.

The yielding of the longitudinal reinforcement led to a long yielding plateau on the load versus deflection curve (Fig. 4-135) that accounted for $80 \%$ of the beam total deflection. Prior to yielding, which occurred at approximately 180 kips, the beam exhibited several inclined cracks that spread over the shear span. After the load of approximately $180 \mathrm{kips}$, crack openings were observed to concentrate in the flexural cracks under the loading point. The beam attained its maximum strength of 190 kips at the mid-length of the yield plateau. The beam failed in flexure (on both sides of the plate) by a sudden crushing of the compression region below the loading plate. It is interesting to note from Fig. 4-134 that deflection was so significant that it caused longitudinal cracking along the reinforcement level (Fig. 4-134). Reinforcement buckling and concrete splitting were observed in the compression zone after failure (Fig. 4-136).

Reinforcement strain and average bond stress. It can be seen from Fig. 4-137 that strain gauges S7 and S8 (see Fig. 4-138 for the location of strain gauges) were strained up to 0.03 and 0.025 just before failure. Strain gauges S5 and S6 were also significantly strained. It should also be noted that there was a jump in strains near the support (S1 and S2, see Fig. 4-139) at the load of 180 kips, as the flexural-shear cracks spread out to the support. The reinforcement strain was seen to be distributed linearly along the shear span up to 180 kips, when yielding occurred. The average bond stress 
also developed significantly for both the upper and lower reinforcing bars from $\mathrm{S} 1$ (S2) to S5 (S6), beyond 400 psi (Fig. 4-140). While a drop in bond stress was observed from S5 to $\mathrm{S} 7$ at the load of $160 \mathrm{kips}$, that from S6 to S8 and that in the region near the support (S1 to S5 and S2 to S6) only occurred at the load of 180 kips (Fig. 4-140).

Concrete strain field. It can be seen from Fig. 4-141a that the beam longitudinal strain mostly concentrated in the region below the loading point. The maximum longitudinal tension strain observed was as large as 0.032 between markers M64 and M69 (see Fig. 4-138 for the location of markers) and 0.037 between M60 and M65. The compressive strain demand was also significant, reaching a value of 0.0046 between M61 and M66.

Significant transverse and shear strain (Fig. 4-141 b and c) demand was placed on the bottom row of the left half of the shear span, reaching the peak value of 0.016 and 0.037, respectively, at Element E48. It should be noted that compared with beams that failed due to diagonal tension (Beams B27-1b and B27-2b), the relative transverse strain and shear strain demands were lower for Beam B27-3a, which failed due to flexure.

The total transverse and shear demand measured over the beam height were less severe than the local demand (Fig. 4-143), reaching the peak value of 0.0047 and 0.0162 , respectively.

At the failure load of $173 \mathrm{kips}$, the compression zone near the loading point was excessively compressed. The maximum longitudinal compressive strains reached a value of up to 0.0059 (Fig. 4-142a). The transverse and shear strains at the reinforcement level were also extremely large. The maximum shear strain was 0.09 radians (or 5.3 degrees), corresponding to the excessive deformed shape of the concrete quadrants in the region. The wide shallow crack above the tension reinforcing bars also contributed to the transverse strains at this level. It should be noted that the compression region also subjected to a significant transverse strains. At this point, the compression region was crushed. Therefore, it was possible that a vertical relative movement occurred.

Fig. 4-144 shows the principal strain field at the peak load of 190 kips and at the end of the load-deflection "yield plateau" where the applied load was 173 kips. It can be seen that the larger strains concentrated on Elements E40, 44, and 48 through 53. At the 
peak load, the principal tensile strains were the largest for E48 (0.0374) and near as large for E50 (0.0304), which was close to the compression region. The principal compressive strain for this element was also significant, up to 0.0051 . At the end of the load-deflection "yield plateau", the strains were extremely large. The principal tensile strain at E48 reached the value of 0.107. It should be emphasized that the strain was that large because two main cracks crossed that element. The principal compressive strain at E50 was also ten times larger than it was at the peak load. It was very interesting that SFRC with a $0.75 \%$ volume fraction of ZP305 fibers could be compressed to such a large strain before failure.

Fig. 4-145a shows the development of principal strains with time during the yield plateau. It can be seen that the strain developed significantly after the applied load of 180 kips, when the beam entered the yield plateau. The strain rate was the largest for the group of reinforcement-level elements (E40, 44, and 48). The group of elements E49 through 53 exhibited a lower strain rate. At the load of 183 kips, the strains throughout the beam increased suddenly (Fig. 4-145a \& b). This can be considered a critical strain state at which concrete crushing initiated. At this load, the concrete compressive strain at the crushed elements E49 and E53 had a magnitude of 0.010. It was not clear why the compressive strain of Element E50 reached a greater value (0.022), but crushing was not seen at that element.

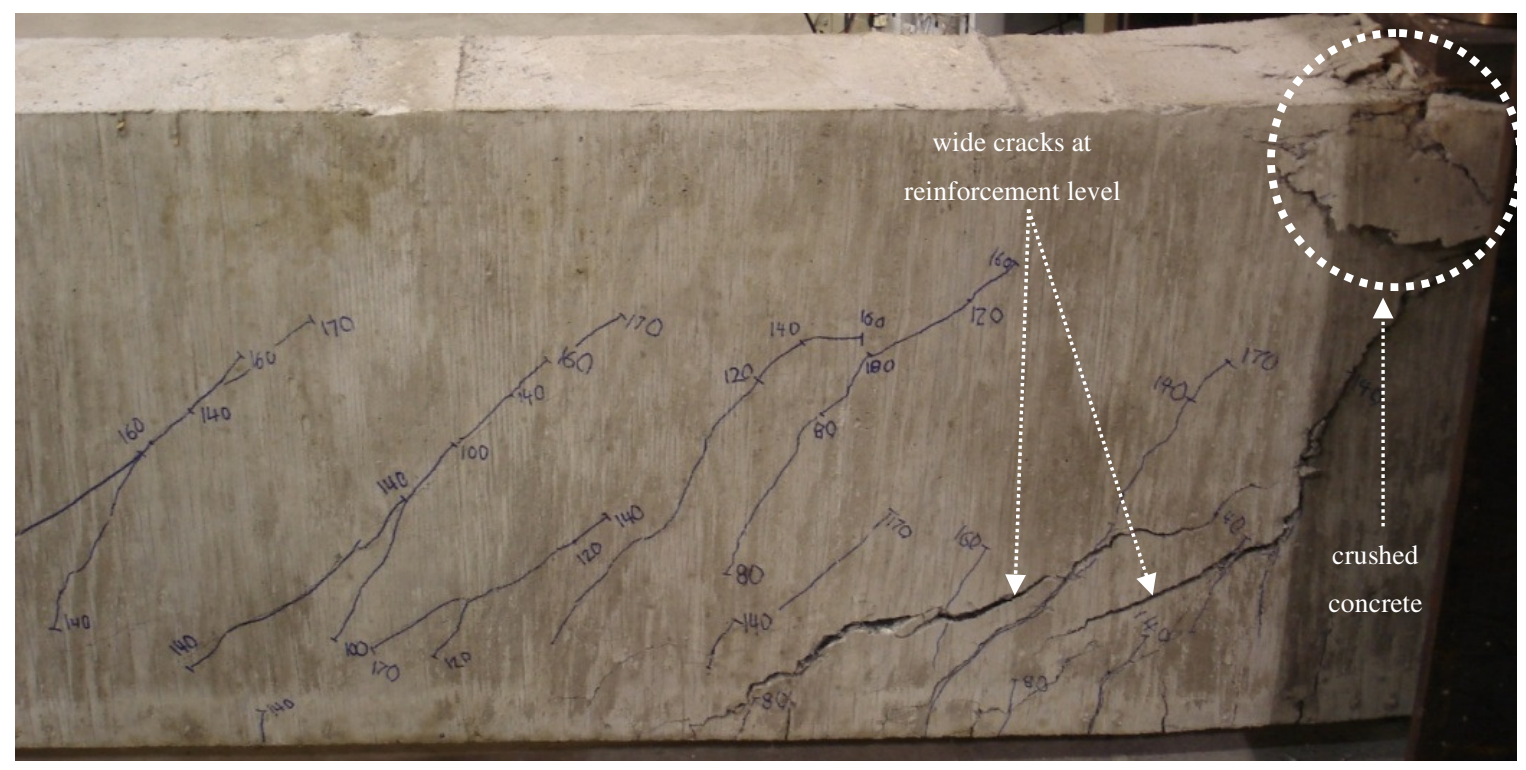

Fig. 4-134: Crack pattern after failure - Beam B27-3a 


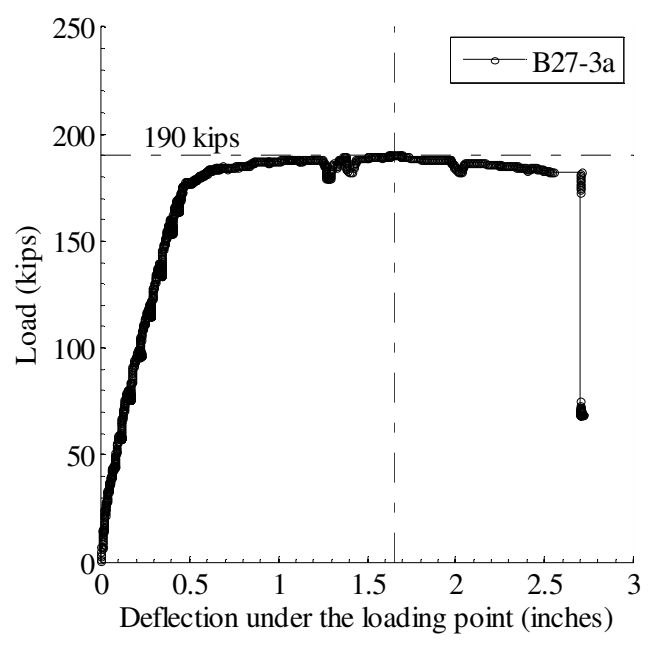

Fig. 4-135: Load versus deflection relationship - Beam B27-3a

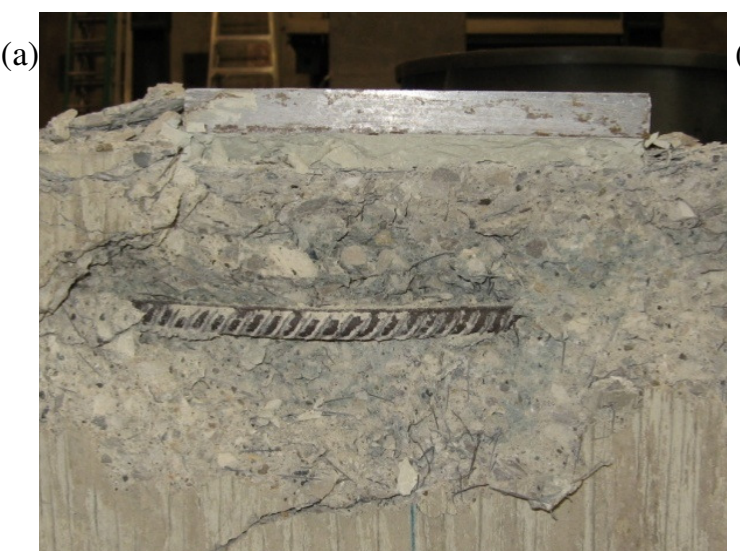

(a)

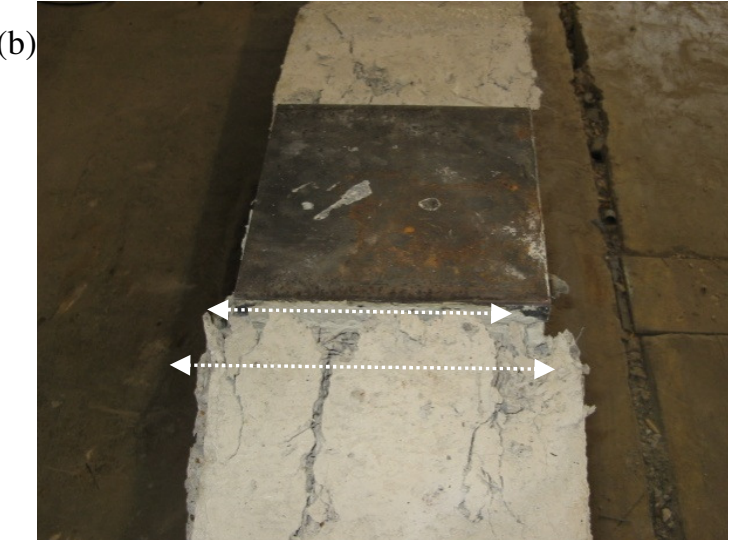

(b)

Fig. 4-136: Reinforcement buckling (a) and transverse expansion (b) - Beam B27-3a

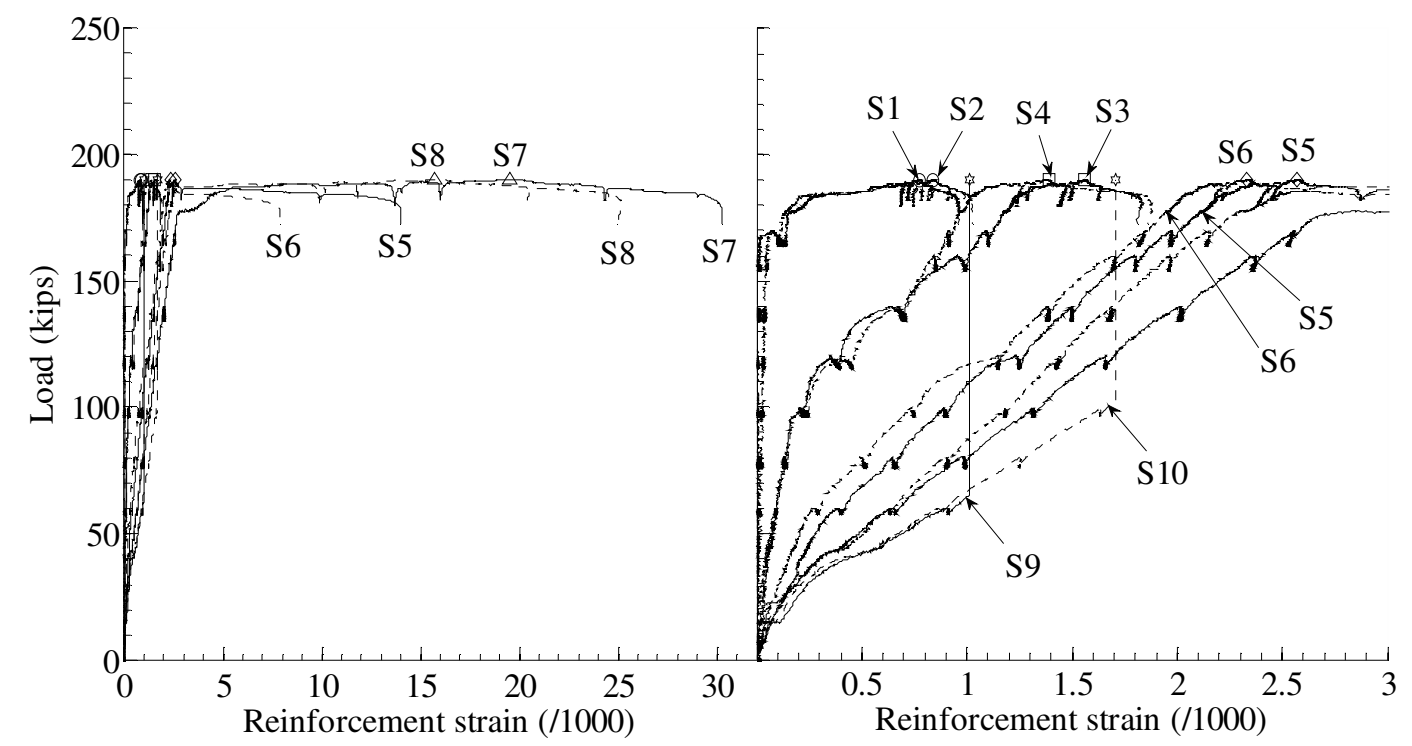

Fig. 4-137: Load versus reinforcement strain relationships - Beam B27-3a 


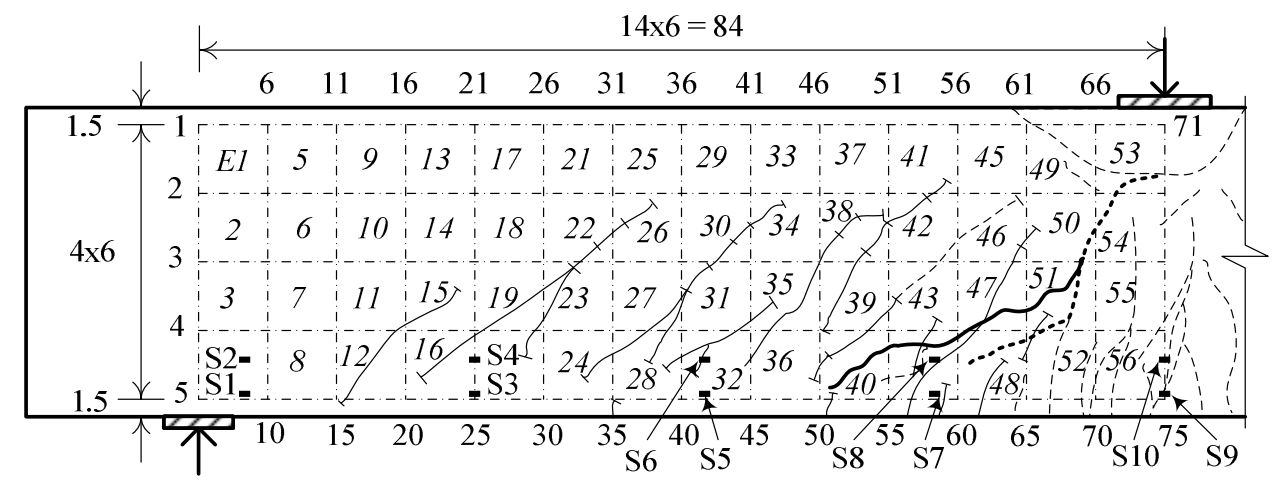

Fig. 4-138: Numbering of markers, elements, and strain gauges - Beam B27-3a

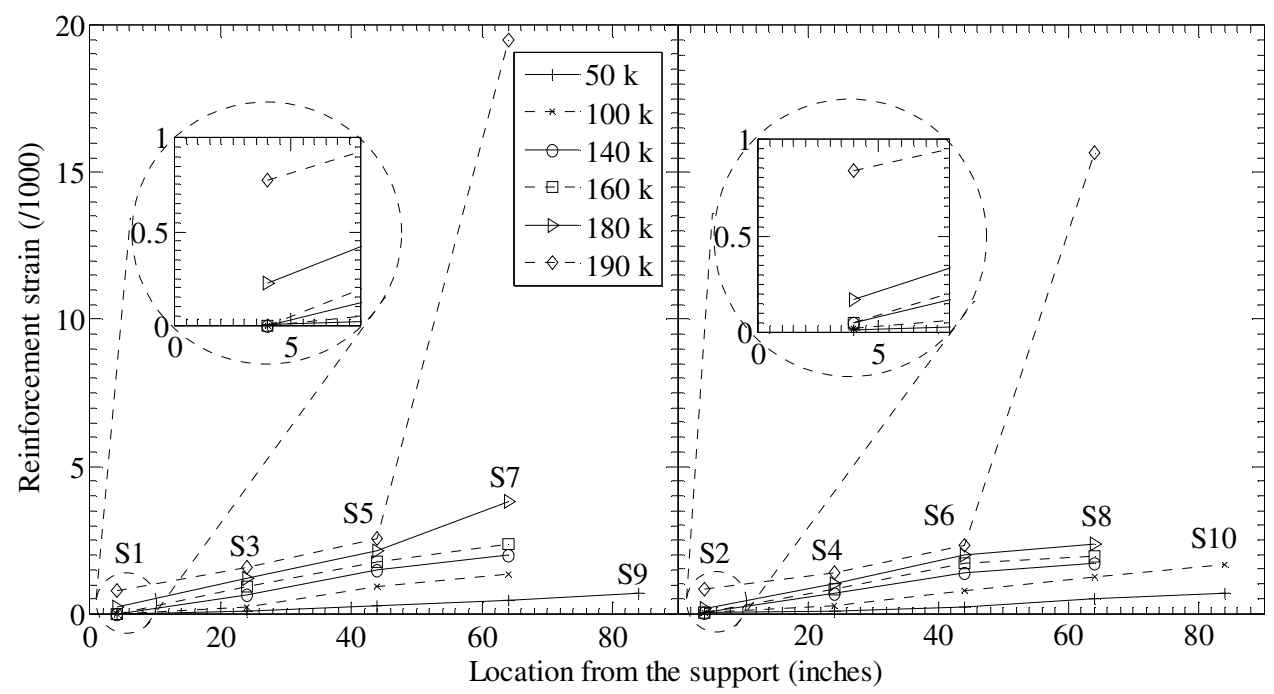

Fig. 4-139: Reinforcement strains along the beam axis - Beam B27-3a

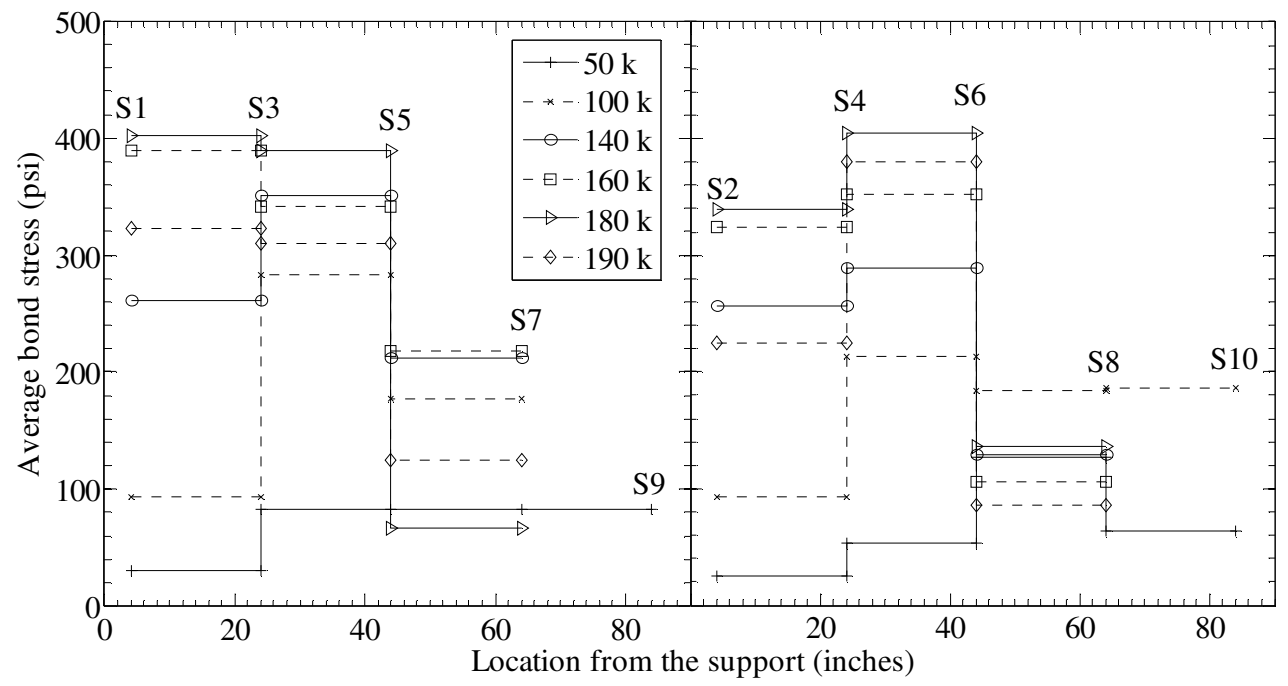

Fig. 4-140: Average bond stress along the beam axis - Beam B27-3a 
(a)

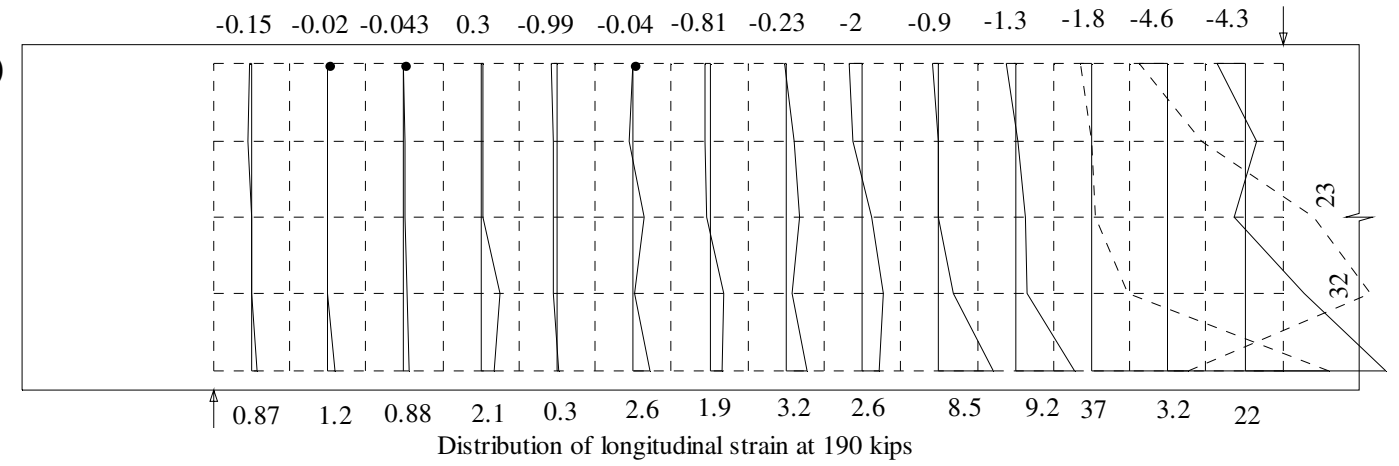

(b)

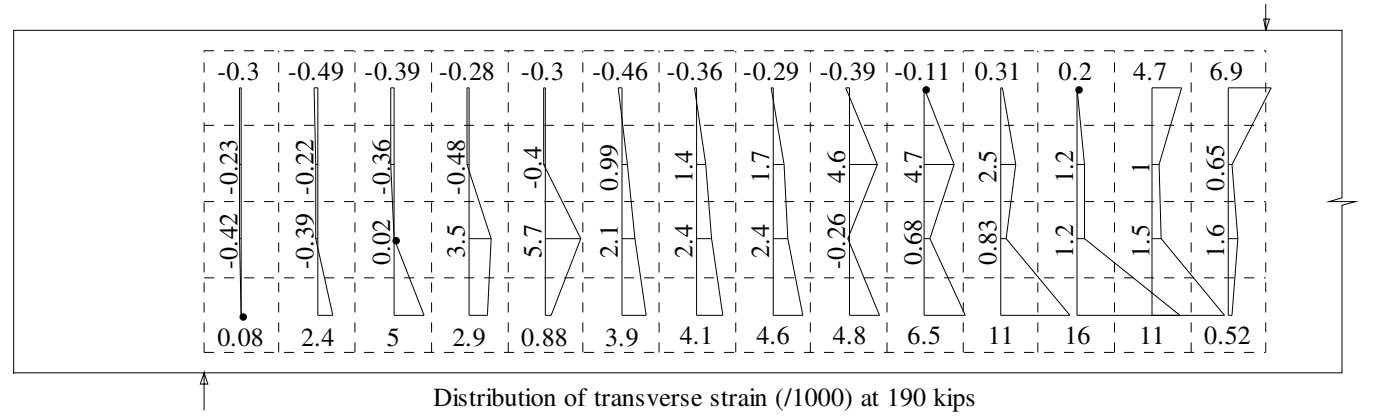

(c)

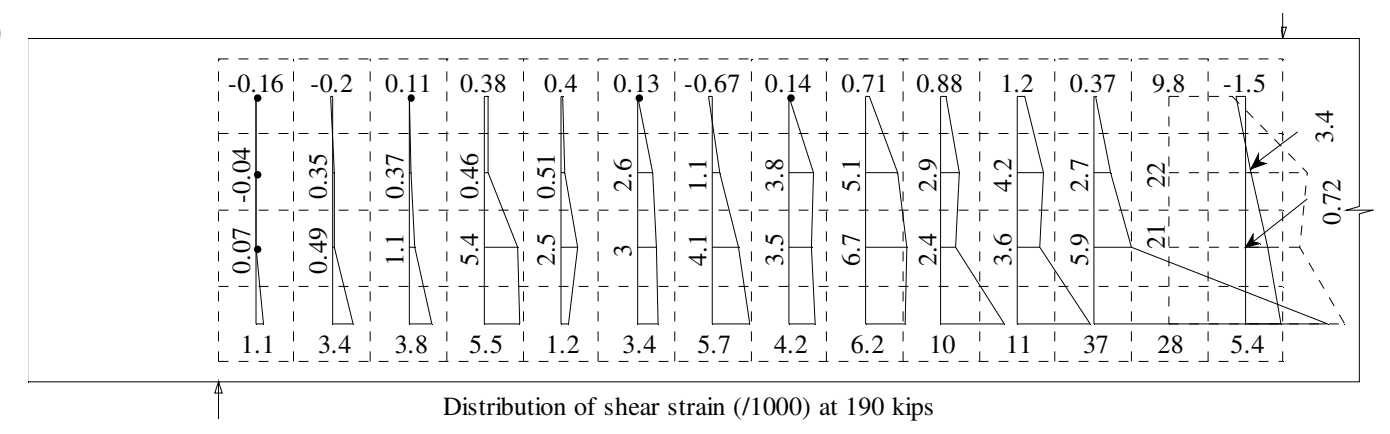

Fig. 4-141: Distribution of longitudinal, transverse, and shear strains at peak load - Beam B27-3a 
(a)

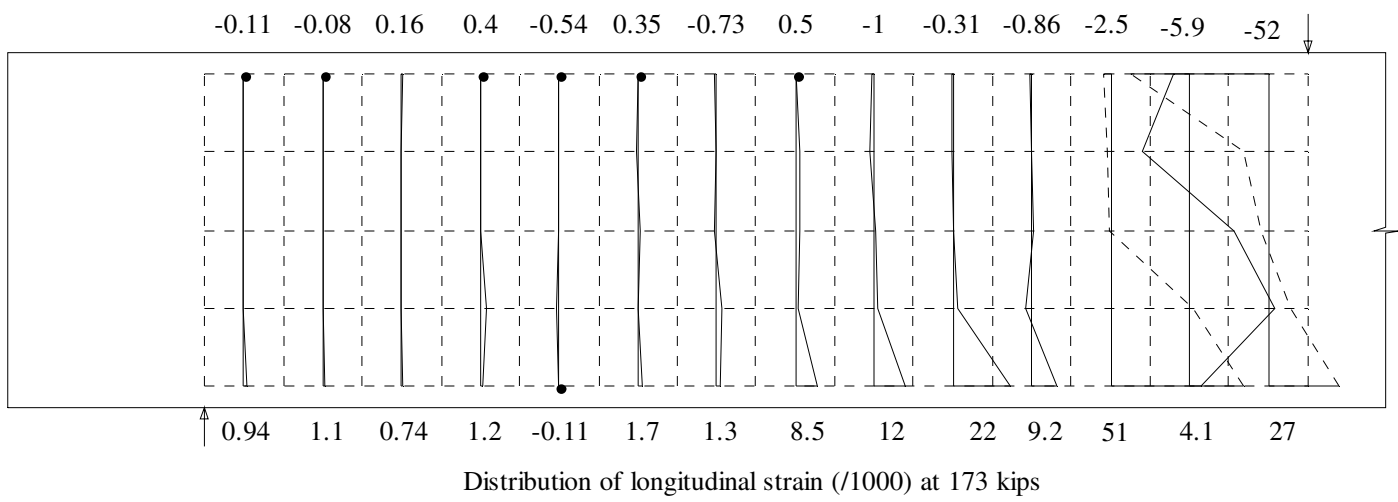

(b)

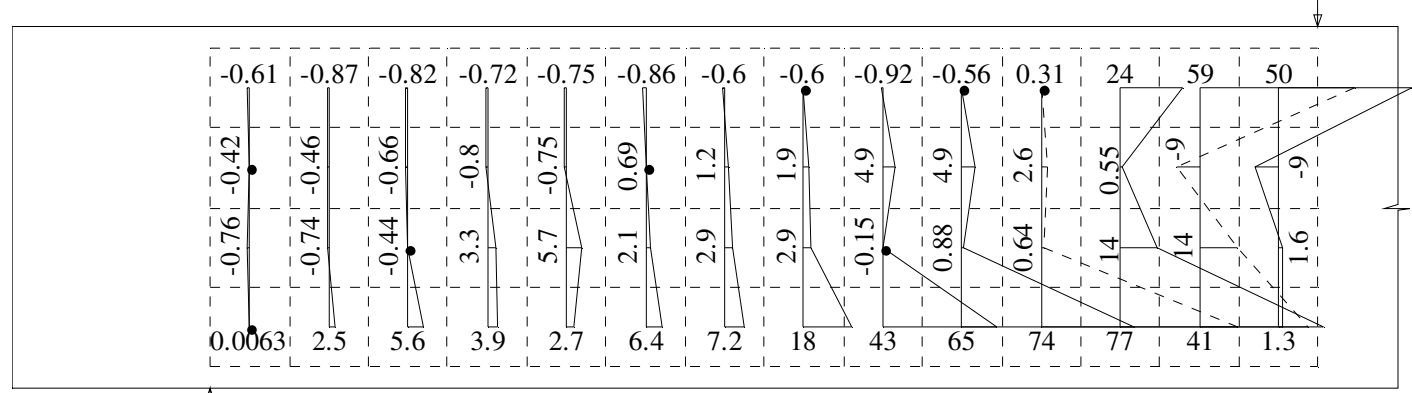

Distribution of transverse strain (/1000) at 173 kips (post-peak)

(c)

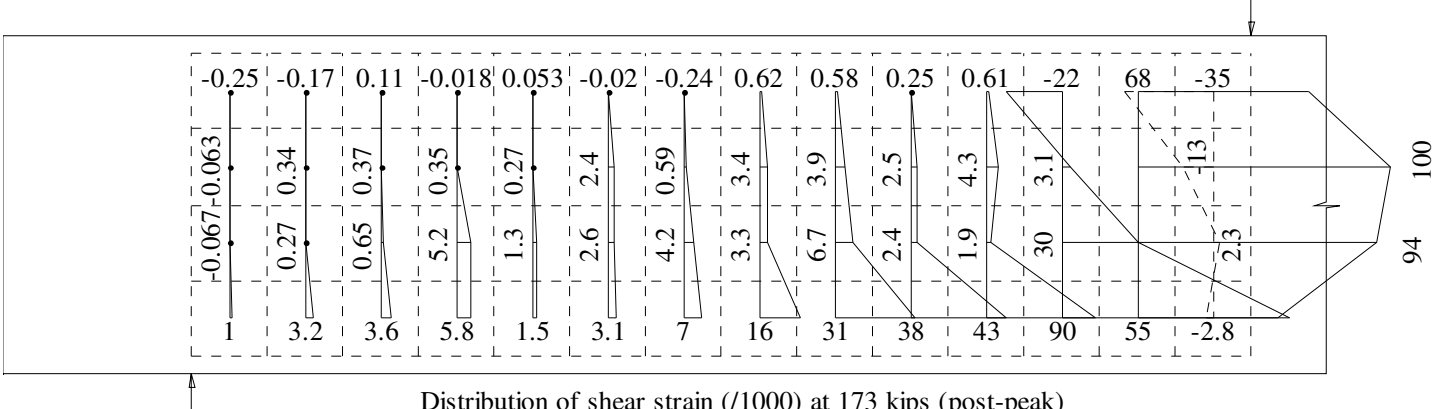

Distribution of shear strain (/1000) at 173 kips (post-peak)

Fig. 4-142: Distribution of longitudinal, transverse, and shear strains at failure load Beam B27-3a 
(a)

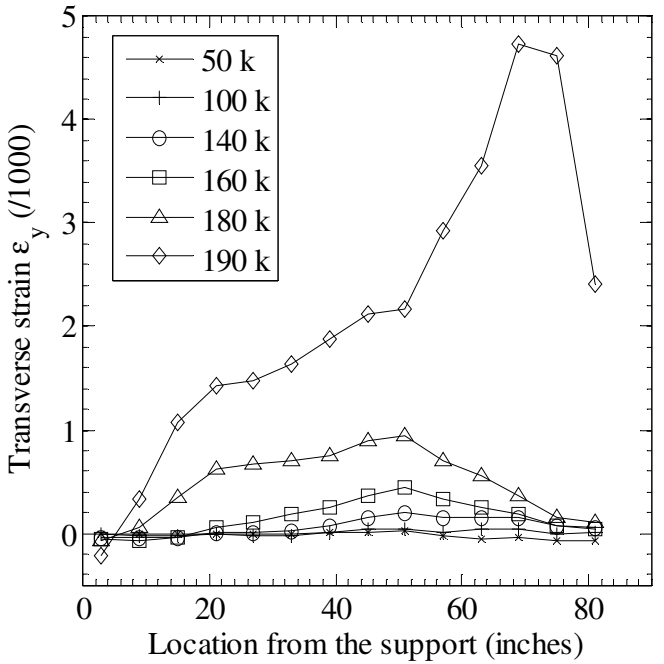

(b)

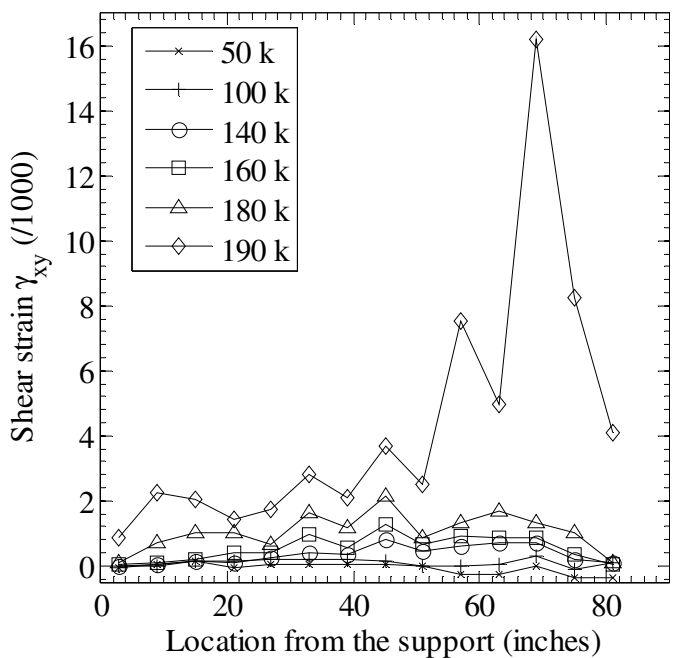

Fig. 4-143: Distribution of transverse and shear strains along the beam axis averaged over the beam depth - Beam B27-3a

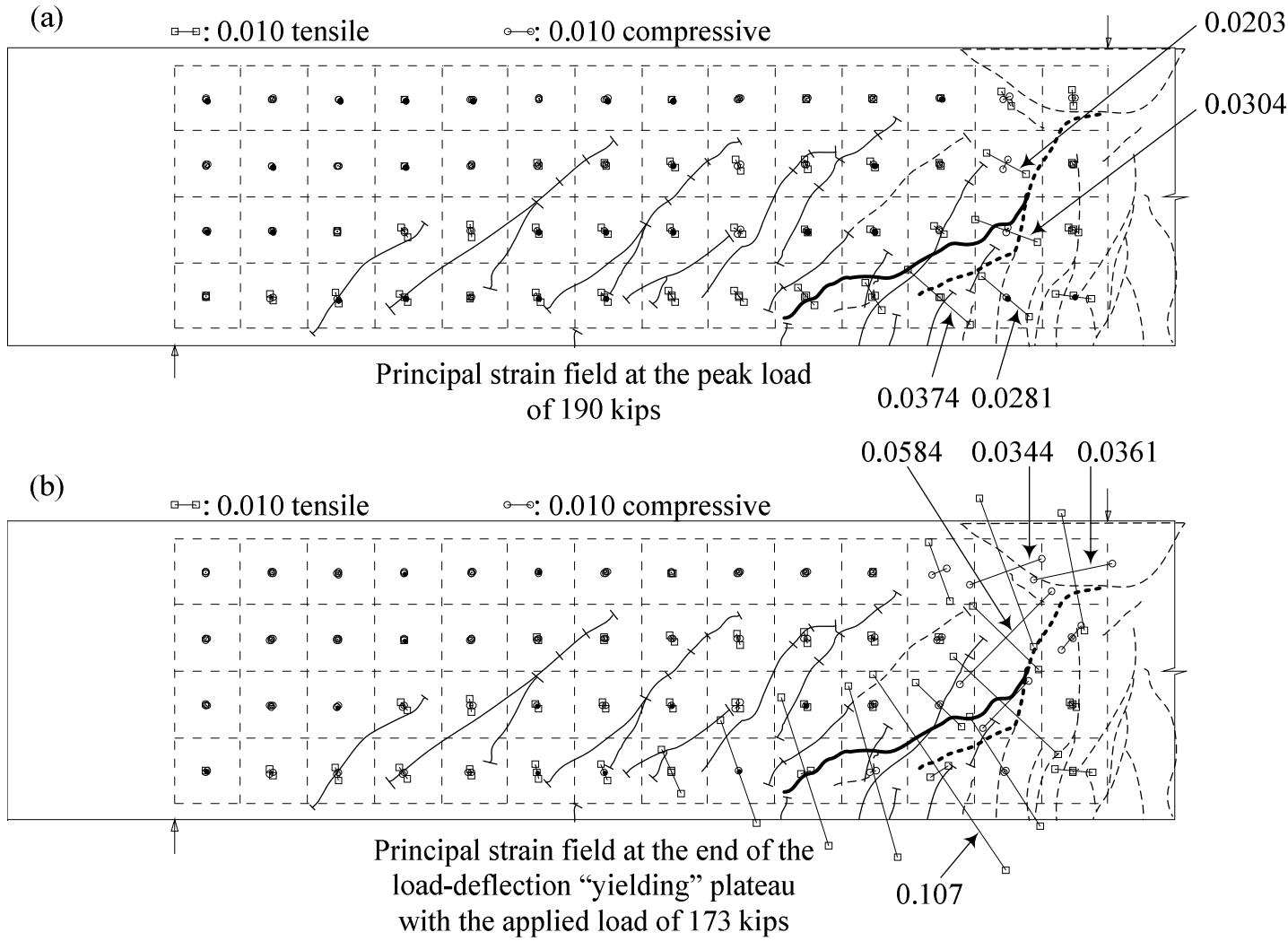

Fig. 4-144: Principal strain field at peak and failure load - Beam B27-3a 
(a)

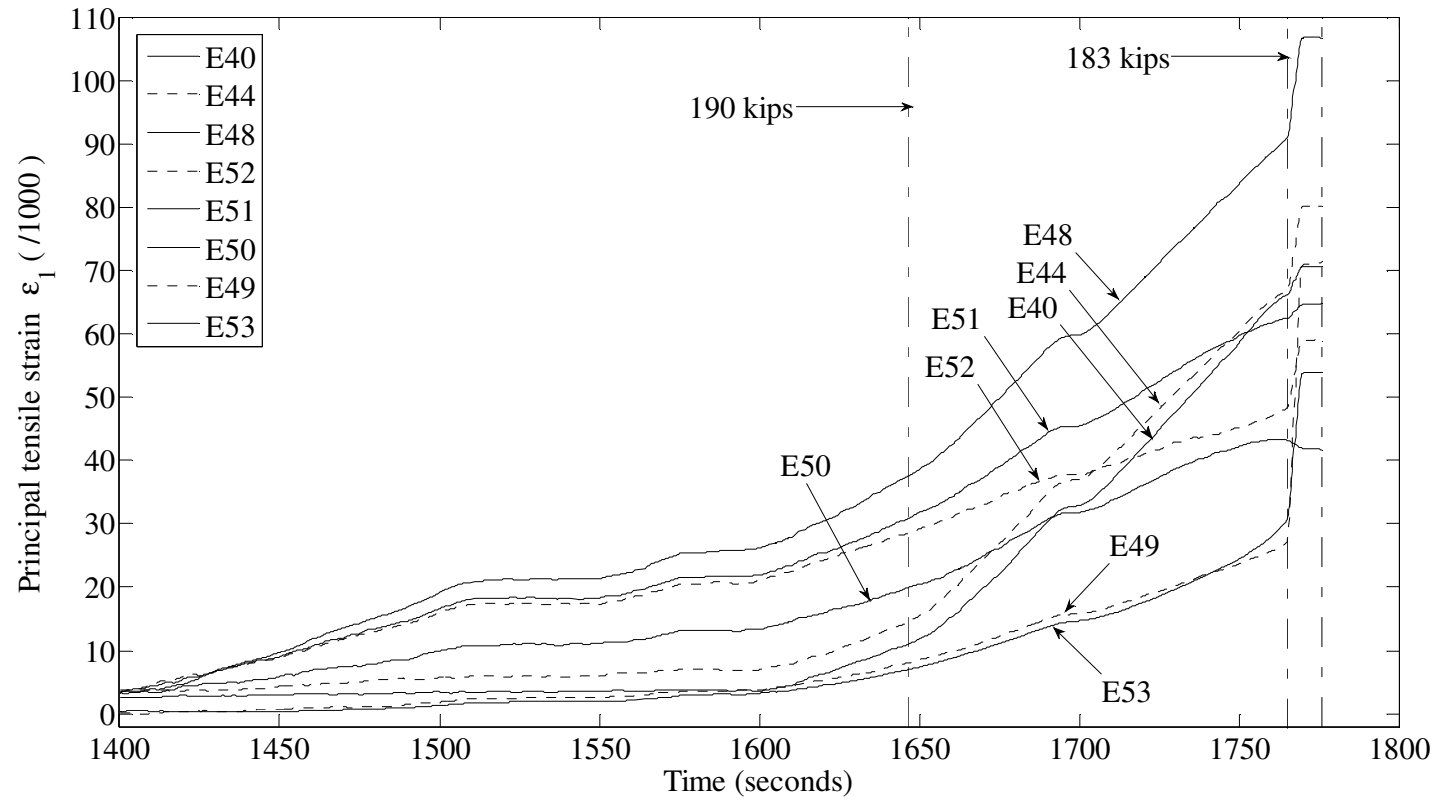

(b)

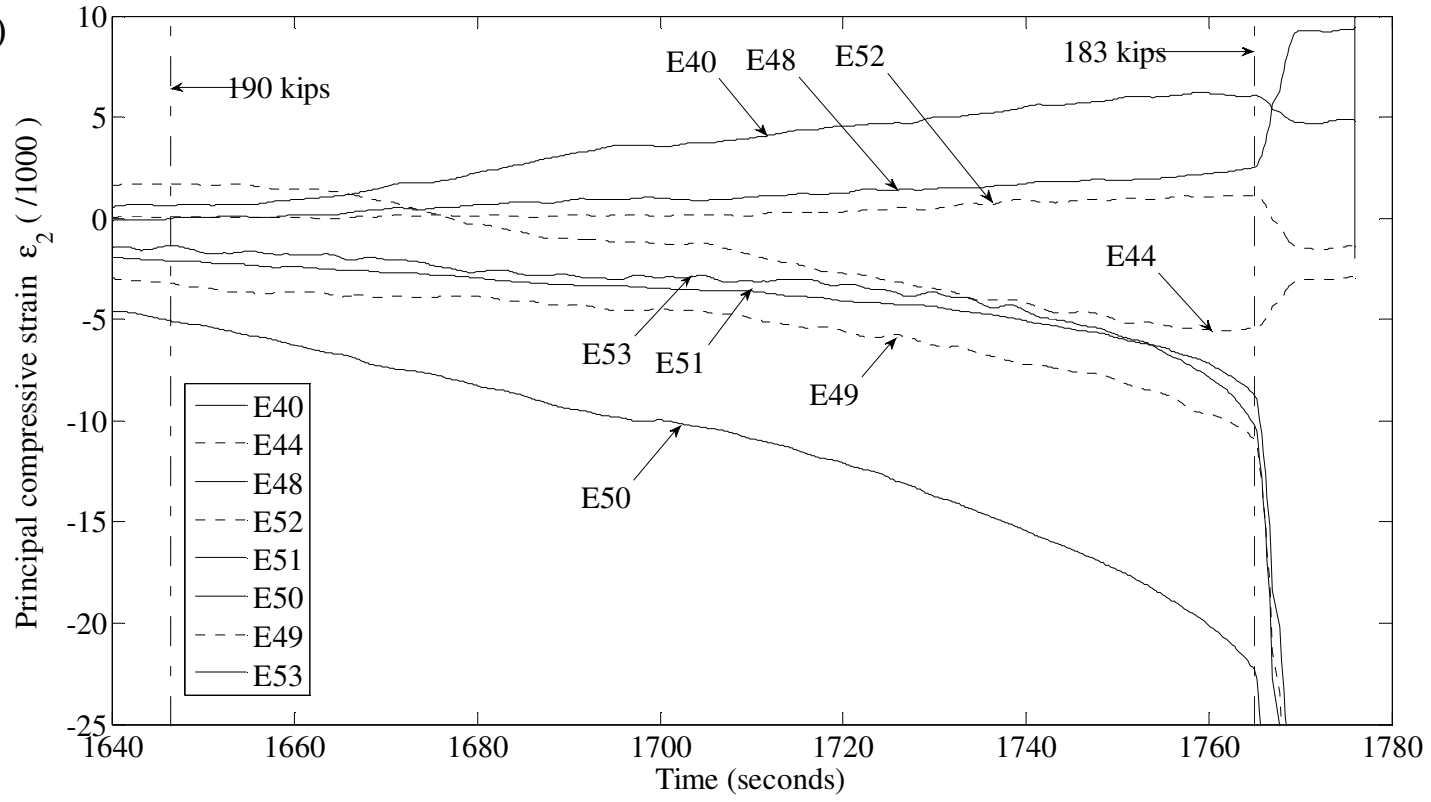

Fig. 4-145: Development of principal tensile and compressive strains along the critical inclined crack - Beam B27-3a 


\subsubsection{Beam B27-3b}

Load versus deflection relationship, crack pattern, and failure mode. Beam B27-3b had similar properties to Beam B27-3a. Therefore, Beam B27-3b behaved similarly to Beam B27-3a, as follows:

(a) The first inclined crack was observed at 100 kips at which no significant load-deflection stiffness reduction was observed (Fig. 4-146).

(b) The flexural-shear cracks spread evenly throughout the shear span (Fig. 4-146).

(c) The beam exhibited a yield plateau, starting at a load of approximately 180 kips, reaching a peak at 194 kips at approximately midway of the plateau, and ending at a load of 180 kips (Fig. 4-147).

While both beam exhibited extensive flexural yielding, the ultimate failure modes were quite different. While Beam B27-3a failed due to flexural action, Beam B27$3 \mathrm{~b}$ experienced a shear-compression failure. Because of the different failure mode, Beam B27-3b had a shorter "yield plateau". Although the beam deformation started to concentrate in the flexural cracks due to the yielding of the reinforcement, the yielding was not sufficient by itself to force a flexural failure of the compression zone. Together with the opening of the critical shear crack that appeared earlier, the yielding and diagonal compression was sufficient to cause crushing of the compression region to the left of the loading plate (Fig. 4-146). Although the failure modes of the two beams of the same pairs were apparently different, their beam strengths were quite similar, governed by the flexural strength of the beam.

Reinforcement strain and average bond stress. Compared with Beam B273a, the reinforcement straining was less severe (Fig. 4-149 and Fig. 4-150) and the bond stresses dropped at smaller loads (Fig. 4-151). For example, strain gauge S7 (instead of $\mathrm{S} 7$ and S8 as in Beam B27-3a) was strained beyond the steel yield plateau at the peak load (Fig. 4-149). At the failure load, both S7 and S8 were in the strain-hardening region, reaching values of 0.020 and 0.015 , respectively. The average bond stress reached a maximum value of 500 psi (Fig. 4-151). 
Concrete strain field. Fig. 4-152 shows that the longitudinal, transverse, and shear strain demand was significant at the peak load, but still less than those in Beam B27-3a at the same load. The maximum longitudinal tensile strain was up to 0.015 at Element E52 (compared with 0.032 in Beam B27-3a), while the maximum compressive strain was up to 0.0057 at Element E49 (compared with 0.0046 in Beam B27-3a). The transverse strain was not very significant, only 0.0045 (compared with 0.016 in Beam B27-3a). Similarly, the maximum shear strain was only 0.012 (compared with 0.037 in Beam B27-3a). The total transverse and shear demand was therefore smaller than that of Beam B27-3a (see Fig. 4-154).

Similar to Beam B27-3a, Beam B27-3b experienced a concrete crushing to the left of the loading point. The longitudinal compressive strain was less severe, reaching the maximum value of 0.0016 near the top fibers. A crushing might have occurred this failure load, but the crushed concrete was still able to transfer some compressive stresses to other region. With this less excessive compressive strain demand, the transverse strain was in the region was also less.

Fig. 4-155a clearly shows that the critical crack was grew from Element E36, to Elements E39 and 42 and penetrated through Element E49. Compared with Beam B273a, the magnitude of principal tensile strains was less. At the final failure load of 190 kips, after the peak load (Fig. 4-155b), the principal compressive strain of Element 49 jumped from 0.010, which is already a significant compressive strain, to 0.0356, indicating a complete failure. From the strain measurements in Beams B27-3a \& b, it can be said that 0.010 is a reasonable estimate of the failure compressive strain for this type of material. The principal tensile strains of all the concrete elements also increased suddenly at the failure load of 190 kips. 


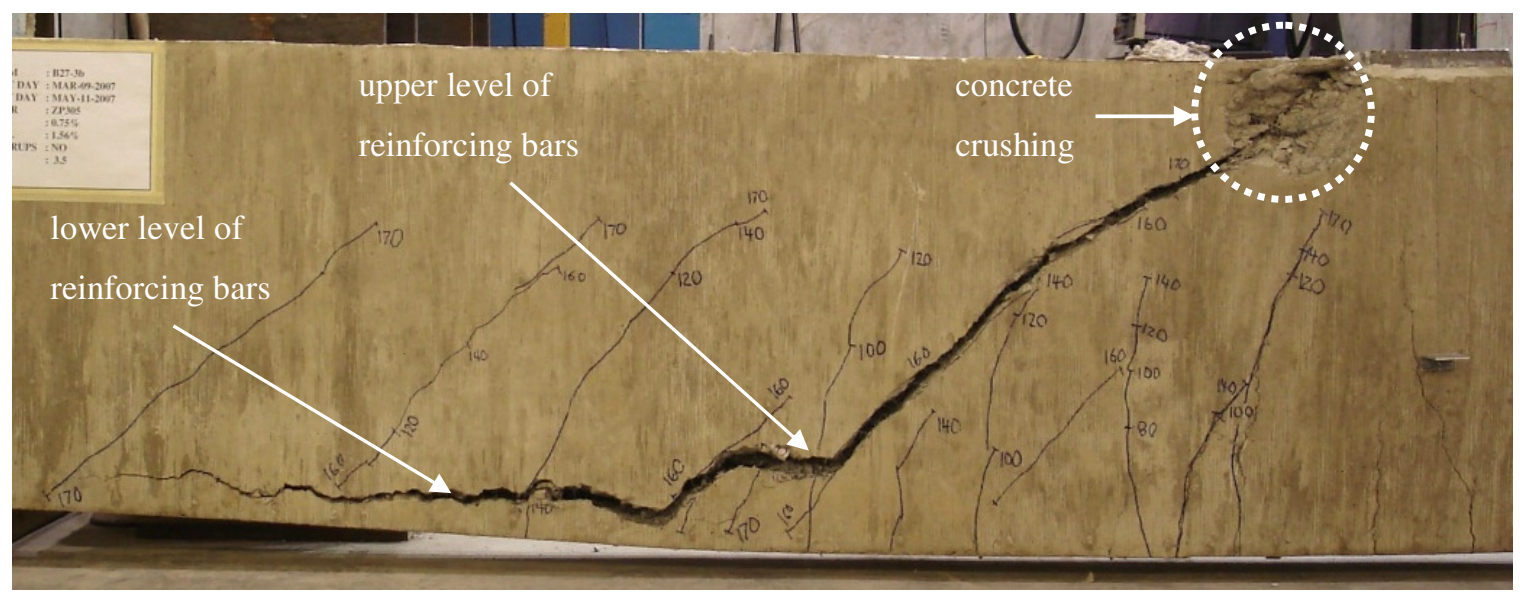

Fig. 4-146: Crack pattern after failure - Beam B27-3b

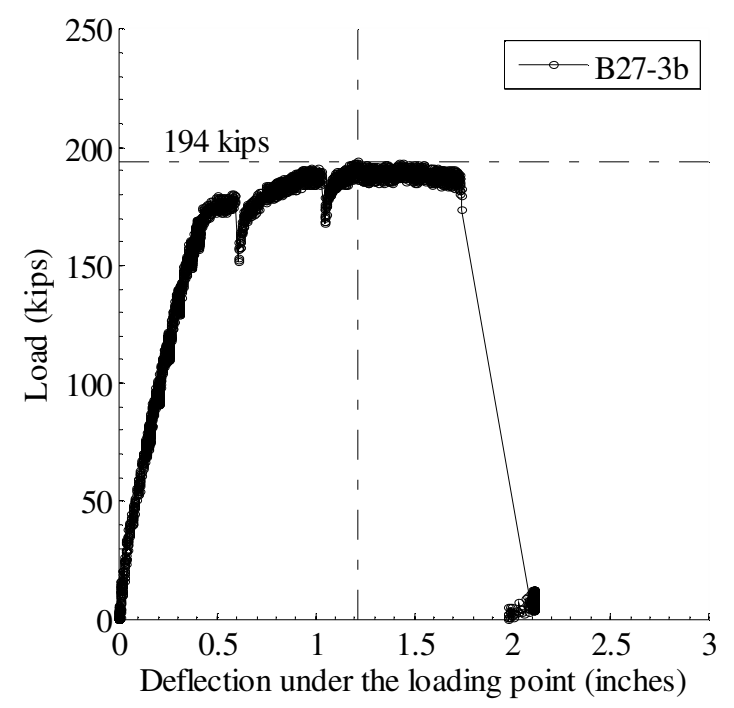

Fig. 4-147: Load versus deflection relationship - Beam B27-3b

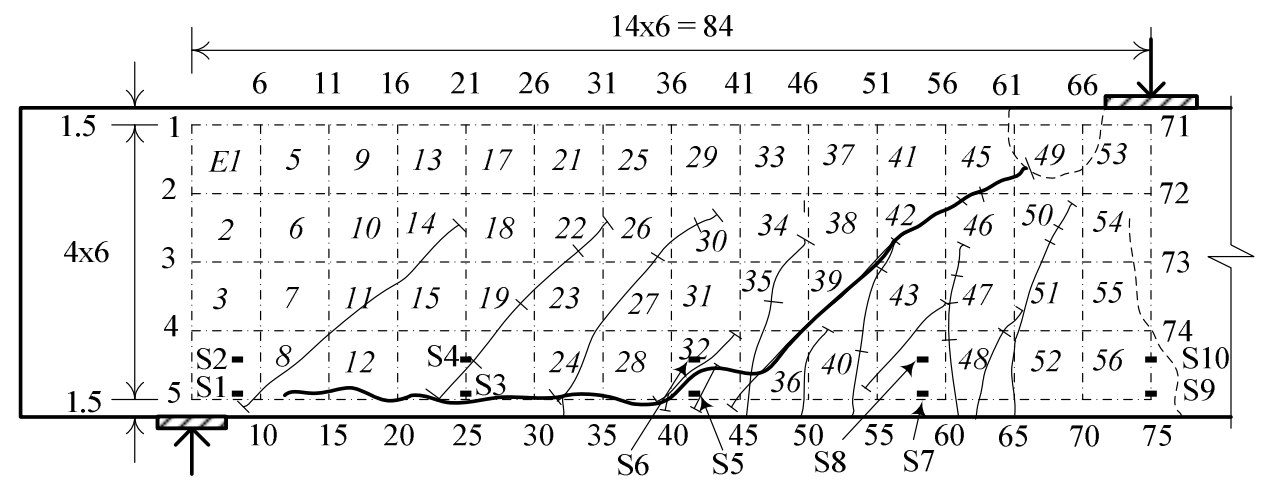

Fig. 4-148: Numbering of markers, elements, and strain gauges - Beam B27-3b 


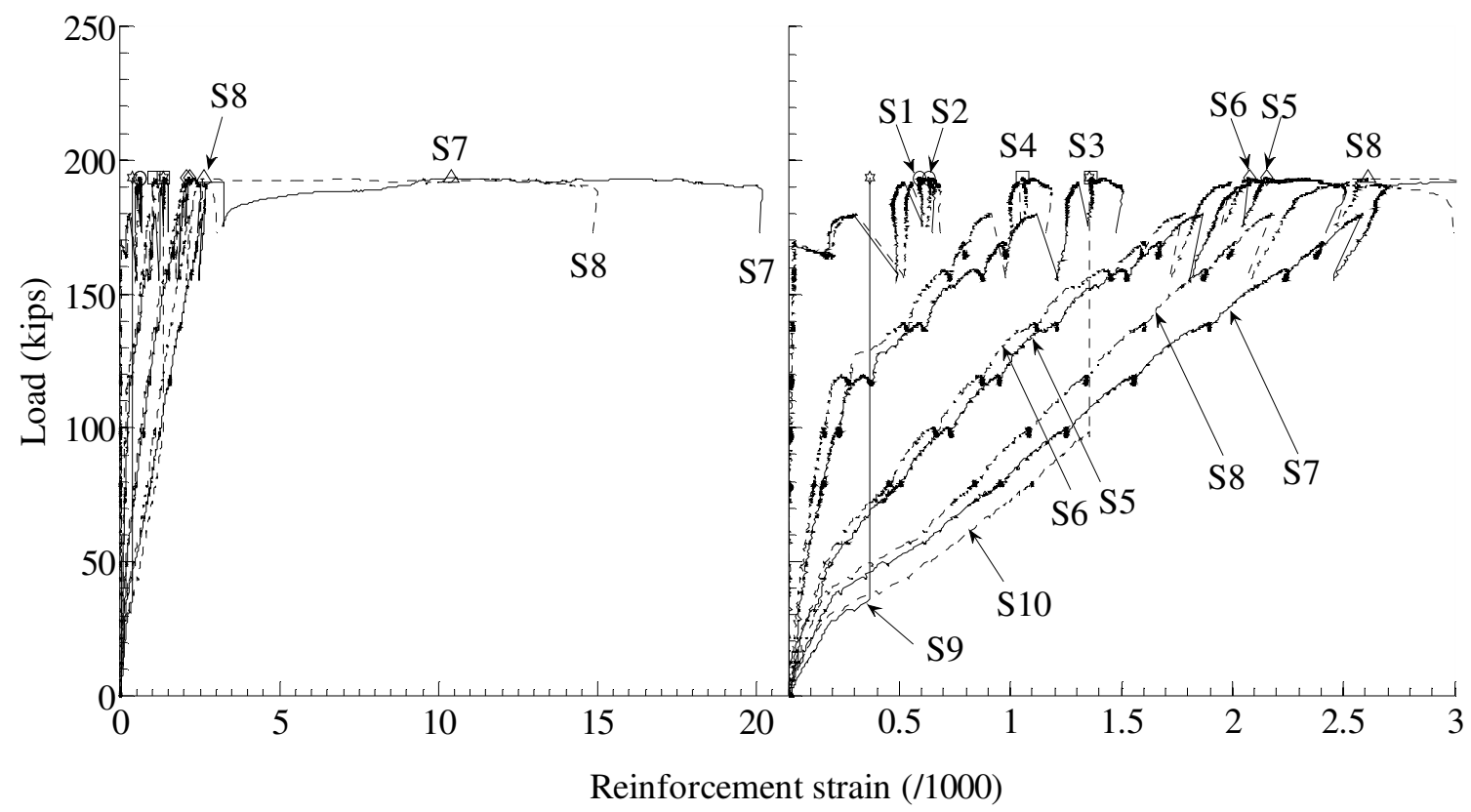

Fig. 4-149: Load versus reinforcement strain relationships - Beam B27-3b

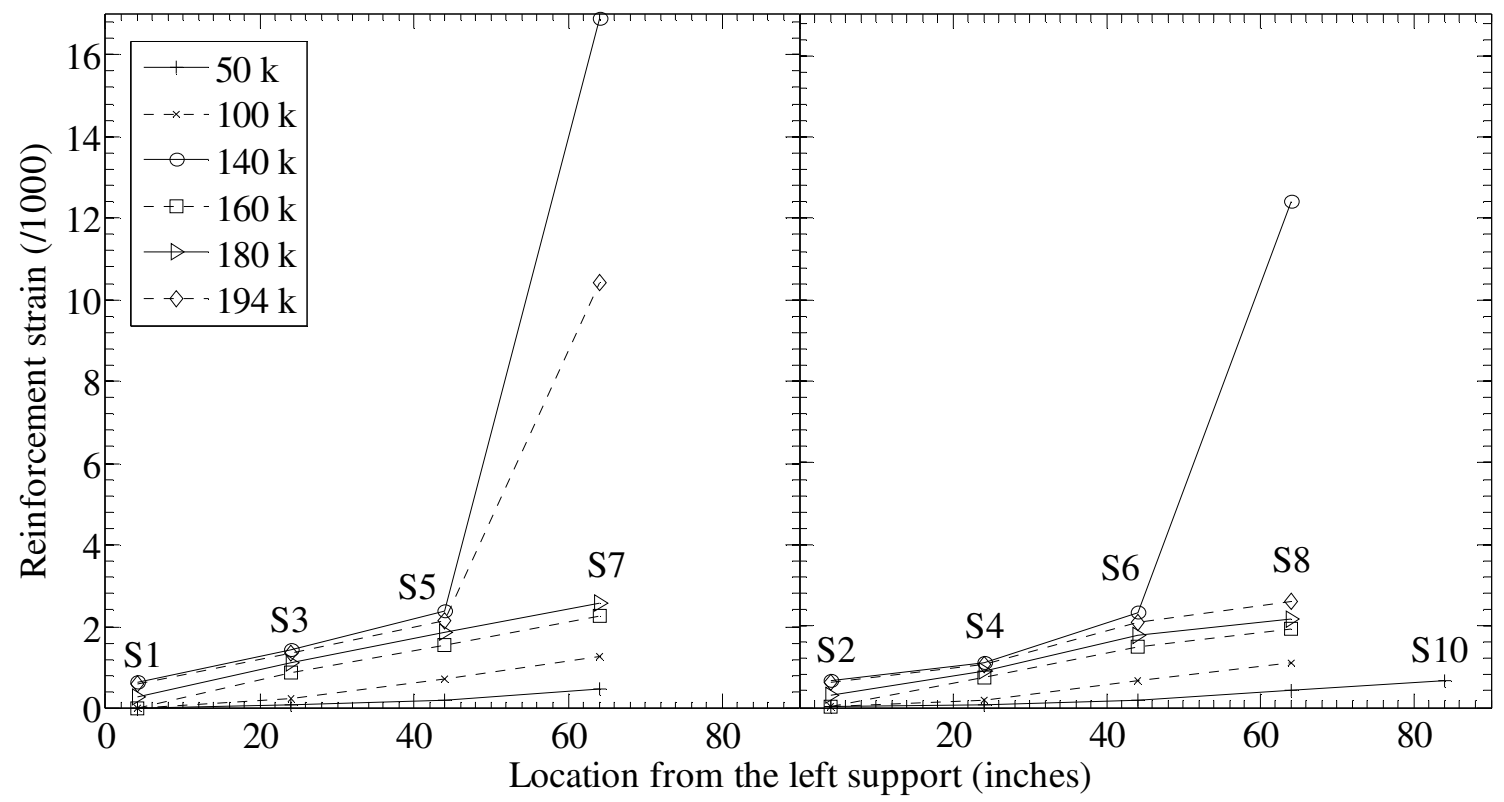

Fig. 4-150: Reinforcement strains along the beam axis - Beam B27-3b 


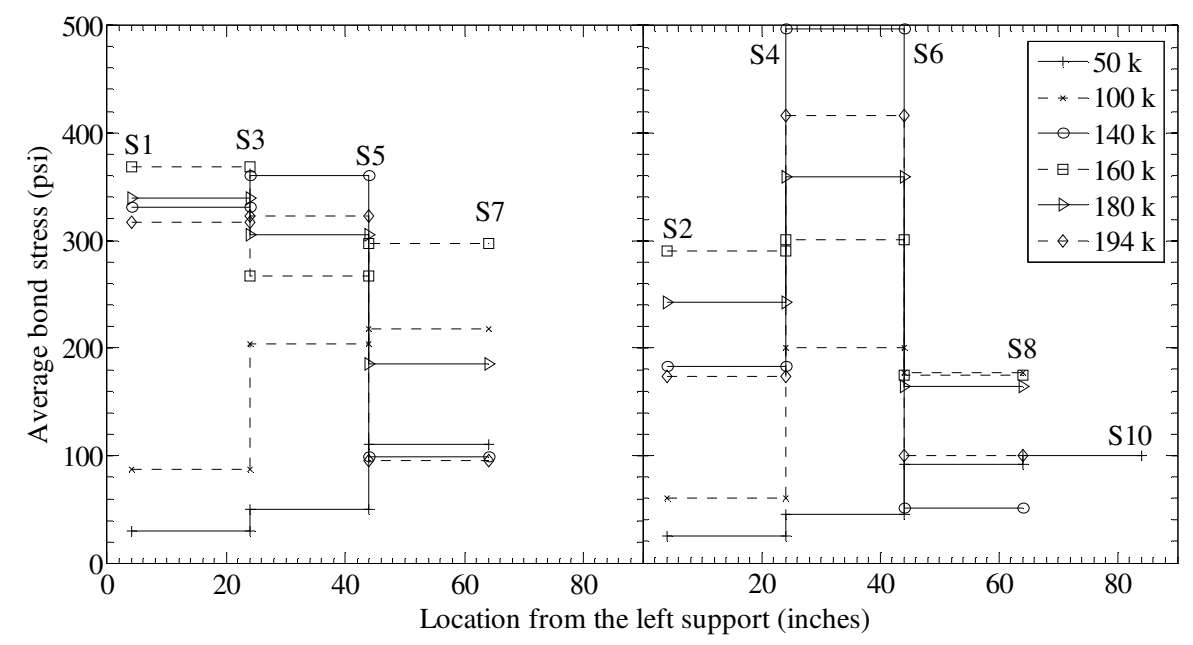

Fig. 4-151: Average bond stress along the beam axis - Beam B27-3b

(a)

$\begin{array}{lllllllllllllll}0.51 & -0.21 & 0.19 & -0.49 & 0.07 & -0.87 & -0.76 & -0.56 & -1.4 & -1.5 & -1.2 & -3.1 & -5.7 & -3.9\end{array}$

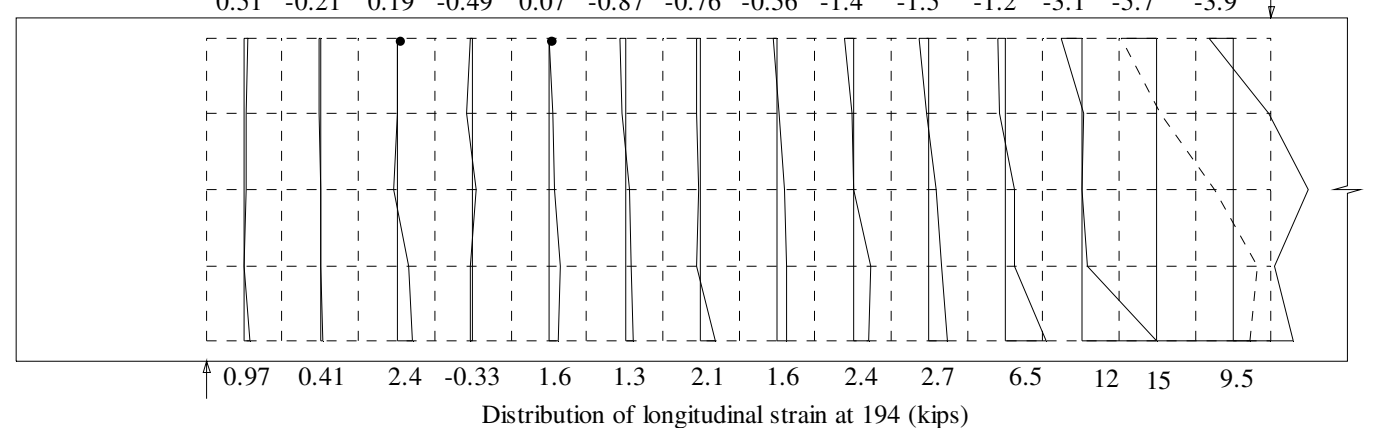

(b)

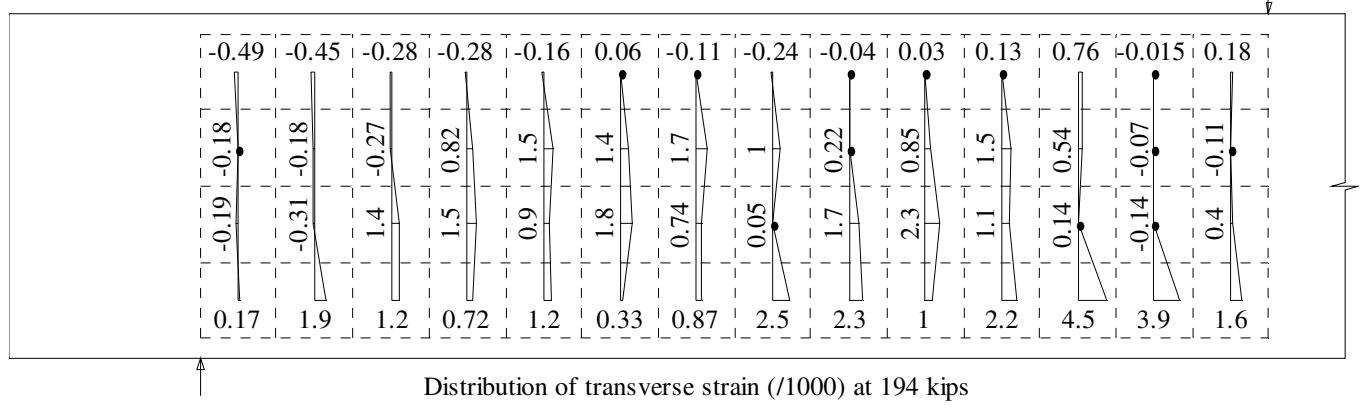

(c)

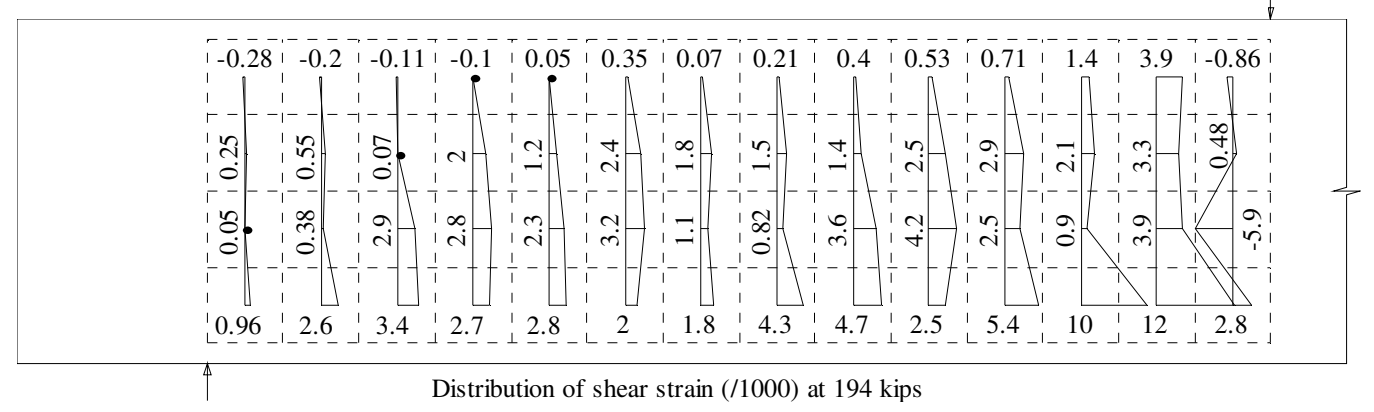

Fig. 4-152: Distribution of longitudinal, transverse, and shear strains at peak load - Beam B27-3b 
(a)

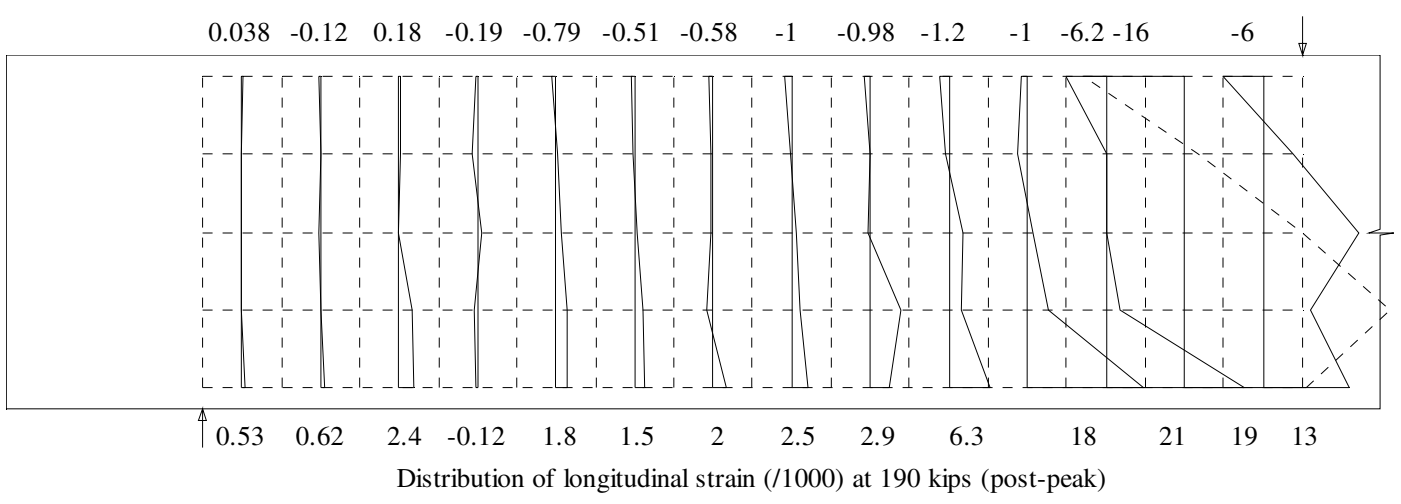

(b)

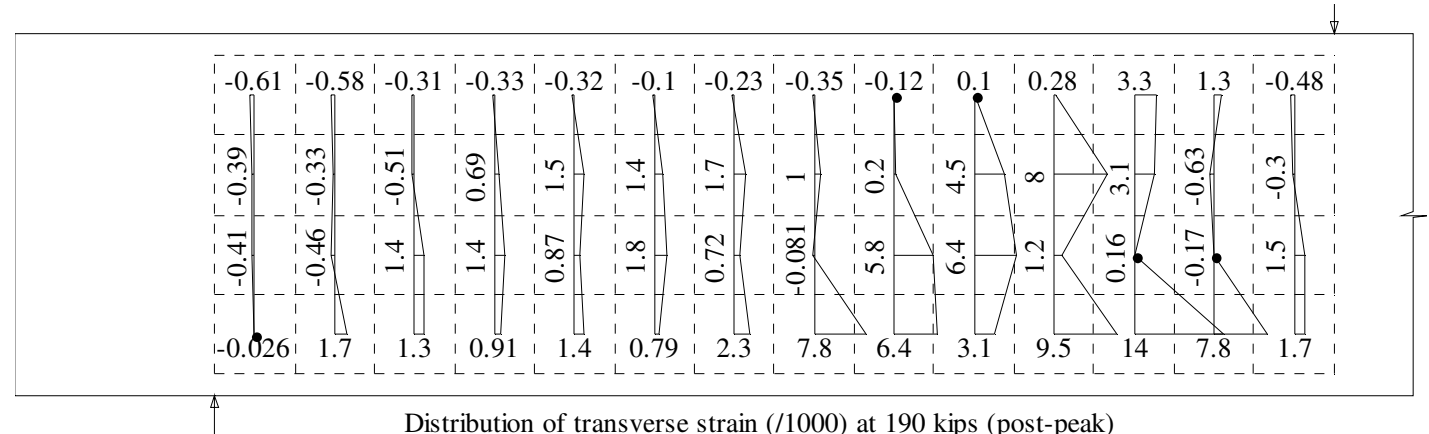

Distribution of transverse strain (/1000) at 190 kips (post-peak)

(c)

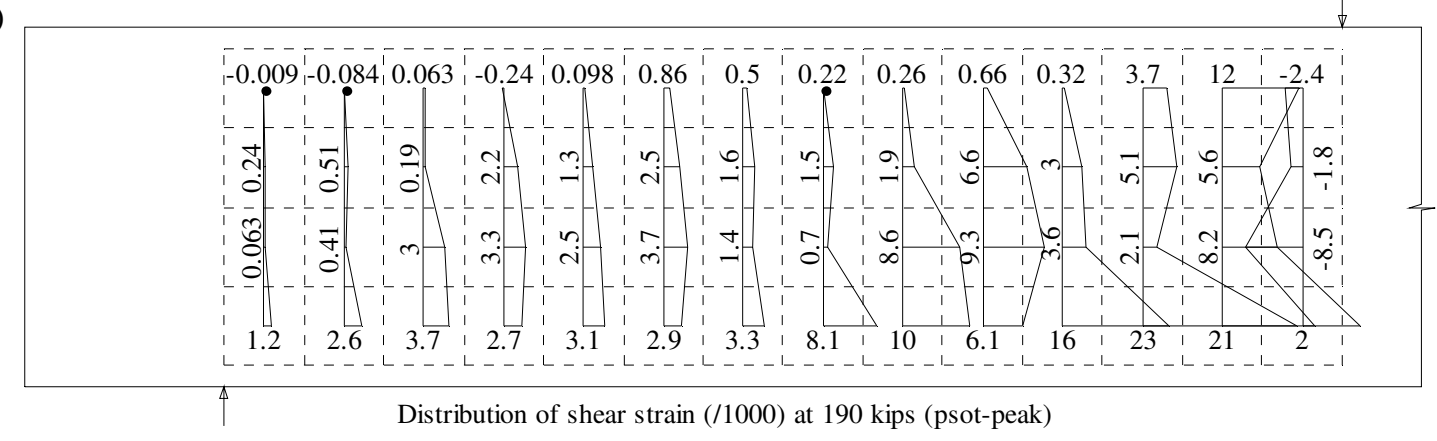

Fig. 4-153: Distribution of longitudinal, transverse, and shear strains at failure load Beam B27-3b 
(a)

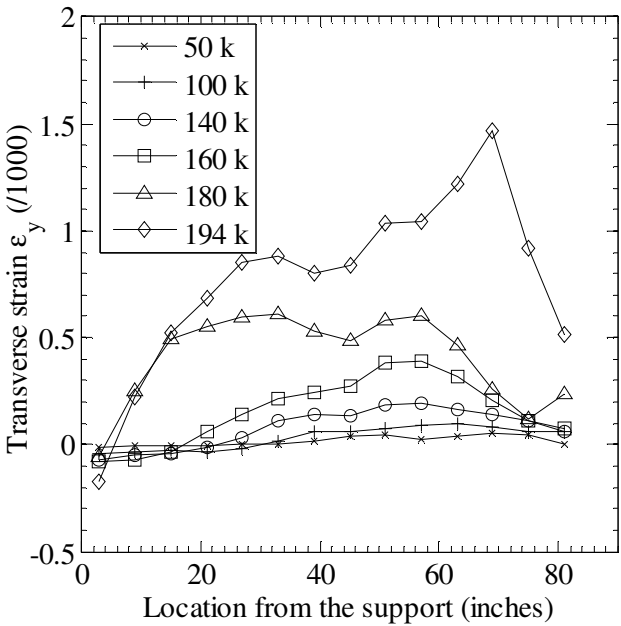

(b)

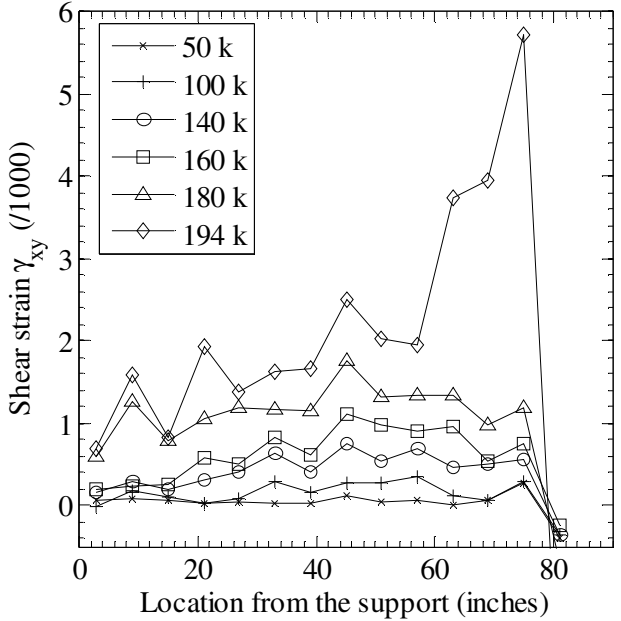

Fig. 4-154: Distribution of transverse and shear strains averaged over the beam depth - Beam B27-3b

(a)

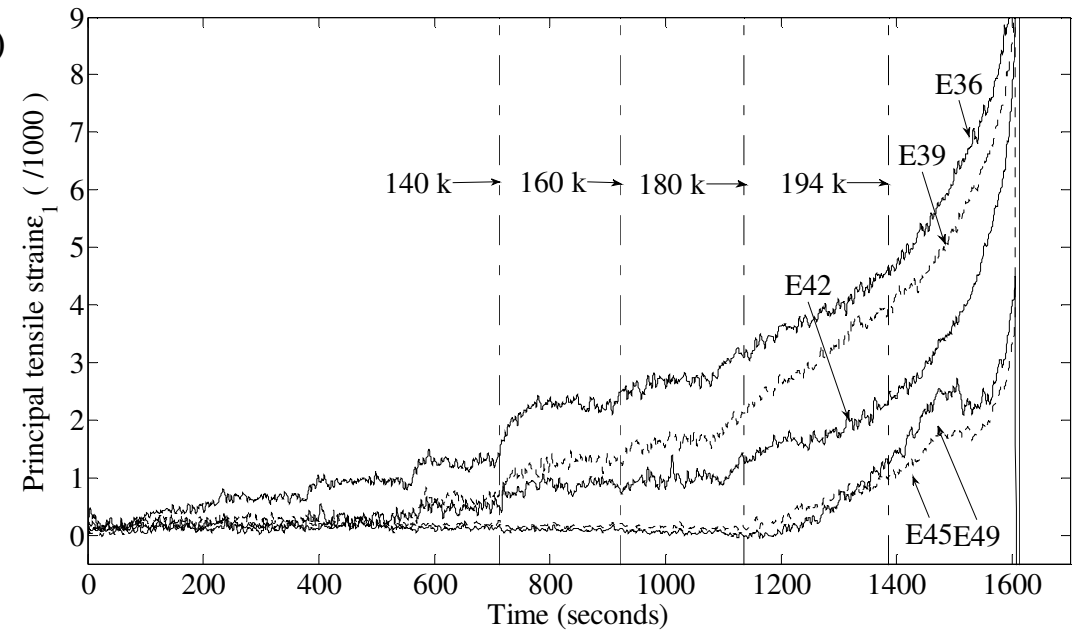

(b)

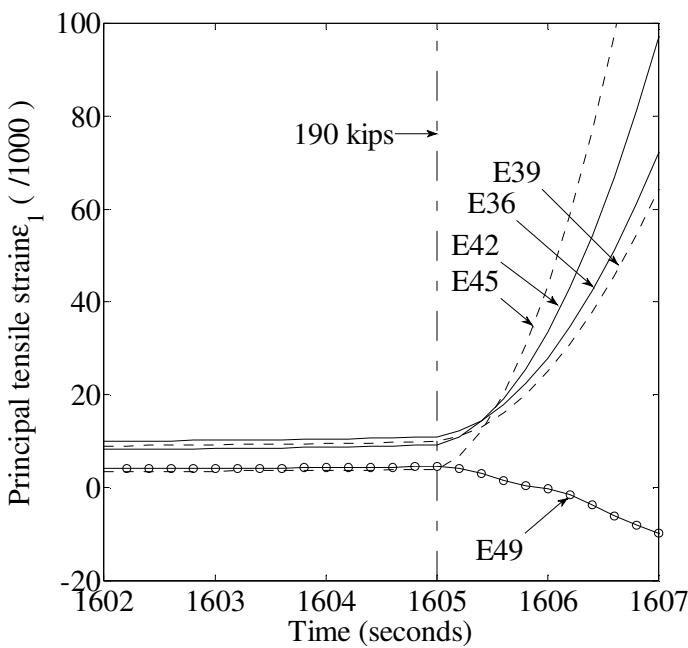

(c)

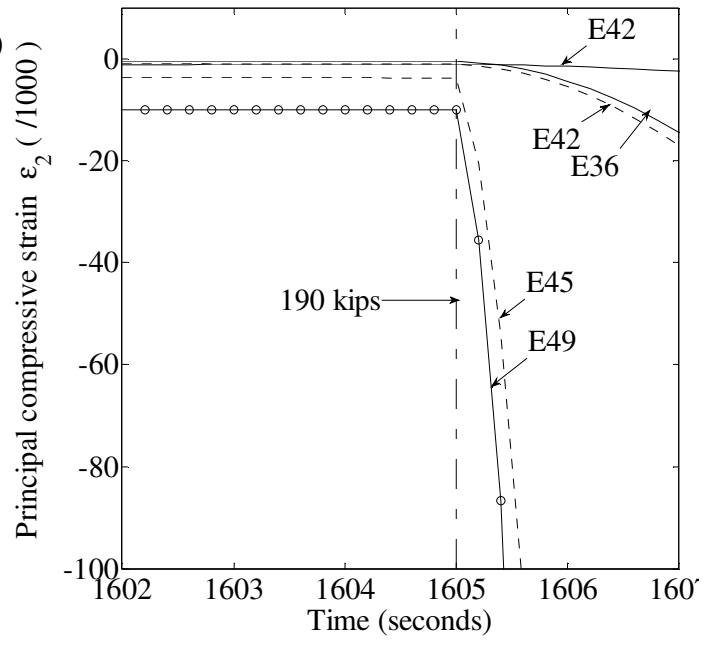

Fig. 4-155: Development of principal tensile and compressive strains along the critical inclined crack - Beam B27-3b 


\subsubsection{Beam B27-4a}

Beam B27-4a was similar to Beams B27-2a \& b $(0.75 \%$ volume fraction of RC80/60BN fibers), except that Beam B27-4a had a lower amount of longitudinal reinforcement (1.56\% compared with 2.06\%). Beam B27-4a was also similar to Beams B27-3a \& b, except that it contained RC80/60BN fibers (instead of ZP305) with a longer length of 2.36 inches and a larger diameter of 0.03 inches, corresponding to an aspect ratio of 80 .

Load versus deflection relationship, crack pattern, and failure mode. Fig. 4-156 shows the crack pattern for Beam B27-4a. Similar to Beams B27-2a \& b, which also contained long fibers $(\mathrm{RC} 80 / 60 \mathrm{BN})$, the number of inclined cracks was less than that in Beams B27-1a \& b and B27-3a \& b, which contained shorter and smaller fibers (ZP305). These inclined cracks did not spread over the shear span region like those in Beams B27-1a \& b and B27-3a \& b. In addition, the inclined cracks had a shallower angle from the beam longitudinal axis.

Fig. 4-157 shows the load versus deflection relationship of Beam B27-4a. It can be seen that there was a smooth decay in load-deflection stiffness throughout the test. This observation was made for Beams B27-2a \& b, which also contained the RC80/60BN fibers. The ultimate strength of Beam B27-4a was significant lower, only 149 kips, compared with the first three pairs of 27-inch deep beams. It should be noted that Beams B27-1a \& b and B27-3a \& b, which contained the same type of fibers (ZP305) but different longitudinal reinforcement ratio, had comparable strengths (approximately 190 kips). This was not the case for Beams B27-2a \& b and Beam B27-4a. They all contained RC80/60BN fibers but had a different longitudinal reinforcement ratio and failed at significantly different loads (194 kips compared with 149 kips).

With cracking along the reinforcing bars and no observed concrete crushing, the beam failure mode was a combination of shear-tension and diagonal-tension.

Reinforcement strain and average bond stress. Compared with Beams B27-

$3 a \& b$, the longitudinal reinforcement strain of Beam B27-4a was very limited, mostly in 
the linear range (Fig. 4-157b). There was a jump in reinforcement strain at S3 and S4 for a load as small as 72 kips. This led to a drop of bond stress between S3-S5 (and S4-S6) and a corresponding jump in bond stress between S1-S3 (and S2-S4)Error! Reference source not found. The bond in the region S1-S3 (S2-S4) kept increasing up to 250 psi (200 psi) prior a drop, which led to an increase in reinforcement strain at S1 (S2) at a load of 110 kips. It can be seen that the developed bond stress was small compared with that of Beams B27-2a and b, which also contained RC80/60BN fibers. This was probably due to the fact that the fibers were so long that they may have restrained good compaction of concrete around the reinforcement. During concrete casting, fibers stuck on top of the reinforcement were noticed. Fig. 4-156 actually shows small voids along the upper layer of tension reinforcement and a concrete strip popped out in between the two layers of reinforcement. These signs were the indication of weak consolidation at the reinforcement level.

Concrete strain field. Fig. 4-159 shows the distribution of longitudinal, transverse, and shear strain. The maximum compressive strain was only 0.0038 , while maximum transverse and shear strains were 0.015 and 0.011 radians, respectively.

Fig. 4-160 shows the principal strain field at the peak load. It can be seen that the principal compressive strain was small throughout the beam, less than 0.002 . The principal tensile strain (Fig. 4-161a) was the largest for the bottom element E25 throughout the test, decreasing toward E28 and E35. At the load of 144 kips, after the peak load, the strains of all concrete elements increased significantly, defining a critical failure state (Fig. 4-161b). 


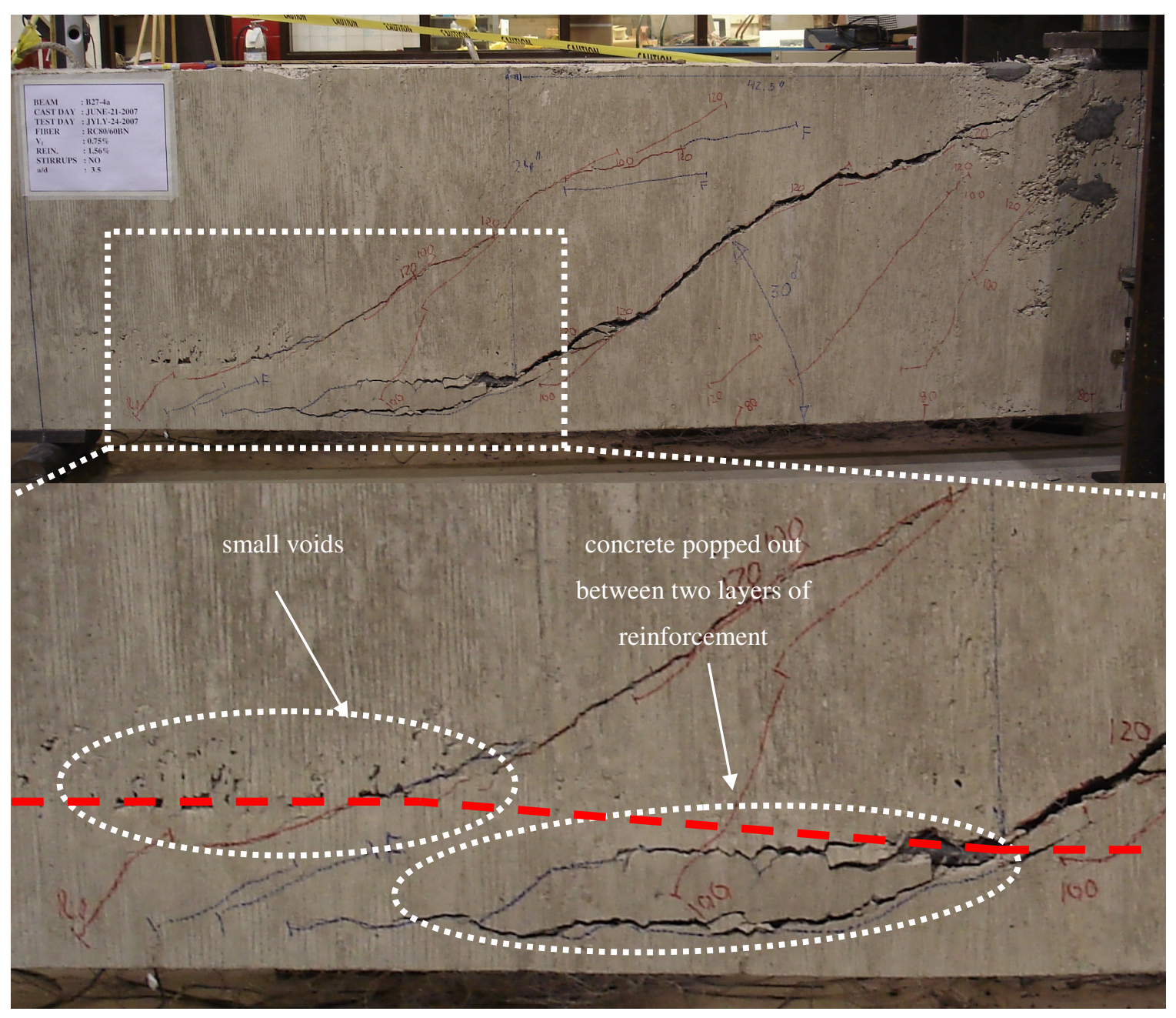

Fig. 4-156: Crack pattern after failure - Beam B27-4a 
(a)

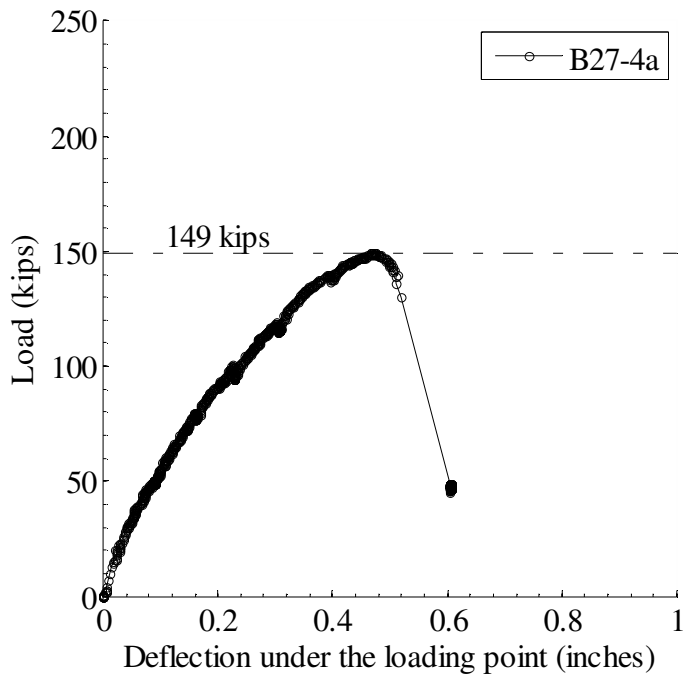

(b)

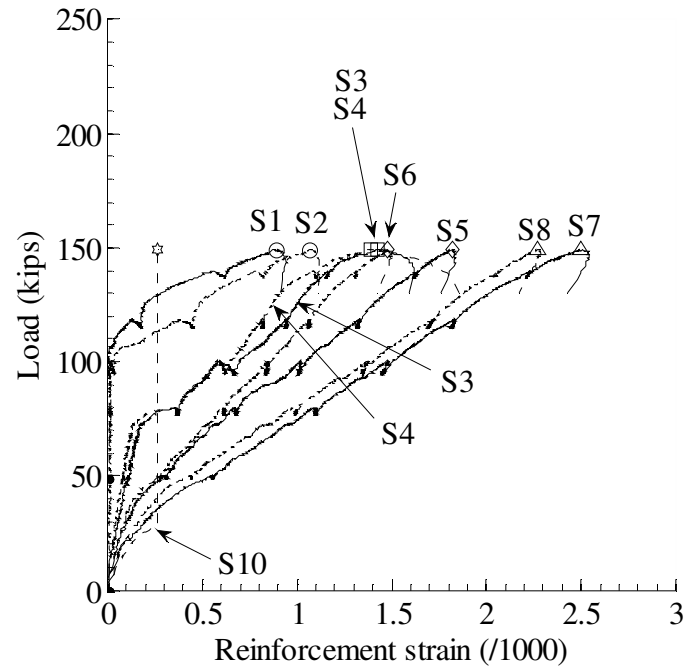

Fig. 4-157: Load versus deflection and reinforcement strain relationships - Beam B27-4a

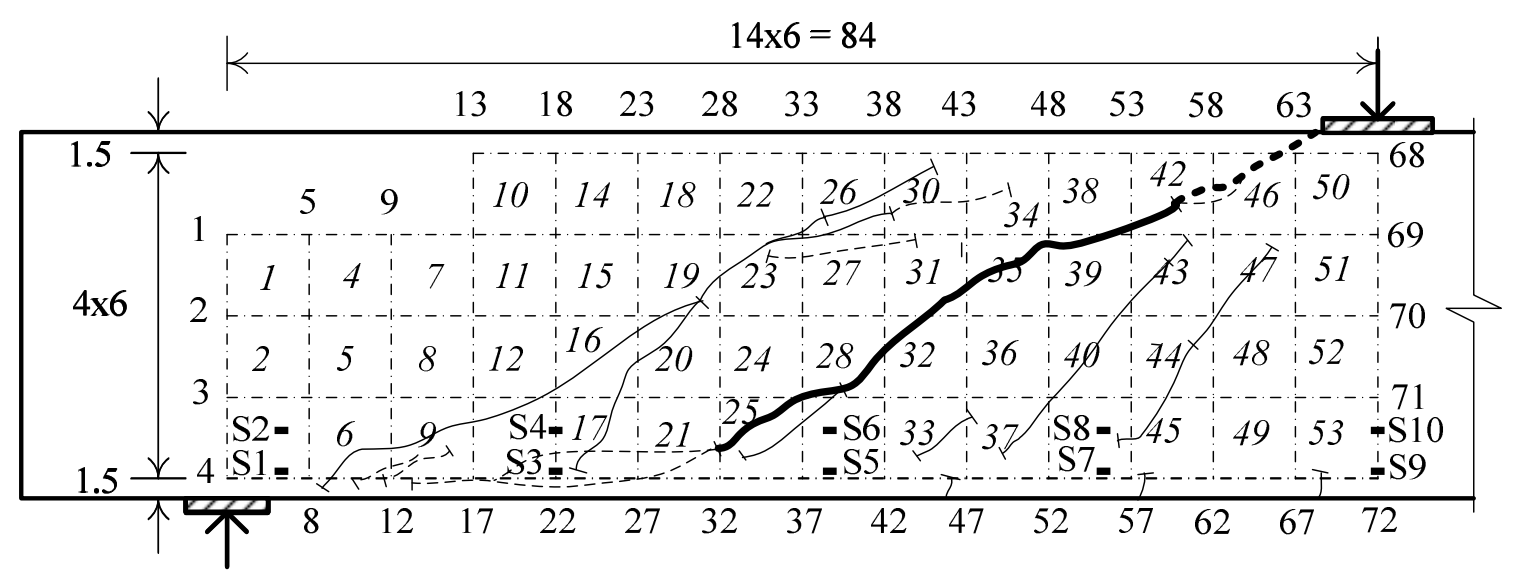

Fig. 4-158: Numbering of markers, elements, and strain gauges - B27-4a 
(a)

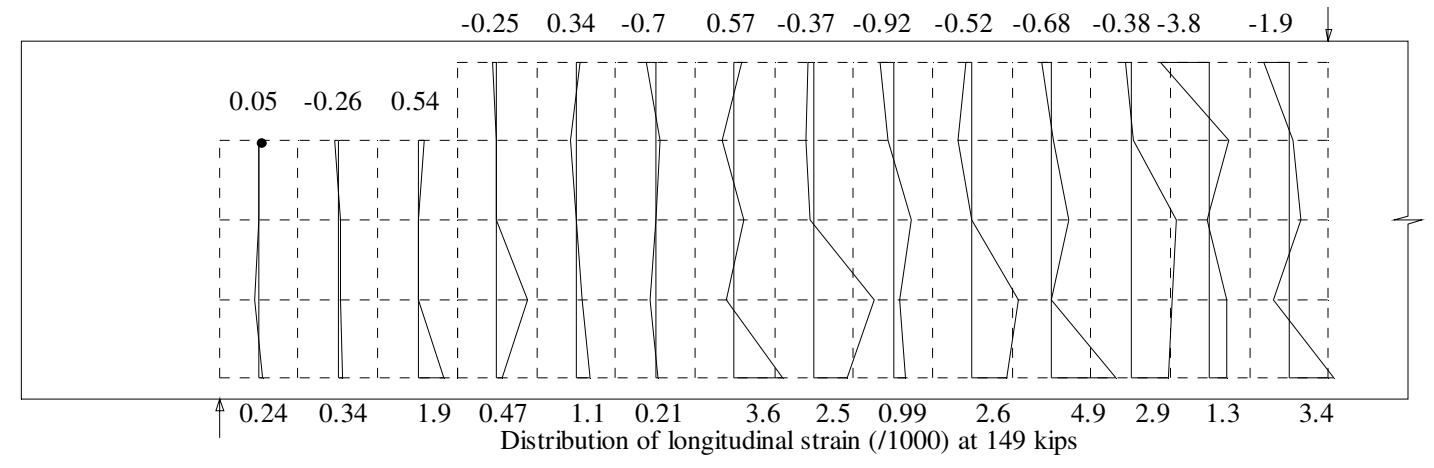

(b)

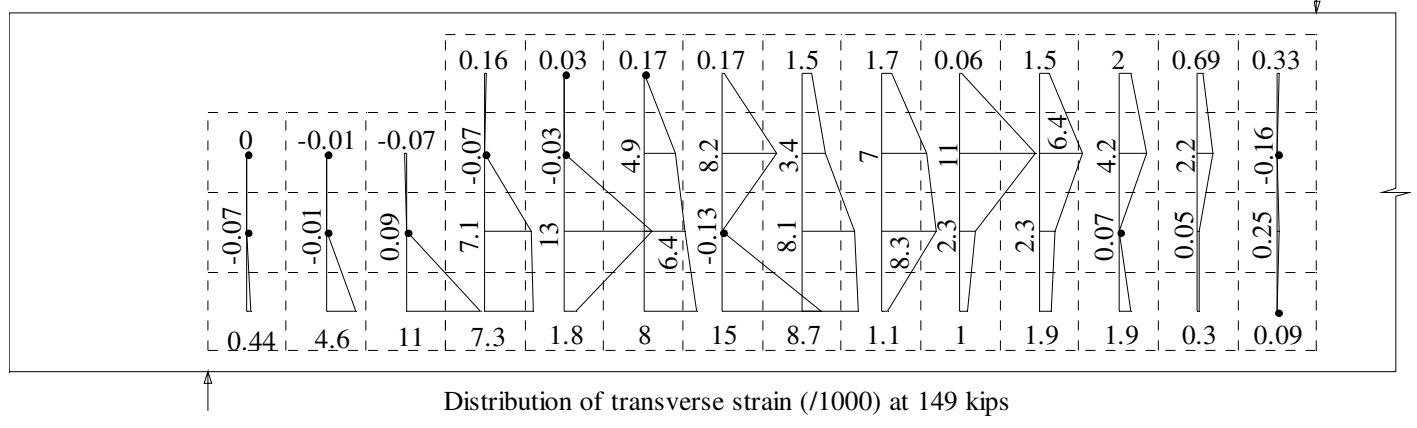

(c)

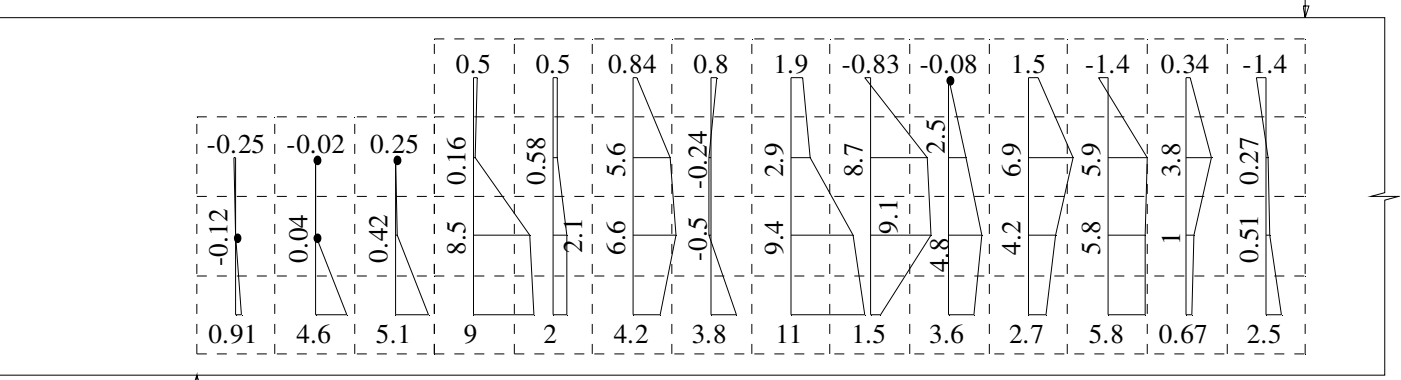

Distribution of shear strain (/1000) at 149 kips

Fig. 4-159: Distribution of longitudinal, transverse and shear strains at $149 \mathrm{k}-$ Beam B27-4a

: 0.001 tensile $\quad 0.001$ compressive

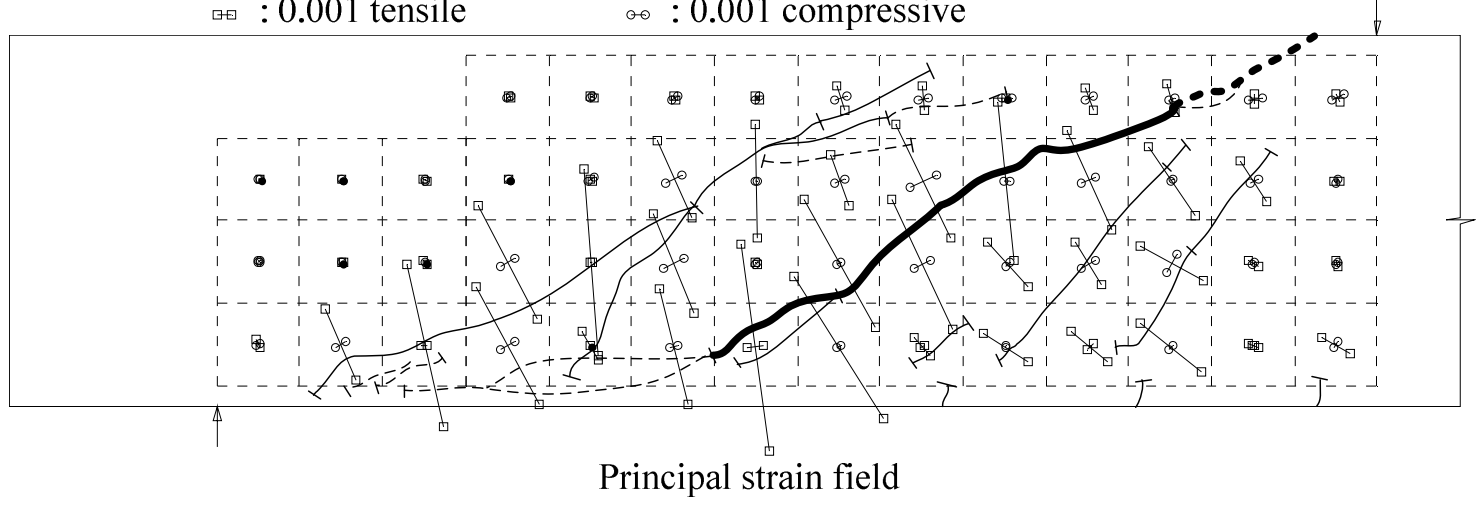

Fig. 4-160: Principal strain field at 149 kips - Beam B27-4a 
(a)

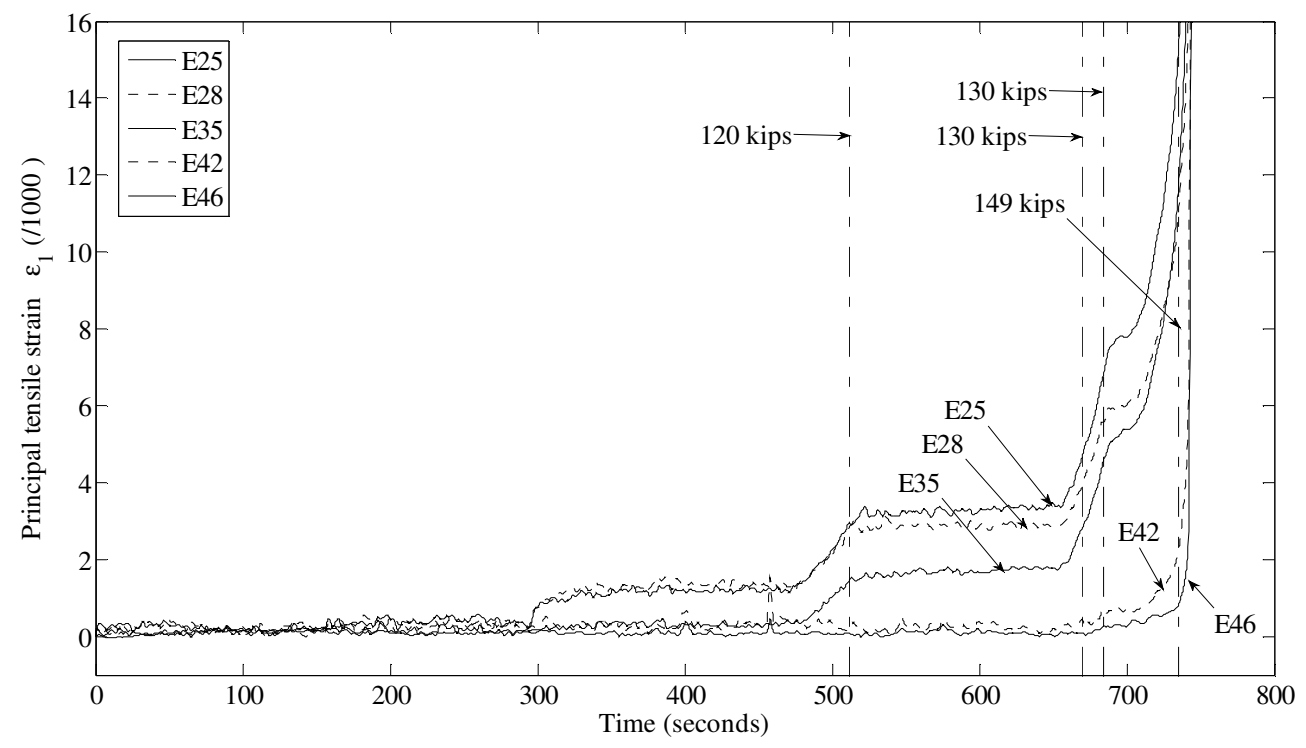

(b)

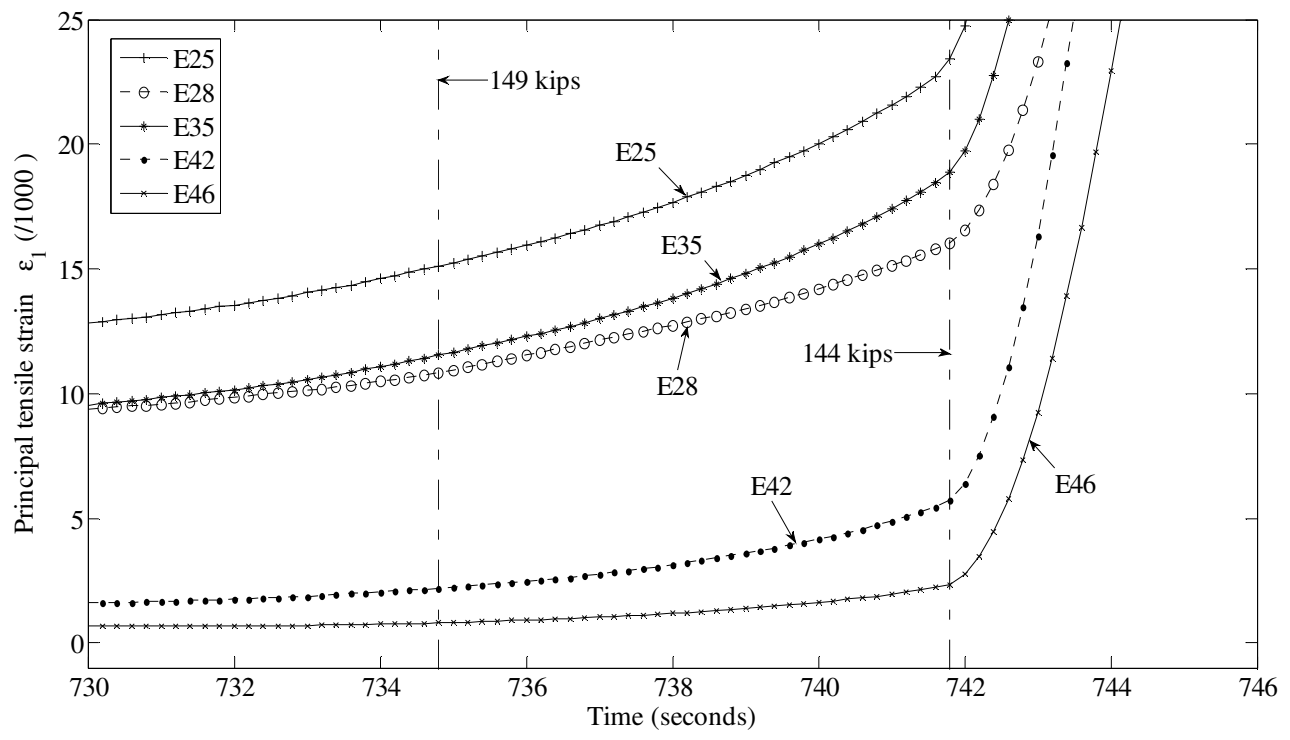

Fig. 4-161: Development of principal tensile strains along the critical inclined crack-

Beam B27-4a 


\subsubsection{Beam B27-4b}

Load versus deflection relationship, crack pattern, and failure mode. Beam B27-4b had identical properties as Beam B27-4a. The behavior of this beam was very similar to that of Beam B27-4a, except that it had the ultimate strength of 125 kips (Fig. 4-162a), which was even lower than that of Beam B27-4a. The reason was that Beam B27-4b had a defect due to a poor concrete consolidation along the longitudinal reinforcement level (Fig. 4-163).

Reinforcement strain and average bond stress. The reinforcement strain was limited, less than 0.002 (Fig. 4-162b). The number of inclined cracks was also less than that of B27-4a. The first diagonal crack developed at a load of 80 kips and the maximum bond stress developed was 300 psi (Error! Reference source not found.). The bond between S3 and S5 (and between S4 and S6) was very small when the beam approached its maximum strength. This was due to the longitudinal splitting crack in this region (Fig. 4-164).

Concrete strain field. The maximum compressive longitudinal strain was 0.0022 (Fig. 4-165a). The maximum transverse and shear strains mostly concentrated in elements along the critical crack, reaching maximum values of 0.019 and 0.016 radians, respectively (Fig. 4-165b \& c).

The principal tensile strain concentrated along the critical inclined crack (Fig. 4-166). Similar to Beam B27-4a, the wide opening of the critical crack proceeded from the reinforcement level to the compression region (see Fig. 4-167a). The beam failure occurred at the load of 124 kips after reaching the peak load (Fig. 4-167b). 
(a)

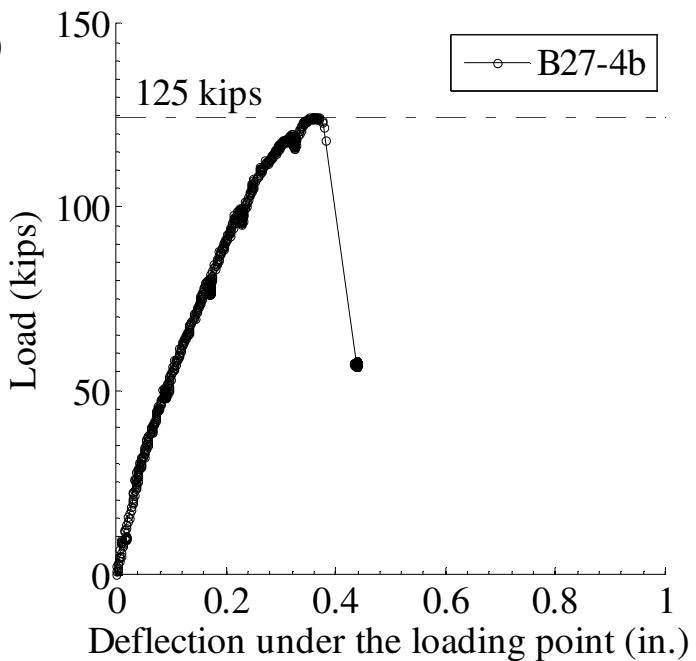

(b)

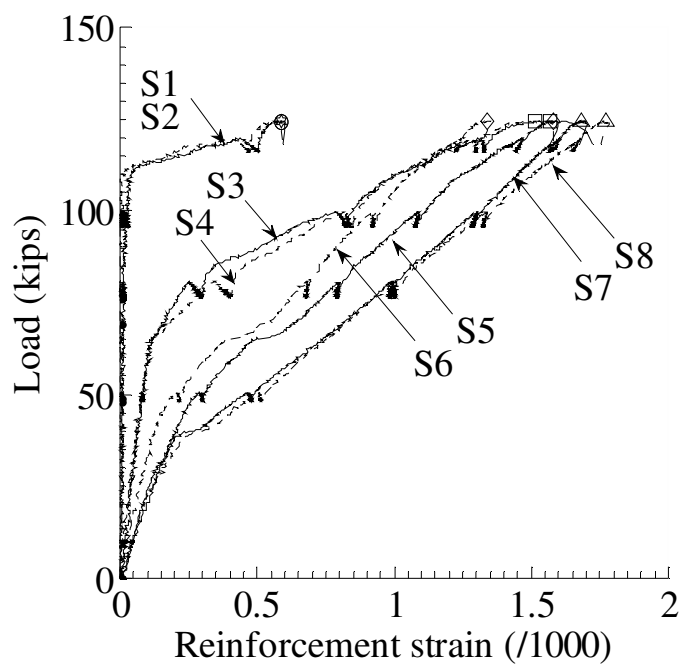

Fig. 4-162: Load versus deflection and reinforcement strain relationships - Beam B27-4b

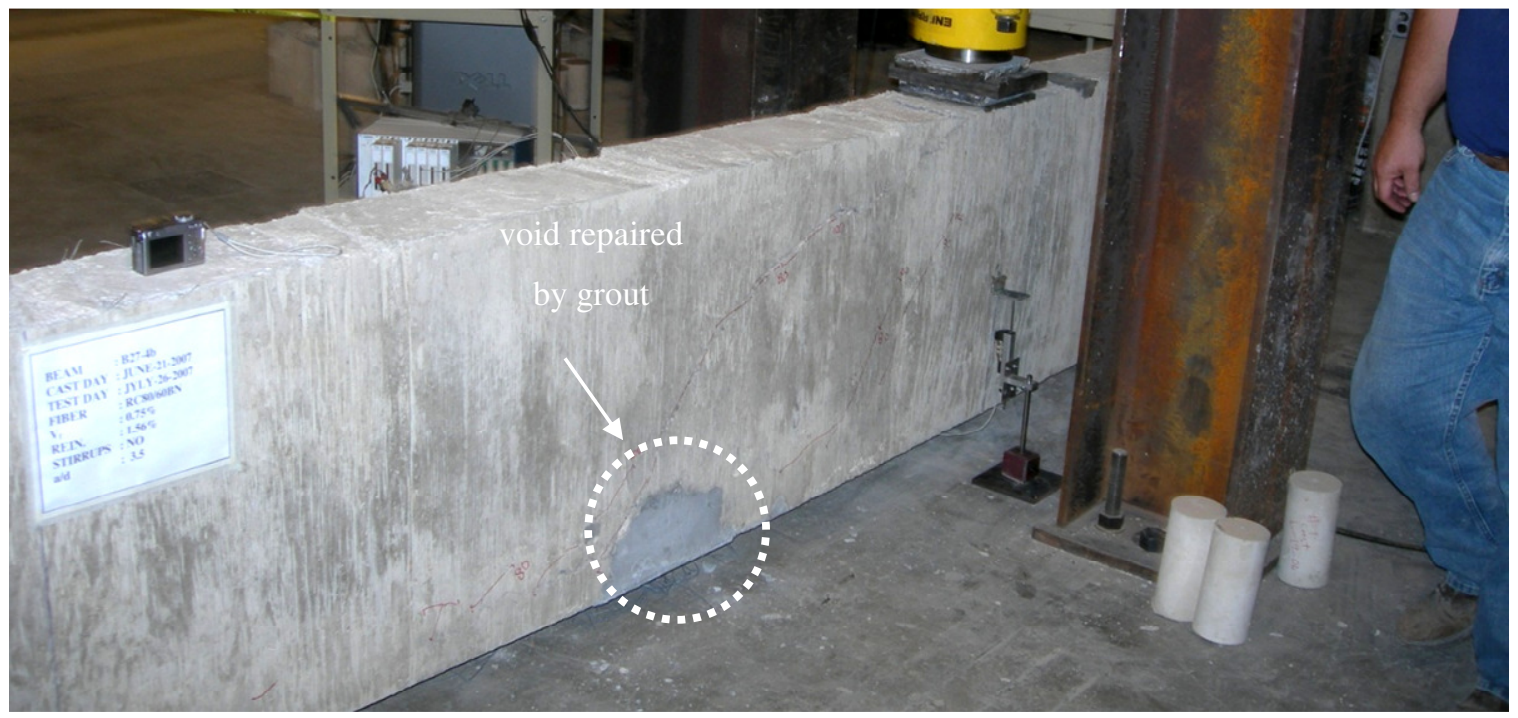

Fig. 4-163: Crack pattern after failure - Beam B27-4b

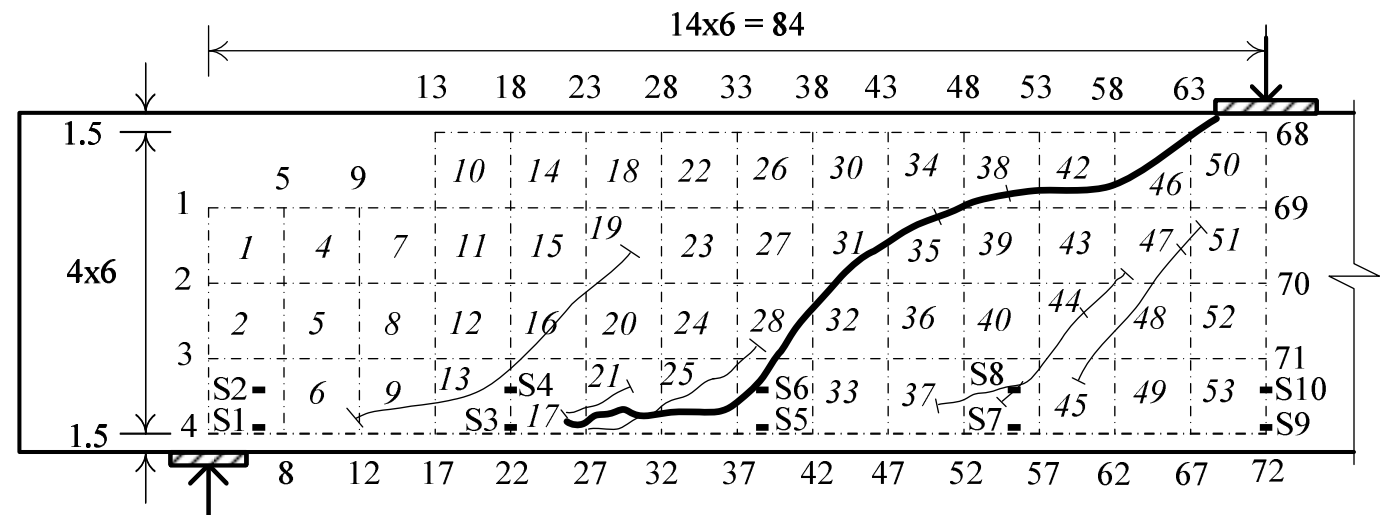

Fig. 4-164: Numbering of markers, elements, and strain gauges - Beam B27-4b 
(a)

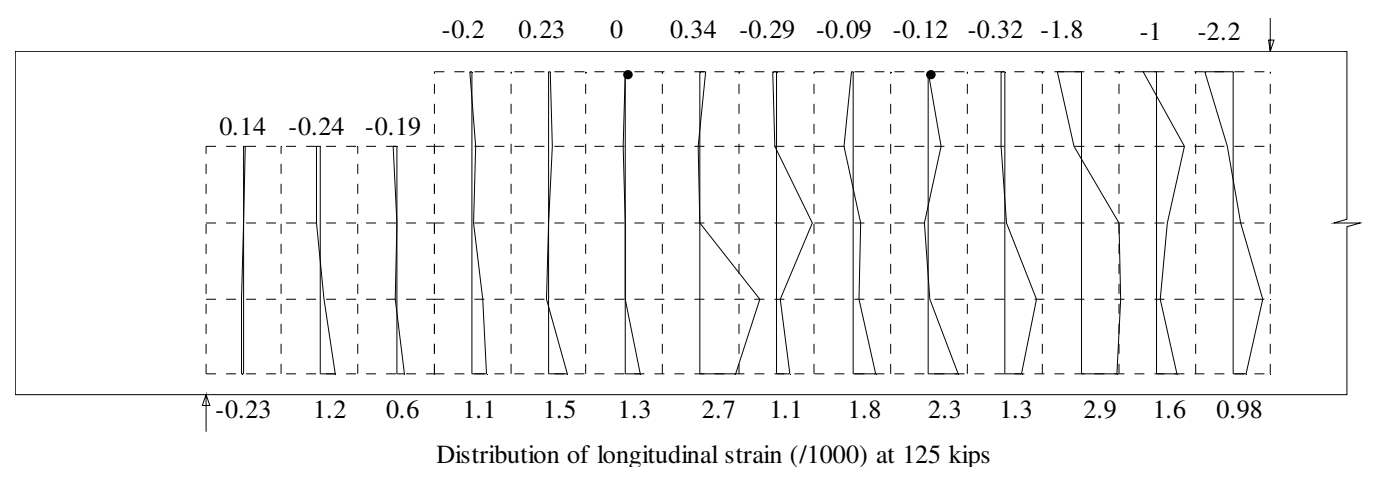

(b)

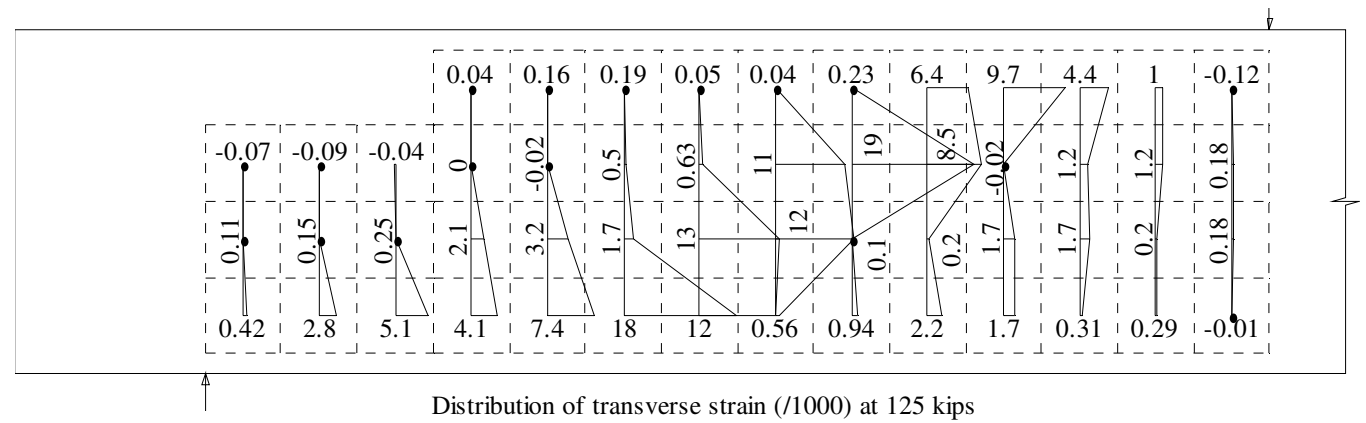

(c)

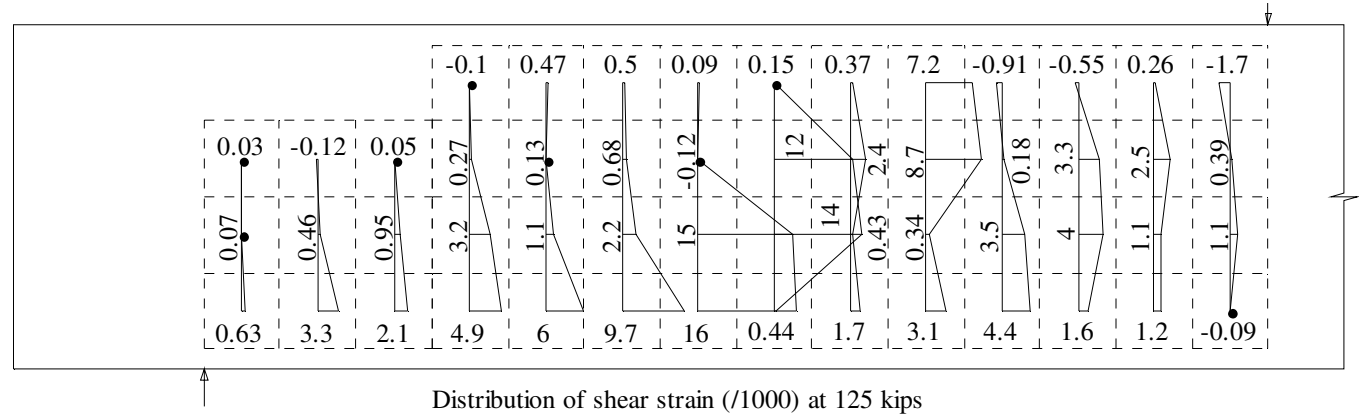

Fig. 4-165: Distribution of longitudinal, transverse, and shear strains at $125 \mathrm{k}-$ Beam B27-4b

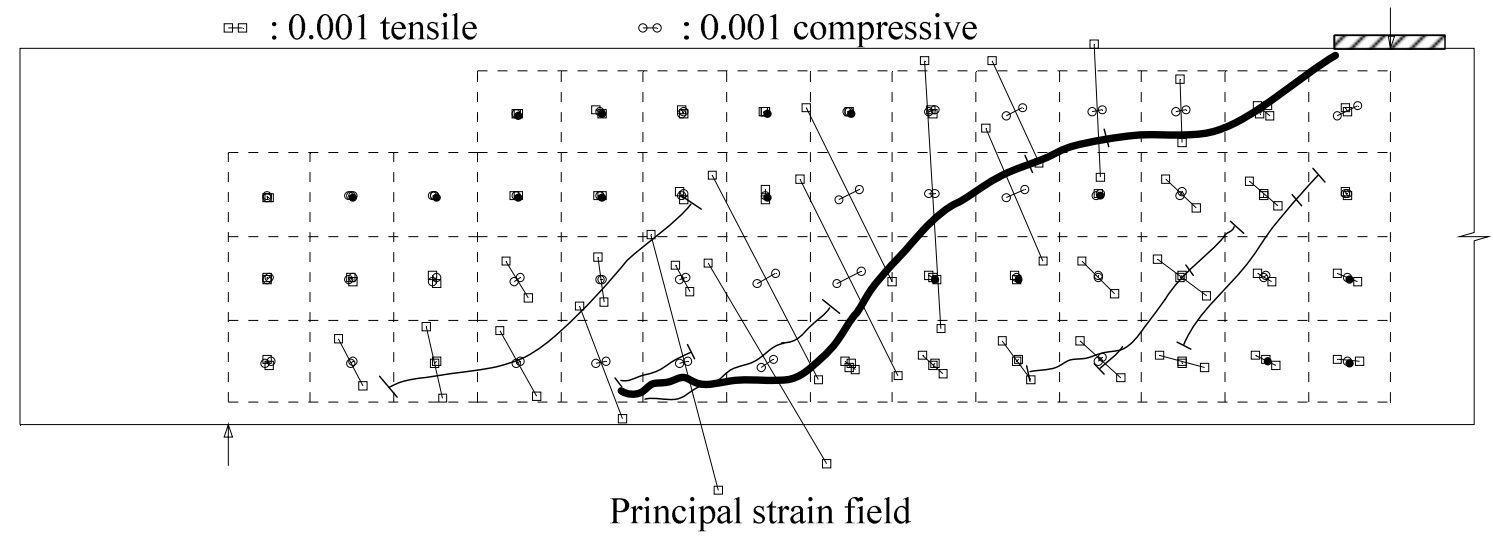

Fig. 4-166: Principal strain field at 125 kips - Beam B27-4b 
(a)

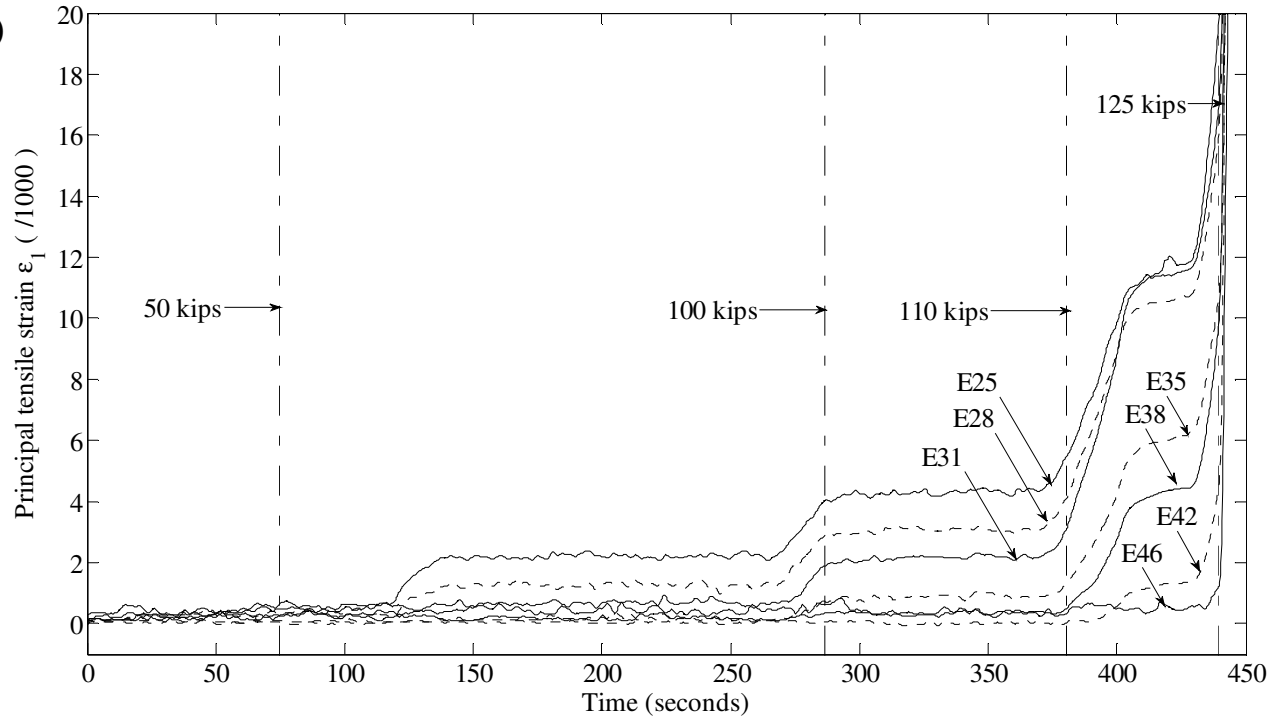

(b)

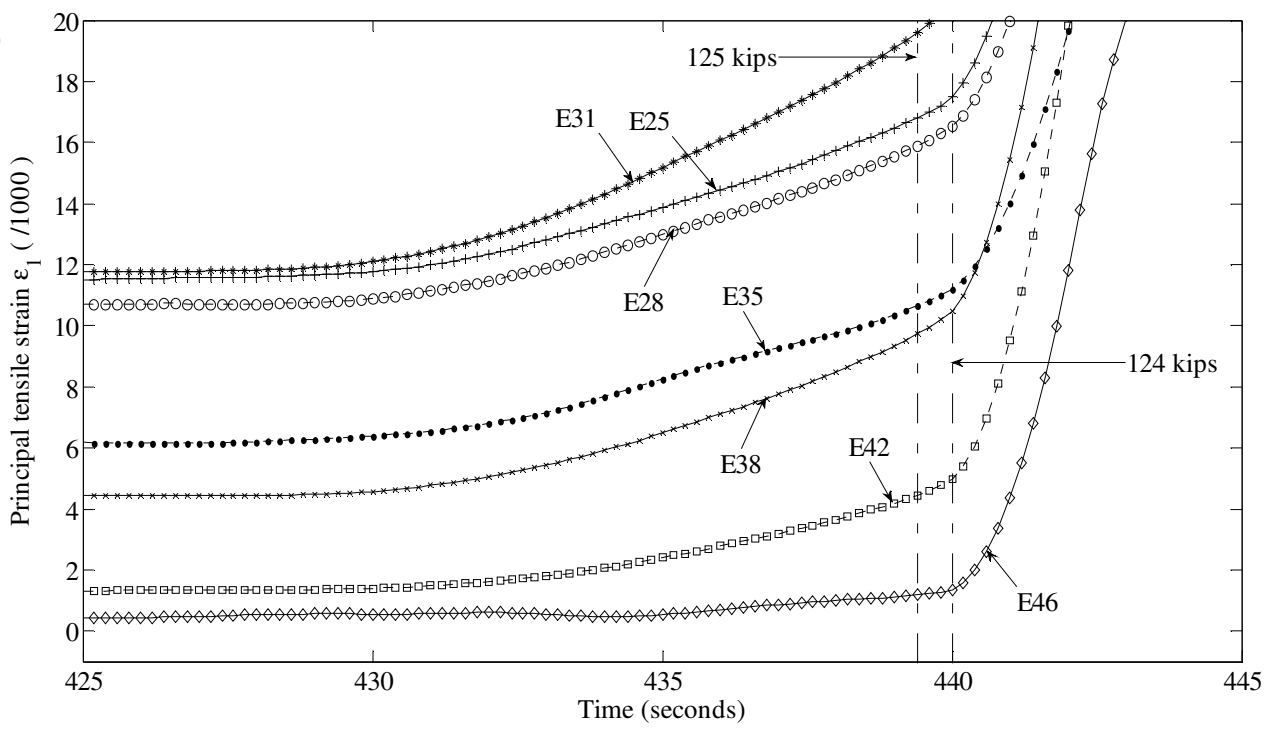

Fig. 4-167: Development of principal tensile strains along the critical inclined crack Beam B27-4b

\subsubsection{Beam B27-5}

Beam B27-5 was similar to Beams B27-1a \& b except that Beam B27-5 contained a higher amount of fibers $(1.5 \%$ by volume compared with $0.75 \%$ for Beams B27-1a \& b). 
Load versus deflection relationship, crack pattern, and failure mode. The crack propagation and failure mode of Beam B27-5 was very similar to that of Beam B27-3b. The beam exhibited the first inclined crack at 140 kips and the inclined cracks spread over the shear span region prior to a load of 240 kips (Fig. 4-168). The beam attained its full strength at 243 kips, followed by a slow decrease in strength until the applied load reached approximately $230 \mathrm{kips}$, and then the beam failed suddenly (Fig. 4-169).

After the load of 240 kips, the reinforcement start to yield (Fig. 4-171) and the beam deformation started to concentrate in the flexural cracks (Fig. 4-168). However, the yielding was not sufficient to cause a flexural failure. Instead, a shear failure occurred prior to the beam reaching its full flexural capacity. The shear failure was initiated by the crushing of the compression region to the left of the loading plate. Together with the concrete crushing, splitting along the reinforcement indicated that a combination of shear-compression and shear-tension failure occurred in this beam. The concrete crushing phenomenon of Beam B27-5 was very similar to that of Beam B27-3b. Compared to Beam B27-3a, Beam 27-5 contained a higher amount of longitudinal reinforcement and fiber content, which respectively increased the moment and shear capacity. Therefore, a shear compression failure was expected for this beam.

Reinforcement strain and average bond stress. Fig. 4-171 indicates that at the peak load the reinforcement strain at S5 and S7 (see Fig. 4-170 for the location of markers) e while that at S9 exceeded the strain at the end of the yield plateau, at the peak load. At approximately 100 kips, the bond between S1 and S3 increased significantly, reaching the value of 430 psi near the peak load (Error! Reference source not found.).

Concrete strain field. The maximum compressive and tensile strain reached 0.0039 and 0.0071 , respectively, at the peak load (Fig. 4-172a). However, these strains jumped to 0.0082 and 0.047 at the failure load of 230 kips (Fig. 4-173a). At this load, the transverse and shear strain were also significant, reaching values of 0.016 and 0.033 , respectively (Fig. 4-173b and c). 
Near the failure load, the compressive strains for Beam B27-5 were similar to Beam B27-3a, reaching the value of 0.0018. The transverse and shear strains were also significant, with similar magnitudes as with the case of Beam 27-3a.

Fig. 4-175a shows the development of principal tensile strains of the elements along the primary critical crack (see Fig. 4-170 for the numbering of elements). Prior to the peak load, the principal tensile strains were largest in the order from the bottom to top elements along the crack (E36, 39, 43, 46, and 49), while that of Element E32 was still small (Fig. 4-175a). It is noted that the principal tensile strain of Element E39 became larger than that of Element E36 from the load of 226 kips (Fig. 4-175a). It was at the load prior to the peak load that the tensile strain of Element E46 jumped up from 0.003 to 0.005 (Fig. 4-175b), while the compressive strain of Element E49 increased suddenly from 0.0017 to 0.0023 (Fig. 4-175c). All these changes could be considered to be due to the formation of the final failure. However, the beam strength was not reduced significantly at this point.

After the peak load, the principal tensile strain of Element E46 increased significantly up to 0.0257 . During this "yielding" period, the compressive strain of Element E49 increased from 0.005 to 0.0083 . After this point, the strains of all elements increased suddenly, signifying a failure. Therefore, the strain state at this point can be considered as critical. It should be noted that Beams B27-3a \& b also failed due to concrete crushing at a strain of a similar magnitude (0.010).

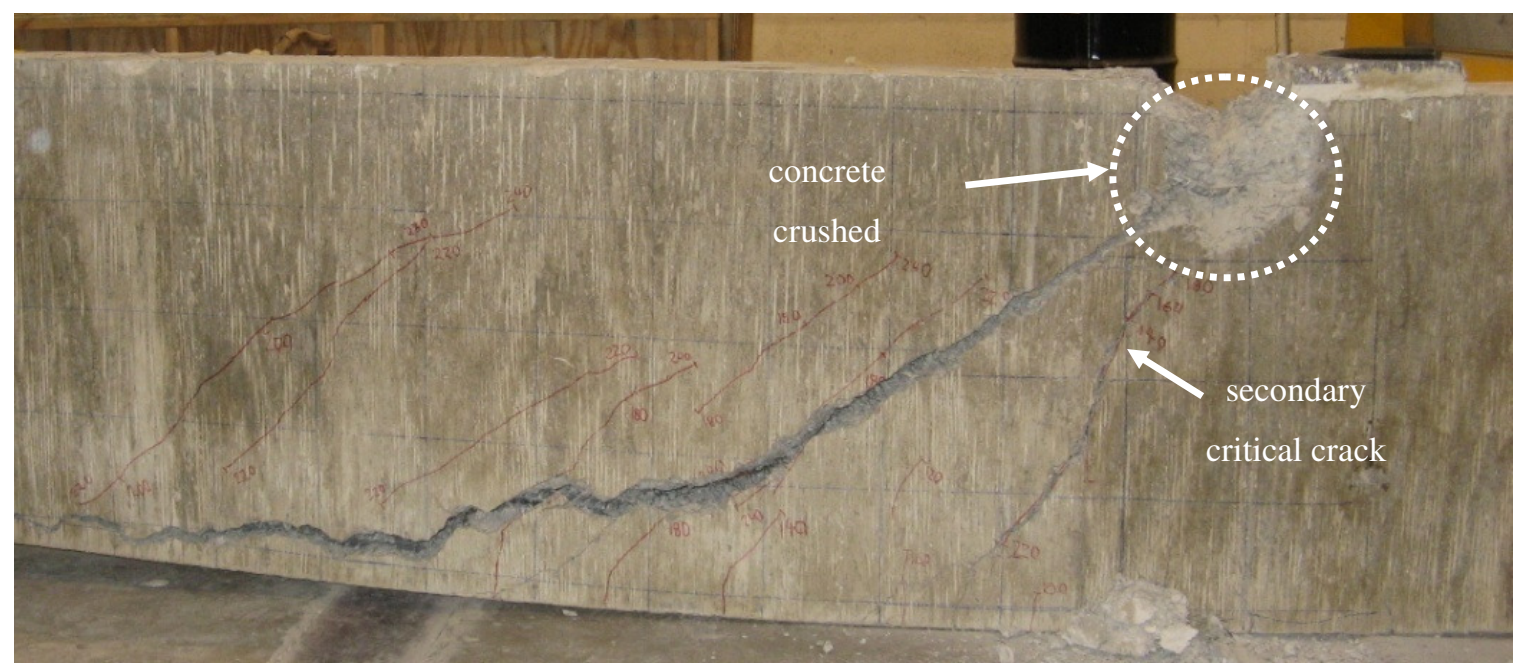

Fig. 4-168: Crack pattern after failure - Beam B27-5 


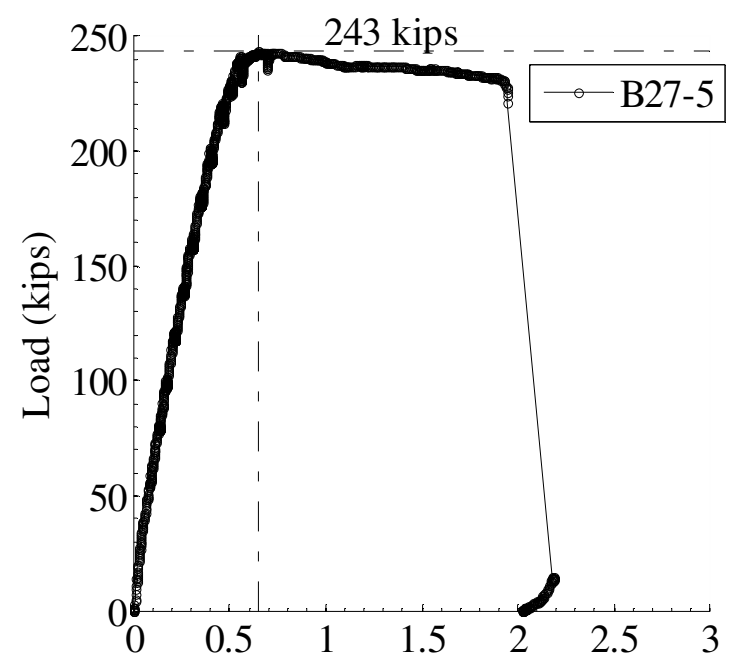

Deflection under the loading point (inche

Fig. 4-169: Load versus deflection relationship - Beam B27-5

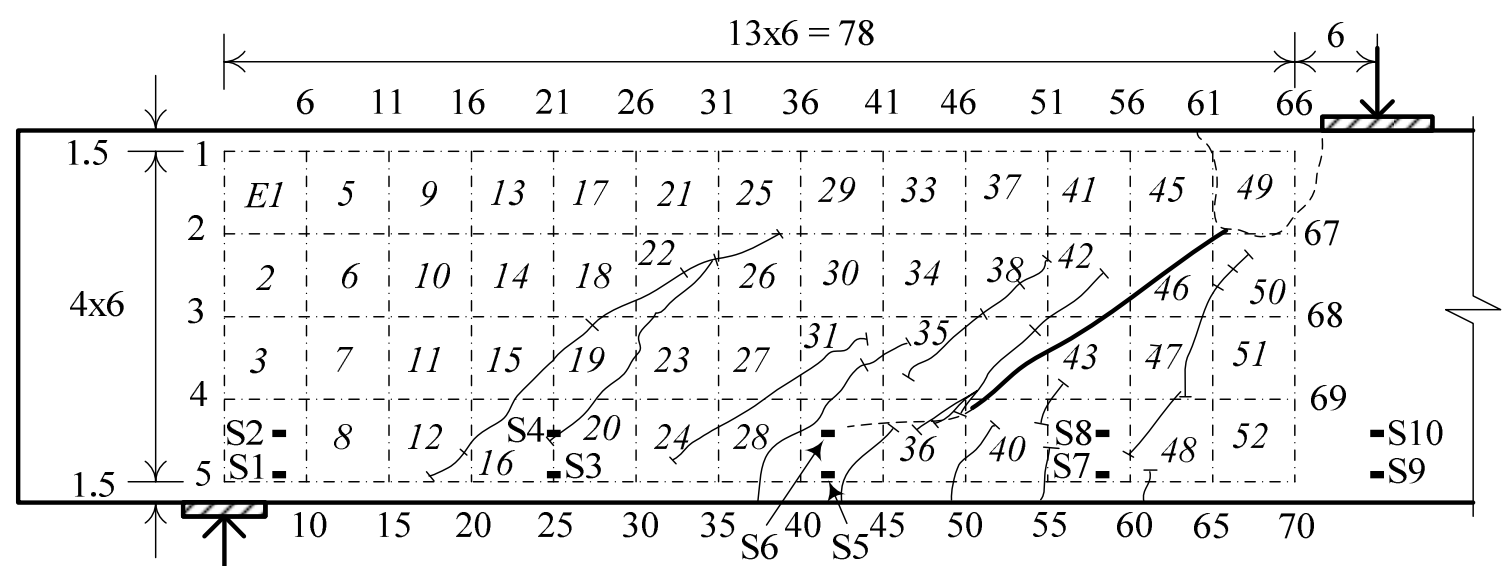

Fig. 4-170: Numbering of markers, elements, and strain gauges - Beam B27-5

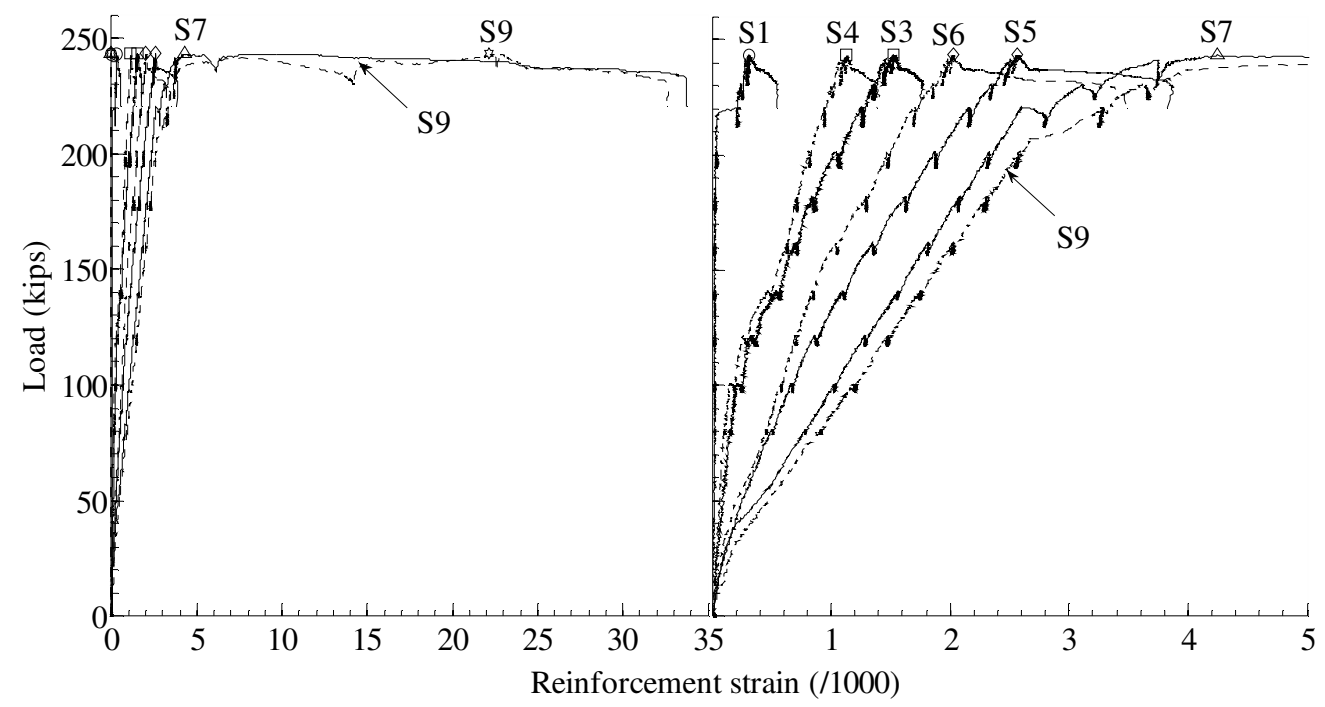

Fig. 4-171: Load versus reinforcement strain relationships - Beam B27-5 
(a)

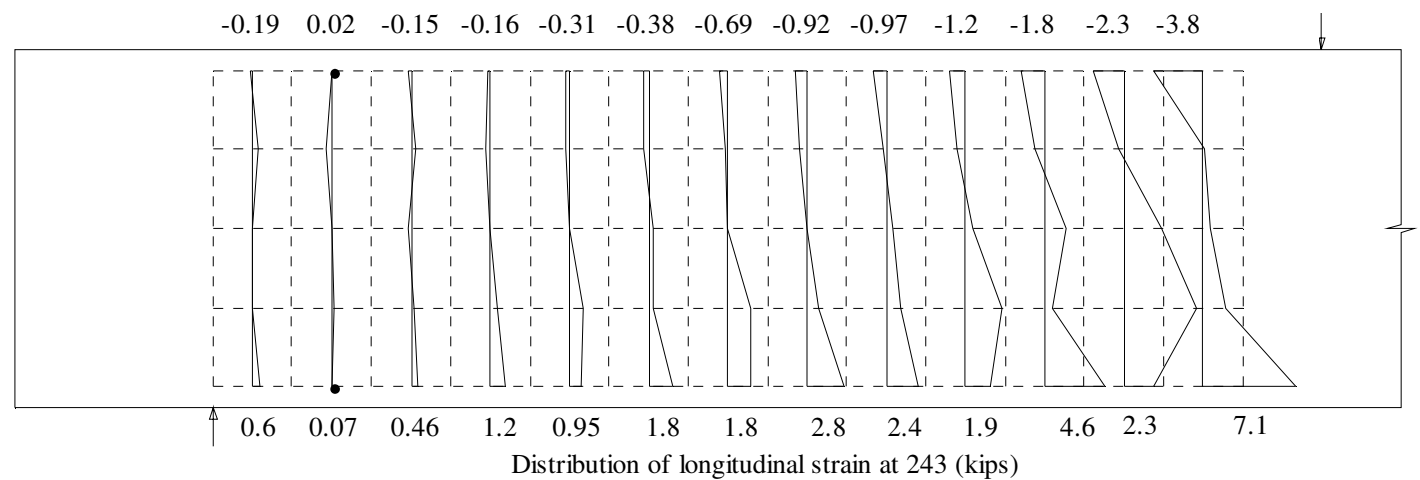

(b)

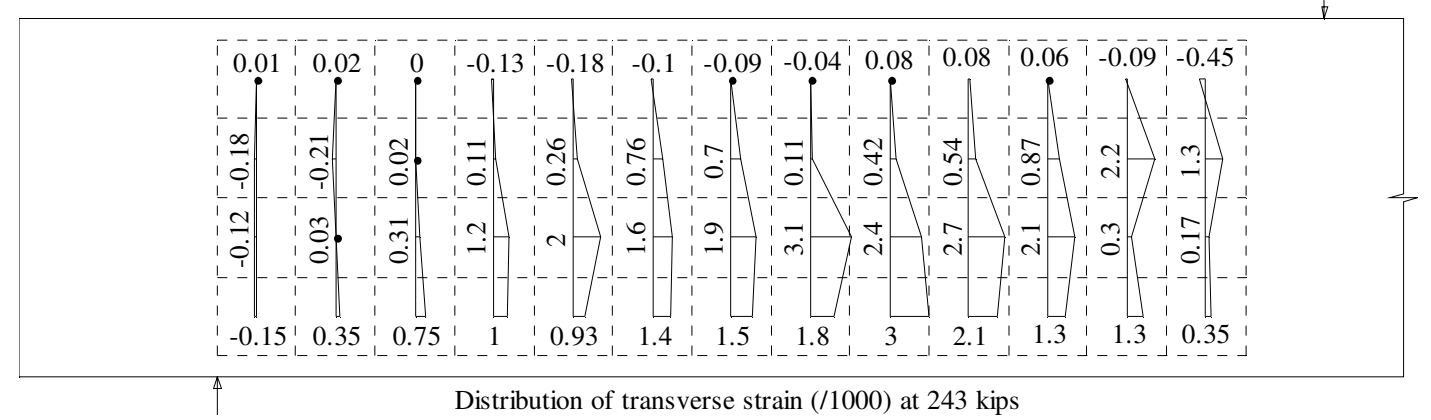

(c)

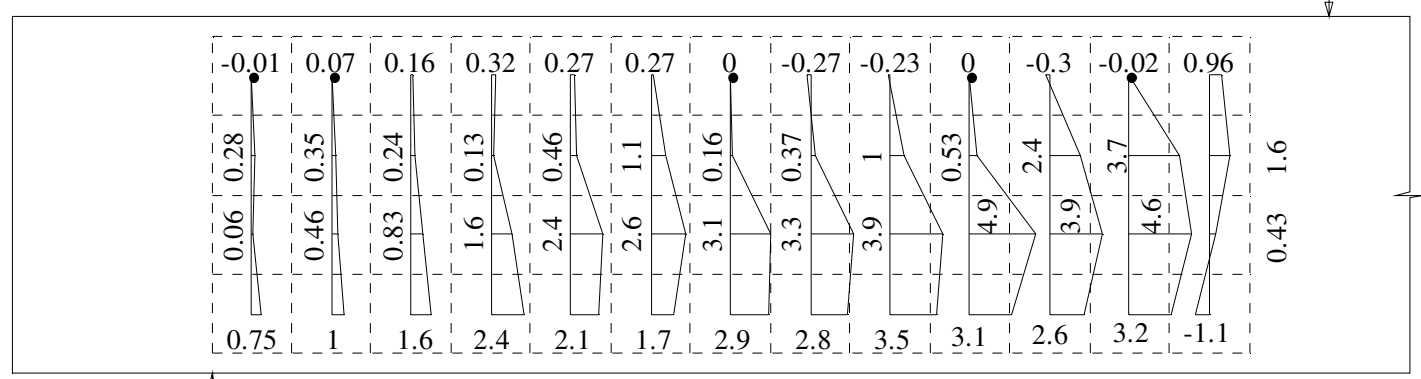

Distribution of shear strain (/1000) at 243 kips

Fig. 4-172: Distribution of longitudinal, transverse, and shear strains at peak load - Beam B27-5 
$\begin{array}{lllllllllllll}0 & -0.36 & 0.04 & -0.08 & -0.19 & -0.38 & -0.55 & -0.7 & -0.79 & -0.73 & -1.8 & -3.9 & -18\end{array}$

(a)

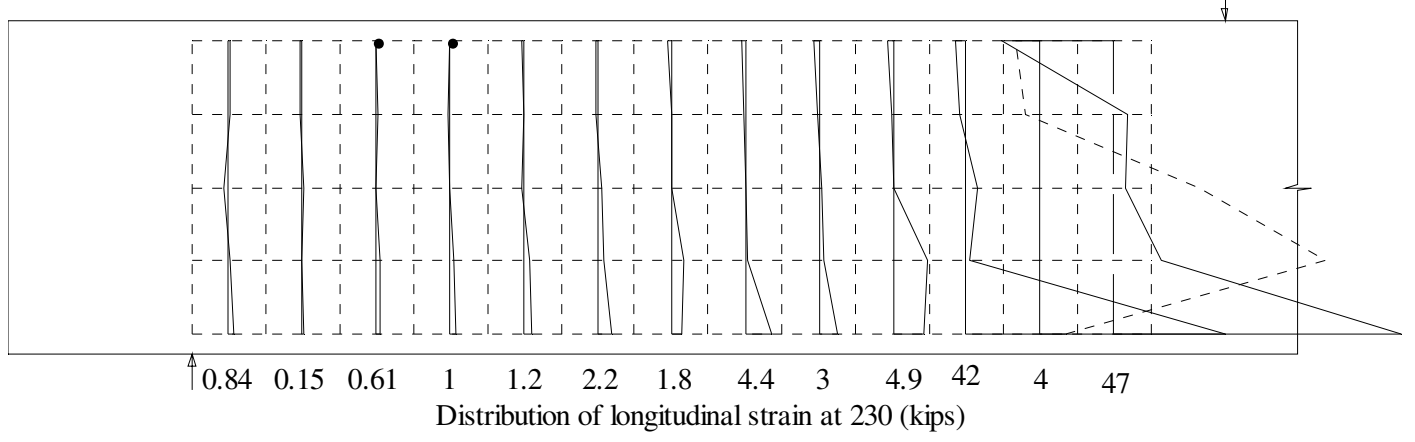

(b)

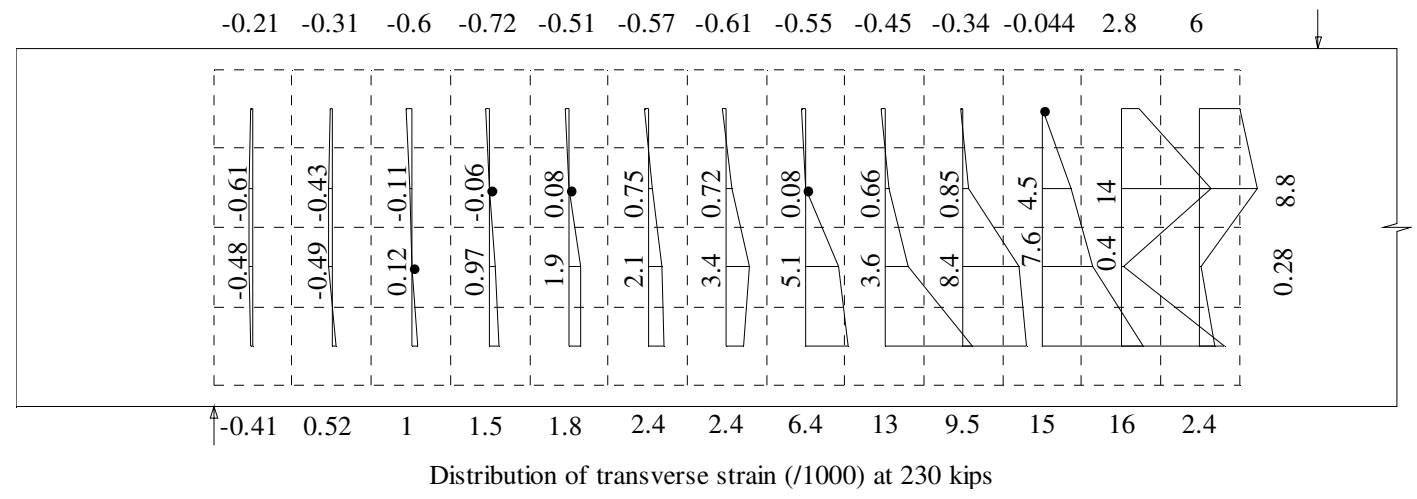

(c)

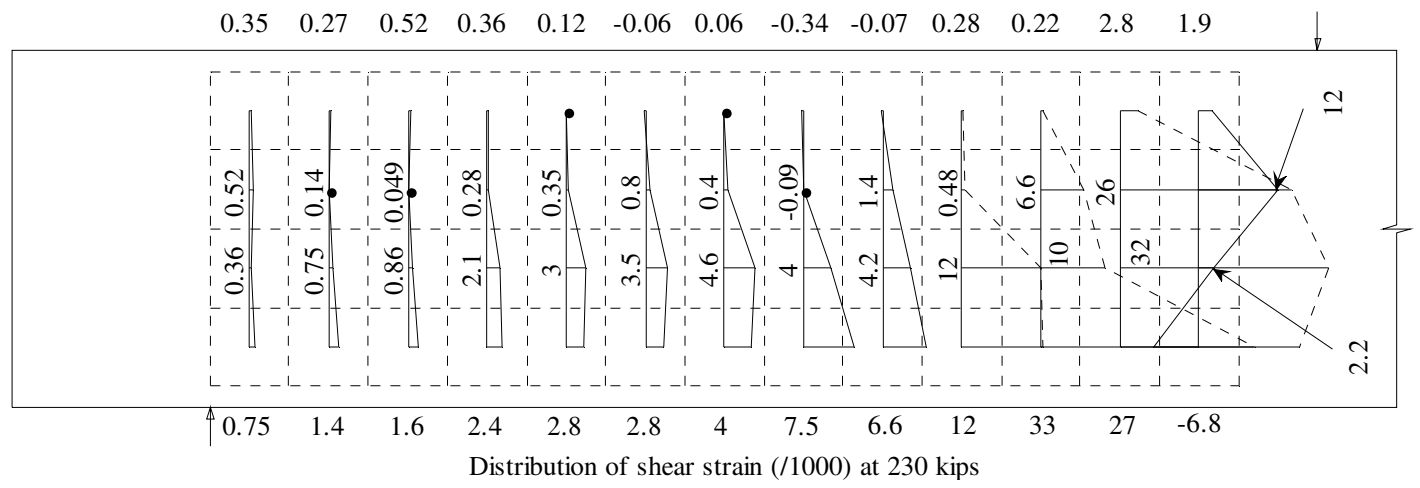

Fig. 4-173: Distribution of longitudinal, transverse, and shear strains failure load - Beam B27-5 
(a)

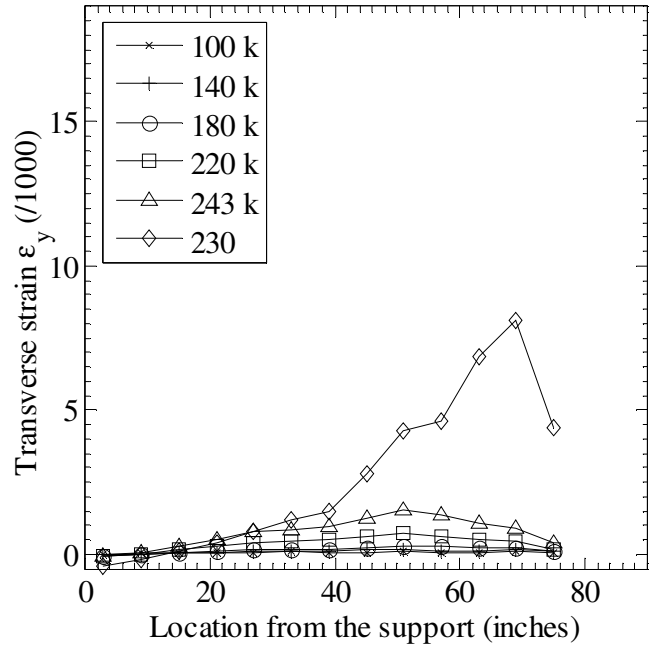

(b)

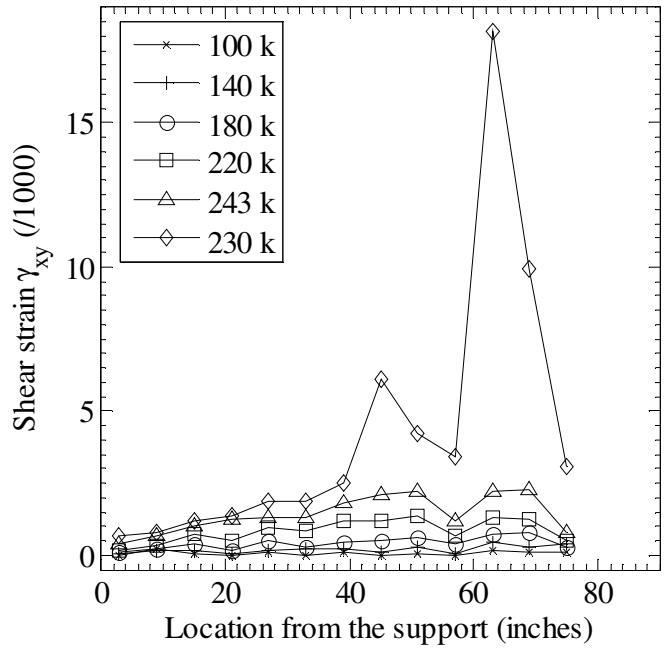

Fig. 4-174: Transverse and shear strains averaged over the beam depth - Beam B27-5 
(a)

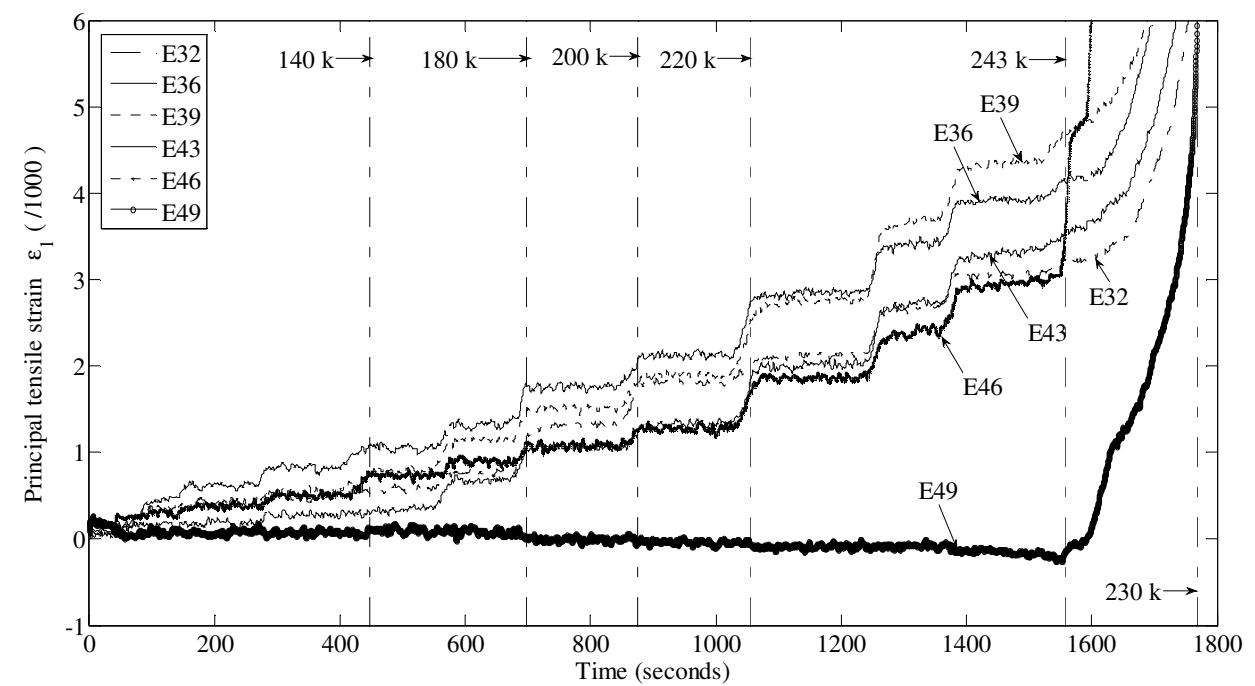

(b)

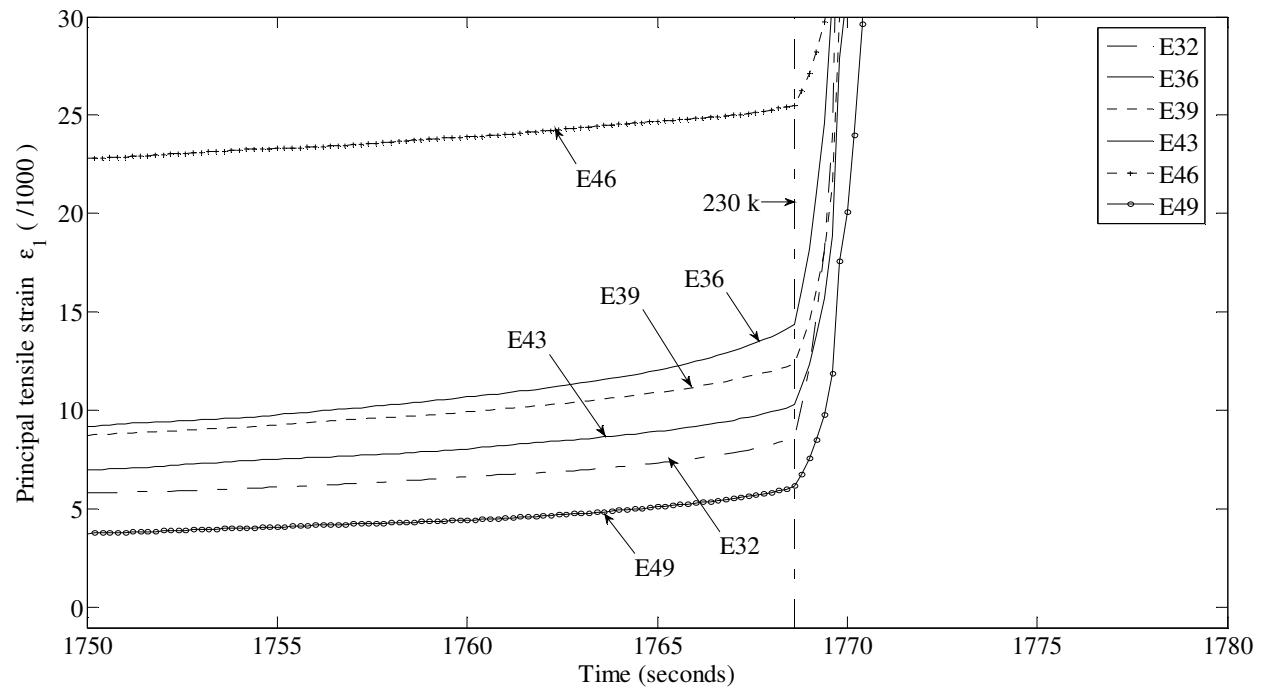

(c)

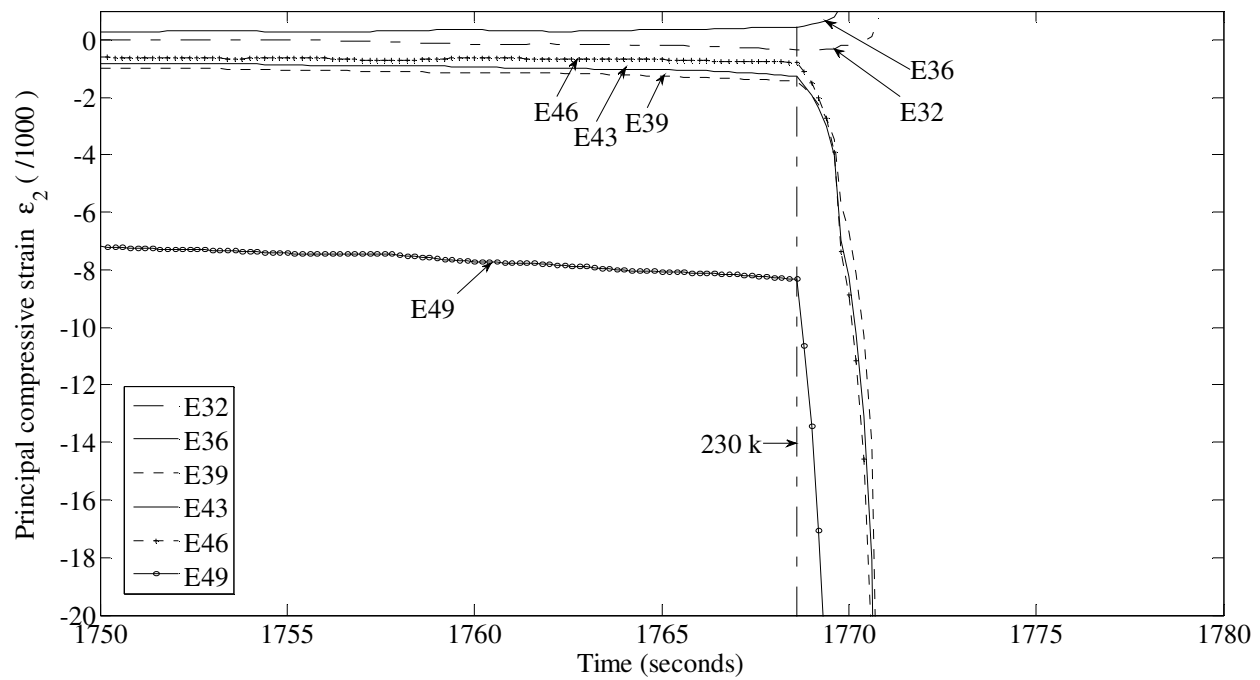

Fig. 4-175: Development of principal tensile and compressive strains along the critical inclined crack - Beam B27-5 


\subsubsection{Beam B27-6}

Beam B27-6 was similar to Beam B27-5 except that it contained RC80/60BN fibers which had an aspect ratio of 80 and a diameter of 0.022 inches.

Load versus deflection relationship, crack pattern, and failure mode. The beam exhibited the first diagonal cracking at 140 kips (Fig. 4-178). The beam exhibited a significant number of inclined cracks throughout the shear span (Fig. 4-179). The angle of the inclined cracks was still shallow, as frequently observed in beams using RC80/60BN. The beam attained its full strength at 235 kips (Fig. 4-176a), comparable to that of Beam B27-5 (243 kips). However, Beam B27-6 did not exhibit the "yield plateau" like Beam B27-5. After the failure at the load of 230 kips, the load cell was able to track various points. This shows that the failure of this beam was "slower" than any other beams analyzed so far.

Reinforcement strain and average bond stress. Strain gauges S7, S8 and S9 were strained beyond the yield point, which is 0.00236 for bars No. 8 while S5 and S6 just reached the yield point (Fig. 4-176b).

Concrete strain field. Fig. 4-183 shows the principal strain field at the peak load and at 230 kips. The principal tensile strains were large and distributed throughout the shear span. At the peak load, the principal tensile strain of Elements E24, 27, 30, 34, 38, 41, 45, and 49 (Error! Reference source not found. for the numbering of elements), which were located along the critical crack, were still small compared with Elements E8, 36, and 43, which approached the principal tensile strain of 0.010. At the load of 230 kips, the principal tensile strains along the critical crack were more significant. The tensile strain of Element E30 was as large as 0.0208. It is interesting to note that the maximum compressive principal strain occurred for Element E27 (0.0048) at the beam mid-depth rather than in an element near the loading point.

At the failure load of 230 kips, the maximum measured compressive strains was 0.005. It is possible that concrete was crushed to a certain degree. Nevertheless, the crack 
opening at the mid-depth and reinforcement level was so excessive that a redistribution of stress to the compressive region did not hold long to impose a deflection demand to the beam.

Fig. 4-184a shows the development of strains along the critical crack with time. The strain of the group Elements E24, 27, and E30 was largest throughout the test. The tensile strain in Element E27 was largest from zero load to $178 \mathrm{kips}$, at which point the tensile strain in E24 surpassed that of Element E27. At the load of 212 kips, the strain of Element E30 exceeded that of Element E27. These observations suggest that the crack was initiated at E27 and propagated through Elements E30 and E24. At the load of 221 kips, prior to the peak, the strains in all elements started to increase significantly. The increase of strains after the peak load was more gradual than observed in other beams (Fig. 4-184b).
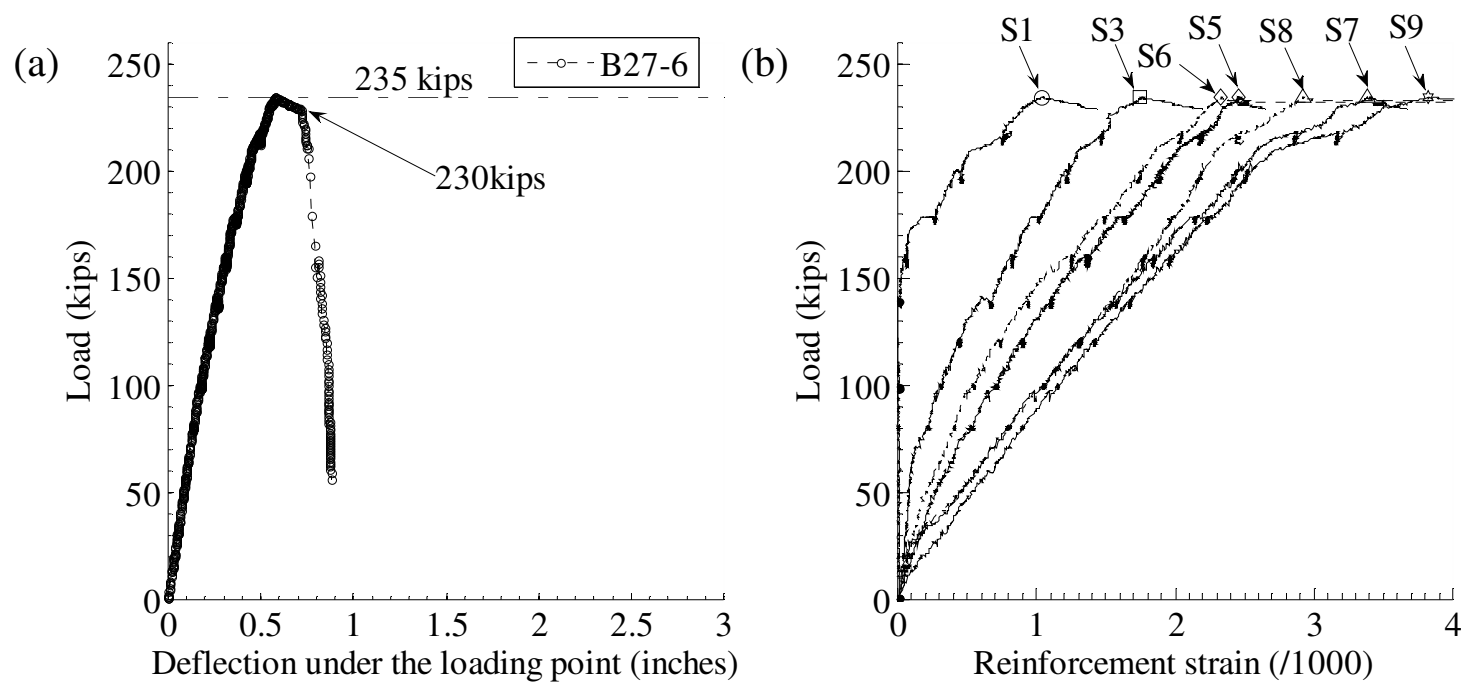

Fig. 4-176: Load versus deflection and reinforcement strain relationships - Beam B27-6

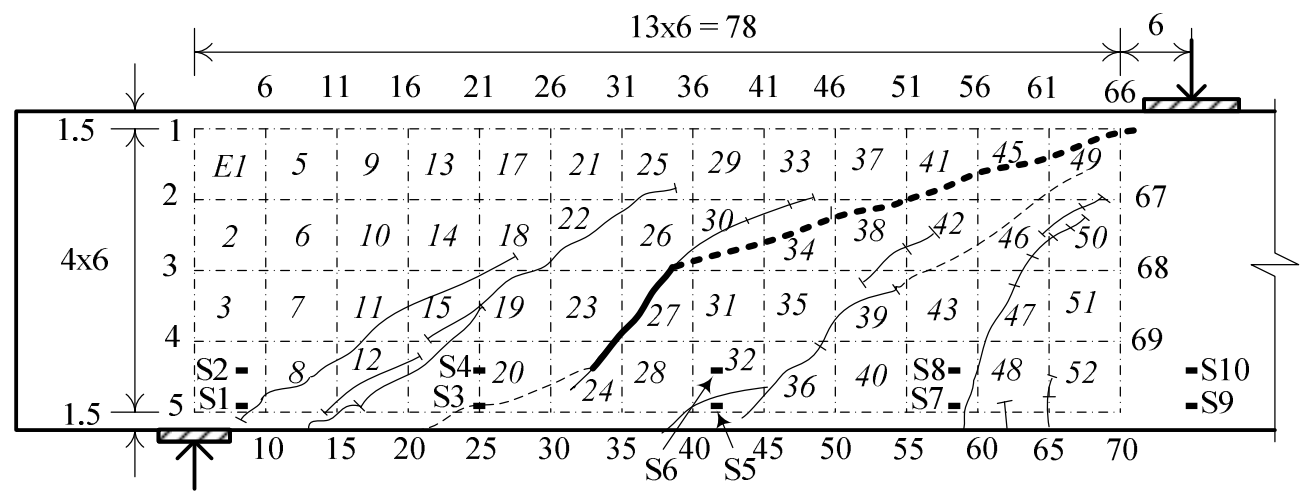

Fig. 4-177: Numbering of markers, elements, and strain gauges - Beam B27-6 


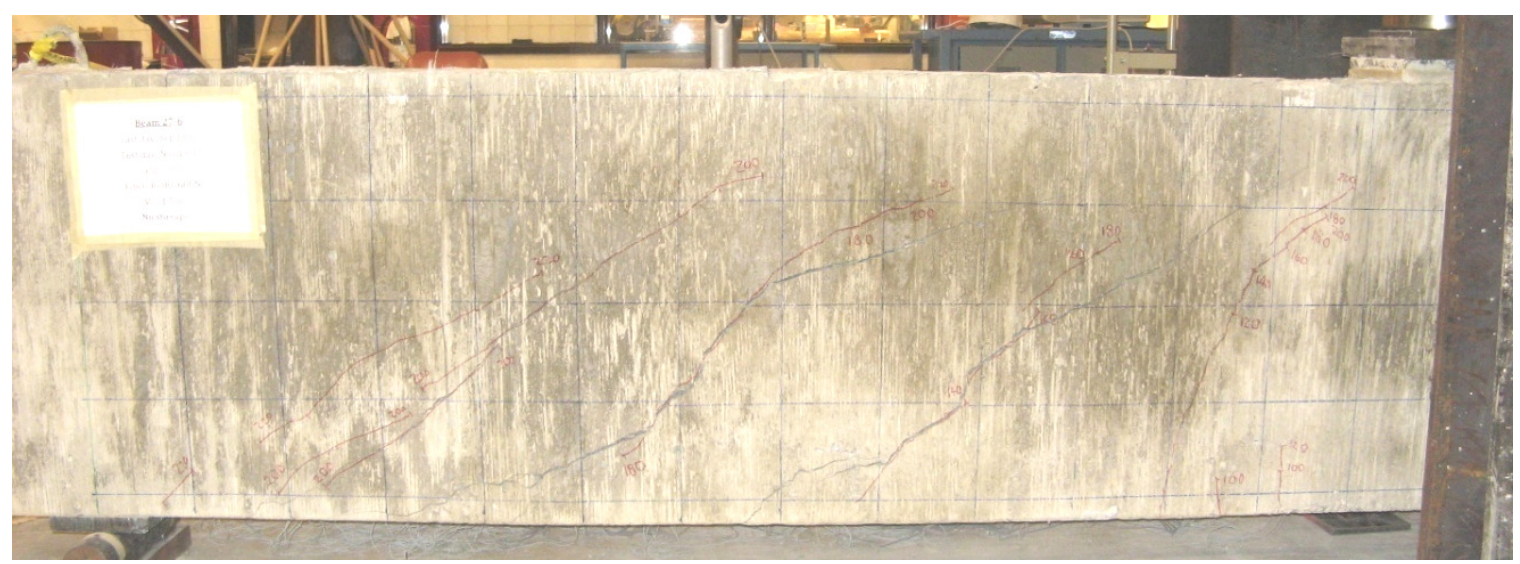

Fig. 4-178: Crack pattern prior to failure - Beam B27-6

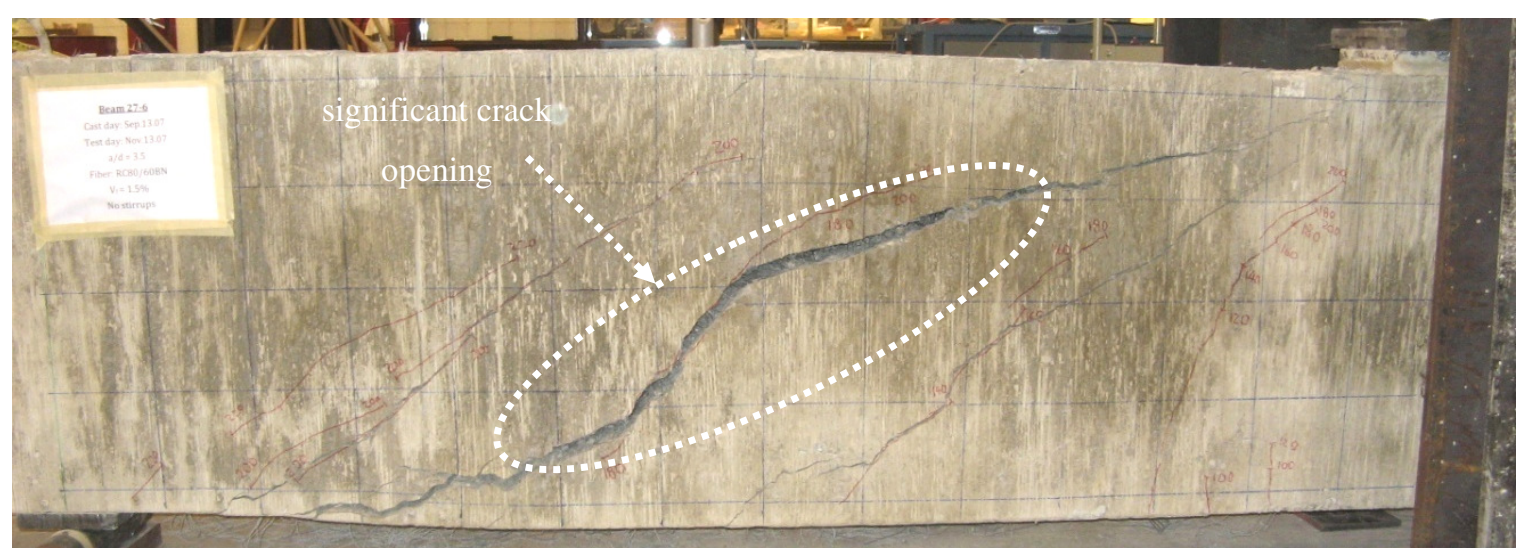

Fig. 4-179: Crack pattern after failure - Beam B27-6

(a)

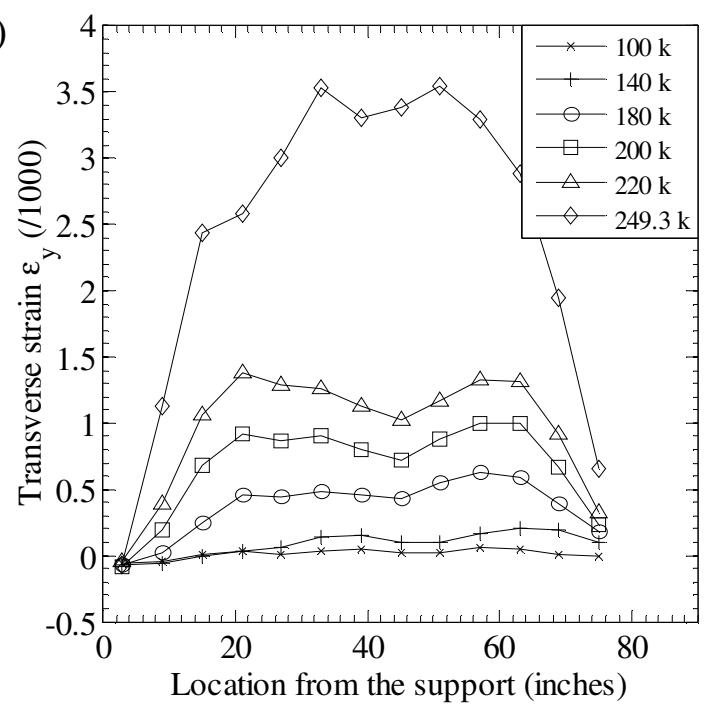

(b)

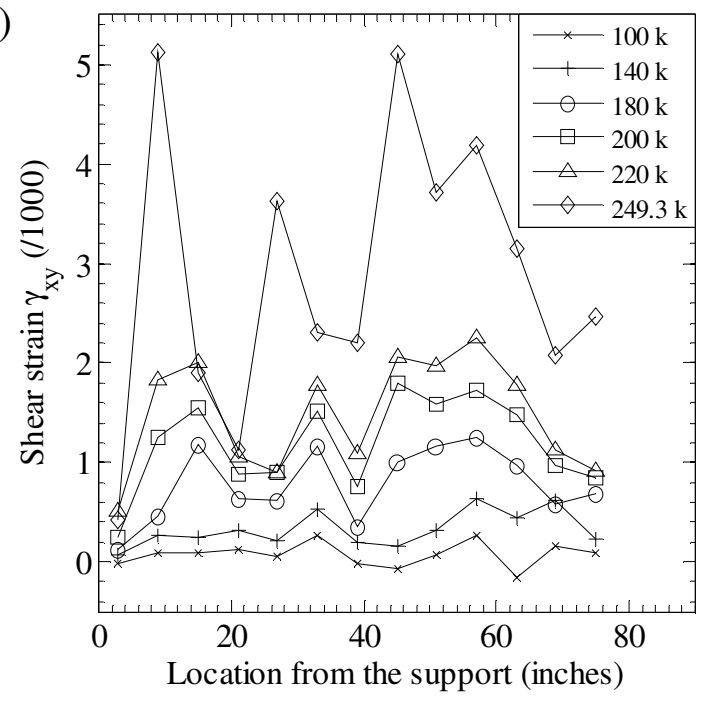

Fig. 4-180: Transverse and shear strains averaged over the beam depth - Beam B27-6 
(a)

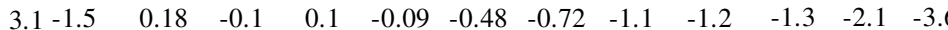

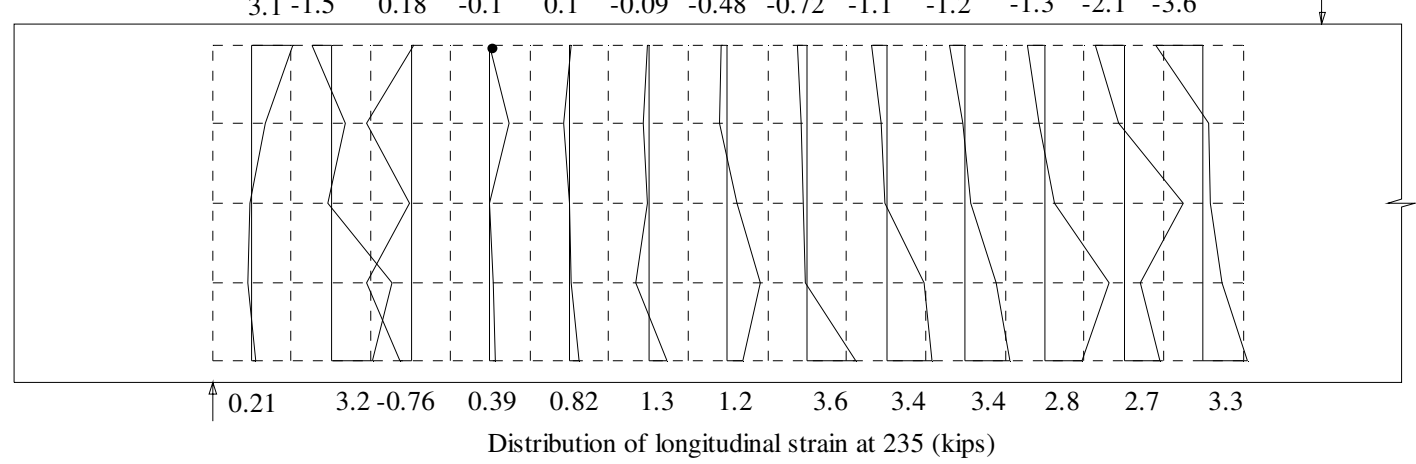

(b)

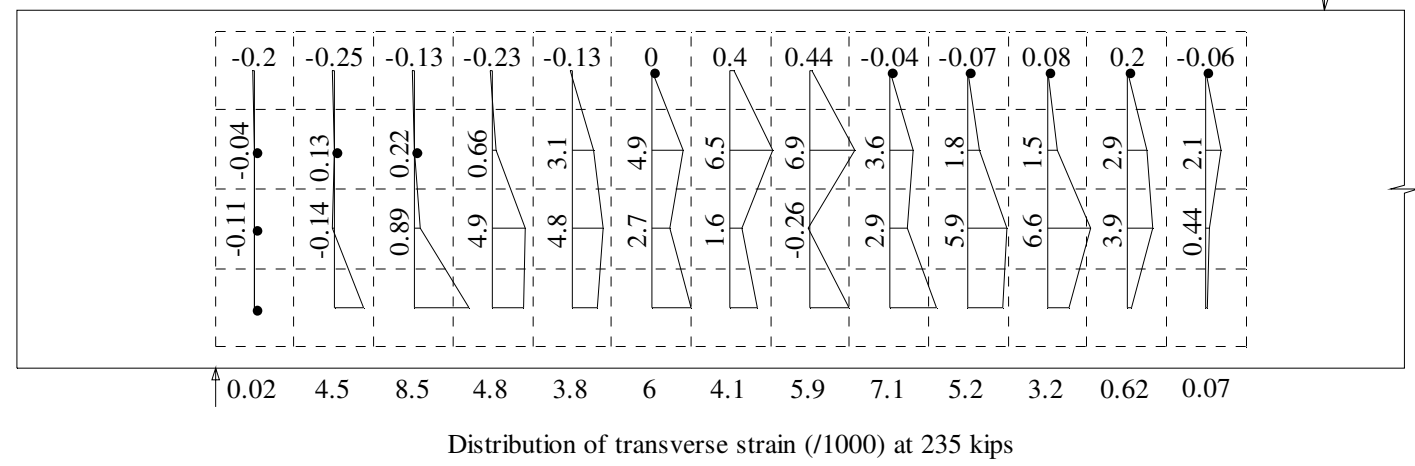

(c)

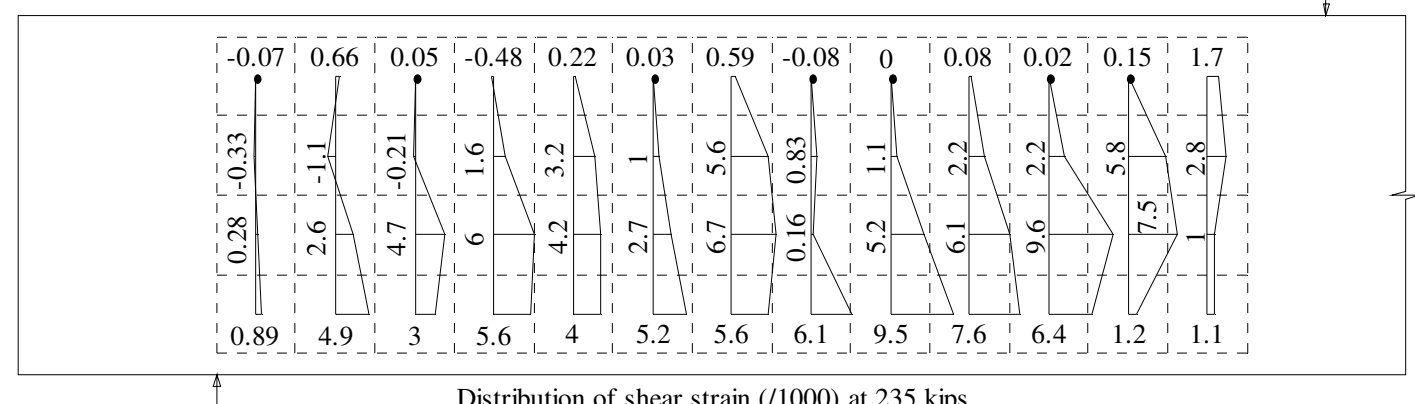

Distribution of shear strain (/1000) at 235 kips

Fig. 4-181: Distribution of longitudinal, transverse, and shear strains at peak load - Beam

B27-6 

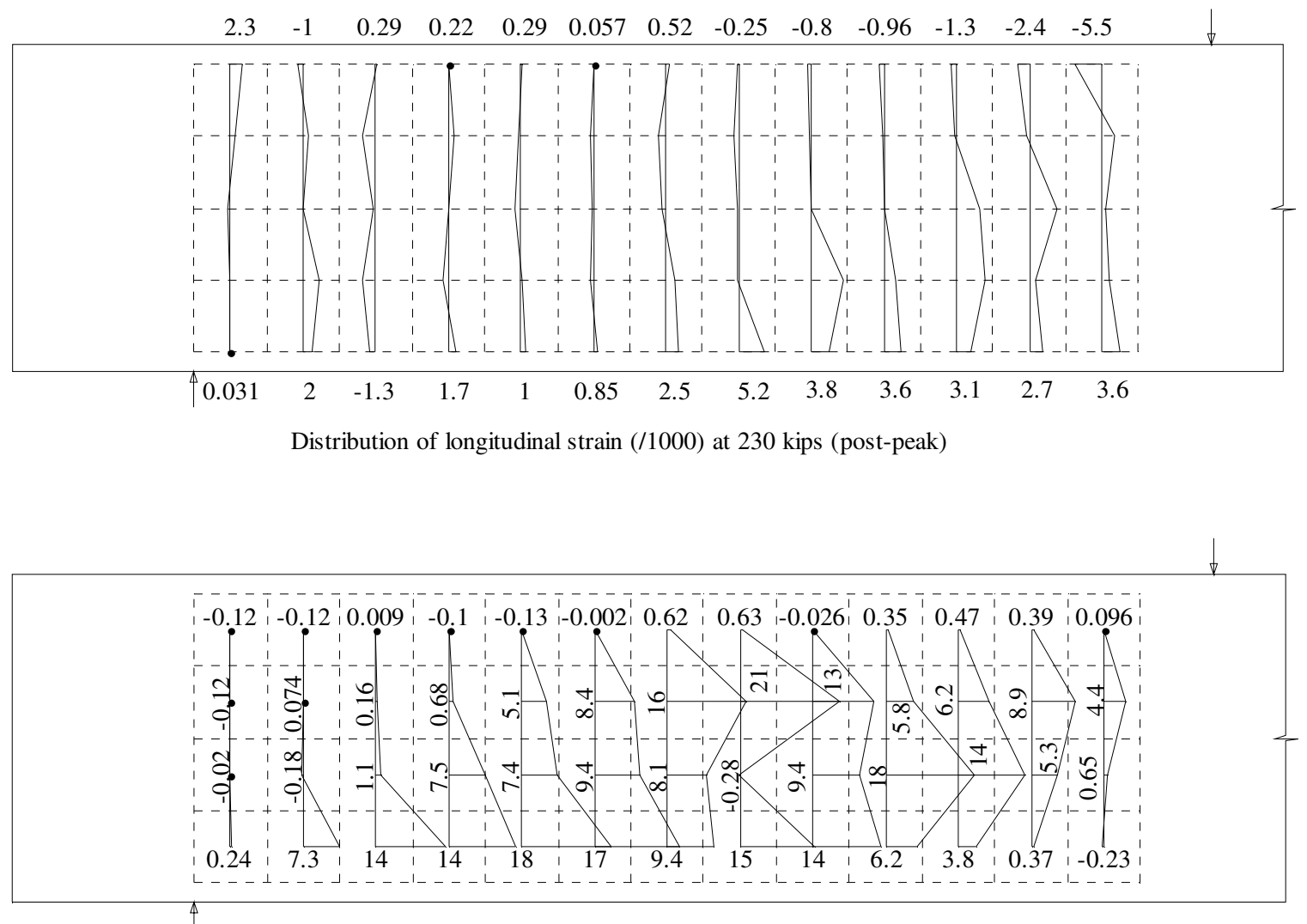

Distribution of transverse strain (/1000) at 230 kips (post-peak)

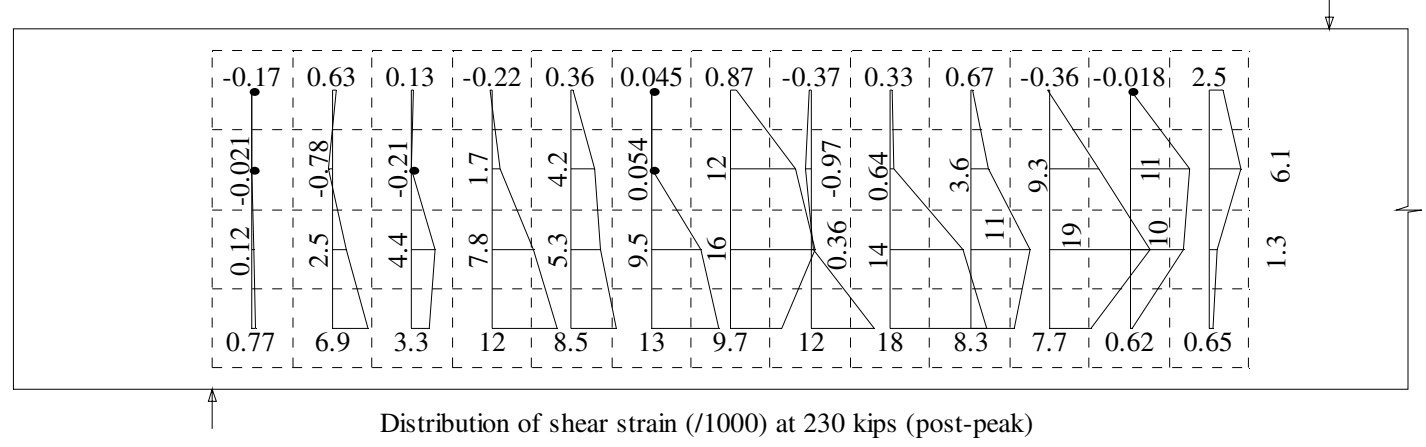

Fig. 4-182: Distribution of longitudinal, transverse, and shear strains at failure load Beam B27-6 


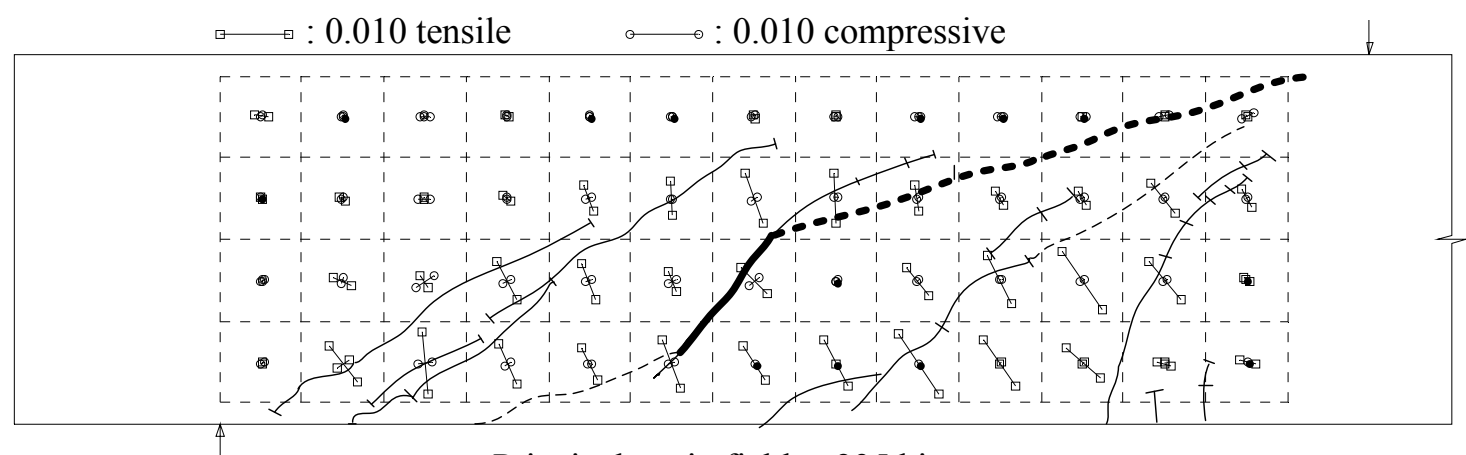

Principal strain field at 235 kips

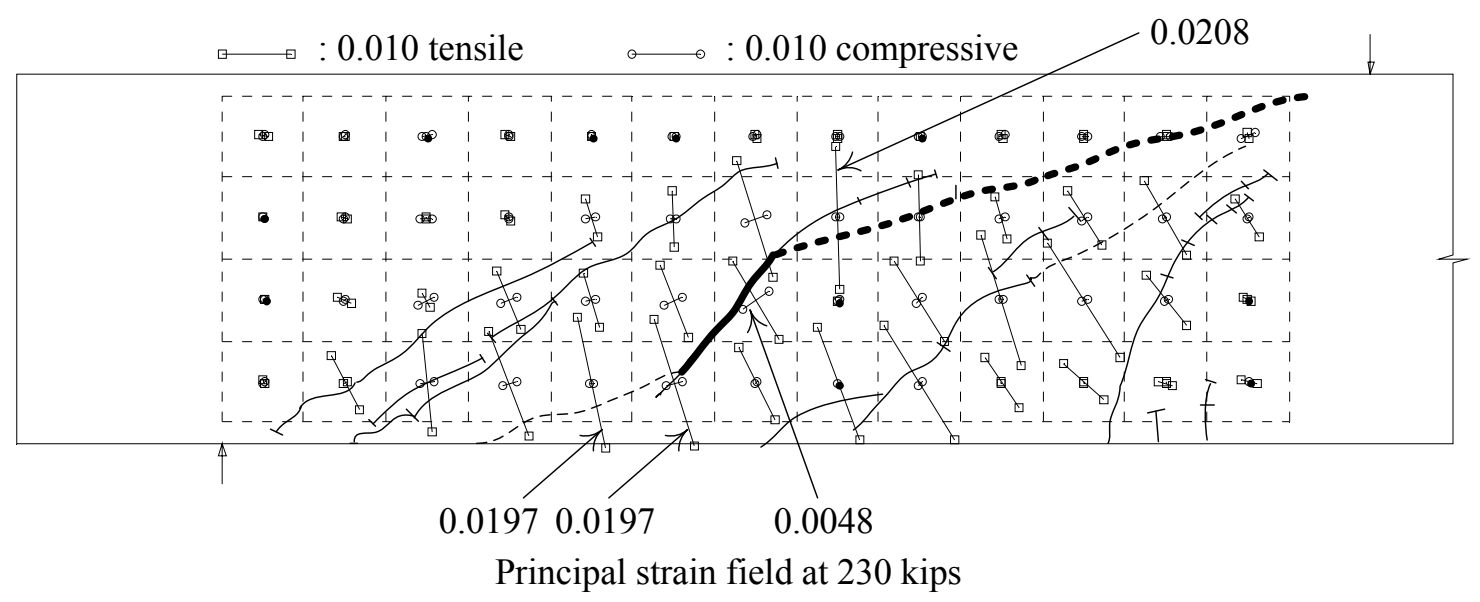

Fig. 4-183: Principal strain field at peak and failure load - Beam B27-6 
(a)

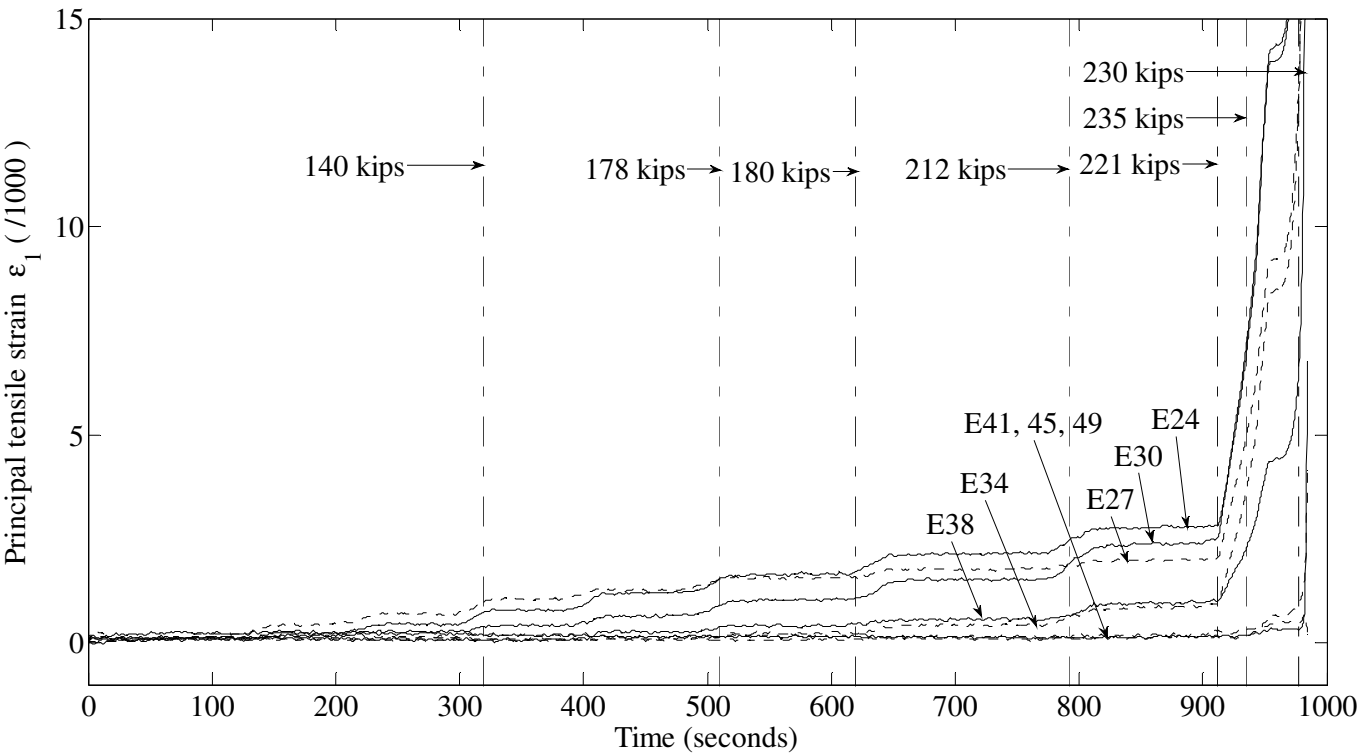

(b)

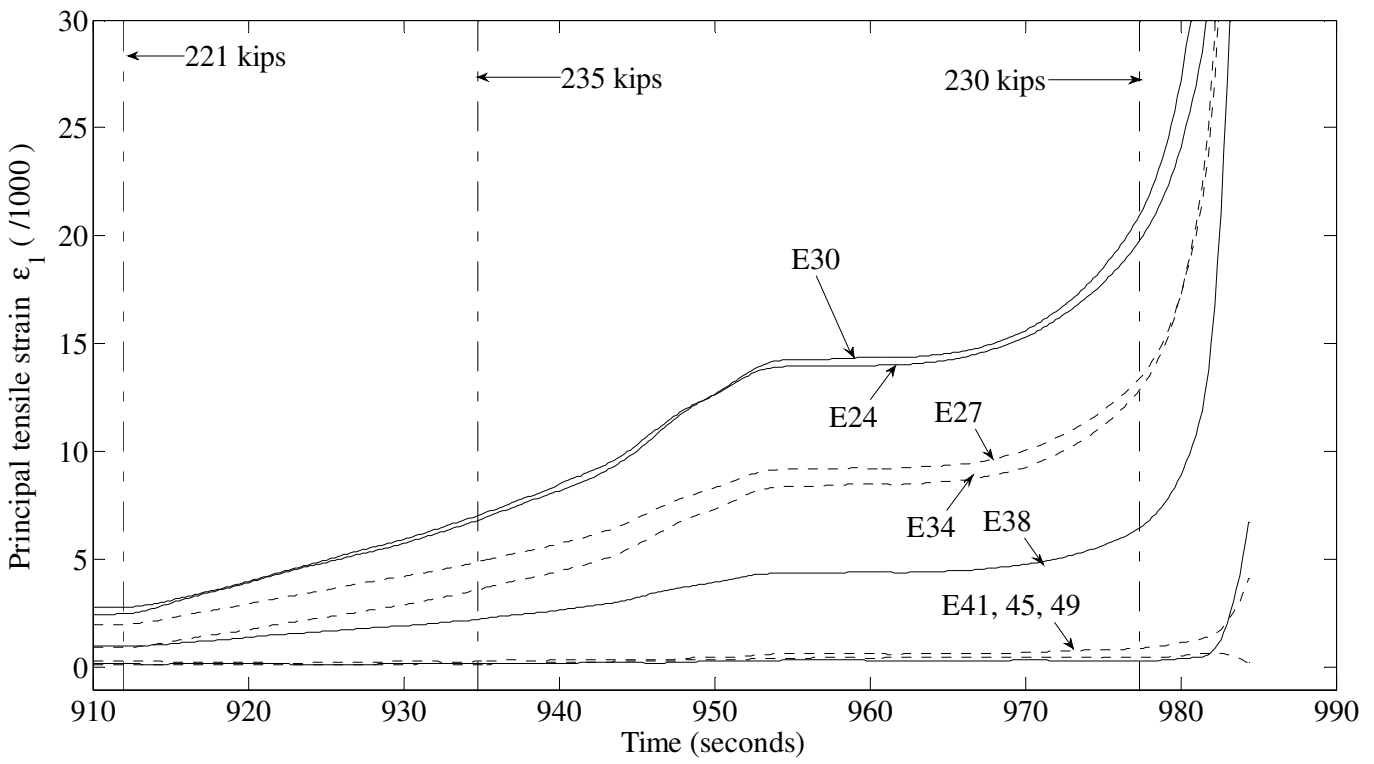

Fig. 4-184: Development of principal tensile strains along the critical inclined crack Beam B27-6 


\subsubsection{Beam B27-7}

Beam B27-7, with no fibers, was the control specimen for Series B27. Its behavior was very similar to any typical RC beam without web reinforcement. The failure therefore followed immediately after the formation of a single inclined crack (Fig. 4-186). The beam attained its full strength at 90.3 kips (Fig. 4-185a) and the maximum beam deflection was small compared to the fiber-reinforced beams. The maximum reinforcement strain (Fig. 4-185b) at S9 was only 0.0012. The maximum average longitudinal compressive and tensile strains were only 0.00077 and 0.0017 , respectively (Fig. 4-188a), while the transverse and shear strains were 0.0006 and 0.001 radians, respectively (Fig. 4-188b and c). The maximum principal tensile strain was very small throughout the test (Error! Reference source not found.). Nevertheless, the OptoTRAK was able to track the crack opening which occurred first at Element E19, then propagated toward bottom Element E16, and finally propagated to the top elements E22, 25, 29, and 37. The principal tensile strain of Element E19 at peak load was only 0.00025 .
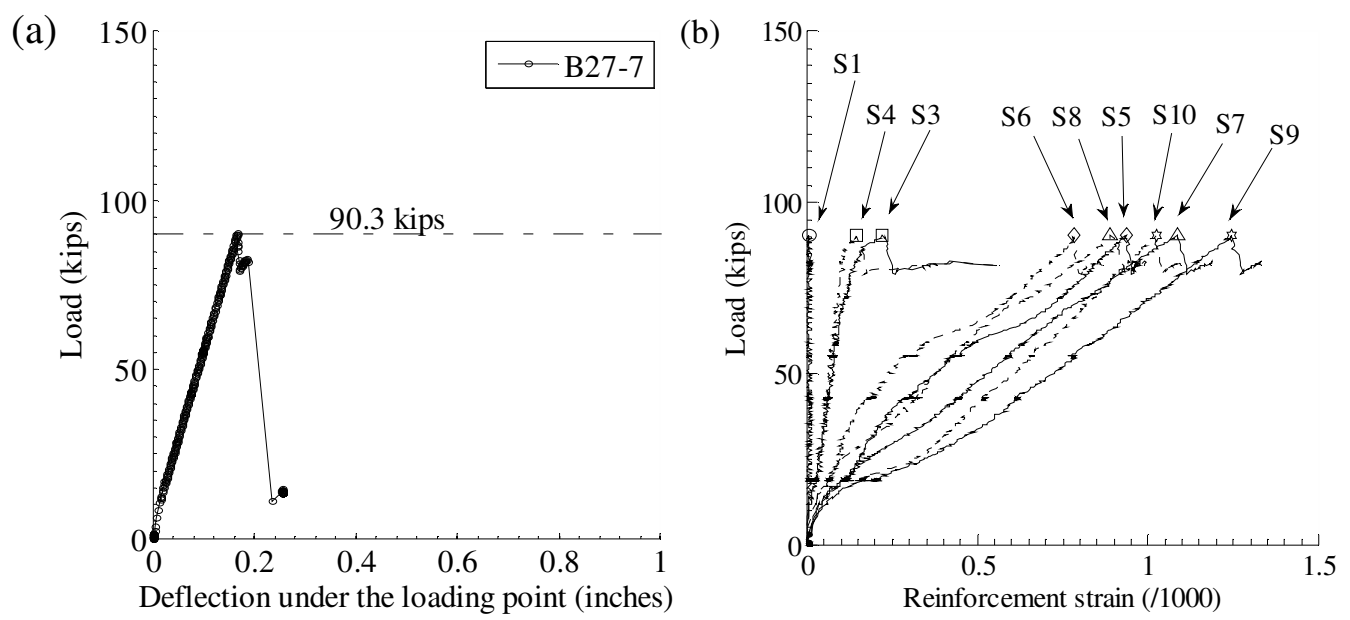

Fig. 4-185: Load versus deflection and reinforcement strain relationships - Beam B27-7

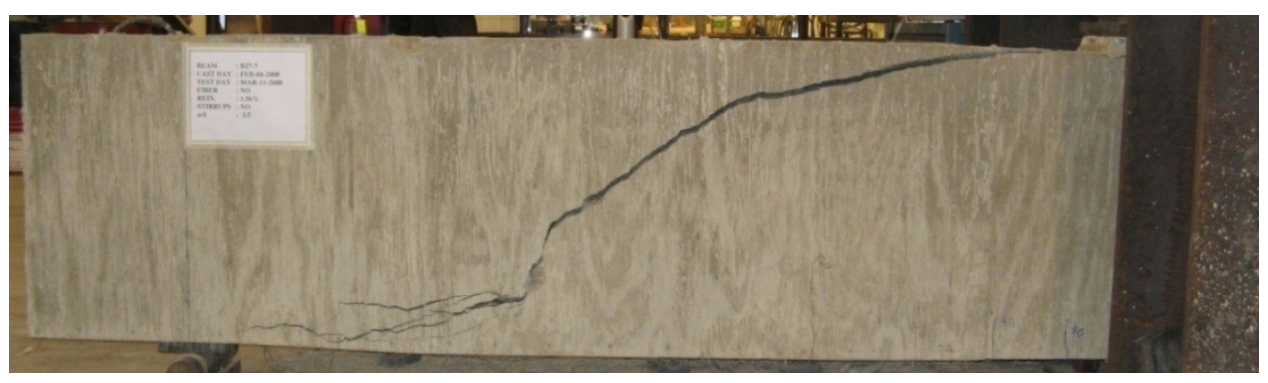

Fig. 4-186: Crack pattern after failure - Beam B27-7 


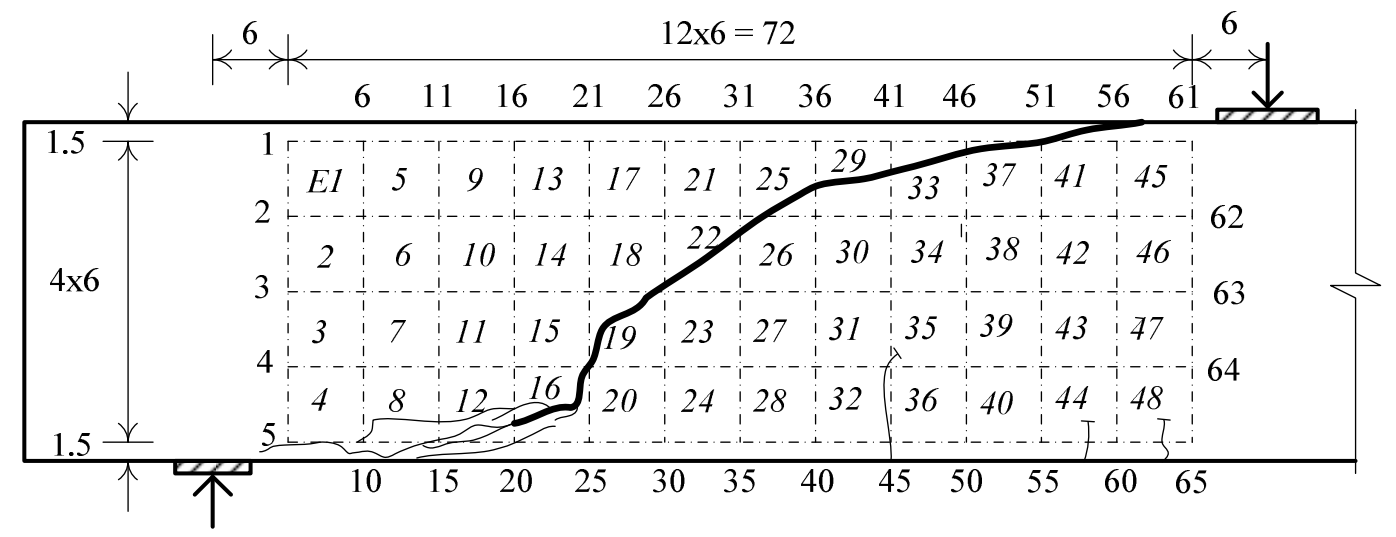

Fig. 4-187: Numbering of markers, elements, and strain gauges - Beam B27-7

(a)

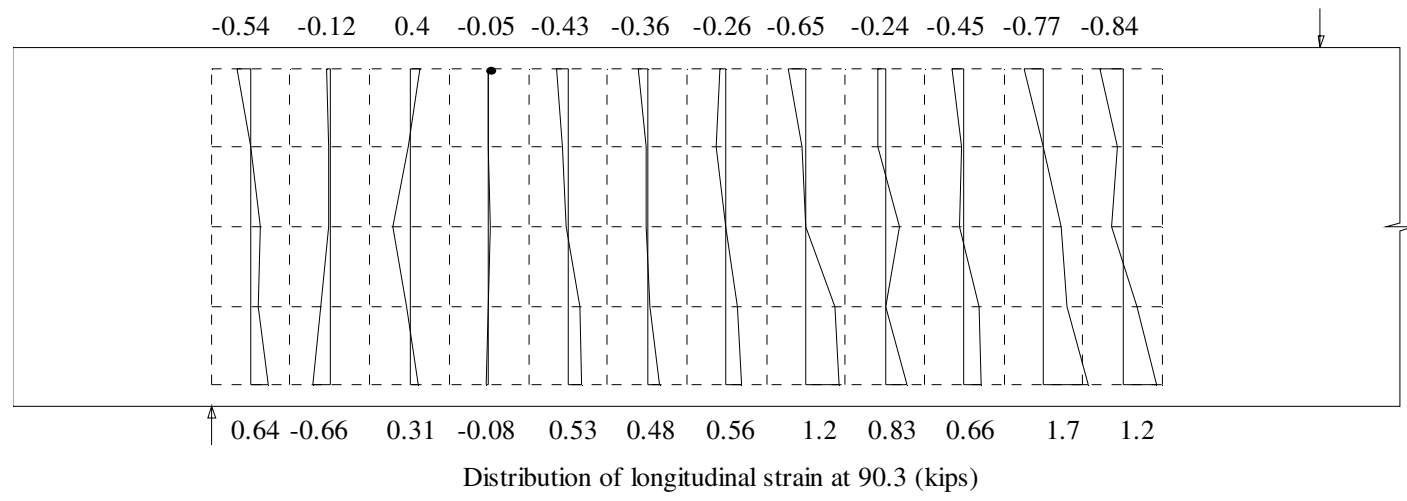

(b)

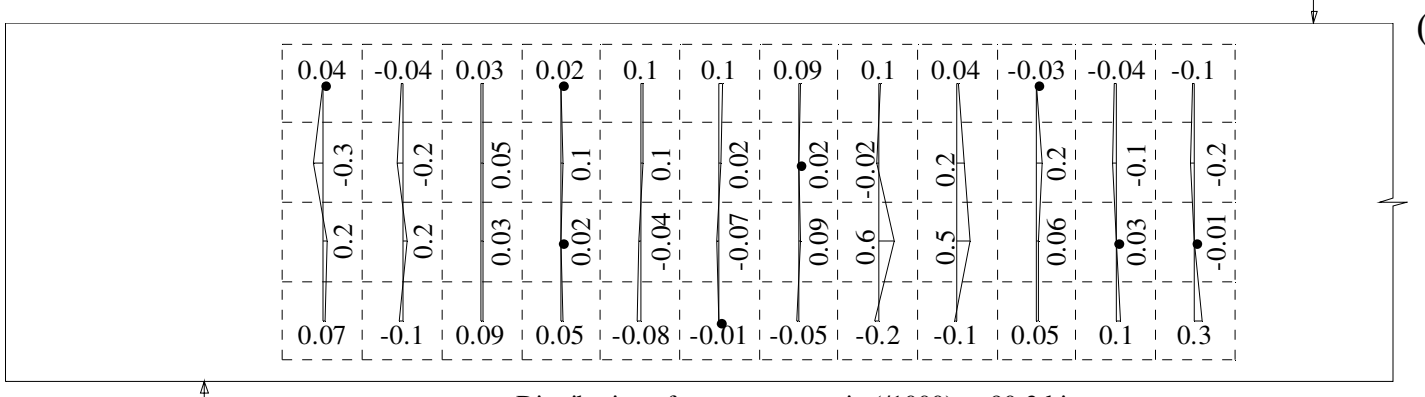

Distribution of transverse strain (/1000) at 90.3 kips

c)

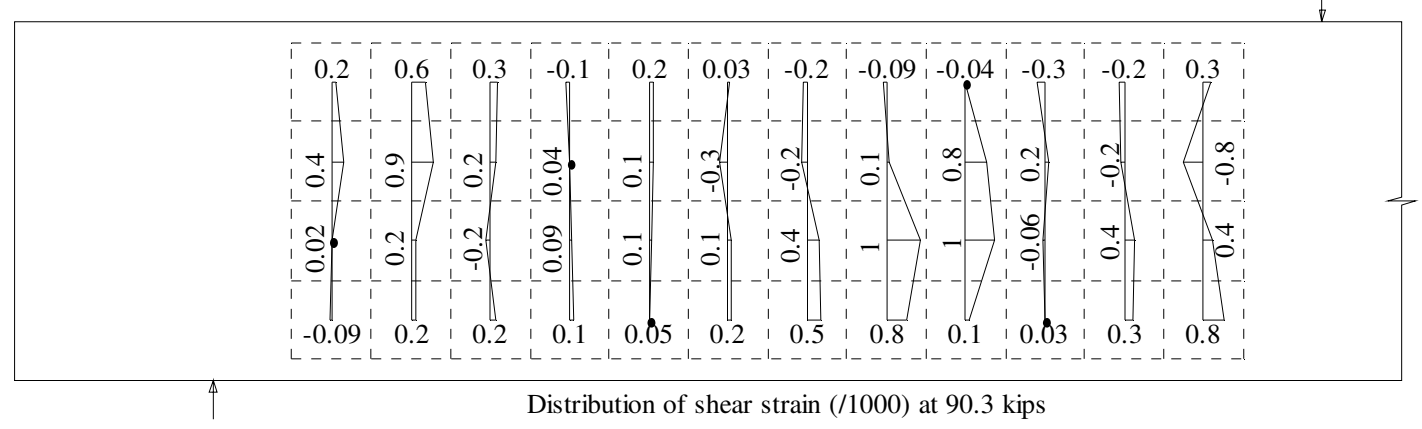

Fig. 4-188: Distribution of longitudinal, transverse, and shear strains at $90.3 \mathrm{k}-$ Beam

B27-7 


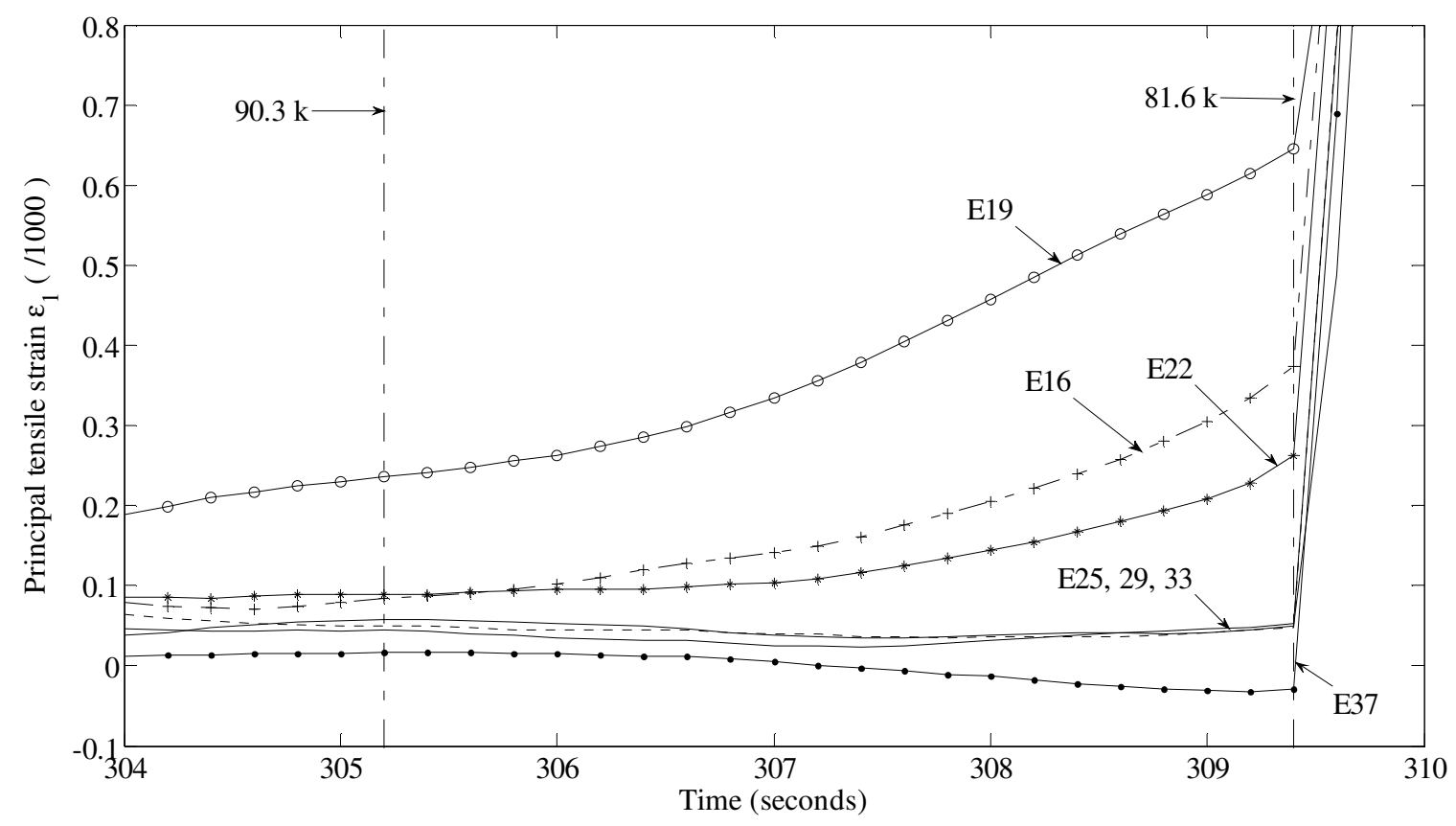

Fig. 4-189: Development of principal tensile strains along the critical inclined crack (close-up at the peak load) - Beam B27-7 


\subsubsection{Beam B27-8}

Beam B27-8 contained an amount of shear reinforcement that was 30\% higher than the minimum requirement stipulated by the ACI 2005 Code. It can be seen from Fig. 4-190, Fig. 4-191, and Fig. 4-193 that Beam B27-8 exhibited only two inclined cracks, which jointed together in the compression region. The fact that the beam contained a minimum amount of shear reinforcement enabled it to resist additional loading after the formation of inclined cracks. The beam failure was considered to be due to diagonal tension.

The beam exhibited its first diagonal crack at approximately 80 kips and attained its full strength at 128 kips (Fig. 4-192a). It should be mentioned that the failure caused a fracture of all the stirrups and crushing in the compression region.

At the peak load, the reinforcement at strain gauges S9 and S10 (Fig. 4-192b) approached the yield point. The longitudinal average tensile strains in the concrete were relatively small, reaching a maximum value of 0.003 (Fig. 4-194a). Also, the magnitude of longitudinal compressive strains was negligible. Compared with Beam B27-7 made of plain concrete, the transverse and shear strains in some of the elements were significant, reaching values of 0.024 and 0.015 , respectively (Fig. 4-194b and c), while the average transverse and shear strain, averaged over the beam depth, at the peak load were also very large (Fig. 4-195).

It can be seen from Error! Reference source not found. that principal tensile strains at peak load were significantly large along the critical inclined crack, compared with the other beam regions. Compared with the SFRC beams, Beam B27-8 was less effective in redistributing the strains to other region. The critical crack first opened at the mid-depth of the beam (Elements E19, 23, 26, and 30), extended to the reinforcement level (Elements E12 and 16, see Fig. 4-193 for numbering of elements), and then propagated into the compression region (Elements E33, 37, and 41), as can be seen in Fig. 4-197. The observation reconfirmed a diagonal tension failure mode, as discussed above. 


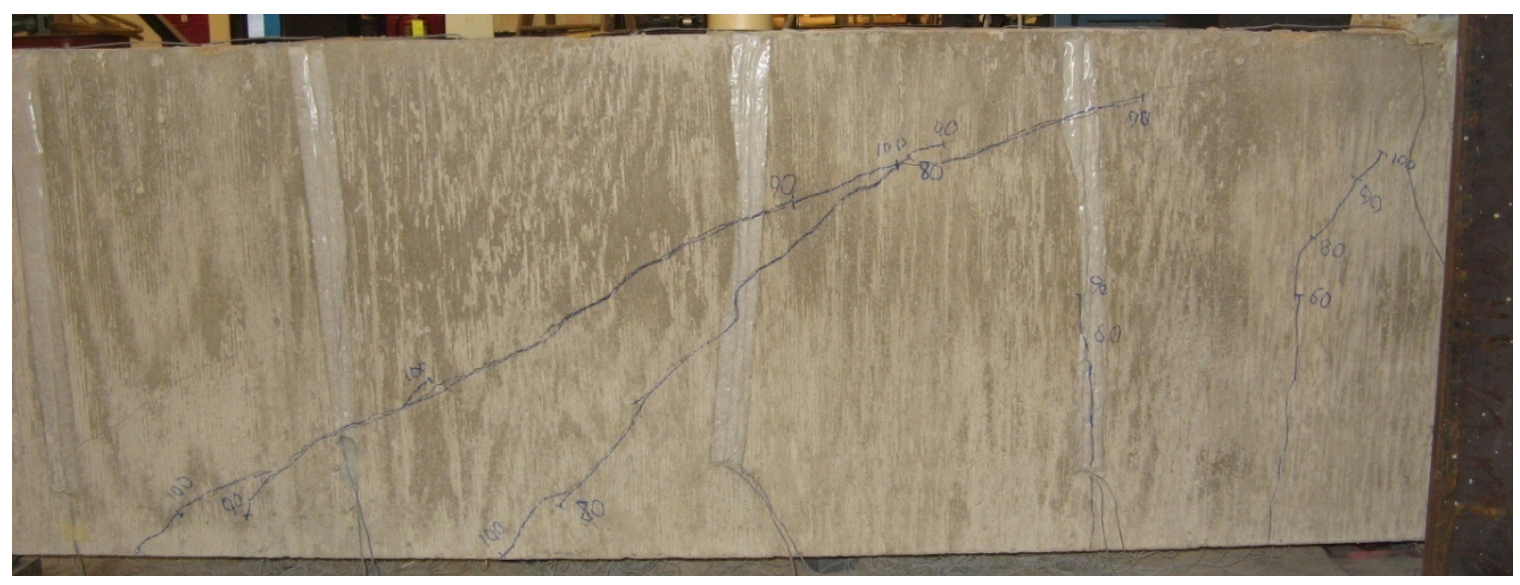

Fig. 4-190: Crack pattern prior to failure - Beam B27-8

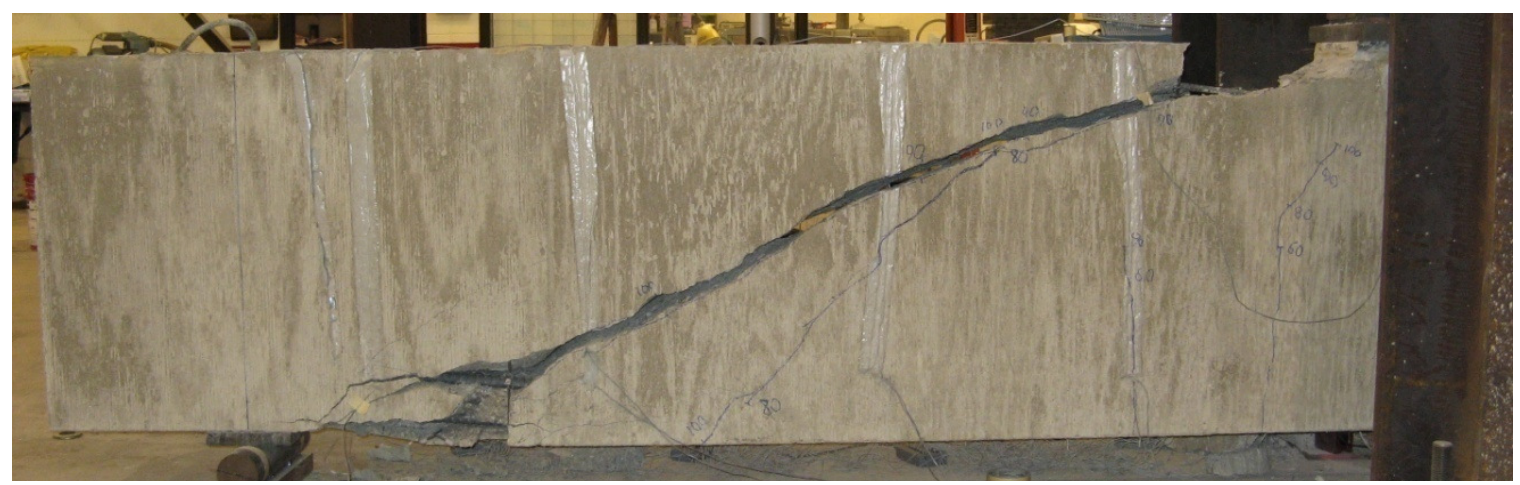

Fig. 4-191: Crack pattern after failure - Beam B27-8

(a)

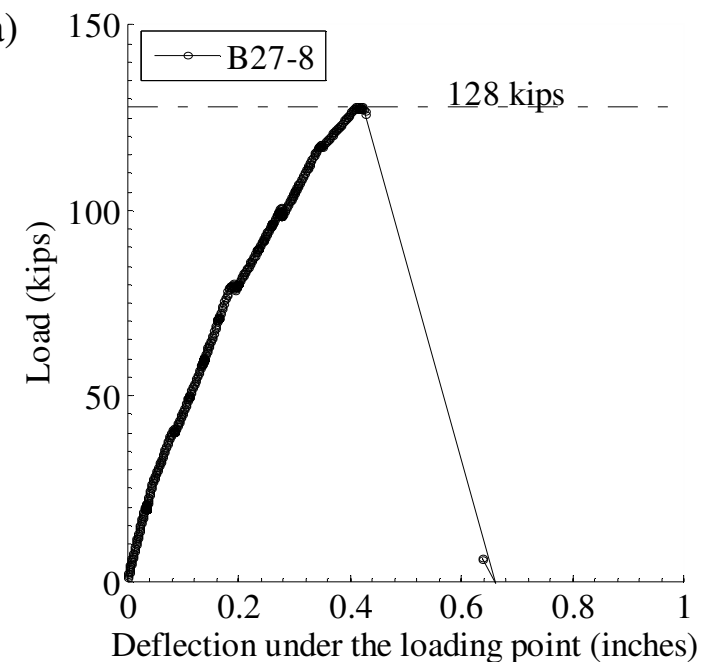

(b)

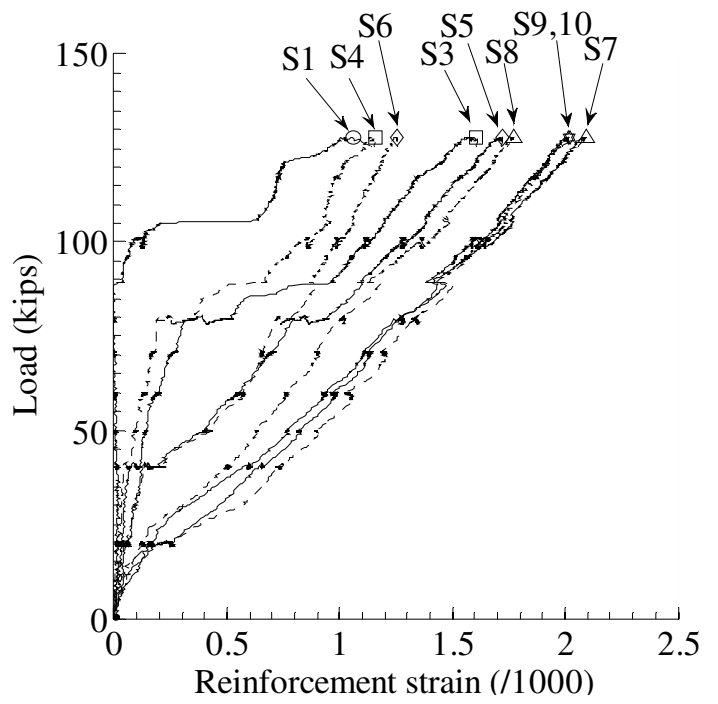

Fig. 4-192: Load versus deflection and reinforcement strain relationships - Beam B27-8 


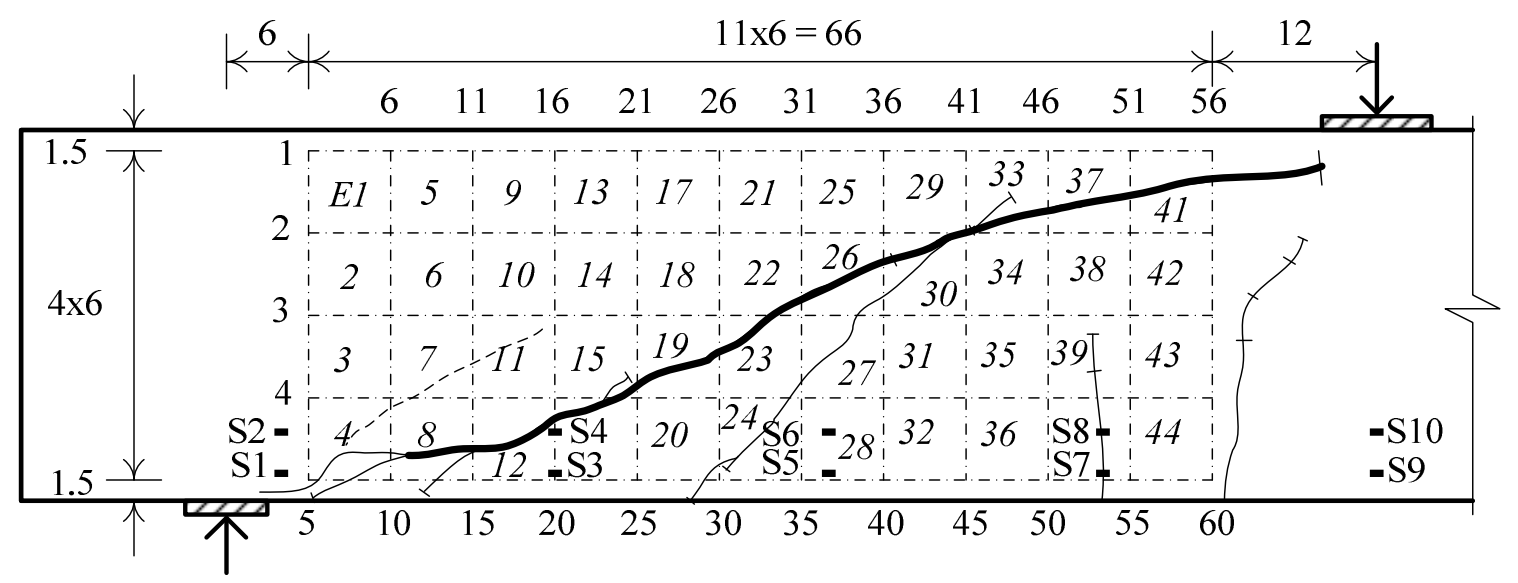

Fig. 4-193: Numbering of markers, elements, and strain gauges - Beam B27-8

(a)

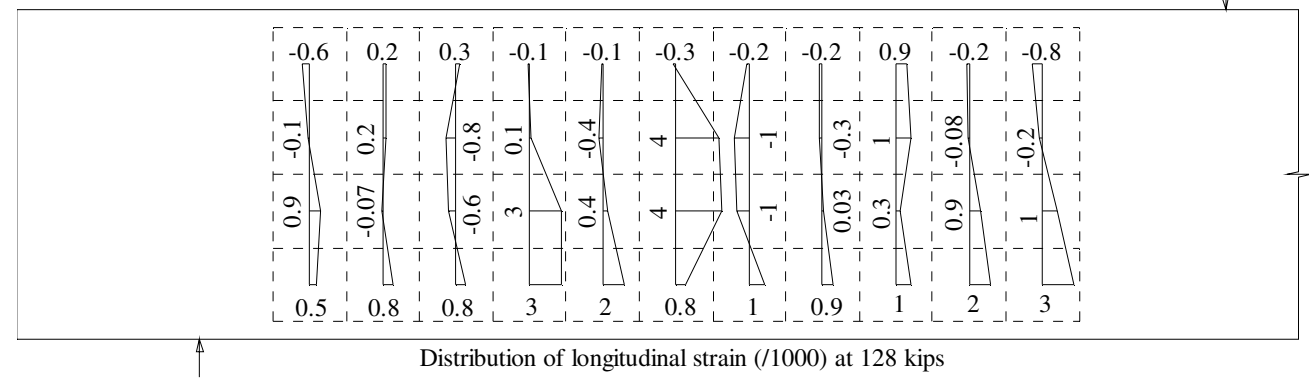

(b)

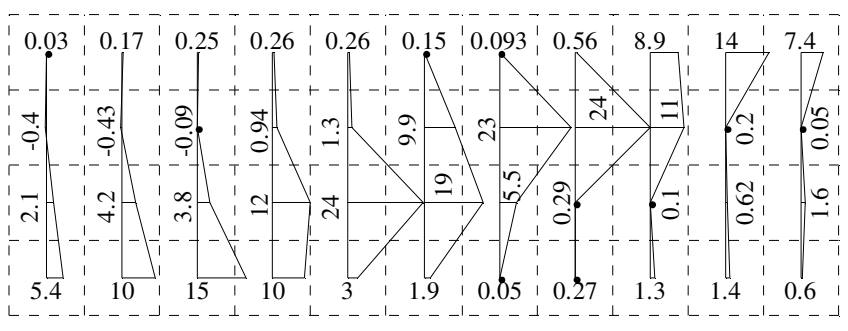

Distribution of transverse strain (/1000) at 128 kips

(c)

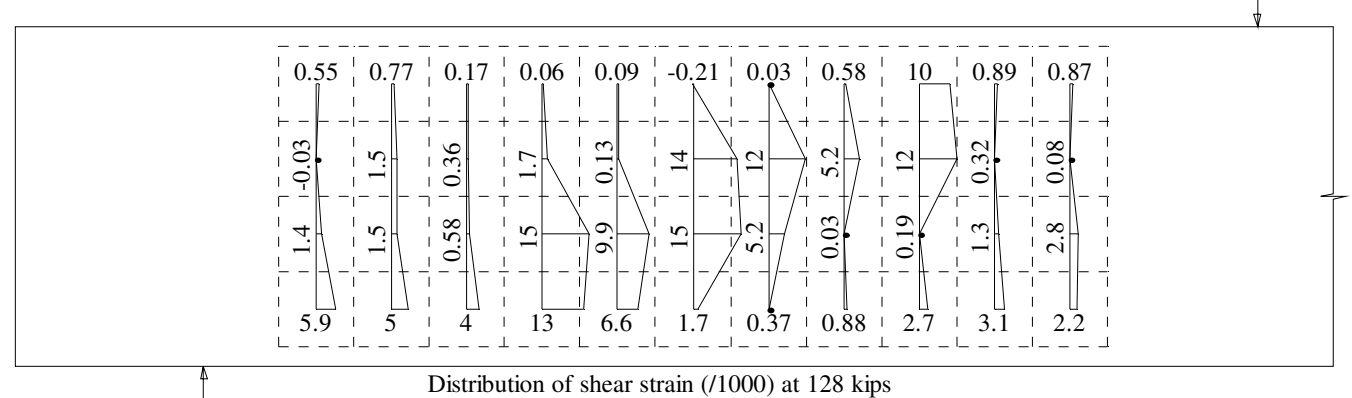

Fig. 4-194: Distribution of longitudinal, transverse, and shear strains at $128 \mathrm{k}-$ Beam 


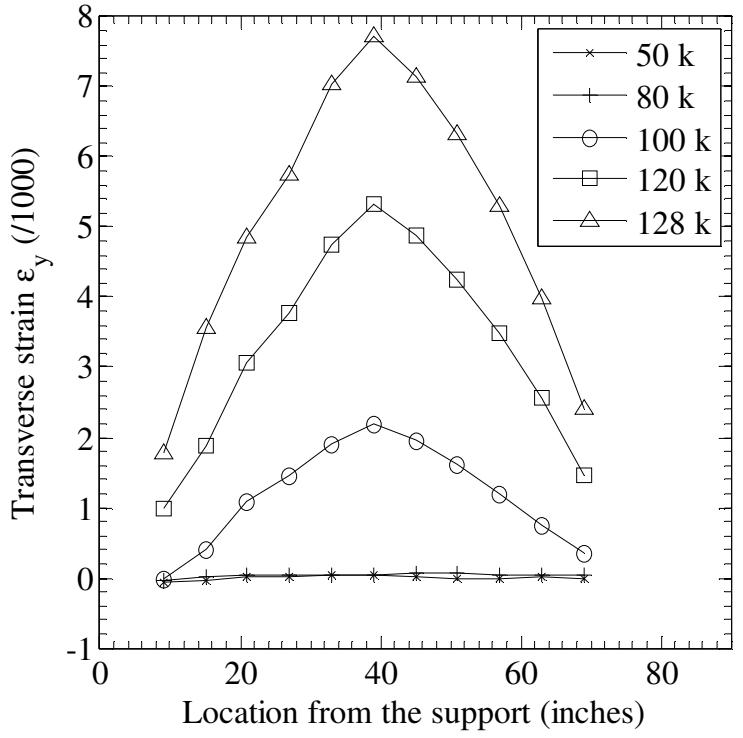

(a)

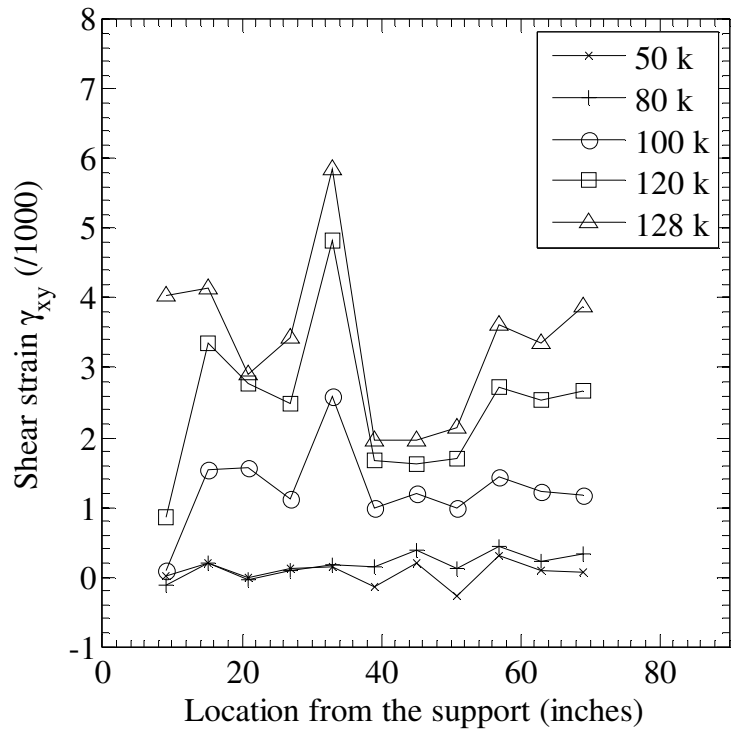

(b)

Fig. 4-195: Transverse and shear strains averaged over the beam depth - Beam B27-8

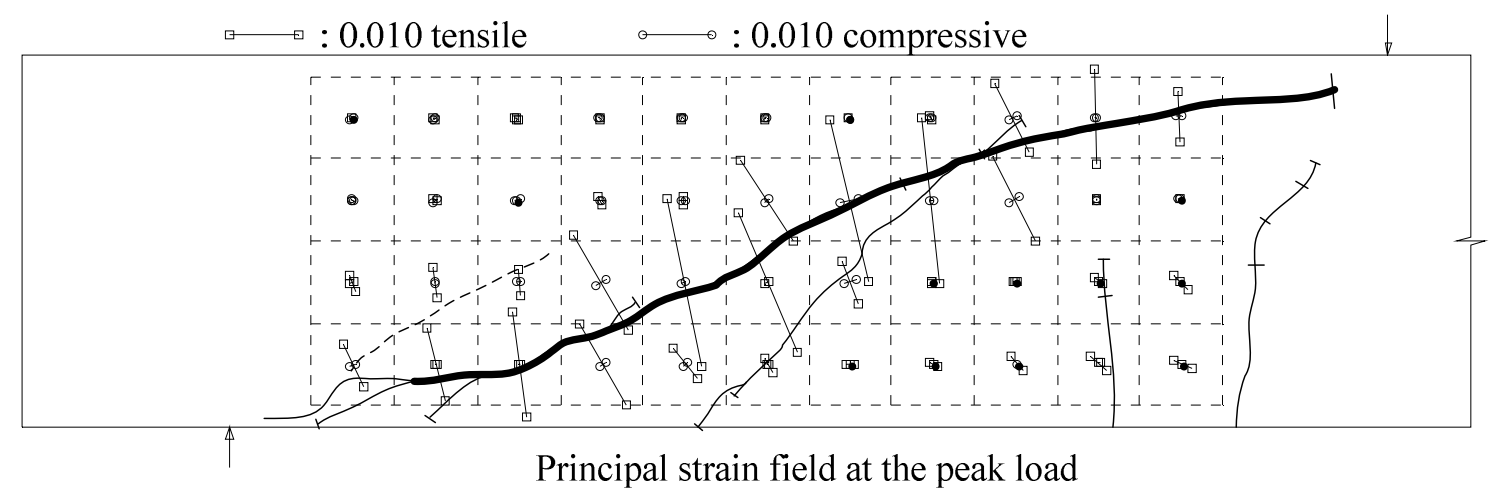

Fig. 4-196: Principal strain field at 128 kips - Beam B27-8 

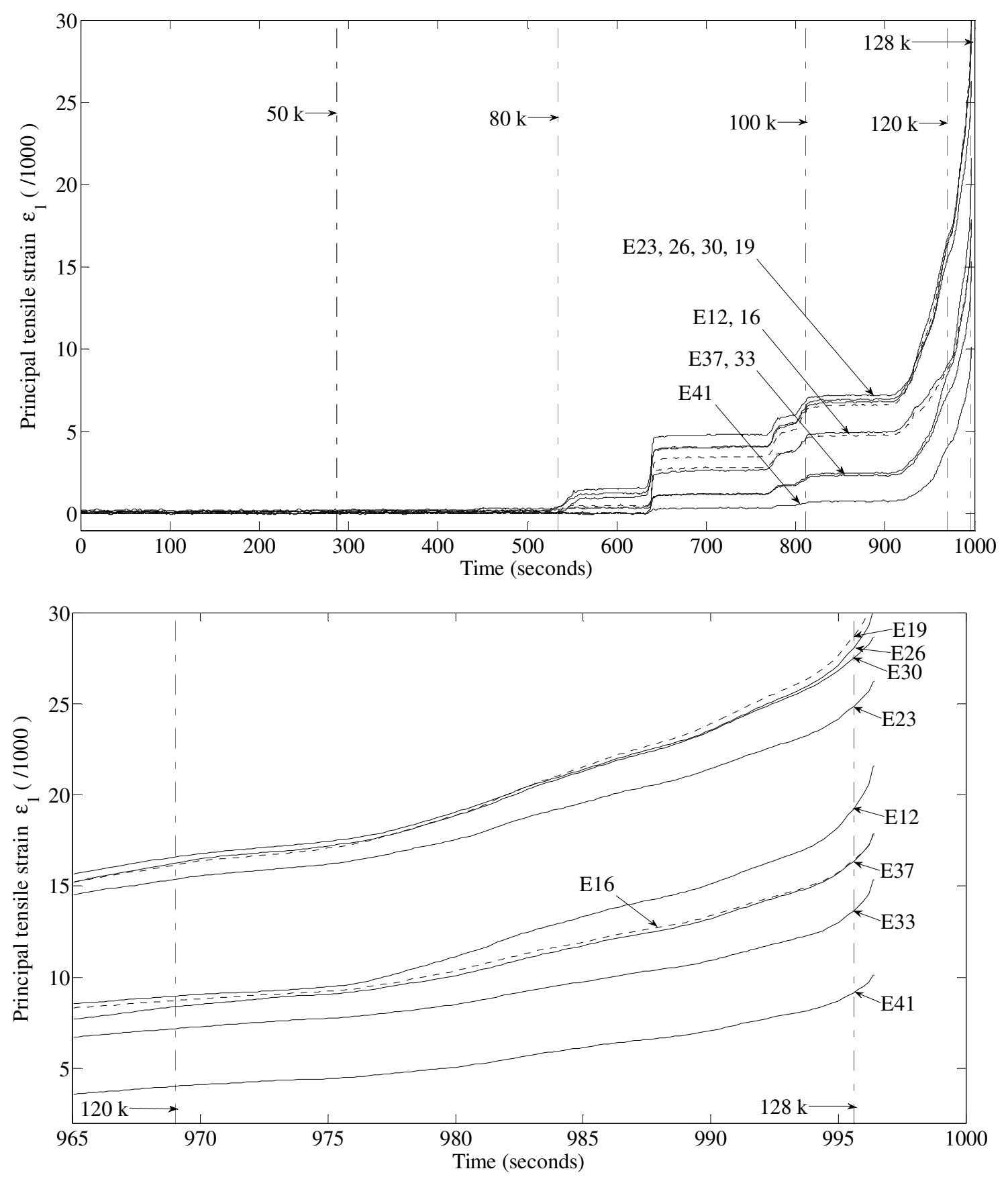

Fig. 4-197: Development of principal tensile strains along the critical inclined crack Beam B27-8 


\section{CHAPTER 5}

\section{ANALYSIS OF}

\section{EXPERIMENTAL RESULTS}

\subsection{OVERALL BEHAVIOR OF RC AND SFRC BEAMS}

\subsubsection{Shear stress and normalized shear stress}

Calculation of shear stress and normalized shear stress. The ultimate shear stress, $v_{u}$, resisted by the test beams in the critical shear span can be calculated on the basis of the peak applied load, $P_{u}$, shear span, $a$, beam span, $\ell$, beam width, $b$, and beam effective depth, $d$, as follows (Fig. 5-1):

$$
v_{u}=\frac{P_{u}(\ell-a)}{\ell \cdot b d} .
$$

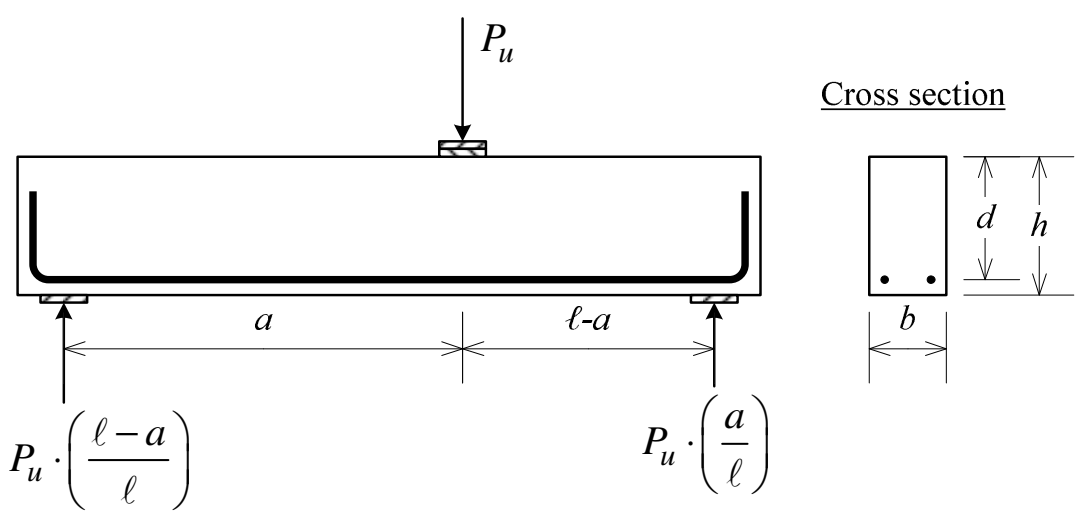

Fig. 5-1: Reaction (shear) forces in test beams 
The peak applied load, ultimate shear stress, and normalized ultimate shear stress, $\frac{v_{u}}{\sqrt{f_{c}^{\prime}}}$, are reported in Table 5-1.

Table 5-1: Summary of test results

\begin{tabular}{|c|c|c|c|c|c|c|c|}
\hline Beam & $\begin{array}{c}P_{u} \\
\text { (kips) }\end{array}$ & $\begin{array}{c}v_{u} \\
(\mathrm{psi})\end{array}$ & $\frac{v_{u}}{\sqrt{f_{c}^{\prime}}}$ & $\begin{array}{c}\text { Variation } \\
(\%)\end{array}$ & $\begin{array}{l}\text { Failure } \\
\text { mode }\end{array}$ & $\begin{array}{l}\text { Bond } \\
\text { failure }\end{array}$ & $\begin{array}{c}\text { Reinf. } \\
\text { yielded? }\end{array}$ \\
\hline (1) & (2) & (3) & (4) & (5) & (6) & (7) & (8) \\
\hline B18-0a & 37.7 & 162 & 2.06 & 1.6 & DT & No & N/A \\
\hline B18-0b & 36.5 & 157 & 1.99 & -1.6 & DT & No & N/A \\
\hline B18-1a & 99.2 & 426 & 5.29 & 3.3 & $\mathrm{SC}+\mathrm{ST}$ & No & Yes \\
\hline B18-1b & 92.8 & 399 & 4.95 & -3.3 & $\mathrm{ST}+\mathrm{DT}$ & No & Yes \\
\hline B18-2a & 98.2 & 440 & 5.92 & -1.0 & $\mathrm{ST}+\mathrm{DT}$ & No & Yes \\
\hline B18-2b & 100 & 449 & 6.04 & 1.0 & $\mathrm{ST}+\mathrm{DT}$ & No & Yes \\
\hline B18-2c & 113 & 507 & 6.81 & 16 & $(*)$ & No & Yes \\
\hline B18-2d & 82.5 & 370 & 4.97 & -16 & $(*)$ & Yes & No \\
\hline B18-3a & 86.4 & 371 & 5.54 & -14 & $\mathrm{ST}+\mathrm{DT}$ & Yes & No \\
\hline B18-3b & 114 & 490 & 7.30 & 14 & $\mathrm{SC}+\mathrm{ST}$ & No & No \\
\hline $\mathrm{B} 18-3 \mathrm{c}$ & 111 & 476 & 5.89 & 0.3 & $\mathrm{ST}+\mathrm{DT}$ & No & No \\
\hline $\mathrm{B} 18-3 \mathrm{~d}$ & 110 & 473 & 5.86 & -0.3 & $\mathrm{ST}+\mathrm{DT}$ & No & No \\
\hline B18-5a & 100 & 431 & 5.10 & -12 & DT & No & No \\
\hline B18-5b & 127 & 547 & 6.48 & 12 & $\mathrm{ST}+\mathrm{DT}$ & No & No \\
\hline B18-7a & 112 & 480 & 6.05 & 0.9 & $\mathrm{ST}+\mathrm{DT}$ & No & Yes \\
\hline B18-7b & 110 & 471 & 5.94 & -0.9 & $\mathrm{ST}+\mathrm{DT}$ & No & Yes \\
\hline B27-1a & 204 & 425 & 4.95 & 4.2 & $\mathrm{ST}+\mathrm{DT}$ & No & No \\
\hline B27-1b & 188 & 391 & 4.55 & -4.2 & DT & No & No \\
\hline B27-2a & 196 & 407 & 6.31 & 0.9 & $\mathrm{SC}+\mathrm{ST}$ & No & No \\
\hline B27-2b & 192 & 400 & 6.20 & -0.9 & DT & No & No \\
\hline B27-3a & 190 & 396 & 5.06 & -0.9 & $\mathrm{~F}$ & No & Yes \\
\hline B27-3b & 194 & 403 & 5.15 & 0.9 & $\mathrm{SC}+\mathrm{ST}$ & No & Yes \\
\hline B27-4a & 149 & 311 & 4.75 & 9.0 & $\mathrm{ST}+\mathrm{DT}$ & Yes & No \\
\hline B27-4b & 125 & 260 & 3.97 & -9.0 & $\mathrm{ST}+\mathrm{DT}$ & Yes & No \\
\hline B27-5 & 243 & 507 & 6.31 & - & $\mathrm{SC}+\mathrm{ST}$ & No & Yes \\
\hline B27-6 & 235 & 489 & 6.20 & - & $\mathrm{ST}+\mathrm{DT}$ & No & Yes \\
\hline B27-7 & 90.3 & 188 & 2.57 & - & DT & No & No \\
\hline B27-8 & 128 & 267 & 3.64 & - & DT & No & No \\
\hline
\end{tabular}


Variation of shear test data. Column 5 in Table 5-1 shows the percentage difference of the ultimate shear stress of each beam from the mean value of the corresponding pair. It can be seen that the ultimate shear stresses deviated in the range of \pm 0.3 to $\pm 16 \%$ from the mean value. Three pairs in Series B18 (B18-2c \& d, B18-3a \& b, and B18-5a \& b) and one pair in Series B27 (B27-4a \& b) showed a variation greater than or equal to 9\%. Potential reasons for such variations were given in Section 4.3.6, 4.3.7, 4.3.9, and 4.4.8. The strength difference with respect to the mean for the remaining beam pairs was less than 5\%. It should be noticed that these variations in shear strength were not large, given the known uncertainty in shear strength of concrete beams.

Ultimate shear stress and normalized ultimate shear stress. The mean ultimate shear stress for RC beams was 159 and 188 psi for Series B18 and B27, respectively, while the maximum measured ultimate shear stress for the SFRC beams in these two series was 547 psi (Beam B18-5b) and 507 psi (Beam B27-5), respectively. It is worth mentioning that these maximum shear stresses occurred, as expected, in the beams with the highest fiber volume fraction and longitudinal reinforcement ratio.

The corresponding normalized ultimate shear stress for these two SFRC beam series was 6.48 and $6.31 \sqrt{f_{c}^{\prime}}(\mathrm{psi})$, respectively. This level of normalized shear stress was 3.2 and 2.5 times greater than that in the control specimens of Series B18 and B27, respectively. It should be noted that a peak normalized ultimate shear stress of $7.3 \sqrt{f_{c}^{\prime}}$ (psi) was measured for Beam B18-3b due to a lower concrete compressive strength compared with that in the other beams in the same series. However, the ultimate shear stress for this beam was $490 \mathrm{psi}$, which is lower than the maximum shear stress value for Beam B18-5b.

The lowest normalized ultimate shear stress for the SFRC beams was still 2.5 and 1.5 times greater than that for the control specimens of Series B18 and B27, respectively. Although the lowest normalized shear strength for the B27 beam series corresponded to one of the test beams with the lowest amount of fibers and reinforcement ratio (Beam B27-4b), this was not the case for the beam in Series B18 (B18-2d). Table 
5-2 compares the maximum and minimum shear strengths of the SFRC beams with the strength of the control RC beams.

Table 5-2: Maximum and minimum shear strengths

\begin{tabular}{|c|c|c|c|c|c|c|c|c|c|c|}
\hline \multirow[b]{2}{*}{ 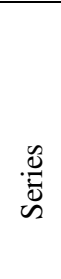 } & \multicolumn{2}{|c|}{$\begin{array}{c}\text { RC beam } \\
\text { (average value) }\end{array}$} & \multicolumn{4}{|c|}{$\begin{array}{c}\text { SFRC beam } \\
\text { (maximum strength) }\end{array}$} & \multicolumn{4}{|c|}{$\begin{array}{c}\text { SFRC beam } \\
\text { (minimum strength) }\end{array}$} \\
\hline & $\begin{array}{c}v_{u} \\
(\mathrm{psi})\end{array}$ & $\frac{v_{u}}{\sqrt{f_{c}^{\prime}}}$ & Beam & $\begin{array}{c}v_{u} \\
(\mathrm{psi})\end{array}$ & $\frac{v_{u}}{\sqrt{f_{c}^{\prime}}}$ & $\frac{\left(\frac{v_{u}}{\sqrt{f_{c}^{\prime}}}\right)_{S F R C}}{\left(\frac{v_{u}}{\sqrt{f_{c}^{\prime}}}\right)_{R C}}$ & Beam & $\begin{array}{c}v_{u} \\
(\mathrm{psi})\end{array}$ & $\frac{v_{u}}{\sqrt{f_{c}^{\prime}}}$ & $\frac{\left.\frac{v_{u}}{\sqrt{f_{c}^{\prime}}}\right)_{S F R C}}{\left(\frac{v_{u}}{\sqrt{f_{c}^{\prime}}}\right)_{R C}}$ \\
\hline B18 & 159 & 2.02 & B18-5b & 547 & 6.48 & 3.2 & B18-2d & 370 & 4.97 & 2.5 \\
\hline B27 & 188 & 2.57 & B27-5 & 507 & 6.31 & 2.5 & B27-4b & 260 & 3.97 & 1.5 \\
\hline
\end{tabular}

\subsubsection{Failure modes}

Column 6 of Table 5-1 lists the failure mode of all beams discussed in Chapter 4. It can be seen that four failure modes were observed: (1) diagonal tension, (2) a combination of diagonal tension and shear-tension, (3) a combination of shearcompression and shear-tension, and (4) flexural failure.

For a diagonal tension failure, the opening of the critical inclined crack occurred in the beam mid-depth region and propagated towards both the reinforcement level and the compression region. At a certain applied load, the principal tensile strain and crack width were largest at mid-depth, smaller for the reinforcement level, and smallest adjacent to the compression region. At failure, the critical crack extended through the beam compression zone without causing crushing of the concrete. The depth of the compression zone above the upper tip of the critical inclined crack was often very small. The crack angle with respect to the beam longitudinal axis was rather shallow, approximately 20 degrees for SFRC beams, as shown in Section 5.1.5. This type of failure occurred for the two RC beams, the RC beam with a minimum stirrup reinforcement, and SFRC Beams B18-5a, B27-1b, and B27-2b. In general, diagonal tension failures were associated with lower shear strengths compared to other failure modes. 
A failure mode somewhat similar to diagonal tension is the combination of diagonal tension and shear-tension. In this failure mode, diagonal tension also caused the diagonal crack to open first, but there were also cracks along the reinforcing bars. In some of the beams that failed in this mode, cracks developing along the top layer of longitudinal reinforcing bars could be seen. The angles of the critical inclined cracks with respect to the beam longitudinal axis were larger than those for the beams that failed in diagonal tension.

For a failure mode which can be considered a combination of shearcompression and shear-tension failures, the widening of the critical crack started at the reinforcement level and extended up, toward the compression region. The crack opening was often a result of reinforcement elongation due to the moment demand. The angle of the crack at the bottom of the beam was therefore steep. The opening of the critical crack resulted in a relative rotation of the two pieces of the beams around the crack upper tip. This rotation, combined with the shear stress carried by the uncracked compressed concrete, imposed a significant compression strain demand on the beam compression zone, which ultimately led to crushing of the concrete and beam failure. The depth of the failed concrete compression zone observed in Series B27 beams varied from 3 to 7 inches, corresponding to 10 to $25 \%$ of the beam depth. This type of failure also led to a significant splitting along the top layer of longitudinal tension reinforcement. This type of failure occurred for Beams B18-1a, B18-3b, B27-2a, B27-3b, and B27-5.

The fact that two different types of shear failures occurred in beams of the same pair is a clear indication of the impossibility to predict one type of shear failure for a given beam. In some cases, a cracking pattern first developed that indicated the possibility of a shear-compression failure, followed later by the opening of a different diagonal crack that led to a diagonal tension failure. Thus, although a distinction has been made with regard to the type of shear failure exhibited by each beam, it is believed that all types of shear failure should be lumped together when evaluating their overall behavior and shear strength.

The last type of failure, flexural failure, was only observed in Beam B27-3a with a lower amount of longitudinal reinforcement. In this case, crushing of the beam 
compression zone near the load point occurred after substantial yielding of the longitudinal tension reinforcement had taken place.

Of all beam specimens, four were found to have exhibited a shear failure triggered by significant deterioration of bond along the longitudinal reinforcement near the support region (Column 7, Table 5-1). These specimens had a lower shear strength compared with their counterparts in the same pairs. The poor bond along the longitudinal reinforcement in these specimens was attributed to either voids during concrete casting that required repair or lumping of fibers along the longitudinal reinforcement, or a combination of both. Column 8 of Table 5-1 lists the specimens that exhibited flexural yielding.

Within a pair of beams, the beam that failed due to combined shear compression and shear tension always had a higher strength than the beam that failed due to combined shear tension and diagonal tension. This was observed in beam pairs B18-1a \& B18-1b and B18-3b \& B18-3a. Similarly, beams that failed due to combined shear tension and diagonal tension had a strength greater than that of beams that failed due to pure diagonal tension. This was evident in beam pairs B18-5b \& B18-5a and B27-1a \& B27-1b.

There were beams which, after opening of a diagonal crack, failed due to a sudden opening of another inclined crack. These beams had a higher strength compared with their counterpart in the same pair, if those other beams did not exhibit the same phenomenon. For example, prior to failure, Beam B18-5b seemed to be headed towards a diagonal tension failure by opening of the diagonal crack closest to the support. Failure in this beam, however, was ultimately caused by the opening of another crack, closer to the loading point, which led to a combined diagonal tension and shear tension failure mode. This beam had a shear strength of 127 kips compared to 100 kips for Beam B18-5a. A similar situation was also observed in Beam B27-5. 


\subsubsection{Crack patterns}

The crack patterns for the RC and SFRC beams were distinctly different. While the RC beams without transverse reinforcement exhibited a single inclined crack followed by a brittle shear failure, all SFRC beams showed at least two diagonal cracks. At first diagonal cracking, a minor reduction in stiffness was observed for the SFRC beams. Prior to failure, these beams may or may not have exhibited flexural yielding. The degree of flexural yielding was primarily defined by the amount of longitudinal reinforcement and fibers. With a minimum amount of stirrup reinforcement, a minor improvement in cracking pattern was observed for Beam B27-8 compared to the RC beam without stirrups. For each beam pair, the SFRC beam with the highest number of flexural and diagonal cracks throughout the critical shear span exhibited a higher shear strength than its counterpart (for example, compare Beam B18-3a with B18-3b, B18-5a with B18-5b, and B27-4b with B27-4a). It should also be mentioned that most of the inclined cracks on beams with higher amount of longitudinal reinforcement and fibers did not propagate beyond the mid-depth level of the beams (compare Beams B18-3a, b, c, \& d with other SFRC beams). 

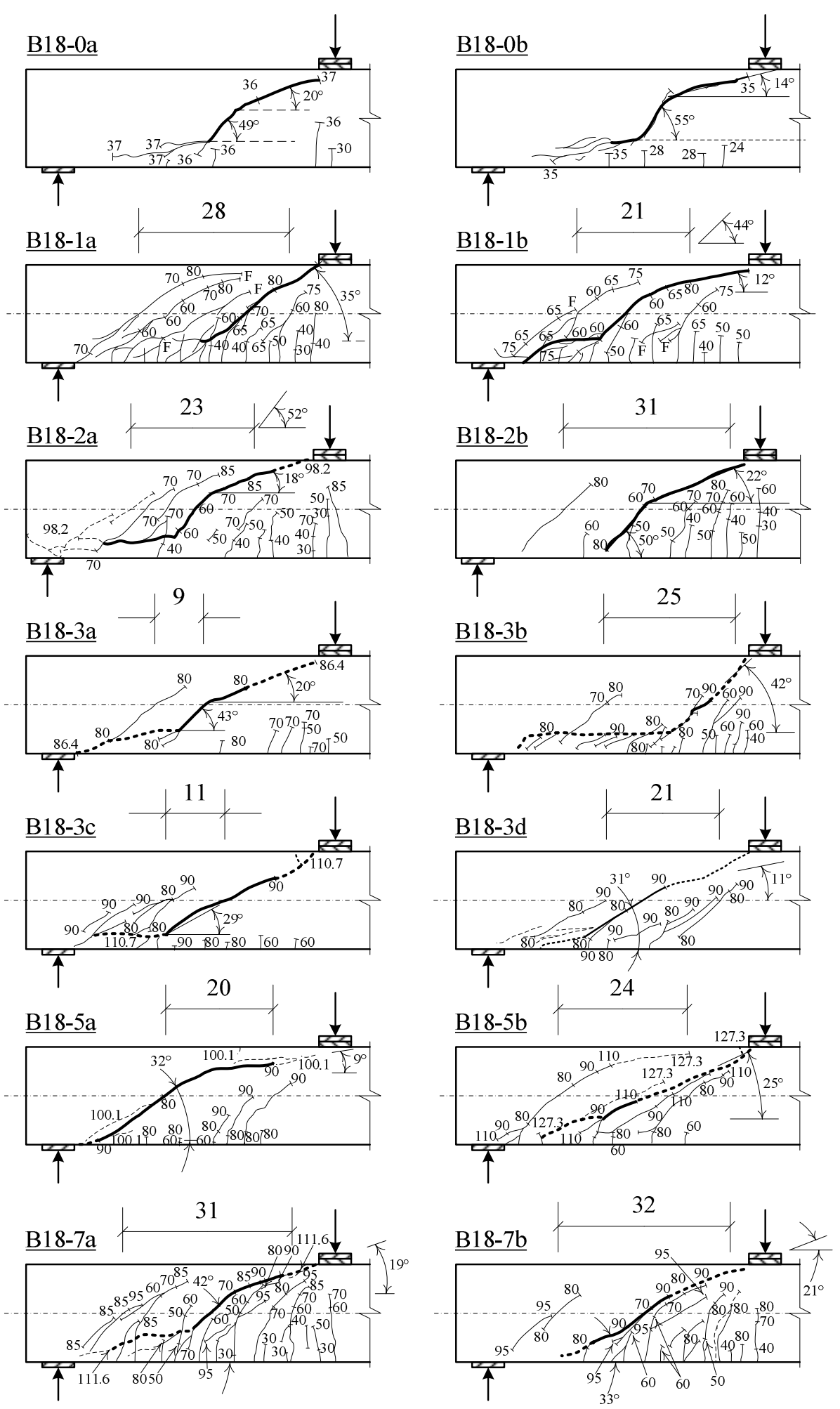

(The dashed lines indicate the cracks marked after failure)

Fig. 5-2: Determination of the number of inclined cracks, average inclined crack spacing, and angle and length of the critical crack (Series B18) 


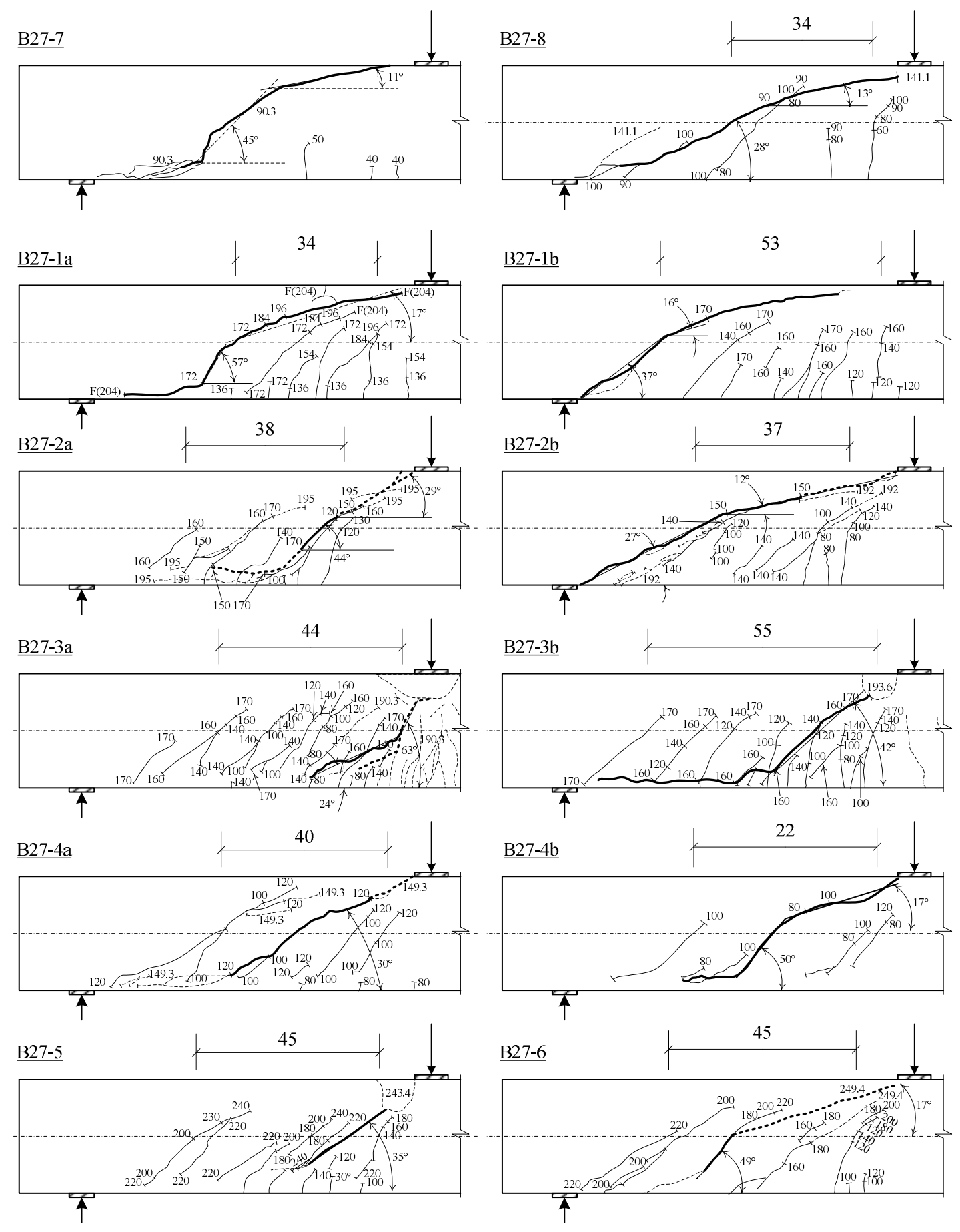

(The dashed lines indicate the cracks marked after failure)

Fig. 5-3: Determination of the number of inclined cracks, average inclined crack spacing, and angle and length of the critical crack (Series B27) 


\subsubsection{Horizontal spacing of inclined cracks}

The number of inclined cracks $(n)$ and the total horizontal distance $\left(\sum s\right)$ at the mid-depth level from the inclined crack nearest to the support to the one closest to the loading point are reported in Table 5-3. An inclined crack was counted only if it was distinct from other inclined cracks, propagated beyond the beam mid-depth level, and was inclined at an angle from 0 to 80 degrees with respect to the beam longitudinal axis. The average inclined crack spacing $(s)$ for each beam is also listed in Table 5-3. This average crack spacing was calculated as follows:

$$
s=\frac{\sum s}{n-1}
$$

Fig. 5-4 shows the relationship between inclined crack spacing and either shear stress or normalized shear stress. In this figure, the data from the beams with known defects (B18-2d, B18-3a, B18-5a, and B27-4b) and those without fibers are excluded. From these plots, it can be seen that beam shear strength increased with a decrease in crack spacing.

Fig. 5-5 shows the relationship between the reinforcement ratio and inclined crack spacing, and between fiber reinforcing index and inclined crack spacing. No clear trend could be observed. However, the inclined crack spacing was larger for beams with larger effective depth. 
Table 5-3: Number of inclined cracks, crack spacing, and crack angles

\begin{tabular}{ccccc}
\hline Beams & $n$ & $\begin{array}{c}\text { } s \\
\text { (inches) }\end{array}$ & $\begin{array}{c}s \\
\text { (inches) }\end{array}$ & $\begin{array}{c}\theta_{c} \\
\left({ }^{\circ}\right)\end{array}$ \\
\hline B18-0a & 1 & - & - & 30 \\
B18-0b & 1 & - & - & 32 \\
B18-1a & 5 & 28 & 7.0 & 33 \\
B18-1b & 4 & 21 & 7.0 & 25 \\
B18-2a & 5 & 23 & 5.8 & 29 \\
B18-2b & 5 & 31 & 7.8 & 30 \\
B18-2c & $(*)$ & $\left(^{*}\right)$ & $(*)$ & $(*)$ \\
B18-2d & $(*)$ & $\left.{ }^{*}\right)$ & $(*)$ & $(*)$ \\
B18-3a & 2 & 9 & 9.0 & 27 \\
B18-3b & 5 & 25 & 6.3 & 42 \\
B18-3c & 3 & 11 & 5.5 & 29 \\
B18-3d & 4 & 21 & 7.0 & 27 \\
B18-5a & 3 & 20 & 10.0 & 21 \\
B18-5b & 5 & 24 & 6.0 & 25 \\
B18-7a & 7 & 31 & 5.2 & 28 \\
B18-7b & 7 & 32 & 5.3 & 25 \\
B27-1a & 5 & 34 & 8.5 & 26 \\
B27-1b & 6 & 53 & 10.6 & 22 \\
B27-2a & 4 & 38 & 12.7 & 35 \\
B27-2b & 5 & 37 & 9.3 & 18 \\
B27-3a & 7 & 44 & 7.3 & 37 \\
B27-3b & 7 & 55 & 9.2 & 42 \\
B27-4a & 4 & 40 & 13.3 & 29 \\
B27-4b & 4 & 22 & 7.3 & 29 \\
B27-5 & 6 & 45 & 9.0 & 35 \\
B27-6 & 6 & 45 & 9.0 & 25 \\
B27-7 & 1 & - & - & 26 \\
B27-8 & 2 & 34 & 17.0 & 21 \\
\hline (*) A record of crack pattern & was not available \\
for these beam specimens. & & \\
\hline & & & &
\end{tabular}



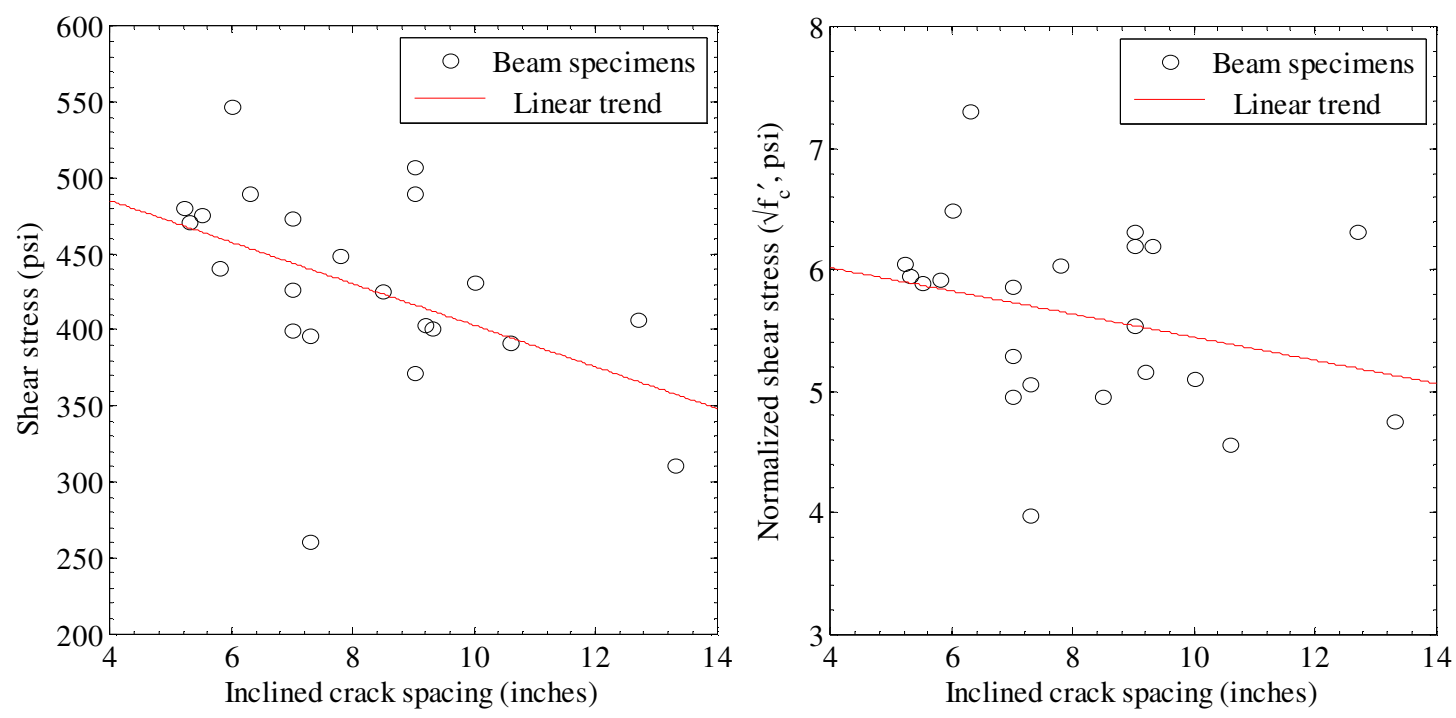

Fig. 5-4: Relationship between inclined crack spacing and either shear stress or normalized shear stress
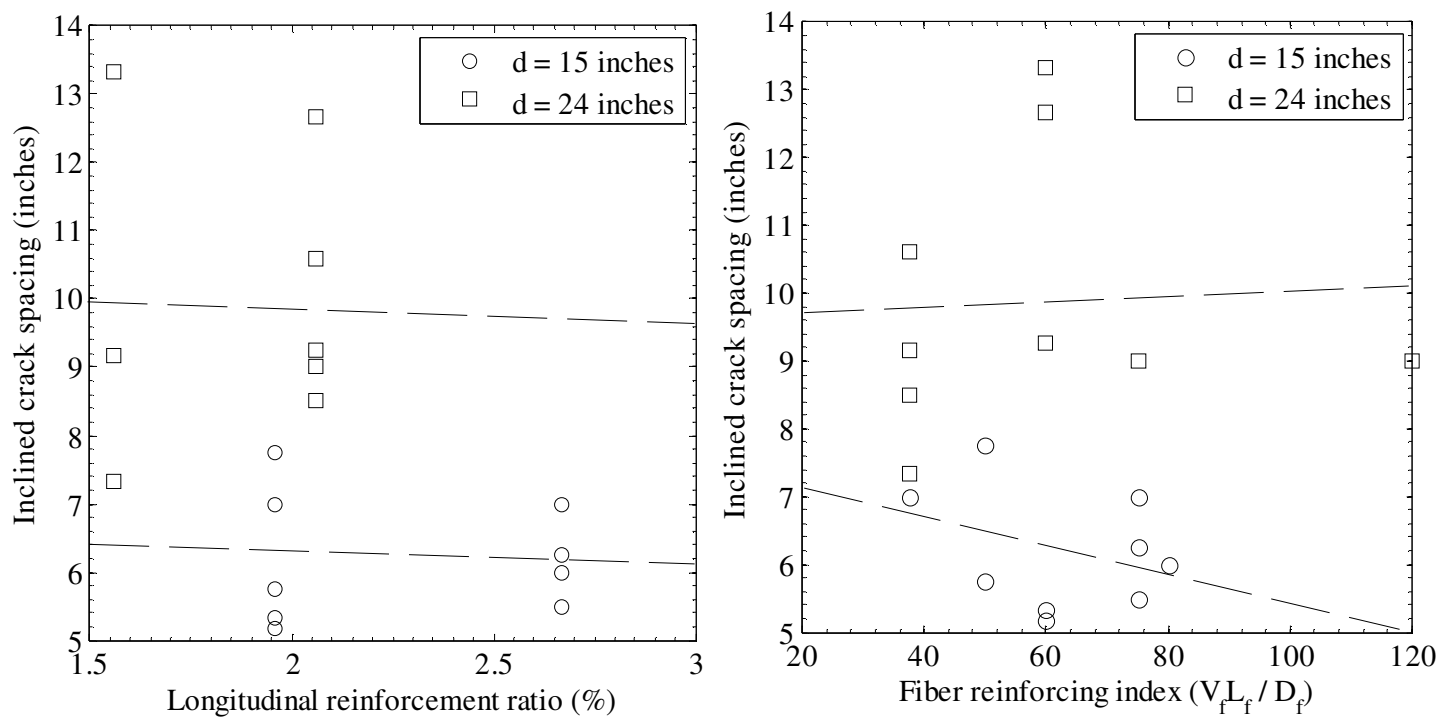

Fig. 5-5: Relationship between inclined crack spacing and longitudinal reinforcement ratio, and between inclined crack spacing and fiber reinforcing index 


\subsubsection{Angles of critical inclined cracks}

The angle of the critical crack with respect to the horizontal axis for each beam is reported in Table 5-3. This angle was measured from the lower to the upper tip of the critical crack. Fig. 5-5 shows the inclined crack angle versus shear stress relationship for all beams with fibers, except for Beams B18-2c \& d (angles not recorded). It can be seen that the crack angle was shallowest for beams with diagonal tension failures and steepest for those with a combined shear-compression and shear-tension failure. The average angles for these two types of failure modes were 20 and 37 degrees, respectively. For beams that failed due to a combination of diagonal tension and shear tension, the average angle for the critical inclined crack was approximately 27 degrees.

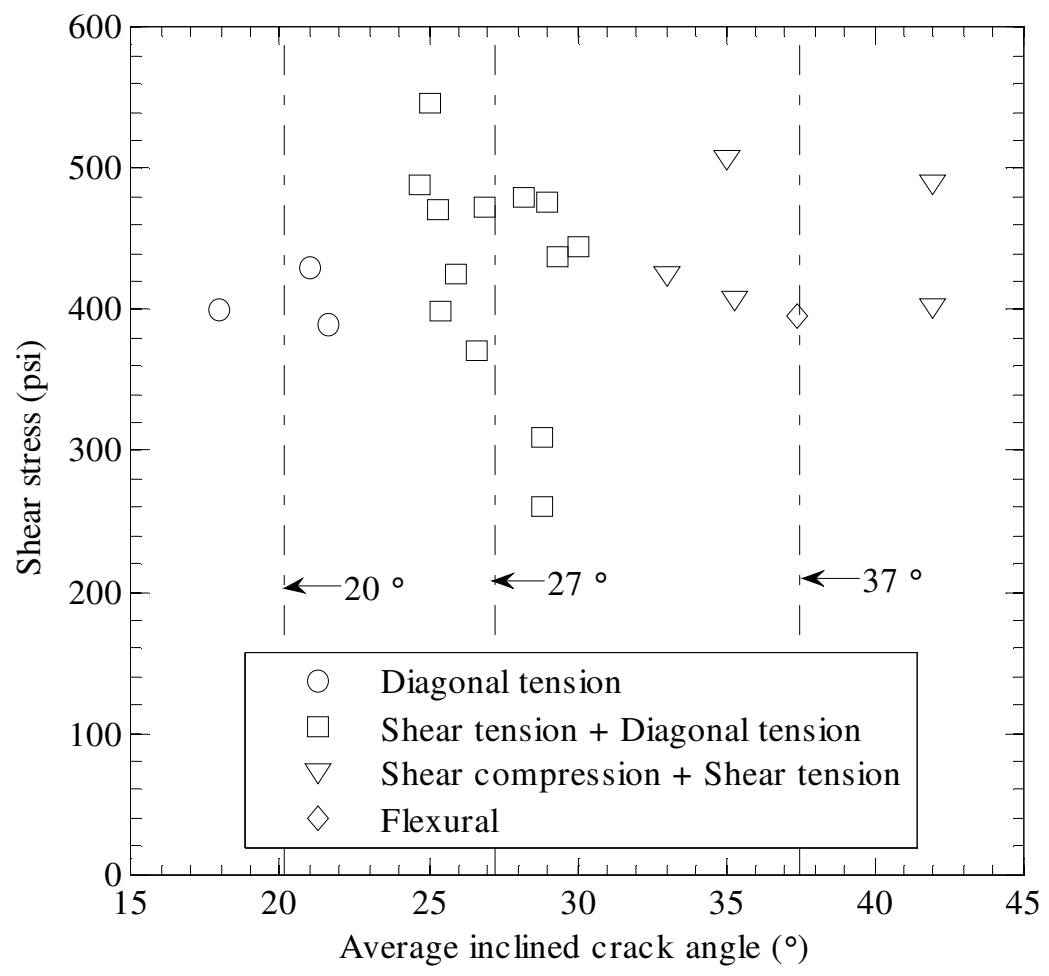

Fig. 5-6: Relationship between average inclined crack angle versus shear stress for beams with fibers 


\subsection{EFFECT OF STUDIED PARAMETERS}

\subsubsection{Effect of fiber type}

Table 5-4 lists the average shear stresses for each beam pair and the strength ratios for pairs of SFRC beams constructed with similar effective depth, fiber volume fraction, and longitudinal reinforcement ratio, but with different types of fibers. It can be seen that the SFRC beams constructed with RC80/30BP fibers exhibited only 17\% higher normalized shear strength compared to those with ZP305 fibers, which have an aspect ratio approximately two thirds that of RC80/30BP fibers. It should be mentioned that ZP305 fibers have a tensile strength less than one-half of that of RC80/30BP fibers. Similarly, the shear strength of the beams with RC80/60BN fibers, which also have a greater aspect ratio, was of similar magnitude to that of beams with ZP305 fibers. The normalized shear stress of the former ranged from 85 to $132 \%$ that of the latter.

Table 5-4: Ratio of shear stresses for SFRC beams with different types of fibers

\begin{tabular}{|c|c|c|c|c|c|c|}
\hline$(\%)$ & $\begin{array}{c}\rho \\
(\%)\end{array}$ & Beam & Fiber & $\begin{array}{l}\text { Fiber } \\
\text { aspect } \\
\text { ratio }\end{array}$ & $\begin{array}{c}\text { Shear stress } \\
\quad(p s i)\end{array}$ & $\begin{array}{c}\text { Normalized shear } \\
\text { stress } \\
\sqrt{f_{c}^{\prime}}(\mathrm{psi})\end{array}$ \\
\hline \multirow{2}{*}{0.75} & \multirow{2}{*}{1.96} & B18-1 & ZP305 & 55 & 413 & 5.12 \\
\hline & & B18-7 & RC80/30BP & 80 & 475 & 6.00 \\
\hline \multirow{3}{*}{1} & \multirow{3}{*}{2.67} & B18-7/B18-1 & & & 1.15 & 1.17 \\
\hline & & B18-2c \& d & ZP305 & 55 & 438 & 5.89 \\
\hline & & B18-5 & $\mathrm{RC} 80 / 60 \mathrm{BN}$ & 80 & 489 & 5.79 \\
\hline \multirow{3}{*}{0.75} & \multirow{3}{*}{2.06} & B18-5/B18-2c \& d & & & 1.12 & 0.98 \\
\hline & & B27-1 & ZP305 & 55 & 408 & 4.75 \\
\hline & & B27-2 & RC80/60BN & 80 & 404 & 6.25 \\
\hline \multirow{3}{*}{0.75} & \multirow{3}{*}{1.56} & B27-2/B27-1 & & & 0.99 & 1.32 \\
\hline & & B27-3 & ZP305 & 55 & 400 & 5.10 \\
\hline & & B27-4 & RC80/60BN & 80 & 285 & 4.36 \\
\hline \multirow{3}{*}{1.5} & & B27-4/B27-3 & & & 0.71 & 0.85 \\
\hline & \multirow{2}{*}{2.06} & B27-5 & ZP305 & 55 & 507 & 6.31 \\
\hline & & B27-6 & $\mathrm{RC} 80 / 60 \mathrm{BN}$ & 80 & 489 & 6.20 \\
\hline & & B27-6/B27-5 & & & 0.96 & 0.98 \\
\hline
\end{tabular}




\subsubsection{Effect of fiber volume fraction}

Table 5-5 lists the average shear stress and normalized shear stress values for beams with similar depths, fiber type, and longitudinal reinforcement ratios. It should be noted that the basic longitudinal reinforcement ratio was approximately $2 \%$ with the exception of Beams B18-2c \& d and B18-3, which had a reinforcement ratio of $2.67 \%$. Also, although the longitudinal reinforcement ratios for the RC beams were not the same as, but close to that of the SFRC beams, the strength comparison should still be valid.

The shear stress and normalized shear stress versus fiber volume fraction relationship shown in Fig. 5-7 indicates that an increase in fiber volume fraction resulted in an increase in shear strength (absolute and normalized). The increase was significant when fibers were added in a $0.75 \%$ volume fraction compared to the beams with no fibers. The efficiency of fiber reinforcement seemed to have diminished when used in higher volume fractions. From Fig. 5-9 it is clear that the shear stress versus fiber volume fraction relationship was more consistent than the normalized shear stress versus fiber volume fraction relationship.

The addition of ZP305 fibers in a $0.75 \%$ volume fraction led to an increase in shear strength of $159 \%$ for beams with a depth of 18 inches and $117 \%$ for beams with a depth of 27 inches. When a $0.75 \%$ volume fraction of RC $80 / 60 \mathrm{BN}$ fibers was used, the increase was 115\% for beams with a depth of 27 inches. When the amount of fibers was doubled from a fiber volume fraction of $0.75 \%$ to $1.5 \%$, the shear stress increased by only $24 \%$ and $21 \%$ for 27 inch-deep beams constructed with ZP305 and RC80/60BN fibers, respectively.

Comparing the large increase in shear strength obtained with the addition of fibers in either a $0.75 \%$ or $1 \%$ volume fraction with the strength increase observed when the fiber volume content was increased to $1.5 \%$, it seems clear that fiber effectiveness decreases as fiber content increases, particularly beyond a $1 \%$ volume fraction. 
Table 5-5: Effect of fiber volume fraction

\begin{tabular}{|c|c|c|c|c|c|c|c|}
\hline \multirow{2}{*}{ Beam } & \multirow{2}{*}{ Fiber } & \multirow{2}{*}{$\begin{array}{c}\rho \\
(\%)\end{array}$} & \multirow{2}{*}{$\begin{array}{c}\mathrm{V}_{\mathrm{f}} \\
(\%)\end{array}$} & \multicolumn{2}{|c|}{ Shear stress } & \multicolumn{2}{|c|}{ Normalized shear stress } \\
\hline & & & & (psi) & SFRC/RC & $\sqrt{f_{c}^{\prime}}(\mathrm{psi})$ & $\mathrm{SFRC} / \mathrm{RC}$ \\
\hline B18-0 & - & 2.67 & 0 & 159 & 1.00 & 2.02 & 1.00 \\
\hline B18-1 & ZP305 & 1.96 & 0.75 & 413 & 2.59 & 5.12 & 2.53 \\
\hline B18-2ab & ZP305 & 1.96 & 1 & 445 & 2.79 & 5.98 & 2.95 \\
\hline B18-2cd & ZP305 & 2.67 & 1 & 438 & 2.75 & 5.89 & 2.91 \\
\hline B18-3 & ZP305 & 2.67 & 1.5 & 452 & 2.84 & 6.15 & 3.04 \\
\hline B27-7 & - & 1.56 & 0 & 188 & 1.00 & 2.57 & 1.00 \\
\hline B27-1 & ZP305 & 2.06 & 0.75 & 408 & 2.17 & 4.75 & 1.85 \\
\hline B27-5 & ZP305 & 2.06 & 1.5 & 507 & 2.70 & 6.31 & 2.46 \\
\hline B27-7 & - & 1.56 & 0 & 188 & 1.00 & 2.57 & 1.00 \\
\hline B27-2 & $\mathrm{RC} 80 / 60 \mathrm{BN}$ & 2.06 & 0.75 & 404 & 2.15 & 6.25 & 2.44 \\
\hline B27-6 & RC80/60BN & 2.06 & 1.5 & 489 & 2.60 & 6.20 & 2.42 \\
\hline
\end{tabular}
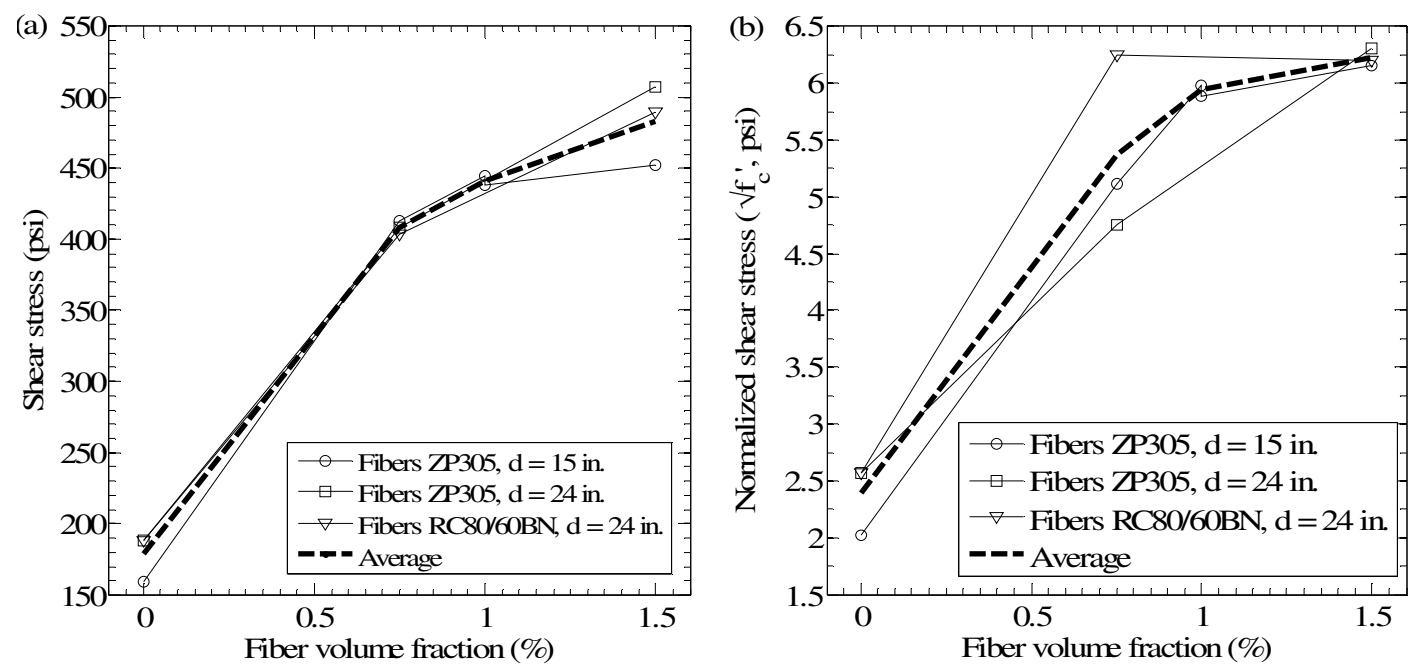

Fig. 5-7: Effect of fiber volume fraction on shear stress and normalized shear stress

\subsubsection{Effect of longitudinal reinforcement ratio}

Table 5-6 lists the average peak shear stress and normalized shear stress for SFRC beams with similar beam depths, fiber type, and fiber volume fraction, but with different longitudinal reinforcement ratios. Fig. 5-8 clearly shows that the shear stress capacity of beams constructed with ZP305 fibers did not essentially change when the reinforcement ratio changed from 1.56 to $2.06 \%$ or from 1.96 to $2.67 \%$. On the other 
hand, the increase in shear stress with increasing reinforcement ratio was significant for beams constructed with RC80/60BN fibers. However, it should be repeated that the strength of Beam B27-4b from pair B27-4 was significantly lower compared with its companion beam.

Table 5-6: Effect of longitudinal reinforcement ratio

\begin{tabular}{cccccc}
\hline Beam & Fiber & $\begin{array}{c}\mathrm{V}_{\mathrm{f}} \\
(\%)\end{array}$ & $\begin{array}{c}\rho \\
(\%)\end{array}$ & $\begin{array}{c}\text { Shear stress } \\
(\mathrm{psi})\end{array}$ & $\begin{array}{c}\text { Normalized shear } \\
\text { stress } \\
\sqrt{f_{c}^{\prime}}(\mathrm{psi})\end{array}$ \\
\hline B18-2a \& b & ZP305 & 1 & 1.96 & 445 & 5.98 \\
B18-2c \& d & ZP305 & 1 & 2.67 & 438 & 5.89 \\
\hline B27-3 & ZP305 & 0.75 & 1.56 & 400 & 5.10 \\
B27-1 & ZP305 & 0.75 & 2.06 & 408 & 4.75 \\
\hline B27-4 & RC80/60BN & 0.75 & 1.56 & 285 & 4.36 \\
B27-2 & RC80/60BN & 0.75 & 2.06 & 404 & 6.25 \\
\hline
\end{tabular}
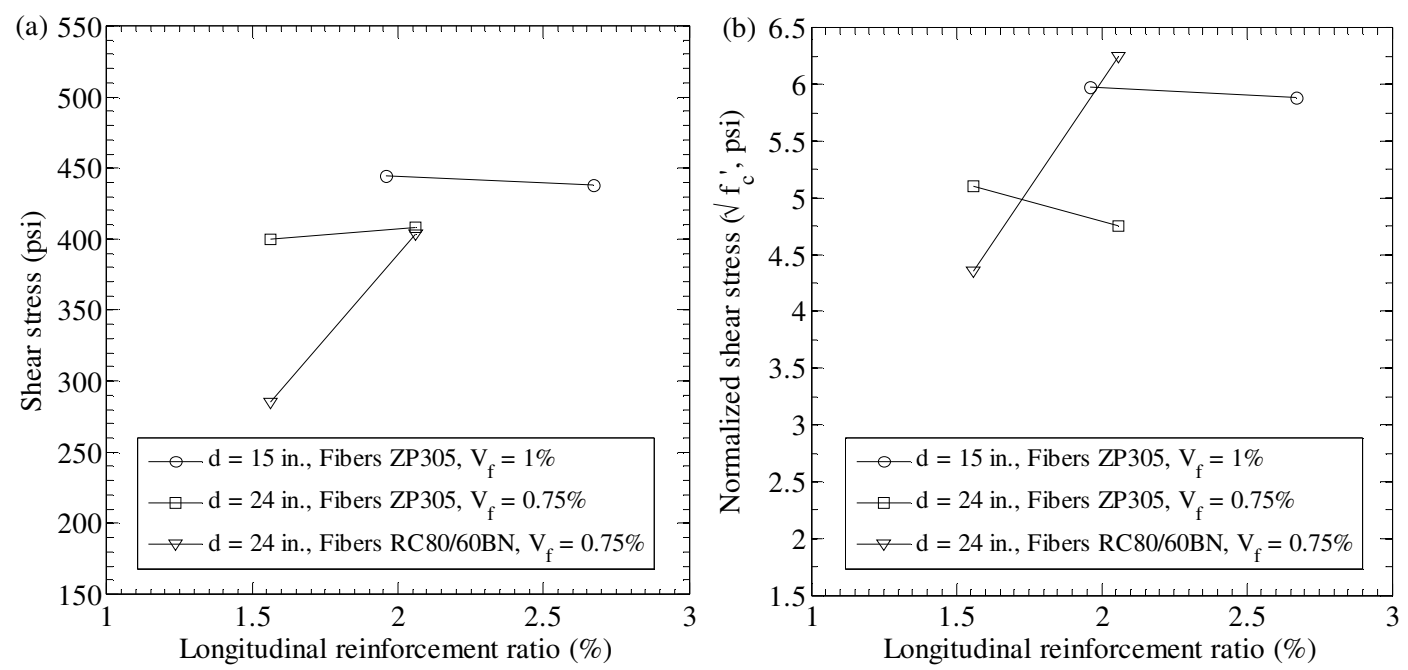

Fig. 5-8: Effect of longitudinal reinforcement ratio on shear stress and normalized shear stress 


\subsubsection{Effect of effective beam depth}

The effect of beam depth on beam shear strength for the range considered was negligible. For example, an increase in effective beam depth from 18 inches to 27 inches resulted in a slight decrease in stress (approximately 7\%) for the beams with ZP305 fibers in a volume fraction of $0.75 \%$ and a longitudinal reinforcement ratio of approximately $2 \%$.

\subsubsection{Replacement of minimum shear reinforcement}

It is clear from Table Fig. 5-1 that the normalized shear strength of all SFRC beams in Series B27 exceeded that of Beam B27-8, reinforced with minimum stirrup reinforcement. It should be noted that the shear reinforcement in Beams B27-8 was 30\% greater than the minimum specified in the 2008 ACI Code. This RC beam also exhibited a displacement ductility approximately equal to or lower than that of the SFRC beams. As mentioned in Section 4.4.12, only two inclined cracks were observed in this beam. Although there was only one test of this type, it should be reasonable to conclude that the strength and ductility of SFRC beams constructed with hooked steel fibers in a volume fraction greater than or equal to $0.75 \%$ exceed those of RC beams with minimum stirrup reinforcement. This indicates that hooked steel fibers of the types considered in this investigation in a volume fraction of $0.75 \%$ can be used in place of the minimum stirrup reinforcement required by ACI Committee 318 for beam depths of up to $27 \mathrm{in}$. 


\subsection{PREDICTION OF SHEAR STRENGTH OF SFRC BEAMS}

\subsubsection{Shear prediction of SFRC beams without stirrups from previous research groups}

Fig. 5-9 shows the relationship between experimental beam shear strengths obtained in this research and the shear strengths predicted using previously proposed expressions (see Section 2.3). If a data point is above the continuous line, the predicted shear strength is greater than the measured shear strength; and vice versa. The dashed lines show the boundary of $20 \%$ offset from the measured shear strength. It can be seen that the use of the expressions proposed by Ashour, Hasanain, and Wafa (Fig. 5-9c), AlTa' an and Al-Feel (Fig. 5-9e), and Khuntia, Stojadinovic and Goel (Fig. 5-9f) led consistently to an under-prediction of shear strength. With the second formulation of Ashour, Hasanain, and Wafa (Fig. 5-9d), the shear strength of seven specimens was greater than $120 \%$ of the corresponding measured shear strength. On the other hand, the shear strength predictions of at least seven specimens, using the expressions proposed by Sharma (Fig. 5-9a) and Narayanan and Darwish (Fig. 5-9b), were below $80 \%$ of the measured strength. The inability of the previous formulations to adequately predict the experimentally obtained shear strengths from this research indicate the need for a reliable method to predict the shear strength of SFRC beams. 


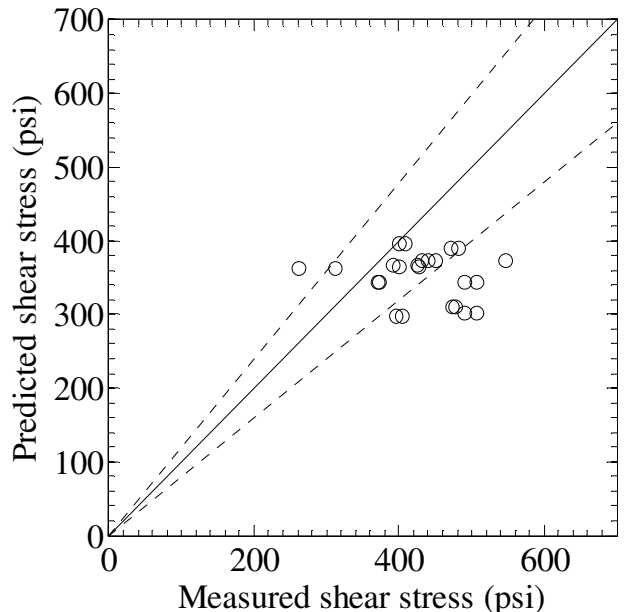

(a) Sharma (1986)

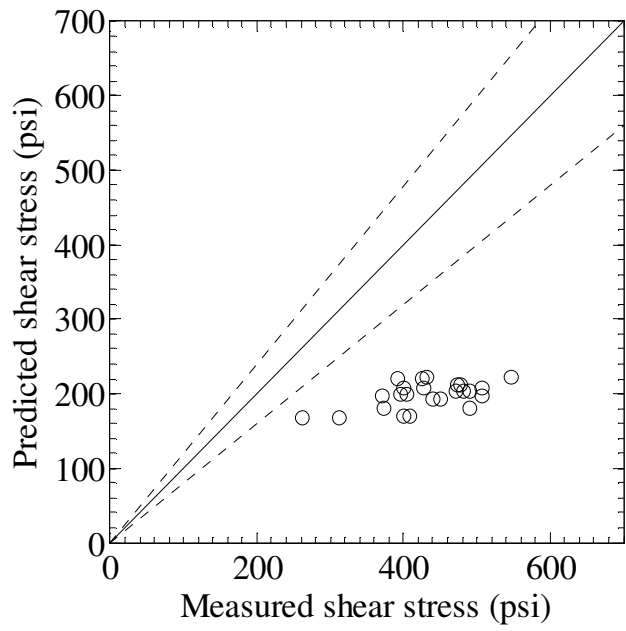

(c) Ashour, Hasanain, and Wafa (1992)

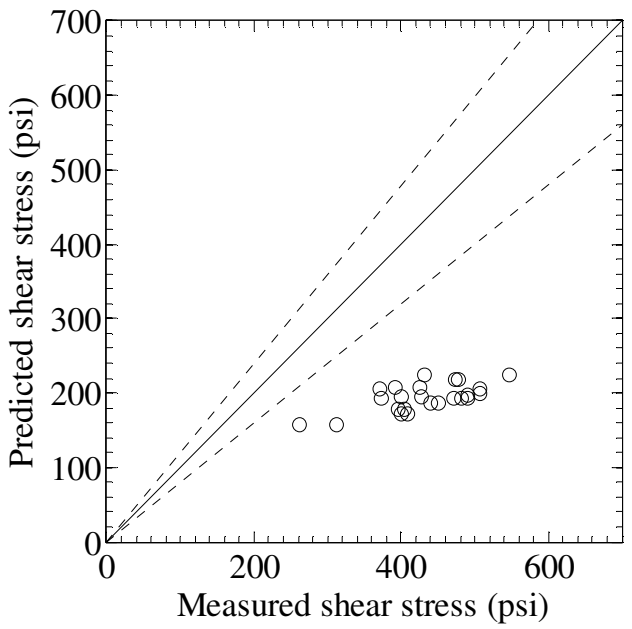

(e) Al-Ta' an and Al-Feel (1990)

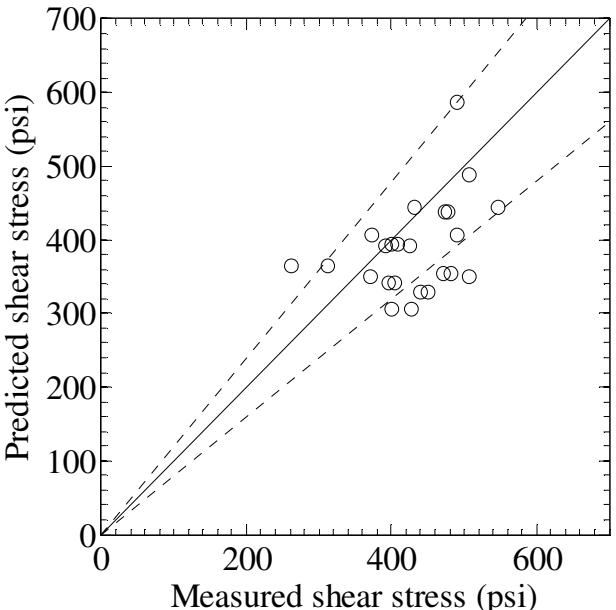

(b) Narayanan and Darwish (1987)

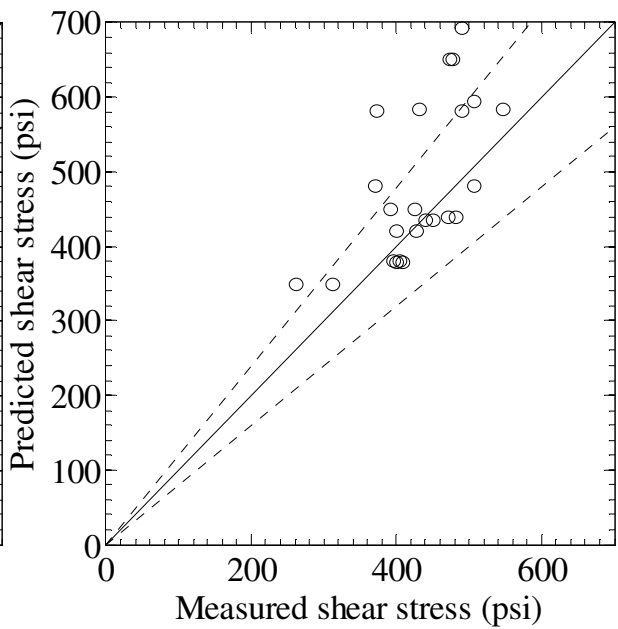

(d) Ashour, Hasanain, and Wafa (1992)

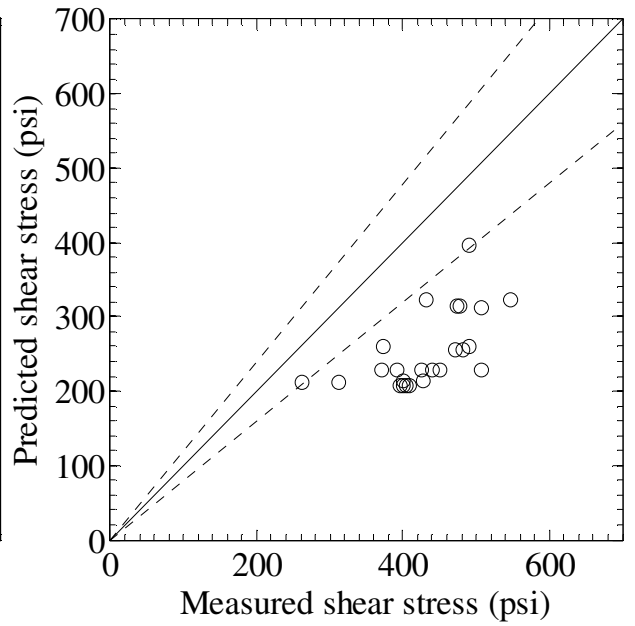

(f) Khuntia, Stojadinovic and Goel (1999)

Fig. 5-9: Shear strength prediction of SFRC beams tested in this research using previously proposed expressions 


\subsubsection{A mechanics-based model for shear prediction of SFRC beams without stirrups}

Overview of the model. This section describes a simple model to predict the shear strength of SFRC beams without stirrup reinforcement, similar to those tested in this experimental program. In this model, an SFRC beam without stirrup reinforcement is assumed to fail along an idealized crack MNP and over the compressed concrete PQ, as shown in Fig. 5-10a. This idealized crack closely represents a critical flexural-shear crack MNN'P, as often observed in the SFRC beams that failed in shear. In many cases, splitting along the reinforcement occurred as a consequence of the beam failure. Therefore, the critical diagonal crack was assumed to extend vertically from the reinforcement level to the bottom (tension) surface of the beam, rather than horizontally along the longitudinal reinforcement.

When the beam reaches its maximum shear strength, the crack width at the reinforcement level is assumed to have a magnitude of $w$. The inclined crack, which forms an angle $\alpha$ with the beam longitudinal axis, is assumed to extend from the reinforcement level up to point $\mathrm{P}$, which defines the neutral axis depth at a section corresponding to the outer face of the loading plate. At this section, the strain in the extreme concrete compression fiber and at the centroid of the reinforcement have a magnitude of $\varepsilon_{c m}$ and $\varepsilon_{s}$, respectively.

As discussed in Chapter 2, at a certain crack width, the beam would resist shear force through the compression region $\mathrm{PQ}$, fiber tension and aggregate interlock across the inclined crack NP, and dowel action. However, the aggregate interlock and dowel action are neglected for various reasons, as will be discussed next.

For the failure case considered, rotation of the beam would take place around point $\mathrm{P}$. This mechanism, combined with the negligible shear deformation of the beam compression zone prior to failure, makes sliding along the critical crack, which is required to develop aggregate interlock, difficult. Further, widening of the critical diagonal crack prior to failure would further diminish any contribution of aggregate interlock to beam shear strength. Therefore, the only assumed contribution to the shear 
resistance along the inclined crack, at ultimate, is assumed to be due to fiber tension. The contribution of the dowel action is neglected because it is believed to be small.

It is therefore appealing, both in light of observed behavior and conservatism, to ignore the contribution of aggregate interlock and dowel action to the beam shear strength. Measured critical crack widths at the level of reinforcement, from 0.0315 to 0.118 inches, further support this assumption. It should be mentioned that several researchers (for example, Walraven, 1981) have experimentally studied shear transfer through aggregate interlock. In these tests, relative sliding between concrete pieces was evident. Applying the findings from those studies to the model proposed in this study was therefore found to be both questionable and unconservative.

According to the proposed model, the shear strength of SFRC beams can be calculated as:

$$
V_{u}=V_{c c}+V_{f}
$$

where $V_{c c}$ and $V_{f}$ are the shear force across the compression region and the shear force due to the fiber tension, respectively. 

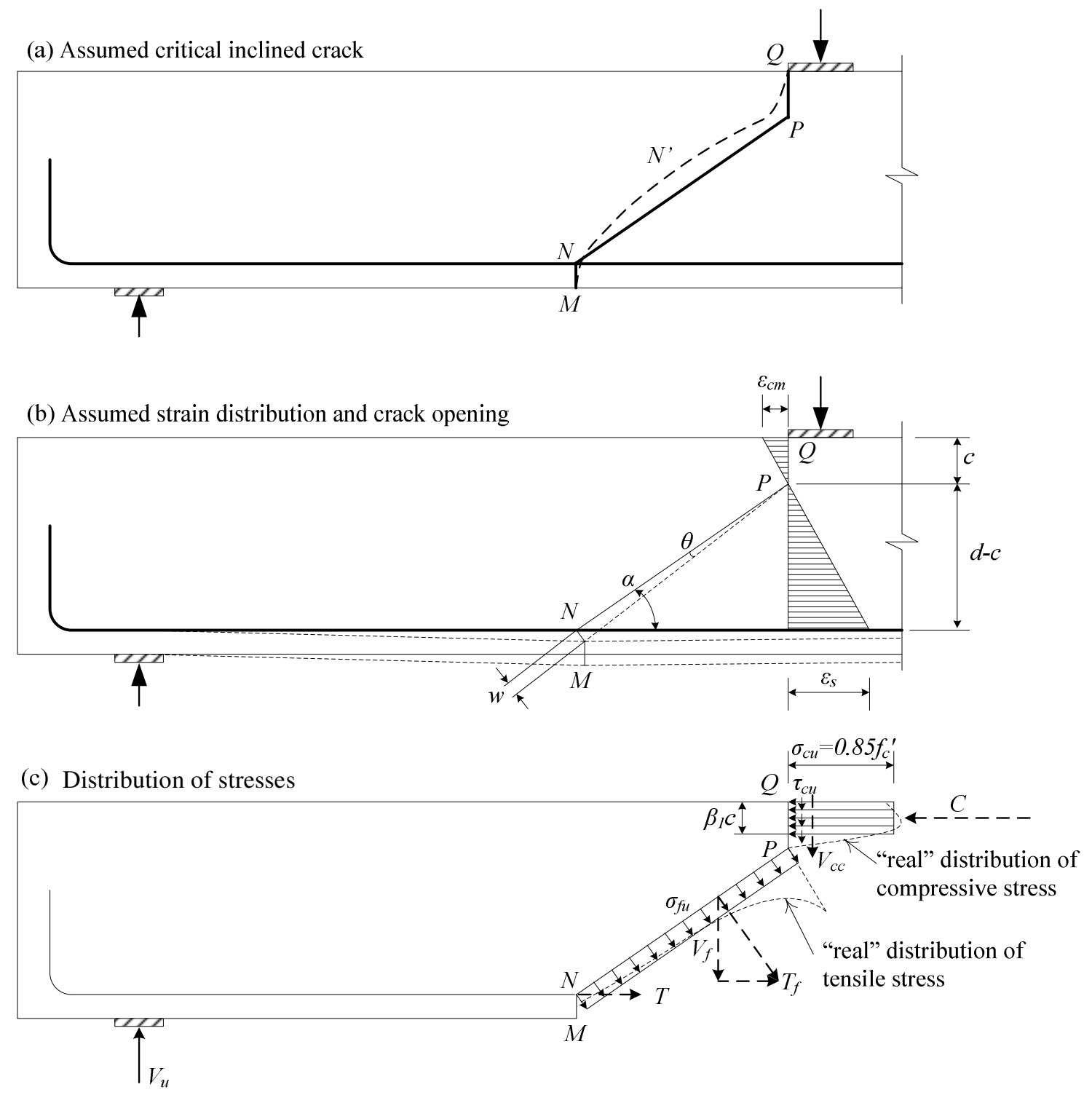

Fig. 5-10: Proposed model to predict shear strength of SFRC beams

\section{Prediction of the shear force resisted by the compression region. An} infinitesimal concrete element in the region above the neutral axis is assumed to be subjected to a stress state that consists of a normal compressive stress in the longitudinal (x) direction and shear stress $v_{x y}$. In the proposed model, crushing of concrete above the neutral is assumed to trigger a shear failure in the beam. The occurrence of concrete crushing can be estimated by using a failure criterion for concrete. 
Based on the significant experimental evidence that shows that steel fibers do not significantly influence the compressive strength of SFRC (Section 2.1.4), the use of a failure criterion for plain concrete is considered to be reasonable.

In this work, the failure criterion proposed by Bresler and Pister (1958) for concrete subjected to combined normal compressive stress and shear stress will be used. This failure criterion is defined as follows:

$$
\frac{\tau_{c u}}{f_{c}^{\prime}}=0.1\left[0.62+7.86\left(\frac{\sigma_{c u}}{f_{c}^{\prime}}\right)-8.46\left(\frac{\sigma_{c u}}{f_{c}^{\prime}}\right)^{2}\right]^{1 / 2} \text {. }
$$

Eq. (5-4) was derived from experimental data of tubular specimens made of concrete with compressive strength ranging from 3000 to 6000 psi. The specimens, which had height, outside diameter, and inside diameter of 30,9, and 6 inches, respectively, were subjected to axial compression and torque at the ends of the specimens.

It can be seen that this failure criterion predicts a normal compressive stress of $f_{c}^{\prime}$ when concrete is in pure compression $\left(\tau_{c u}=0\right)$ and a nearly constant shear strength of $0.16 f_{c}^{\prime}$ in a relatively wide range of compressive stress, from 0.39 to $0.54 f_{c}^{\prime}$ (Fig. 5-11).

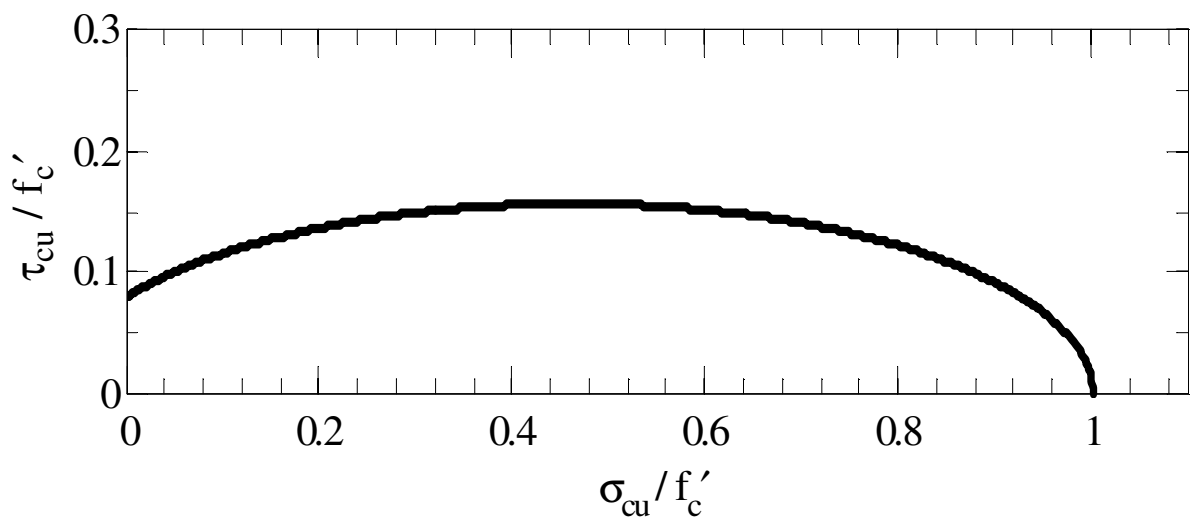

Fig. 5-11: Normal compressive and shear stress relationship used in this study (Bresler and Pister, 1958)

This failure criterion was then applied by Bresler and Pister (1958) to predict the shear strength of reinforced concrete beams without stirrup reinforcement. In their model, a uniform compressive stress at shear failure, which was adapted from the study of Hognestad et al. (1955), was assumed, as follows: 


$$
\sigma_{c u}=\frac{3900+0.35 f_{c}^{\prime}}{3200+f_{c}^{\prime}} f_{c}^{\prime} .
$$

This uniform compressive stress was assumed to act over the total depth of the compression region ( $c$ in Fig. 5-10b), which was calculated from the equation of equilibrium at the section considered and assuming yielding of the longitudinal reinforcement. The calculated shear strength was reported to correlate well with available test data of beams without stirrup reinforcement that failed in shear. It is believed that Bresler and Pister's model is robust because of the independence of the material failure criterion from the beam tests on which the model was applied.

Based on the success of the Bresler and Pister's model to estimate the shear strength of regular concrete beams without stirrup reinforcement, a similar method is proposed to calculate the shear carried across the compression region in SFRC beams. As in the work by Bresler and Pister, yielding of the longitudinal tension reinforcement will be assumed at shear failure of SFRC beams. This is supported by experimental evidence from this program. An upper limit to the reinforcement ratio $\left(A_{s} /\left(b_{w} d\right)\right)$ used in the calculation of the tensile force in the longitudinal reinforcement of $2 \%$ is proposed in order to avoid overestimation of the tensile force developed at shear failure for beams with excessive amount of flexural reinforcement. With this assumption, the depth of the compression zone $c$ can be calculated based on equilibrium of normal forces at the section considered, as follows:

$$
c=\frac{A_{s} f_{y}}{0.85 \beta_{1} f_{c}^{\prime} b} .
$$

In Eq. (5-6), a uniform stress block with a stress intensity of $0.85 f_{c}^{\prime}$ is used (Fig. 5-10c), acting over a depth $\beta_{1} c$. The factor $\beta_{1}$, proposed by Mattock, Kriz, and Hognestad (1961) and improved for the case of high strength concrete by Kaar, Hanson, and Capell (1978), is used:

$$
\beta_{1}=\left\{\begin{array}{l}
0.85 \text { if } f_{c}^{\prime} \leq 4000 \mathrm{psi} \\
1.05-0.05 f_{c}^{\prime} / 1000 \text { if } 4000 \mathrm{psi}<f_{c}^{\prime} \leq 8000 \mathrm{psi} . \\
0.65 \text { if } f_{c}^{\prime}>8000 \mathrm{psi}
\end{array}\right.
$$


In order to reasonably predict the shear force carried across the compression region, it is important to make a reasonable selection for the ratio of uniform compressive stress to the concrete compressive strength. The following example demonstrates that the assumption of a constant compressive stress distributed over the full depth of the compression zone provides a higher shear strength than the shear strength from a "real" distribution of compressive stresses.

As an example, Curve (1) in Fig. 5-12 illustrates a modified Hognestad compressive stress-strain relationship (Hognestad, 1951) for a concrete compressive strength of $6000 \mathrm{psi}$ and a maximum allowable concrete strain of 0.003 at the top surface of the beam. Line (2) shows the equivalent uniform concrete stress of $0.69 f_{c}^{\prime}$, which corresponds to a uniform shear stress of $0.142 f_{c}^{\prime}$ shown by Line (3) calculated using Bresler and Pister failure criterion (Eq. 5-4). Curve (4) shows the "real" distribution of shear stress with each point calculated from Curve (1) and using equation Eq. 5-4. The equivalent uniform shear stress corresponding to this "real" shear stress distribution is $0.123 f_{c}^{\prime}$, as shown by Line (5). This represents $87 \%$ of the shear stress calculated assuming an average normal stress acting over the depth of the compression zone. 


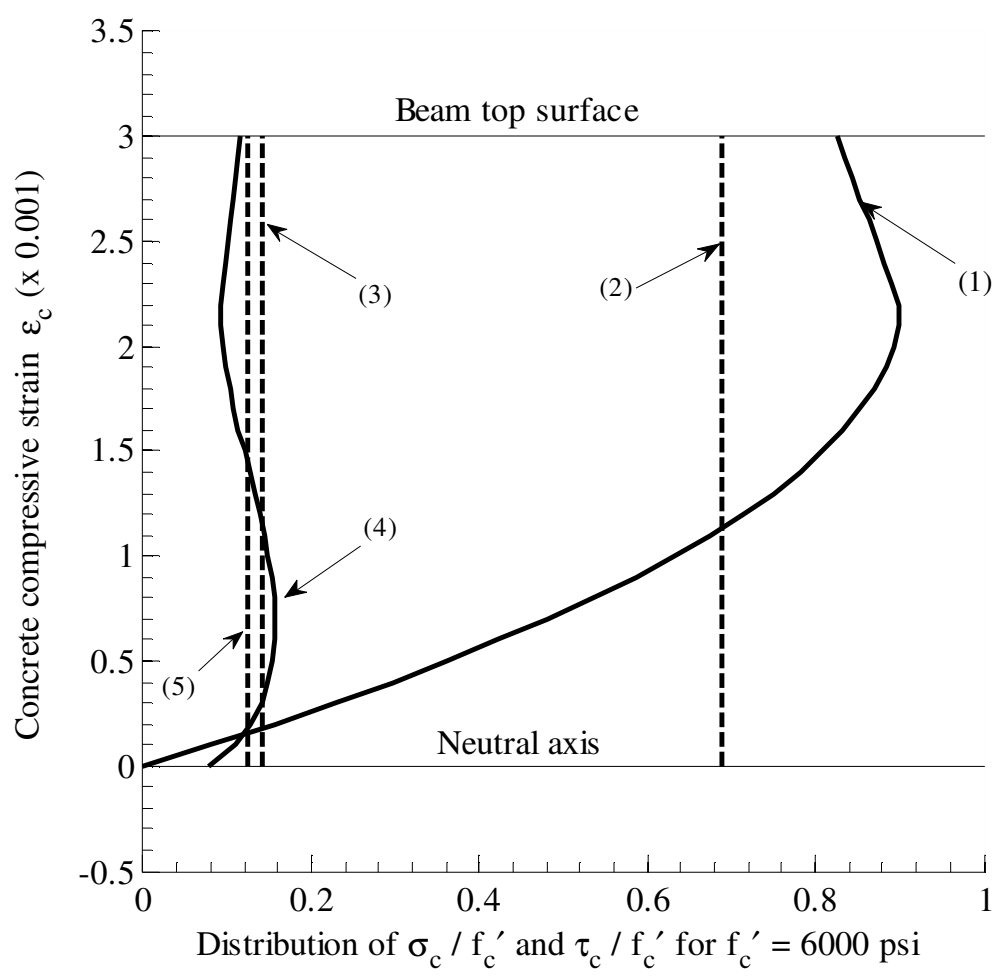

Fig. 5-12: Comparison of two methods to obtain a uniform shear stress

From the observation above, the use of a uniform compressive stress acting over a reduced depth seems to be attractive and conservative. In this research, a uniform shear stress of $0.11 f_{c}^{\prime}$, which corresponds to a normal stress of $0.85 f_{c}^{\prime}$, is proposed. This shear stress is assumed then to act over a reduced depth of $\beta_{1} c$. The shear carried by the beam compression zone is thus calculated as:

$$
V_{c c}=\left(0.11 f_{c}^{\prime}\right) \cdot\left(\beta_{1} c\right) \cdot b=0.11 \beta_{1} f_{c}^{\prime} b c=0.13 A_{s} f_{y} .
$$

For instance, the predicted shear force for a concrete compressive strength of 6000 psi is:

$$
V_{c c}=0.11 \times 0.75 \times f_{c}^{\prime} b c=0.083 f_{c}^{\prime} b c .
$$

In the absence of a normal stress-shear stress failure criterion for SFRC and based on the fact that the addition of fibers to the concrete increases compression ductility, the use of Eq. (5-8) is believed to provide a conservative estimate of the shear force carried in the compression zone of SFRC beams. 
Prediction of the shear force due to fiber tension. The tensile force transferred across the critical crack through fiber tension depends on the crack width. In order to estimate the magnitude of this force, the use of a constant tension stress is desirable, rather than trying to estimate its "actual" distribution. Fig. 5-10c illustrates the proposed approach, in which the "actual" tension stress distribution (dashed curve along line NP) is replaced by an equivalent uniform tensile stress with the same tensile force resultant. It follows that the contribution of fiber tension to the beam shear strength has the following form:

$$
V_{f}=T_{f} \cos \alpha=\left[\sigma_{f u} b\left(\frac{d-c}{\sin \alpha}\right)\right] \times \cos \alpha=\sigma_{f u} b(d-c) \operatorname{cotan} \alpha .
$$

Considering the fact that the proposed model relies on a crack of varying width, the equivalent uniform tensile stress $\sigma_{f u}$ may be obtained from material bending tests following ASTM 1609 or a similar method. An analogy can thus be made between the single crack that typically occurs in such tests and the critical diagonal crack assumed in the proposed shear strength model. This would allow the determination of an average tensile stress for a given crack width at the crack bottom end (Fig. 5-13e) from a fourpoint bending test (Fig. 5-13b). This average tensile stress is then assumed to be representative of that along the inclined crack in Fig. 5-10 for the same crack width, taken at the level of the reinforcement.

Substituting Eqs. (5-8) and (5-10) into Eq. (5-3) and dividing the resulting shear strength by the product $b d$, the predicted shear stress has the following form:

$$
v_{u}=\frac{V_{c c}+V_{f}}{b d}=0.11 \beta_{1} f_{c}^{\prime} \frac{c}{d}+\sigma_{f u}\left(1-\frac{c}{d}\right) \operatorname{cotan} \alpha
$$

\section{Derivation of uniform tensile stress versus crack width relationship from}

four-point bending tests. This discussion is limited to beams exhibiting a single flexural crack (i.e. deflection softening behavior). Once a flexural crack occurs in the beam, beam behavior can be modeled as two rigid blocks rotating an angle $\theta$ with respect to each other (Fig. 5-13c and d). The assumed stress distribution is shown in Fig. 5-13e. The angle $\theta$ can be calculated from the beam deflection at the crack location, as follows: 


$$
\theta=\theta_{1}+\theta_{2}=\frac{\delta_{c}}{a}+\frac{\delta_{c}}{\ell-a}=\frac{\delta_{c} \ell}{a(\ell-a)}
$$

with $2 \ell / 3 \geq a \geq \ell / 2$. The beam deflection at the crack location can be obtained from the beam deflection at mid-span as:

$$
\delta_{c}=\delta \cdot\left(\frac{a}{\ell / 2}\right)
$$

Therefore,

$$
\theta=\delta \cdot\left(\frac{a}{\ell / 2}\right) \frac{\ell}{a(\ell-a)}=\frac{2 \delta}{(\ell-a)} .
$$
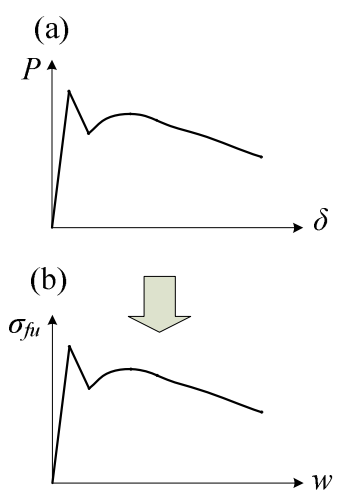
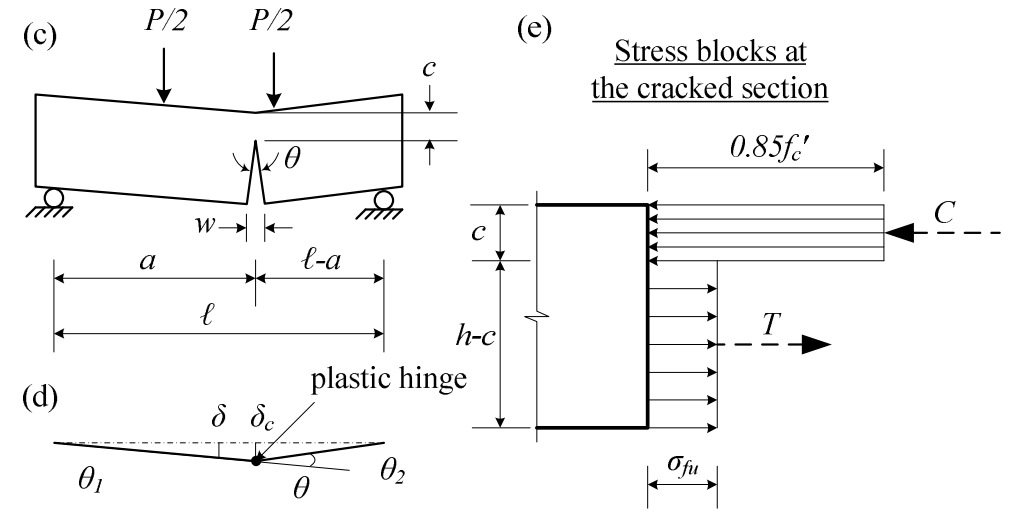

(e)

$\underline{\text { Stress blocks at }}$ the cracked section

Fig. 5-13: Derivation of uniform tensile stress versus crack width relationship from fourpoint bending tests

The crack width at the beam bottom surface is then estimated as follows:

$$
w=\theta(h-c)=\frac{2 \delta(h-c)}{(\ell-a)} .
$$

Therefore, at any given deflection, the maximum crack width is a function of the depth of the compression region, the crack location $a$, and the beam height $h$ and span length $\ell$.

In order to determine the neutral axis depth, a uniform compressive stress of $0.85 f_{c}^{\prime}$ can be assumed, regardless of the beam deflection. Even though this assumption is questionable over a wide range of deflections, the error incurred in the estimation of the neutral axis depth has a negligible effect on the determination of the average tensile stress, given the fact that it represents a very small percentage of the total beam depth. The moment at the cracked section can then be calculated as follows: 


$$
M=C \times\left(\frac{h}{2}\right)=\left(0.85 f_{c}^{\prime} b c\right) \times\left(\frac{h}{2}\right)
$$

Thus, $\quad c=\frac{2 M}{0.85 f_{c}^{\prime} b h}$.

This moment is also used to calculate the uniform tensile stress, as follows:

$$
M=T \times\left(\frac{h}{2}\right)=\left[\sigma_{f u} b(h-c)\right] \times\left(\frac{h}{2}\right)
$$

so: $\quad \sigma_{f u}=\frac{2 M}{b h(h-c)}$.

The average tensile stress versus crack width (at beam bottom) relationships, obtained from material beam tests corresponding to several of the SFRC beam specimens through the method described above, are shown in Fig. 5-14. For each SFRC test beam, the average results from the material four-point bending tests are also shown. 


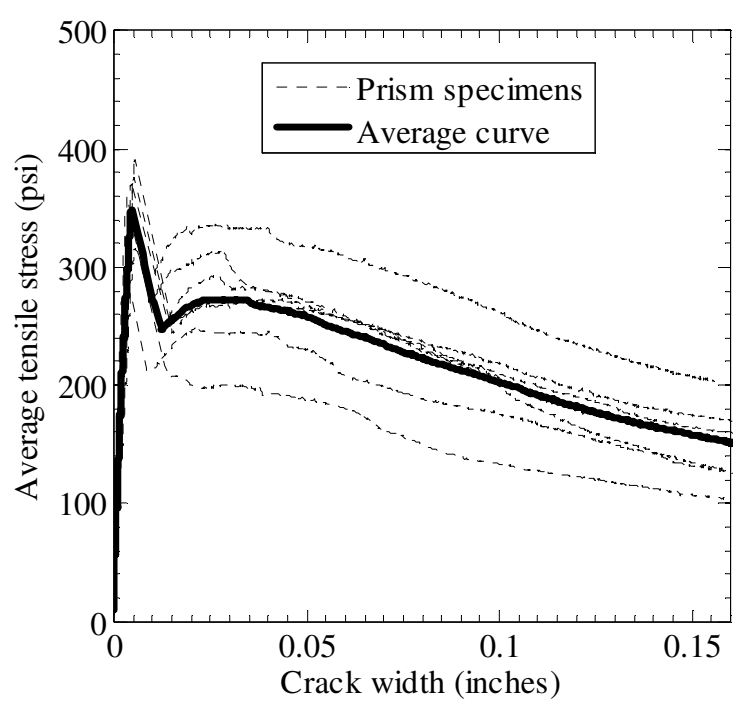

(a) B18-1a \& b (ZP305 fibers, $\left.V_{f}=0.75 \%\right)$

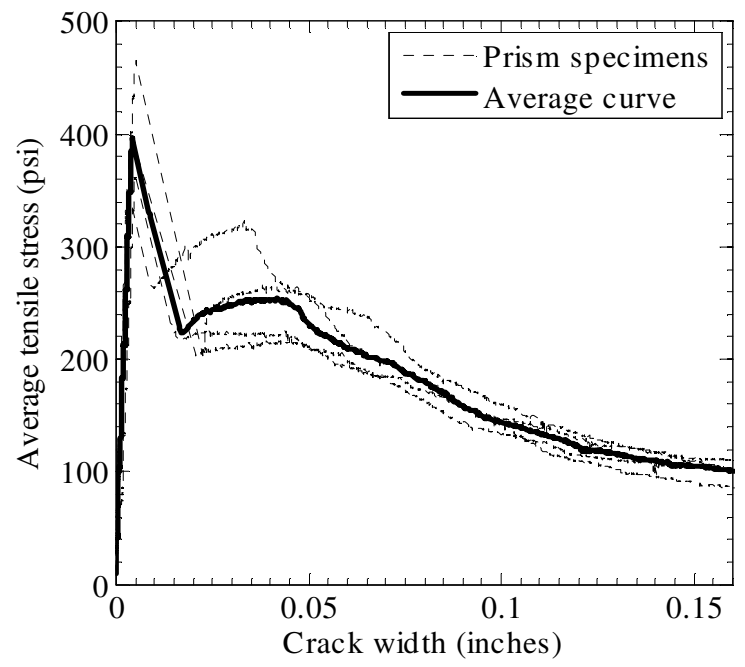

(c) B27-1a \& b (ZP305 fibers, $\left.V_{f}=0.75 \%\right)$

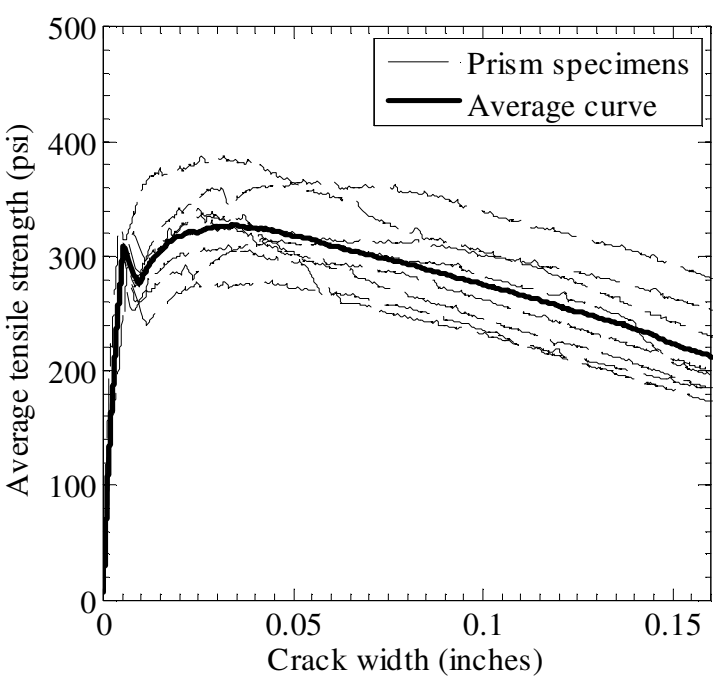

(b) B $18-7 \mathrm{a} \& \mathrm{~b}\left(\mathrm{RC} 80 / 30 \mathrm{BP}\right.$ fibers, $\left.V_{f}=0.75 \%\right)$

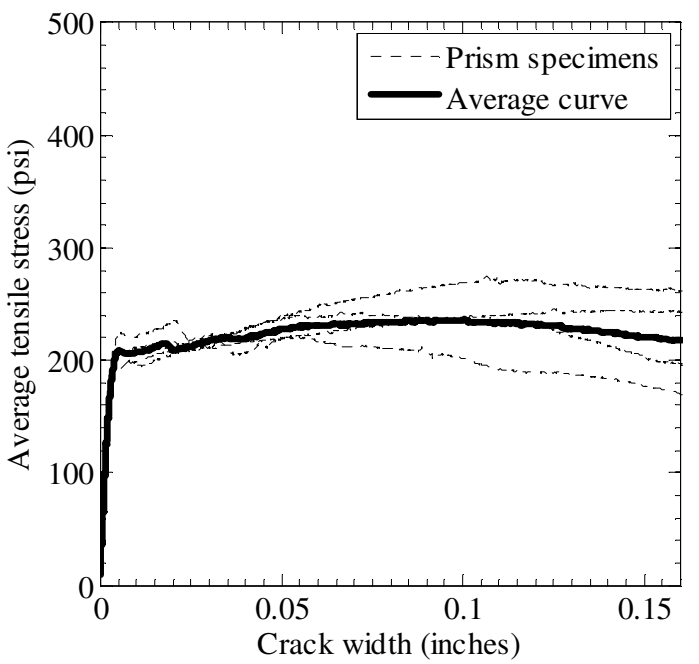

(d) B27-2a \& b (RC80/60BN fibers, $\left.V_{f}=0.75 \%\right)$

Fig. 5-14: Average tensile stress versus crack width relationship 


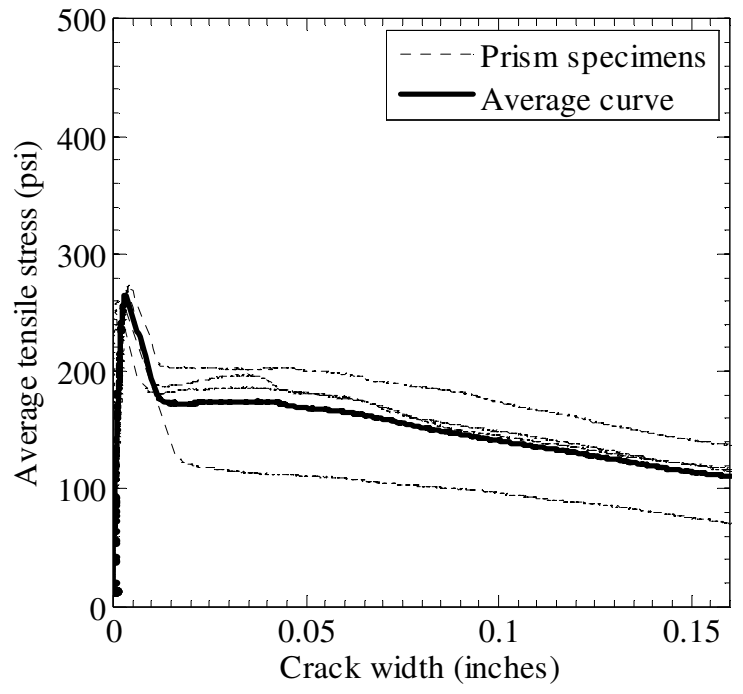

(e) B27-3 (ZP305 fibers, $V_{f}=0.75 \%$ )

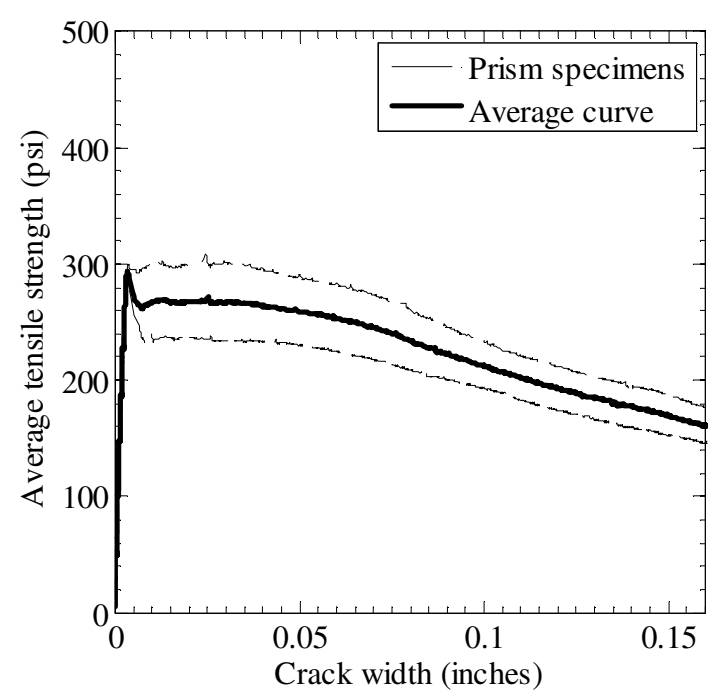

(g) B27-5 (ZP305 fibers, $V_{f}=1.5 \%$ )

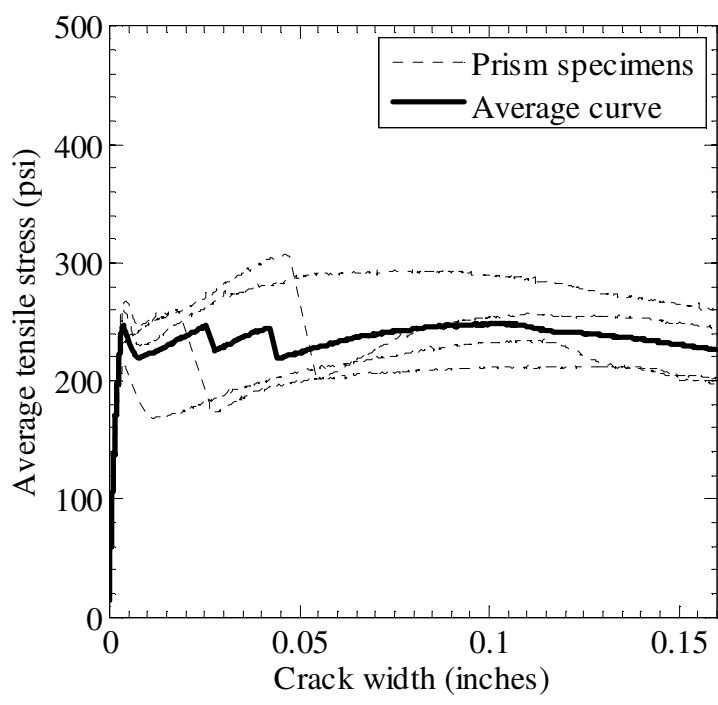

(f) B27-4 (RC80/60BN fibers, $\left.V_{f}=0.75 \%\right)$

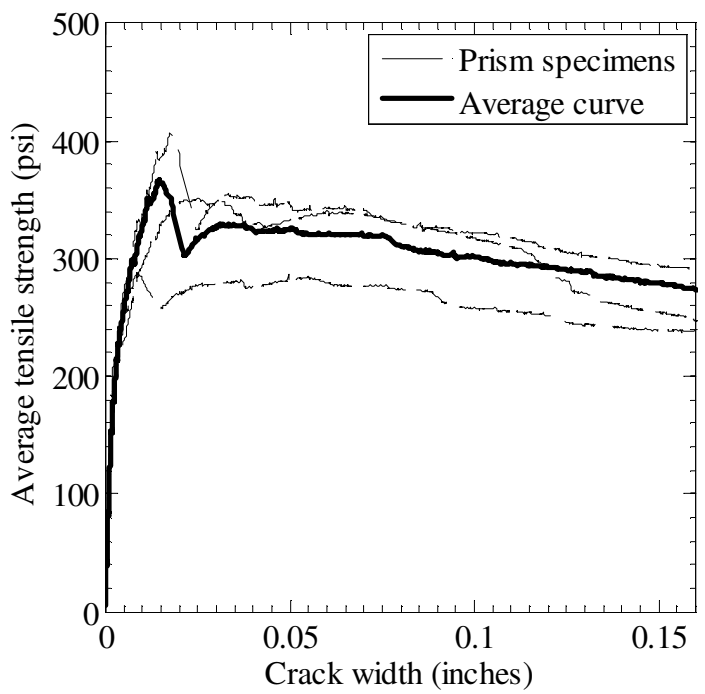

(h) B27-6 (RC80/60BN fibers, $V_{f}=1.5 \%$ )

Fig. 5-14: Average tensile stress versus crack width relationship (continued) 
Proposed angle for critical inclined crack. In order to be conservative, and considering that the average angle of the critical inclined cracks for the beams that failed due to shear-compression was 37 degrees, an angle of 40 degrees is proposed.

\section{Prediction of shear strength of beams in this experimental program. With} an assumed failure criterion for the concrete in the compression region, the depth of the compression zone calculated based on the assumption of reinforcement yielding (calculated based on $\rho \leq 2 \%$ ), and a uniform tensile stress determined as a function of crack width, knowledge of this crack width at beam ultimate state would be sufficient to calculate the shear strength of the beam following the proposed model. The following example illustrates the application of the method to the calculation of the shear strength of Beam B18-1a.

(1) The $\beta_{1}$ factor for a concrete compressive strength of $6500 \mathrm{psi}$ is 0.725 and the depth of the compression zone is:

$$
c=\frac{A_{s} f_{y}}{0.85 \beta_{1} f_{c}^{\prime} b}=\frac{1.76 \mathrm{in}^{2} \times 72 \mathrm{ksi}}{0.85 \times 0.725 \times 6.5 \mathrm{ksi} \times 6 \mathrm{in}}=5.27 \mathrm{in} .
$$

(2) The shear force resisted by the compression region is then calculated using the Bresler and Pister failure criterion as follows:

$V_{c c}=0.11 \beta_{1} f_{c}^{\prime} b c=0.11 \times 0.725 \times 6.5 \mathrm{ksi} \times 5.27 \mathrm{in} . \times 6 \mathrm{in} .=16.3 \mathrm{kips}$

(3) A critical inclined crack departing at the neutral axis (5.27 in. below the top surface of the beam) at the beam section passing through the left edge of the loading plate corresponds to concrete element E21. The crack width at ultimate for this element was 0.0437 in. $(1.11 \mathrm{~mm})$, which leads to a uniform tensile stress of $264 \mathrm{psi}$ (read from Fig. 5-14a). The shear resistance due to fiber tension is then:

$$
\begin{aligned}
V_{f} & =\sigma_{f u} b(d-c) \operatorname{cotan} \alpha=264 \mathrm{psi} \times 6 \text { in. } \times(15 \mathrm{in} .-5.27 \text { in. }) \operatorname{cotan} 40^{\circ} \\
& =18400 \mathrm{lb}=18.4 \mathrm{kips}
\end{aligned}
$$

(4) The predicted shear strength is then:

$$
V_{u}=V_{c c}+V_{f}=16.3+18.4=34.7 \mathrm{kips}
$$


The predicted shear strength is equal to $90 \%$ of the experimental shear strength $(38.4$ kips). The same steps were followed to calculate the shear strength for the other SFRC beams in this experimental program and the strength predictions are listed in Table 5-7. It should be mentioned that for beams that have a crack width smaller than the crack width corresponding to the peak uniform tensile stress, the peak tensile stress value was selected. This is based on the belief that at crack widths smaller than that at peak stress, some contribution from aggregate interlock should have occurred, which added to the contribution of the fibers, should have been greater than or equal to the sole contribution of the fiber tension to shear strength at peak stress. As schematically illustrated in Fig. 5-15, the shear resistance of fiber tension across the critical crack for crack widths smaller than that at peak stress is believed to be supplemented by aggregate interlock, which decreases as the crack width increases. In the proposed model, this resistance is assumed to have vanished at a crack width corresponding to the peak tensile stress. Based on this assumption and for a critical crack width smaller than that at peak tensile stress, the calculation of beam shear strength based on the peak stress while neglecting any contribution from aggregate interlock should lead to conservative results.

For the cases of Beams B27-3a \& b, B27-5, and B27-6, in which reinforcement strains were large enough to cause the steel to behave in the strain-hardening region, the calculation of resultant tension force and corresponding neutral axis depth was performed based on the acting moment at ultimate measured during the tests.

For SFRC beams with no bending test results available, an average tensile stress was determined from the bending test results of other SFRC beams. Specifically, the average tensile stress for Beams B18-3, which contained ZP305 fibers in a volume fraction of $1.5 \%$, was taken to be equal to the post-peak average tensile stress of Beam B27-5. On the other hand, the average tensile stress for Beams B18-2, constructed with ZP305 fibers in a volume fraction of 1\%, was assumed to be equal to the post-peak average tensile stress of Beams B18-1, which contained a $0.75 \%$ volume fraction of ZP305 fibers. Similarly, the average tensile stress for Beams B18-5 was assumed to be equal to the post-peak average tensile stress of Beams B27-4, which contained $\mathrm{RC} 80 / 60 \mathrm{BN}$ fibers with a lower amount of fibers ( 0.75 compared to $1 \%)$. 
Fig. 5-16 shows a plot of the ratios between predicted and experimental shear strengths for the SFRC test beams. It can be seen that most of the predicted shear strengths varied from 74 to $103 \%$ of the experimental shear strength, except for Beam B27-4b, which had a very low shear strength due to construction related issues, as was discussed in Section 4.4.8. It is worth mentioning that in some cases the measured shear strengths within a beam pair deviated from the mean value up to $16 \%$. Therefore, this shear predictive model is considered to give an adequate prediction of the shear strength of SFRC beams.

Table 5-7: Prediction of beam shear strength

\begin{tabular}{|c|c|c|c|c|c|c|c|c|c|c|}
\hline Beam & $\beta_{1}$ & $\begin{array}{l}c(*) \\
\text { (in.) } \\
\end{array}$ & $\begin{array}{c}\tau_{c} \\
(\mathrm{psi}) \\
\end{array}$ & $\begin{array}{c}V_{c c} \\
\text { (kips) } \\
\end{array}$ & Element & $\begin{array}{c}w \\
\text { (in.) }\end{array}$ & $\begin{array}{c}\sigma_{f} \\
\text { (psi) } \\
\end{array}$ & $\begin{array}{c}V_{f} \\
\text { (kips) } \\
\end{array}$ & $\begin{array}{c}V_{u} \\
\text { (kips) }\end{array}$ & $\frac{\underline{\mathrm{V}}_{\mathrm{u}}(\text { predicted })}{\mathrm{V}_{\mathrm{u}}(\text { measured })}$ \\
\hline B18-1a & 0.73 & 5.27 & 709 & 16.3 & E21 & 0.0437 & 264 & 18.4 & 34.6 & 0.90 \\
\hline B18-1b & 0.73 & 5.27 & 709 & 16.3 & E21 & 0.0393 & 266 & 18.5 & 34.8 & 0.97 \\
\hline B18-2a & 0.77 & 5.81 & 603 & 16.3 & - & - & 274 & 18.0 & 34.3 & 0.86 \\
\hline B18-2b & 0.77 & 5.81 & 603 & 16.3 & - & - & 274 & 18.0 & 34.3 & 0.85 \\
\hline B18-2c & 0.77 & 5.36 & 603 & 15.0 & - & - & 274 & 18.9 & 33.9 & 0.74 \\
\hline B18-2d & 0.77 & 5.36 & 603 & 15.0 & - & - & 274 & 18.9 & 33.9 & 1.02 \\
\hline B18-3a & 0.83 & 6.18 & 491 & 15.0 & - & - & 310 & 19.6 & 34.6 & 1.03 \\
\hline B18-3b & 0.83 & 6.18 & 491 & 15.0 & - & - & 310 & 19.6 & 34.6 & 0.78 \\
\hline B18-3c & 0.72 & 4.86 & 711 & 15.0 & - & - & 310 & 22.5 & 37.5 & 0.88 \\
\hline B18-3d & 0.72 & 4.86 & 711 & 15.0 & - & - & 310 & 22.5 & 37.5 & 0.88 \\
\hline B18-5a & 0.69 & 4.64 & 778 & 15.0 & - & - & 288 & 21.3 & 36.3 & 0.94 \\
\hline B18-5b & 0.69 & 4.64 & 778 & 15.0 & - & - & 288 & 21.3 & 36.3 & 0.74 \\
\hline B18-7a & 0.74 & 5.37 & 686 & 16.3 & E21 & 0.0665 & 334 & 23.0 & 39.3 & 0.91 \\
\hline B18-7b & 0.74 & 5.37 & 686 & 16.3 & E21 & 0.0088 & 365 & 25.1 & 41.4 & 0.98 \\
\hline B27-1a & 0.68 & 7.42 & 804 & 32.5 & E14 & 0.0135 & 253 & 40.0 & 72.5 & 0.89 \\
\hline B27-1b & 0.68 & 7.42 & 804 & 32.5 & $\mathrm{E} 32+\mathrm{E} 36 / 2$ & 0.0192 & 253 & 40.0 & 72.5 & 0.97 \\
\hline B27-2a & 0.84 & 10.62 & 455 & 32.5 & E44 & 0.0184 & 280 & 35.7 & 68.2 & 0.87 \\
\hline B27-2b & 0.84 & 10.62 & 455 & 32.5 & E44 & 0.0098 & 280 & 35.7 & 68.2 & 0.89 \\
\hline B27-3a & 0.74 & 7.74 & 669 & 30.8 & E44 & 0.1016 & 175 & 27.1 & 57.9 & 0.76 \\
\hline B27-3b & 0.74 & 7.74 & 669 & 30.8 & E44 & 0.0347 & 175 & 27.1 & 57.9 & 0.75 \\
\hline B27-4a & 0.84 & 8.00 & 468 & 25.0 & E37 & 0.0298 & 225 & 34.3 & 59.3 & 0.99 \\
\hline B27-4b & 0.84 & 8.00 & 468 & 25.0 & E37 & 0.0274 & 225 & 34.3 & 59.3 & 1.19 \\
\hline B27-5 & 0.73 & 9.63 & 703 & 39.4 & E44 & 0.0236 & 244 & 33.4 & 72.8 & 0.75 \\
\hline B27-6 & 0.74 & 8.12 & 677 & 32.5 & E44 & 0.0539 & 320 & 48.5 & 81.0 & 0.86 \\
\hline
\end{tabular}




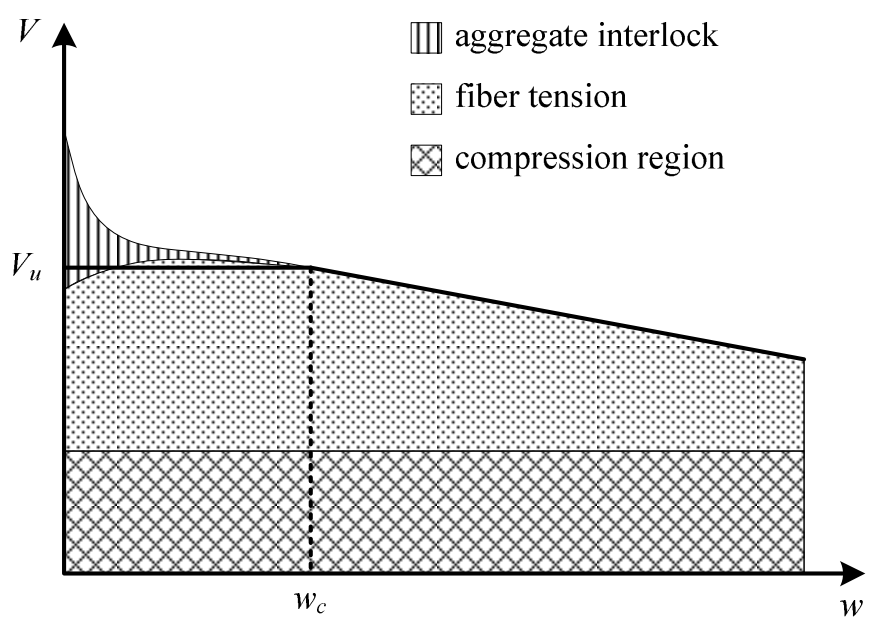

$w_{c}=$ crack width corresponding to peak tensile stress in SFRC

Fig. 5-15: Assumed contribution from different shear resisting mechanisms at different crack widths

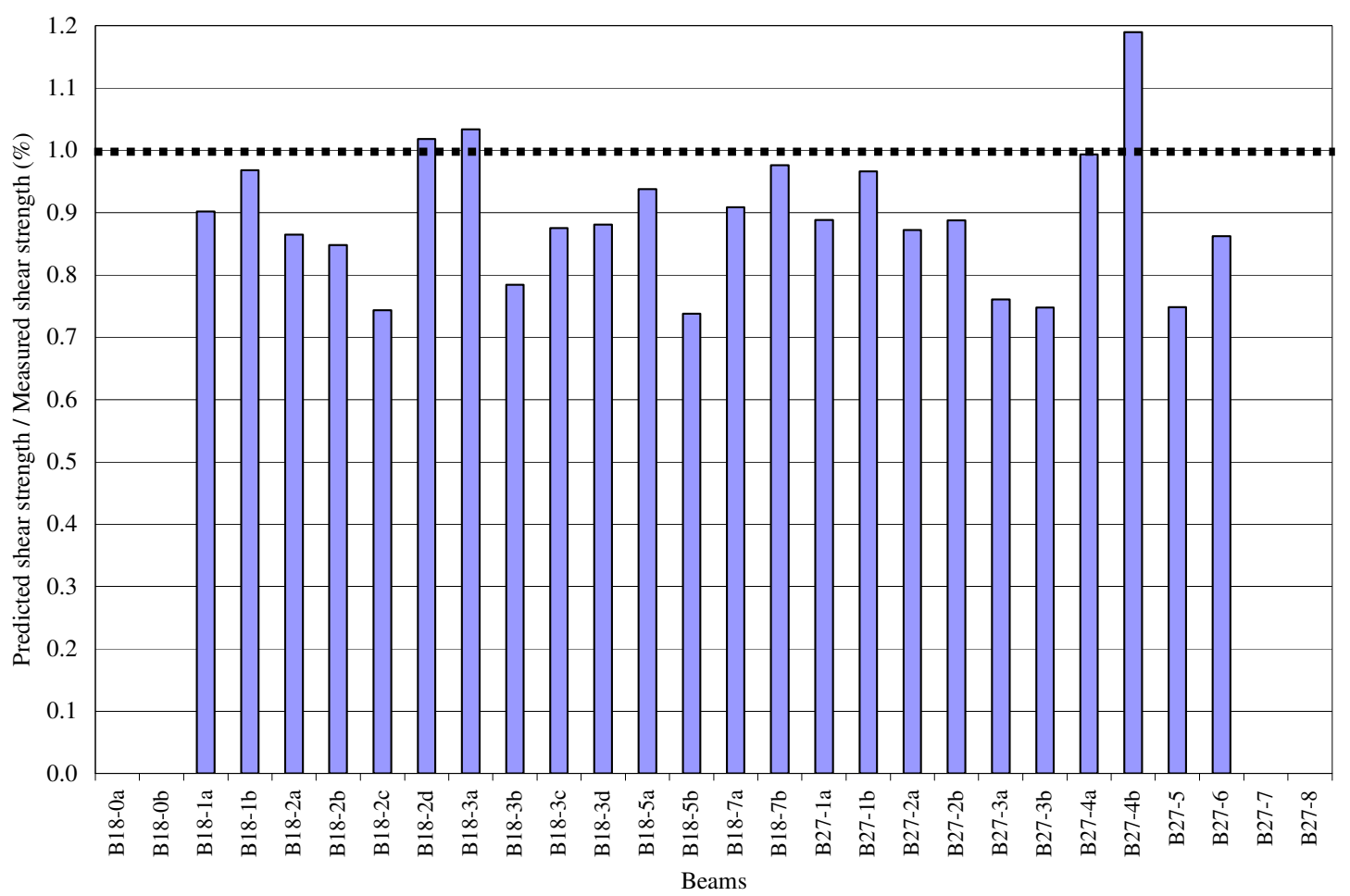

Fig. 5-16: Predicted shear strengths of test beams using the proposed model 


\subsubsection{Design recommendation for average tensile stress}

Design recommendation for average tensile stress. For the purpose of design, it is more beneficial to determine the average tensile stress without the knowledge of crack width corresponding to the beam ultimate shear strength. For this purpose, the average tensile stress can be assumed to have the following form:

$$
\sigma_{f u}=K \cdot \frac{L_{f}}{D_{f}} \cdot 0.0075 \cdot \sqrt{\frac{V_{f}}{0.0075}}=K \cdot \frac{L_{f}}{D_{f}} \cdot \sqrt{0.0075 V_{f}}
$$

From unpublished data of a large number of four-point bending tests conducted at The University of Michigan Structural Engineering Laboratory, $K$ had a mean value of 587 psi (private communication with Gustavo J. Parra-Montesinos and Terrence M. McGovern, March, 2009) and a standard deviation of 185 psi, measured at a deflection of $L / 600$ (0.03 inches) for fiber volume fractions of up to approximately $0.75 \%$. The test prisms had a size of 6 in. x 6 in. $x 20$ in. and contained hooked steel fibers with an aspect ratio of 80 .

A comparison of the average tensile stresses calculated based on Eq. (5-16) and those available from the experimental program is shown in Fig. 5-17. The average tensile stresses are plotted against the deflection rather than the crack width. The plot also shows the deflections corresponding to the observed crack widths, which ranged from 0.035 to 0.063 inches for ZP305 and $\mathrm{RC} 80 / 30 \mathrm{BP}$ fibers, and from 0.047 to 0.157 inches for $\mathrm{RC} / 60 \mathrm{BN}$ fibers. It can be seen that Eq. (5-16) with a $K$ value of 400 , mean minus one standard deviation, at a deflection of $L / 600$ gives a conservative prediction for concrete reinforced with either $\mathrm{ZP} 305$, or $\mathrm{RC} 80 / 30 \mathrm{~PB}$, or $\mathrm{RC} 80 / 60 \mathrm{BN}$ fibers in a volume fraction of $0.75 \%$. For the case of Beams B27-5a \& b and B27-6a \& b, reinforced with ZP305 or RC80/60BN fibers in a volume fraction of $1.5 \%$, Eq. (5-16) still gives a reasonable prediction of the average tensile strength (Fig. 5-17g and h).

From the discussion above, Eq. (5-16) with a $K$ value of 400 psi is recommended for the purpose of estimating the average tensile stress along the critical crack for beams reinforced with $\mathrm{ZP} 305$ or $\mathrm{RC} 80 / 60 \mathrm{BN}$ fibers in volume fractions ranging from $0.75 \%$ to $1.5 \%$. This equation should be applicable to SFRC with other types of 
hooked steel fibers with a similar aspect ratio (from 55 to 80) and strength (greater than or equal to $160 \mathrm{ksi}$ ) in a similar content (from 0.75 to $1.5 \%$ by volume).

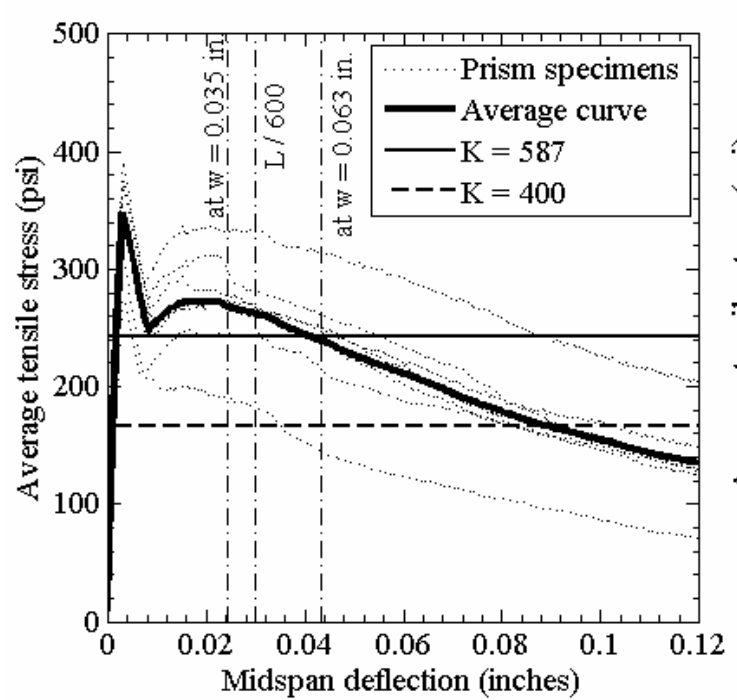

(a) B18-1a \& b (ZP305 fibers, $\left.V_{f}=0.75 \%\right)$

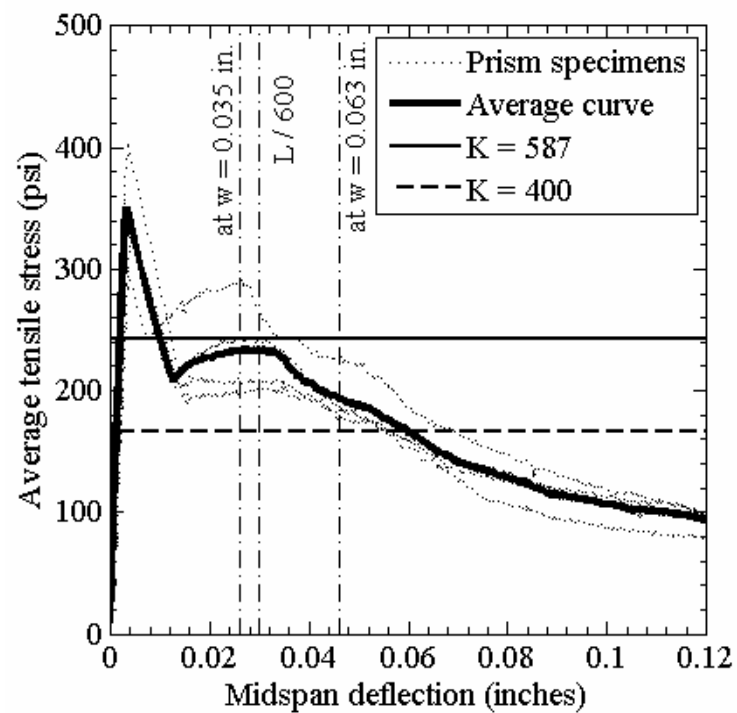

(c) B27-1a \& b (ZP305 fibers, $\left.V_{f}=0.75 \%\right)$

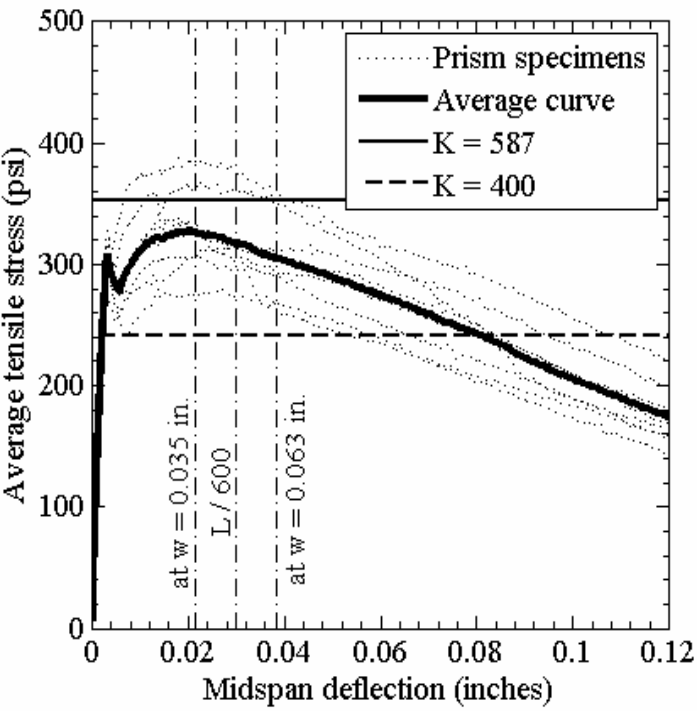

(b) B18-7a \& b (RC80/30BP fibers, $\left.V_{f}=0.75 \%\right)$

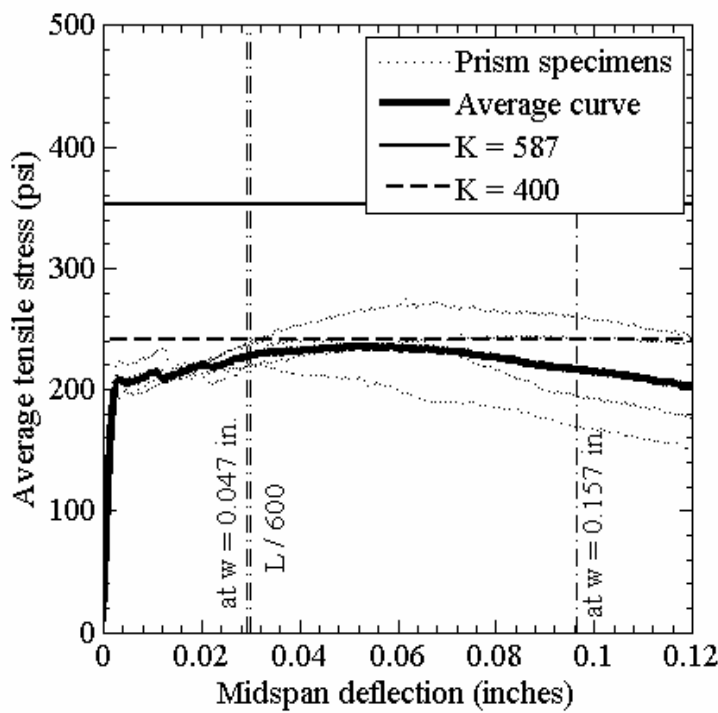

(d) B27-2a \& b (RC80/60BN fibers, $\left.V_{f}=0.75 \%\right)$

Fig. 5-17: Comparison of experimentally obtained SFRC average tensile stress and recommended tensile stress 


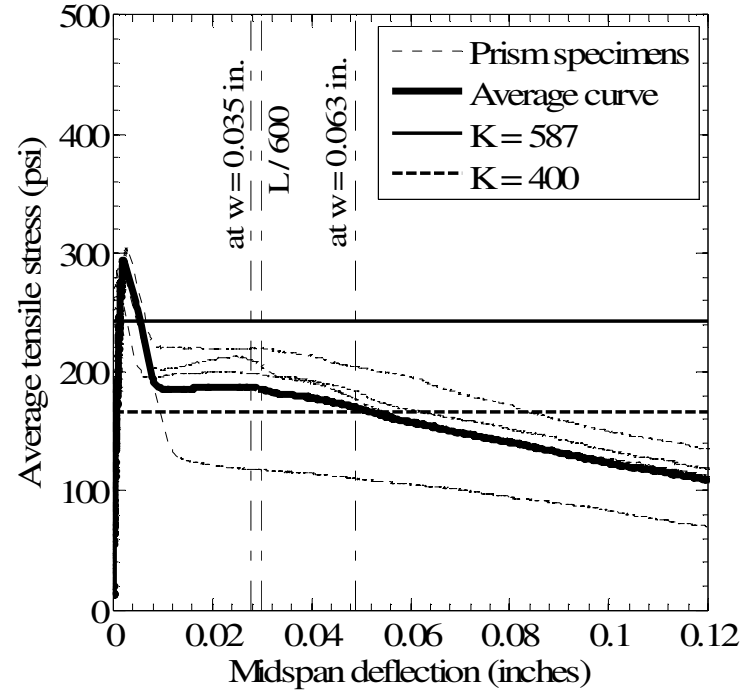

(e) B27-3 (ZP305 fibers, $V_{f}=0.75 \%$ )

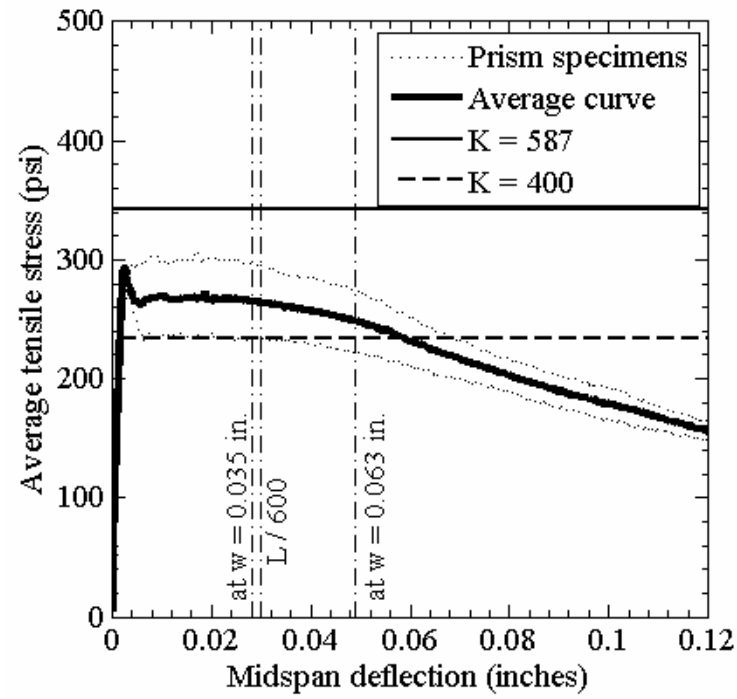

(g) B27-5 (ZP305 fibers, $\left.V_{f}=1.5 \%\right)$

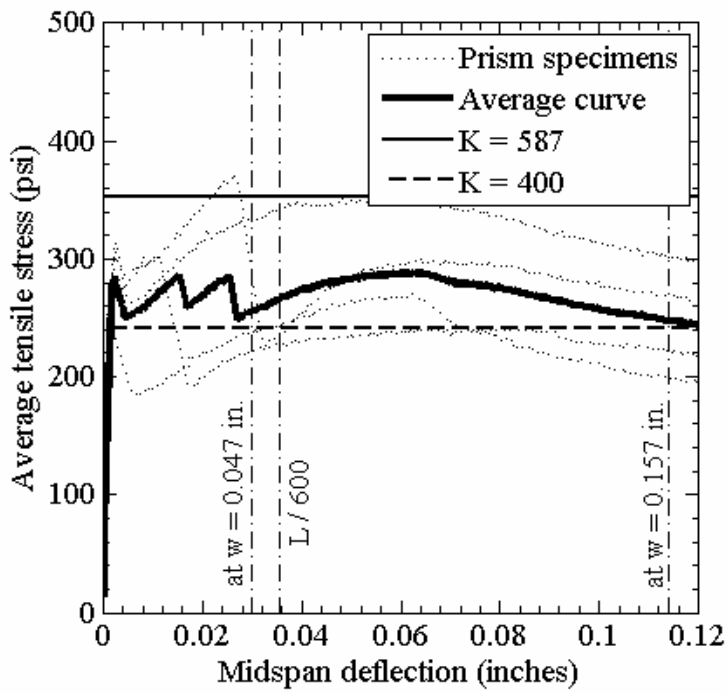

(f) $\mathrm{B} 27-4\left(\mathrm{RC} 80 / 60 \mathrm{BN}\right.$ fibers, $\left.V_{f}=0.75 \%\right)$

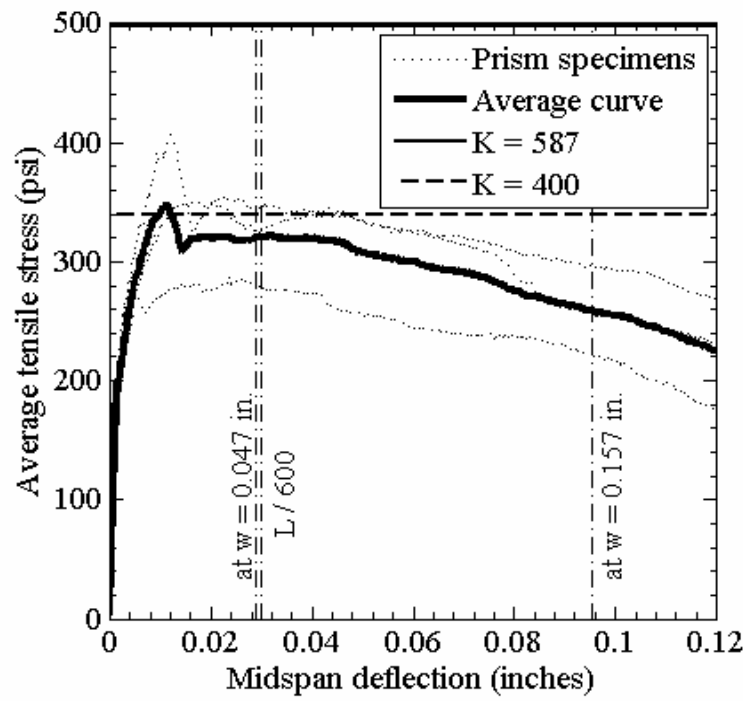

(h) $\mathrm{B} 27-6\left(\mathrm{RC} 80 / 60 \mathrm{BN}\right.$ fibers, $\left.V_{f}=1.5 \%\right)$

Fig. 5-17: Comparison of experimentally obtained SFRC average tensile stress and recommended tensile stress (continued) 


\subsubsection{Validation of the proposed method for estimating the shear strength of SFRC beams with data obtained from previous experimental programs}

In order to evaluate the accuracy of the proposed formulation to predict the shear strength of SFRC beams, a database of SFRC beams without stirrup reinforcement from previous research was assembled.

The database used is a subset of a larger shear database compiled by ParraMontesinos (2006), based on the following criteria:

(1) Cylinder compressive strength ranged from 3000 to 8000 psi. Data from beams constructed with higher strength concrete were excluded because the proposed model uses the Bresler and Pister failure criterion for concrete subjected to combined compression and shear, which was based on concrete with strength ranging between 3000 and 6000 psi.

(2) Only beams constructed with hooked steel fibers in volume fractions greater than or equal to $0.5 \%$ were considered because the $K$ factor used in Eq. (5-16) was based on results from flexural tests of beams with hooked steel fibers. The lower limit on fiber volume fraction is set based on safety concerns.

(3) Only beams considered to be "slender" were considered. Thus, beams with shear-span-to-depth ratios smaller than 2.5 were not included in the database.

(4) Only beams which were reported to fail due to shear were considered.

The key parameters for each SFRC beam included in the database are reported in Table 5-8. In absence of the yield strength for flexural reinforcing bars, a value of $60 \mathrm{ksi}$ was assumed. As mentioned earlier, an upper limit of $2 \%$ for the longitudinal reinforcement ratio was used to estimate the depth of the compression region. The predicted shear strengths and the ratios of predicted to experimental shear strengths are reported in Columns 13 and 14 of Table 5-8. Fig. 5-18 plots the ratios of predicted to experimental shear strengths versus the shear-span-to-effective-depth ratio. As can be seen, the method was able to predict the shear strengths within the range of 75 to $120 \%$ of the measured values. This is considered to be acceptable given the wide range of the parameters considered and the uncertainty related to beam shear strength. 


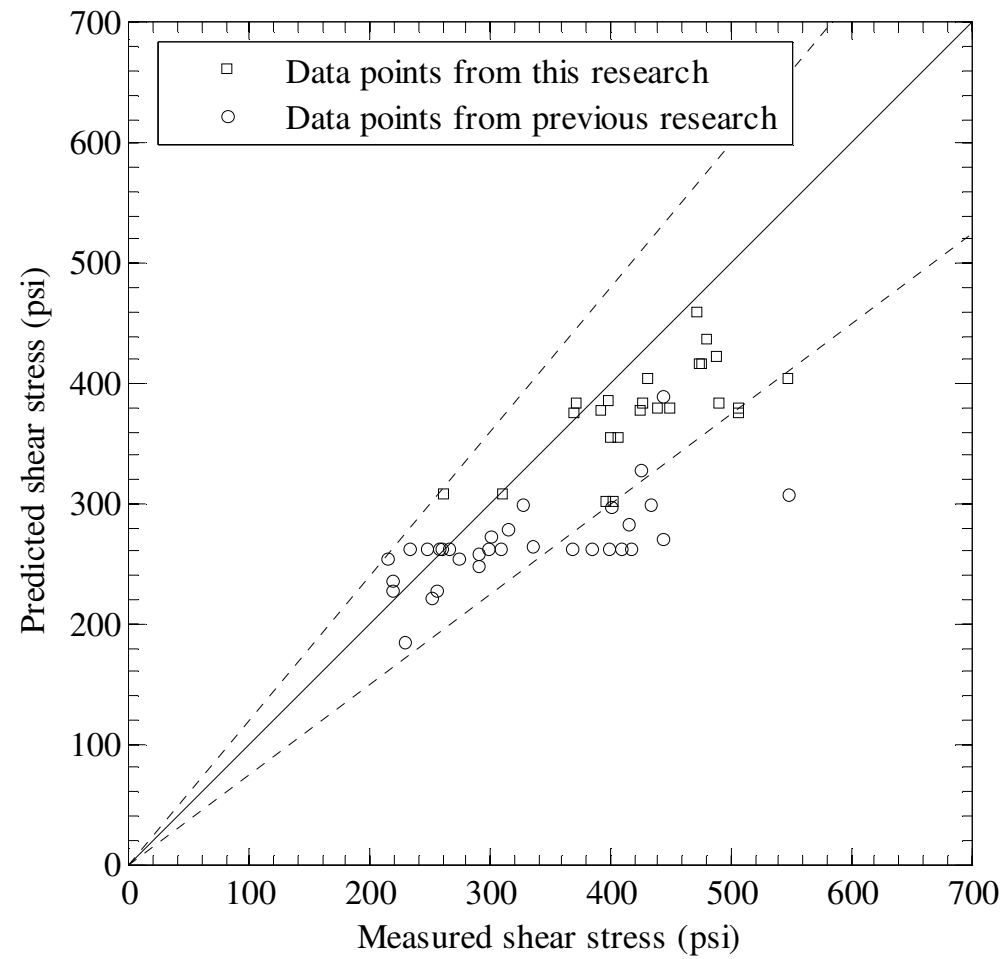

Fig. 5-18: Shear strength prediction for SFRC beams tested by this and other research groups 
Table 5-8: Shear strength prediction for SFRC beams tested by previous research groups

\begin{tabular}{|c|c|c|c|c|c|c|c|c|c|c|c|c|c|}
\hline 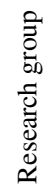 & ఐే & $\underset{0}{\stackrel{\Xi}{\Xi}}$ & $\underset{\Xi}{\Xi}$ & $\underset{\circlearrowright}{\stackrel{\Xi}{\Xi}}$ & స్త & $\underbrace{d e}_{a}$ & 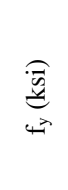 & $\begin{array}{l}\text { 离 } \\
\dot{y}\end{array}$ & $\stackrel{\check{ص}}{\varrho}$ & $\underbrace{2}_{>}$ & 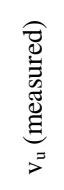 & 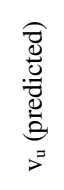 & 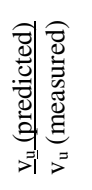 \\
\hline (1) & (2) & (3) & (4) & (5) & (6) & (7) & (8) & (9) & (10) & (11) & (12) & (13) & (14) \\
\hline 1 & A10 & 5.90 & 9.8 & 8.6 & 2.80 & 0.0190 & 88.5 & 5.93 & 60 & 1 & 425 & 328 & 0.77 \\
\hline 2 & & 5.00 & 9.0 & 8.0 & 3.00 & 0.0220 & N/A & 3.29 & 60 & 1 & 443 & 272 & 0.62 \\
\hline 2 & & 5.00 & 9.0 & 8.0 & 3.00 & 0.0220 & N/A & 3.77 & 100 & 1 & 443 & 389 & 0.88 \\
\hline 3 & $2 / 0.5 / 2.5$ & 6.00 & 10 & 8.7 & 2.50 & 0.0110 & 65.0 & 4.93 & 60 & 0.5 & 250 & 223 & 0.89 \\
\hline 3 & $4 / 0.5 / 2.5$ & 6.00 & 10 & 8.7 & 2.50 & 0.0220 & 65.0 & 4.93 & 60 & 0.5 & 274 & 255 & 0.93 \\
\hline 3 & $4 / 0.5 / 3.5$ & 6.00 & 10 & 8.7 & 3.50 & 0.0220 & 65.0 & 4.93 & 60 & 0.5 & 213 & 255 & 1.20 \\
\hline 4 & B2 & 6.00 & 9.0 & 7.8 & 2.80 & 0.0134 & 67.1 & 4.22 & 60 & 0.5 & 254 & 228 & 0.90 \\
\hline 4 & B3 & 6.00 & 9.0 & 7.8 & 3.60 & 0.0134 & 67.1 & 4.22 & 60 & 0.5 & 217 & 228 & 1.05 \\
\hline 4 & $\mathrm{C} 2$ & 6.00 & 9.0 & 7.8 & 2.80 & 0.0134 & 67.1 & 4.34 & 60 & 0.75 & 290 & 258 & 0.89 \\
\hline 4 & C6 & 6.00 & 9.0 & 7.8 & 2.80 & 0.0200 & 67.1 & 4.34 & 60 & 0.75 & 314 & 280 & 0.89 \\
\hline 4 & E2 & 6.00 & 9.0 & 7.8 & 2.80 & 0.0134 & 67.1 & 2.99 & 60 & 0.75 & 217 & 237 & 1.09 \\
\hline 4 & E3 & 6.00 & 9.0 & 7.8 & 2.80 & 0.0200 & 67.1 & 2.99 & 60 & 0.75 & 290 & 248 & 0.85 \\
\hline 4 & F3 & 6.00 & 9.0 & 7.8 & 2.80 & 0.0200 & 67.1 & 4.85 & 60 & 0.75 & 415 & 284 & 0.68 \\
\hline 5 & $1.2 / 3$ & 7.87 & 11.8 & 10.2 & 3.46 & 0.0356 & N/A & 6.35 & 67 & 0.51 & 335 & 266 & 0.79 \\
\hline 5 & $1.2 / 4$ & 7.87 & 11.8 & 10.2 & 3.46 & 0.0356 & N/A & 7.00 & 67 & 0.76 & 432 & 299 & 0.69 \\
\hline 5 & $2.3 / 2$ & 7.87 & 11.8 & 10.3 & 2.48 & 0.0115 & N/A & 5.81 & 67 & 0.25 & 228 & 186 & 0.81 \\
\hline 5 & $2.3 / 3$ & 7.87 & 11.8 & 10.3 & 2.48 & 0.0115 & N/A & 5.61 & 67 & 0.76 & 299 & 273 & 0.91 \\
\hline 5 & $2.4 / 3$ & 7.87 & 11.8 & 10.2 & 2.50 & 0.0356 & N/A & 5.61 & 67 & 0.76 & 401 & 298 & 0.74 \\
\hline 5 & $2.6 / 3$ & 7.87 & 11.8 & 10.2 & 4.04 & 0.0356 & N/A & 5.85 & 67 & 0.76 & 326 & 299 & 0.92 \\
\hline 5 & $20 \times 30 S 1$ & 7.87 & 11.8 & 10.2 & 3.50 & 0.0283 & N/A & 5.47 & 67 & 0.5 & 308 & 264 & 0.86 \\
\hline 5 & $20 \times 45 S 1$ & 7.87 & 17.7 & 16.1 & 3.34 & 0.0308 & N/A & 5.47 & 67 & 0.5 & 257 & 264 & 1.03 \\
\hline 5 & T10x50S1 & 7.87 & 19.7 & 18.1 & 3.37 & 0.0280 & N/A & 5.47 & 67 & 0.5 & 266 & 264 & 0.99 \\
\hline 5 & $\mathrm{~T} 15 \times 50 \mathrm{~S} 1$ & 7.87 & 19.7 & 18.1 & 3.37 & 0.0280 & N/A & 5.47 & 67 & 0.5 & 416 & 264 & 0.63 \\
\hline 5 & $\mathrm{~T} 15 \times 75 \mathrm{~S} 1$ & 7.87 & 19.7 & 18.1 & 3.37 & 0.0280 & N/A & 5.47 & 67 & 0.5 & 408 & 264 & 0.65 \\
\hline 5 & $\mathrm{~T} 15 \times 100 \mathrm{~S} 1$ & 7.87 & 19.7 & 18.1 & 3.37 & 0.0280 & N/A & 5.47 & 67 & 0.5 & 384 & 264 & 0.69 \\
\hline 6 & $20 \times 30 S 2$ & 7.87 & 11.8 & 10.2 & 3.50 & 0.0283 & N/A & 5.63 & 67 & 0.5 & 367 & 264 & 0.72 \\
\hline 6 & $20 \times 50 S 2$ & 7.87 & 19.7 & 18.1 & 3.37 & 0.0241 & N/A & 5.63 & 67 & 0.5 & 233 & 264 & 1.13 \\
\hline 6 & $20 \times 60 S 2$ & 7.87 & 23.6 & 21.3 & 3.50 & 0.0273 & N/A & 5.63 & 67 & 0.5 & 298 & 264 & 0.89 \\
\hline 6 & $\mathrm{~T} 10 \times 50 \mathrm{~S} 2$ & 7.87 & 19.7 & 18.1 & 3.37 & 0.0280 & N/A & 5.63 & 67 & 0.5 & 247 & 264 & 1.07 \\
\hline 6 & $\mathrm{~T} 15 \times 50 \mathrm{~S} 2$ & 7.87 & 19.7 & 18.1 & 3.37 & 0.0280 & N/A & 5.63 & 67 & 0.5 & 258 & 264 & 1.03 \\
\hline 6 & $\mathrm{~T} 23 \times 50 \mathrm{~S} 2$ & 7.87 & 19.7 & 18.1 & 3.37 & 0.0280 & N/A & 5.63 & 67 & 0.5 & 398 & 264 & 0.66 \\
\hline 7 & 5 & 2.36 & 14.8 & 13.4 & 2.50 & 0.0344 & 66.7 & 5.22 & 60 & 1 & 548 & 308 & 0.56 \\
\hline \multicolumn{14}{|c|}{ Research groups: } \\
\hline & $\begin{array}{ll}\text { 1. } & \text { Cuchiara } \\
\text { 2. } & \text { Li, Ward } \\
\text { 3. } & \text { Lim, Par } \\
\text { 4. } & \text { Mansur, } \\
\text { 5. } & \text { Rosenbus } \\
\text { 6. } & \text { Rosenbus } \\
\text { 7. } & \text { Tan, Mur }\end{array}$ & $\begin{array}{l}\text { lendola } \\
\text { id Han } \\
\text { asivam } \\
g \text {, and } \\
\text { and T } \\
\text { and T } \\
\text { appan, }\end{array}$ & $\begin{array}{l}\text { and Pa } \\
\text { (1992 } \\
\text { and Lee } \\
\text { aramas } \\
\text { tsch (2 } \\
\text { tsch (2 } \\
\text { ad Para }\end{array}$ & $\begin{array}{l}\text { a }(200 \\
(1987) \\
\text { am (1s } \\
02 a) \\
\text { O2b) } \\
\text { hasivar }\end{array}$ & (1993) & & & & & & & & \\
\hline
\end{tabular}




\section{CHAPTER 6}

\section{SUMMARY AND CONCLUSIONS}

\subsection{SUMMARY}

Summary of the experimental program. A comprehensive experimental program to study the shear behavior of large-scale SFRC beams without stirrup reinforcement was conducted. A total of 28 simply-supported beams were tested to failure under a monotonically increased concentrated load. All beams had a moderate slenderness with a shear span-to-effective depth ratio of 3.5. Cylinder strengths for the concrete used in the test beams varied from 4170 to 7370 psi. The test beams contained moderate to high longitudinal reinforcement ratios, ranging from 1.56 to $2.67 \%$. The yield strength of the reinforcing bars varied from 65 to $72 \mathrm{ksi}$.

The four studied parameters were beam depth, type of hooked steel fibers, fiber volume fraction, and longitudinal reinforcement ratio. Of the 28 beams, 16 beams had a depth of 18 inches (Series B18) and 12 beams had a depth of 27 inches (Series B27). A pair of control RC beams was built for Series B18. For Beam Series B27, one control RC beam without stirrups and one RC beam with stirrup reinforcement that satisfied the minimum ACI Code requirement were tested.

Three types of hooked steel fibers manufactured by Bekaert Corporation, namely ZP305, RC80/60BN, and RC80/30BP, were used. The first two types of fibers had a specified tensile strength of $160 \mathrm{ksi}$ with aspect ratio of 55 and 80, respectively. The third type of fiber had a higher strength of $330 \mathrm{ksi}$ and an aspect ratio of 80 . For Series B18 beams, all the three types of fibers were used, while only the first two types of fibers were used for Series B27 beams. Three fiber volume fractions, namely $0.75,1$, and 
$1.5 \%$, were evaluated in Series B18, while the SFRC beams of Series B27 contained fibers in either a 0.75 or $1.5 \%$ volume fraction.

Summary of the test results. The measured peak shear stress in the SFRC beams varied from 391 to 547 psi, which corresponded to a normalized shear stress range of 4.55 to $6.48 \sqrt{f_{c}^{\prime}}$ (psi). The beam with the lowest strength, which had clearly visible construction defects (air voids), still exhibited a peak shear stress of 260 psi, which corresponded to a normalized shear stress of $3.97 \sqrt{f_{c}^{\prime}}(\mathrm{psi})$. Noteworthy, there was one beam with very high peak normalized shear stress of $7.3 \sqrt{f_{c}^{\prime}}$ (psi), but its absolute shear peak stress value was not the highest.

Of the four parameters evaluated, fiber volume fraction had the strongest influence on the shear strength of SFRC beams. Significant shear strength improvement from a concrete beam without fibers was observed when fibers in a volume fraction of $0.75 \%$ were included. Excluding the beam specimens with known defects, the shear strength with this amount of fibers ranged from 391 to 426 psi (4.55 to $6.31 \sqrt{f_{c}^{\prime}}$, psi). Doubling the amount of fibers from a 0.75 to a $1.5 \%$ volume fraction did not lead to a significant increase in shear strength. Normalized shear strength values ranging from 5.86 to $6.31 \sqrt{f_{c}^{\prime}}(\mathrm{psi})$ were observed for the beams constructed with $1.5 \%$ fiber volume fraction.

If good concrete consolidation was obtained, no significant difference in shear strength for the beams with ZP305 and RC80/60BN fibers was observed. However, consolidation difficulties were encountered in some of the beams with RC80/60BN fibers (2.36 in. long), particularly at the level of the reinforcement, which in turn led to significant lower shear strength due to premature bond failure. The beam specimens reinforced with RC80/60BN fibers for which good concrete consolidation was observed exhibited an enhanced strength and ductility. Although wider crack widths were observed in these beams compared to those in beams reinforced with shorter fibers (ZP305 or $\mathrm{RC} 80 / 30 \mathrm{BP})$, their shear strength was comparable. The use of high strength RC80/30BP 
fibers led to a more evident enhancement of shear strength compared to the other two fibers evaluated (ZP305 and RC80/60BN fibers).

\subsection{CONCLUSIONS}

The following conclusions were drawn from the results of the research program:

(1) The use of hooked steel fibers in a volume fraction greater than or equal to 0.75\% led to: (a) an enhanced inclined cracking pattern (multiple cracks) and more ductile shear failure, (b) negligible shear size effect for beam depths of up to 27 in., and (c) improved shear strength, greater than or equal to $4 \sqrt{f_{c}^{\prime}}$ (psi).

(2) Any of the three types of hooked steel fibers evaluated in this investigation, when used in a volume fraction greater than or equal to $0.75 \%$, can be used in place of the minimum stirrup reinforcement required by ACI Committee 318.

(3) Compared with longitudinal reinforcement ratio and effective beam depth, fiber volume fraction had a stronger influence on the shear strength of SFRC beams.

(4) For the hooked steel fibers used in this research, the increase in shear strength due to the addition of fiber reinforcement diminished when a volume fraction greater than or equal to $1 \%$ was used.

(5) Longer hooked steel fibers with a length of 2.36 inches allowed a greater inclined crack opening before failure compared to that observed in beams with a fiber length of 1.18 inches, but were prone to unfavorable consolidation. A horizontal clear spacing between reinforcing bars no less than the fiber length is suggested to obtain reasonable consolidation. 
(6) Larger beam depths led to wider spacing between inclined cracks. In general, beams with smaller crack spacing exhibited a higher shear strength compared to beams with wider spacing between cracks.

(7) The combination of the results from bending tests of SFRC prisms following the ASTM C1609-05 and the failure criterion proposed by Bresler and Pister for concrete subjected to normal compression and shear stresses, can be used to reliably predict the shear strength of SFRC beams. 


\section{REFERENCES}

AASHTO (1998). LRFD Bridge Specifications and Commentary, 2nd Edition, American Association of State Highway and Transportation Officials, Washington, D.C., 1216.

ACI Committee 318 (2008). Building Code Requirements for Structural Concrete and Commentary, American Concrete Institute, Farmington Hills, MI, 465.

ACI-ASCE Committee 426 (1973). "The Shear Strength of Reinforced Concrete Members." ACI Journal Proceedings, 70(7), 471-473.

ACI-ASCE Committee 544 (1988). "Design Considerations for Steel Fiber Reinforced Concrete." ACI Journal Proceedings, 85(5), 563-579.

Adebar, P., Mindess, S., St.-Pierre, D., and Olund, B. (1997). "Shear Tests of Fiber Concrete Beams without Stirrups." ACI Structural Journal, 94(1), 68-76.

Allen, H. G. (1972). "The Strength of Thin Composites of Finite Width, with Brittle Matrices and Random Discontinuous Reinforcing Fibres (Tensile Strength Estimation for Two Dimensional Composite with Brittle Matrix and Randomly Orientated Discontinuous Elastic Fibrous Reinforcement)." Journal of Physics D (Applied Physics), 5, 331-343.

Al-Ta'an, S. A., and Al-Feel, J. R. (1990). "Evaluation of Shear Strength of FibreReinforced Concrete Beams." Cement \& Concrete Composites, 12(2), 87-94.

Anderson, B. G. (1957). "Rigid Frame Failures." ACI Journal Proceedings, 53(1), 625636.

Ashour, S. A., Hasanain, G. S., and Wafa, F. F. (1992). "Shear Behavior of HighStrength Fiber Reinforced Concrete Beams." ACI Structural Journal, 89(2), 176184.

ASTM A 370 (2003). "Standard Test Methods and Definitions for Mechanical Testing of Steel Products." ASTM International, West Conshohocken, PA.

ASTM C31/C31M (2003). "Standard Practice for Making and Curing Concrete Test Specimens in the Field." ASTM International, West Conshohocken, PA.

ASTM C39/C39M (2003). "Standard Test Method for Compressive Strength of 
Cylindrical Concrete Specimens." ASTM International, West Conshohocken, PA.

ASTM C172 (1999). "Standard Practice for Sampling Freshly Mixed Concrete." ASTM International, West Conshohocken, PA.

ASTM C617 (2003). "Standard Practice for Capping Cylindrical Concrete Specimens." ASTM International, West Conshohocken, PA.

ASTM C1609/1609M (2006). "Standard Test Method for Flexural Performance of FiberReinforced Concrete (Using Beam with Third-Point Loading)." ASTM International, West Conshohocken, PA.

Aveston, J., Mercer, R. A., and Sillwood, J. M. "Fibre Reinforced Cements - Scientific Foundation for Specifications." Proceedings of National Physical Laboratory Conference, UK, 93-103.

Banthia, N., and Trottier, J.-F. (1994). "Concrete Reinforced with Deformed Steel Fibers, Part I: Bond-Slip Mechanisms." ACI Materials Journal, 91(5), 435-446.

Batson, G., Jenkins, E., and Spatney, R. (1972). "Steel Fibers as Shear Reinforcement in Beams." ACI Journal Proceedings, 69(10), 640-644.

Bernaert, S., and Siess, C. P. (1956). "Strength in Shear of Reinforced Concrete Beams under Uniform Load." Civil Engineering Studies, Structural Research Series No. 120, University of Illinois.

Comitée euro-international du béton (1993). CEB-FIP model code 1990, Thomas Telford, Ltd., London.

Bresler, B., and K.S., Pister (1958). "Strength of Concrete under Combined Stresses." ACI Journal Proceedings, 55(9), 321-345.

Brown, M. D., Bayrak, O., and Jirsa, J. O. (2006). "Design for Shear Based on Loading Conditions." ACI Structural Journal, 103(4), 541-550.

Casanova, P., and Rossi, P. (1999). "High-Strength Concrete Beams Submitted to Shear: Steel Fibers versus Stirrups." ACI Special Publication, 182, 53-68.

Cho, S.-H., and Kim, Y.-I. (2003). "Effects of Steel Fibers on Short Beams Loaded in Shear." ACI Structural Journal, 100(6), 765-774.

Cox, H. L. (1952). "The Elasticity and Strength of Paper and other Fibrous Materials." British Journal of Applied Physics, 3, 72-79.

Cucchiara, C., La Mendola, L., and Papia, M. (2004). "Effectiveness of Stirrups and Steel Fibres as Shear Reinforcement." Cement and Concrete Composites, 26(7), 777- 
786.

Technical Committee of Reinforced Concrete Design (1994). Design of Concrete Structures, A23.3-94, Canadian Standards Association, Rexdale, Ontario.

Dwarakanath, H. V., and Nagaraj, T. S. (1991). "Comparative Study of Predictions of Flexural Strength of Steel Fiber Concrete." ACI Structural Journal, 88(6), 714720.

Enerpac (2001). Hydraulic Power for all Industrial Applications.

Ezeldin, A., and Balaguru, P. (1989). "Bond Behavior of Normal and High-Strength Fiber Reinforced Concrete." ACI Materials Journal, 86(5), 515-524.

Fanella, D. A., and Naaman, A. E. (1985). "Stress-Strain Properties of Fiber Reinforced Mortar in Compression." ACI Journal Proceedings, 82(4), 475-483.

Ghalib, M. A. (1980). "Moment Capacity of Steel Fiber Reinforced Concrete Slabs." ACI Journal Proceedings, 77(4), 247-257.

Harajli, M.H., Hout, M., and Jalkh, W. (1995). "Local Bond Stress-Slip Behavior of Reinforcing Bars Embedded in Plain and Fiber Concrete." ACI Materials Journal, 92(4), 343-354.

Han, S.-M., Kong, J.-S., Kim, S.-W., Kang, S.-T., and Park, H.-G. (2004). "Shear and Flexural Behavior of I-Shaped RC Beams Made of Steel Fiber Reinforced Cementious."

Hannant, D. J. (1978). Fibre Cements and Fibre Concretes, John Wiley and Sons Ltd.

Hognestad, E. (1951). "A Study of Combined Bending and Axial Load in Reinforced Concrete Members." Bulletin 399, Univeristy of Illinois Engineering Experiment Station, Urbana, IL, 128.

Hognestad, E., Hanson, N. W., and McHenry, D. (1955). "Concrete Stress Distribution in Ultimate Strength Design." ACI Journal Proceedings, 52(12), 455-480.

Hota, S., and Naaman, A. E. (1997). "Bond Stress-Slip Response of Reinforcing Bars Embedded in FRC Matrices under Monotonic and Cyclic." ACI Structural Journal, 90(5), 525-537.

Hsu, T. T. C., Mau, S. T., and Chen, B. (1987). "Theory of Shear Transfer Strength of Reinforced Concrete." ACI Structural Journal, 84(2), 149-160.

Kaar, P. H., Hanson, N. W., and Capell, H. T. (1978). "Stress-Strain Characteristics of High-Strength Concrete." ACI Special Publication, 55, 161-186. 
Khaloo, A. R., and Kim, N. (1996). "Mechanical Properties of Normal to High-Strength Steel Fiber-Reinforced Concrete." Cement, Concrete and Aggregates, 18(2), 9297.

Khaloo, A. R., and Kim, N. (1997). "Influence of Concrete and Fiber Characteristics on Behavior of Steel Fiber Reinforced Concrete Under Direct Shear." ACI Materials Journal, 94(6), 592-601.

Khuntia, M., Stojadinovic, B., and Goel, S. C. (1999). "Shear Strength of Normal and High-Strength Fiber Reinforced Concrete Beams without Stirrups." ACI Structural Journal, 96(2), 282-289.

Kim, S.-W., Kang, S.-T., Koh, K.-T., Kim, D.-G., and Han, S.-M. (2004). "Shear and Flexural Behavior of Rectangular Beams Made of Steel Fiber Reinforced Cementitious Composite."

Kronenberg, J. (2006). "Sliding Arch Construction Method Used on the Gotthard Base Tunnel." Concrete Engineering International, 10(1), 19-20.

Kwak, Y.-K., Eberhard, M. O., Kim, W.-S., and Kim, J. (2002). "Shear Strength of Steel Fiber-reinforced Concrete Beams without Stirrups." ACI Structural Journal, 99(4), 530-538.

Li, V. C., Ward, R., and Hamza, A. M. (1992). "Steel and Synthetic Fibers as Shear Reinforcement." ACI Materials Journal, 89(5), 499-508.

Lim, D. H., and Oh, B. H. (1999). "Experimental and Theoretical Investigation on the Shear of Steel Fibre Reinforced Concrete Beams." Engineering Structures, 21(10), 937-944.

Lim, T. Y., Paramasivam, P., and Lee, S. L. (1987). "Analytical Model for Tensile Behavior of Steel-Fiber Concrete." ACI Materials Journal, 84(4), 286-298.

Lim, T. Y., Paramasivam, P., and Lee, S. L. (1987). "Shear and Moment Capacity of Reinforced Steel-Fibre-Concrete Beams." Magazine of Concrete Research, 39(140), 148-160.

Lok, T. S., and Xiao, J. R. (1999). "Flexural Strength Assessment of Steel Fiber Reinforced Concrete." ASCE Journal of Materials in Civil Engineering, 11(3), 188-196.

Mansur, M. A., Ong, K. C. G., and Paramasivam, P. (1986). "Shear Strength of Fibrous Concrete Beams Without Stirrups." ASCE Journal of Structural Engineering, 112(9), 2066-2079. 
Mass, G. R. (1997). "SFRC Lining for an Embankment Dam." Concrete International, 19(6), 24-27.

Mattock, A. H., Kriz, L. B., and Hognestad, E. (1961). "Rectangular Concrete Stress Distribution in Ultimate Strength Design." ACI Journal Proceedings, 57(2), 875928.

Mitchell, D., and Collins, M. P. (1974). "Diagonal Compression Field theory-A Rational Model for Structural Concrete in Pure Torsion." ACI Journal Proceedings, 71(8), 396-408.

Moretto, O. (1945). "An Investigation of the Strength of Welded Stirrups in Reinforced Concrete Beam." ACI Journal Proceedings, 42(11), 141-162.

Murty, D. S. R., and Venkatacharyulu, T. (1987). "Fibre Reinforced Concrete Beams Subjected to Shear Force." International Symposium on Fibre Reinforced Concrete, Vol. I, Madras, India, 1.125-1.132.

Naaman, A. E. (1985). "Fiber Reinforcement for Concrete." Concrete International, 7(3), 5.

Naaman, A. E., and Najm, H. (1991). "Bond-Slip Mechanisms of Steel Fibers in Concrete." ACI Materials Journal, 88(2), 135-145.

Naaman, A. E., and Reinhardt, H. W (1995). "Characterization of High Performance Fiber Reinforced Cement Composites." Proceedings of the Second International Workshop ' High Performance Fiber Reinforced Cement Composites', Ann Arbor, USA, 528.

Narayanan, R., and Darwish, I. Y. S. (1987). "Use of Steel Fibers as Shear Reinforcement." ACI Structural Journal, 84(3), 216-227.

Narayanan, R., and Darwish, I. Y. S. (1988). "Fiber Concrete Deep Beams in Shear." ACI Structural Journal, 85(2), 141-149.

Narayanan, R., and Green, K. R. (1981). "Fiber Reinforced Concrete Beams in Combined Bending and Torsion." Indian Concrete Journal, 55(8), 222-228.

Noghabai, K. (2000). "Beams of Fibrous Concrete in Shear and Bending: Experiment and Model." ASCE Journal of Structural Engineering, 126(2), 243-251.

Northern Digital Inc. (2005). NDI OptoTRAK Certus User Guide.

Parameswaran, V. S., and Rajacopalan, K. S. "Strength of Concrete Beams with Aligned and Random Steel Fiber Micro Reinforcement." Rilem Symposium of Fibre Reinforced Cement and Concrete, 95-103. 
Parra-Montesinos, G. J. (2006). "Shear Strength of Beams with Deformed Steel Fibers." Concrete International, 28(11), 57-66.

Placas, A., Regan, P. E., and Baker, A. L. L. (1971). "Shear Failure of Reinforced Concrete Beams." ACI Journal Proceedings, 68(10), 763-773.

Ramakrishnan, V., Brandshaug, T., Coyle, W. V., and Schrader, E. K. (1980). "Comparative Evaluation of Concrete Reinforced with Straight Steel Fibers and Fibers with Deformed Ends Glued Together into Bundles." ACI Journal Proceedings, 77(3), 135-143.

Richart, F. E. (1927). "An Investigation of Web Stresses in Reinforced Concrete Members." Bulletin 166, Univeristy of Illinois Engineering Experiment Station, Urbana, IL.

Robinson, C., Colasanti, A., and Boyd, G. (1991). "Steel Fibers Reinforced Auto Assembly Plant Floor." Concrete International, 13(4), 30-35.

Rodriguez, J. J., Bianchini, A. C., Viest, I. M., and Kesler, C. E. (1959). "Shear Strength of Two-Span Continuous Reinforced Concrete Beams." ACI Journal Proceedings, 55(4), 1089-1130.

Romualdi, J. P., and Batson, G. B. (1963). "Mechanics of Crack Arrest in Concrete." ASCE Journal of the Engineering Mechanics, 89(EM3), 147-168.

Rosenbusch, J., and Teutsch, M. (2002). "Trial Beams in Shear (Series 1 and 2)."

Rosenbusch, J., and Teutsch, M. (2002). "Trial Beams in Shear : Test Programme 3."

Schantz, B. A. (1993). "The Effect of Shear Stress on Full Scale Steel Fiber Reinforced Concrete Beams," MS Thesis, Clarkson University, Potsdam.

Shah, S., and Rangan, B. V. (1971). "Fiber Reinforced Concrete Properties." ACI Journal Proceedings, 68(2), 126-35.

Sharma, A. K. (1986). "Shear Strength of Steel Fiber Reinforced Concrete Beams." ACI Journal Proceedings, 83(4), 624-628.

Shin, S.-W., Oh, J.-G., and Ghosh, S. K. (1994). "Shear Behavior of Laboratory-Sized High-Strength Concrete Beams Reinforced with Bars and Steel Fibers." ACI Special Publication, 142, 181-200.

Song, P. S., and Hwang, S. (2004). "Mechanical properties of high-strength steel fiberreinforced concrete." Construction and Building Materials, 18(9), 669-673. 
Soroushian, P., and Bayasi, Z. (1991). "Fiber Type Effects on the Performance of Steel Fiber Reinforced Concrete." ACI Materials Journal, 88(2), 129-134.

Soroushian, P., Mirza, F., and Alhozaimy, A. (1994). "Bond of Confined Steel Fiber Reinforced Concrete to Deformed Bars." ACI Materials Journal, 91(2), 141-149.

Swamy, R. N., and AI-Ta'an, S. a. A. (1981). "Deformation and Ultimate Strength in Flexure of Reinforced Concrete Beams Made with Steel Fiber Concrete." ACI Journal Proceedings, 78(5), 395-405.

Swamy, R. N., and Bahia, H. M. (1985). "Effectiveness of Steel Fibers as Shear Reinforcement." Concrete International, 7(3), 35-40.

Swamy, R. N., Jones, R., and Chiam, A. T. P. (1993). "Influence of Steel Fibers on The Shear Resistance of Lightweight Concrete I- Beams." ACI Structural Journal, 90(1), 103-114.

Swamy, R. N., and Mangat, P. S. (1974). "Theory for the Flexural Strength of Steel Fiber Reinforced Concrete." Cement and Concrete Research, 4(2), 313-325.

Tan, K. H., Murugappan, K., and Paramasivam, P. (1993). "Shear Behavior of Steel Fiber Reinforced Concrete Beams." ACI Structural Journal, 90(1), 3-11.

Thomas, J., and Ramaswamy, A. (2007). "Mechanical Properties of Steel Fiberreinforced Concrete." ASCE Journal of Materials in Civil Engineering, 19(5), 385-392.

Tureyen, A. K., and Frosch, R. J. (2003). "Concrete Shear Strength: Another Perspective." ACI Structural Journal, 100(5), 609-615.

Valle, M., and Buyukozturk, O. (1993). "Behavior of Fiber Reinforced High-strength Concrete under Direct Shear." ACI Materials Journal, 90(2), 122-133.

Vecchio, F. J. (2000). "Disturbed Stress Field Model for Reinforced Concrete: Formulation." ASCE Journal of Structural Engineering, 126(9), 1070-1077.

Vecchio, F. J. (2001). "Disturbed Stress Field Model for Reinforced Concrete: Implementation." ASCE Journal of Structural Engineering, 127(1), 1070-1077.

Vecchio, F. J., and Collins, M. P. (1986). "The Modified Compression Field Theory for Reinforced Concrete Elements Subjected to Shear." ACI Structural Journal, 83(2), 219-231.

Wafa, F. F., and Ashour, S. A. (1992). "Mechanical Properties of High-strength Fiber Reinforced Concrete." ACI Materials Journal, 89(5), 449-455. 
Walraven, J. C. (1981). "Fundamental Analysis of Aggregate Interlock." ASCE Journal of Structural Engineering, 107(11), 2245-2270.

Wight, J. K., and MacGregor, J. G. (2009). Reinforced Concrete: Mechanics and Design, Pearson Prentice Hall.

Williamson, G. R., and Knab, L. I. "Full Scale Fiber Concrete Beam Tests." Rilem Symposium 'Fiber Reinforced Cement Concrete'.

Wright, P. J. F. (1955). "Comments on Indirect Tensile Test on Concrete Cylinders." Magazine of Concrete Research, 7(20), 87-96.

Zsutty, T. C. (1968). "Beam Shear Strength Prediction by Analysis of Existing Data." ACI Journal Proceedings, 65(11), 943-951. 